\title{
FLEX FUEL OPTIMIZED SI AND HCCI ENGINE
}

(DOE Project Officer: Nicholas D’Amico)

FINAL PROJECT REPORT

DOE Award \#: DE-EE0000211

December 20, 2013

Guoming (George) Zhu, Harold Schock, Xiaojian Yang, Andrew Huisjen, Tom Stuecken, Kevin Moran, Ren Zhen, and Shupeng Zhang

Mechanical Engineering

Michigan State University

East Lansing, MI 48824

John Opra and Ron Reese

Chrysler Corporation

Auburn Hill, MI 48326 


\section{TABLE OF CONTENTS}

1 EXECUTIVE SUMMARY ............................................................................................ 5

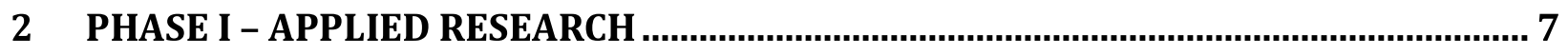

2.1 Confirmation of SI and HCCI dual-mode engine design and specifications ........... 7

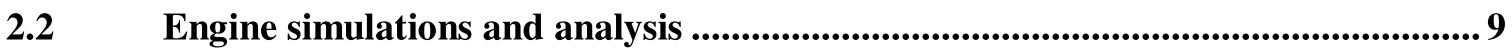

2.3 Control-oriented engine model development........................................................... 11

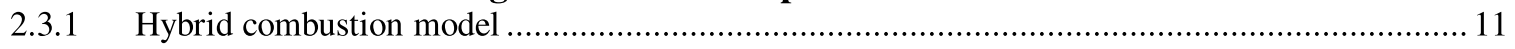

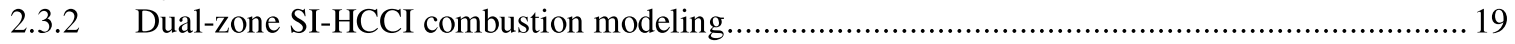

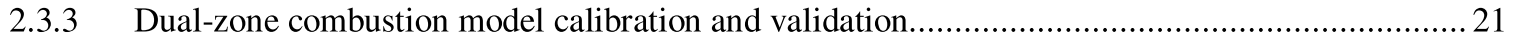

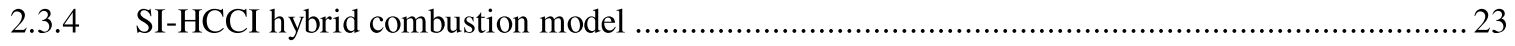

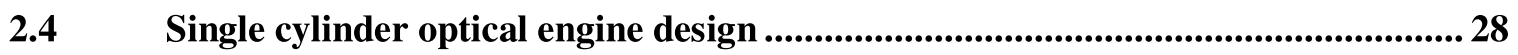

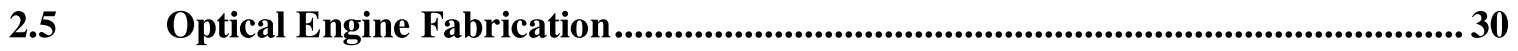

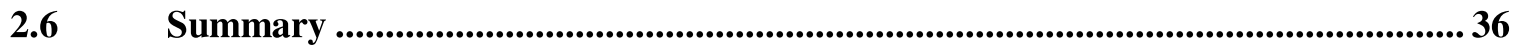

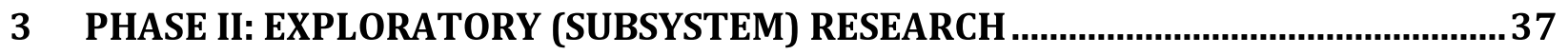

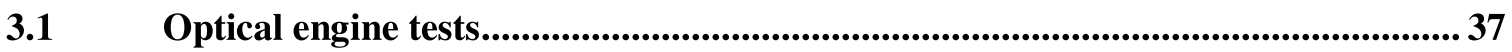

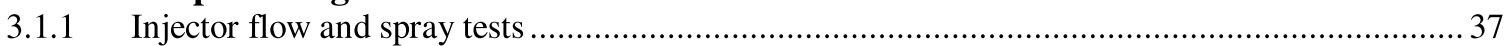

3.1.2 Electrical cam phase actuator and lift control test on optical engine \#2 ......................................43

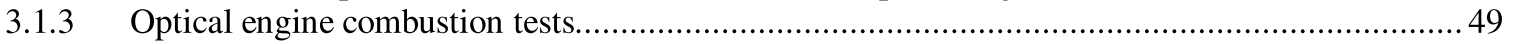

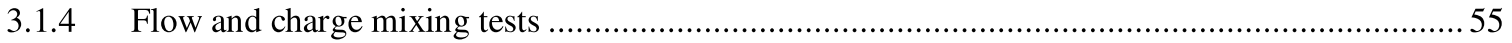

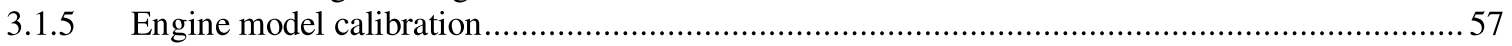

3.2 Two-step lift and electrical variable valve timing control ..................................557

3.2.1 Two-step valve lift and phase system range determination .......................................................5 57

3.2.2 Two-step valve supply selection and cylinder head modification ................................................ 61

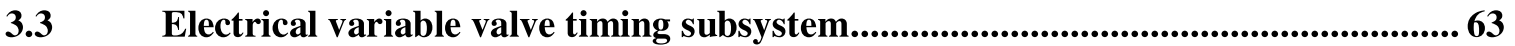

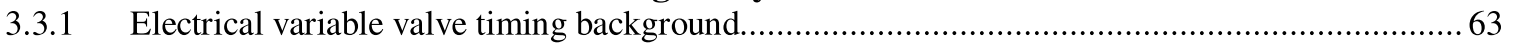

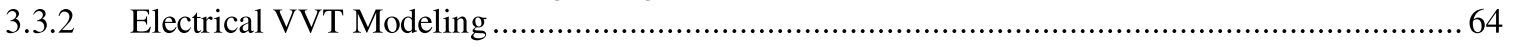

3.3.3 System identification of the electrical cam phasing system .......................................................69

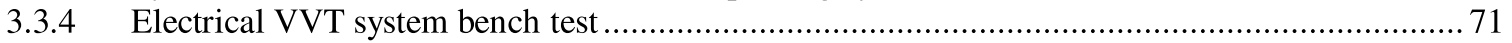

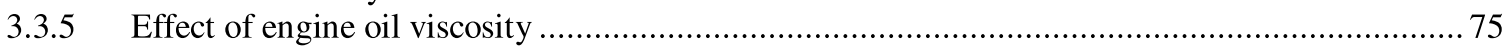

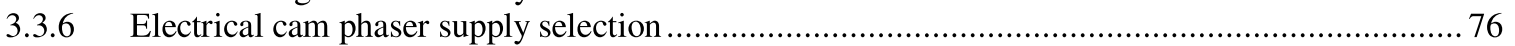

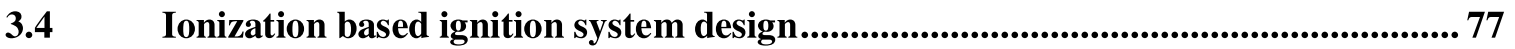

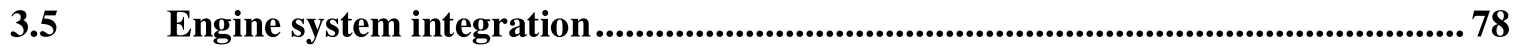

3.6 Implementation of control-oriented engine model into HIL simulator ................. 78

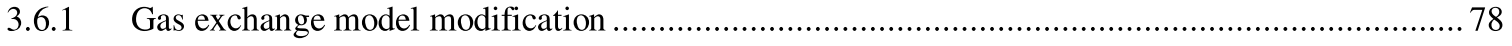

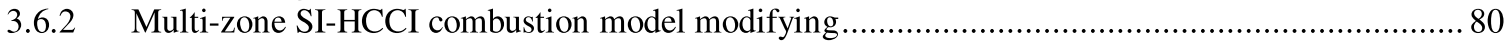

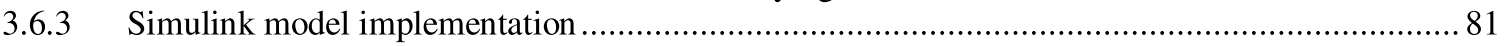


3.7 Engine prototype controller design and fabrication

3.8 Summary

4 PHASE III: CLOSED-LOOP COMBUSTION CONTROL FOR SI AND HCCI MODES 84

4.1 SI and HCCI dual-mode combustion engine fabrication ....................................... 84

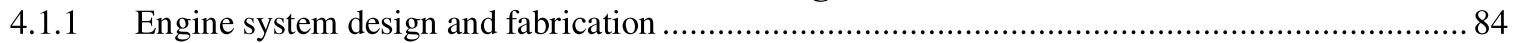

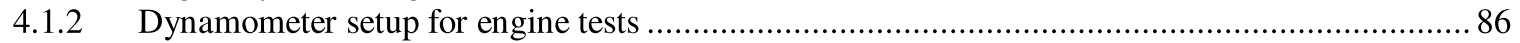

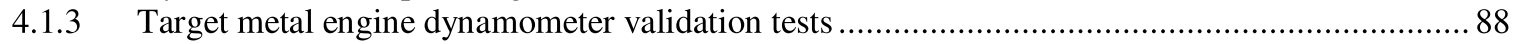

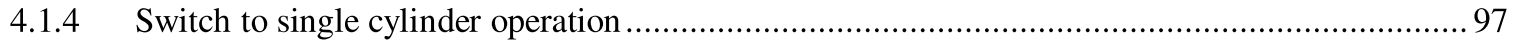

4.2 HCCI and combustion mode transition control ......................................................98

4.2.1 HCCI Capable SI engine model implemented in an HIL simulator ......................................... 98

4.2.2 Engine setup for SI to HCCI combustion mode transition...................................................... 99

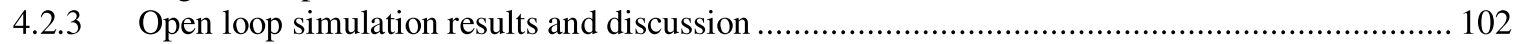

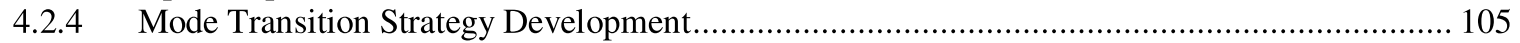

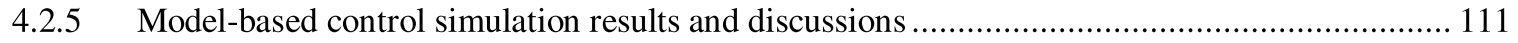

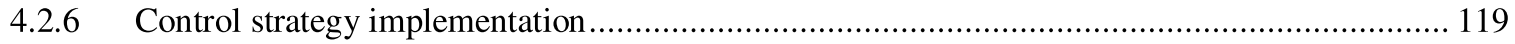

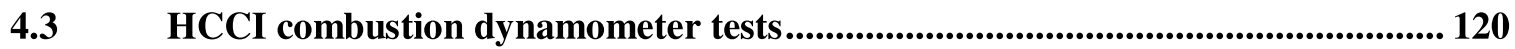

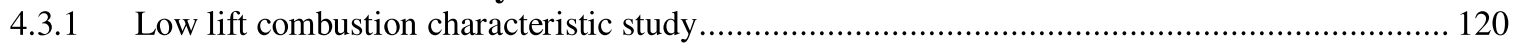

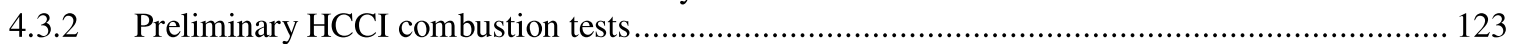

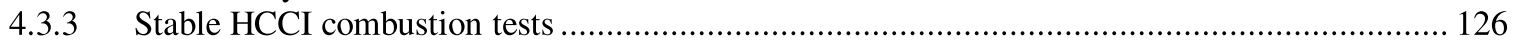

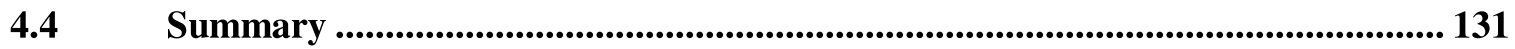

5 PHASE IV: FINAL PERFORMANCE EVALUATION ....................................................... 132

5.1 Motoring tests of SI-to-HCCI transition .............................................................. 132

5.2 Hybrid combustion mode during SI-to-HCCI mode transition............................. 135

$5.3 \quad$ Successful fast SI-to-HCCI mode transition...................................................... 136

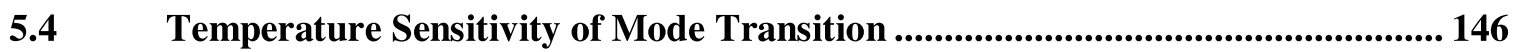

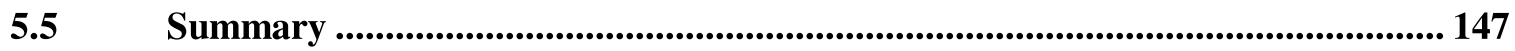

6 CONCLUSIONS AND RECOMMENDATIONS .......................................................... 148

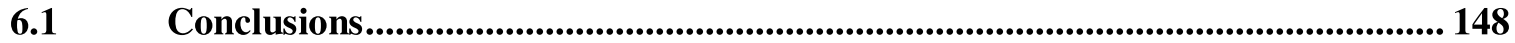

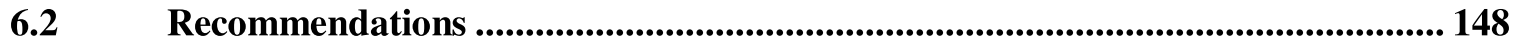

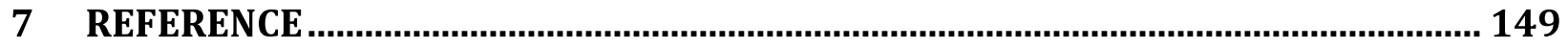

8 APPENDIX B - PUBLICATION LIST ASSOCIATED WITH THIS AWARD ................ 152

9 APPENDIX C - PUBLISHED JOURNAL AND CONFERENCE (JC) PAPERS ................ 153 
9.1 Published JC Paper \#1 ……….................................................................................. 154

$9.2 \quad$ Published JC Paper \#2 …………………...................................................................... 161

$9.3 \quad$ Published JC Paper \#3 …………............................................................................. 173

$9.4 \quad$ Published JC Paper \#4 ………………………............................................................ 181

9.5 Published JC Paper \#5 ................................................................................................. 207

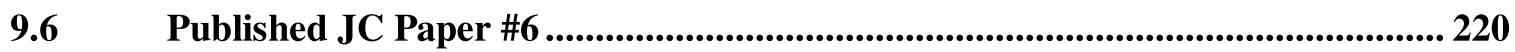

$9.7 \quad$ Published JC Paper \#7 ................................................................................................ 237

9.8 Published JC Paper \#8 ................................................................................................ 245

$9.9 \quad$ Published JC Paper \#9 ......................................................................................... 252

$9.10 \quad$ Published JC Paper \#10 ........................................................................................ 261

9.11 Published JC Paper \#11 ........................................................................................... 268

$9.12 \quad$ Published JC Paper \#12 .............................................................................................. 275 


\section{Executive Summary}

The central objective of the proposed work is to demonstrate an HCCI (homogeneous charge compression ignition) capable SI (spark ignited) engine that is capable of fast and smooth mode transition between SI and HCCI combustion modes. The model-based control technique was used to develop and validate the proposed control strategy for the fast and smooth combustion mode transition based upon the developed control-oriented engine; and an HCCI capable SI engine was designed and constructed using production ready two-step valve-train with electrical variable valve timing actuating system. Finally, smooth combustion mode transition was demonstrated on a metal engine within eight engine cycles.

The Chrysler turbocharged 2.0L I4 direct injection engine was selected as the base engine for the project and the engine was modified to fit the two-step valve with electrical variable valve timing actuating system. To develop the model-based control strategy for stable HCCI combustion and smooth combustion mode transition between SI and HCCI combustion, a control-oriented realtime engine model was developed and implemented into the MSU HIL (hardware-in-the-loop) simulation environment. The developed model was used to study the engine actuating system requirement for the smooth and fast combustion mode transition and to develop the proposed mode transition control strategy. Finally, a single cylinder optical engine was designed and fabricated for studying the HCCI combustion characteristics.

Optical engine combustion tests were conducted in both SI and HCCI combustion modes and the test results were used to calibrate the developed control-oriented engine model. Intensive GTPower simulations were conducted to determine the optimal valve lifts (high and low) and the cam phasing range. Delphi was selected to be the supplier for the two-step valve-train and Denso to be the electrical variable valve timing system supplier. A test bench was constructed to develop control strategies for the electrical variable valve timing (VVT) actuating system and satisfactory electrical VVT responses were obtained. Target engine control system was designed and fabricated at MSU for both single-cylinder optical and multi-cylinder metal engines. Finally, the developed control-oriented engine model was successfully implemented into the HIL simulation environment.

The Chrysler 2.0L I4 DI engine was modified to fit the two-step vale with electrical variable valve timing actuating system. A used prototype engine was used as the base engine and the cylinder head was modified for the two-step valve with electrical VVT actuating system. Engine validation tests indicated that cylinder \#3 has very high blow-by and it cannot be reduced with new pistons and rings. Due to the time constraint, it was decided to convert the four-cylinder engine into a single cylinder engine by blocking both intake and exhaust ports of the unused cylinders. The model-based combustion mode transition control algorithm was developed in the MSU HIL simulation environment and the Simulink based control strategy was implemented into the target engine controller. With both single-cylinder metal engine and control strategy ready, stable HCCI combustion was achieved with COV of $2.1 \%$.

Motoring tests were conducted to validate the actuator transient operations including valve lift, electrical variable valve timing, electronic throttle, multiple spark and injection controls. After the actuator operations were confirmed, 15-cycle smooth combustion mode transition from SI to HCCI combustion was achieved; and fast 8-cycle smooth combustion mode transition followed. With a fast electrical variable valve timing actuator, the number of engine cycles required for 
mode transition can be reduced down to five. It was also found that the combustion mode transition is sensitive to the charge air and engine coolant temperatures and regulating the corresponding temperatures to the target levels during the combustion mode transition is the key for a smooth combustion mode transition.

As a summary, the proposed combustion mode transition strategy using the hybrid combustion mode that starts with the SI combustion and ends with the HCCI combustion was experimentally validated on a metal engine. The proposed model-based control approach made it possible to complete the SI-HCCI combustion mode transition within eight engine cycles utilizing the well controlled hybrid combustion mode. Without intensive control-oriented engine modeling and HIL simulation study of using the hybrid combustion mode during the mode transition, it would be impossible to validate the proposed combustion mode transition strategy in a very short period. 


\section{Phase I - Applied Research}

This phase includes computer modeling and simulation of the target SI (spark-ignited) and HCCI (homogeneous charge compression ignition) dual-mode engine to assess the technical and economic viability of the proposed research and the optical engine will be designed and constructed.

\subsection{Confirmation of SI and HCCI dual-mode engine design and specifications}

Selection of the SI and HCCI dual-mode engine that represents the future production engine platform was narrowed down to two Chrysler engine platforms: turbocharged I4 with dual-VVT (variable valve timing) and naturally aspired V6.

- Turbocharged 2.0L I4 direct injection engine with dual over head cams and dual VVT (variable valve timing)

- Naturally aspired 3.0L V6 direct injection engine with dual over head cams and dual VVT.

The advantage of the proposed I4 engine is that both engine intake and exhaust ports and combustion chamber are redesigned recently by Chrysler and will be suitable for both SI and HCCI combustion operations; and the disadvantage is that no new hardware is available for the proposed research and the used prototype hardware needs to be reused, which adds constraints to the target engine design. On the other hand, the advantage of the V6 engine is the availability of the hardware and the disadvantage is that the engine design is not up to date. The purpose of this selection process is to make sure that the selected SI and HCCI dual mode engine platform will be suitable for future production. After careful considerations, the selected target engine by the Chrysler and MSU team is

- Turbocharged 2.0L I4 direct injection engine with dual over head cams and dual VVT (variable valve timing) cam phase systems. The engine cam systems will be modified to be capable of two-step valve lift with electrical variable valve timing systems.

Figure 1 shows the target engine head supplied by Chrysler to be used for constructing the optical engine.

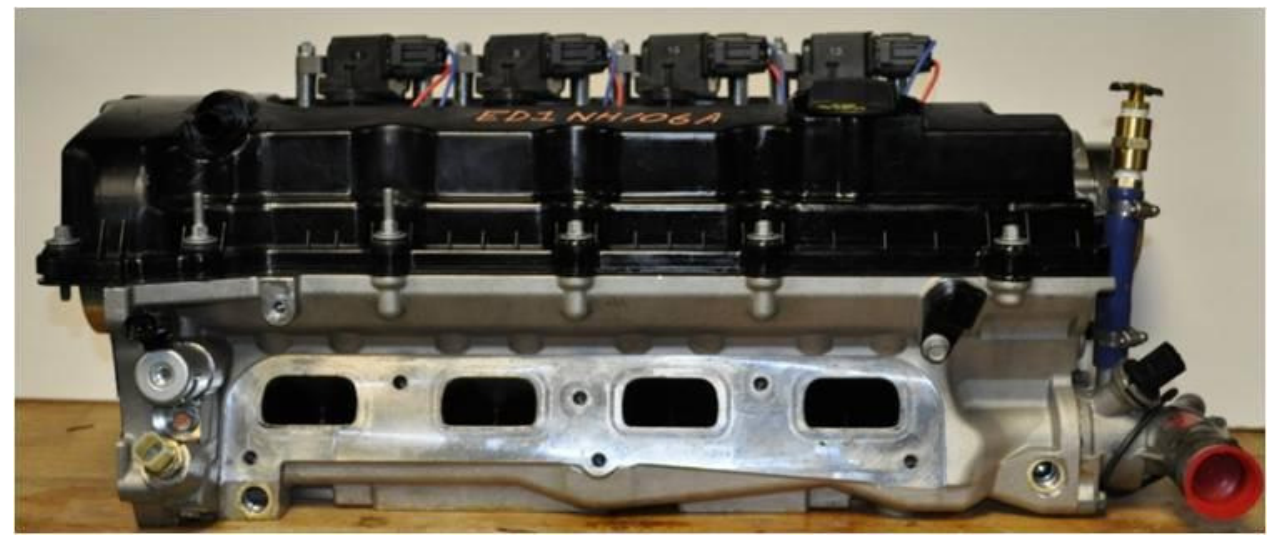

Figure 1: Target engine head provided by Chrysler for optical engine construction 
In order to make SI and HCCI mode transition control feasible, the conventional hydraulic dualcam VVT systems need to be upgrade to the electrical dual-cam variable valve timing systems with two-step valve lift capability. Note that the details of the two-step VVT valvetrain are discussed in Subsection 3.2. The upgraded electrical VVT system reduces cam phase response time which is necessary for the SI-HCCI mode transition. The dual-step valve lift subsystems are also essential for the HCCI operation. Chrysler conducted the feasibility study of fitting the twostep valve lift hydraulic systems into both I4 and V6 engine heads, there was no major issues found. Figure 2 shows the modification of the exhaust valve lash and the exhaust valve length required to fit the two-step valve systems onto both cylinder heads.

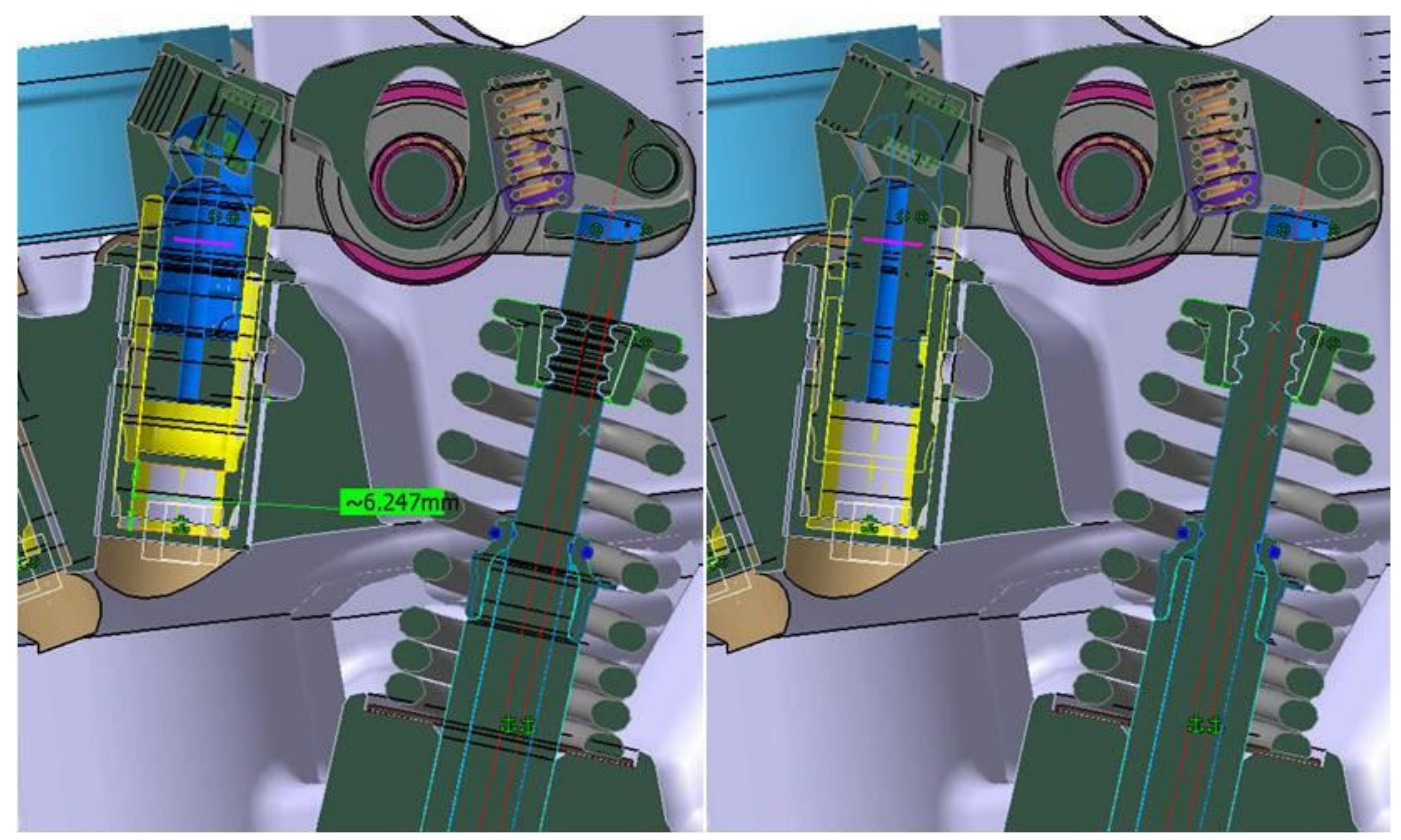

Figure 2: Feasibility study of two-step valve lift implementation

The upgraded electrical VVT systems reduce cam phase response time significantly, which is necessary for the fast SI-HCCI mode transition operation. The dual-step valve lift subsystems are also essential for the HCCI operation. Figure 3 shows the target valve lift profile for the target cam system. 


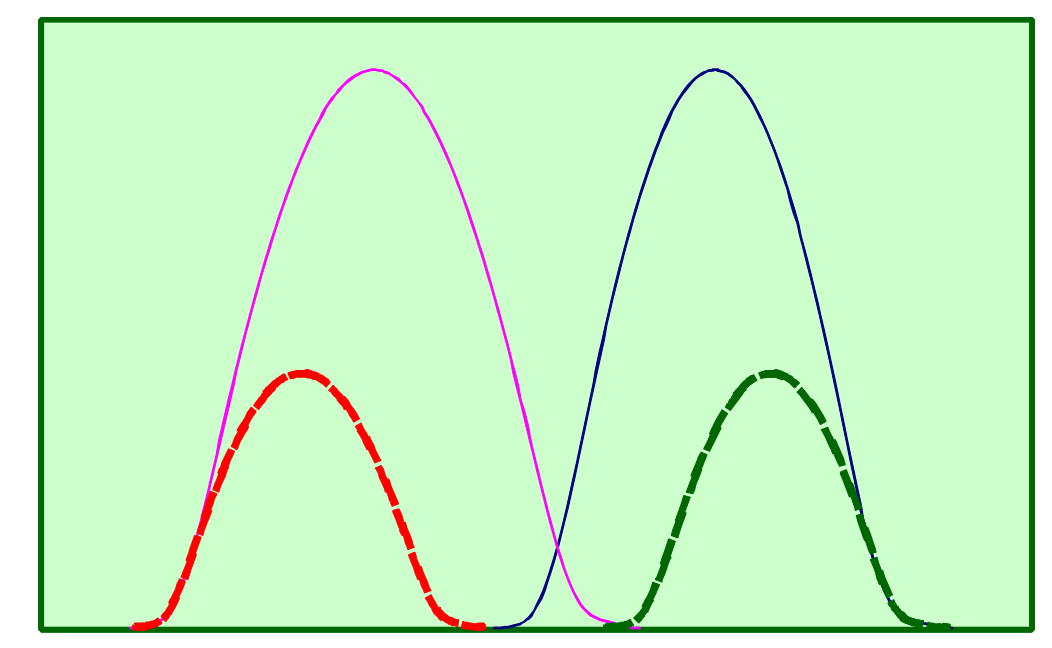

Figure 3: Target intake and exhaust valve lift profiles of the target cam

\subsection{Engine simulations and analysis}

The purpose of this study is to develop a control-oriented engine model to study the SI, HCCI, and SI-HCCI hybrid combustion modes, where the GT-Power model was used as the baseline model to complete the initial evaluation of the specifications for SI and HCCI dual-mode combustion system that can meet performance and fuel economy targets.

Before the engine platform selection was finalized, a GT-Power engine model of a three-cylinder engine with both cooled EGR (exhaust gas recirculation) and trapped EGR through exhaust valve timing adjustment was developed, see Figure 4. This engine model was used to simulate an I3 engine in both SI and HCCI combustion modes. The simulation results were used to calibrate the Simulink based control-oriented real-time engine model.

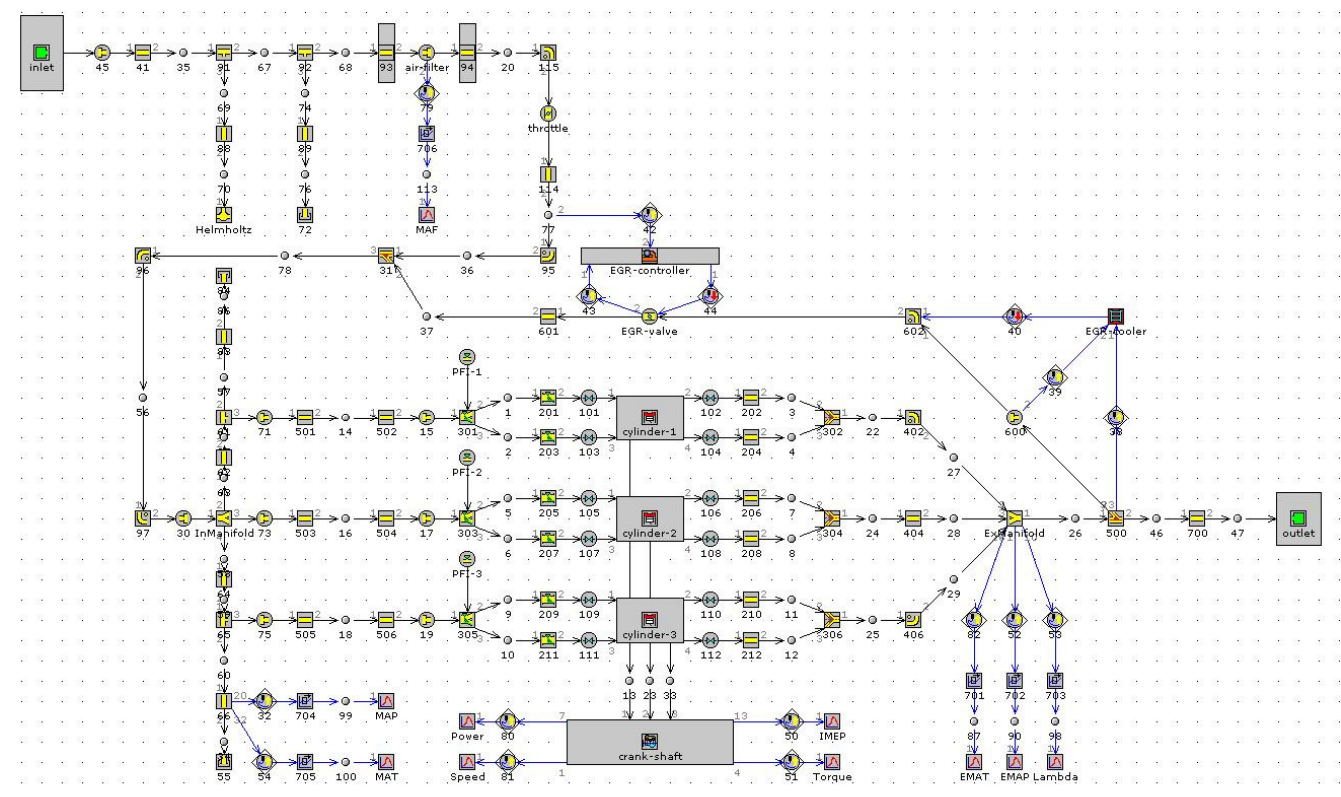

Figure 4: An engine model (I3) operated at either SI or HCCI combustion mode 
Since one of the GT-Power modeling objectives is to simulate the SI-HCCI dual (hybrid) combustion mode for the target engine. The hybrid combustion mode starts with the SI combustion mode and ends with the HCCI combustion. The hybrid combustion model is a very important tool for developing smooth mode transition strategies between SI and HCCI combustion modes. Unfortunately, the existing GT-Power modeling tool does not allow us to model the hybrid SI and HCCI combustion within the same engine cycle, and we decided to model the hybrid combustion mode in Subsection 2.3.

\section{GT-Power simulation for SI and HCCI combustion}

After the I4 engine platform was selected, Chrysler provided a single-cylinder GT-Power model for the target engine. The engine model was calibrated using dynamometer testing data from Chrysler. The HCCI combustion mode was simulated at MSU using the provided GT-Power model and the simulation results were used for calibrating the control-oriented engine model in Subsection 2.3. The GT-Power simulation results can also be found in the publication due to the funded research in Subsections 9.7 and 9.10. Figure 4 shows the diagram of the single cylinder engine GT-Power model. This GT-Power model was also used to simulate the engine in HCCI mode to figure out the valve-train (cam) requirement for the two-step lift and electrical VVT range.

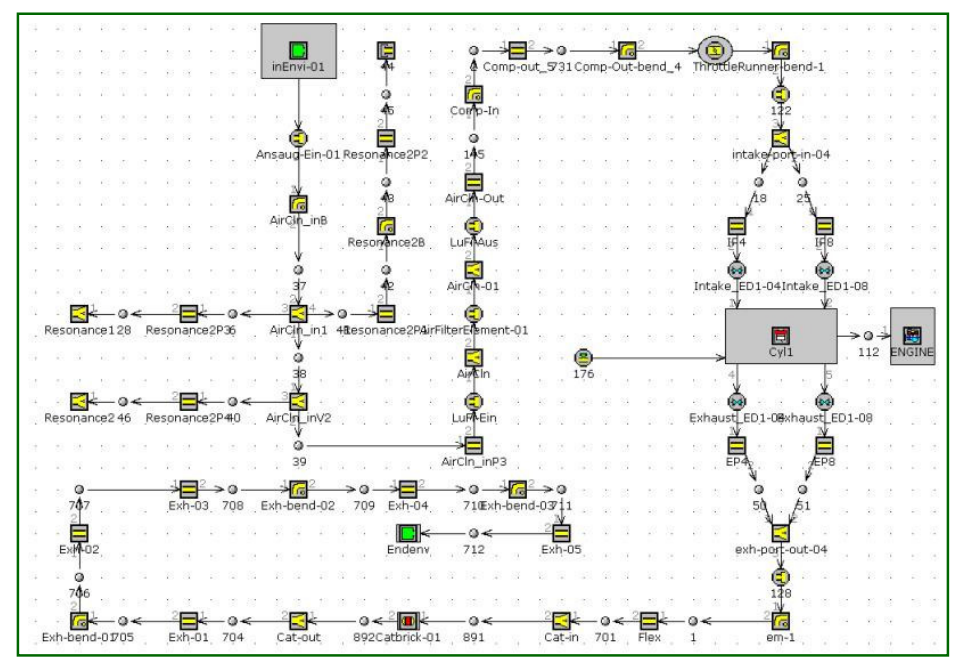

Figure 5: A single cylinder target engine GT-Power model

\section{$\underline{\text { Numerical simulation of injector flow and spray dynamics }}$}

In order to insure accurate fuel injection timing and quantity, fuel injector dynamics during the fuel injection process is also modeled in GT-Power; see Figure 6. This guarantees the control accuracy of the DI (direct injection) fuel injection for both hybrid SI-HCCI and HCCI combustion operations, and it is especially important for SI-HCCI hybrid combustion control during the combustion mode transition between SI and HCCI combustion. 


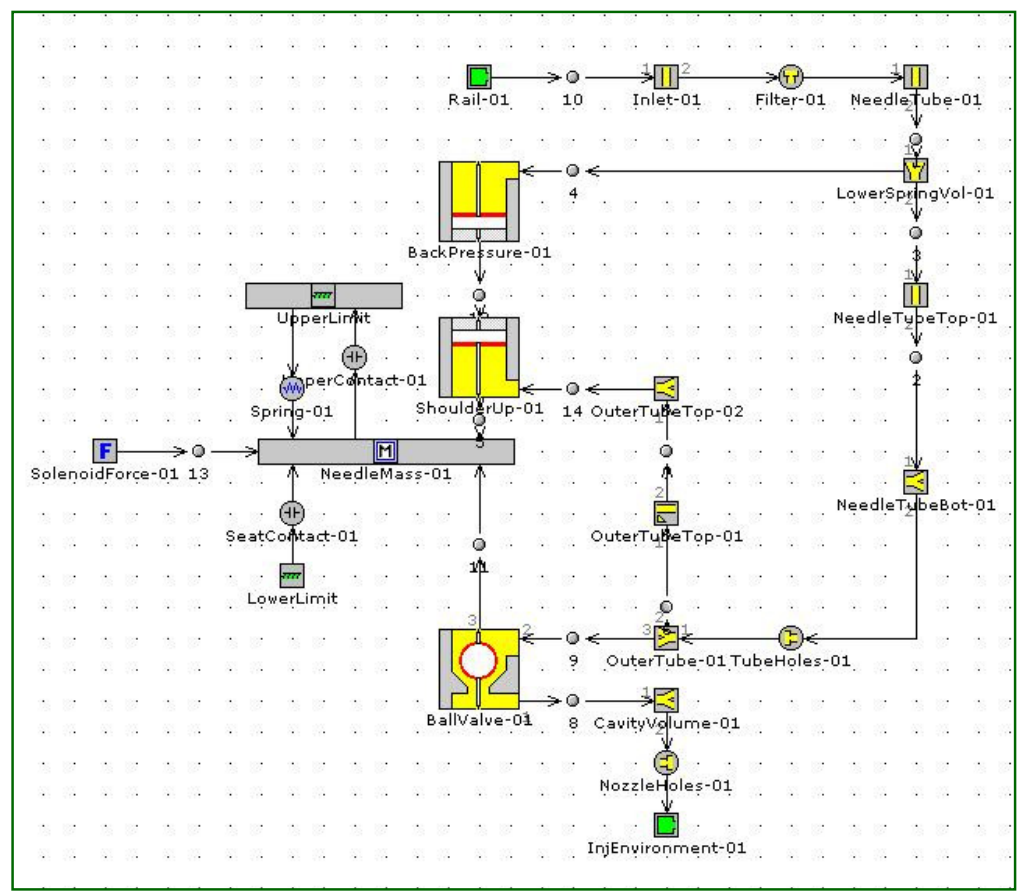

Figure 6: Fuel injector dynamics model in GT-Power

\subsection{Control-oriented engine model development}

A control-oriented engine model of SI and HCCI dual combustion modes was developed in Matlab/Simulink. The developed model was implemented into the dSPACE HIL (hardware-inthe-loop) simulation environment at MSU and the validation simulations for a combustion mode transition from SI to HCCI combustion have been conducted. This control-oriented model will be used for developing model-based combustion mode transition control strategies for mode transition between SI and HCCI combustion.

\subsubsection{Hybrid combustion model}

To accurately control the HCCI combustion process, especially the model transition between SI and HCCI combustion, a precise combustion model is a necessity. This is mainly due to the fact that not all key control parameters of an HCCI combustion process can be measured directly. For instance, the temperature of the in-cylinder gas mixture after the intake valve is closed, which is a key parameter for estimating start of combustion (SOC), cannot be measured directly using existing sensor technology. It is well known that the existing engine modeling tools, such as GTPower and WAVE, can only be used for off-line simulations, and therefore, cannot be used for model-based control. The other HCCI combustion models ([1], [2], and [3]) describe only the mean-value (averaged) HCCI combustion without crank-based engine combustion information such as in-cylinder pressure and temperature signals. As discussed in [4], to control the mode transition between SI and HCCI combustion mode, we need to have a mixed mean-value and crank-based SI-HCCI hybrid combustion model that can be used for model-based control purpose. This model shall be able to be executed in real-time for HIL simulations. This is the modeling research proposed originally. 


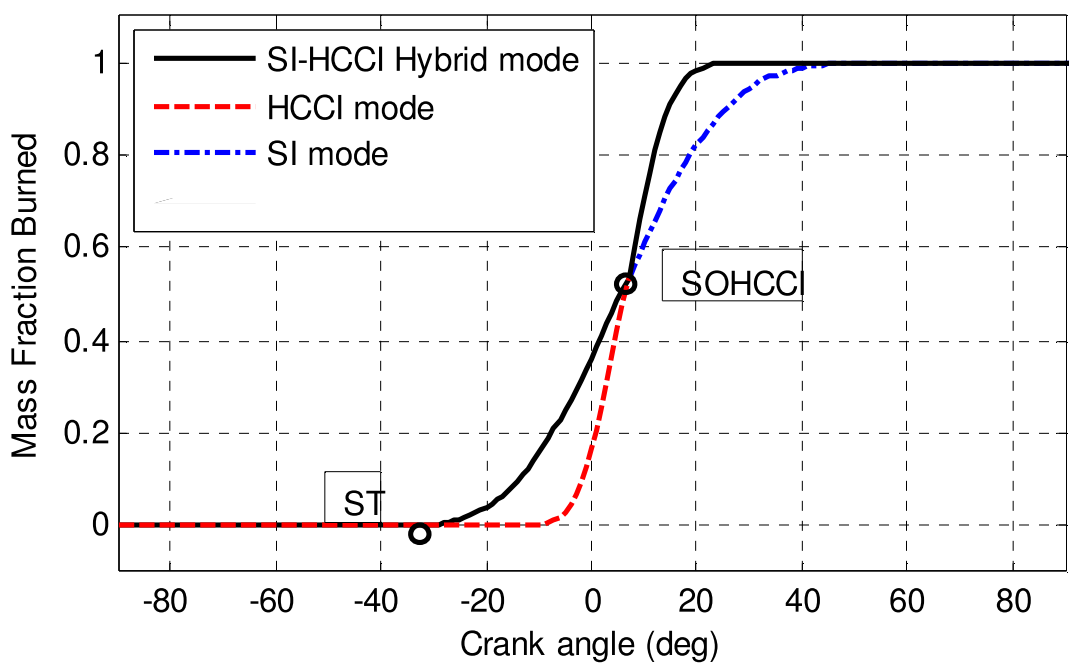

Figure 7: MFB in SI-HCCI Hybrid mode transition process

Also, to have smooth transition between SI and HCCI combustion, accurate intake flow with good mixing is the key. References [5] and [6] describe an intake strategy entailing opening the intake valves at different timings to create improved flow characteristics, and therefore better mixing. The advantage of this strategy is the ability to use NVO (negative valve overlap) while avoiding the inherent mixing problems associated with it. Since the NVO strategy alters the existing valve timings, it is much simpler than re-induction strategies. Re-induction strategies need to open the exhaust valves at non-traditional timings, thereby complicating the control system. Furthermore, since the exhaust gas does not leave the cylinder (as it does with reinduction strategies), there is less heat lost to the environment and the overall efficiency of the engine is greater. To fully utilize this method, we must have accurate information about the system behavior, particularly the fluid flow during the mixing process. Therefore, an intake mixing model needs to be integrated with the SI-HCCI combustion model.

The main goal of mode transition control is to ensure smooth and robust transition between SI and HCCI combustion modes to avoid engine misfire at low load or knocking at mediate load. The main challenge for the mode transition is that a stable steady state HCCI operating condition is not necessarily stable and robust during mode transitions. This is primarily due to the fact that the HCCI combustion is heavily influenced by the residual gas properties (quantity and temperature) from previous cycles. Also given the practical constraints of short transition period ( 5 to 6 combustion cycles) and smooth transition torque, a cycle-to-cycle intervention of the combustion process is a necessity. We propose to develop an SI-HCCI combustion model, shown in Figure 7, where SI and HCCI combustion modes are its special cases. The input to this combustion model will be the output of the intake mixing model developed in [7]. The purpose of modeling the SI-HCCI hybrid combustion process is to be able to correlate the trapped incylinder gas properties (such as air-to-fuel ratio, trapped gas re-circulation, and temperature) to the combustion characteristics such as misfire, knock and IMEP (indicated mean effective pressure). The developed model will be used for model-based transition operation from SI operation to HCCI or vice versa.

For modeling the hybrid SI-HCCI combustion, we used a two-zone approach: one zone for burned gas mixture and the other for unburned one. Figure 8 illustrate a two-zone hybrid SI- 
HCCI combustion architecture, where the center zone is the burned gas due to spark ignited combustion with a radius $r_{l}$ and the outer zone is the unburned gas mixture with the outside radius $r_{2}$ equal to half of bore. For the engine operated at an SI-HCCI hybrid combustion mode, the combustion initiates by the ignition system, and the combustion continues, leading to increased $r_{l}$ until the auto-ignition condition of HCCI combustion is satisfied with inner zone radius $r_{l}$ equals to $r_{H C C I}$. There are two special cases: one is $r_{H C C I}$ equal to zero when engine is operated at HCCI combustion mode and the other is $r_{H C C I}$ equal to $r_{2}$ when the engine is operated at SI combustion model. Therefore, the SI-HCCI hybrid combustion model is a generalization of both SI and HCCI combustion modes.

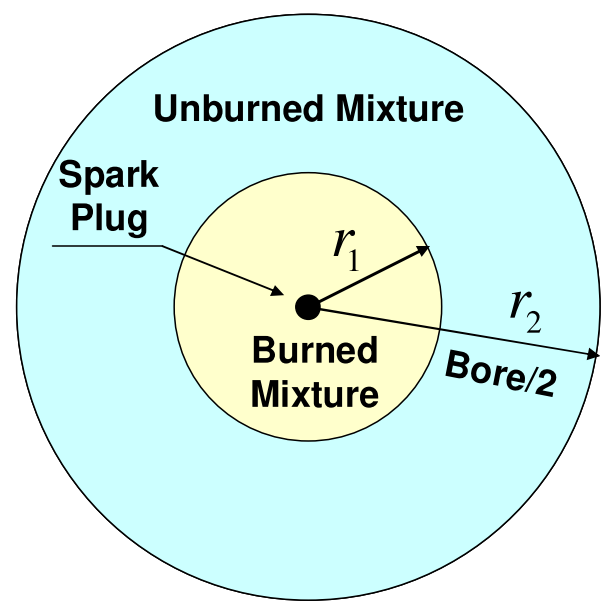

Figure 8: Two zone SI-HCCI hybrid combustion model

The thermodynamic characteristics of in-cylinder gas, such as in-cylinder pressure and temperature, are of great interest in the SI-HCCI combustion modeling research. This is especially important at certain critical combustion phases such as the intake valve closing.

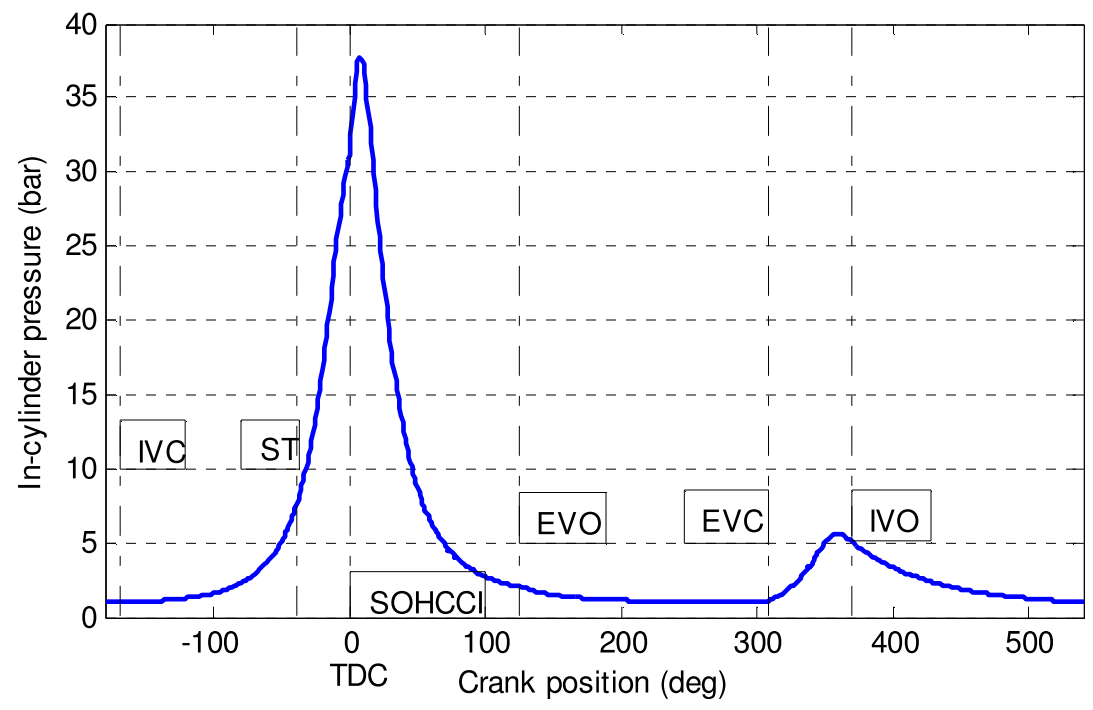

Figure 9: Combustion phases within an engine cycle

Figure 9 shows all key combustion phases of an SI to HCCI hybrid combustion process within one engine cycle. We consider six key combustion phases in the SI-HCCI hybrid combustion 
process. They are intake valve closing (IVC), spark timing (ST), start of HCCI combustion (SOHCCI), exhaust valve opening (EVO), exhaust valve closing (EVC), and intake valve opening (IVO). For each combustion phase, the in-cylinder behaviors (such as pressure, temperature, etc.) are modeled using thermodynamic governing equations. The governing equations of six combustion phases described above were developed and the corresponding Simulink engine models were constructed for real-time simulations.

The SI and HCCI combustion modes and the SI-HCCI hybrid mode were simulated based upon the engine configuration shown in Figure 10, where the engine parameters are given in Table 1.

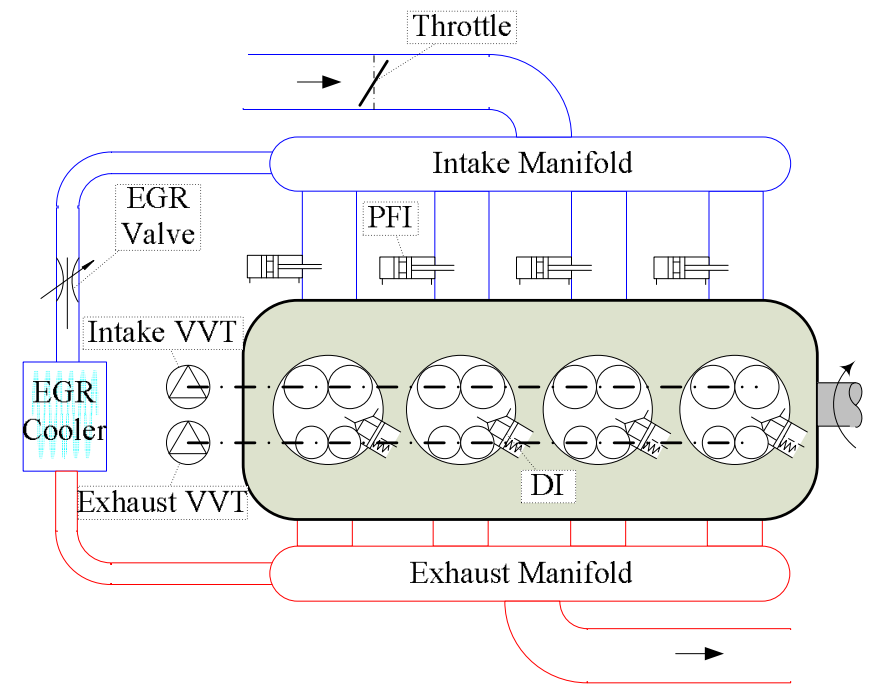

Figure 10: Engine configuration of control-oriented model

Table 1: Engine specifications

\begin{tabular}{c|c}
\hline \hline PARAMETER & Model value \\
\hline bore/stroke/con-rod length & $88 \mathrm{~mm} / 82.2 \mathrm{~mm} / 132 \mathrm{~mm}$ \\
\hline compression ratio & $11: 1$ \\
\hline $\begin{array}{c}\text { intake valve opening } \\
\text { duration }\end{array}$ & $180 \mathrm{crank}$ degree \\
\hline $\begin{array}{c}\text { exhaust valve opening } \\
\text { duration }\end{array}$ & $180 \mathrm{crank}$ degree \\
\hline $\begin{array}{c}\text { intake manifold/exhaust } \\
\text { manifold volume }\end{array}$ & $2.5 \mathrm{~L} / 2.3 \mathrm{~L}$ \\
\hline
\end{tabular}

This engine is also equipped with the EGR cooler and associated control valve to adjust cooled external EGR gas, which is important to provide an additional degree of freedom to adjust the incylinder EGR fraction independent of the in-cylinder gas mixture temperature. The engine also features dual intake and exhaust valves with independent variable valve timing (VVT) control for both intake and exhaust valves. The exhaust and intake VVT controls are mainly used to generate a recompression of the residue gas during the exhaust stroke (see the recompression process between EVC and IVO shown in Figure 9). The residue gas recompression can affect the 
in-cylinder gas mixture temperature at IVC $\left(T\left(\theta_{I V C}\right)\right)$ significantly, and $T\left(\theta_{I V C}\right)$, along with the IVC timing, determines the SOHCCI timing. Higher compression ratio is also selected to make the HCCI combustion possible. For this simulation, the engine compression ratio is $11: 1$, see Table 1.

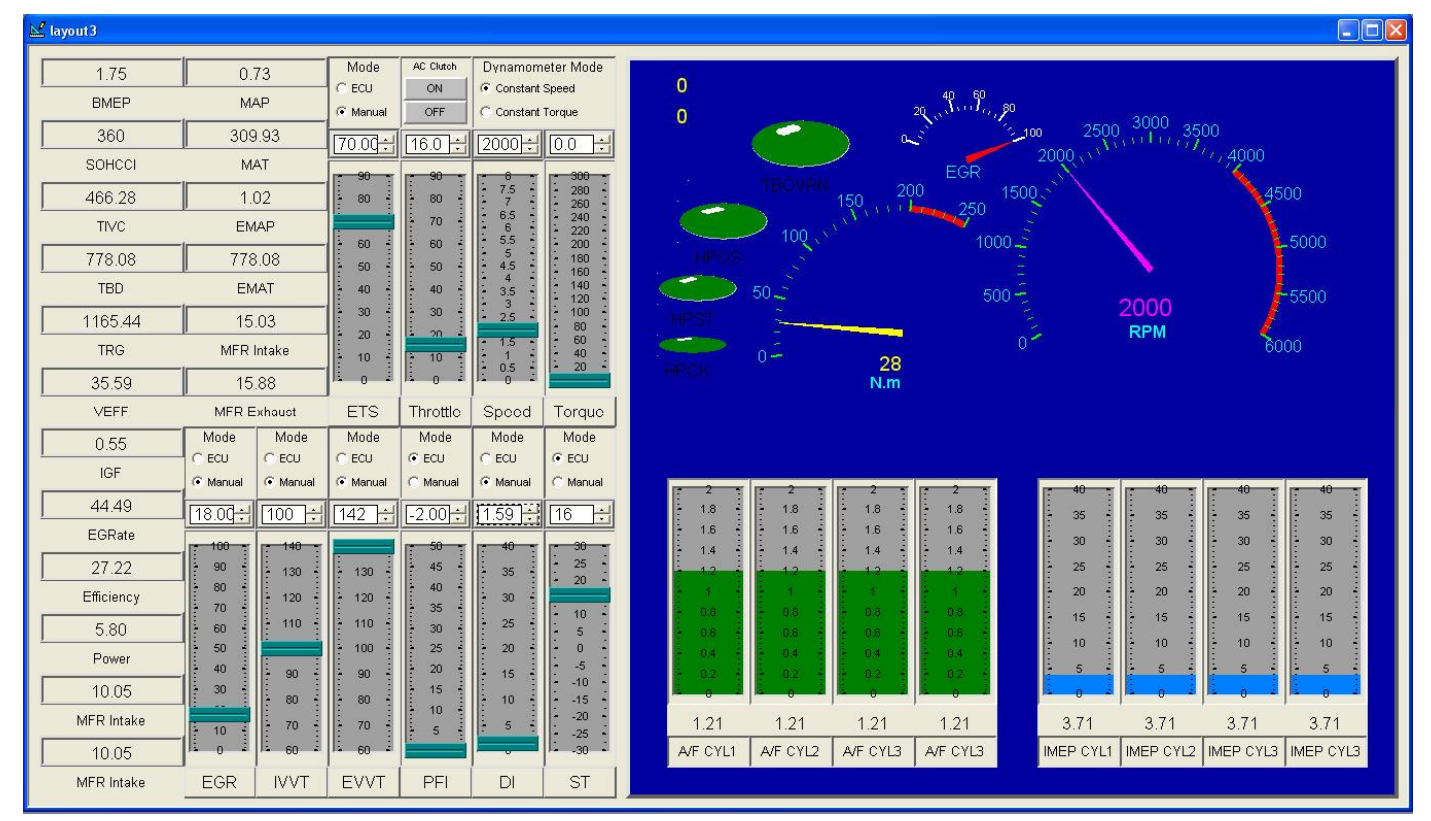

Figure 11: Hybrid combustion HIL simulation GUI

HIL simulations based upon the developed engine model were conducted in the dSPACE HIL simulation environment (see Figure 11) to simulate the SI-HCCI hybrid combustion. Note that both SI and HCCI combustion modes are special cases of the SI-HCCI hybrid combustion. When the HCCI combustion did not occur, the engine is operated at the SI combustion mode; when the HCCI combustion occurs at the start of SI combustion, the engine can be considered to be operated at the HCCI combustion mode; while the SI-HCCI combustion occurs inter-between the HCCI and SI combustion modes.

In the simulations, the engine combustion started with SI combustion mode, and then the engine exhaust valve timing were advanced to generate recompression that leads to high in-cylinder mixture temperature, which leads to HCCI combustion after spark ignition. This is so called SIHCCI hybrid combustion mode. As the exhaust valve timing advanced, the in-cylinder gas mixture temperature at intake valve closing timing $T\left(\theta_{I V C}\right)$ increased, see Figure 13, and the SOHCCI timing advances as the temperature increases. This can be observed in Figure 14, where after SI combustion initiated, the start of HCCI combustion advanced, leading to increased fraction of fuel burned in the HCCI mode. At last when the exhaust valve timing advanced to certain location, the spark ignition was turned off and engine transited into pure HCCI combustion mode. Six cycles of combustion data were measured for the combustion mode transition from SI to HCCI combustion, Figure 12 to Figure 14 show the in-cylinder profiles of pressure, temperature and MFB (mass fraction burned). 


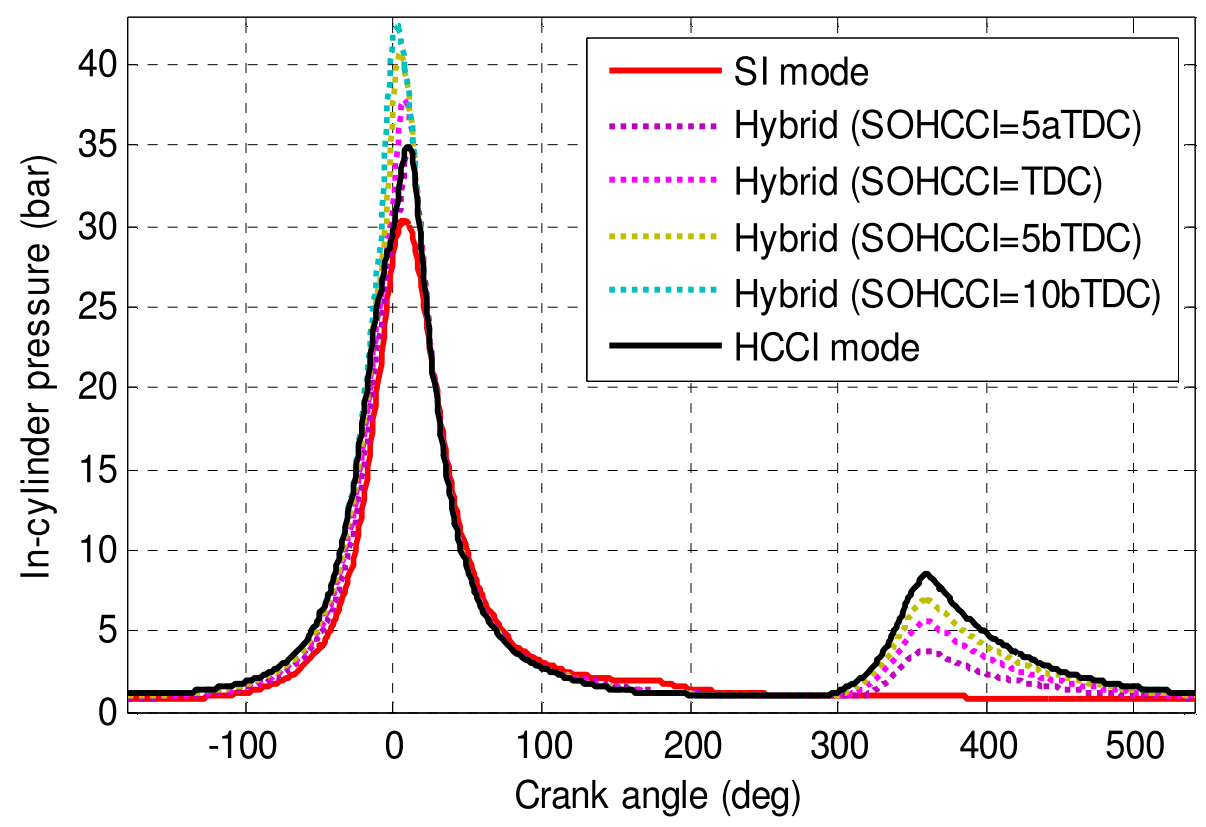

Figure 12: In-cylinder pressure profiles during combustion mode transition

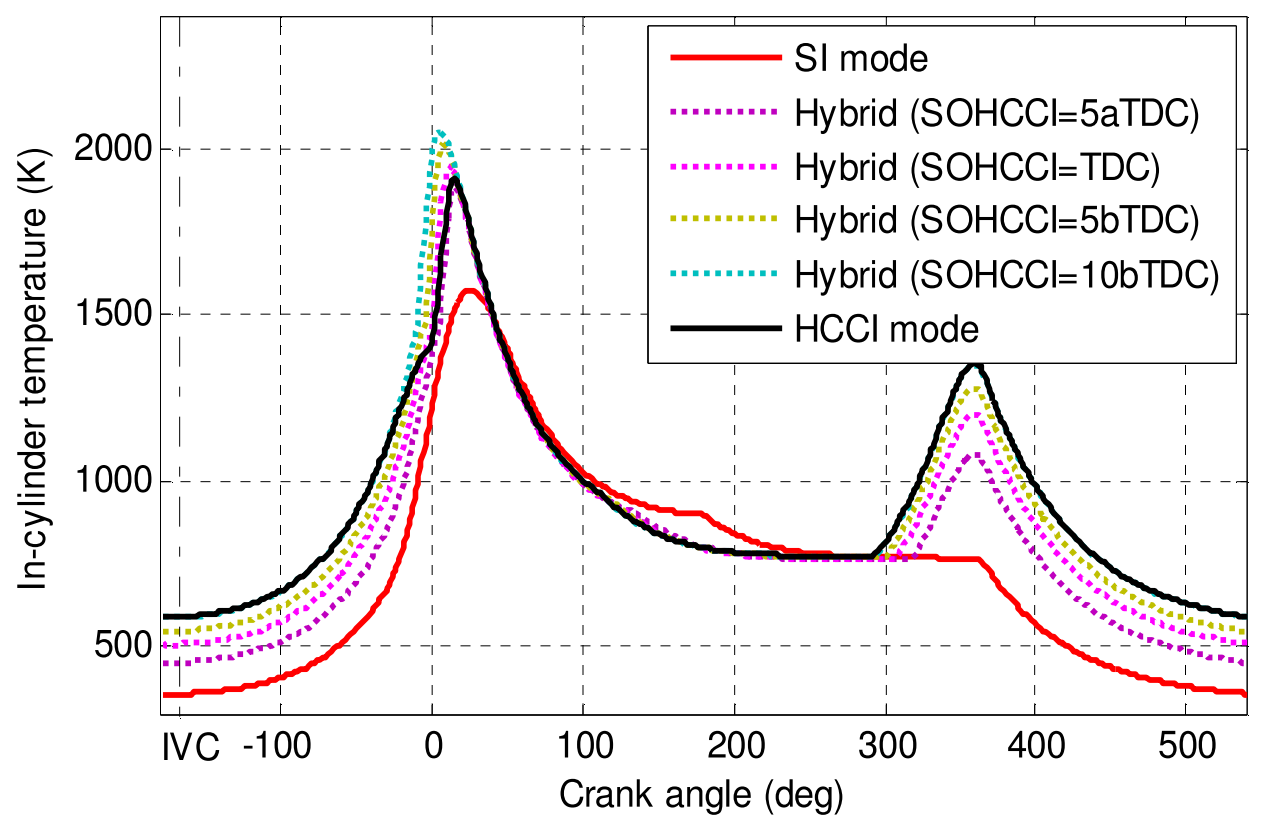

Figure 13: In-cylinder temperature profiles during combustion mode transition 


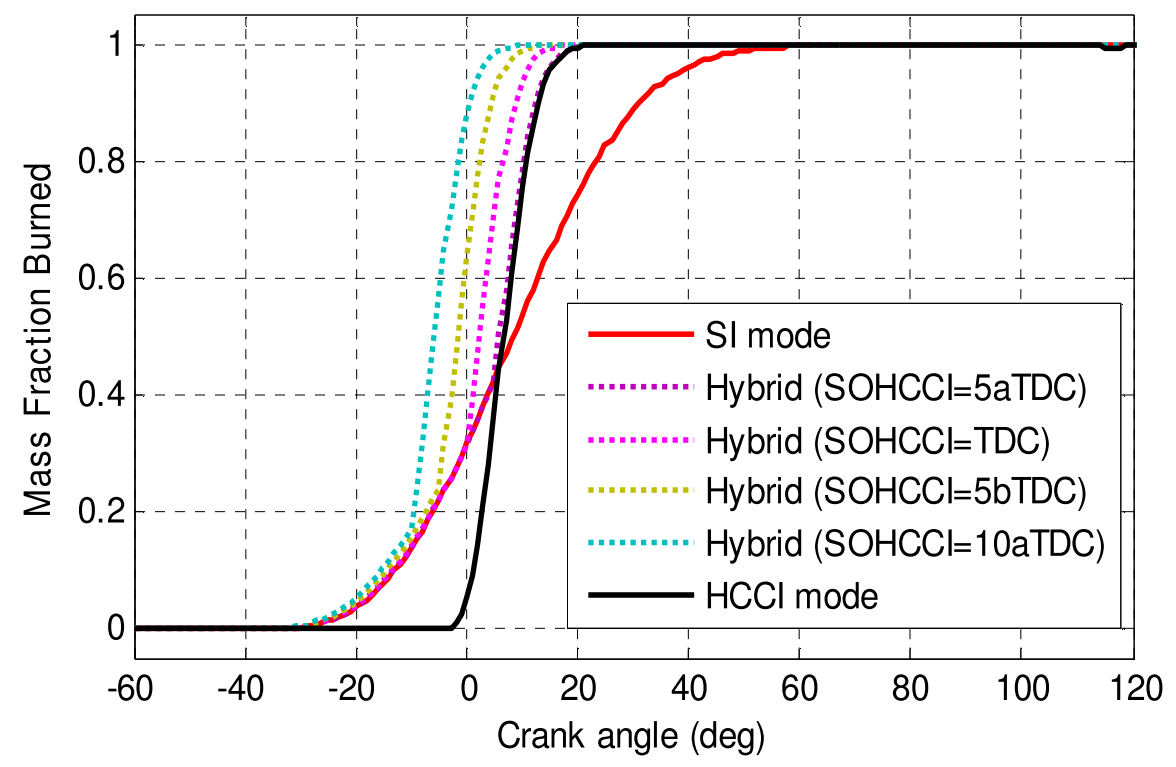

Figure 14: MFB profiles during combustion mode transition

In these simulations, engine IMEP was maintained at 3.7 bar as shown in Figure 15; intake valve timing was also held at 95 crank degrees after gas exchange TDC. In order to hold a constant IMEP (3.7 bar) when the exhaust valve timing advanced, the amount of fuel injected was decreased, accordingly, leading to increased air-to-fuel ratio due to improved combustion efficiency (fuel economy). From these simulations, we found that the HCCI fuel consumption is about $28 \%$ less than that of SI combustion when the IMEP was held constant.
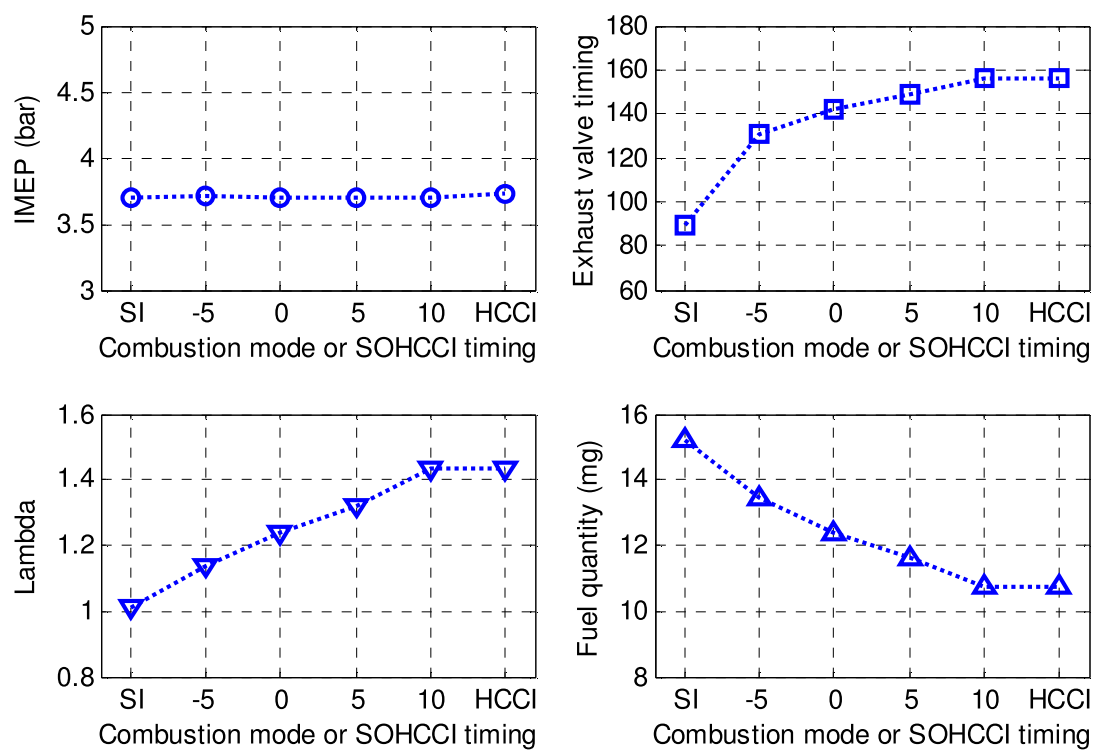

Figure 15: Engine performance of the combustion mode transition 
The hybrid SI-HCCI combustion modeling and simulation results were summarized into three papers; and they were published in 2010 ASME DSCC (Dynamic System Control Conference) [8] (see Subsection 9.12), 2011 American Control Conference [9] (see Subsection 9.10), and IMechE Journal of Automobile Engineering [10] (see Subsection 9.6).

The transient performance of the combustion mode transition is of great interest in the controloriented modeling. The SI to HCCI mode transition with hybrid combustion mode was simulated. The exhaust valve timing and fuel quantity were adjusted every consecutive engine cycle (cycles 1 to 6 in Figure 16). Two different strategies were adopted for the hybrid mode control. One used the steady-state simulation parameters for each transitional operation condition; the other used control parameters tuned for the transient operation. For comparison, the SI to HCCI mode transition without the hybrid mode was also simulated. Note that in this one cycle mode transition was used for simulation. For all cases, the engine spark was cut at cycle 7 to achieve pure HCCI combustion. Figure 17 shows the engine transient responses of IMEP (indicated mean effective pressure) and SOHCCI (start of HCCI) timing for 20 consecutive engine cycles. The engine was operated at 2000 RPM and note that 20 engine cycles cover 1.25 second time period.
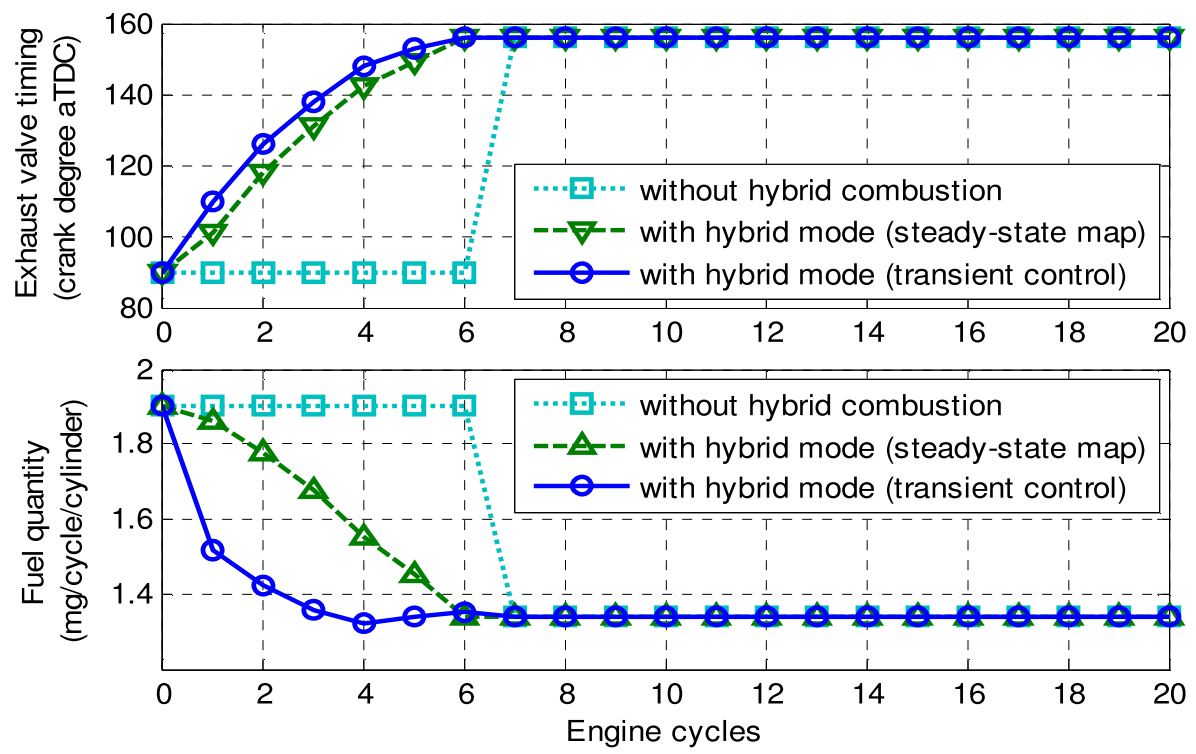

Figure 16: Engine control parameters used for mode transition 

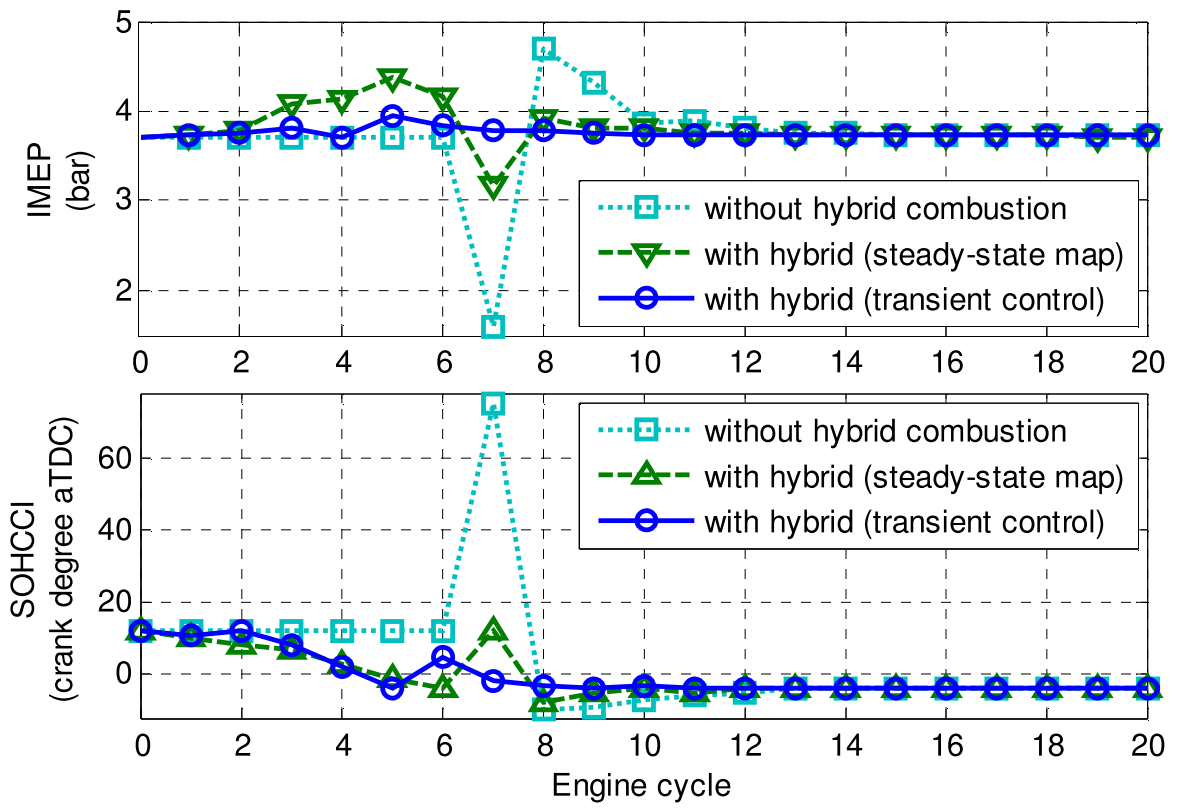

Figure 17: Engine Transient performance during mode transition

From Figure 17, we can observe that the engine transient performance was improved by utilizing the hybrid combustion mode during the combustion mode transition. Without hybrid combustion mode, the IMEP dropped far below the target value $(3.7$ bar) and SOHCCI was dramatically retarded to almost 80 crank degrees after gas-exchange TDC, which indicates partial-burn combustion (or close to misfire) at cycle 7 . In both cases with hybrid combustion mode, the controller using the tuned transient control parameters provides less IMEP and SOHCCI variations during the transient operation than the one using steady state parameters or one-step transition control. This indicates that using steady-state mapping in the mode transition cannot provide optimal mode transition performance and model-based transient control strategy has potential to provide optimal mode transition, which is the key contribution of this project. Notice that the engine IMEP using the tuned transient control parameters was kept close to 3.7 bar during the transient operation and SOHCCI was smoothly transient from 14 degrees before TDC to 3 degrees after TDC.

The simulation results, using the developed real-time engine model, show that the transient performances of the mode transition between SI and HCCI combustion is heavily dependent of the mode transition control strategy that is based upon the developed real-time model. This is due to the fact that the steady state control parameters for SI-HCCI hybrid combustion are not optimal for the transient SI-HCCI combustion operation and the model-based control is a necessity.

\subsubsection{Dual-zone SI-HCCI combustion modeling}

It is assumed that the flame front due to SI combustion separates the in-cylinder gas mixture into two zones, the burned zone and unburned zone, as shown in Figure 18. The temperature of the unburned zone is very different from that of burned zone. However, in the one-zone model, this difference is ignored and the average temperature is used for both zones. This may not cause 
large modeling error for the SI combustion, in which the temperature difference doesn't impact much on the combustion. However, for the SI-HCCI hybrid combustion, the expansion of burned zone during the initial SI combustion process applies work to the unburned zone; as a result, it makes the unburned zone temperature rise faster, and finally causes HCCI combustion in the unburned zone. The unburned zone gas properties are the key parameters for modeling the start of HCCI combustion. Also the dual-zone model approximates spark ignited combustion better than the one-zone model. These are the key motivations for developing this dual-zone combustion model.

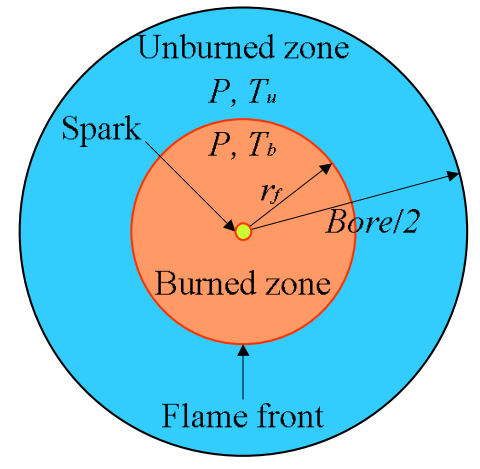

Figure 18: Dual-zone combustion

In the dual-zone model, the combustion process is also divided into five phases, shown in Figure 19.

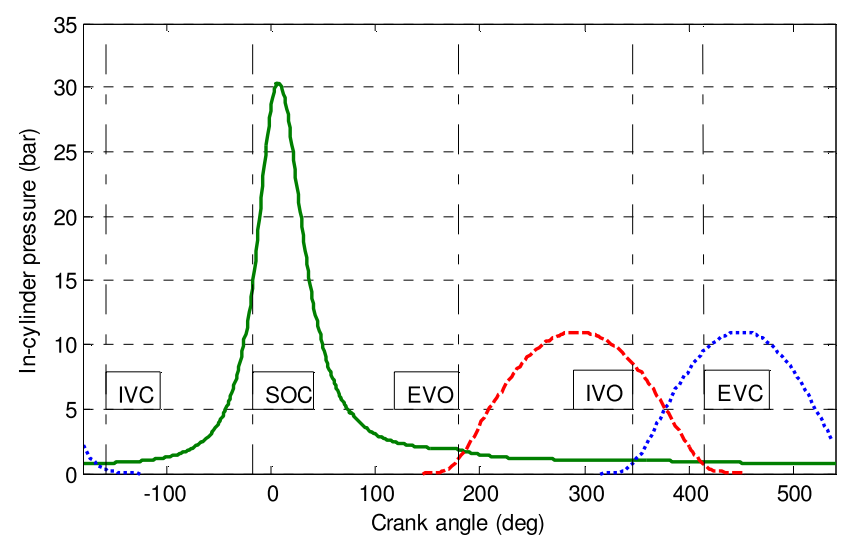

Figure 19: Combustion related events and phases

Except for the combustion phase (between SOC and EVO), the governing equations in the onezone model are used in the dual-zone model, whereas in the combustion phase (between SOC and EVO) first law of thermodynamics and ideal gas equation are used for modeling both zones. New energy balance equations were developed for the dual-zone SI-HCCI combustion model, which is published in 2011 American Control Conference [9]; see Subsection 9.9.

The newly developed dual-zone model was implemented into the dSPACE HIL system for realtime simulations. Due to additional computing throughput requirement, the dual-zone model real-time simulations was limited to 3700 RPM engine speed to avoid overrun, whereas the onezone model can operated up to 10000 RPM. Fortunately the top engine speed for the dual-zone model (operated in the SI-HCCI hybrid combustion mode) is limited to around 3500 RPM, and 
therefore, currently the throughput issue doesn't affect the application of the dual-zone combustion model.

\subsubsection{Dual-zone combustion model calibration and validation}

The dual-zone and one-zone combustion models were calibrated by the simulation results of the GT-Power combustion model. In order to calibrate the combustion models without influence from the engine air handling system, the engine air handling dynamics was not included in the GT-Power combustion model, see Figure 20. The inlet and outlet thermo conditions of the engine cylinder were assumed to be constant in this model. The setup is the same for Simulink based combustion models.

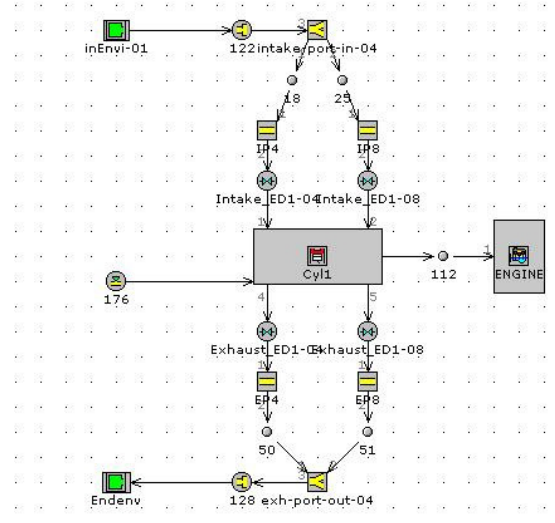

Figure 20: GT-Power combustion model

Calibrations were generated by sweeping the engine speed and load within the engine operating range during simulations. For each test condition, GT-Power simulation results are used as baseline.

Table 2: Model setup parameters for validation

\begin{tabular}{||l|c|c||}
\hline Parameter & Low load & High load \\
\hline Engine speed & $2000 \mathrm{RPM}$ & $2000 \mathrm{RPM}$ \\
\hline Intake manifold pressure & $0.5 \mathrm{bar}$ & $1.0 \mathrm{bar}$ \\
\hline Intake manifold temperature & $303 \mathrm{~K}$ & $303 \mathrm{~K}$ \\
\hline Exhaust manifold pressure & $1.0 \mathrm{bar}$ & $1.0 \mathrm{bar}$ \\
\hline Burn duration $(10 \% \sim 90 \%$ MFB) & $28 \mathrm{deg}$ & $20 \mathrm{deg}$ \\
\hline $50 \%$ MFB location & $5 \mathrm{deg}$ ATDC & $5 \mathrm{deg}$ ATDC \\
\hline
\end{tabular}

The pressure traces of the dual-zone and one-zone combustion models were also compared using the GT-Power simulation results, shown in Figure 21. Two engine operational conditions were chosen, the low (4.7 bar) and high (12.0 bar) load conditions. The model parameters of each condition were shown in Table 2. 


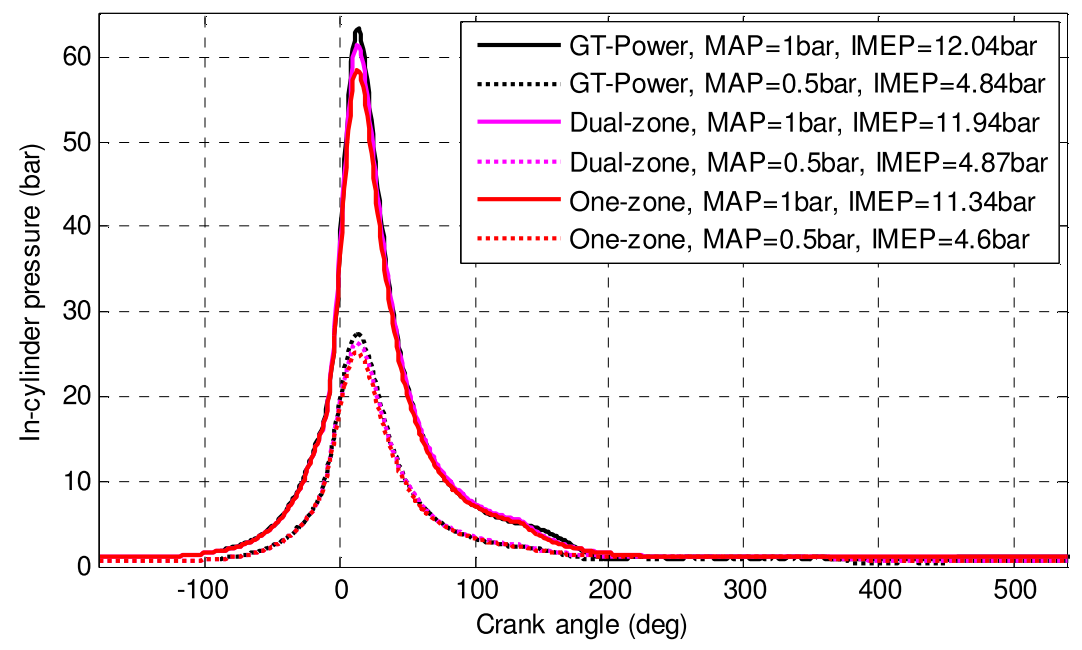

Figure 21: In-cylinder pressure traces

Good correlations of in-cylinder pressure signals over entire engine cycle were found in Figure 21 for both combustion models. The pressure signal of the dual-zone model has a better match with the GT-Power pressure curve, and the IMEP values are closer to the GT-Power results for both low and high load conditions than one-zone ones. This demonstrated that the dual-zone combustion model leads to higher modeling accuracy than the one-zone one.

The averaged one-zone and dual-zone in-cylinder temperatures were shown in Figure 22 for the convenience of comparison. Both temperatures of the dual-zone and one-zone models follow the GT-Power model temperature signals very well in all phases, except during the exhaust phase (between EVO and EVC). The maximum error is about $250 \mathrm{~K}$ or $15 \%$. This error is mainly due to the isentropic assumption of the expanding process of in-cylinder burned gas into the exhaust manifold. The heat loss through exhaust valve and manifold was ignored, which makes the calculated temperature higher than the actual one. Fortunately, the heat loss will be considered in the time based mean-value model of exhaust manifold when the combustion model is integrated with it, so it will not affect the overall engine performance.

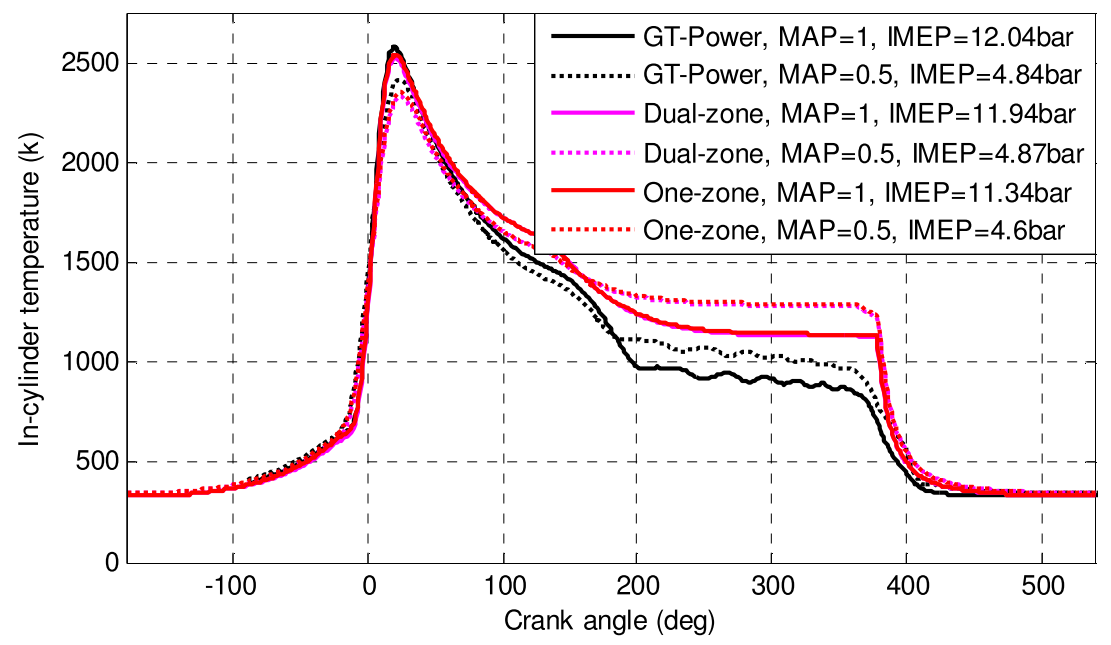

Figure 22: In-cylinder temperature curves 


\subsubsection{SI-HCCI hybrid combustion model}

Another important simulation result is shown in Figure 23, where the MFB traces produced by both GT-Power and the developed dual-zone models match very well. The error of the normalized flame radius between GT-Power and dual-zone model result is peaked at the early stage of combustion. This is mainly due to the fact that in the dual-zone model the flame radius calculation starts after MFB is great than $0.1 \%$, which creates several crank degree delay in the result. Both flame radius curves are above the MFB curves. This indicates that the flame front pushes toward the unburned zone and applies work to it, which shows that the initial stage SI combustion supplies energy to the unburned zone gas mixture and helps to reach the conditions of the start of HCCI combustion.

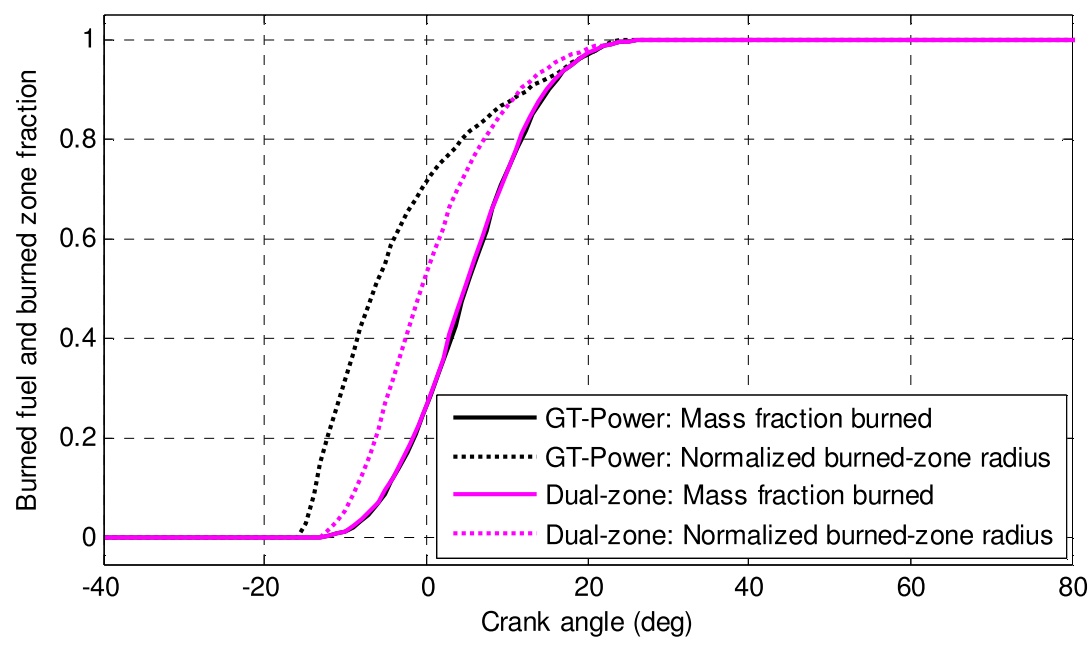

Figure 23: MFB and normalized burned zone radius curves

Note that the proposed SI-HCCI hybrid combustion is especially useful for modeling combustion mode transition between the SI and HCCI combustion. Also, as discussed before both SI and HCCI combustion modes are special cases of the SI-HCCI combustion. When the HCCI combustion did not occur, the engine is operated at the SI combustion mode; and when the HCCI combustion occurs at the start of SI combustion (SOC), the engine is operated at the HCCI combustion mode; while the SI-HCCI hybrid combustion occurs inter-between the HCCI and SI combustion modes.

To model the hybrid combustion, both one-zone and dual-zone modeling approaches are needed, where dual-zone model is used during SI combustion and the one-zone model is used after start of the HCCI combustion. We divided the entire engine cycle into six phases shown in Figure 24. The existence of ST (spark timing) and SOHCCI depends on the engine operating condition and control strategy. Without SI combustion, one-zone model is used and engine is operated at pure HCCI mode. With the initial SI combustion, dual-zone model is used during the SI combustion while the SOHCCI condition is checked every crank degree after SI combustion starts. After the SOHCCI condition is satisfied, one-zone model is used to model the rest of combustion process in HCCI mode. If the condition is not satisfied until the combustion is completed, the resulting combustion is an SI one. 


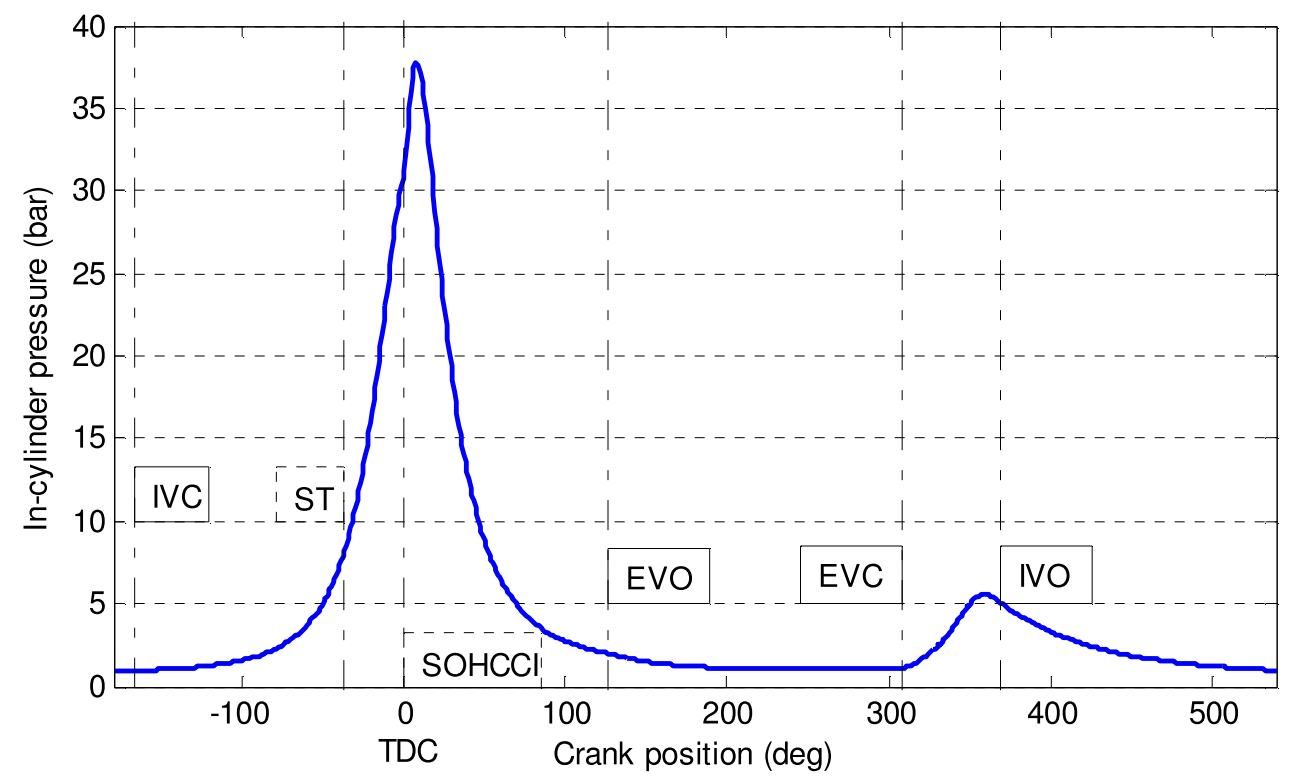

Figure 24: Combustion related phases for SI-HCCI hybrid combustion

Based upon references [1] and [11], the Arrhenius integral can be used to estimate the crank position of start of HCCI combustion (SOHCCI). The SOHCCI crank position, defined as the crank angle for $1 \%$ fuel burned under HCCI combustion, can be determined as the crank angle when the Arrhenius integral reaches one, see references [1] and [11] for definition. Once the Arrhenius integral reaches one; the HCCI combustion starts. The MFB of the HCCI combustion is still computed by Wiebe function as in [9]. The predicted combustion duration can also be calculated based upon [9]. The developed hybrid combustion model has been implemented in Simulink and the HIL simulator.

Two simulations were conducted to demonstrate the necessity of dual-zone model for the SIHCCI combustion. One simulation uses the one-zone model only, while the other uses the dualzone model, where the Arrhenius criterion is based on unburned zone temperature. The temperature and MFB traces, in Figure 25 and Figure 26, show 5 crank degree difference in SOHCCI timing. This difference is significant and cannot be ignored since in some operational conditions this estimation error could lead to engine misfire. 


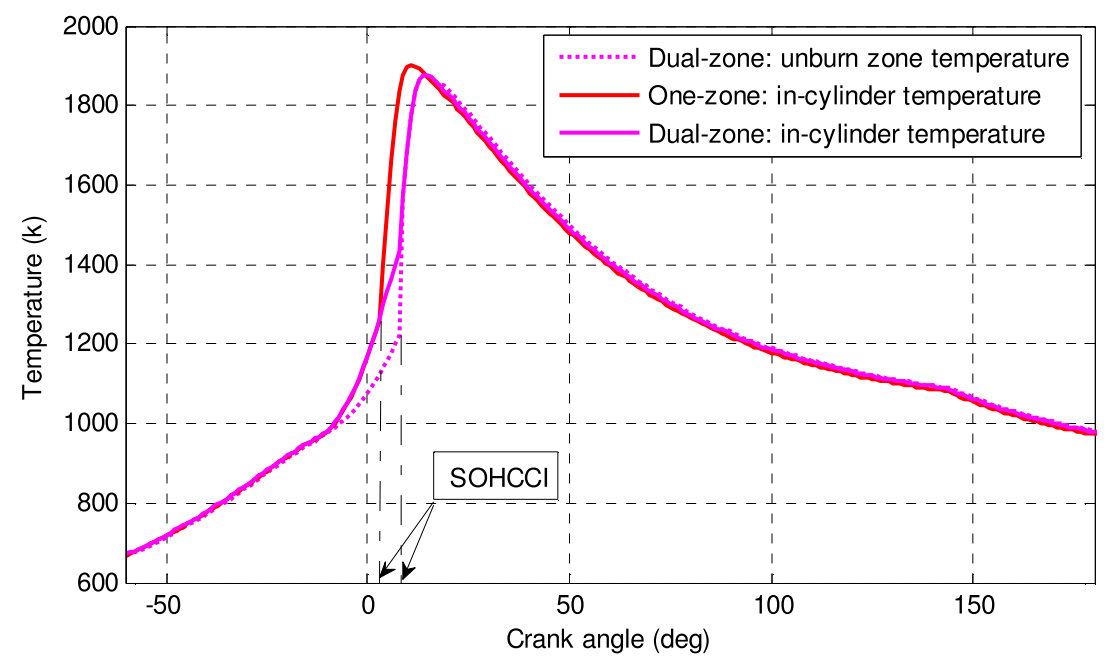

Figure 25: Temperature curves of SI-HCCI hybrid combustion

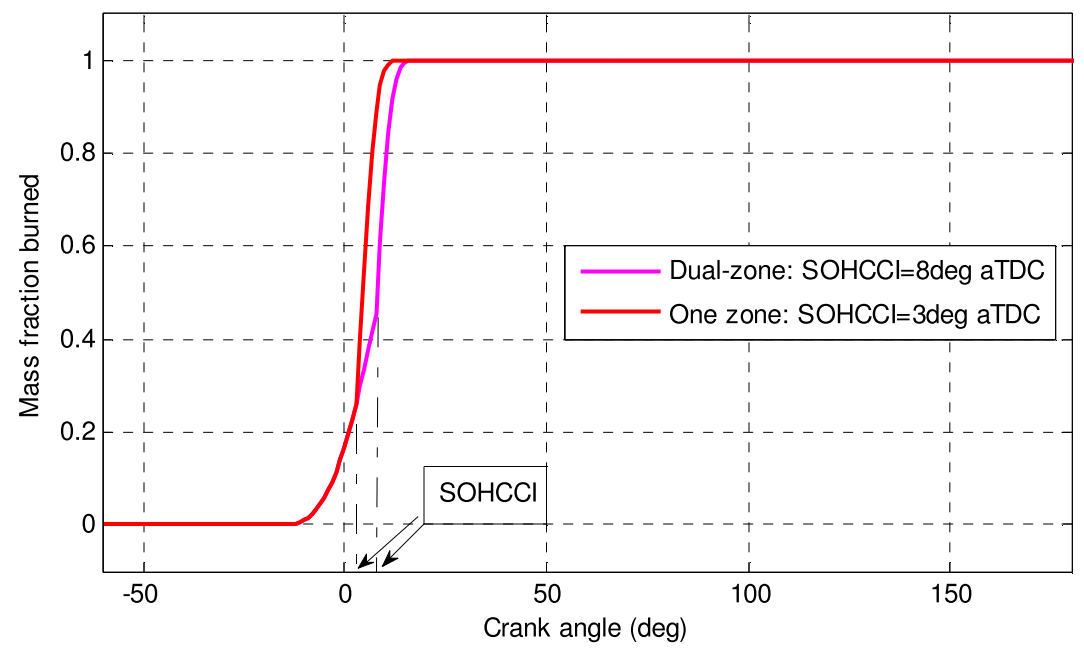

Figure 26: MFB curves of SI-HCCI hybrid combustion

To demonstrate the effectiveness of using the SI-HCCI hybrid combustion mode for mode transition, an SI-HCCI mode transition process was simulated by two different control strategies. One uses the hybrid combustion mode and the other switches from SI to HCCI combustion mode in one step (engine cycle). Figure 27 shows the in-cylinder pressure traces of two strategies generated during the simulation evaluation. The pressure profiles vary significantly from cycleto-cycle during the mode transition for both strategies. The engine control parameters used for the simulations were spark timing, fuel quantity, intake-exhaust valve timings and lifts. For the simulation results presented in Figure 27, these control parameters were adjusted manually to have the best (smooth) transition. To obtain smooth combustion mode transition for the entire engine operating range, advanced control strategy will be developed. 


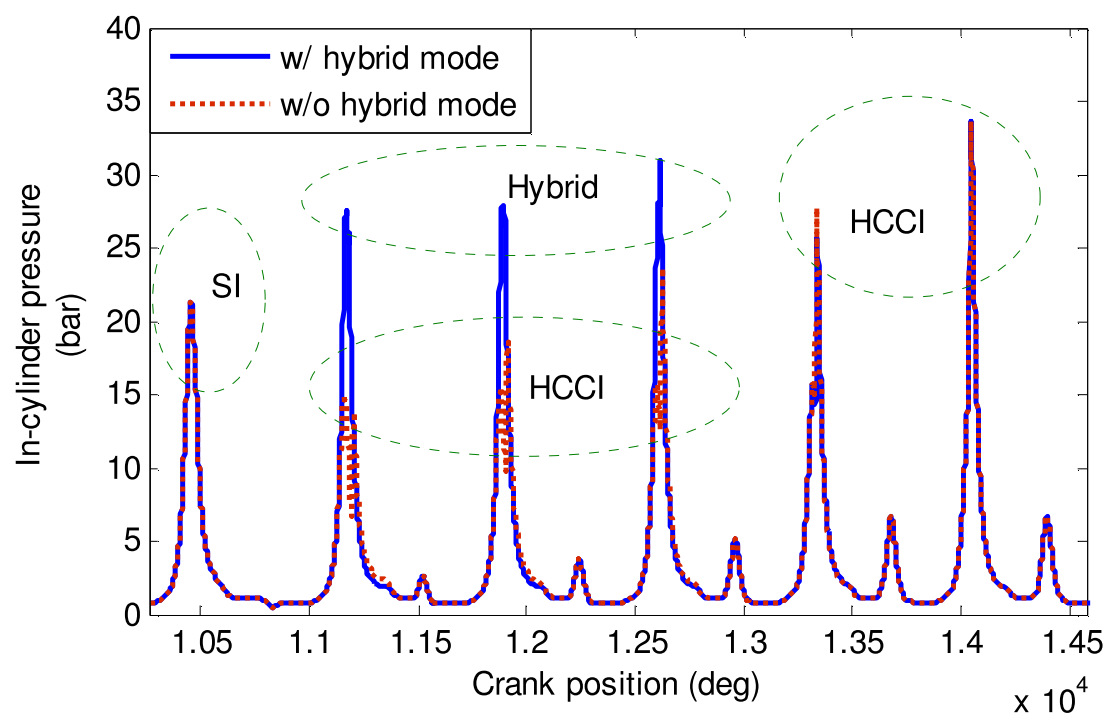

Figure 27: In-cylinder pressure traces during SI-HCCI transition

In the mode transition simulations, 26 engine cycles of combustion were simulated for both control strategies. Engine speed was set to 2000 RPM throughout the entire process. In the first 15 cycles, engine was operated in SI combustion mode and $12 \mathrm{mg}$ (per cycle) of gasoline was injected to each cylinder; spark timing was set to be at 23 crank degrees before TDC (top dead center); intake and exhaust valve timings (at peak lift position) were set to 440 and 270 degrees after TDC, respectively; and the lifts of both valves were in the high stage. Combustion mode transition started from SI to HCCI at engine cycle 16. At cycle 16 both intake and exhaust valves switched to low stage (lift) mode, but the valve timing change was limited to 10 crank degrees per engine cycle due to the continuing operation of the electrical variable valve timing actuating system. Note that 10 crank degrees per cycle were for the engine operated at 2000 RPM. The valve speed limitation varies as a function of engine speed. Also at cycle 16, spark was cut for the one-step transition control strategy without hybrid combustion mode and the control parameters were adjusted to make HCCI combustion possible with minimal IMEP variation. On the other hand, for the hybrid combustion mode transition, spark was kept on in cycles 16, 17 and 18, and it was even advanced for smooth mode transition. After cycle 19 the spark was cut. Note that at cycle 19 both intake and exhaust valves also reached their target cam phase positions and remain at the target valve timing. Throughout the entire mode transition process, fuel mass was adjusted to minimize the IMEP fluctuation. All selected control parameters can be found in Figure 28. 

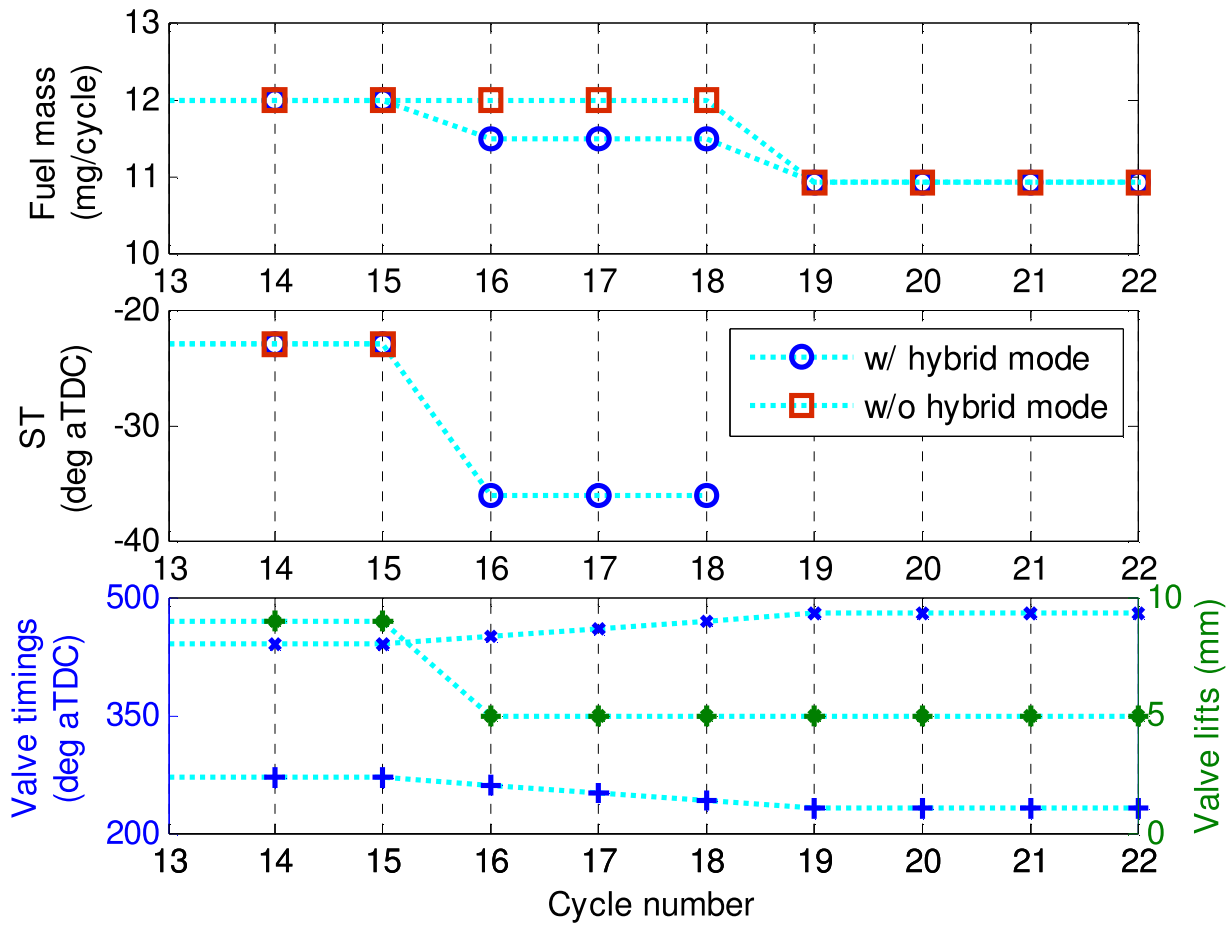

Figure 28: Combustion parameter settings in SI-HCCI transition

Figure 29 shows a few key combustion performance parameters. IMEP is the most important performance parameters of the mode transition since the less IMEP variation is; the less the engine brake torque variation or the smoother the combustion mode transition. With the one step combustion transition, the IMEP drop is significant right after the mode transition when the spark was cut, see cycles 16 and 17. Note that during mode transition, the in-cylinder gas mixture can't reach appropriate temperature for HCCI combustion at IVC with or without hybrid mode of combustion, see $T_{I V C}$ trace in Figure 29. If spark were cut at this engine cycle, the SOHCCI timing would be retarded significantly. This can be found in Figure 27 and Figure 29. The retarded SOHCCI timing leads to large IMEP reduction, or even misfires under certain extreme conditions. Therefore, spark assistant HCCI combustion or the hybrid HCCI combustion is a necessary tool for smooth mode transition. Based upon Figure 27 the hybrid combustion mode exists in cycles 16, 17 and 18 and the IMEP variation is significantly reduced, comparing to the one-step mode transition. Figure 30 shows the MFB profiles of the SI-HCCI transition with hybrid mode and illustrate how the combustion timing changes during the process.

The simulation results show that the SI-HCCI hybrid combustion mode can be used to reduce engine output torque variation during combustion mode transition. For SI and HCCI combustion modes, the model has also been calibrated and validated by GT-Power model. The model can also be implemented into HIL simulator for real-time engine simulation due to low computational throughput of the model. Unlike the SI and HCCI combustion modes, the hybrid combustion cannot be validated using GT-Power simulation results since GT-Power doesn't have hybrid combustion model currently. 

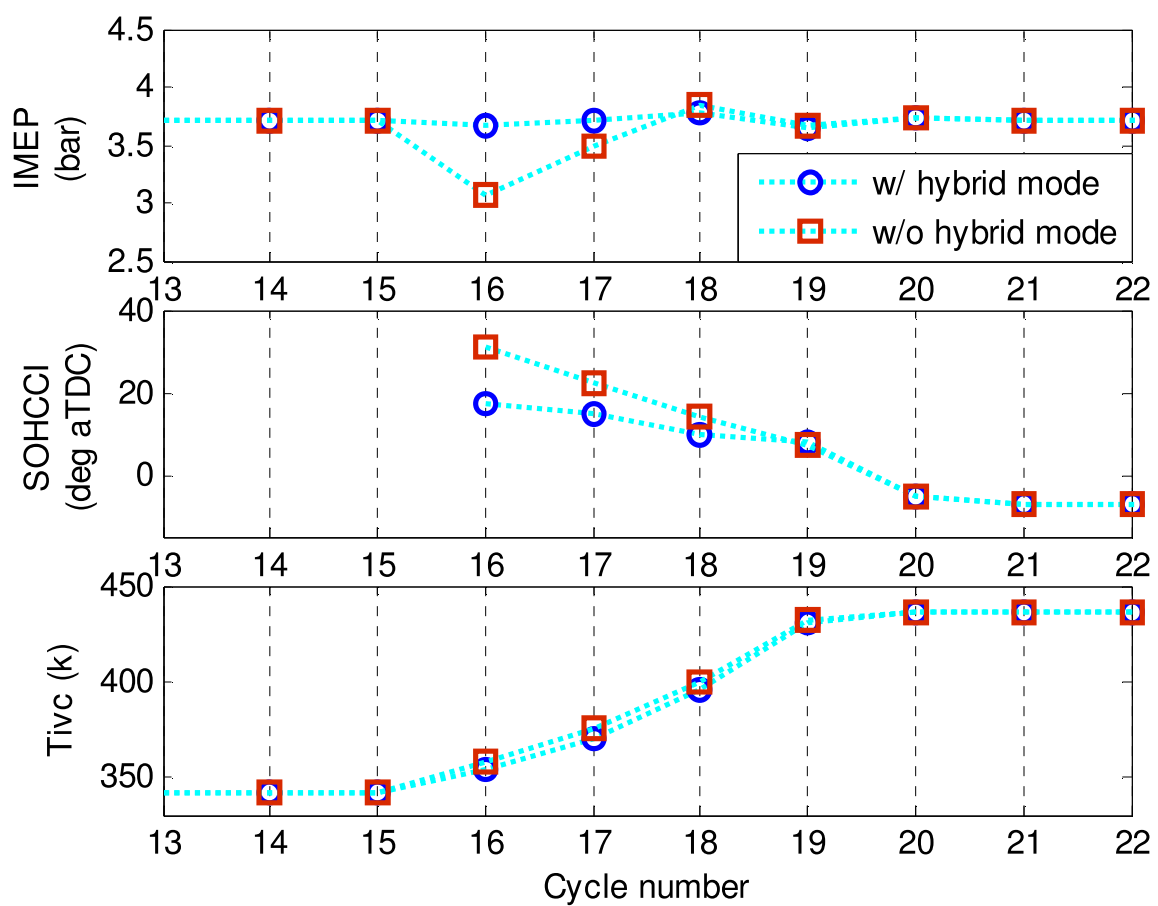

Figure 29: Combustion performance in SI-HCCI transition

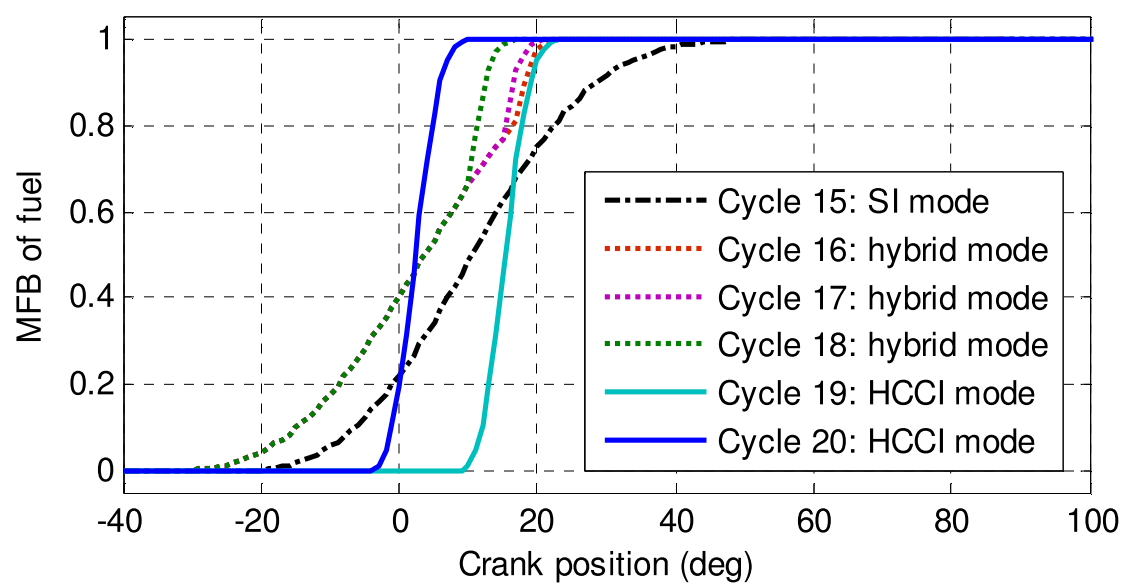

Figure 30: MFB profiles in SI-HCCI transition with hybrid mode

\subsection{Single cylinder optical engine design}

The optical engine design is shown in Figure 31 and the following is a list of design details:

1. Chrysler multi-cylinder engine head was modified to fit on the designed optical engine, where the existing dual hydraulic cam phase subsystems were modified into the electrical variable valve timing systems.

2. Both optical cylinder and piston are used in the optical engine, where the optical cylinder configuration was used for fuel spray and mixing tests and optical piston configuration was used for combustion tests of SI, HCCI, and SI-HCCI combustion.

3. A Hatz crank case was used as the base of the optical engine. The crank shaft was modified to match the stroke of the target engine and the connecting rod was also modified to match 
the engine cylinder volume profile.

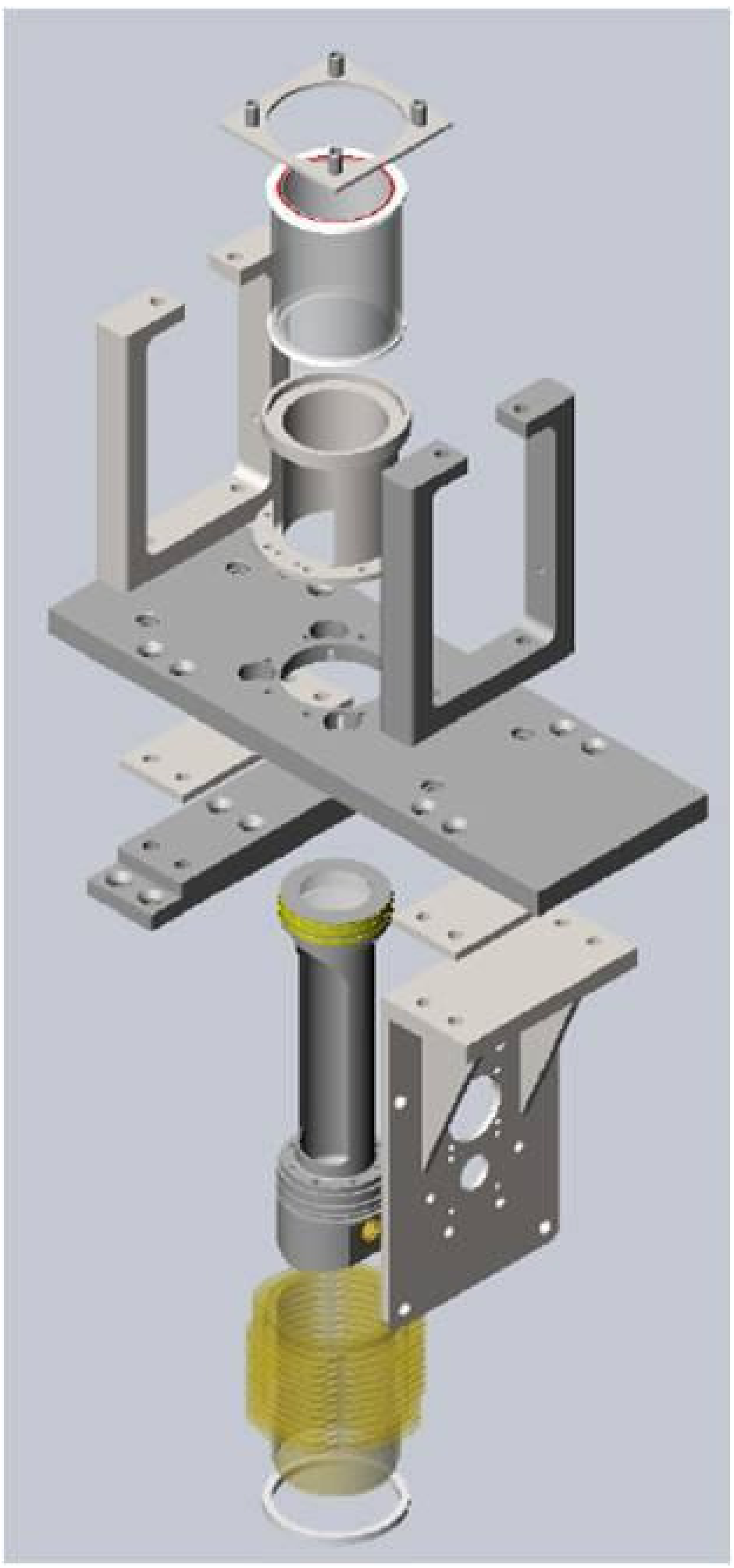

Figure 31: Optical engine integration diagram 


\subsection{Optical Engine Fabrication}

Total of two optical engines were fabricated: one without the target two-step valve and electrical cam phaser due to the late availability of the valve-train hardware. Both optical engines use the following components:

1. The Hatz crank shaft was redesigned; see Figure 32 for the manufactured part.

2. The connecting rod of the optical engine was fabricated by modifying an existing Chrysler connecting rod, see Figure 32.

3. Optical cylinder was also designed and fabricated.

4. Optical piston lens (flat piston) was designed and fabricated; see Figure 33 for the piston assembly with optical lens.

5. The Bowditch optical piston for the target engine was designed, fabricated and assembled with the optical lens, see Figure 33.

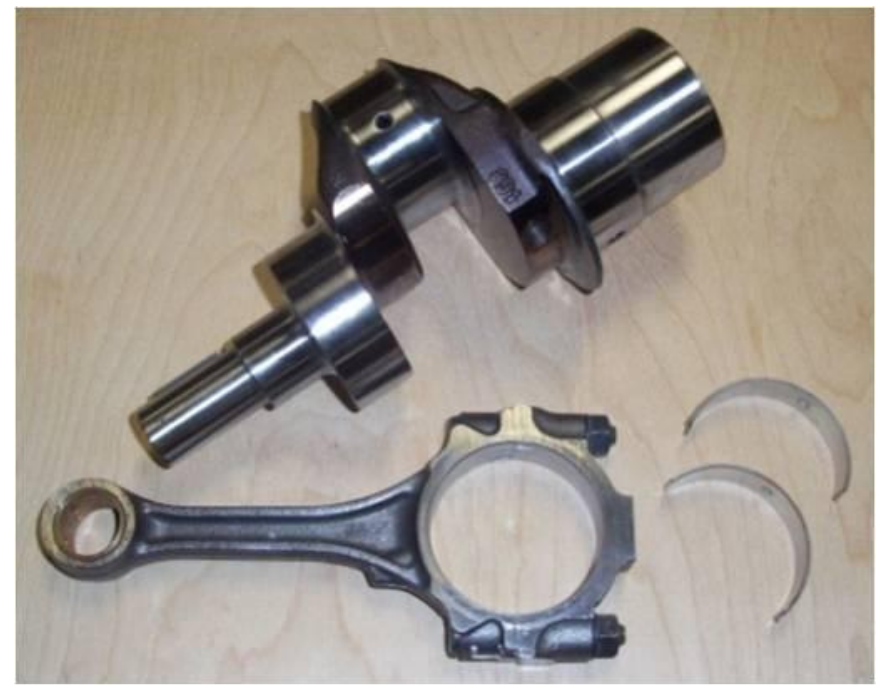

Figure 32: Optical engine components fabricated

All the optical engine components were designed at MSU for the baseline (SI combustion) optical engine (optical engine \#1) and the optical engine was assembled and installed in the MSU optical engine dynamometer. Figure 34 shows the assembled optical engine (optical engine \#1) for baseline SI combustion research. Fuel injection cold flow visualization and combustion tests were conducted on this engine. After the two-step valve and electrical phaser systems were available, the valvetrain was upgraded to allow HCCI combustion for optical engine \#2. SI optical combustion tests were conducted and the results were reported in Subsection 3.1. 


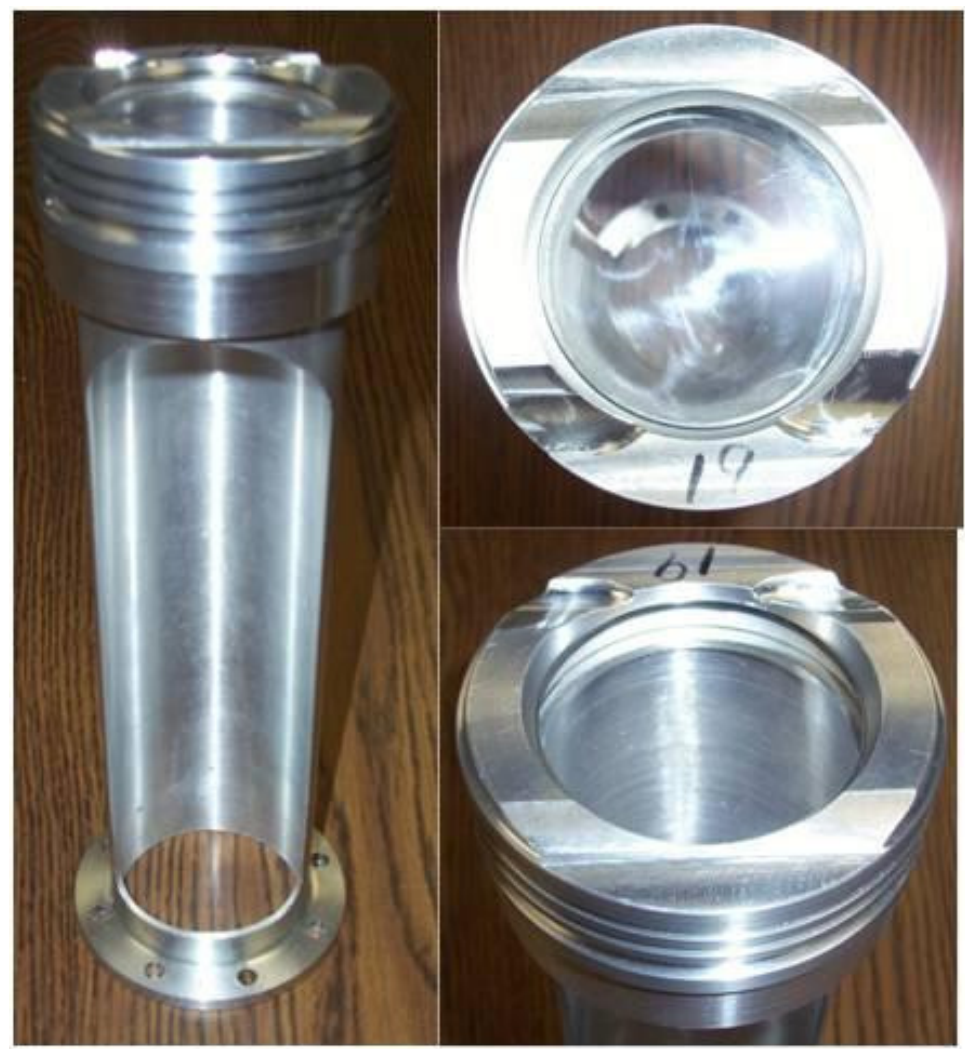

Figure 33: Constructed optical engine piston with optical lens

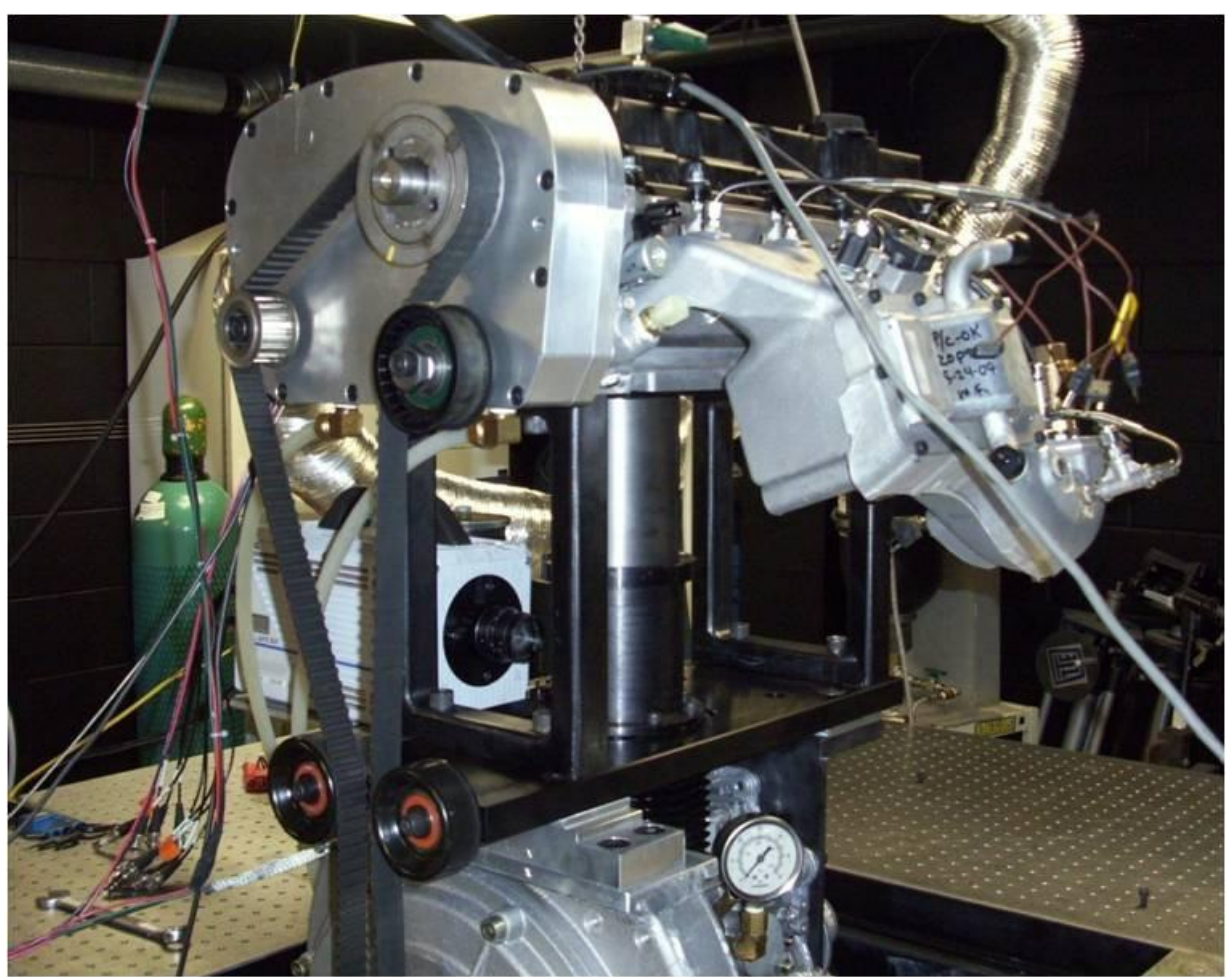

Figure 34: Completed baseline (SI combustion) optical engine assembly (optical engine \#1) 
For studying the HCCI combustion, the cylinder and cylinder head of the optical engine \#2 (HCCI optical engine) needs to be heated using an external heater. Figure 34 shows the designed and fabricated the engine coolant temperature control unit and coolant heated cylinder. To simulate the turbocharger effect on the combustion, compressed air was used in the optical engine tests, an intake charging damper was also designed and constructed; see Figure 34.

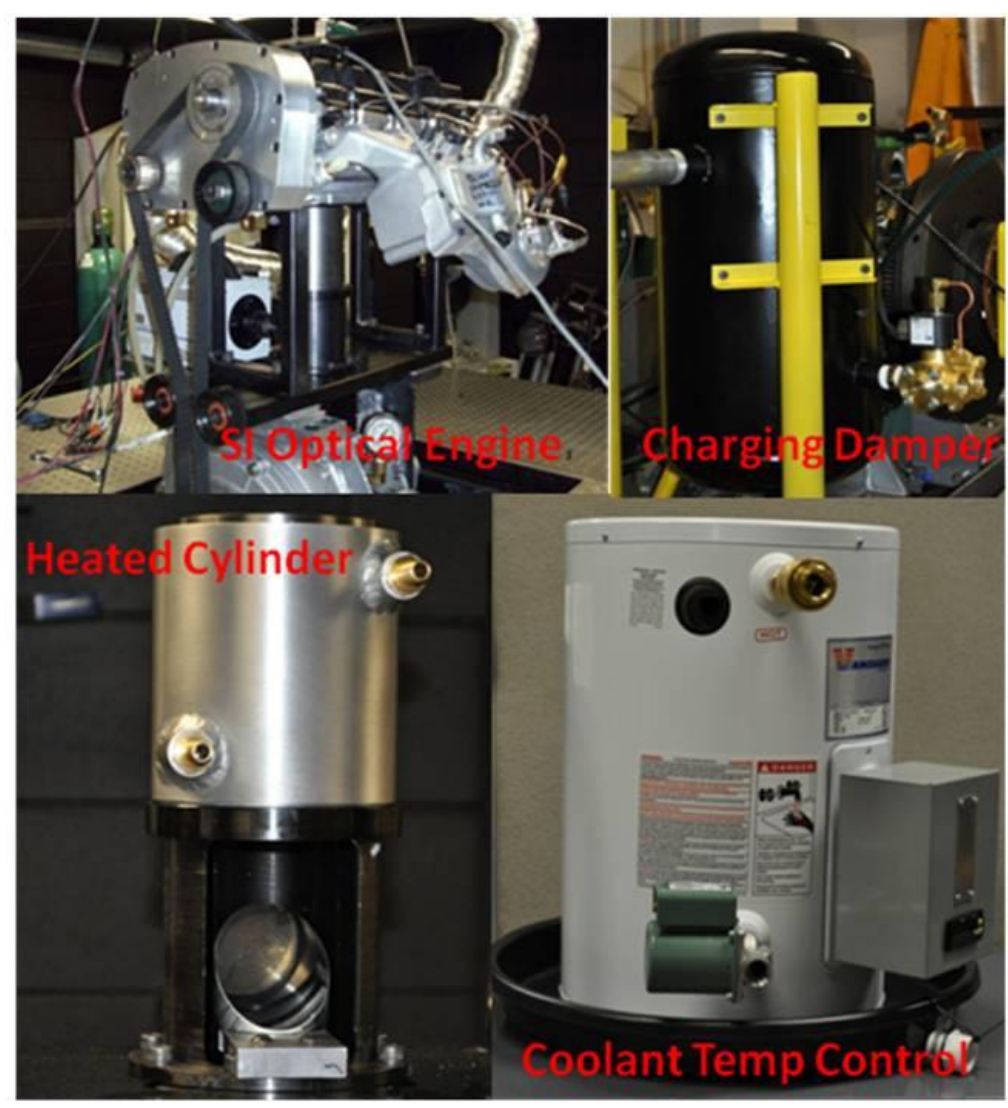

Figure 35: Completed baseline (SI combustion) optical engine assembly

The optical engine electrical variable valve timing (EVVT) system and timing belt-gear system design are shown in Figure 36 with the demonstrated assembly in Figure 37. The machined parts of the timing-belt-gear system are shown in Figure 38, and the assembled optical engine is shown in Figure 39. 


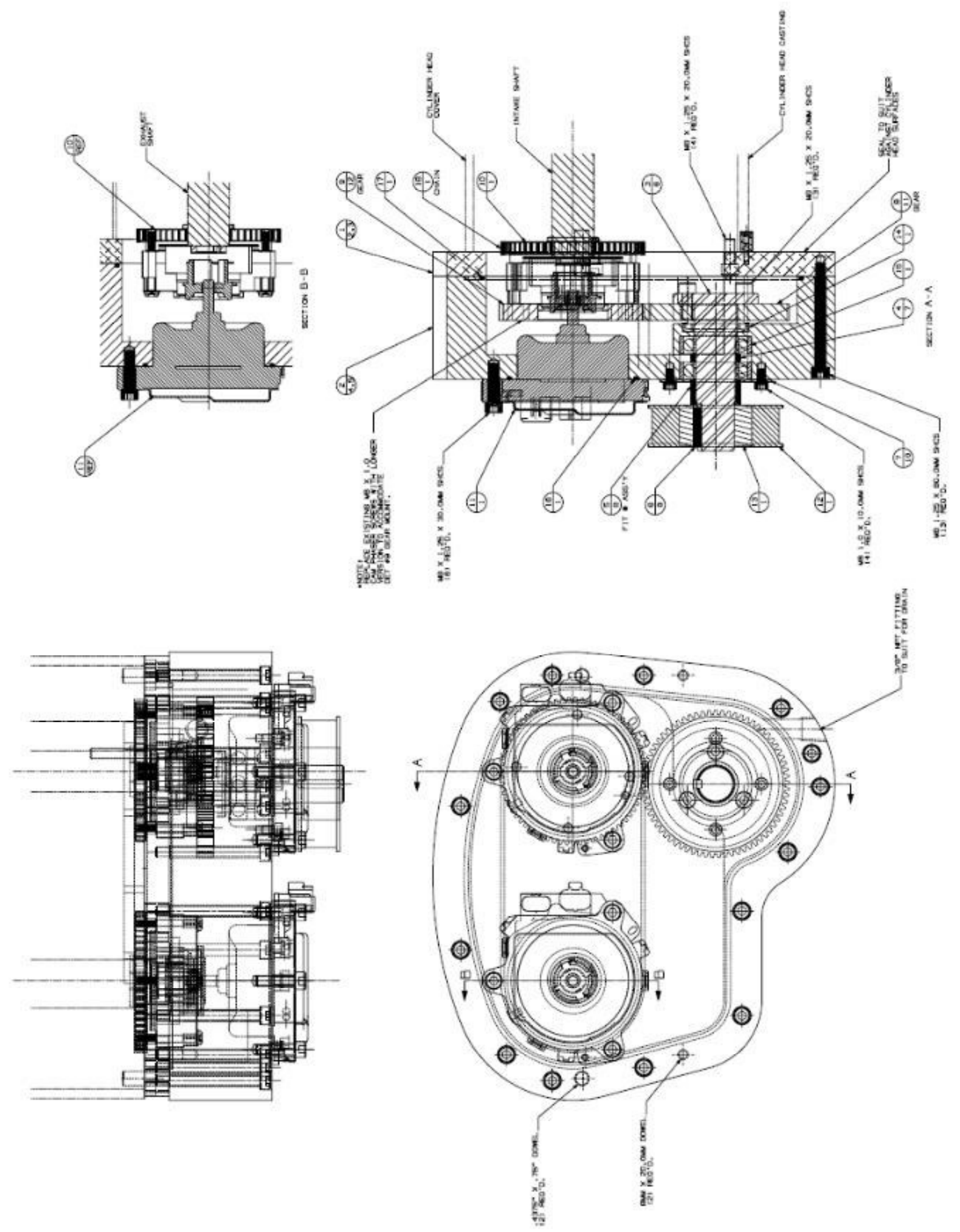

Figure 36: Electrical cam phasing system and timing belt design 


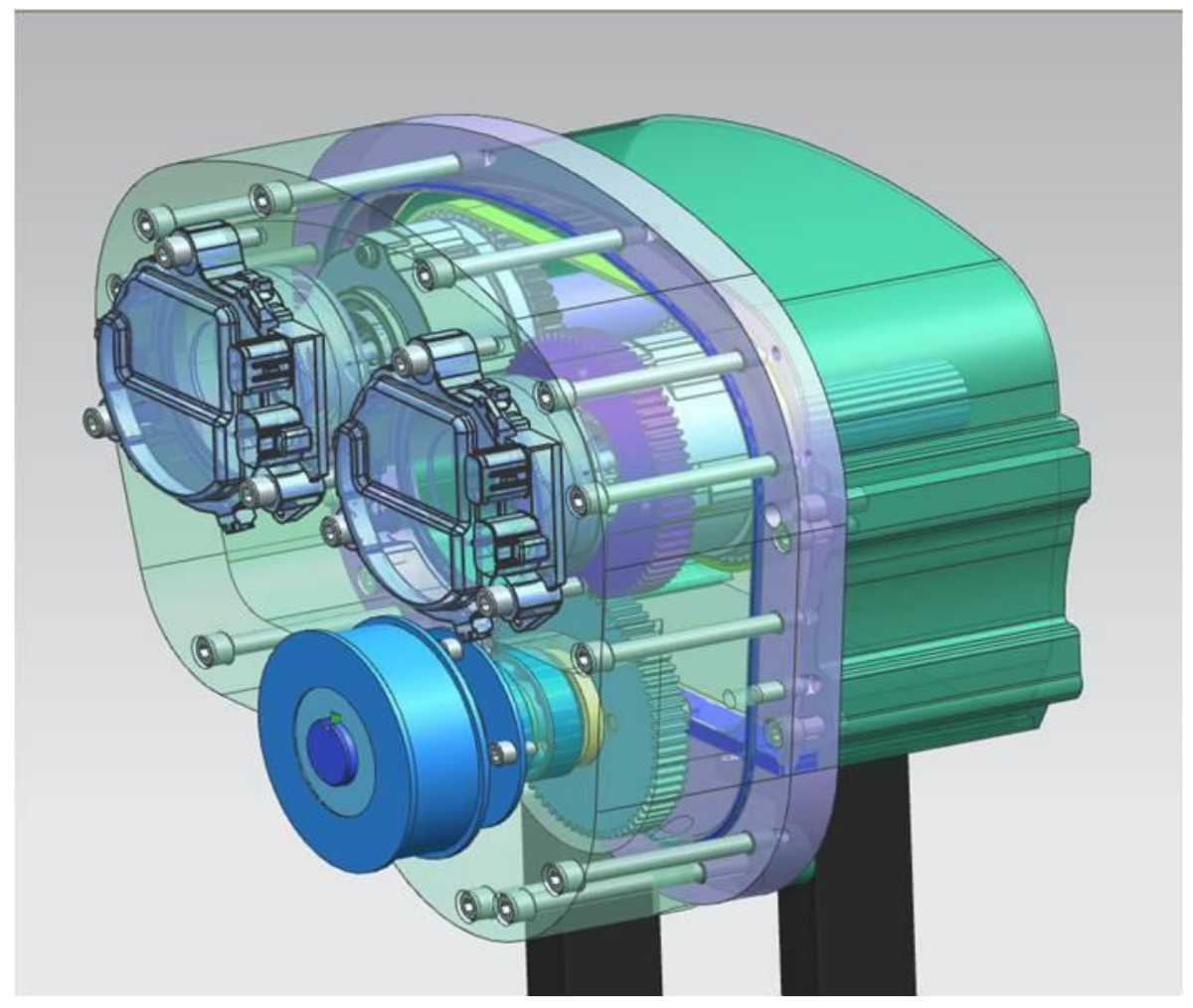

Figure 37: Electrical cam phasing system and timing belt assembly

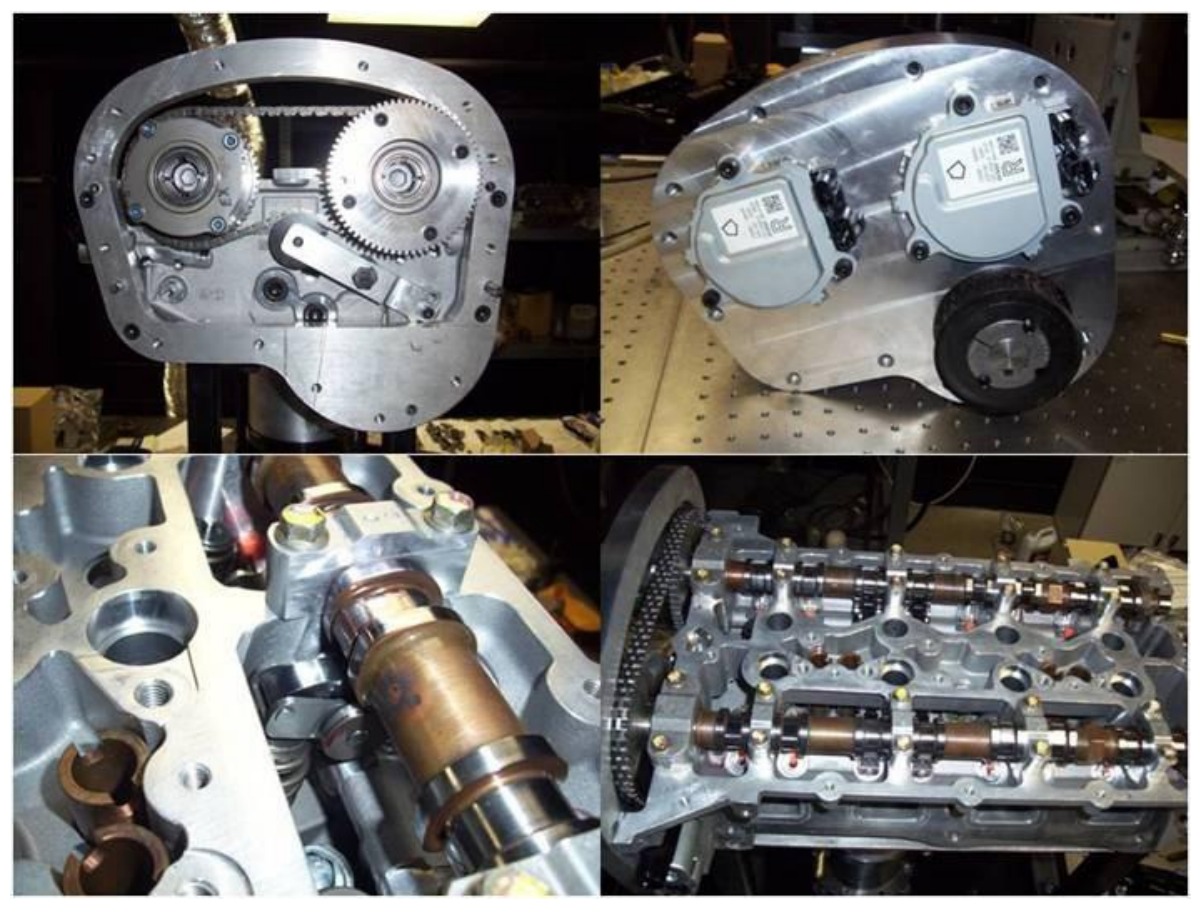

Figure 38: Cam phasing system and cylinder head with two step valve system 
The optical engine \#2, see Figure 39, was assembled using the modified target engine head equipped with the target two-step valve and electrical VVT (variable valve timing) systems. The following is a list of the optical engine parameters.

a) 0.5 liter per cylinder

b) Direct fuel injection system with fuel rail pressure at $10 \mathrm{MPa}$

c) Dual-lift intake/exhaust valves with dual-cam profiles

d) Fast electrical cam phasing with phasing range of \pm 40 degrees

e) Fast electronic throttle with response time less than $100 \mathrm{~ms}$

f) Heated engine head and cylinder

g) Heated charge air with closed-loop temperature control

h) Key engine sensors for closed-loop control: in-cylinder pressure and ionization, AFR (airto-fuel ratio), manifold pressure and temperature, mass-air-flow, dual cam positions, etc.

Note that the optical engine \#2 was modified from the optical engine \#1 fabricated early for this project, where the original target engine head was used. To make the engine capable of the HCCI combustion, the following changes were made.

Firstly, the engine head was modified to fit with the Delphi's two-step valve system and Denso's electrical VVT actuators. We worked closely with both Delphi and Chrysler, redesigned and fabricated the engine head. Delphi also helped validating the operation of the two-step valve system. We also worked closely with Denso and Chrysler and extended the electrical VVT operational range to \pm 40 degrees.

Secondly, an electrical throttle was fabricated that uses a motor cycle throttle and a servo actuator with very fast response time (time constant less than $50 \mathrm{~ms}$ ). This actuator meets the requirement of the SI and HCCI mode transition operation.

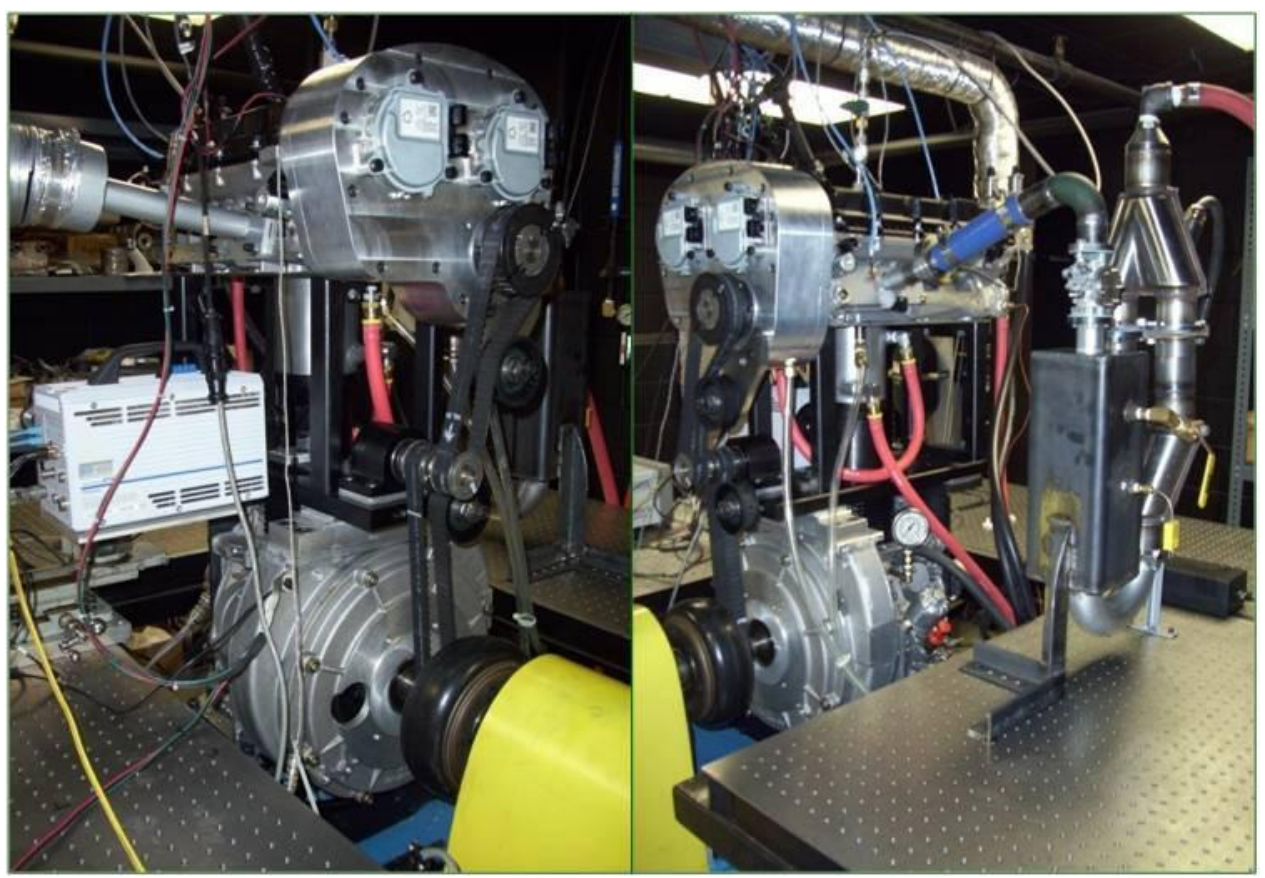

Figure 39: Final assembled optical engine capable of SI and HCCI combustion (optical engine \#2) 
Finally, due to the relatively low compression ratio of the optical engine, both engine head and cylinder were fabricated in such a way to use the engine coolant to heat them up to 200 degree F. Also, a six KW intake heater was also used to heat the intake charge air to make the HCCI combustion possible.

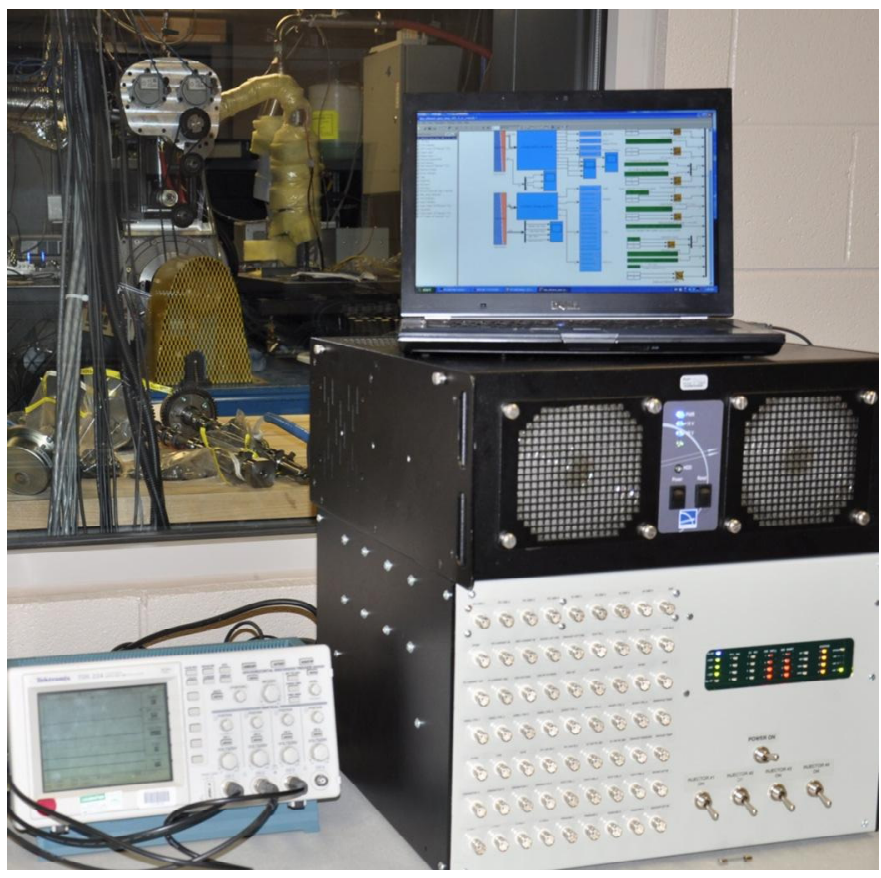

Figure 40: Optical and metal engine prototype control system

The engine prototype controller, see Figure 40, was finalized in this quarter, where the laptop on the top is the host computer running the Simulink in real-time so that the engine control parameters can be changed or modified in real-time; the middle is the Opal-RT PC based realtime controller that is capable of generating " $\mathrm{C}$ " code from Simulink and compiling it into the PC microprocessor; and the bottom one is the customer engine controller I/O box (designed and fabricated at MSU) used to amplify the control signals for actuators (DI injectors, two-step valve control solenoids, electrical VVT motors, throttle, etc.) and to condition the sensor signals (throttle position, cam phases, in-cylinder pressure, etc.)

\subsection{Summary}

The Chrysler turbocharged 2.0L I4 direct injection engine was selected as the base engine for the project and the engine was modified to fit the two-step valve with electrical variable valve timing actuating system. To develop the model-based control strategy for stable HCCI combustion and smooth combustion mode transition between SI and HCCI combustion, a control-oriented realtime engine model was developed and implemented into the MSU HIL (hardware-in-the-loop) simulation environment. The developed model was used to study the actuating system requirement for the smooth and fast combustion mode transition and to develop the proposed mode transition control strategy. Finally, a single cylinder optical engine was designed and fabricated for studying the HCCI combustion characteristics. 


\section{Phase II: Exploratory (Subsystem) Research}

This phase includes research and development in support of enabling technology at the component and subcomponent levels. Existing technologies and research results were utilized, where possible. All subsystem components such as ignition and ionization subsystem, PFI and DI dual fuel subsystem, two-step variable valve timing subsystem were finalized in this phase.

\subsection{Optical engine tests}

This section discusses the injector bench tests, two-step valve and electrical variable valve timing actuating system test, the optical engine test for optical engines \#1 and \#2, and flow and mixing test.

\subsubsection{Injector flow and spray tests}

In order to study the HCCI combustion characteristics, the fuel injector drop size property was tested using a Malvern Spraytec system. This subsection summarizes the fuel spray bench tests of the fuel injectors under different fuel blends and fuel rail pressures. The line-of-sight spatiallyintegrated drop size characterizations were conducted using a laser diffraction instrument called Spraytec/Malvern. In order to have a comparison evaluation, a Bosch production DI injector was tested and compared with the target injector to be used in our HCCI engine noted as the "Chrysler injector."

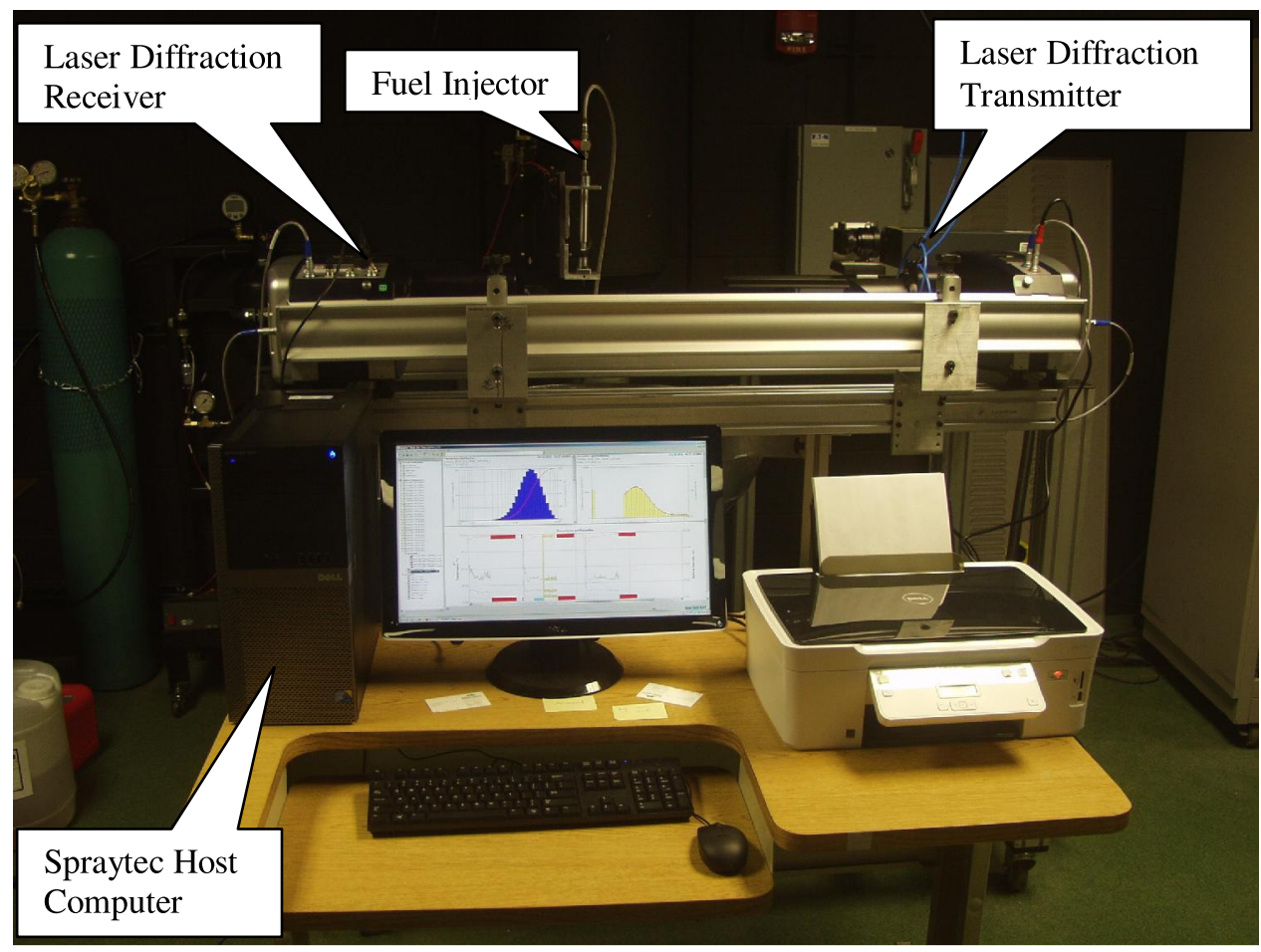

Figure 41: Spraytec/Malvern laser diffraction drop sizing measurement setup

Figure 41 shows the overall test setup for the laser diffraction drop sizing measurement system. The instrument consists of a $5 \mathrm{~mW}$ and $623.8 \mathrm{~nm}$ wavelength laser light transmitter with a 10 $\mathrm{mm}$ diameter beam and a receiver of 36 detectors. The receiver was fitted with a lens of $300 \mathrm{~mm}$ focal length, so that drop sizes between 0.1 and 900 microns can be measured. The injector was mounted on a test rig and the laser beam was positioned in the line of measurement centered at 
$50 \mathrm{~mm}$ below the injector tip. To avoid vignetting and fuel sprayed onto the receiver, the injector was placed at a distance of $120 \mathrm{~mm}$ from the receiver's Fourier lens. The injection frequency was set at $12.5 \mathrm{~Hz}$.

All injector drop size measurements were performed for sprays under an atmospheric condition. The Spraytec is capable of sampling at a maximum rate of $10 \mathrm{kHz}$. The trigger was setup to enquire an event every 4 times, leading to a sample rate of $2.5 \mathrm{kHz}$. The results were recorded and temporally averaged. When the laser passed through the spray structure, the diffracted light pattern was received by the Fourier lens mounted behind of the spray on the receiver unit. The light intensities from the detectors were processed by the Spraytec software to derive the spray drop size distribution and characteristic diameters. The injector spray drop size characteristics obtained in drop sizing measurement tests are:

1. Drop size distribution

2. Statistical Drop Diameters: DV10, DV50, DV90, Sauter Mean Diameter and D43

Table 3 lists the values of the representative diameters in the spray drop size measurements. Among these key diameters are the Sauter Mean Diameter (SMD, also known as D32) and DV90, in which they are considered as the mean drop diameter and maximum drop diameter of the spray, respectively.

Table 3: Drop size comparison data

\begin{tabular}{|c|c|c|c|c|c|c|c|c|}
\hline Injector & Fuel & $\begin{array}{c}\text { Pressure } \\
(\mathrm{MPa})\end{array}$ & $\begin{array}{c}\text { PW } \\
\text { (ms) }\end{array}$ & $\begin{array}{l}\text { Dv10 } \\
(\mu \mathrm{m}) \\
\end{array}$ & $\begin{array}{l}\text { Dv50 } \\
(\mu \mathrm{m}) \\
\end{array}$ & $\begin{array}{l}\text { Dv90 } \\
(\mu \mathrm{m}) \\
\end{array}$ & $\begin{array}{l}\text { SMD } \\
(\mu \mathrm{m}) \\
\end{array}$ & $\begin{array}{l}\text { D43 } \\
(\mu \mathrm{m}) \\
\end{array}$ \\
\hline \multirow{8}{*}{$\begin{array}{l}\text { Bosch Production } \\
\text { Injector }\end{array}$} & \multirow{4}{*}{ Gasoline } & \multirow{2}{*}{5} & 2.5 & 12.64 & 27.0 & 53.14 & 20.41 & 30.32 \\
\hline & & & 5 & 12.83 & 29.12 & 58.39 & 21.19 & 32.79 \\
\hline & & \multirow{2}{*}{10} & 2.5 & 9.48 & 19.48 & 38.67 & 15.28 & 22.14 \\
\hline & & & 5 & 9.40 & 18.68 & 35.63 & 14.66 & 20.88 \\
\hline & \multirow{4}{*}{ E85 } & \multirow{2}{*}{5} & 2.5 & 13.91 & 31.74 & 65.61 & 22.97 & 36.43 \\
\hline & & & 5 & 14.38 & 31.53 & 63.14 & 23.46 & 35.68 \\
\hline & & \multirow{2}{*}{10} & 2.5 & 8.63 & 16.6 & 31.72 & 14.68 & 18.71 \\
\hline & & & 5 & 9.44 & 17.63 & 32.43 & 15.73 & 19.56 \\
\hline \multirow{8}{*}{ Chrysler Injector } & \multirow{4}{*}{ Gasoline } & \multirow{2}{*}{5} & 2.5 & 12.09 & 24.43 & 46.7 & 18.87 & 27.25 \\
\hline & & & 5 & 12.46 & 24.25 & 45.97 & 21.27 & 27.13 \\
\hline & & \multirow{2}{*}{10} & 2.5 & 8.32 & 16.57 & 33.19 & 14.49 & 19.05 \\
\hline & & & 5 & 8.58 & 16.51 & 31.52 & 14.6 & 18.6 \\
\hline & \multirow{4}{*}{ E85 } & \multirow{2}{*}{5} & 2.5 & 12.32 & 24.15 & 44.62 & 18.17 & 26.52 \\
\hline & & & 5 & 12.46 & 24.42 & 44.66 & 19.95 & 26.71 \\
\hline & & \multirow{2}{*}{10} & 2.5 & 8.31 & 16.29 & 31.82 & 14.31 & 18.53 \\
\hline & & & 5 & 8.11 & 15.5 & 29.04 & 13.71 & 17.3 \\
\hline
\end{tabular}

The level of atomization is dependent upon many factors such as fuel fluid properties, injector nozzle design, and most importantly, the injection pressure. In this report, it can be concluded from the drop size data:

1) It is very clear that the drop size decreases when the fuel injection pressure increased. Higher injection pressure provides a larger inertia force to overcome the viscous effect of the fuel, leading to better atomization. Figure 42 and Figure 43 show the effect of fuel injection pressure to the fuel spray drop size. 
2) The Chrysler target injector demonstrates good robustness of the fuel drop size to the fuel blend variations, see Figure 44 and Figure 45 . Figure 46 and Figure 47 show the fuel blend effect on the fuel drop size of the Bosch production injector. However, for the Chrysler target injector, as shown in Figure 44 and Figure 45, the fuel drop size of E85 is very close to that of gasoline.

3) When the fuel pressure is at $5 \mathrm{MPa}$, the fuel drop size of the Chrysler target injector is smaller than that of the Bosch injector as illustrated by Figure 46. Even more, the E85 drop size of the Chrysler target injector is smaller than the gasoline drop size of Bosh production injector at this pressure. Only when the fuel pressure increases to $10 \mathrm{MPa}$, the gasoline drop size of the Bosch injector is close to that of the Chrysler target injector as shown in Figure 47.

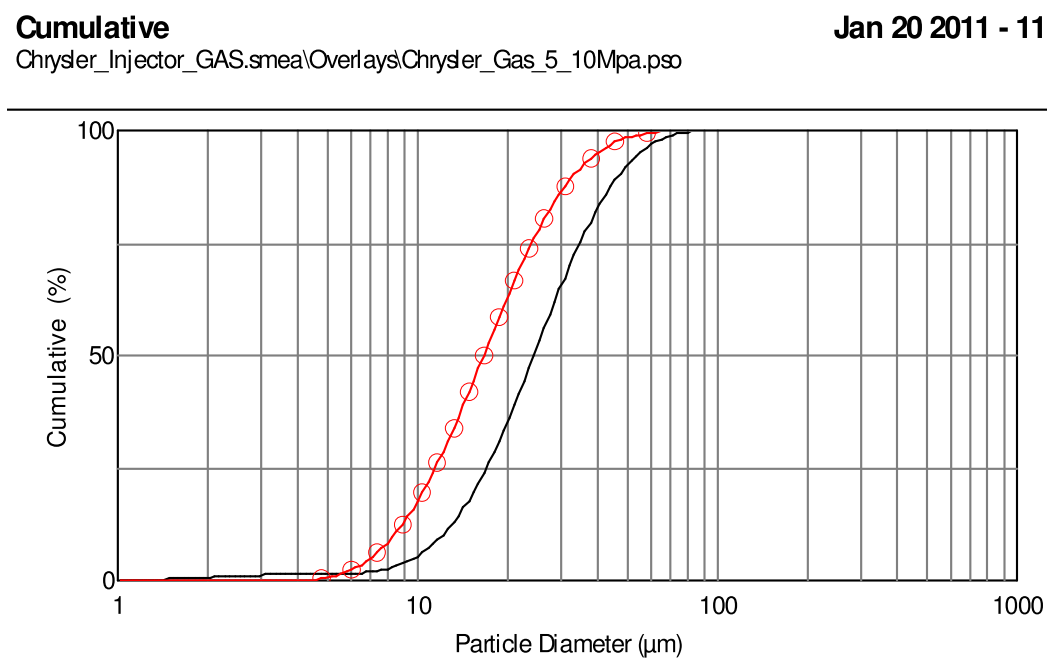

\begin{tabular}{|l|l|l|l|l|l|l|}
\hline & Date-Time & File & Sample & Dx(10) & Dx(50) & Dx(90) \\
\hline \hline VV & Oct 20 2010... & Crs Ga.... & Crs Gas 5Mpa 2p5Pulse 4 & 12.09 & 24.43 & 46.70 \\
\hline$-[V]$ & Oct 20 2010... & Crs Ga.... Crs Gas 10Mpa 2p5Pulse... & 8.32 & 16.57 & 33.19 \\
\hline
\end{tabular}

$[\mathrm{V}]=$ Volume $[\mathrm{N}]=$ Number

Figure 42: Cumulative drop size: Chrysler target injector (5 and $10 \mathrm{MPa}$, gasoline) 


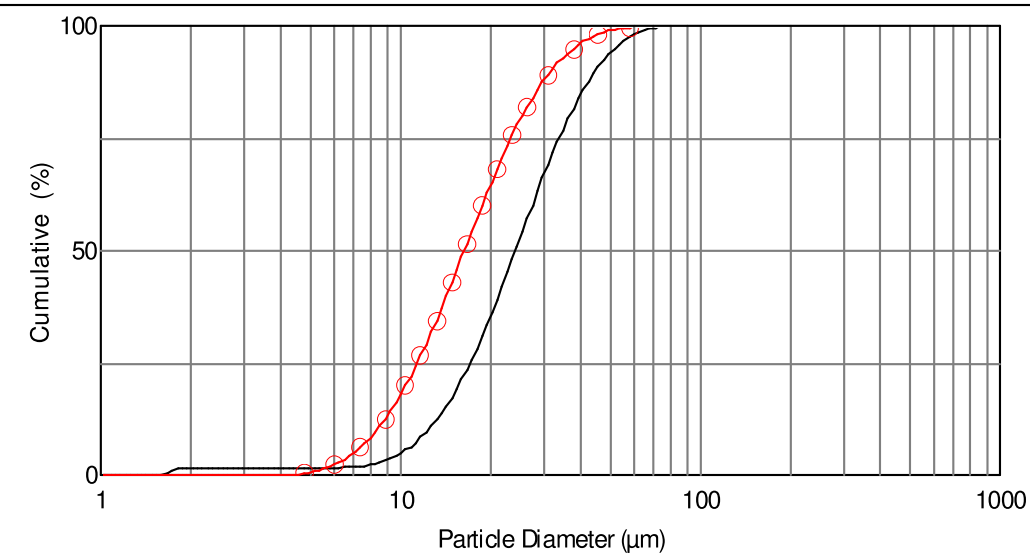

\begin{tabular}{|l|l|l|l|l|l|l|}
\hline & Date-Time & File & Sample & Dx(10) & Dx(50) & Dx(90) \\
\hline$-[V]$ & Oct 22 2010... & Crs E8... & Crs E85 5Mpa 2p5 2 & 12.32 & 24.15 & 44.62 \\
\hline \hline$[$ V] & Oct 22 2010... & Crs E8.... & Crs E85 10Mpa 2p5 2 & 8.31 & 16.29 & 31.82 \\
\hline
\end{tabular}

$[\mathrm{V}]=$ Volume $[\mathrm{N}]=$ Number

Figure 43: Cumulative drop size: Chrysler target injector (5 and $10 \mathrm{MPa}, \mathrm{E} 85$ )

\section{Cumulative}

Jan 202011 - 11:28:57

Chrysler_Injector_GAS.smealOverlaysiChryster_Gas_E85_5Mpa.pso

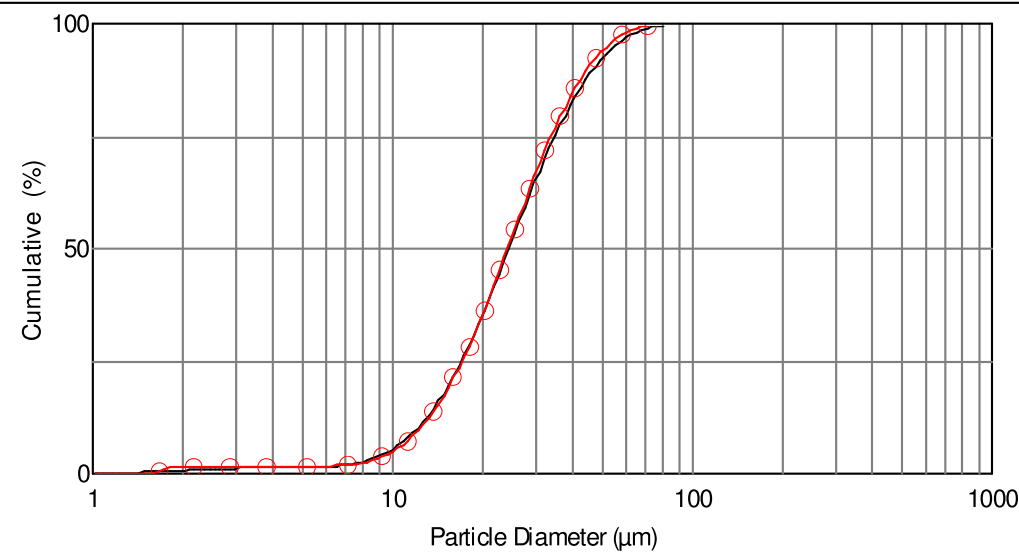

\begin{tabular}{|l|l|l|l|l|l|l|}
\hline & Date-Time & File & Sample & Dx(10) & Dx(50) & Dx(90) \\
\hline \hline$-[V]$ & Oct 20 2010... & Crs Ga... & Crs Gas 5Mpa 2p5Pulse 4 & 12.09 & 24.43 & 46.70 \\
\hline \hline$-[V]$ & Oct 22 2010... & Crs E8... Crs E85 5Mpa 2p5 2 & 12.32 & 24.15 & 44.62 \\
\hline \hline
\end{tabular}

$[\mathrm{V}]=$ Volume $[\mathrm{N}]=$ Number

Figure 44: Cumulative drop size: Chrysler target injector (5 MPa, gasoline/E85) 


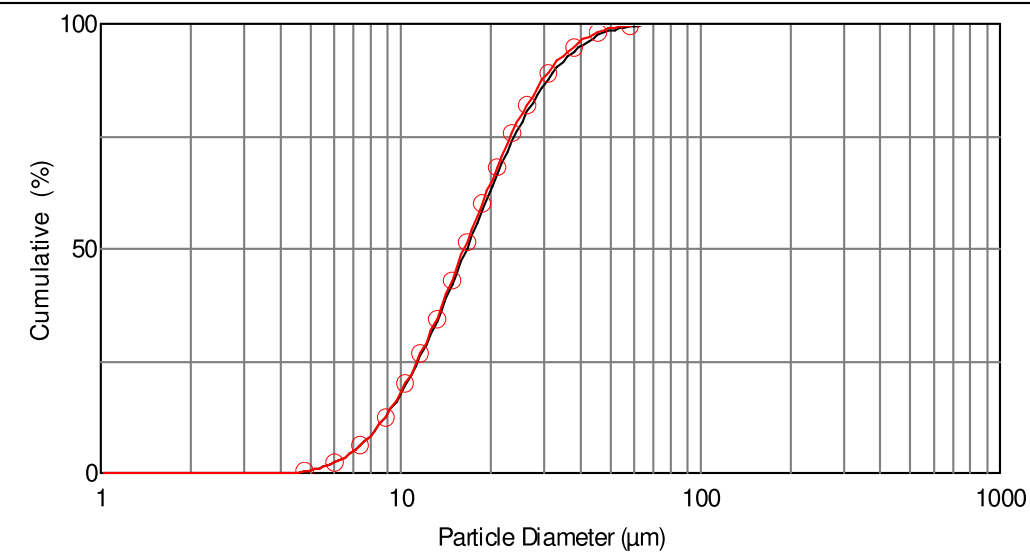

\begin{tabular}{|l|l|l|l|l|l|l|}
\hline & Date-Time & File & Sample & Dx(10) & Dx(50) & Dx(90) \\
\hline [V] & Oct 20 2010... & Crs Ga... & Crs Gas 10Mpa 2p5Pulse... & 8.32 & 16.57 & 33.19 \\
\hline \hline$[V]$ & Oct 22 2010... & Crs E8.... & Crs E85 10Mpa 2p5_2 & 8.31 & 16.29 & 31.82 \\
\hline
\end{tabular}

$[\mathrm{V}]=$ Volume $[\mathrm{N}]=$ Number

Figure 45: Cumulative drop size: Chrysler target injector (10 MPa, gasoline/E85)

\section{Cumulative}

Jan 202011 - 11:28:57

Chrysler_Injector_GAS.smealOverlaysiChryser_Bosch_5Mpa.pso

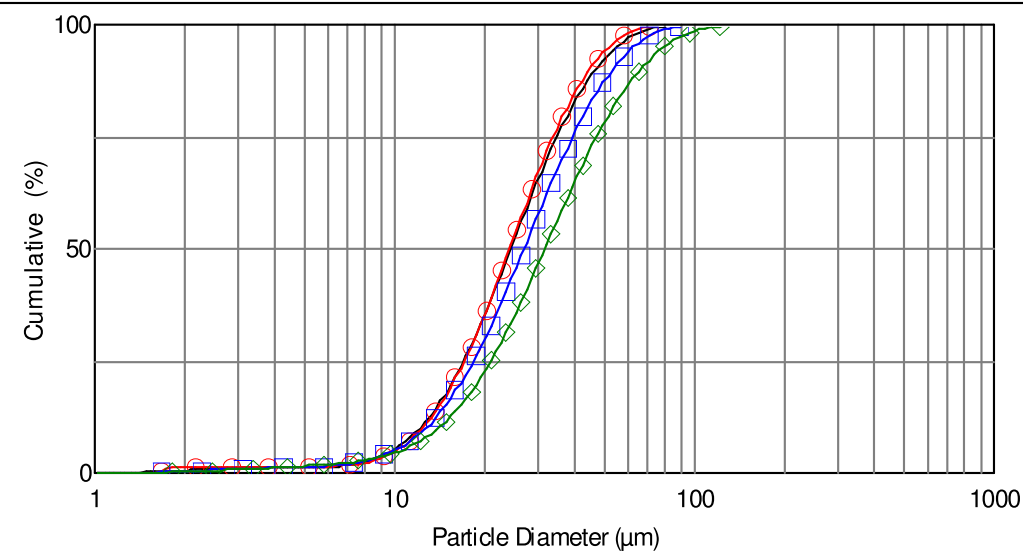

\begin{tabular}{|r|l|l|l|l|l|l|}
\hline & Date-Time & File & Sample & Dx(10) & Dx(50) & Dx(90) \\
\hline$-[V]$ & Oct 20 2010... & Crs Ga... & Crs Gas 5Mpa 2p5Pulse 4 & 12.09 & 24.43 & 46.70 \\
\hline \hline$[$ V] & Oct 22 2010... & Crs E8... & Crs E85 5Mpa 2p5 2 & 12.32 & 24.15 & 44.62 \\
\hline \hline$[$ V] & Oct 21 2010... & Bosh_... & Bosh Prdu Gas 5Mpa 2p... & 12.64 & 27.00 & 53.14 \\
\hline$-[V]$ & Oct 21 2010... & Bosh_... & Bosh Prdu E85 5MPa 2p... 13.91 & 31.74 & 65.61 \\
\hline
\end{tabular}

$[\mathrm{V}]=$ Volume $[\mathrm{N}]=$ Number

Figure 46: Chrysler target vs. Bosch production injectors (5 MPa, gasoline/E85) 
Cumulative

Jan 202011 - 11:31:08

Chryster_Injector_GAS.smealOverlaysiChryser_Bosch_10Mpa.pso

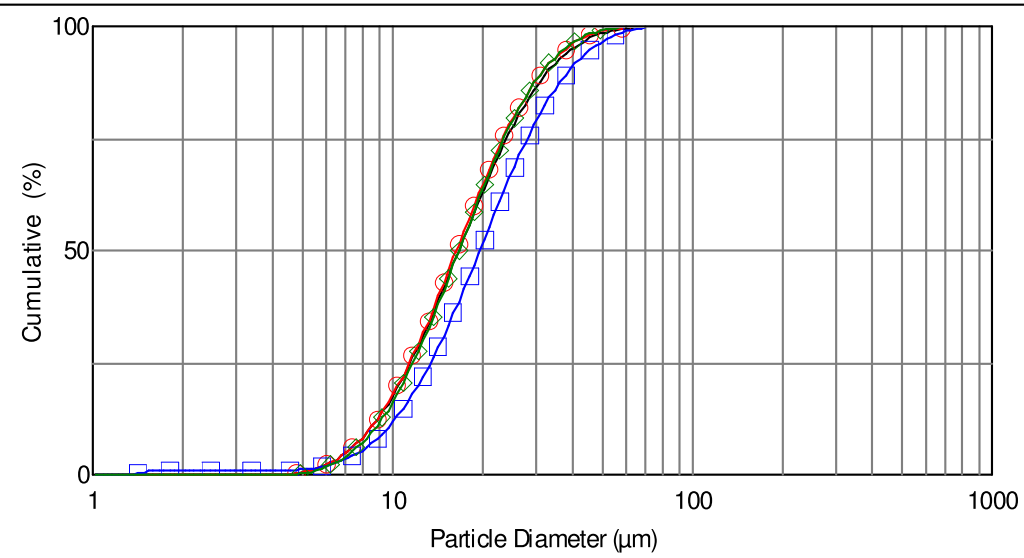

\begin{tabular}{|c|c|c|c|c|c|c|}
\hline & Date-Time & File & Sample & Dx(10) & Dx(50) & $\mathrm{Dx}(90)$ \\
\hline$-[\mathrm{V}]$ & Oct 202010 .. & Crs Ga. & Crs Gas 10Mpa 2p5Pulse. & 8.32 & 16.57 & 33.19 \\
\hline - $[\mathrm{V}]$ & Oct 222010. & Crs E8.. & Crs E85 10Mpa 2p5 2 & 8.31 & 16.29 & 31.82 \\
\hline$-\mathrm{Q}-[\mathrm{V}]$ & Oct 212010. & Bosh ... & Bosh Prdu Gas 10Mpa 2. & 9.48 & 19.48 & 38.67 \\
\hline$\leadsto[\mathrm{V}]$ & Oct 212010. & Bosh & Bosh Prdu E85 10Mpa 2. & 8.63 & 16.60 & 31.72 \\
\hline
\end{tabular}

$[\mathrm{V}]=$ Volume $[\mathrm{N}]=$ Number

Figure 47: Cumulative drop size: Chrysler vs. Bosch injectors (10 MPa, gasoline/E85) 


\subsubsection{Electrical cam phase actuator and lift control test on optical engine \#2}

The electrical cam phasing (or variable valve timing) actuating system was validated for both intake and exhaust cams. We also studied the cam phasing behavior under both heated and room temperature to study the temperature influence to the electrical cam phase response velocity. Finally, both combustion and motoring cases were also tested. The test matrix is summarized in Table 4.

Table 4: Electrical cam phasing actuator test matrix

\begin{tabular}{|l|l|l|l|l|}
\hline & CAD & Cycles & Combustion/motoring & Heated/cold \\
\hline Advance & 70 & $10-11$ cycles & motoring & cold \\
\hline Advance & 70 & $8-9$ cycles & motoring & heated \\
\hline Advance & 70 & $8-9$ cycles & combustion & heated \\
\hline Advance & 35 & $6-7$ cycles & motoring & cold \\
\hline Advance & 35 & $5-6$ cycles & motoring & heated \\
\hline Advance & 35 & $5-6$ cycles & combustion & heated \\
\hline Retard & 70 & $6-7$ cycles & motoring & cold \\
\hline Retard & 70 & $5-6$ cycles & motoring & heated \\
\hline Retard & 35 & $4-5$ cycles & motoring & cold \\
\hline Retard & 35 & $3-4$ cycles & motoring & heated \\
\hline
\end{tabular}

a) $70 \mathrm{CAD}$ exhaust advance and retard with heated head and no combustion (motoring)

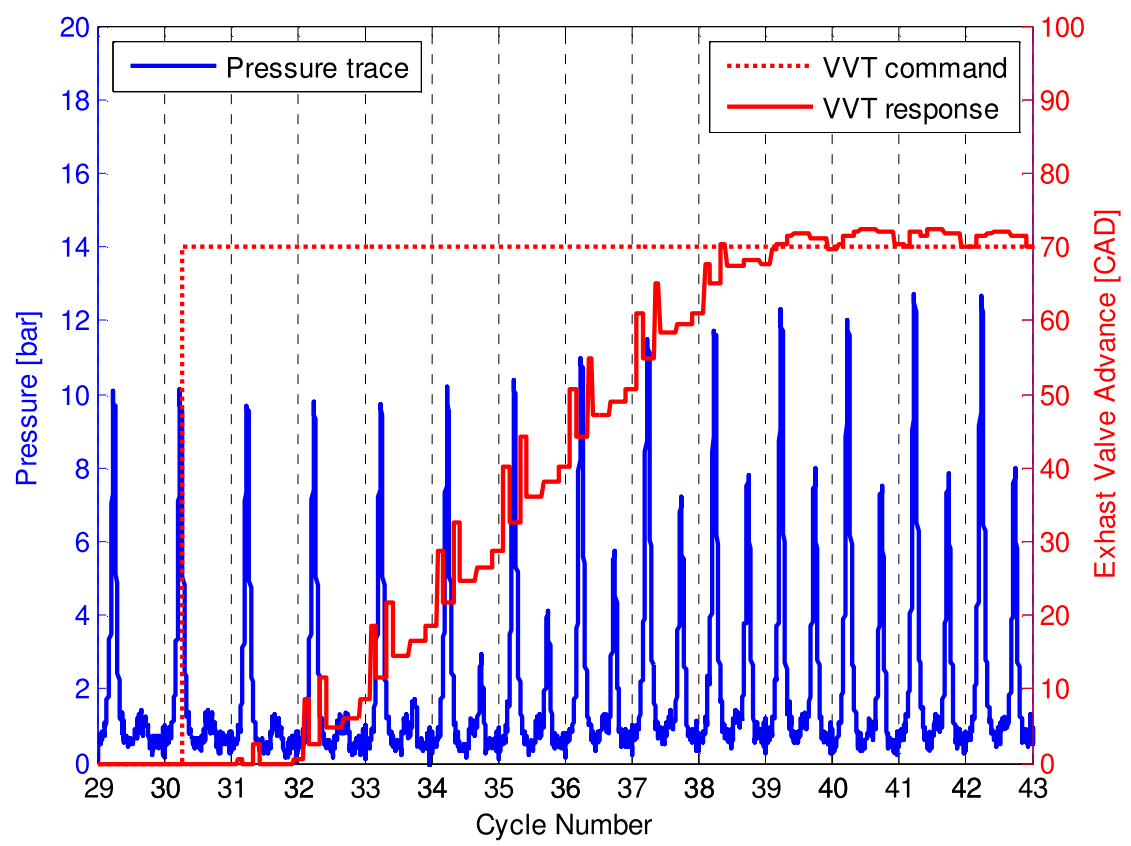

Figure 48: 70 CAD exhaust advance with heated head and no combustion (motoring) 


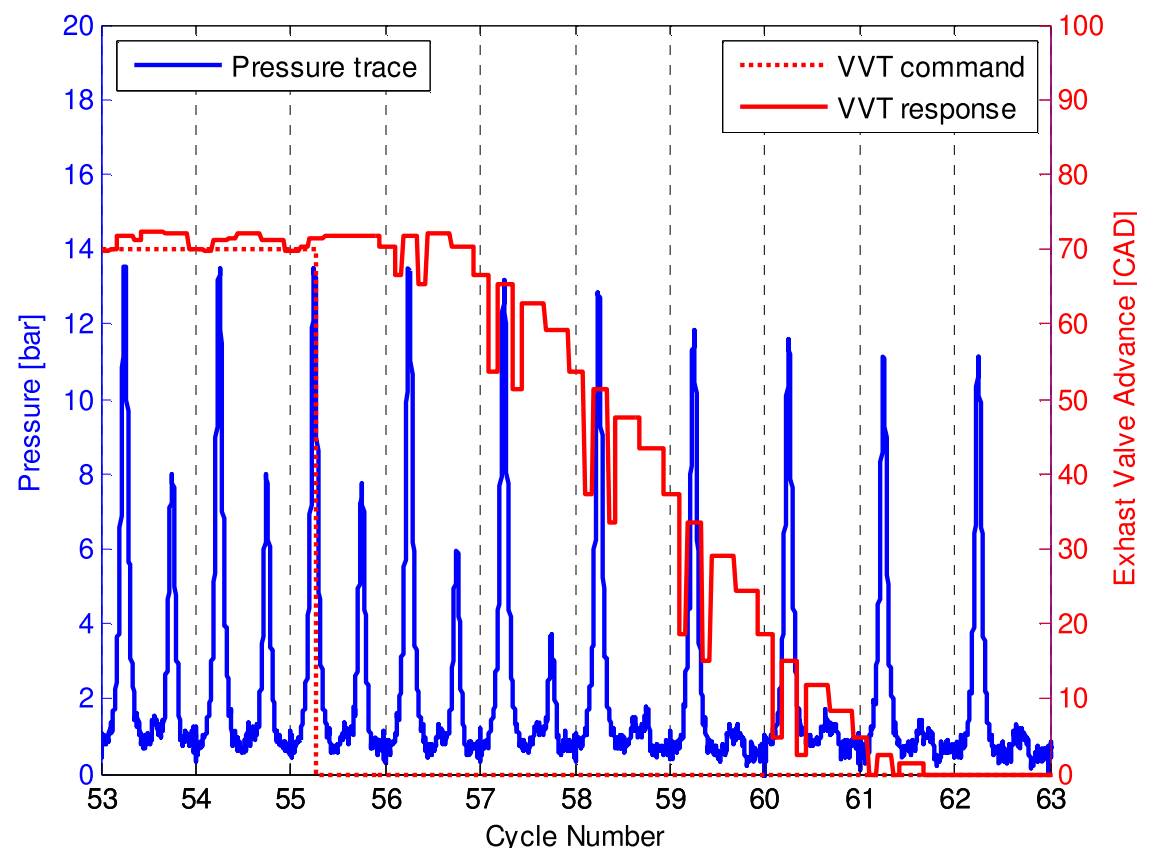

Figure 49: $70 \mathrm{CAD}$ exhaust retard with heated head and no combustion (motoring)

b) $70 \mathrm{CAD}$ exhaust advance and retard with cold head and no combustion (motoring)

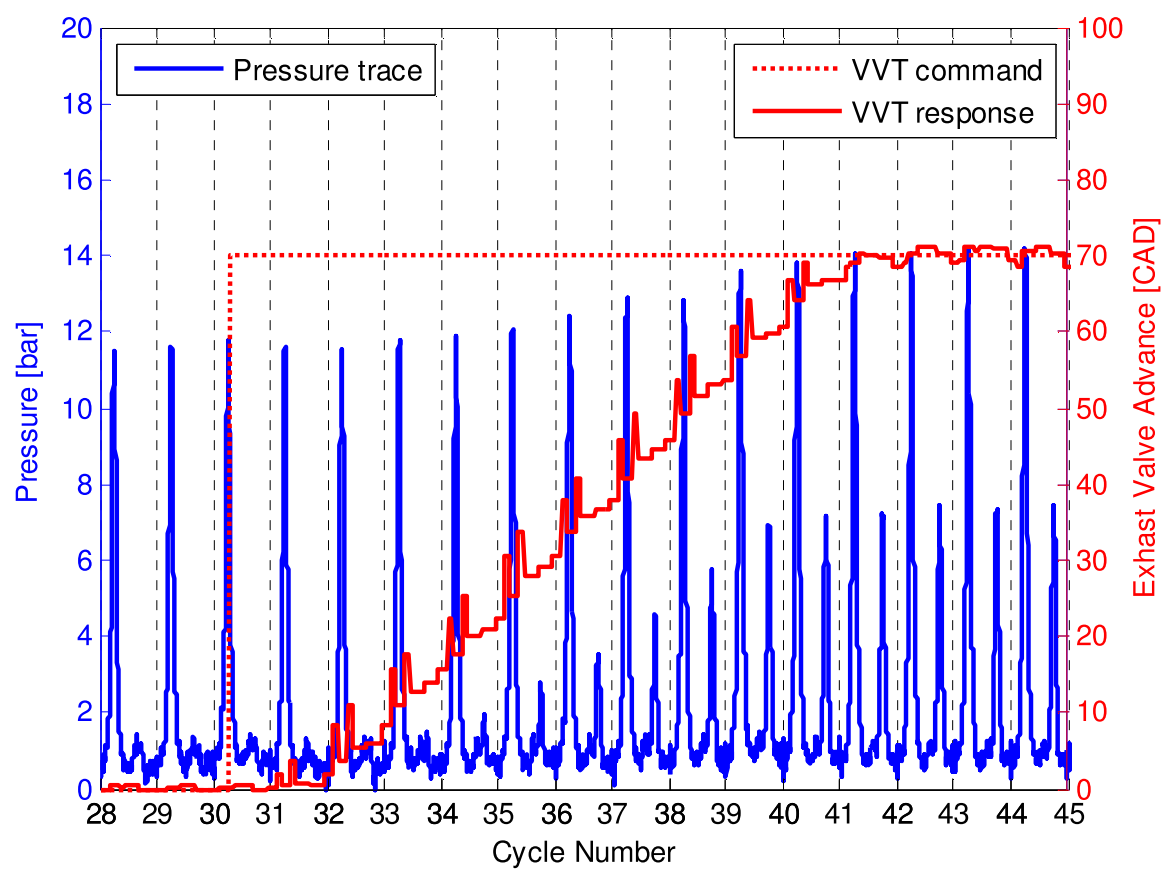

Figure 50: $70 \mathrm{CAD}$ exhaust advance with cold head and no combustion (motoring) 


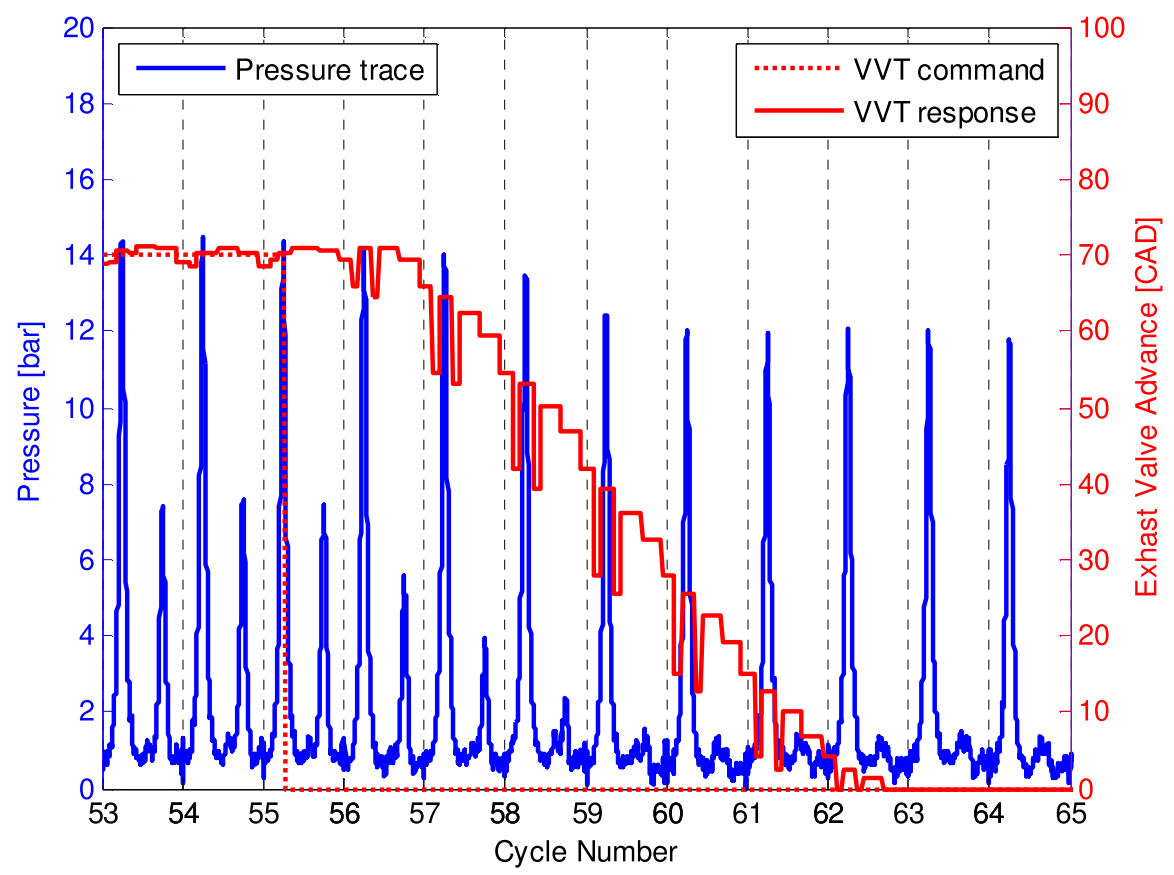

Figure 51: $70 \mathrm{CAD}$ exhaust retard with cold head and no combustion (motoring)

c) Combustion and motoring comparison test (70 CAD advance)

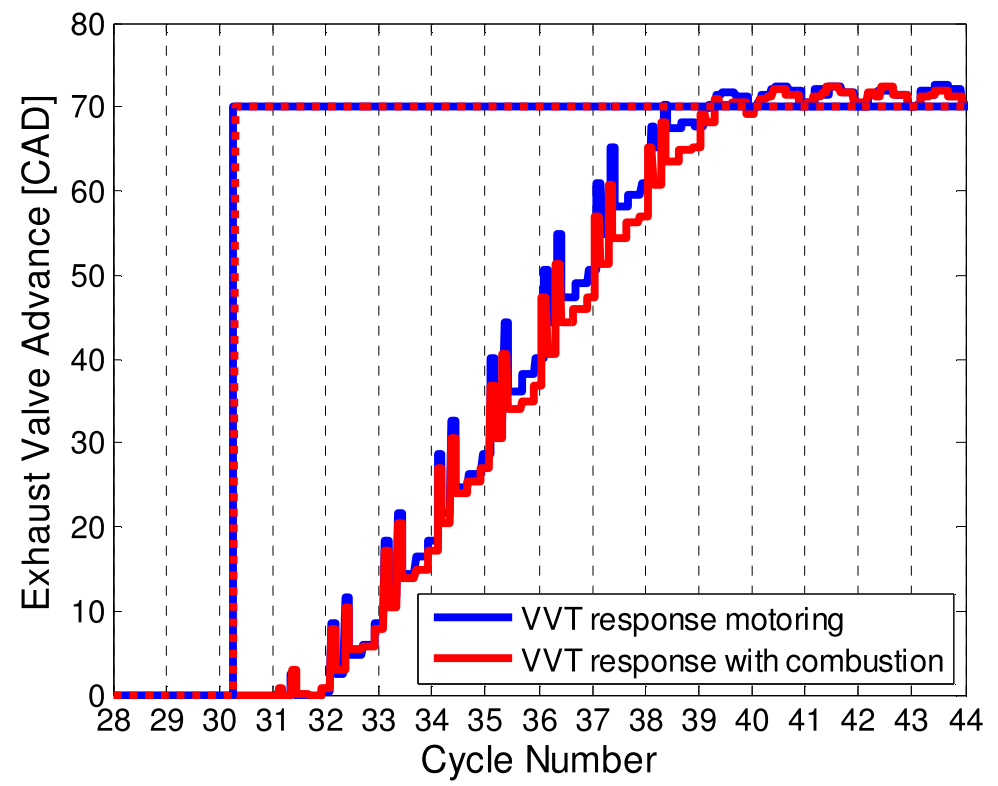

Figure 52: Combustion and motoring comparison (70 CAD advance) 
d) $35 \mathrm{CAD}$ exhaust advance and retard with heated head and no combustion (motoring)

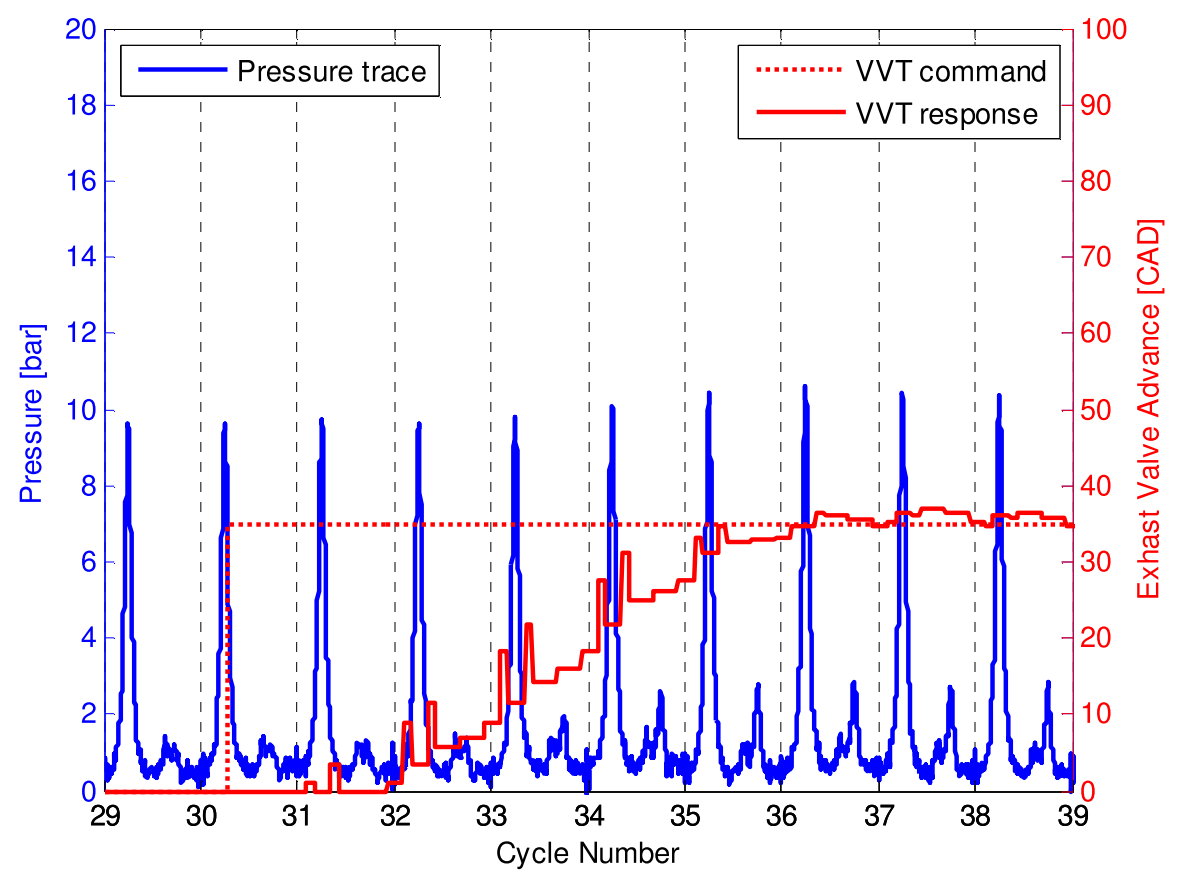

Figure 53: 35 CAD exhaust advance with heated head and combustion

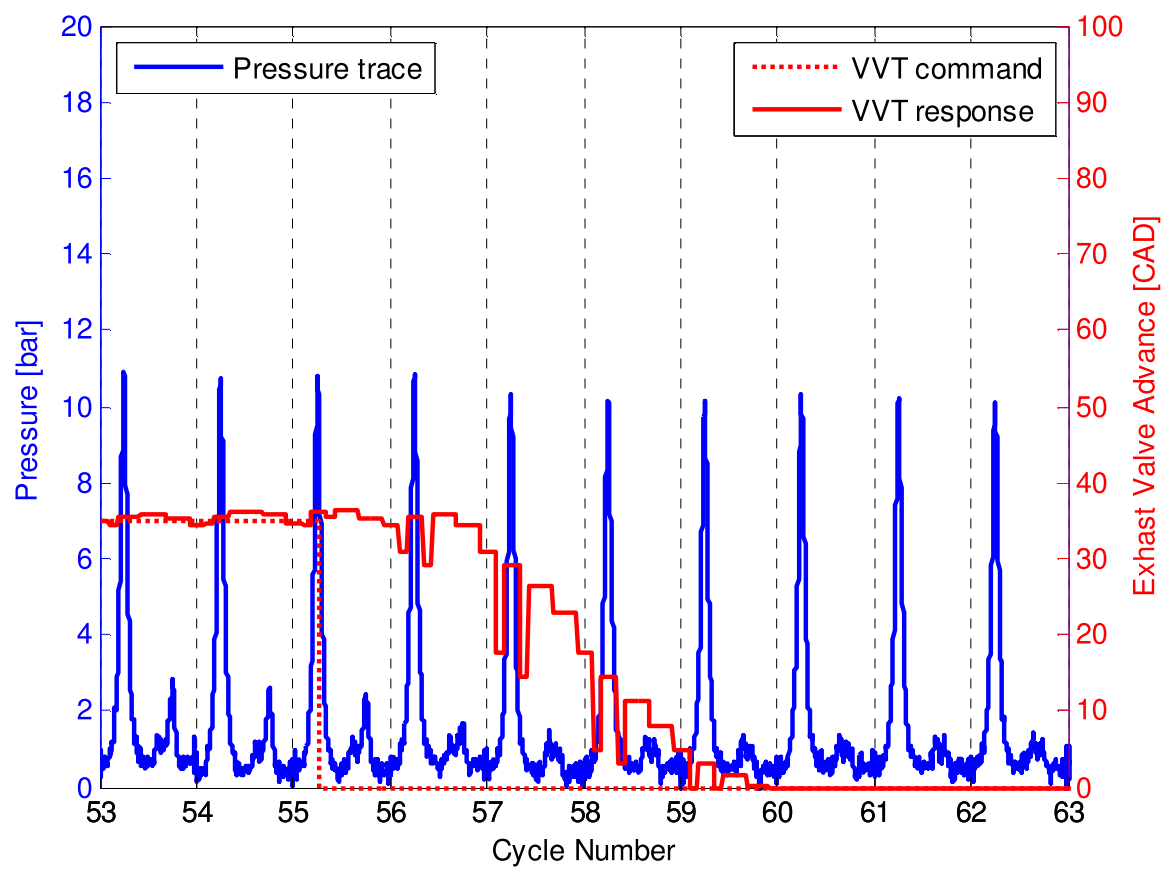

Figure 54: 35 CAD exhaust retard with heated head and combustion 


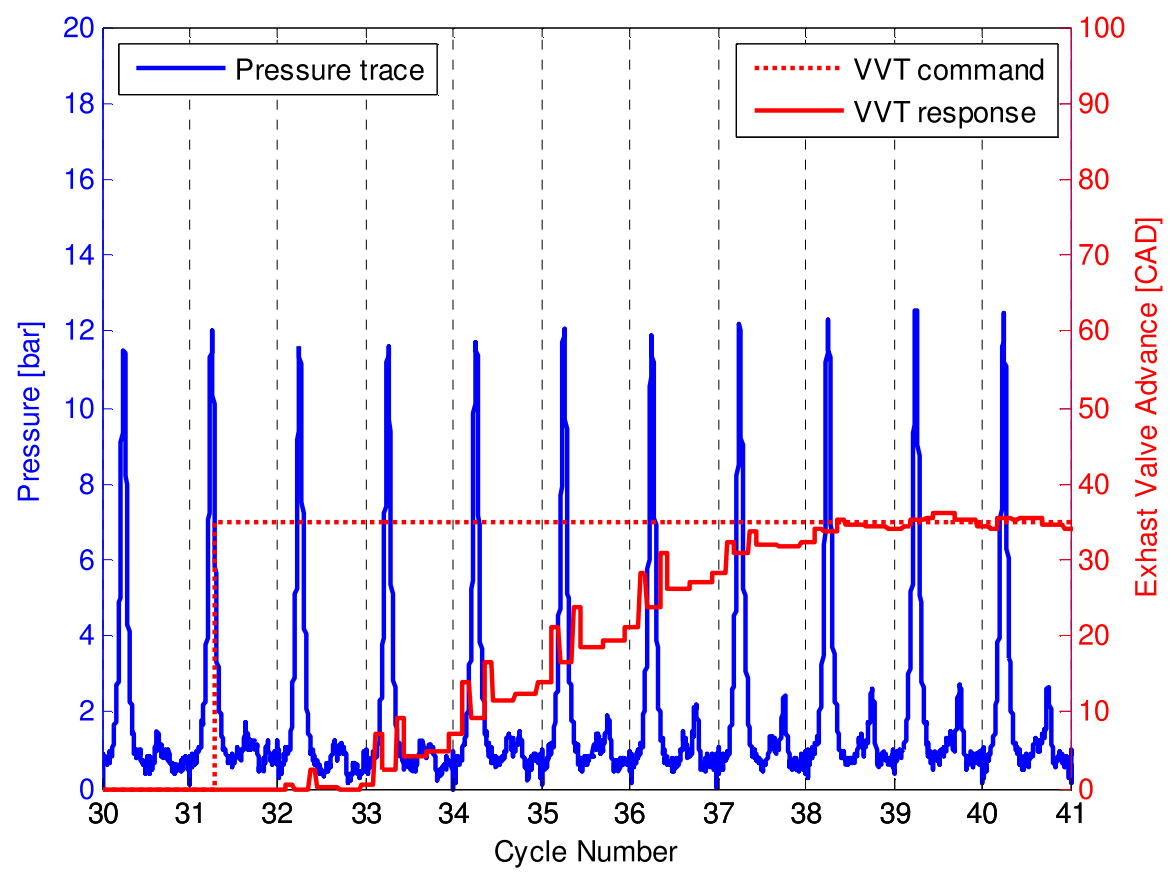

Figure 55: 35 CAD exhaust advance with cold head and no combustion (motoring)

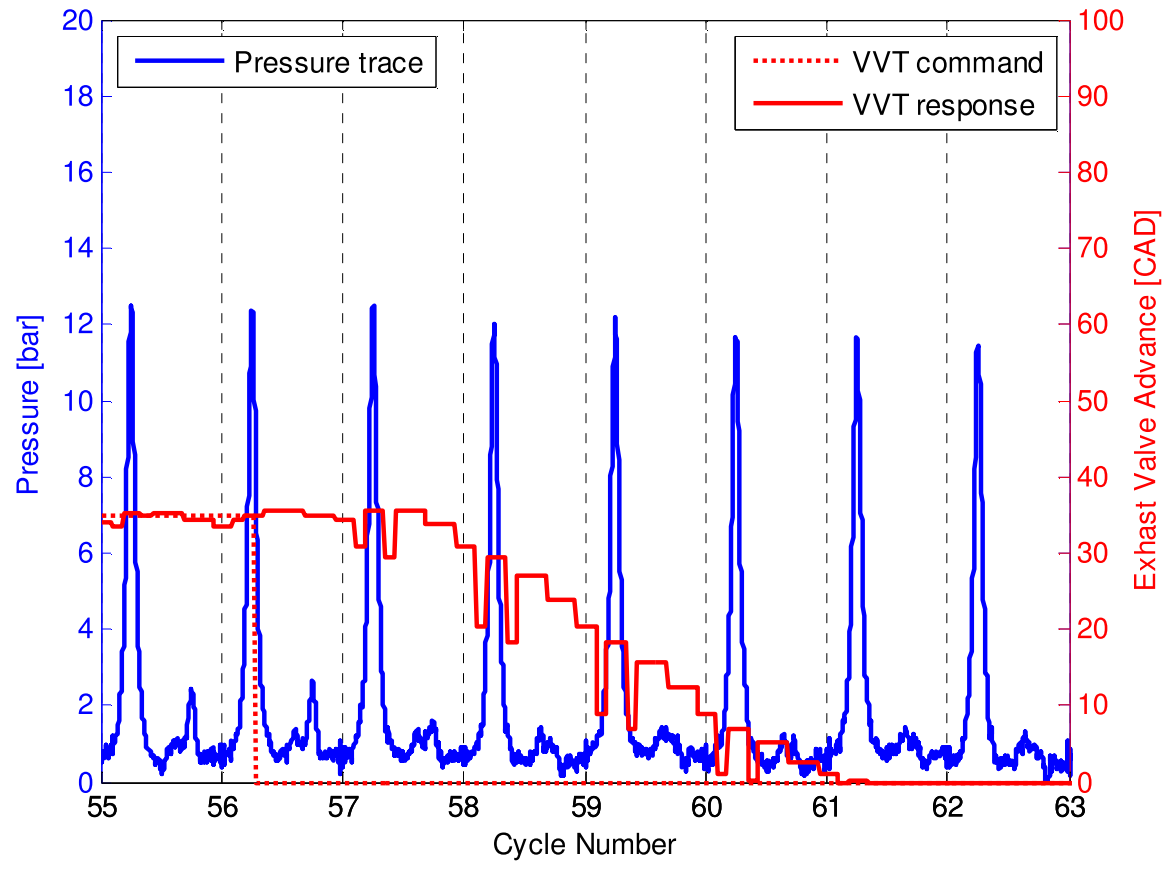

Figure 56: $35 \mathrm{CAD}$ exhaust retard with cold head and no combustion (motoring)

From the electrical cam phasing tests the following conclusions were made:

- The engine temperature does influence the electrical cam phaser response time. The response time with heated oil is one engine cycle shorter than that of the cold one.

- Combustion also slows down the electrical cam phase slightly. 
The two-step valve lift tests were also conducted and the results are shown in Figure 57 and Figure 58. Both intake and exhaust actuating systems meet the design specifications.

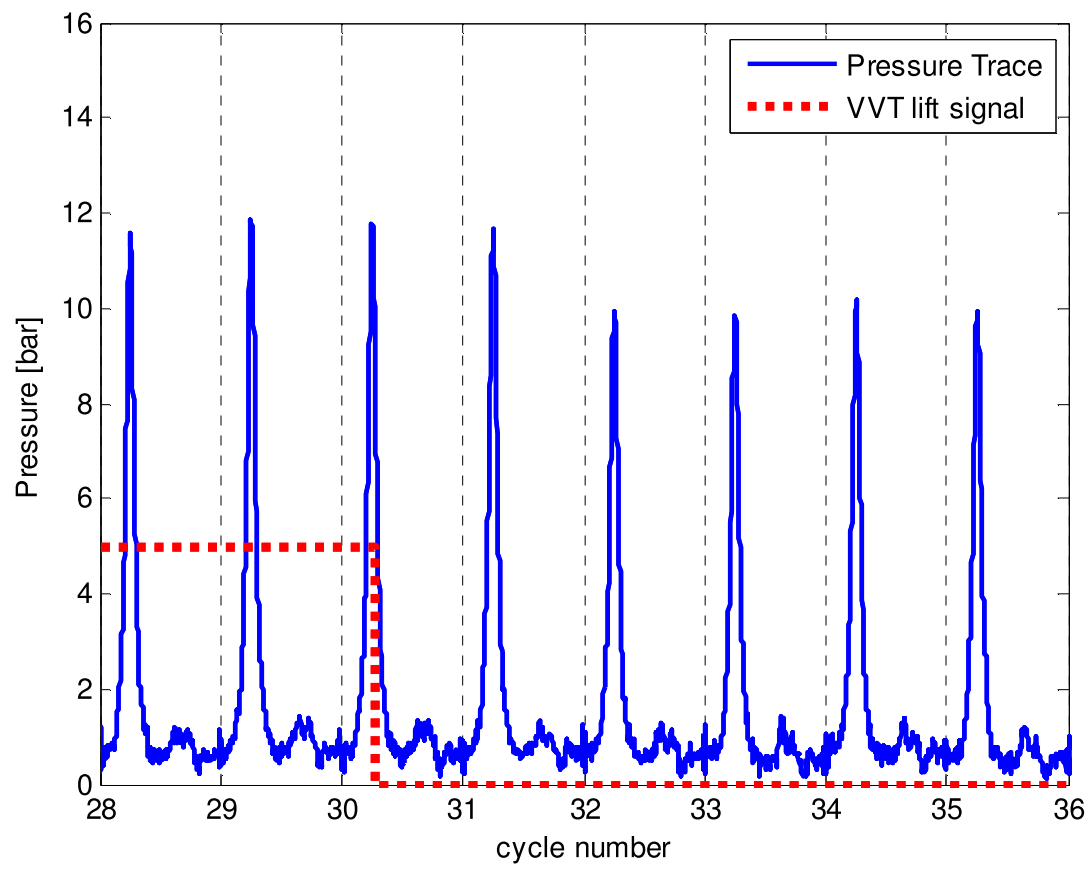

Figure 57: Exhaust valve from high to low in one engine cycle

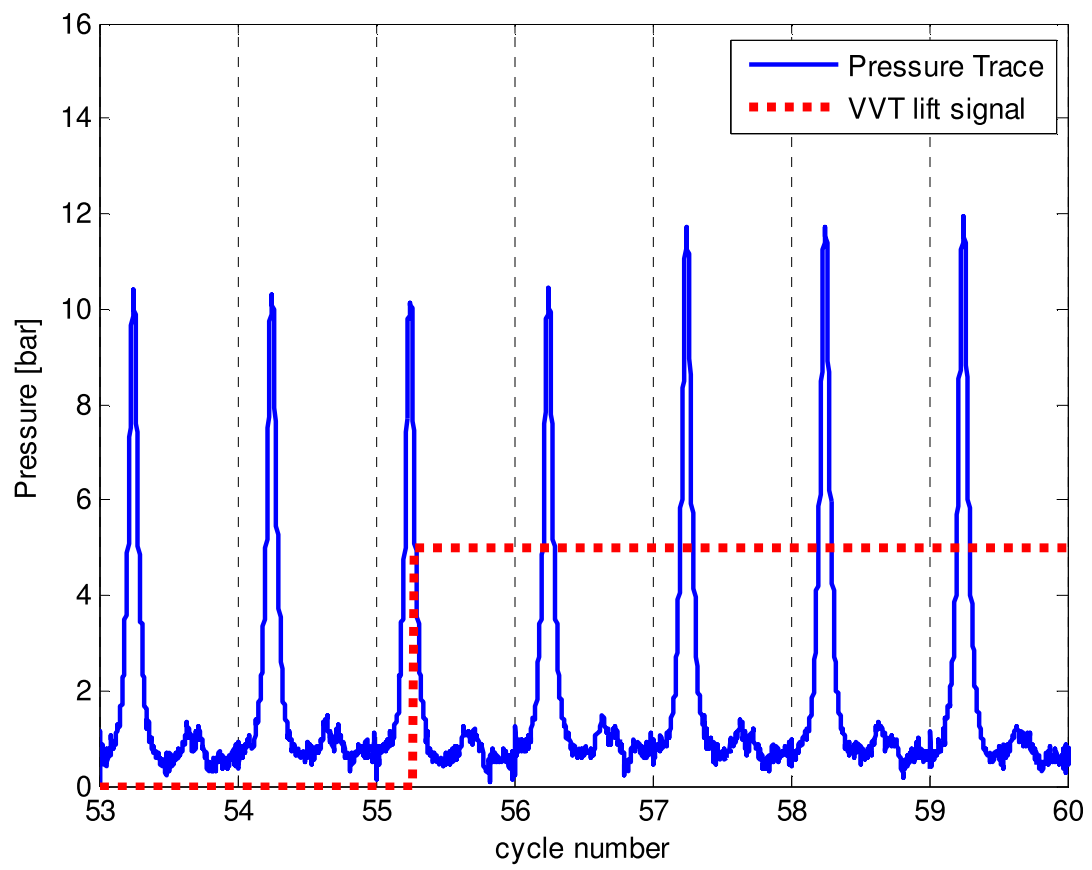

Figure 58: Exhaust valve from low to high in one engine cycle 


\subsubsection{Optical engine combustion tests}

After the SI optical engine (optical engine \#1) was assembled, the SI combustion tests were conducted at 1500 RPM with about 4 bar IMEP. The details about the test condition are shown in Table 5. Note that 4 bar IMEP is close to the SI and HCCI combustion mode operational condition and it will be interesting to compare the optical test results of HCCI combustion next.

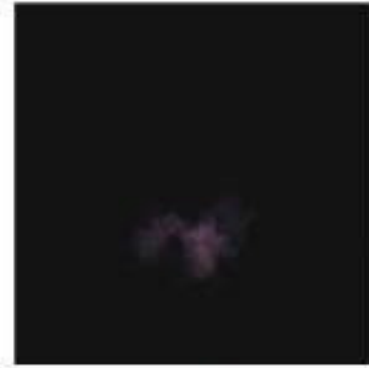

$-19.7^{\circ}$ ATDC

$\left(15.3^{\circ} \mathrm{AIT}\right)$

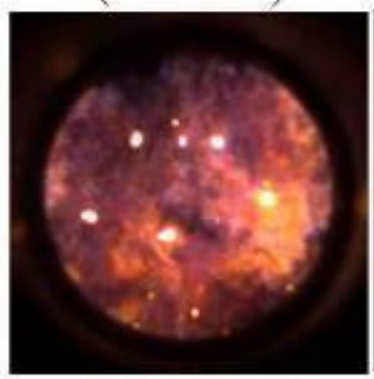

$-19.7^{\circ}$ ATDC

$\left(15.3^{\circ} \mathrm{AIT}\right)$

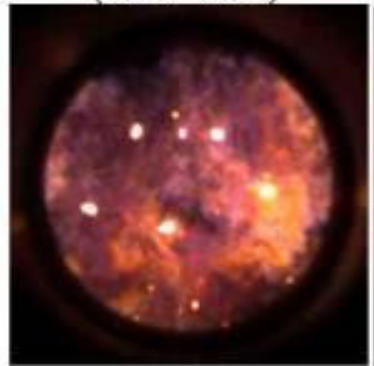

$0.1^{\circ} \mathrm{ATDC}$

$\left(35.1^{\circ} \mathrm{AIT}\right)$

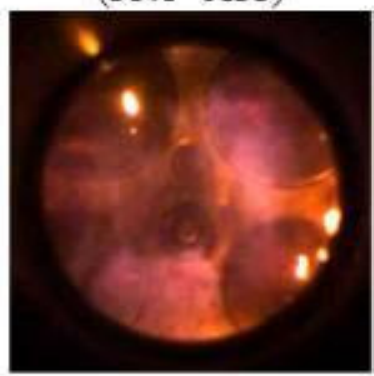

$19.9^{\circ} \mathrm{ATDC}$

$\left(54.9^{\circ} \mathrm{AIT}\right)$

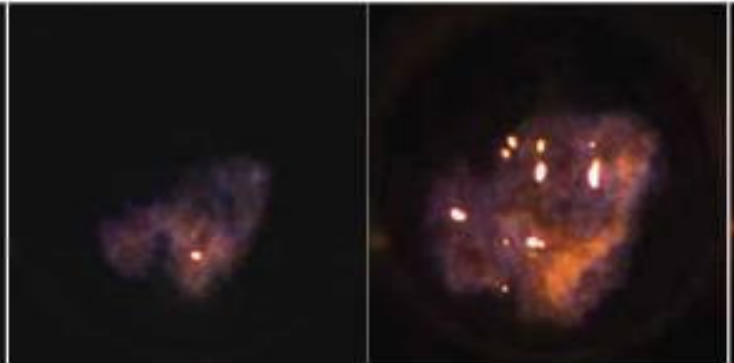

$-15.2^{\circ}$ ATDC

(19. $\left.8^{\circ} \mathrm{AIT}\right)$

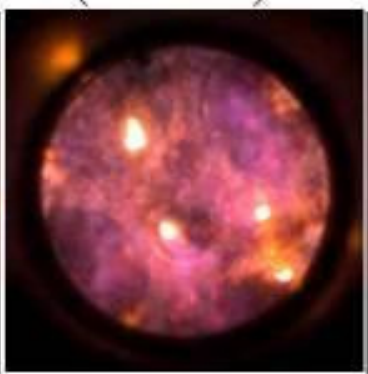

$-15.2^{\circ}$ ATDC

(19. $8^{\circ}$ AIT)

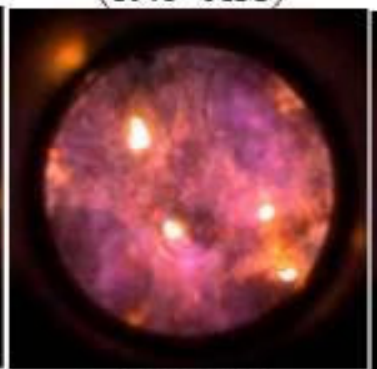

$4.6^{\circ}$ ATDC

$\left(39.6^{\circ} \mathrm{AIT}\right)$

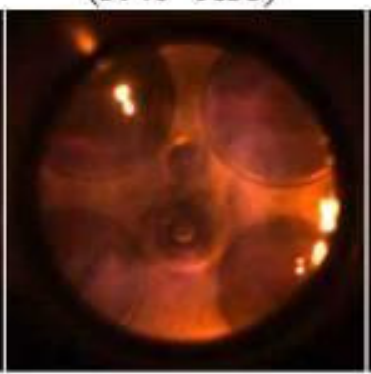

$25.3^{\circ}$ ATDC

$\left(60.3^{\circ} \mathrm{AIT}\right)$ $-9.8^{\circ}$ ATDC

$\left(25.2^{\circ} \mathrm{AIT}\right)$

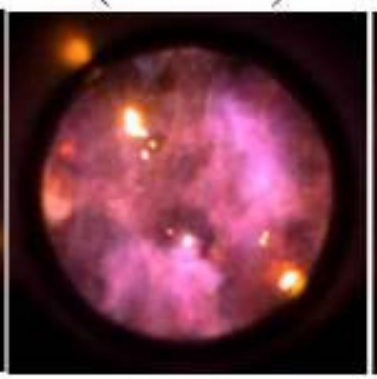

$-9.8^{\circ}$ ATDC

(25.2 $\left.2^{\circ} \mathrm{AIT}\right)$

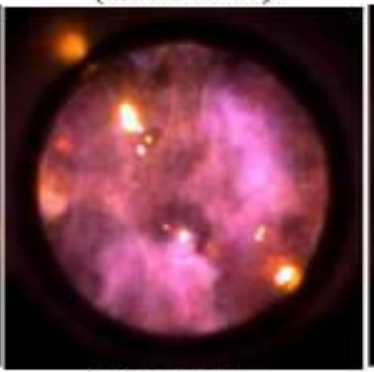

$10^{\circ}$ ATDC

$\left(45^{\circ} \mathrm{AIT}\right)$

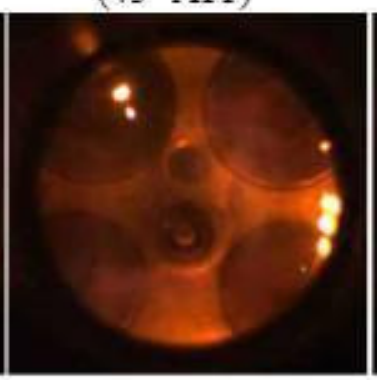

$29.8^{\circ}$ ATDC

$\left(64.8^{\circ} \mathrm{AIT}\right)$

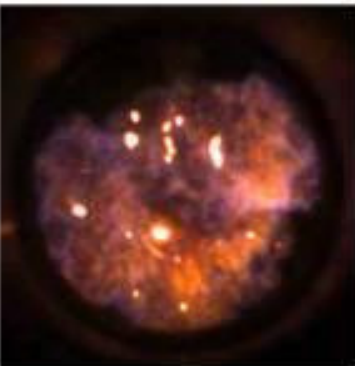

$-5.3^{\circ}$ ATDC

$\left(29.7^{\circ} \mathrm{AIT}\right)$

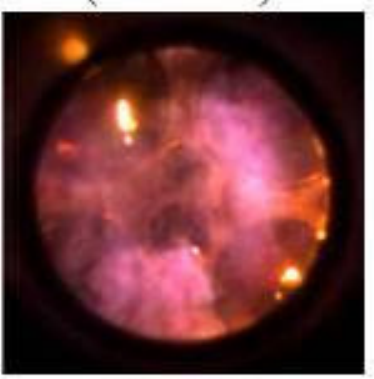

$-5.3^{\circ}$ ATDC

(29. $\left.7^{\circ} \mathrm{AIT}\right)$

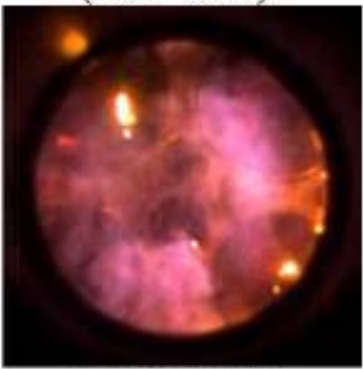

$14.5^{\circ} \mathrm{ATDC}$

$\left(49.5^{\circ} \mathrm{AIT}\right)$

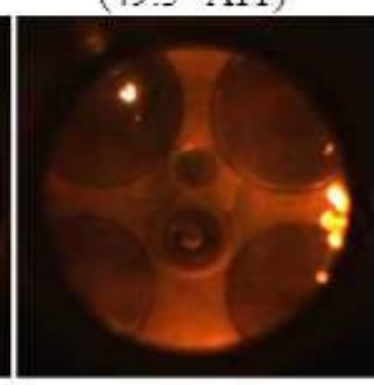

$35.2^{\circ}$ ATDC

$\left(70.2^{\circ} \mathrm{AIT}\right)$

Figure 59: Combustion images as a function of crank angle 
Table 5: Test parameters and results for firing test

\begin{tabular}{|c|c|}
\hline Speed: & 1500 RPM \\
\hline IMEP: & 3.89 bar \\
\hline MAP: & 0.43 bar \\
\hline Relative Air-Fuel ratio $(\lambda):$ & 1 \\
\hline Spark Timing: & $35^{\circ}$ BTDC \\
\hline Peak Pressure: & 16 bar \\
\hline Peak Pressure timing: & $19^{\circ}$ ATDC \\
\hline 10\% Burn location & $-4^{\circ}$ ATDC \\
\hline $50 \%$ burn location & $7^{\circ}$ ATDC \\
\hline 90\% burn location & $21^{\circ}$ ATDC \\
\hline Burn duration & 25 CAD \\
\hline
\end{tabular}

The related optical combustion images are shown in Figure 59, where ATDC represents for After Top Dead Center and AIT represents for After Ignition Timing.

Figure 60 and Figure 61 show the averaged in-cylinder pressure and its associated mass fraction burn (MFB) curves. It can be observed that the 10 to $90 \%$ burn duration is about 23 crank degrees. This burn duration is going to be compared with these of the HCCI and SI-HCCI hybrid combustion.

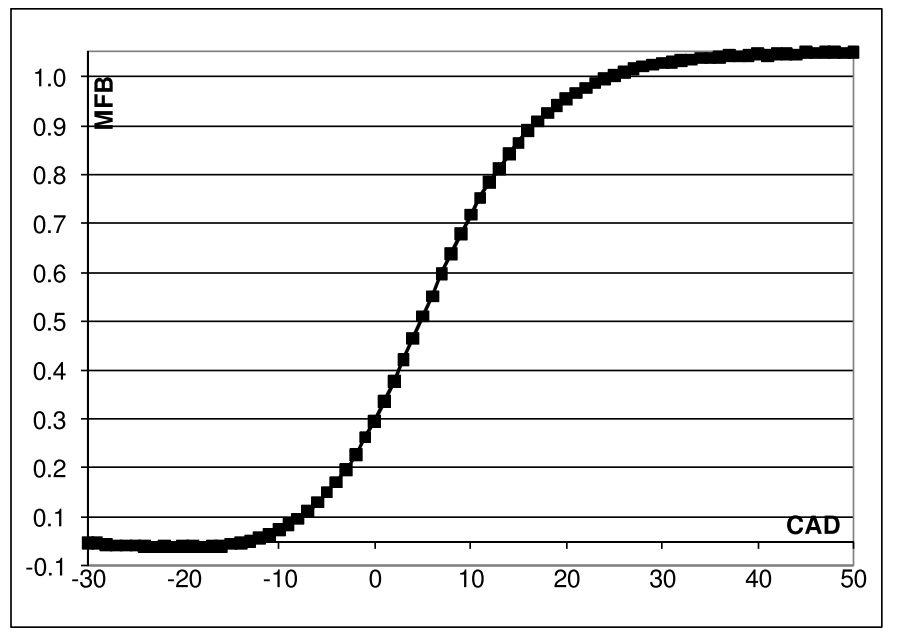

Figure 60: Mass fraction burned curve for combustion image data

Two kinds of optical combustion tests were conducted using the optical engine \#1. They are a) the SI air-to-fuel ratio (AFR) transient test and b) the HCCI combustion tests.

For the SI AFR combustion test, the optical engine was operated for 48 consecutive combustion cycles with the fixed spark timing at 28 degree before TDC. The engine fuel injection pulse width was fixed for the first 25 engine cycles and then reduced linearly to have a transient air-tofuel ratio from rich to lean, see Figure 62 for the detailed engine test configuration and operational parameters. 


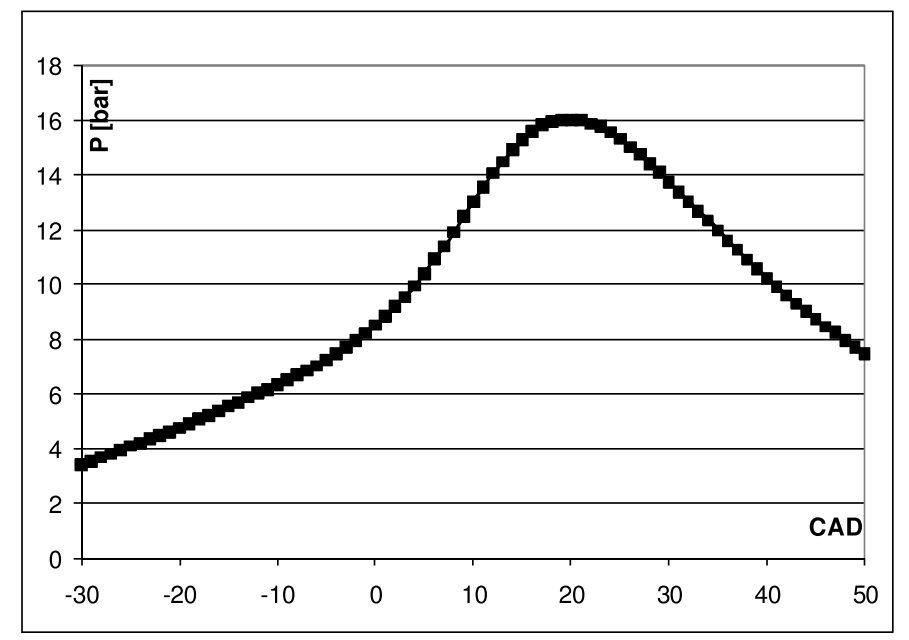

Figure 61: In-cylinder pressure trace for combustion image data

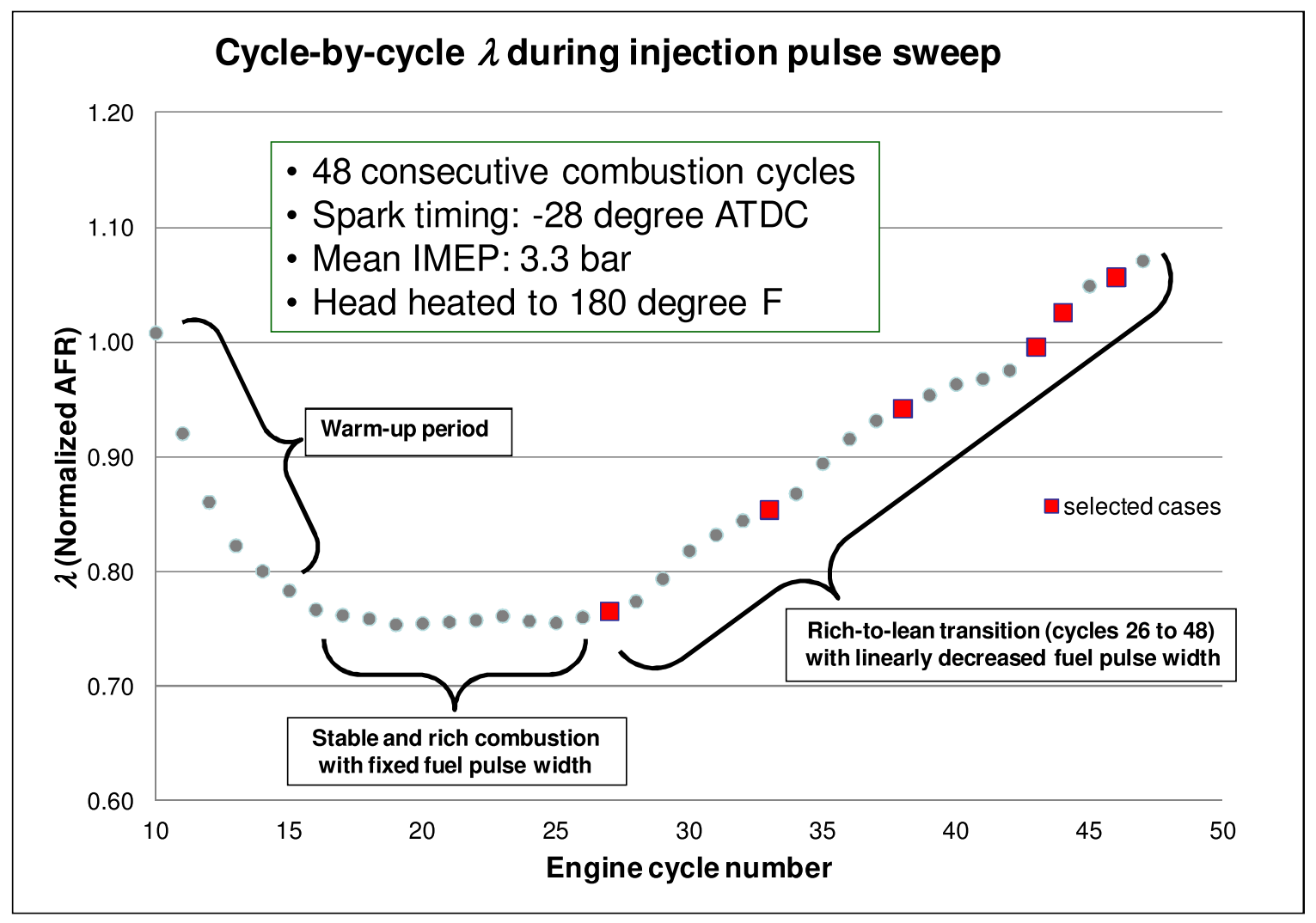

Figure 62: Rich to lean air-to-fuel ratio test configuration

Figure 63 to Figure 67 show the recorded optical engine combustion images at different crank angles form 8 degree before TDC (top dead center) to 31.6 degree after TDC, and each figure shows six combustion images at different AFR (normalized) from 0.77 to 1.06 . It can be clearly observed that the richest combustion (at 0.77 ) provides the brightest combustion images, and as 
the AFR gets lean, the combustion image turns to light blue. This test demonstrated that the modified SI and HCCI optical engine is capable of operating under the SI transient combustion.

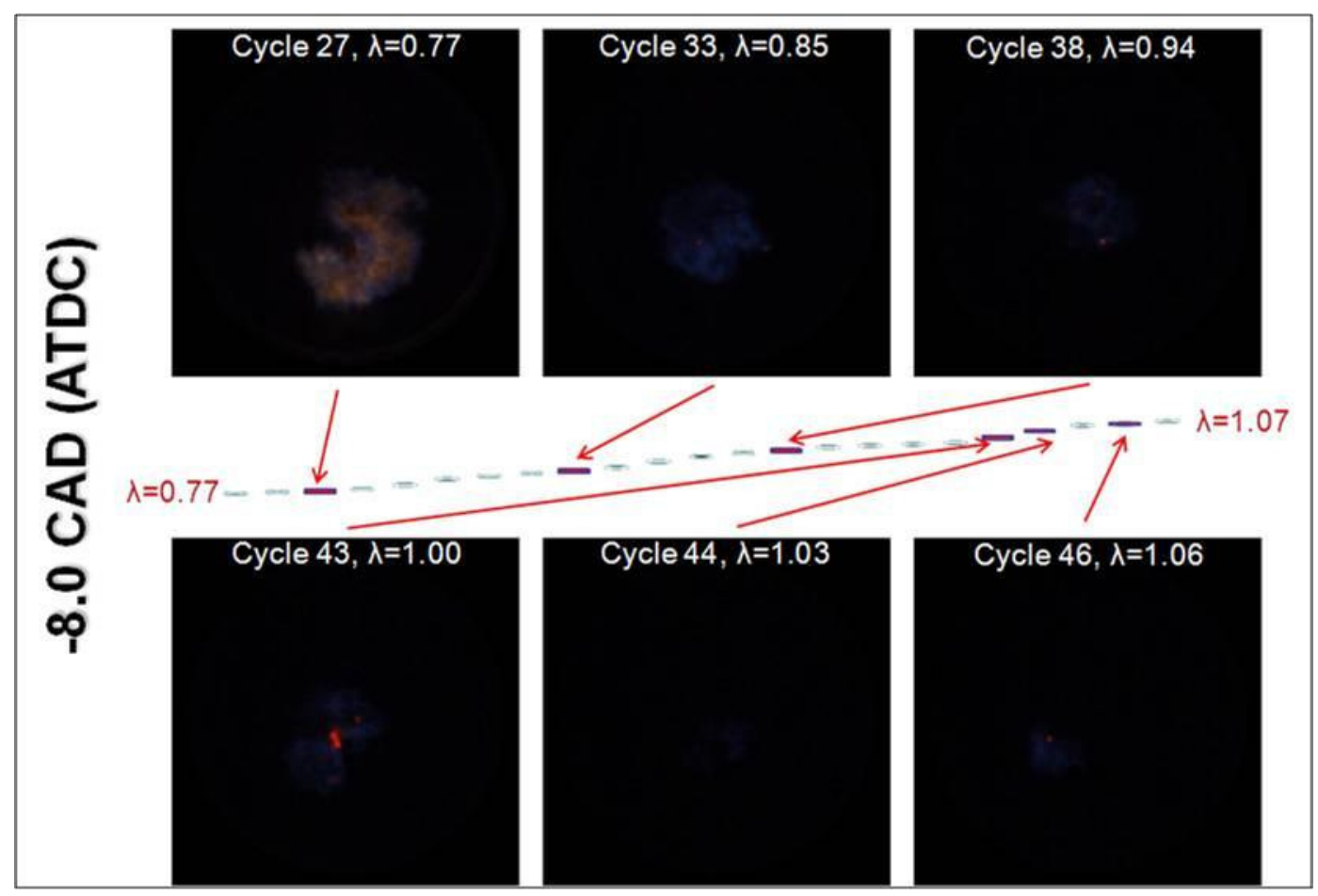

Figure 63: Optical engine combustion images at -8 degree ATDC under different AFRs

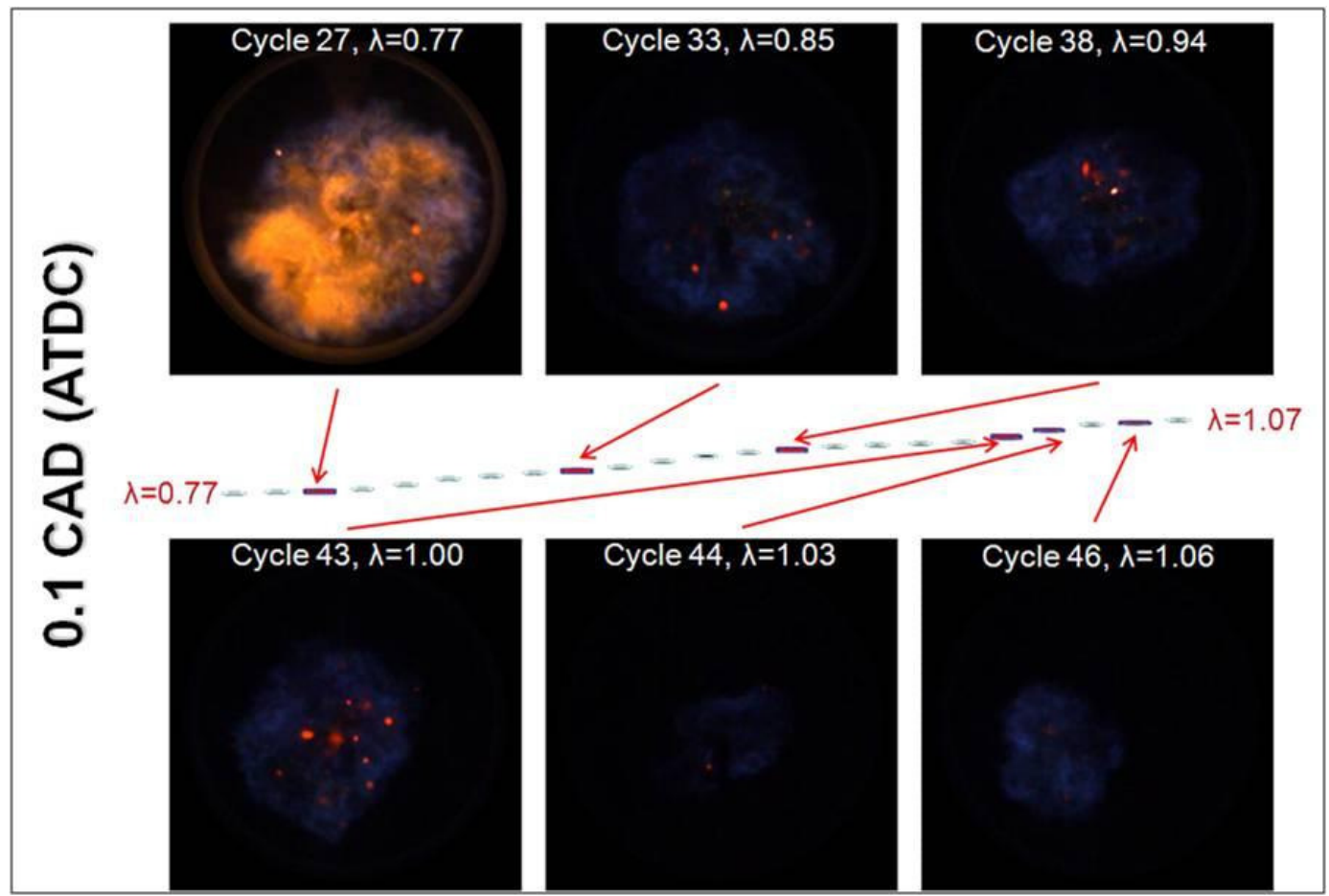

Figure 64: Optical engine combustion images at 0.1 degree ATDC under different AFRs 


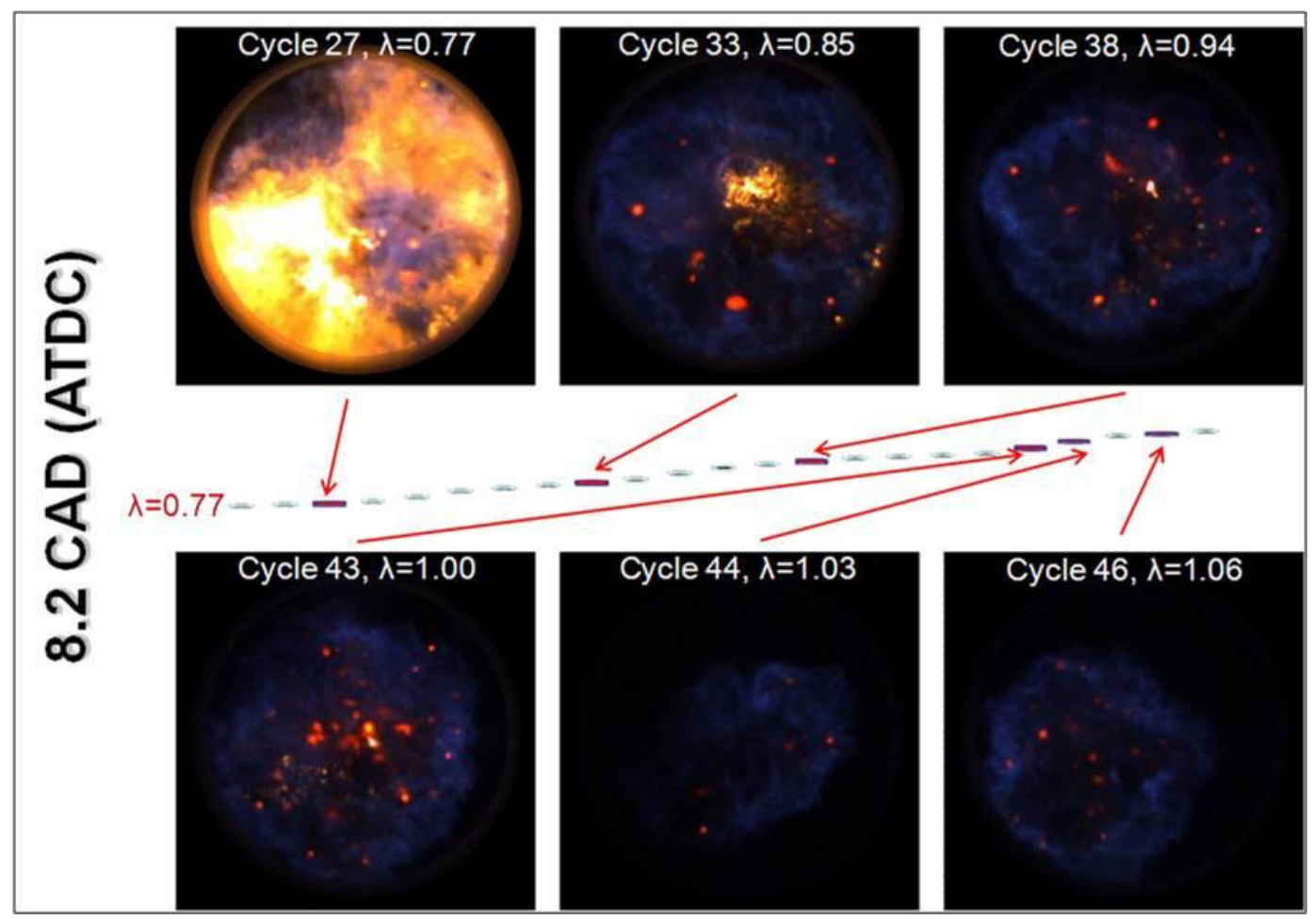

Figure 65: Optical engine combustion images at 8.2 degree ATDC under different AFRs

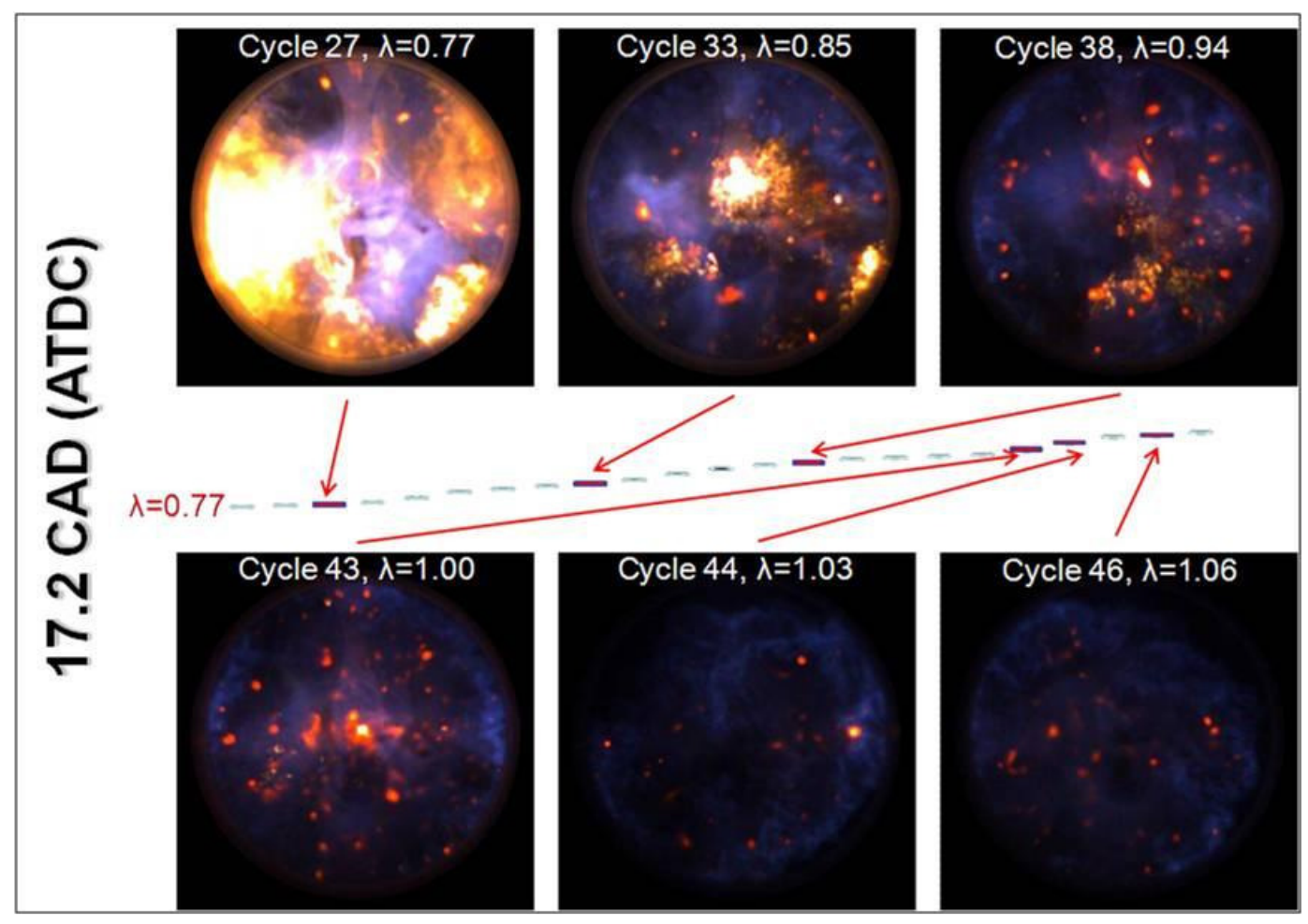

Figure 66: Optical engine combustion images at 17.2 degree ATDC under different AFRs 


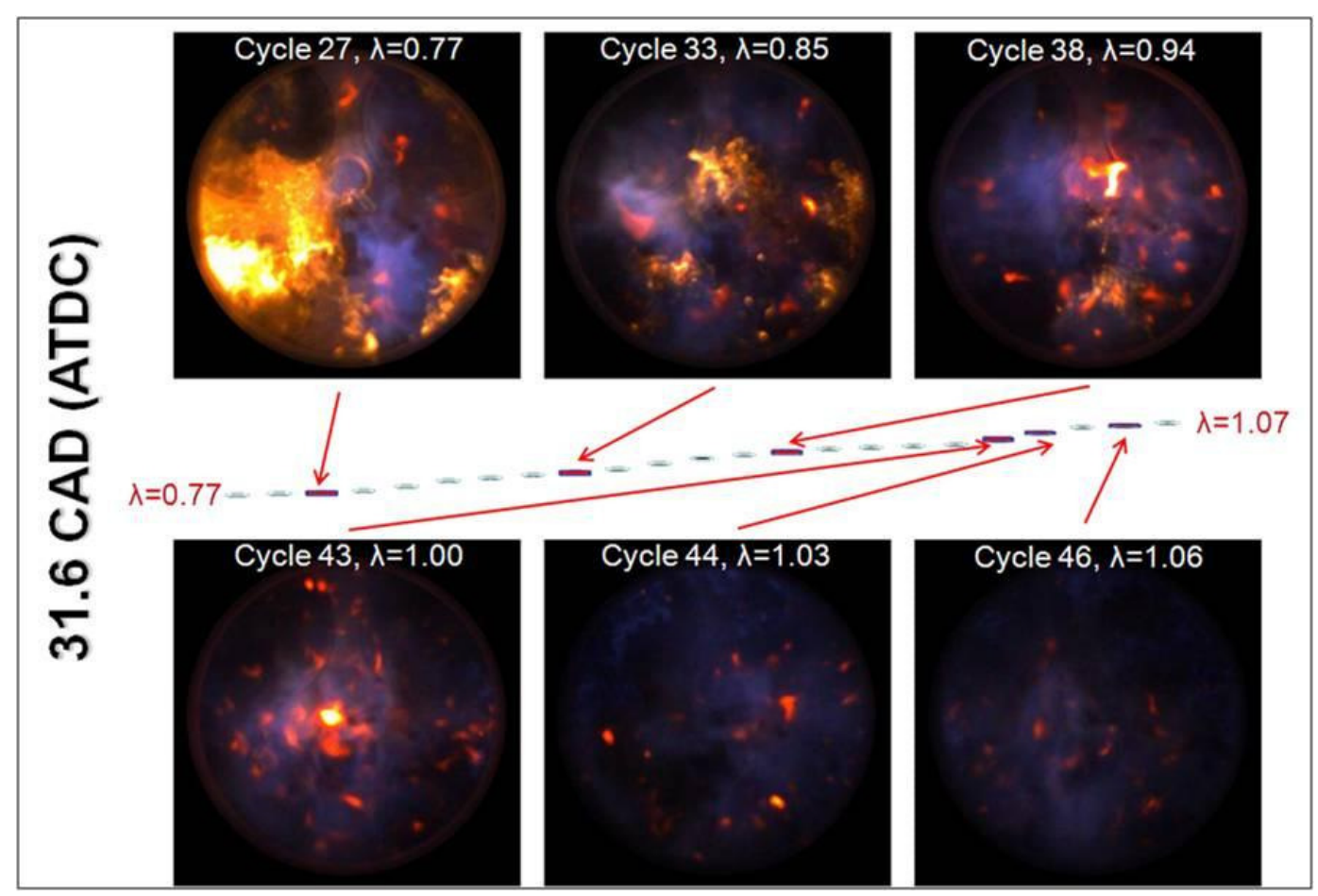

Figure 67: Optical engine combustion images at 31.6 degree ATDC under different AFRs

After the optical engine \#1 was modified for the HCCI combustion mode, the resulting optical engine \#2 was used for the HCCI combustion tests. Figure 68 shows the HCCI combustion images over single combustion event at different crank angles. It can be clearly observed from the first combustion image that the combustion initiated from multiple locations with very short combustion duration (less than 10 crank degrees). The engine coolant was heated to $96 \mathrm{C}^{\circ}$ and the intake charge was heated to $240 \mathrm{C}^{\circ}$.

As a summary, two optical combustion tests were conducted and they are a) the SI air-to-fuel ratio (AFR) transient test using optical engine \#1 and b) the HCCI combustion test using optical engine \#2. The purpose of the SI transient AFR combustion test is to demonstrate the transient operation of the optical engine and its prototype controller; and the purpose of the HCCI combustion test is to confirm the HCCI combustion capability. The collected data were also used to calibrate the developed HIL (hardware-in-the-loop) engine model. 


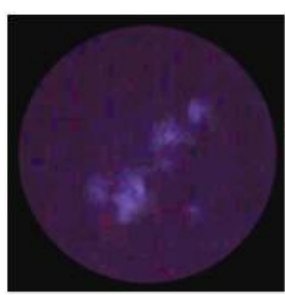

$-16.3^{\circ}$

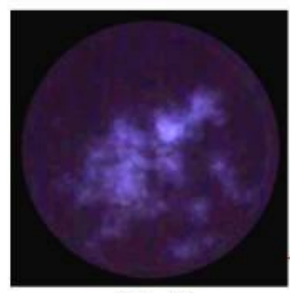

$-15.4^{\circ}$

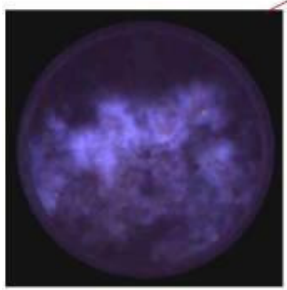

$-14.5^{\circ}$

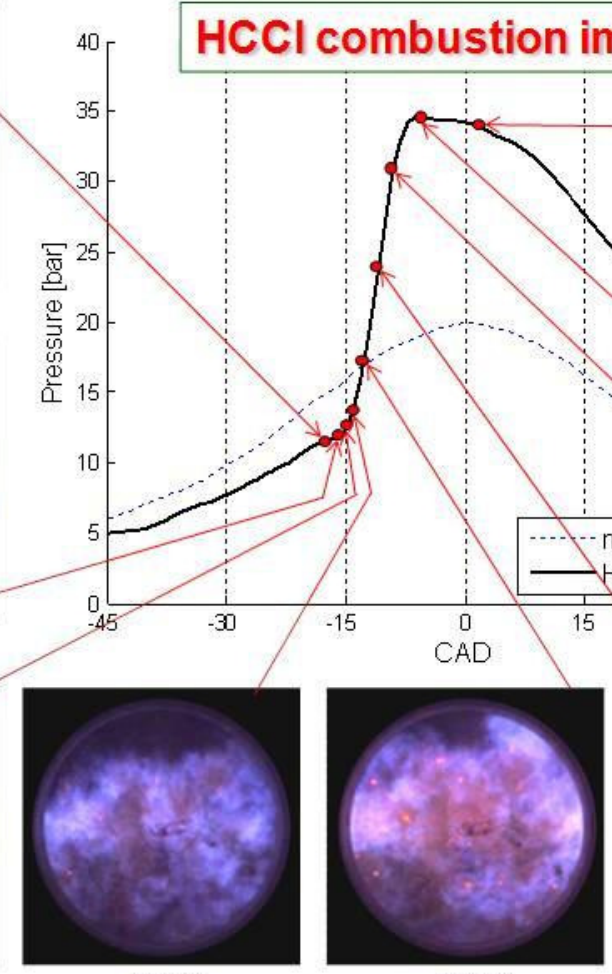

$-13.6^{\circ}$

$-12.7^{\circ}$

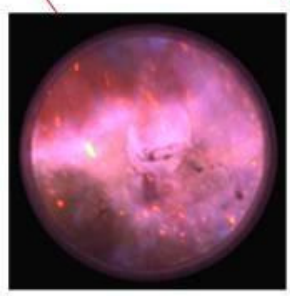

$-10.9^{\circ}$

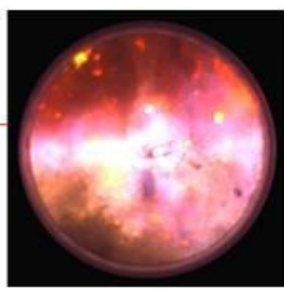

$2.6^{\circ}$

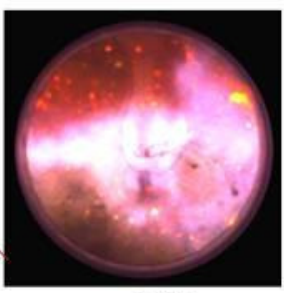

$-6.4^{\circ}$

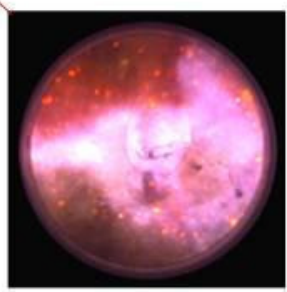

$-9.1^{\circ}$

Figure 68: Optical engine HCCI combustion test

\subsubsection{Flow and charge mixing tests}

It was learned from the optical HCCI combustion tests that the level of recompression is a key factor to induce HCCI combustion in the optical engine. Therefore, the future HCCI tests will focus on the intake valve timing and the fuel injection control during the recompression phase to stabilize the HCCI combustion.

From the optical combustion tests, we also learned that cycle-to-cycle charge mixing is quiet different, which motivates us to explore the capabilities of using IR imaging to analyze mixing of intake air and trapped exhaust gas. Five sets of 10 IR images were taken with each set of 10 being from a different CAD location near IVO. Each set of 10 images was taken on consecutive cycles, as the camera is only capable of taking one IR image per engine cycle. These 10 images from the same $\mathrm{CAD}$ location were then averaged to get an idea of the averaged heat distribution of the gases at that moment in the engine cycle. Figure 69 shows 10 raw IR images (in grayscale) over 10 engine cycles and the averaged image of the 10 IR image is shown in color. This is to demonstrate the cycle-to-cycle variations since the 10 IR images from different cycles are quite different. This makes the mode transition control even more difficult.

Figure 70 shows five images obtained by averaging the 10 IR images at each CAD location. These were taken at 330, 320, 310, 300, and 290 CAD BTDC. Note that, for the images shown, the exhaust valves are on top and intake valves are on the bottom. 
- IR imaging can be used to capture mixing of fresh intake air with hot recompressed gas IVO timing

a 10 images were taken at the same CAD on ten consecutive cycles. An average image of these ten image can be useful for showing larger mixing trends

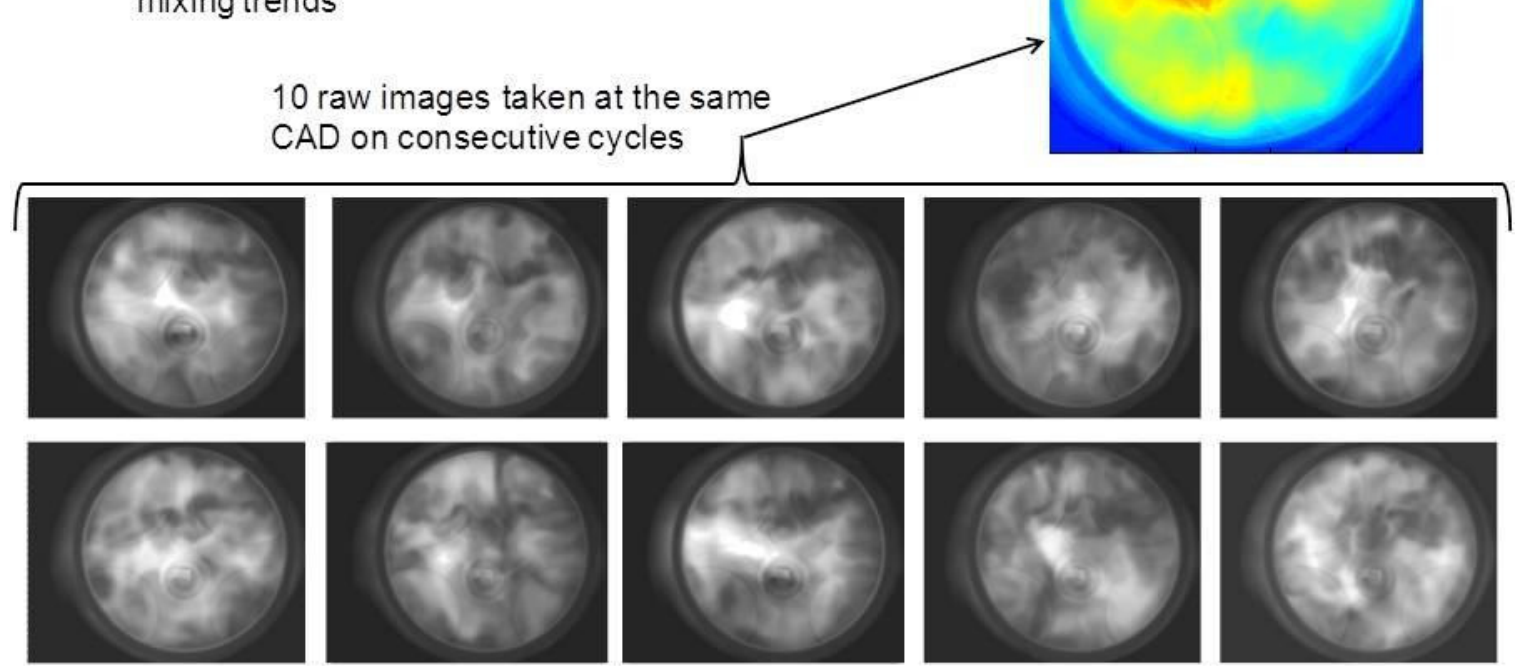

Figure 69: IR images of the charge mixing for 10 engine cycles

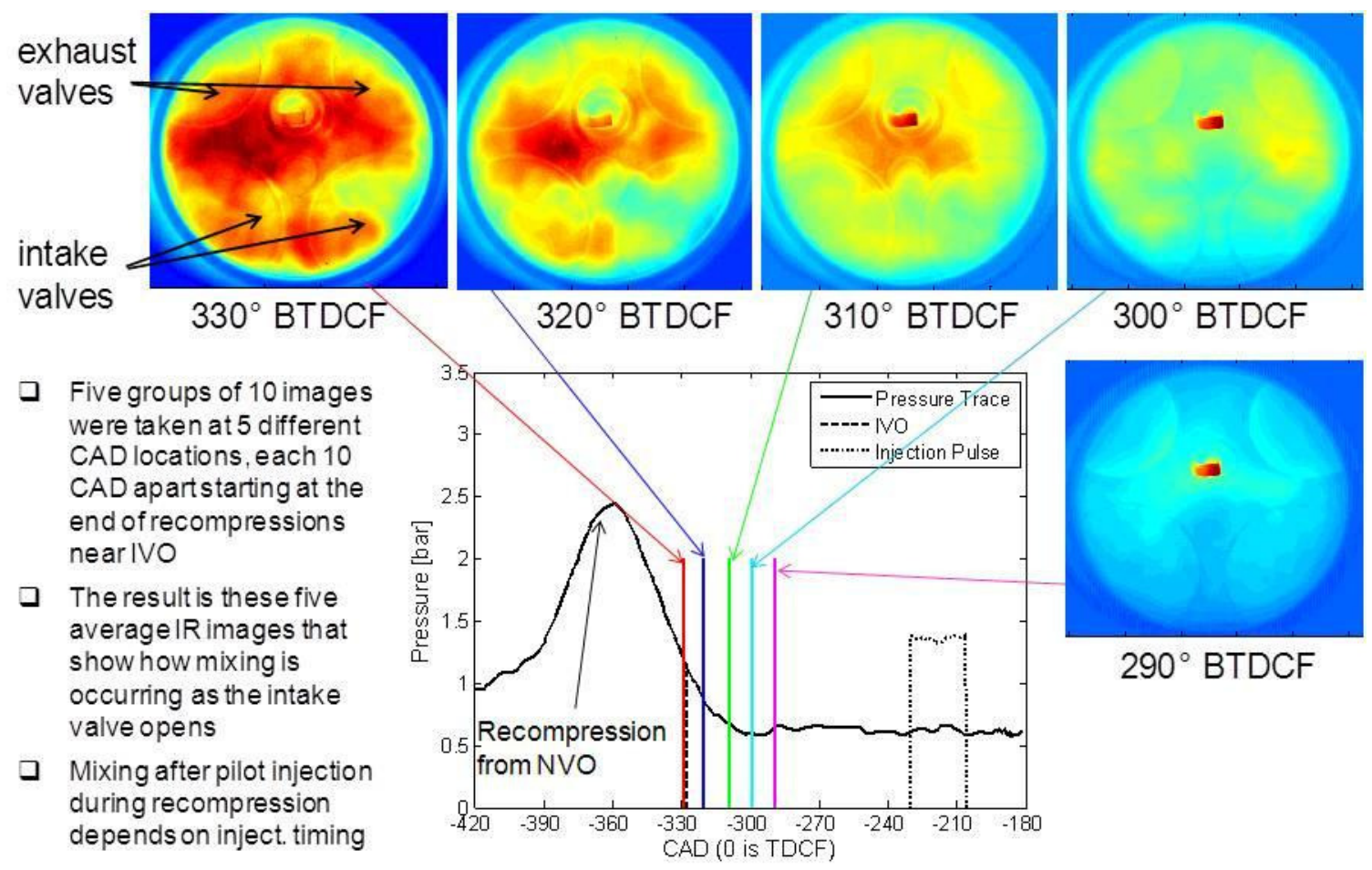

Figure 70: Averaged IR images of the charge mixing for a given engine cycle 
From the optical combustion tests it is learned that cycle-to-cycle charge mixing is quiet different and the IR imaging technique can be used to analyze mixing of intake air and trapped exhaust gas. IR images show that at a given engine crank location these images are quite different, indicating large cycle-to-cycle charge mixing variations. This makes the mode transition control quite difficult.

\subsubsection{Engine model calibration}

The developed engine HIL model was also recalibrated based upon the optical engine data and the engine in-cylinder pressure and temperature model was also calibrated for the intake and exhaust processes. The results were summarized into a paper and published in 2012 ASME DSCC (Dynamic System Control Conference) [38], see Subsection 9.7. Based upon optical test data, the charge mixing IR images from the optical engine tests were used to improve the accuracy of the charge mixing model and to calibrate the developed model.

\subsection{Two-step lift and electrical variable valve timing control}

\subsubsection{Two-step valve lift and phase system range determination}

For a so-called HCCI combustion capable engine, SI combustion mode is still required at high engine load conditions with high valve lift and wide valve opening duration of both intake and exhaust valves are required when the engine is operated at SI combustion mode. However, once the engine is operated at the HCCI combustion mode at medium and low load conditions, the valve profiles (both lift and width) of both intake and exhaust valves needs to be reduced. The reduced exhaust valve lift and duration create engine "recompression" to increase the internal residual gas and gas mixture temperature, which is critical to make the HCCI combustion possible and control the HCCI combustion timing and duration. The reduced intake valve lift and duration make it possible for throttless combustion. Therefore, the optimal engine performance can be achieved by optimizing the intake and exhaust valve lift, timing, and duration.

To make our engine capable of operating at both SI and HCCI combustion modes, we need to find the proper operational range for valve lift and duration of both intake and exhaust valves. Figure 71 demonstrates that an engine operated at the HCCI combustion mode with mediate and light load could have improved combustion efficiency with proper valve lift, timing, and duration. In three simulations, fuel quantity of $12 \mathrm{mg}$ for each cylinder per engine cycle was injected and the engine speed was operated at 2000 RPM. The SI combustion, in blue lines, was simulated with 3.58 bar IMEP. The engine was operated at stoichiometric air-to-fuel ratio that was achieved through throttled operation with high pumping loss comparing with throttless operation. The HCCI combustion, in red and green lines, was also simulated with different valve timings but with fixed lift and duration. The optimized valve timing produce 4.02 bar IMEP and non-optimized 3.52 bar, see Figure 71.

In this study, the intake and exhaust valve lifts were fixed at $9 \mathrm{~mm}$ for SI combustion with the optimized valve timing. However, for HCCI and SI-HCCI transient combustion modes, the valve lift, timing, and duration need to be determined so proper cam phase and lift ranges can be selected. Since we have determined to use a continuous variable electrical variable valve timing system with dual lift capability, our target is to determine the cam phase range and second lift (low) since the other lift (high) was determined by the SI combustion at $9 \mathrm{~mm}$. A GT-Power engine model was developed to find the best valve lift and timing ranges of the intake and exhaust valves. 

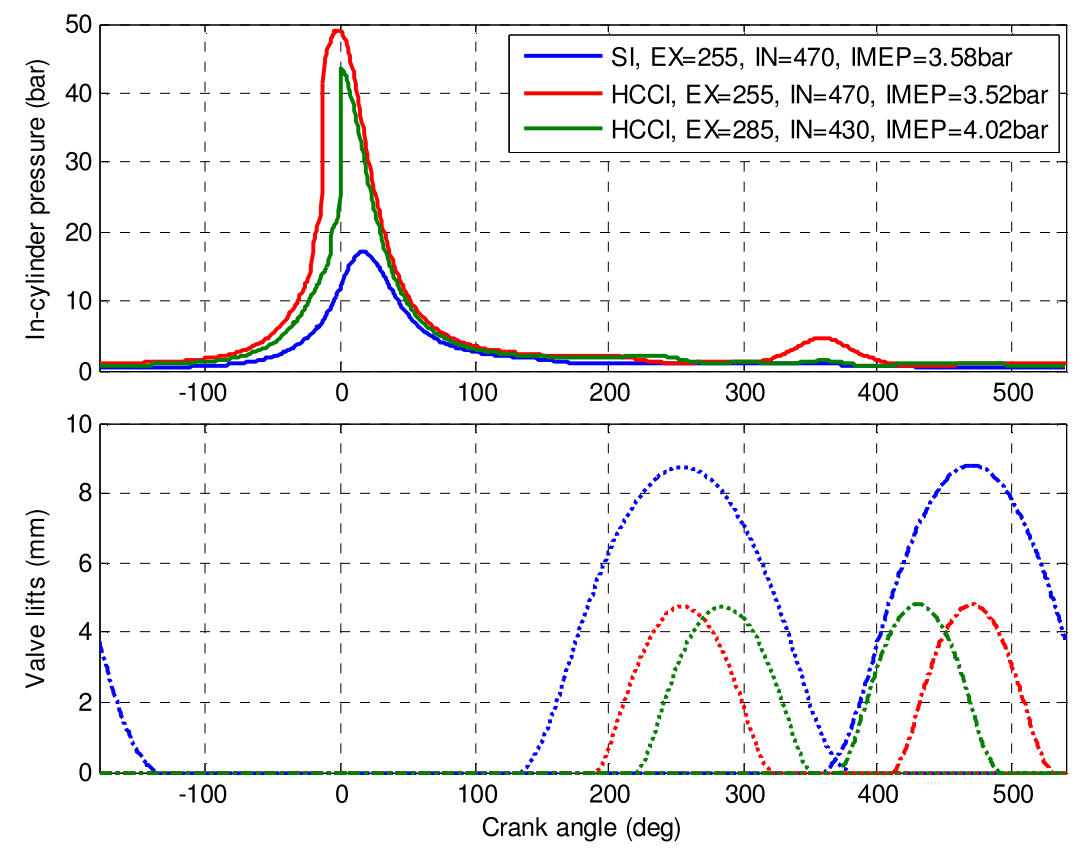

Figure 71: Engine performance for different valve profiles

Figure 72 shows the GT-Power engine model with specifications listed in Table 6. Only one cylinder was modeled, since this research focuses on the engine combustion and not on the entire engine performance. For the optimal valve lift and timing study, HCCI combustion model was developed based upon the so-called "ChemGas" method provided by GT-Power, in which 29 chemical species, including the intermediate species, and 52 chemical reactions were simulated.

Simulations have been conducted using the developed GT-Power model. The engine IMEP was recorded to compare engine performance at different valve timings and lifts. All simulations were conducted at 2000 RPM of engine speed. Engine fueling of $5 \mathrm{mg}$ per cycle was used to simulate the low engine load bound of the HCCI combustion; and engine fueling of $16 \mathrm{mg}$ per cycle was used for upper load bound. With the given fuel quantity, engine valve lift and timing were swept. As a result, different quantity of air and residue gas was trapped into cylinder, and different IMEP was obtained. Engine IMEP for each simulation was shown in Table 7 to Table 14. Note that for all tables, the entry with blue background is for intake valve timing in degree after firing TDC; the red entry for exhaust valve timing in degree after firing TDC; the yellow entry indicates misfired combustion; and the green entry is the optimal operational valve timing combination at the given load and valve lift.

Table 6: Engine specifications

\begin{tabular}{l|l}
\hline \hline Parameter & Model value \\
\hline Bore / stroke & $86 \mathrm{~mm} / 86 \mathrm{~mm}$ \\
\hline Connecting rod length & $143.6 \mathrm{~mm}$ \\
\hline Compression ratio & $9.8: 1$ \\
\hline
\end{tabular}




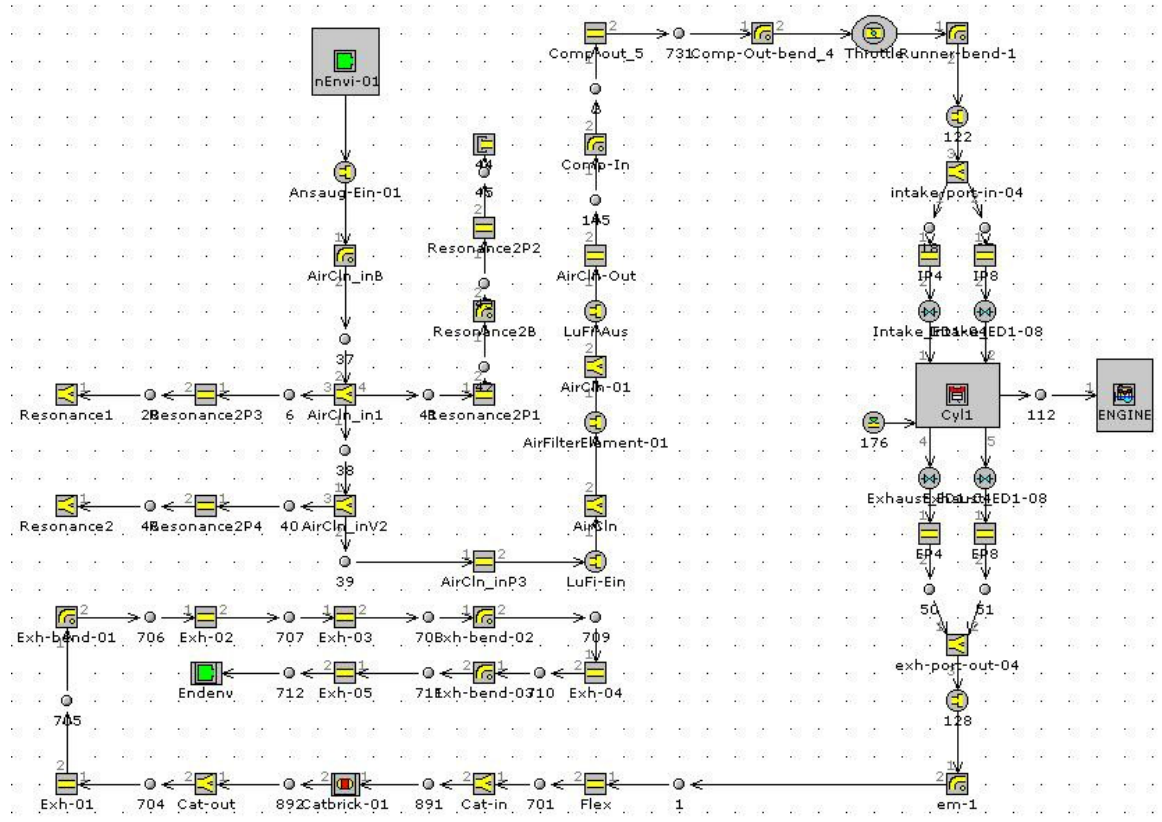

Figure 72: GT-Power engine model layout

Table 7: IMEP with FUEL $=16 \mathrm{mg} /$ cycle, $\mathrm{LIFT}=4 \mathrm{~mm}$

\begin{tabular}{|r|r|r|r|r|r|r|r|r|r|r|r|}
\hline & 400 & 405 & 410 & 415 & 420 & 425 & 430 & 435 & 440 & 460 & 480 \\
\hline 230 & -1.597 & -1.523 & -1.311 & -1.045 & -0.773 & -0.559 & -0.445 & -0.545 & -0.27 & 1.003 & 2.352 \\
\hline 250 & -0.906 & -0.842 & -0.739 & -0.415 & -0.604 & -0.521 & -0.449 & -0.389 & -0.334 & 2.94 & 3.144 \\
\hline 270 & -0.539 & -0.297 & 1.258 & 0.477 & 2.222 & 2.59 & 3.108 & 3.443 & 3.75 & 3.62 & 3.444 \\
\hline 275 & -0.453 & 1.521 & 1.68 & 1.154 & 2.75 & 3.068 & 3.497 & 3.867 & 4.013 & 3.674 & 3.397 \\
\hline 280 & -0.38 & 0.345 & 0.784 & 1.395 & 3.316 & 3.65 & 4.015 & 4.164 & 4.195 & 3.657 & 3.317 \\
\hline 285 & -0.32 & 0.814 & 1.315 & 1.04 & 3.883 & 4.175 & 4.313 & 4.334 & 4.285 & 3.61 & 3.216 \\
\hline 290 & -0.271 & 0.576 & 1.451 & 3.986 & 4.279 & 4.431 & 4.443 & 4.388 & 4.281 & 3.518 & 3.083 \\
\hline 295 & -0.233 & -0.215 & 1.375 & 4.238 & 4.448 & 4.516 & 4.47 & 4.365 & 4.218 & 3.404 & 2.933 \\
\hline 300 & -0.205 & -0.195 & 1.171 & 4.284 & 4.434 & 4.484 & 4.412 & 4.281 & 4.121 & 3.263 & 2.77 \\
\hline
\end{tabular}

Table 8: IMEP with FUEL $=16 \mathrm{mg} / \mathrm{cycle}, \mathrm{LIFT}=5 \mathrm{~mm}$

\begin{tabular}{|r|r|r|r|r|r|r|r|r|r|r|r|}
\hline & 400 & 405 & 410 & 415 & 420 & 425 & 430 & 435 & 440 & 460 & 480 \\
\hline 230 & -0.063 & 0.234 & 0.375 & 0.472 & -0.151 & 1.107 & 1.613 & 2.113 & 2.456 & 3.414 & 3.339 \\
\hline 250 & 1.17 & 0.406 & 0.732 & 2.283 & 2.798 & 3.343 & 3.73 & 3.92 & 4.036 & 4.253 & 4.09 \\
\hline 270 & 5.589 & 5.625 & 5.628 & 4.543 & 4.668 & 4.733 & 4.779 & 4.803 & 4.815 & 4.553 & 4.015 \\
\hline 275 & 5.597 & 5.635 & 5.644 & 4.811 & 4.875 & 4.909 & 4.929 & 4.932 & 4.912 & 4.513 & 3.929 \\
\hline 280 & 5.575 & 5.613 & 5.62 & 4.985 & 5.023 & 5.031 & 5.027 & 5.007 & 4.961 & 4.45 & 3.83 \\
\hline 285 & 5.519 & 5.562 & 5.574 & 5.085 & 5.102 & 5.102 & 5.079 & 5.033 & 4.953 & 4.361 & 3.722 \\
\hline 290 & 5.43 & 5.481 & 5.505 & 5.099 & 5.128 & 5.115 & 5.077 & 5.007 & 4.905 & 4.259 & 3.599 \\
\hline 295 & 5.315 & 5.381 & 5.416 & 5.033 & 5.094 & 5.081 & 5.028 & 4.944 & 4.833 & 4.142 & 3.474 \\
\hline 300 & 5.198 & 5.257 & 5.295 & 4.913 & 5.016 & 5.002 & 4.946 & 4.852 & 4.734 & 4.02 & 3.351 \\
\hline
\end{tabular}


Table 9: IMEP with FUEL $=16 \mathrm{mg} / \mathrm{cycle}, \mathrm{LIFT}=6 \mathrm{~mm}$

\begin{tabular}{|r|r|r|r|r|r|r|r|r|r|r|r|}
\hline & 400 & 405 & 410 & 415 & 420 & 425 & 430 & 435 & 440 & 460 & 480 \\
\hline 230 & -0.115 & 1.784 & 1.918 & 2.163 & 2.43 & 2.76 & 3.146 & 3.39 & 3.575 & 4.001 & 4.102 \\
\hline 250 & 4.057 & 4.345 & 4.472 & 4.524 & 4.544 & 4.562 & 4.599 & 4.643 & 4.684 & 4.786 & 4.585 \\
\hline 270 & 5.305 & 5.388 & 5.408 & 5.396 & 5.371 & 5.343 & 5.317 & 5.293 & 5.273 & 5.018 & 4.493 \\
\hline 275 & 5.347 & 5.439 & 5.477 & 5.475 & 5.454 & 5.425 & 5.387 & 5.359 & 5.319 & 4.983 & 4.434 \\
\hline 280 & 5.276 & 5.441 & 5.492 & 5.502 & 5.485 & 5.453 & 5.41 & 5.375 & 5.319 & 4.933 & 4.371 \\
\hline 285 & 5.232 & 5.374 & 5.481 & 5.493 & 5.474 & 5.439 & 5.399 & 5.348 & 5.284 & 4.875 & 4.296 \\
\hline 290 & 5.091 & 5.275 & 5.436 & 5.453 & 5.431 & 5.387 & 5.337 & 5.287 & 5.225 & 4.814 & 4.237 \\
\hline 295 & 4.893 & 5.231 & 5.373 & 5.394 & 5.366 & 5.315 & 5.26 & 5.208 & 5.143 & 4.736 & 4.163 \\
\hline 300 & 5.039 & 5.196 & 5.294 & 5.313 & 5.282 & 5.229 & 5.168 & 5.107 & 5.048 & 4.65 & 4.095 \\
\hline
\end{tabular}

Table 10: IMEP with FUEL $=16 \mathrm{mg} / \mathrm{cycle}, \mathrm{LIFT}=7 \mathrm{~mm}$

\begin{tabular}{|r|r|r|r|r|r|r|r|r|r|r|r|}
\hline & 400 & 405 & 410 & 415 & 420 & 425 & 430 & 435 & 440 & 460 & 480 \\
\hline 230 & 4.171 & 4.328 & 4.374 & 4.367 & 4.337 & 4.308 & 4.299 & 4.291 & 4.316 & 4.587 & 4.681 \\
\hline 250 & 5.309 & 5.346 & 5.362 & 5.352 & 5.324 & 5.289 & 5.255 & 5.233 & 5.219 & 5.251 & 5.023 \\
\hline 270 & 5.589 & 5.625 & 5.628 & 5.611 & 5.596 & 5.576 & 5.549 & 5.529 & 5.504 & 5.345 & 4.94 \\
\hline 275 & 5.597 & 5.635 & 5.644 & 5.633 & 5.603 & 5.57 & 5.535 & 5.505 & 5.48 & 5.319 & 4.905 \\
\hline 280 & 5.575 & 5.613 & 5.62 & 5.61 & 5.585 & 5.551 & 5.505 & 5.467 & 5.434 & 5.276 & 4.877 \\
\hline 285 & 5.519 & 5.562 & 5.574 & 5.573 & 5.55 & 5.51 & 5.468 & 5.424 & 5.379 & 5.228 & 4.84 \\
\hline 290 & 5.43 & 5.481 & 5.505 & 5.507 & 5.493 & 5.459 & 5.413 & 5.365 & 5.316 & 5.165 & 4.802 \\
\hline 295 & 5.315 & 5.381 & 5.416 & 5.424 & 5.414 & 5.388 & 5.347 & 5.297 & 5.244 & 5.094 & 4.755 \\
\hline 300 & 5.198 & 5.257 & 5.295 & 5.314 & 5.316 & 5.297 & 5.266 & 5.216 & 5.161 & 5.008 & 4.692 \\
\hline
\end{tabular}

Table 11: IMEP with FUEL $=5 \mathrm{mg} / \mathrm{cycle}, \mathrm{LIFT}=4 \mathrm{~mm}$

\begin{tabular}{|r|r|r|r|r|r|r|r|r|r|r|r|}
\hline & 390 & 400 & 410 & 420 & 430 & 440 & 450 & 460 & 470 & 480 & 490 \\
\hline 220 & -2.175 & -2.132 & -1.814 & -1.046 & -0.74 & -0.032 & 1.006 & 1.178 & 1.19 & 1.313 & 1.374 \\
\hline 230 & -1.804 & -1.779 & -1.314 & -0.757 & -0.515 & 0.871 & 1.126 & 1.238 & 1.376 & 1.433 & 1.461 \\
\hline 240 & -1.478 & -1.292 & -0.864 & -0.619 & 0.314 & 1.104 & 1.301 & 1.433 & 1.485 & 1.511 & 1.508 \\
\hline 250 & -0.991 & -0.724 & -0.275 & 0.329 & 1.479 & 1.366 & 1.49 & 1.533 & 1.555 & 1.541 & 1.491 \\
\hline 260 & -0.679 & 0.239 & 0.499 & 1.529 & 1.767 & 1.553 & 1.58 & 1.591 & 1.561 & 1.491 & 1.41 \\
\hline 270 & -0.577 & 1.083 & 1.304 & 1.496 & 1.568 & 1.572 & -0.096 & -0.101 & -0.097 & 1.37 & 1.267 \\
\hline
\end{tabular}

Table 12: IMEP with FUEL $=5 \mathrm{mg} / \mathrm{cycle}, \mathrm{LIFT}=5 \mathrm{~mm}$

\begin{tabular}{|c|c|c|c|c|c|c|c|c|c|c|c|}
\hline & 390 & 400 & 410 & 420 & 430 & 440 & 450 & 460 & 470 & 480 & $7=$ \\
\hline 220 & -0.873 & -0.543 & .155 & 0.324 & 0.021 & 944 & .105 & 1.312 & .418 & 1.486 & . \\
\hline 230 & -0 & 1 & & 4 & 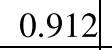 & & & & & 0 & (5) \\
\hline 240 & 0.388 & 0.769 & 0.802 & 0.975 & 19 & 365 & 1.45 & 1.589 & 1.632 & 1.643 & 1.61 \\
\hline 250 & & 1.117 & 1.176 & 1.306 & $1.42 \mathrm{~J}$ & & & & & & $\cdots$ \\
\hline 26 & 1.431 & 1.451 & 1.456 & 1.49 & -0.196 & 0.128 & 0.084 & 0.069 & 0.064 & 553 & 4 \\
\hline
\end{tabular}


Table 13: IMEP with FUEL $=5 \mathrm{mg} /$ cycle, $\mathrm{LIFT}=6 \mathrm{~mm}$

\begin{tabular}{|c|c|c|c|c|c|c|c|c|c|c|c|}
\hline & 390 & 400 & 410 & 420 & 430 & 440 & 450 & 460 & 470 & 480 & 49 \\
\hline 220 & 0.986 & 0.841 & 0.688 & 0.662 & 0.804 & 1.021 & 1.238 & .416 & 1.539 & .613 & .653 \\
\hline 230 & 9 & 14 & & & & & & 58 & 666 & 05 & 71. \\
\hline 240 & 1.498 & 1.454 & 1.375 & 1.344 & 1.406 & 529 & 1.641 & 1.701 & 1.713 & 1.718 & 1.701 \\
\hline 250 & -0.301 & -0.339 & -0.288 & -0.268 & -0.214 & -0.148 & 0.094 & 0.065 & -0.061 & -0.073 & 1.622 \\
\hline 260 & -0.219 & -0.318 & -0.253 & -0.162 & -0.135 & -0.101 & -0.077 & -0.077 & -0.104 & -0.14 & 1.5 \\
\hline
\end{tabular}

Table 14: IMEP with FUEL $=5 \mathrm{mg} /$ cycle, LIFT $=7 \mathrm{~mm}$

\begin{tabular}{|r|r|r|r|r|r|r|r|r|r|r|r|}
\hline & 390 & 400 & 410 & 420 & 430 & 440 & 450 & 460 & 470 & 480 & 490 \\
\hline 220 & 1.539 & -0.273 & 1.316 & 1.188 & 1.121 & 1.154 & 1.306 & 1.49 & 1.615 & 0.031 & -0.006 \\
\hline 230 & -0.303 & -0.241 & 1.526 & 1.469 & 1.428 & 1.461 & 1.557 & 1.634 & -0.084 & -0.051 & -0.056 \\
\hline 240 & -0.278 & -0.102 & -0.177 & -0.199 & -0.195 & -0.16 & -0.116 & -0.094 & -0.076 & -0.071 & -0.101 \\
\hline 250 & -0.25 & -0.017 & -0.092 & -0.105 & -0.109 & -0.096 & -0.073 & -0.066 & -0.075 & -0.113 & -0.164 \\
\hline 260 & -0.044 & 0.017 & -0.038 & -0.049 & -0.048 & -0.044 & -0.042 & -0.056 & -0.096 & -0.164 & -0.216 \\
\hline
\end{tabular}

The criteria of finding optimal valve lift and timing from the simulation results shown in Table 7 to Table 14 are the engine IMEP (combustion efficiency) and the distance of the optimal IMEP condition to the unstable combustion region (the yellow area). We know that the higher the IMEP, the better the combustion efficiency; and the longer the distance, the more the stability. Based on these criteria, we believe that $5 \mathrm{~mm}$ lift is the best for HCCI combustion and the intake valve timing should be centered at 450 degrees after TDC and exhaust valve at 250 degrees. Both intake and exhaust valve phase should have a minimum range of 60 degrees. Therefore, 80 degree cam phase range is selected.

\subsubsection{Two-step valve supply selection and cylinder head modification}

After Delphi was selected as the two-step valve system supplier, Chrysler engineers worked closely with Delphi engineers and integrated the selected two-step valve system onto the Chrysler target engine head. Due to the compact packaging of the engine head, integrating the two-step valve system is not trivial. Intensive GT-Power engine simulations were conducted at MSU to optimize the intake and exhaust valve timing under both SI and HCCI combustion; see Subsection 3.2.1. Optimal valve lift levels for both SI and HCCI operations were also finalized based upon the simulation results. Delphi then conducted the integration feasibility study based upon the desired intake and exhaust valve timings and lifts, and Chrysler validated the final design. Figure 73 shows the designs of cam shaft and integration of the two-step valves. 


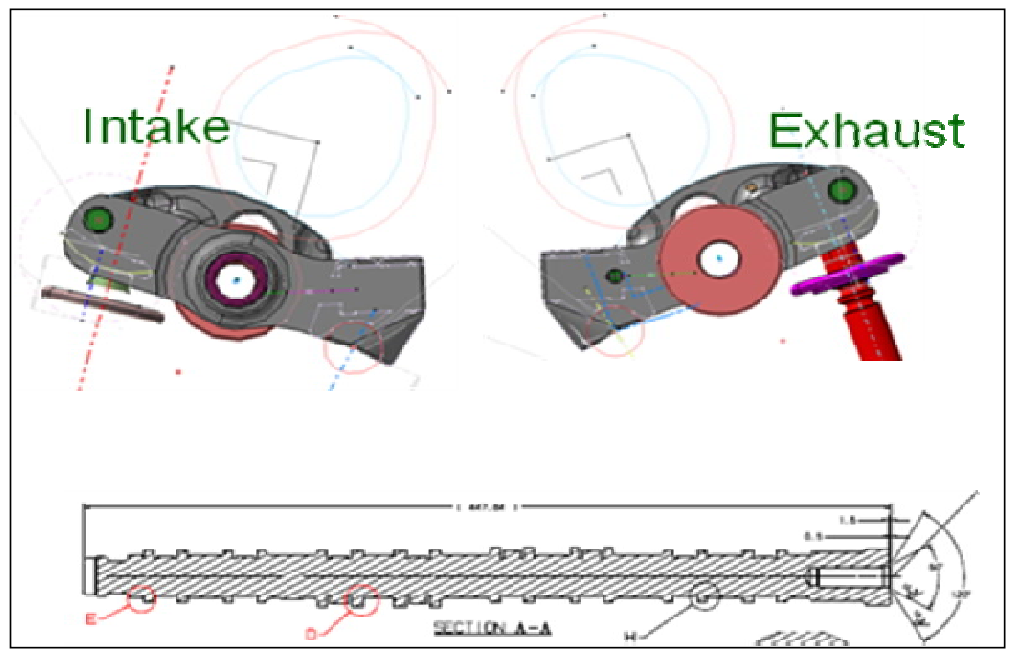

Figure 73: Two-step valve integration design

Two target cylinder heads, one for the optical engine and one for the multi-cylinder engine, were machined to fit with the Delphi's two-step valve systems, see Figure 74. In order to fit the twostep valvetrain on to the target cylinder head the following modification were made:

a) Sixteen two-step valve seats were machine for sixteen two-step valve hydraulic actuators.

b) The cam shaft bearing seats were narrowed to fit double cam profiles for each valve.

c) The oil path of the target cylinder head were re-routed to make the two-step valve actuation possible.

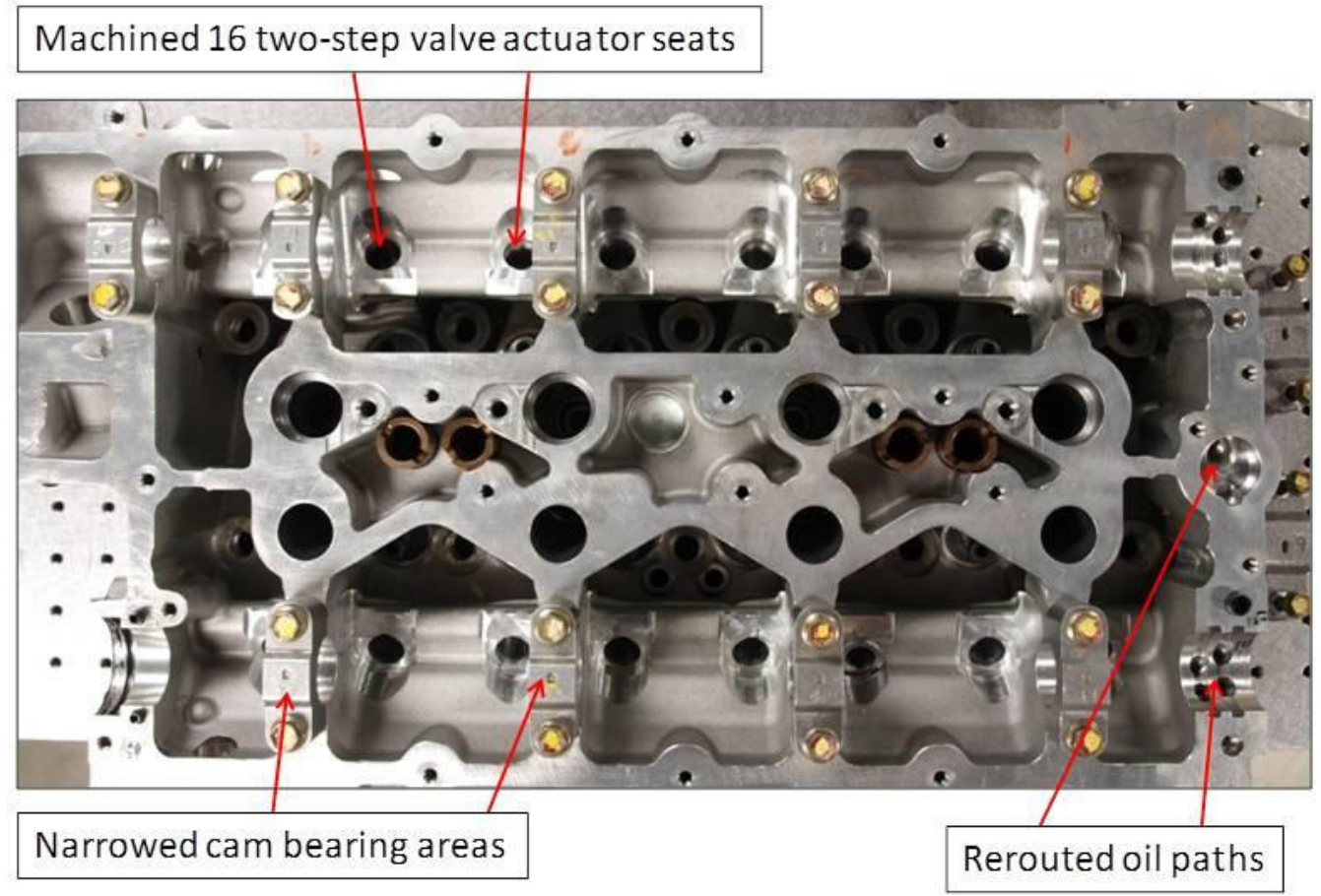

Figure 74: Machined target engine head 


\subsection{Electrical variable valve timing subsystem}

\subsubsection{Electrical variable valve timing background}

Continuously variable valve timing (VVT) system used in an internal combustion engine was developed in nineties [12] and has since been widely used due to the growing fuel economy demands and emission regulations. VVT system improves fuel economy and reduces emissions at low engine speed, as well as improves engine power and torque at high engine speed. Conventional electronic-hydraulic VVT ([12] and [13]), also called hydraulic VVT, is the most widely used in the automotive industry today. The hydraulic VVT system requires minor changes when applied to a previously non-VVT valve-train [12], making design and engineering relatively easy. However due to its mechanism, the hydraulic VVT system also has its limitations [14]. The response and performance of the hydraulic VVT system are significantly affected by the engine operating conditions such as engine oil temperature and pressure. For instance, at low engine temperature, the hydraulic VVT system cannot be activated and remains at the default position, instead of maintaining at optimal position, so that the cold start performance and emissions cannot be improved [14]. This leads to the study of other variable valve-train system, such as electromagnetic [15], and electrical motor driven planetary gear system ([16] and [17]). Electric motor driven VVT operational performance is independent of engine oil temperature and pressure [14]. Comparing to hydraulic VVT system, electric motor driven VVT system is less limited to engine operating conditions and therefore gives better performance and better emission in a wider range. Especially, since the electrical VVT is independent of the oil pressure, the response time is greatly improved.

The major advantage of HCCI (homogeneous charge compression ignition) combustion is realized by eliminating the formation of flames and results in much lower combustion temperature. As a consequence of the low temperature, the formation of NOx (nitrogen oxides) is greatly reduced. The lean burn nature of the HCCI engine also enables un-throttled operation to improve engine fuel economy. Unfortunately, HCCI combustion is feasible only over a limited engine operational range due to knock and misfire. To make a HCCI engine work in an automotive, the internal combustion engine has to be capable of operating at both SI combustion mode at high load and HCCI combustion mode at low and mediate load ([4] and [21]). This makes it necessary to have a smooth transition between SI and HCCI combustion modes.

Achieving the HCCI combustion and controlling the mode transition between SI and HCCI combustion in a practical engine require implementation of enabling devices and technologies. There are a number of options, and the necessary prerequisite for considering any of them is their ability to provide control of thermodynamic conditions in the combustion chamber at the end of compression. The range of devices under consideration includes variable valve actuation (cambased or camless), variable compression ratio, dual fuel systems (port and direct fuel injection with multiple fuel injections), supercharger and/or turbocharger, exhaust energy recuperation and fast thermal conditioning of the intake charge mixture, spark-assist, etc. Variable Valve Actuation can be used for control of the effective compression ratio (via the intake valve closing time), the internal (hot) residual fraction via the negative valve overlap (recompression) ([2] and [3]), or secondary opening of the exhaust valve (residual re-induction) ([2] and [3]). In addition 
to providing the basic control of the HCCI combustion, i.e., ignition timing and burn rate or duration, the VVT systems will play a critical role in accomplishing smooth mode transitions from SI to HCCI and vice versa ([18], [19], and [20]). In this paper, the electrical VVT system is selected to control the engine valve timings when it is operated at SI and HCCI combustion modes and also during the combustion mode transition.

In order to control the electrical planetary VVT system, a feedback controller was introduced in [17]. Due to the steady state and transient control accuracy requirement of the HCCI combustion, the closed-loop electrical VVT system needs to not only meet steady-state performance requirement but also follow a desired trajectory during the combustion mode transition. Therefore, a feedback controller with feedforward control is developed. For the selected electrical VVT system, the cam phase is the integration of speed difference between the electrical VVT motor and crankshaft. This leads to the proposal of using the rate of the reference phase as feedforward command. Output covariance control (OCC) strategy ([22], [23], and [24]) is proposed for feedback control to reduce the tracking error.

\subsubsection{Electrical VVT Modeling}

The planetary gear VVT system studied in this paper consists of four major components (see Figure 37). Ring gear, serves as VVT pulley, is driven directly by crankshaft through a timing belt at half crankshaft speed. Planet gear carrier is driven by an electrical VVT motor. Planet gears engage both ring and sun gears. Sun gear is connected to the camshaft. The sun and planet gears are passive components that obtain kinetic energy from carrier and ring gears. Comparing to other components, the inertia of engine fly wheel and crank shaft is very large. As a result, dynamics of the ring gear is ignored in this study. All other components have known mechanical properties and their dynamics are considered in the modeling.

\section{Planetary Gear System Kinematics}

In a planetary gear system [25] shown in Figure 75, angular velocities of components are determined by:

$$
\frac{\omega_{s}(t)-\omega_{c}(t)}{\omega_{r}(t)-\omega_{c}(t)}=-\frac{n_{r}}{n_{s}}
$$

where $\omega_{s}, \omega_{c}$ and $\omega_{r}$ are angular velocities of the sun, carrier, and ring gears, respectively. $n_{r}$ and $n_{s}$ are the teeth numbers of ring and sun gears. Laplace transformation of (1) can be written as:

$$
\Omega_{s}(s)=-\frac{n_{r}}{n_{s}} \Omega_{r}(s)+\frac{n_{r}}{n_{s}} \Omega_{c}(s)+\Omega_{c}(s)
$$




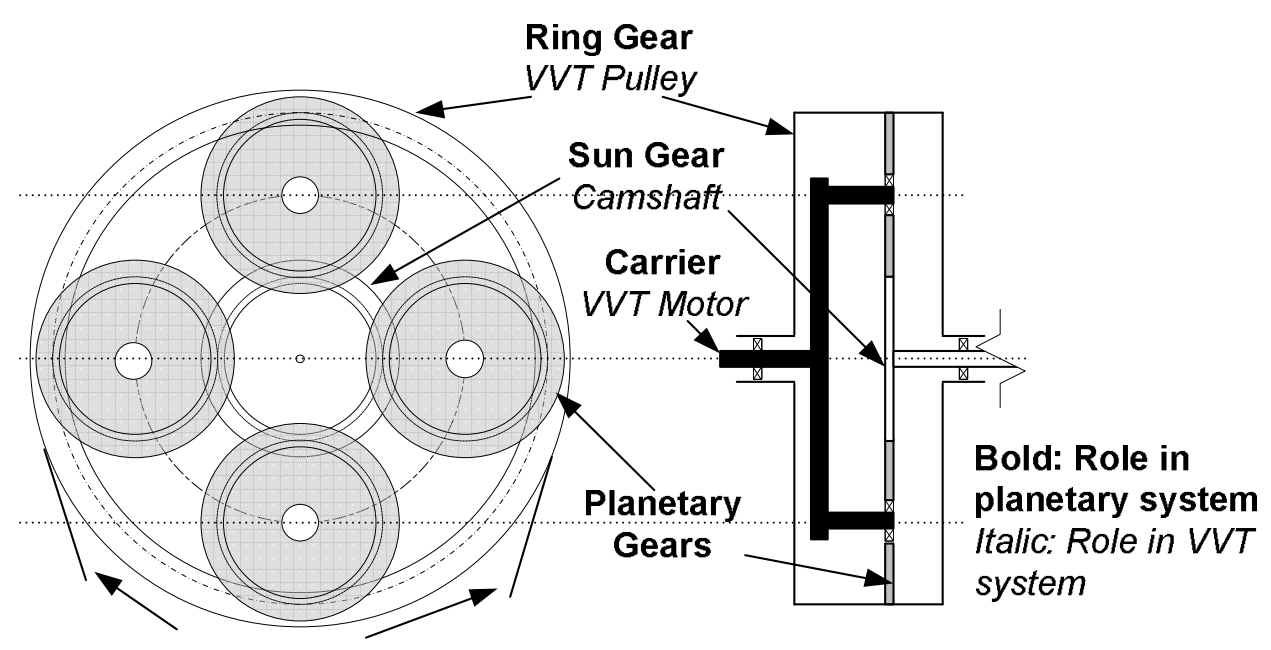

Figure 75: Planetary VVT system driven by electric motor

Cam phase angle $\phi$ is two-time integration of the difference between camshaft and crankshaft speeds:

$$
\phi=2 \int_{0}^{t}\left[\omega_{s}(\tau)-\omega_{r}(\tau)\right] d \tau
$$

and its Laplace transformation is:

$$
\Phi(s)=\frac{2}{s}\left[\Omega_{s}(s)-\Omega_{r}(s)\right]
$$

Using (2) in (4), we have:

$$
\Phi=\frac{2}{s}\left(\frac{n_{s}+n_{r}}{n_{s}}\right)\left(\Omega_{c}-\Omega_{r}\right)
$$

Equation (5) shows that the cam phase is an integral function of speed difference between carrier and ring gears. In other word, by controlling the VVT motor speed with respect to the engine speed, cam phase can be adjusted. When the carrier speed is equal to the ring speed, cam phase is held; when the carrier speed is greater than the ring speed, cam phase is advancing; and when the carrier speed is slower than the ring speed, cam phase is retarding. Notice that equation (5) has an integrator, so steady-state value of the reference signal cannot be used for feedforward control directly.

\section{Planetary Gear System Dynamics}

Planetary gear system dynamics with an electric motor are modeled in this section. In this study, it is assumed that the gear system does not have friction. Figure 76 shows free body diagrams of planetary gear components. 

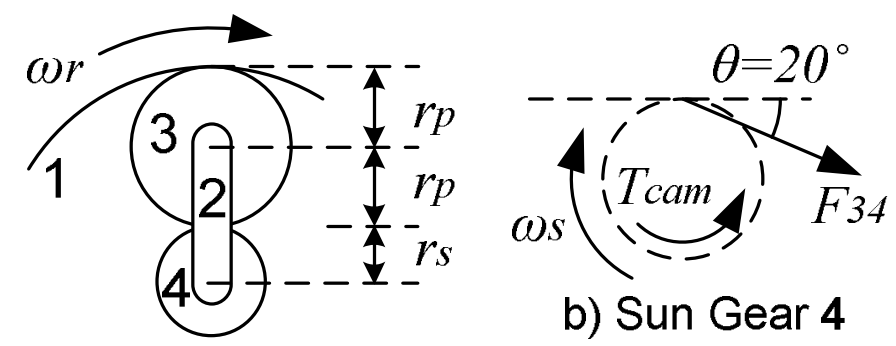

a) Planetary System

b) Sun Gear 4
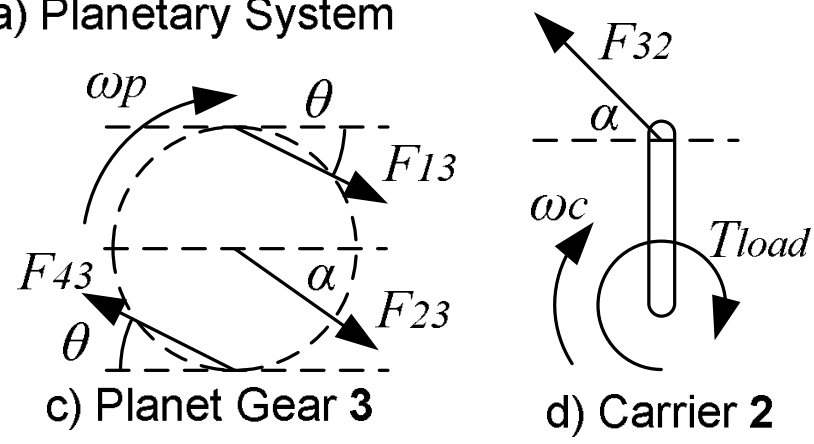

d) Carrier 2

Figure 76: Body diagrams of planetary gear components

Without loss of generality comparing with the system in Figure 75, the system is treated as having only one planet gear (Figure 76a). Since all the gears are properly engaged:

$$
\frac{n_{s}}{r_{s}}=\frac{n_{p}}{r_{p}}=\frac{n_{r}}{r_{r}}, \quad r_{r}=2 r_{p}+r_{s}
$$

where $n_{p}$ is planet gear number of teeth. $r_{s}, r_{p}$, and $r_{r}$ are pitch circle radius of sun, planet, and ring gears. In this study, the gears use a standard pressure angle of 20 degrees. Since the ring has a very large inertia comparing to other components, angular velocity of the ring $\omega_{r}$ is assumed to be constant during the phase shift. From equation (2):

$$
\dot{\omega}_{s}=\frac{n_{r}+n_{s}}{n_{s}} \dot{\omega}_{c}
$$

There are two torques acting on sun gear (Figure 76b), camshaft load $T_{\text {cam }}$ and torque from tooth force $F_{34}$.

$$
F_{34} \cdot r_{s} \cdot \cos \theta-T_{c a m}=J_{s} \dot{\omega}_{s}
$$

where $J_{s}$ is sun gear's moment of inertia with respect to its center of gravity.

Two tooth forces $\left(F_{43}\right.$ and $\left.F_{13}\right)$ and one bearing force from carrier $F_{23}$ are applied to planet gear 
(Figure 76C) that rotates around the bearing on the carrier at $\omega_{p}$ :

$$
\frac{\omega_{p}(t)-\omega_{c}(t)}{\omega_{s}(t)-\omega_{c}(t)}=-\frac{n_{s}}{n_{p}}
$$

and from torque balance with respect to bearing point:

$$
\left(F_{43}+F_{13}\right) \cdot r_{p} \cdot \cos \theta=J_{p} \dot{\omega}_{p}
$$

where $J_{p}$ is planet gear's moment of inertia with respect to its center of gravity. The planet gear also rotates about the center of sun gear:

$$
\left[F_{13}\left(2 r_{r}\right)-F_{43} r_{s}\right] \cos \theta+F_{23}\left(r_{p}+r_{s}\right) \cos \alpha=J_{p s} \dot{\omega}_{c}
$$

where the direction and magnitude of bearing force $F_{23}$ are unknown. The planet gear's moment of inertia with respect to the center of sun gear $J_{p s}$ can be calculated:

$$
J_{p s}=J_{p}\left[1+m_{p}\left(r_{s}+r_{p}\right)^{2}\right]
$$

Since the carrier is driven directly by the motor shaft, the carrier's inertia is also considered as part of motor shaft inertia, and modeled in the next sub-section. Torque balance of carrier is:

$$
F_{32} \cos \alpha\left(r_{p}+r_{s}\right)=T_{l o a d}
$$

where $T_{l o a d}$ is the mechanical load to the motor shaft and $F_{32}$ is the bearing force from planet gear.

Equations (6) to (13) can be simplified to:

$$
T_{\text {load }}=J_{\text {gears }} \dot{\omega}_{c}+k T_{\text {cam }}
$$

where constant $J_{\text {gears }}$ is an equivalent inertia of the planetary gear system, and $k$ is a factor of gear ratio. Details for their value calculations are included the appendix.

\section{Electrical VVT Motor Dynamics}

An electric motor is used to drive carrier in the planetary system. A local closed-loop speed governor is used to control both the motor speed and direction. The input to the local motor controller is the reference speed and direction. In this study, the motor and its controller are treated as an actuator. It is modeled with two inputs of motor velocity command and cam load, and one output of motor shaft speed. 
The mechanical load of the motor can be modeled [25] as:

$$
J_{c} \dot{\omega}_{c}=\tau-B \omega_{c}-T_{\text {load }}
$$

where $J_{c}$ is moment of inertia of motor shaft and carrier, $B$ is friction coefficient, $\tau$ is the motor torque. Using (14) in (15) leads to

$$
\left(J_{c}+J_{\text {gears }}\right) \dot{\omega}_{c}+B \omega_{c}=\tau-k T_{\text {cam }}
$$

and the associated transfer function can be written as:

$$
\Omega_{c}(s)=\frac{1}{\left(J_{c}+J_{\text {gears }}\right) s+B}\left[T(s)-k T_{\text {cam }}(s)\right]
$$

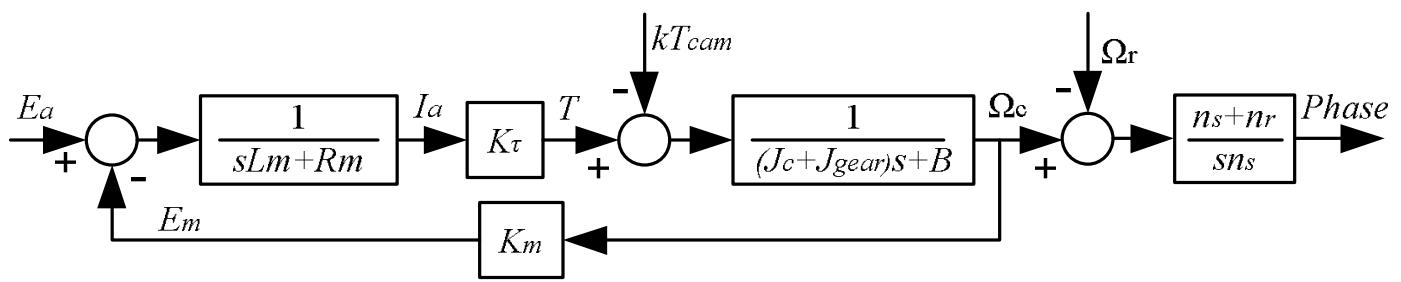

Figure 77: diagram of electric motor with planetary gear system

Modeling procedure of the electrical portion can be found in [26]. Let $J=J_{c}+J_{\text {gears }}$, the electric motor with planetary gear load (Figure 77) can then be represented by:

$$
\Omega_{c}(s)=G_{e}(s) E_{a}(s)+G_{m}(s) T_{c a m}(s)
$$

where the voltage input transfer function:

$$
G_{e}=\frac{K_{\tau}}{\left(L_{m} s+R_{m}\right)(J s+B)+K_{\tau} K_{m}} \cong \frac{K_{\tau}}{R_{m}\left(J_{s}+B\right)+K_{\tau} K_{m}}
$$

and the mechanical input transfer function:

$$
G_{m}=\frac{-\left(L_{m} s+R_{a}\right)}{\left(L_{m} s+R_{m}\right)\left(J_{s}+B\right)+K_{\tau} K_{m}} \cong \frac{-R_{m} k}{R_{m}\left(J_{s}+B\right)+K_{\tau} K_{m}}
$$

$K_{\tau}, K_{m}, L_{m}, R_{m}$ are the motor parameters representing motor torque constant, back EMF constant, armature inertia and armature resistance, respectively [26].

As a summary, a dynamic model of the electrical variable valve timing systems has been 
developed in this quarter. This model is going to be used for model-based control development and validation.

\subsubsection{System identification of the electrical cam phasing system}

Consider a general form of linear time-invariant closed-loop system shown in Figure 78, where signal $r$ is the reference; $n$ is the measurement noise; $u$ and $y$ are input and output, respectively. There are many approaches for the closed-loop system identification, which are categorized as direct, indirect, and joint input-output approaches. In this study, we utilize the knowledge of the closed-loop controller to calculate the open-loop plant model from identified closed-loop plant model, which is called indirect approach. To ensure the quality of identified plant, the closedloop controller in this paper is selected to be proportional due to [27] and [28].

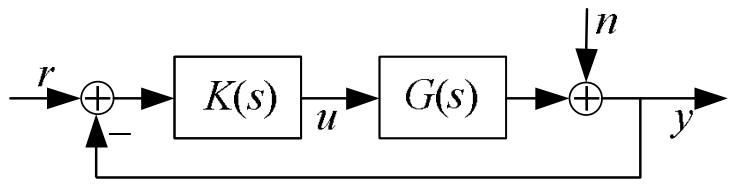

Figure 78: Closed-loop identification framework

The input and output relationship of the generalized closed-loop system, shown in Figure 78, can be expressed below

$$
\mathbf{y}=\mathbf{H} \cdot \mathbf{r}=\mathbf{G K}(\mathbf{I}+\mathbf{G K})^{-1} \mathbf{r}
$$

Let $\hat{\mathbf{H}}$ be identified closed-loop transfer functions from $r$ to $y$. The open-loop system model $\mathbf{G}_{I D}$ can be calculated using the identified $\hat{\mathbf{H}}$, assuming that $(\mathbf{I}-\hat{\mathbf{H}})^{-1}$ is invertible. The closedloop controller transfer function is used to solve for the open-loop system models. We have

$$
\mathbf{G}_{I D}=\hat{\mathbf{H}}(\mathbf{I}-\hat{\mathbf{H}})^{-1} \mathbf{K}^{-1}
$$

PRBS (pseudo-random binary signal) is used as an input excitation for identifying the closedloop system model. The most commonly used PRBSs are based on maximum length sequences (called m-sequences) [29] for which the length of the PRBS signals is $m=2 n-1$, where $\mathrm{n}$ is an integer (order of PRBS). Let $z^{-1}$ represent a delay operator, and define $\hat{p}\left(z^{-1}\right)$ and $p\left(z^{-1}\right)$ to be polynomials

$$
p\left(z^{-1}\right)=a_{n} z^{-n+1} \oplus \cdots \oplus a_{2} z^{-1} \oplus a_{1}=\hat{p}\left(z^{-1}\right) z^{-1} \oplus 1
$$

where $a_{i}$ is either 1 or 0 , and $\oplus$ obeys binary addition, i.e.,

$$
1 \oplus 1=0=0 \oplus 0 \& 0 \oplus 1=1=1 \oplus 0
$$

and the non-zero coefficients $a_{\mathrm{i}}$ of the polynomial are defined in the following table and also in 
[29].

Table 15: Nonzero Coefficients of PRBS Polynomial

\begin{tabular}{|c|c|c|}
\hline $\begin{array}{c}\text { Polynomial } \\
\text { order }(\mathrm{n})\end{array}$ & $\begin{array}{c}\text { Period of } \\
\text { sequence }(\mathrm{m})\end{array}$ & $\begin{array}{c}\text { Non-zero } \\
\text { Coefficient }\end{array}$ \\
\hline 2 & 63 & $\mathrm{a}_{5}, \mathrm{a}_{6}$ \\
\hline 3 & 127 & $\mathrm{a}_{4}, \mathrm{a}_{7}$ \\
\hline 4 & 255 & $\mathrm{a}_{2}, \mathrm{a}_{3}, \mathrm{a}_{4}, \mathrm{a}_{8}$ \\
\hline 5 & 511 & $\mathrm{a}_{5}, \mathrm{a}_{9}$ \\
\hline 6 & 1023 & $\mathrm{a}_{7}, \mathrm{a}_{10}$ \\
\hline 7 & 2047 & $\mathrm{a}_{9}, \mathrm{a}_{11}$ \\
\hline
\end{tabular}

Then the PRBS can be generated by the following formula

$$
\hat{u}(k+1)=\hat{p}\left(z^{-1}\right) \hat{u}(k), \quad k=0,1,2, \ldots
$$

where $\hat{u}(0)=1$ and $\hat{u}(-1)=\hat{u}(-2)=\cdots=\hat{u}(-n)=0$. Defined the following sequence

$$
s(k)= \begin{cases}a ; & \text { If } k \text { is even } \\ -a ; & \text { If } k \text { is odd }\end{cases}
$$

Then, the signal

$$
u(k)=s(k) \otimes[-a+2 a \hat{u}(k)]
$$

is called the inverse PRBS, where $\otimes$ obeys

$$
\mathrm{a} \otimes \mathrm{a}=-\mathrm{a}=-\mathrm{a} \otimes-\mathrm{a} \& \quad \mathrm{a} \otimes-\mathrm{a}=\mathrm{a}=-\mathrm{a} \otimes \mathrm{a}
$$

It is clear after some analysis that $u$ has a period of $2 m$ and $u(k)=-u(k+m)$. The mean of the inverse PRBS is

$$
m_{u}=E_{2 m} u(k) \equiv \frac{1}{2 m} \sum_{k=0}^{2 m-1} u(k)=0
$$

and the autocorrelation $\left(R_{u u}(\tau)=E_{2 m} u(k+\tau) u(k)\right)$ of $u$ is

$$
R_{u u}(\tau) \equiv \frac{1}{2 m} \sum_{k=0}^{2 m-1} u(k+\tau) u(k)= \begin{cases}a^{2}, & \tau=0 \\ -a^{2}, & \tau=m ; \\ -a^{2} / m, & \tau \text { even } \\ a^{2} / m, & \tau \text { odd }\end{cases}
$$

Note that the first and second order information of the inverse PRBS signal is very close to these of white noise with $m$ large enough. The inverse PRBS is used in the $q$-Markov Cover identification algorithm [30]. 
Consider an unknown (presumed nonlinear) system:

$$
\begin{aligned}
& \mathbf{x}(k+1)=f(\mathbf{x}(k), \mathbf{w}(k)) \\
& \mathbf{y}(k)=g(\mathbf{x}(k), \mathbf{w}(k))
\end{aligned}
$$

subject to an input sequence $\{w(0), w(1), w(2), \ldots\}$ generating the output sequence $\{y(0), y(1), y(2), \ldots\}$. The unknown system is $q$-identifiable, if there exists a linear model of the form:

$$
\begin{aligned}
& \mathbf{x}(k+1)=\mathbf{A x}(k)+\mathbf{D} \mathbf{w}(k) \\
& \mathbf{y}(k)=\mathbf{C x}(k)+\mathbf{H w}(k)
\end{aligned}
$$

that can reproduced the same output sequence $\{y(0), y(1), y(2), \ldots, y(q-1)\}$ subject to the same input sequence $\{w(0), w(1), w(2), \ldots, w(q-1)\}$. In case that the system is not $q$-identifiable, it is possible for $q$-Markov cover to construct the least square fit using linear model for the input-output sequence ([30] and [31]).

\subsubsection{Electrical VVT system bench test}

The bench test consists of a) closed-loop system identification and b) control system performance. The EVVT model identified uses the speed difference between the motor and half engine speed as the input. Since the engine speed has much slower dynamics than that of the EVVT system, and can be considered as a constant during the cam phasing. The resulting indentified model has the following form

$$
\Phi=G_{\text {evvt }}(s)\left(u-\frac{1}{2} R P M_{\text {engine }}\right)
$$

where $\Phi$ is the cam phase, $G_{\text {evvt }}(s)$ is the identified EVVT model, $u$ is the speed command from the controller and the constant $1 / 2 \cdot R P M_{\text {engine }}$ is half engine speed. The PRBS was used as reference signal for the closed-loop identification and its amplitude was selected to be 10 degrees centered at 20 degrees from the most retarded position. A proportional controller with gain of 70 was used for the system identification.

Table 16: Closed-loop identification parameters for EVVT bench

\begin{tabular}{|c|c|c|}
\hline Engine Speed (RPM) & 1000 & 1500 \\
\hline Input Sample Rate (ms) & 5 & 5 \\
\hline Output Sample Rate (ms) & 30 & 20 \\
\hline Output/Input Sample Ratio & 0.167 & 0.25 \\
\hline PRBS order & 13 & 13 \\
\hline Signal length (s) & 81.88 & 81.88 \\
\hline Markov parameter. \# & 100 & 100 \\
\hline ID open-loop model order & 4 & 4 \\
\hline
\end{tabular}

Due to the speed limitation on the test bench, the EVVT system was identified at 1000 and 1500RPM. A nominal system model was obtained as 


$$
G_{\text {evvt }}(s)=\frac{-9.7 s^{3}+139 s^{2}+5760 s+5785}{s^{4}+12.2 s^{3}+159 s^{2}-62 s+11}(\% / 1000 r p m)
$$

An OCC controller [24] was designed for the EVVT system bench. The system plant matrices of the nominal model were obtained from equation (14)

$$
\begin{aligned}
& \mathbf{A p}=\mathbf{A}=\left[\begin{array}{cccc}
-12.2 & -159 & 62 & -11 \\
1 & 0 & 0 & 0 \\
0 & 1 & 0 & 0 \\
0 & 0 & 1 & 0
\end{array}\right], \mathbf{B p}=\mathbf{D p}=\mathbf{B}=\left[\begin{array}{l}
1 \\
0 \\
0 \\
0
\end{array}\right] \\
& \mathbf{C p}=\mathbf{M p}=\mathbf{C}=\left[\begin{array}{lll}
-9.6 & 139.9 & 5756 \quad 5783
\end{array}\right], \mathbf{D} \cong \mathbf{0}
\end{aligned}
$$

Controller design parameters were selected as

$$
\mathbf{W}_{\mathbf{p}}=1, \quad \mathbf{V}=0.01, \quad \mathbf{R}=[1]
$$

Using the OCC iterative control design algorithm in [24], an OCC controller can be obtained

$$
K_{\text {OCC }}(s)=\frac{6 \times 10^{4} s^{3}+8.5 \times 10^{5} s^{2}+1 \times 10^{7} s+6.7 \times 10^{7}}{s^{4}+148 s^{3}+6937 s^{2}+8.6 \times 10^{4} s+8.2 \times 10^{4}} r p m /^{\circ}
$$

A proportional controller (38) was tuned for performance comparison. The proportional controller was tuned to achieve similar balance between fast response time and low overshoot at both 1000 and 1500 RPM as the OCC controller, while using a square wave as reference signal, see Figure 79.

$$
K_{P}(s)=80\left(r p m /^{\circ}\right)
$$
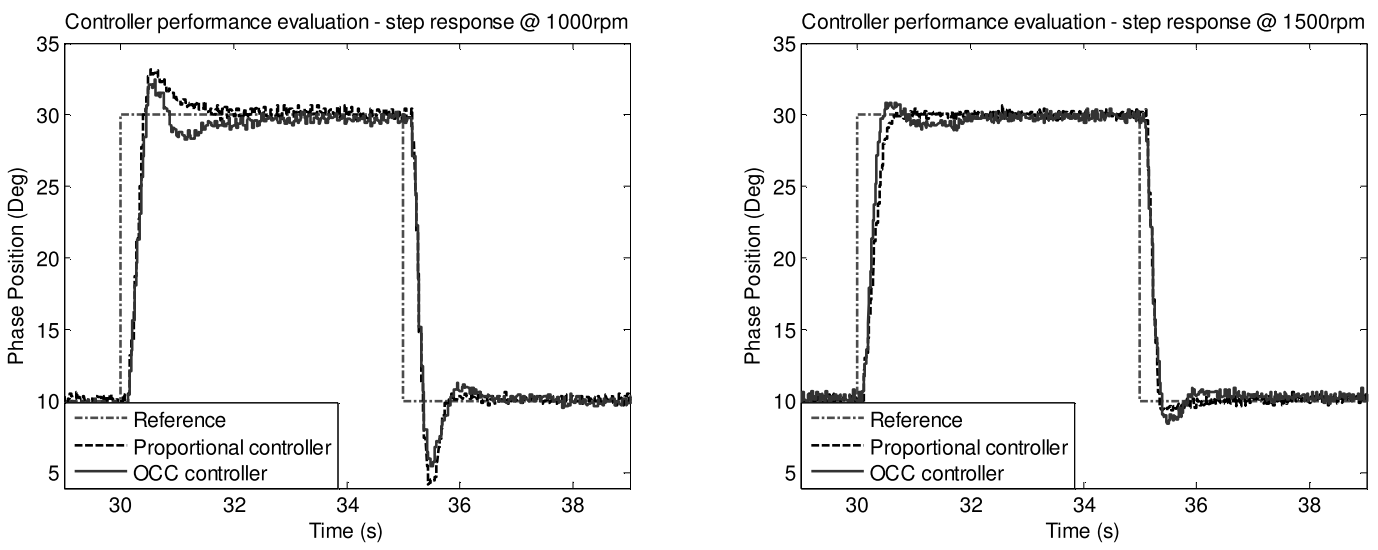

Figure 79: Step response comparison on EVVT bench

The controllers were tested at 1000 and 1500 RPM. Both proportional and OCC controllers have feedforward portions from engine speed shown in (39) Different reference signals were used to validate the trajectory tracking performance of the EVVT system. 


$$
r \dot{e} f=\frac{s}{0.05 s+1} r e f
$$

A 20 crank degree phase advance/retard that completes in 3 engine cycles, with the phase changed by $10(50 \%), 6.67(33 \%)$ and $3.33(16.7 \%)$, respectively, was also used as reference signal to simulate the phase change during the SI-HCCI mode transition (Figure 80). From the plot, it is shown that the OCC controller has less overshoot than the proportional controller at 1000 RPM. At 1500 RPM, overshoots for both controllers are very low. The OCC controller has a faster response time than the proportional controller in both engine speeds. Settling times for the two controllers are also very close to each other.
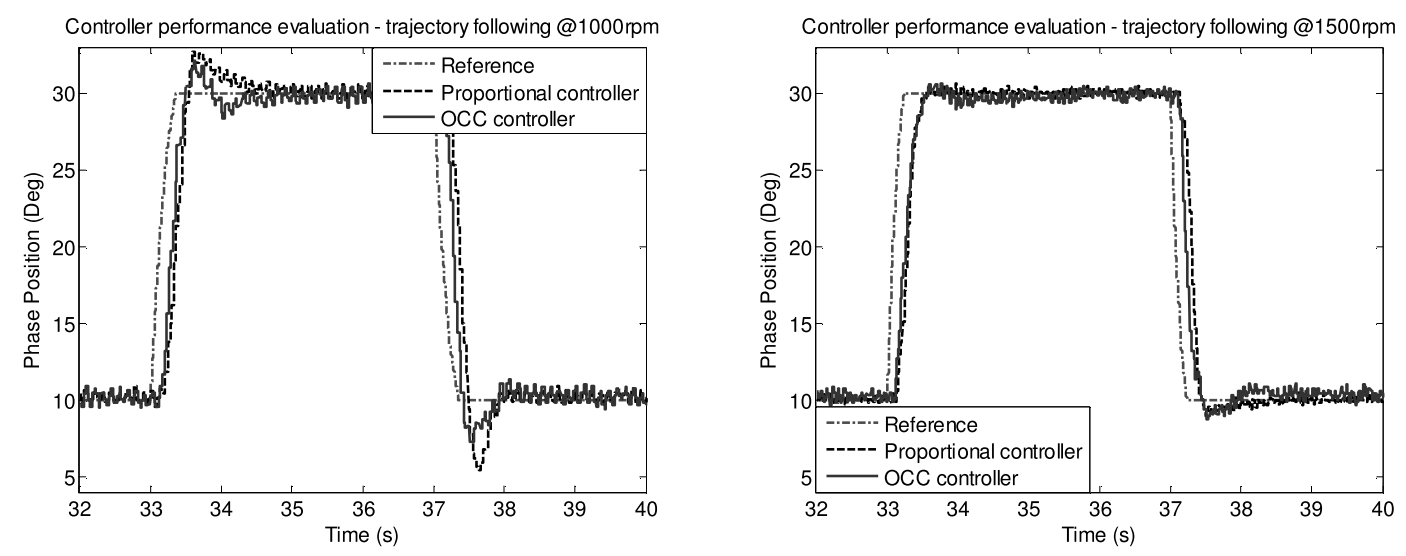

Figure 80: Trajectory tracking comparison on EVVT bench

In order to further investigate trajectory tracking performance for the EVVT controllers, a series of sinusoidal waves was used to test the frequency response of the closed-loop systems. The amplitude of the sinusoidal signal was set to be 10 degrees and centered at 20 degrees from the most retarded position, and the frequencies of the signals vary from $0.01 \mathrm{~Hz}$ to $2 \mathrm{~Hz}$. The test results show that both controllers have very good tracking performance at low frequency (Figure 81 , Figure 82 and Table 17). When the excitation frequency increases, both controllers have performance decay. The two controllers have almost identical gains at different frequencies, but the OCC has a lower phase delay compared to the proportional controller.

It is observed that when the engine is running at $1000 \mathrm{RPM}$, the closed-loop EVVT system has overshoots when the excitation frequency is closed to $1 \mathrm{~Hz}$. After $1.5 \mathrm{~Hz}$, the system gain quickly decays. The identified fourth order nominal model in (14) does not show similar behavior at $1 \mathrm{~Hz}$. A seventh order model was obtained using closed-loop identification at 1000 RPM and its predicted performance was compared to the measured data (Figure 83:). The overshoot of the closed-loop system is able to be duplicated under simulation environment using the identified model at 1000 RPM. However the physical dynamics behind the phenomenon are still unknown and will be investigated in the future. 
Table 17: Frequency response of close-loop EVVT system

\begin{tabular}{|c||c|c|c|c||c|c|c|c|}
\hline \multirow{2}{*}{\multicolumn{1}{|c||}{$\begin{array}{c}\text { Input } \\
\text { Freq. } \\
(\mathrm{Hz})\end{array}$}} & \multicolumn{4}{c||}{$1000 \mathrm{RPM}$} & \multicolumn{4}{c|}{1500 RPM } \\
\cline { 2 - 9 } & Magnitude & \multicolumn{2}{c|}{ Phase (deg) } & \multicolumn{2}{c|}{ Magnitude } & \multicolumn{2}{c|}{ Phase (deg) } \\
\hline \hline 0.01 & 1 & 1 & 6 & 5 & 1 & 1 & 4 & 4 \\
\hline 0.1 & 0.98 & 0.98 & 21 & 16 & 0.97 & 0.97 & 21 & 9 \\
\hline 0.2 & 0.97 & 0.96 & 30 & 19 & 0.95 & 0.96 & 33 & 19 \\
\hline 0.4 & 0.95 & 0.97 & 46 & 30 & 0.93 & 0.95 & 50 & 21 \\
\hline 0.8 & 1.18 & 1.18 & 74 & 49 & 0.96 & 1.02 & 66 & 46 \\
\hline 1 & 1.33 & 1.35 & 94 & 58 & 0.95 & 0.98 & 87 & 58 \\
\hline 1.5 & 1.05 & 1.04 & 161 & 108 & 0.82 & 0.78 & 119 & 91 \\
\hline 2 & 0.70 & 0.72 & 202 & 137 & 0.60 & 0.61 & 144 & 115 \\
\hline
\end{tabular}
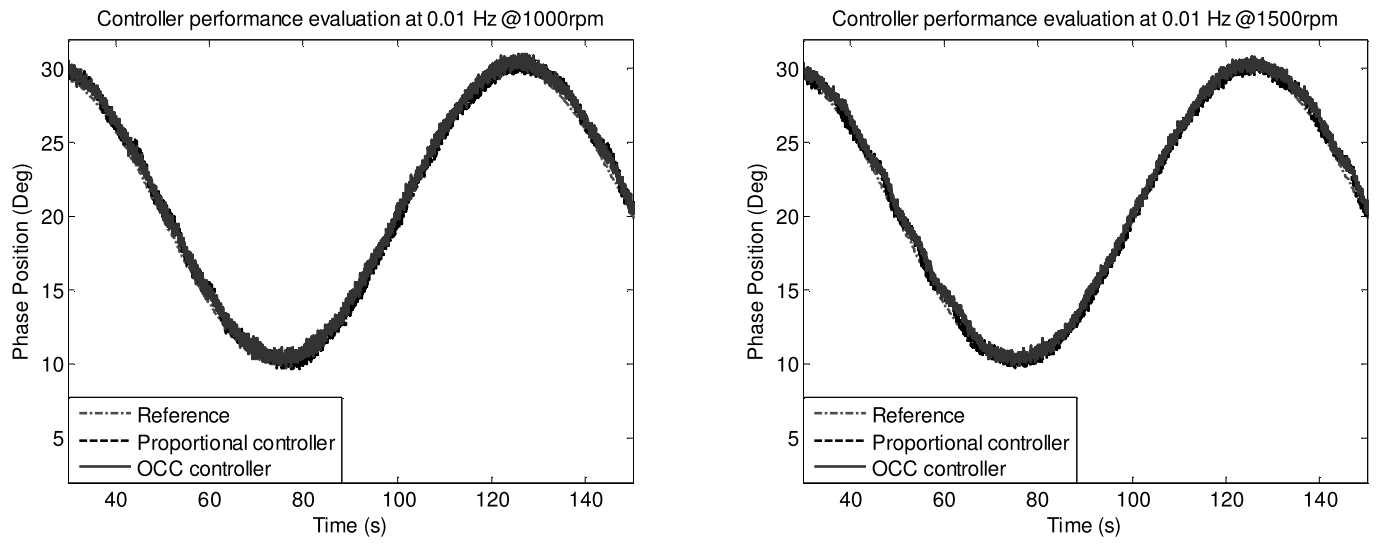

Figure 81: Frequency response of the closed-loop EVVT system
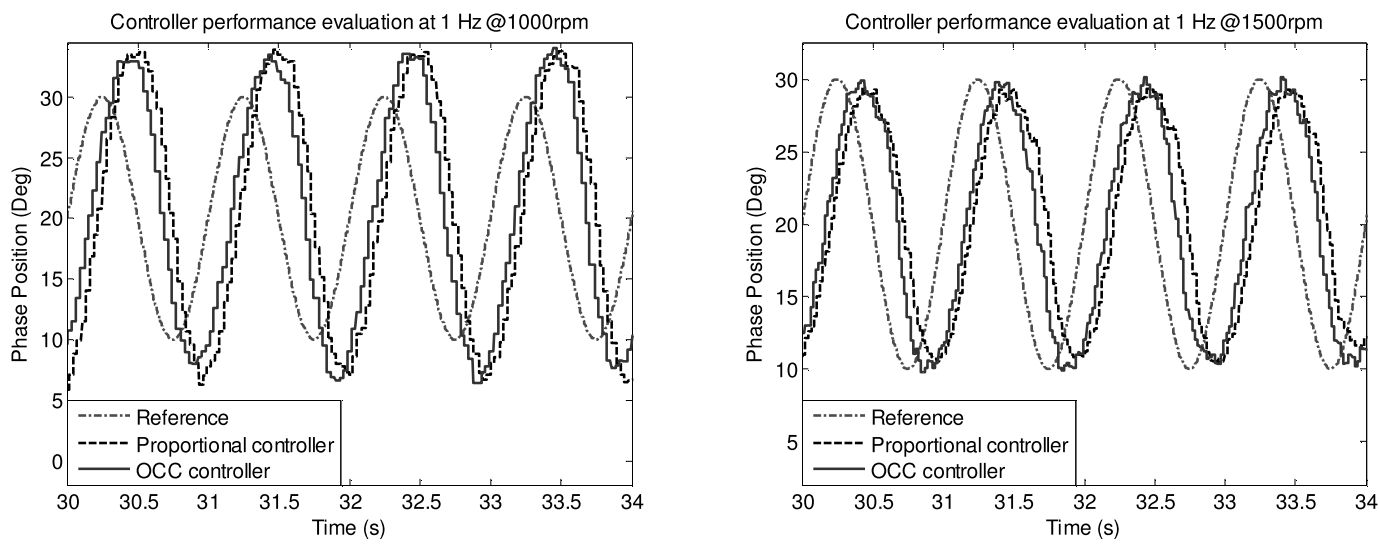

Figure 82: Frequency response of the closed-loop EVVT system (continued) 


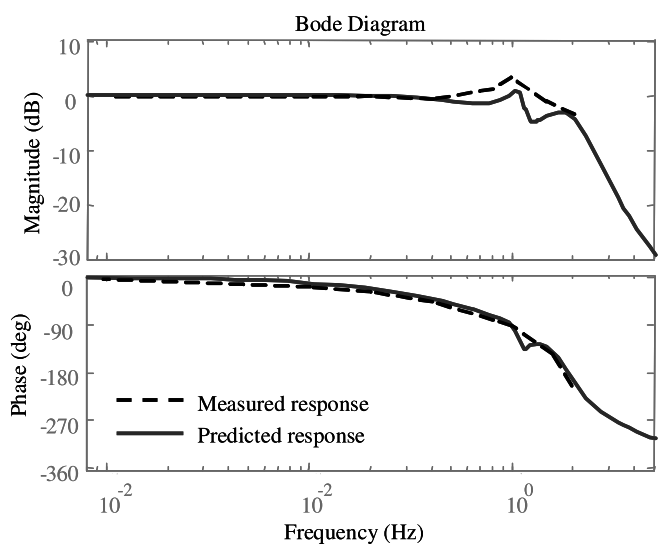

Figure 83: Measured and predicted VVT frequency response at 1000 RPM

\subsubsection{Effect of engine oil viscosity}

Two different types of engine oil, SAE 5W20 and SAE 30, were used during the bench test. The engine was running at $1500 \mathrm{RPM}$ at room temperature $\left(25^{\circ} \mathrm{C}\right.$ ), and $\mathrm{OCC}$ controller (37) was used in both cases. The reference signal was a 20 degree advance step. The 10 to 90 percent rising-time was 0.3 second with SAE 5W20, and 0.48 second with SAE 30 engine oil. The response time difference is due to the friction in the planetary gear system caused by the engine oil viscosity. Figure 84 shows the system response and normalized speed difference between the EVVT motor and half engine speed (750 RPM). The VVT motor speed was saturated at 30 units above 750 RPM with SAE 30. With SAE 5W20, the motor speed was saturated at about 50 units above 750 RPM. The system response time is inversely proportional to the difference between the EVVT motor speed and half of the engine speed. Notice that the EVVT motor speed is measured by calculating PWM frequency from the EVVT local speed controller and has some error (spikes) during the calculation process.

The test result also suggests that the no-friction assumption during the simulation study is not true. On an engine, the nominal operating temperature is much higher than room temperature of the test bench and the engine oil has lower viscosity as a result. The EVVT system will respond much faster when it is installed on an engine than on the test bench.
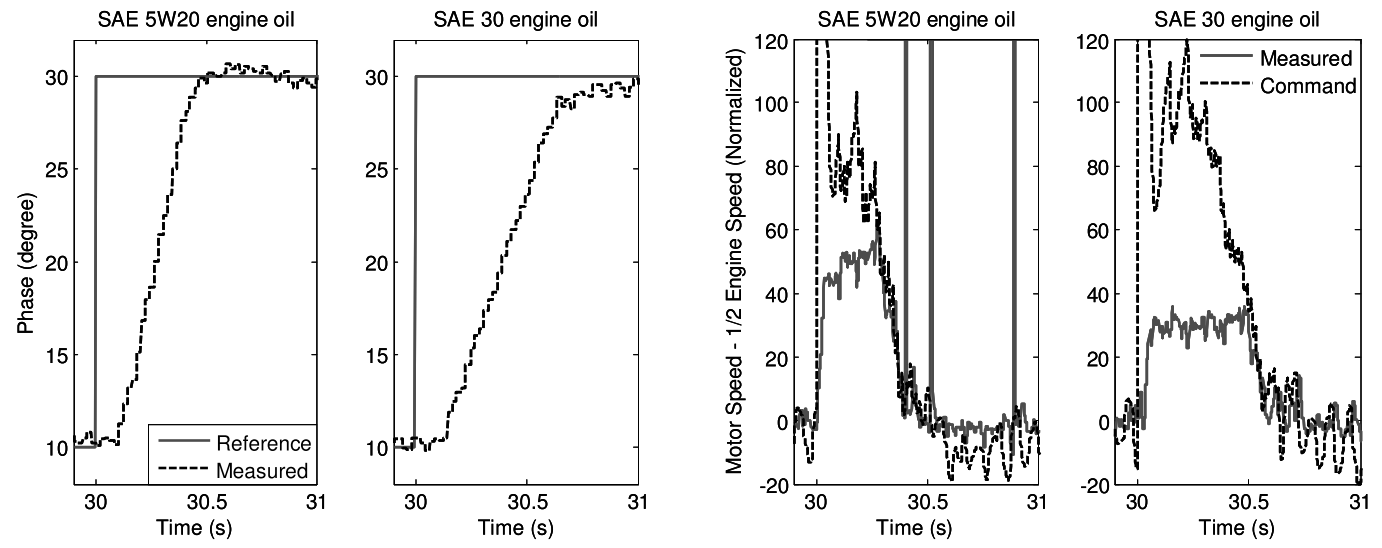

Figure 84: Impact of engine oil viscosity to EVVT response time

As a summary, a test bench of the EVVT (electrical variable valve timing) system was developed and the EVVT system plant model was obtained by using closed-loop system identification. The 
model has very similar closed-loop response compared to the physical system. An OCC (output covariance constraint) controller was developed based on the identified model. Different reference signals were used to test the controller performance. The test results showed that the OCC controller has a faster response time compared to a well-tuned proportional controller with reduced phase delay. The bench test results also show that the EVVT response time is fast enough for the SI and HCCI combustion mode transition (20 degree phasing within three engine cycles).

The impact of engine oil viscosity to the response time of an EVVT system was also investigated. The test results showed that the engine viscosity has a heavy impact on the EVVT response time. The results suggested that it is necessary to use low viscosity engine oil to achieve the maximum performance. This could mean either operating at a high oil temperature or using low viscosity engine oil.

The system identification and EVVT control design results have been summarized into two papers published in 2011 American Control Conference [39] (see Subsection 9.11) and 2013 ASME Journal of Dynamic System, Measurement and Control [40] (see Subsection 9.4).

\subsubsection{Electrical cam phaser supply selection}

MSU and Chrysler also finalized the integration of the electrical cam phaser onto the target engine head and the final selection of Denso electrical VVT actuator. Figure 85 shows the electrical cam phasing actuator design. The finalized design was sent to Denso.

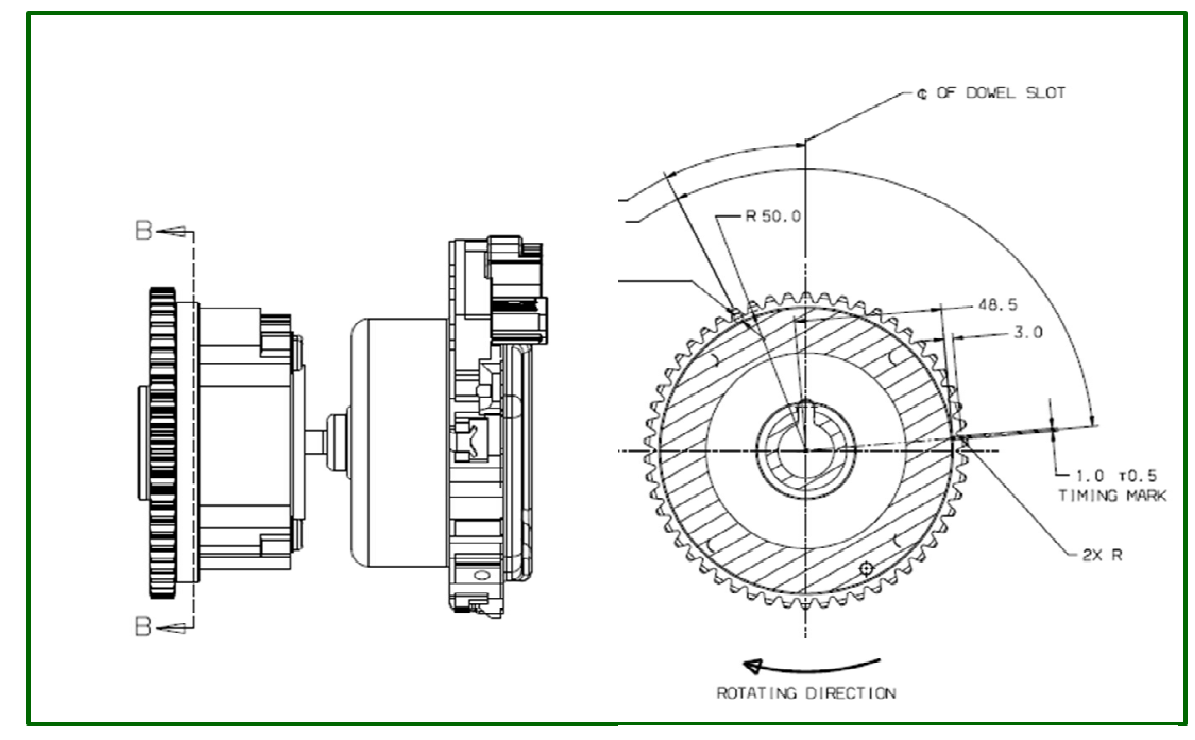

Figure 85: Prototype engine controller I/O box in progress

Denso delivered the electrical VVT actuator and it was installed on a test bench for the tests conducted in Subsections 3.34 and 3.35. 


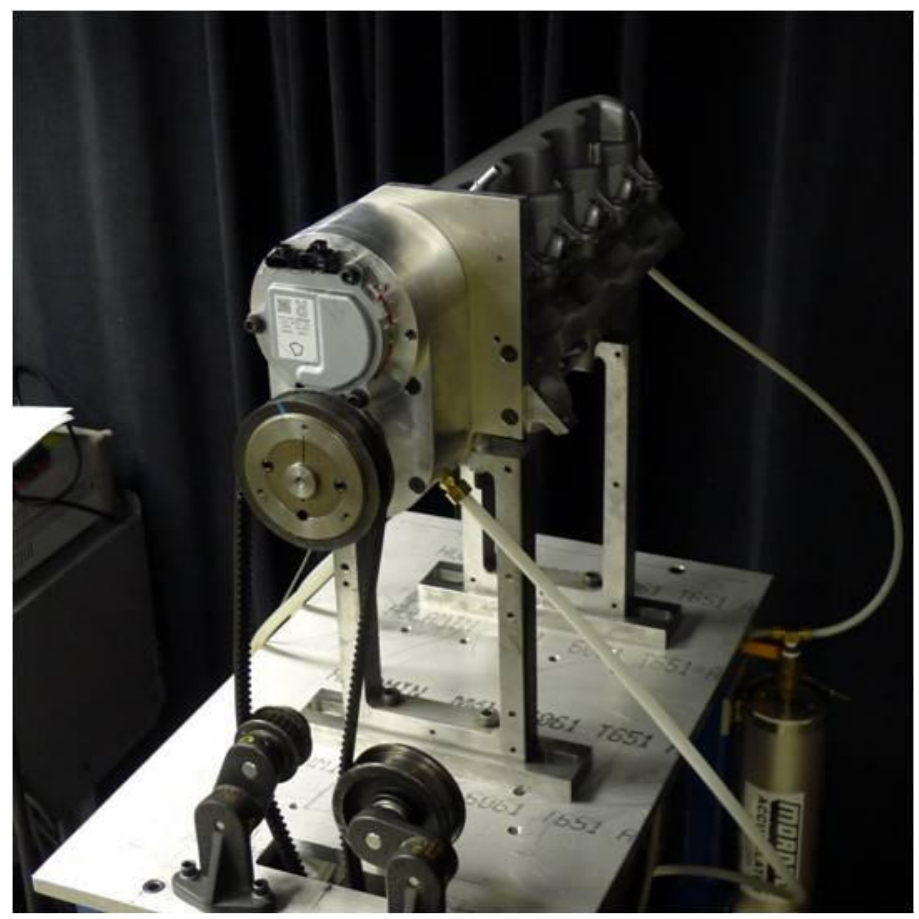

Figure 86: Electrical cam phasing system test bench

\subsection{Ionization based ignition system design}

The original ignition system of the target engine was modified due to the fact that ionization detection requires the ignition coil without the embedded diode. Chrysler worked with their ignition coil supply and provided MSU with ignition coil without the embedded diode.

Due to the high ignition energy requirement of the SI-HCCI operations, the ionization system for high energy ignition system was redesign and fabricated. The test results show significant improvement on ionization signal bandwidth. Figure 87 shows the fabricated ionization ignition drive box. 


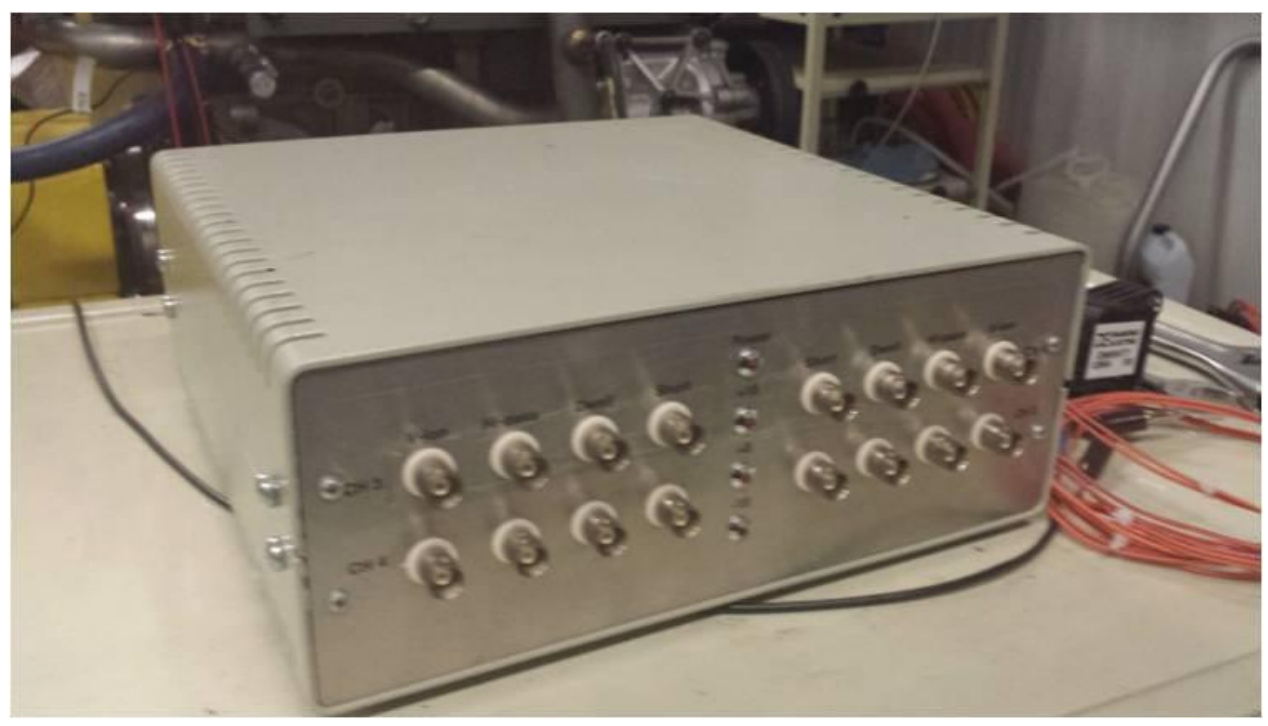

Figure 87: Prototype ionization capable ignition drive box

\subsection{Engine system integration}

The engine system integration was completed by modifying the metal engine configuration based upon the optical engine valve-train design. We concentrated to modify the engine pistons to increase the compression ratio to around 12.5 and worked closely with Chrysler and Mahle to fabricate these blank pistons so that they can be machined to reach the desired compression ratio.

The bentch validation of the ptototype engine control system, capable of controlling the EVVT and two-step valve systems, intake heater, electronic throttle, ignition and fuel in jection systems, was completed. This prototype controller will be used to control the optical engine system and multi-cylinder engine system.

\subsection{Implementation of control-oriented engine model into HIL simulator}

Before implementing the developed control-oriented engine model into the MSU HIL simulation environment, gas exchange and combustion model was updated; see Subsections 3.6.1 and 3.6.2. The detailed results have been summarized in a paper published in 2013 IEEE Transaction on Vehicle Technology [41]; see Subsection 9.2.

\subsubsection{Gas exchange model modification}

During the last reporting period, a control-oriented dual-zone SI-HCCI hybrid combustion model was developed. Model simulation validation shows that this model performs very well during the combustion process. However, in the gas exchange phase, it leads to large error of the in-cylinder pressure signal. The main reason is that the gas exchange process is very complicated and difficult to model. A CFD (computational fluid dynamics) model could achieve fairly accurate simulation results, but it is impossible to be used in the control-oriented model due to the realtime simulation requirement. Thus, a simple first-order tracking approach was used to approximate the in-cylinder pressure. Although this does not affect the accuracy of the IMEP (indicated mean effective pressure) calculation, it leads to large in-cylinder pressure and engine 
torque errors, which could affect the estimation of the residual EGR and temperature. Especially in NVO (negative valve overlap) case, using the first-order approximation for the in-cylinder pressure leads to higher in-cylinder pressure than the manifold pressure during the intake process, therefore, no fresh gas can enter into the cylinder. This is the main motivation for us to modify the model-based upon the fluid mechanics as follows.

The widely used equation for the air flow rate can be described as follows:

$$
\begin{cases}\dot{m}=\frac{C_{D} A_{R} p_{0}}{\left(R T_{0}\right)^{1 / 2}}\left(\frac{p_{T}}{p_{0}}\right)^{1 / \gamma}\left\{\frac{2 \gamma}{\gamma-1}\left[1-\left(\frac{p_{T}}{p_{0}}\right)^{(\gamma-1) / \gamma}\right]\right\}^{1 / 2} & p_{T} / p_{0}>[2 /(\gamma+1)]^{\gamma /(\gamma-1)} \\ \dot{m}=\frac{C_{D} A_{R} p_{0}}{\left(R T_{0}\right)^{1 / 2}} \gamma^{1 / 2}\left(\frac{2}{\gamma+1}\right)^{(\gamma+1) /(\gamma-1)} & p_{T} / p_{0} \leq[2 /(\gamma+1)]^{\gamma /(\gamma-1)}\end{cases}
$$

The intake process of NVO can be divided into the following three stages:

1) At the beginning of the intake valve opening, the in-cylinder pressure is greater than the manifold pressure. Hence, some fraction of the residual gas escapes into the manifold through the intake valve, where $p_{0}$ is the in-cylinder pressure; $p_{T}$ is the manifold pressure; and $T_{0}$ is the in-cylinder temperature. During this process, the in-cylinder pressure reduces significantly.

2) Once the in-cylinder pressure is lower than the manifold pressure, the air charge enters the cylinder. In order to simplify this physical process, it is assumed that the escaped residual gas will not be mixed with the fresh air in the manifold and will come back into the cylinder completely. Then, $p_{0}$ is the manifold pressure; $p_{T}$ is the in-cylinder pressure; and $T_{0}$ is the escaped gas temperature that is lower than the gas temperature before the intake valve opening due to the heat transfer with the fresh air.

3) When all the escaped residual gas goes back to the cylinder, the fresh air charge starts. During this stage, $p_{0}$ is the manifold pressure; $p_{T}$ is the in-cylinder pressure; and $T_{0}$ is the manifold temperature.

The continuous time differential equation is complicated to solve since the pressure and temperature are coupled. We used the mass conservation equation below.

$$
\frac{d}{d t} \iiint \rho d V=\frac{\partial}{\partial t} \iiint \rho d V+\iint \rho \vec{v} \cdot \vec{n} d A=0
$$

By using the previous crank-degree's pressure to calculate the mass flow $\dot{m}$ approximately, the mass conservation equation becomes

$$
p=(\tilde{p} \tilde{v}+\dot{m} R \tilde{t} \cdot 60 / r p m) \cdot t / v
$$


where $\tilde{p}, \tilde{v}$, and $\tilde{t}$ denote the previous crank-degree's values, $\dot{m}$ is negative in the first stage and positive in the second and third stages. Note that the in-cylinder temperature $t$ also satisfies

$$
t=\frac{p v}{m R}
$$

The successive approximation method was used to solve $p$ and $t$ using an iterative approach. Figure 88 shows the online simulation results, comparing with that form the previous engine model. One can see that the in-cylinder pressure during intake process is much more close to actual one, comparing with that of the first-order approximation method.

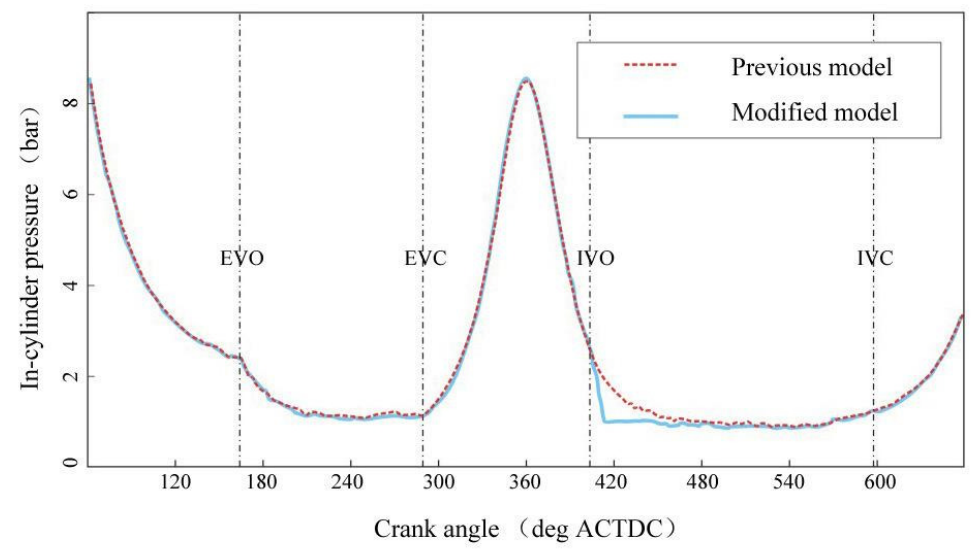

Figure 88: Real-time simulation results using HIL

\subsubsection{Multi-zone SI-HCCI combustion model modifying}

In the previous work a dual-zone combustion model was used for the SI combustion mode and a one-zone model was used for the HCCI combustion mode. It was based on the assumption that the fuel, air, and residual gas charges are uniformly premixed at IVC. Therefore, it was assumed that there are two zones in the cylinder, burned and unburned zones, for the SI combustion (as shown on left side of Figure 89 and only one zone for HCCI combustion. However, at the beginning of combustion, some portion of the residual gas will not be well mixed with the fresh charge air, and it is called the fraction of the unmixed residual gas (usually among 2\% 10\%). Ignoring the fraction of the unmixed residual gas could result in certain modeling error and hence leading to poor model-based control. This is due to the fact that this fraction has quite different temperature from the other zones and the volume and AFR (air-to-fuel ratio) of the mixed zone would be affected. The dual-zone combustion model was modified into a three-zone model for the SI combustion mode (as shown on right side of Figure 89) and a two-zone model for the HCCI combustion mode. In Figure 89, one can see that at the bottom of the cylinder is assumed for the unmixed residual gas zone and at the top are the burned and unburned zones. 


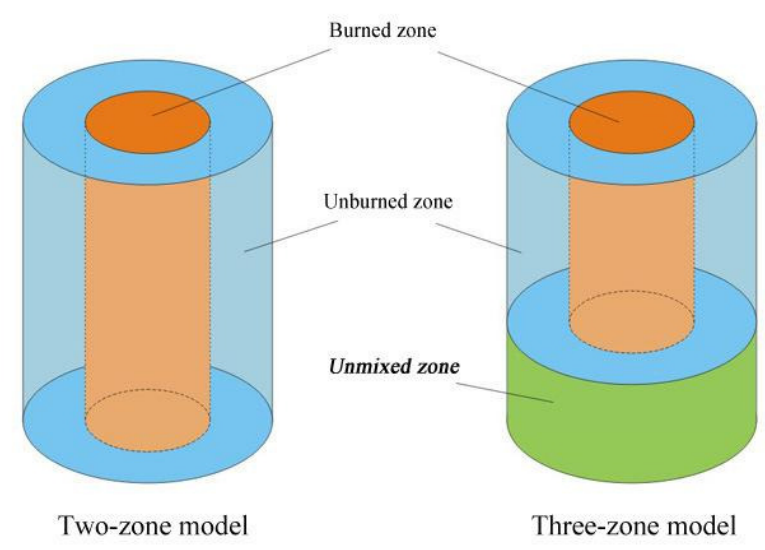

Figure 89: Two-zone model and three-zone model

For the unmixed zone, the residual gas is considered as the ideal gas and the entire combustion process is approximated by a combination of an isentropic volume change process of ideal gas in a closed system and a heat transfer process. The previous crank-degree's pressure was used to approximate the current pressure to solve the unmixed zone volume, and the summation of the two volumes for the burned and unburned zones is the total cylinder volume minus the unmixed zone volume. All the parameters of the burned and unburned zones are solved using the five governing equations described in the previous report.

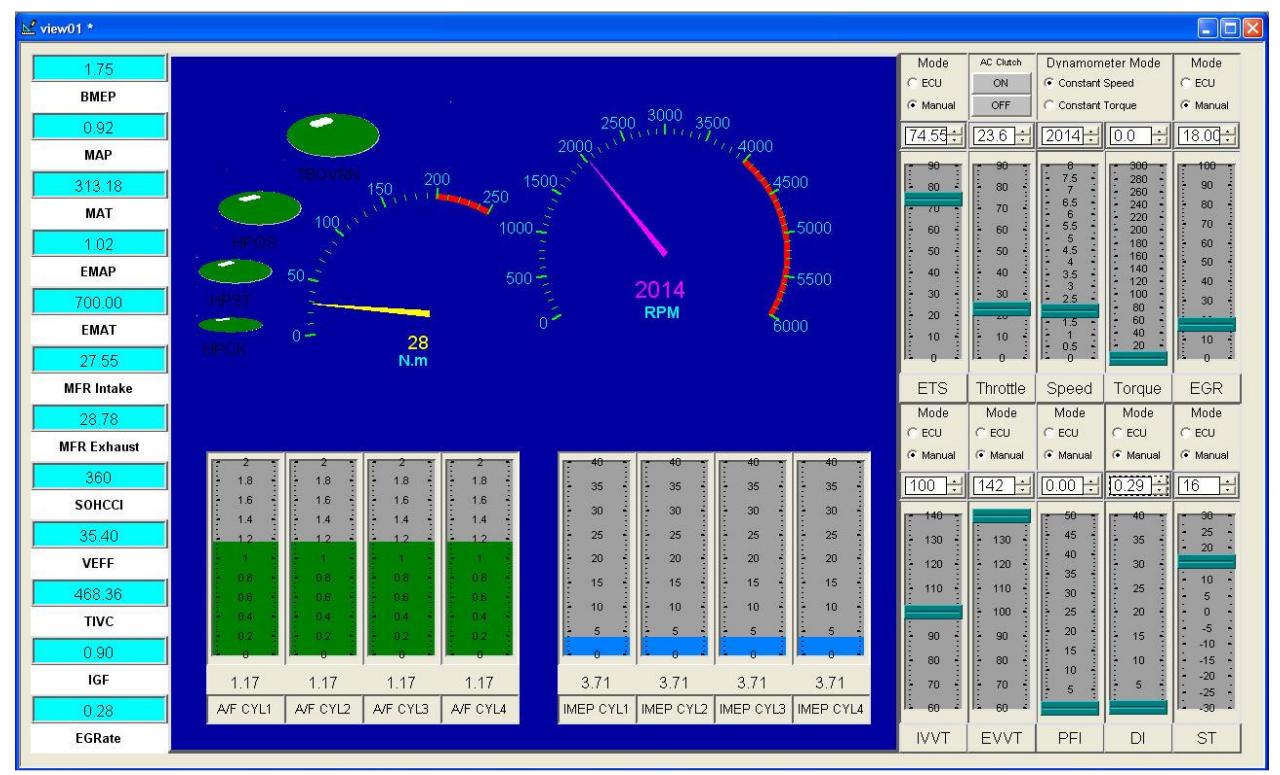

Figure 90: GUI of the dSPACE HIL system

\subsubsection{Simulink model implementation}

The finalized control-oriented engine model has also be implmented into Matlab/Simulink environmen validated by off-line simulation using calibrations obtained from both the GT-Power 
simulations and optical engine tests. The validated Simulink engine model was implemenetd into MSU dSPACE HIL simulation environment, see Figure 121 for the HIL GUI interface.

\subsection{Engine prototype controller design and fabrication}

The prototype engine controller was based upon a low industrial cost PC-based Opal-RT realtime simulator with a customized engine control I/O box. The following is a list of work conducted:

a) MSU worked closely with the prototype engine controller supplier, Opal-RT, and designed an engine controller that is suitable for controlling the SI and HCCI dual model combustion of both optical and metal engines, where the engine controller is able to sample the combustion information (such as in-cylinder pressure) every crank degree for closed-loop combustion control.

b) After the engine controller was designed, the engine controller I/O box design was completed and fabricated. Figure 91 shows the designed and partially assembled engine controller I/O box.

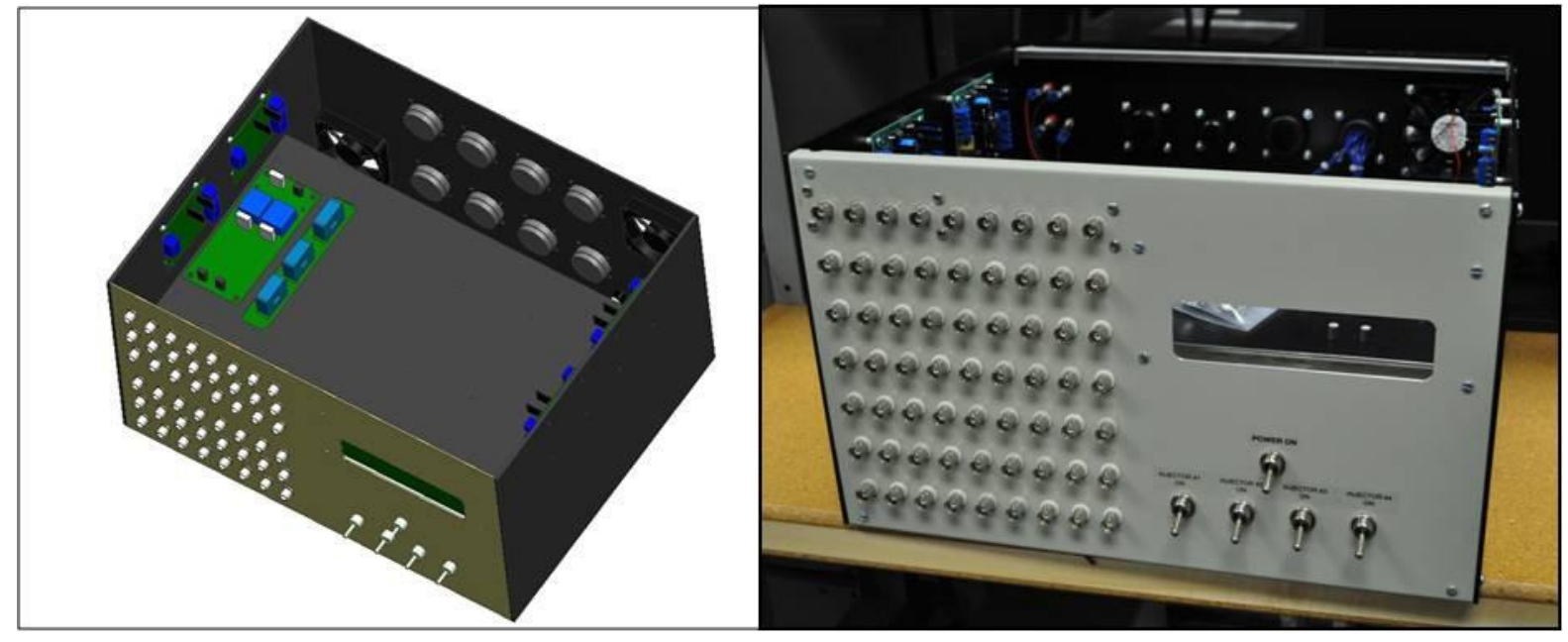

Figure 91: Prototype engine controller I/O box design

The engine controller $\mathrm{I} / \mathrm{O}$ box is capable of multiple fuel injections (four channels), multi-strike spark (four channels), electrical cam phasing controls (two channels), two-step lift controls (two channels), external EGR control, throttle control ([42] and [43]), etc. Figure 91 shows the finalized I/O box and the Opal-RT based engine controller and its I/O box is shown in Figure 40. The key feature of the Opal-RT based engine prototype controller is that it is able to sample the in-cylinder pressure signal every crank degree, which is the key for in-cylinder pressure signal based closed-loop combustion control. The engine control strategy is programmed in Matlab/Simulink so that the control strategy developed in Matlab/Simulink can be imported into the Opal-RT based engine controller directly. 


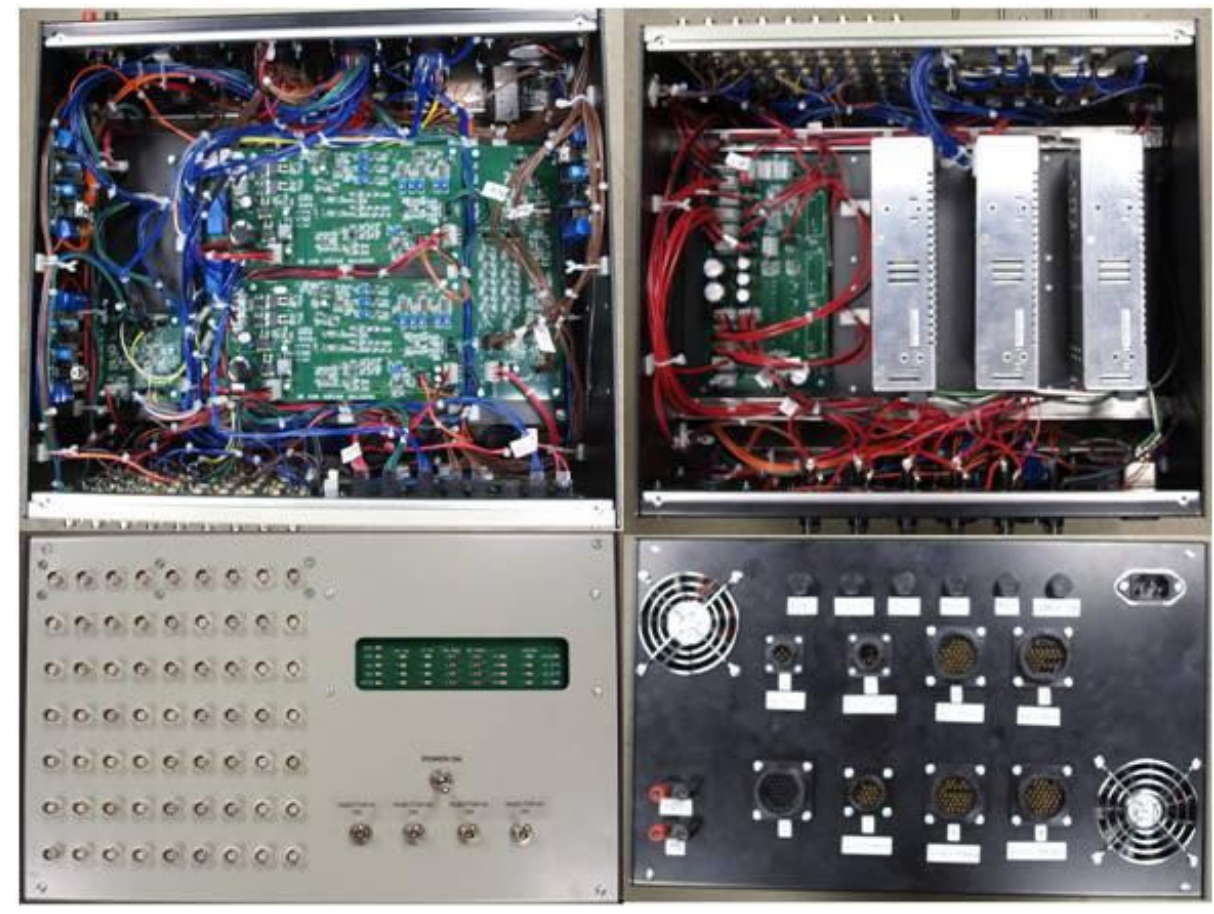

Figure 92: Prototype engine controller I/O box completed

\subsection{Summary}

Optical engine combustion tests were conducted in both SI and HCCI combustion modes and the test results were used to calibrate the developed control-oriented engine model. Intensive GTPower simulations were conducted to determine the optimal valve lifts (high and low) and the cam phasing range. Delphi was selected to be the supplier for the two-step valve-train and Denso to be the electrical variable valve timing system supplier. A test bench was constructed to develop control strategies for the electrical variable valve timing (VVT) actuating system and satisfactory electrical VVT responses were obtained. Target engine control system was designed and fabricated at MSU for both single-cylinder optical and multi-cylinder metal engines. Finally, the developed control-oriented engine model was successfully implemented into the HIL simulation environment. 


\section{Phase III: Closed-loop Combustion Control for SI and HCCI Modes}

This maturation phase includes development of closed-loop combustion control strategies, where the developed strategies were validated in HIL simulation environment and dynamometer tests. As a parallel effort, the mode transition closed-loop combustion control strategies was be developed and evaluated in HIL simulations.

\subsection{SI and HCCI dual-mode combustion engine fabrication}

\subsubsection{Engine system design and fabrication}

The compression ratio of the original engine provided by Chrysler is around 9.8, in order to increase the compression ratio from 9.8 to around 12.5, the original engine piston top was redesigned to achieve compression ratio of 12.5 and blank pistons were also ordered in case that different compression ratio is required. The following figure shows both pistons manufactured by Mahle.

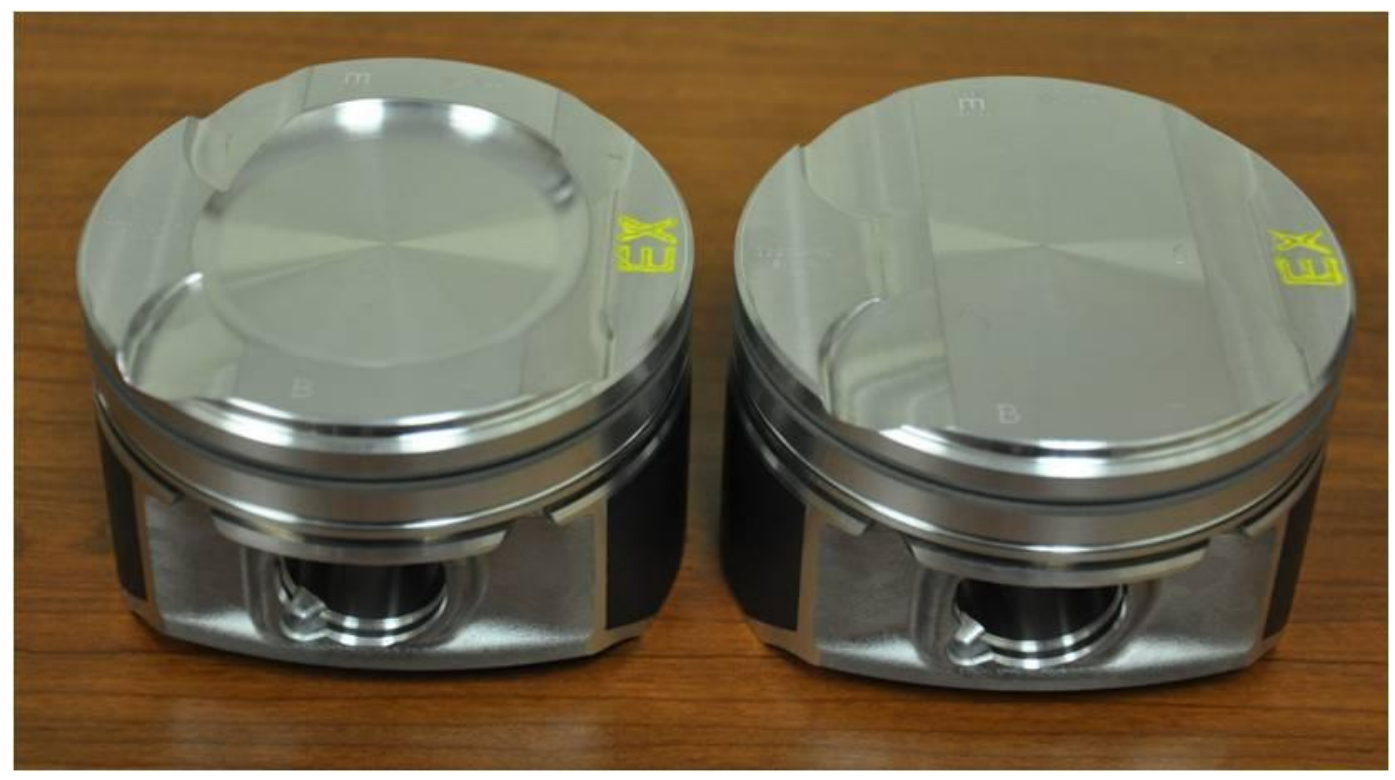

Figure 93: Modified engine piston with compression ratio of 12.5 and blank piston

In order to install the two-step valve system, the original cylinder head was machined to fit the two-step lift hydraulic actuators and lift control solenoid actuators. In order to enable the closedloop combustion control, each cylinder was also instrumented with Kistler in-cylinder pressure sensor. Also, to protect the two-step lift actuators, an auxiliary oil pump was installed to generate the oil pressure when the engine is cranked so that the valve lift will be locked at low lift position. The auxiliary oil pump will be shut down after the engine is motored to the desired speed so that the engine oil pressure will be maintained by the engine oil pump.

The engine cam gear housing was redesigned (see Figure 94) and machined to fit the electrical cam phaser for both intake and exhaust cam shafts. The original cam sensors on the engine were used for detecting cam position four times every engine cycle. The developed closed-loop electrical cam phasing control system for the single cylinder optical engine was retuned for the 
four-cylinder metal engine to ensure good tracking performance. The tuned cam timing control system was validated on the engine.

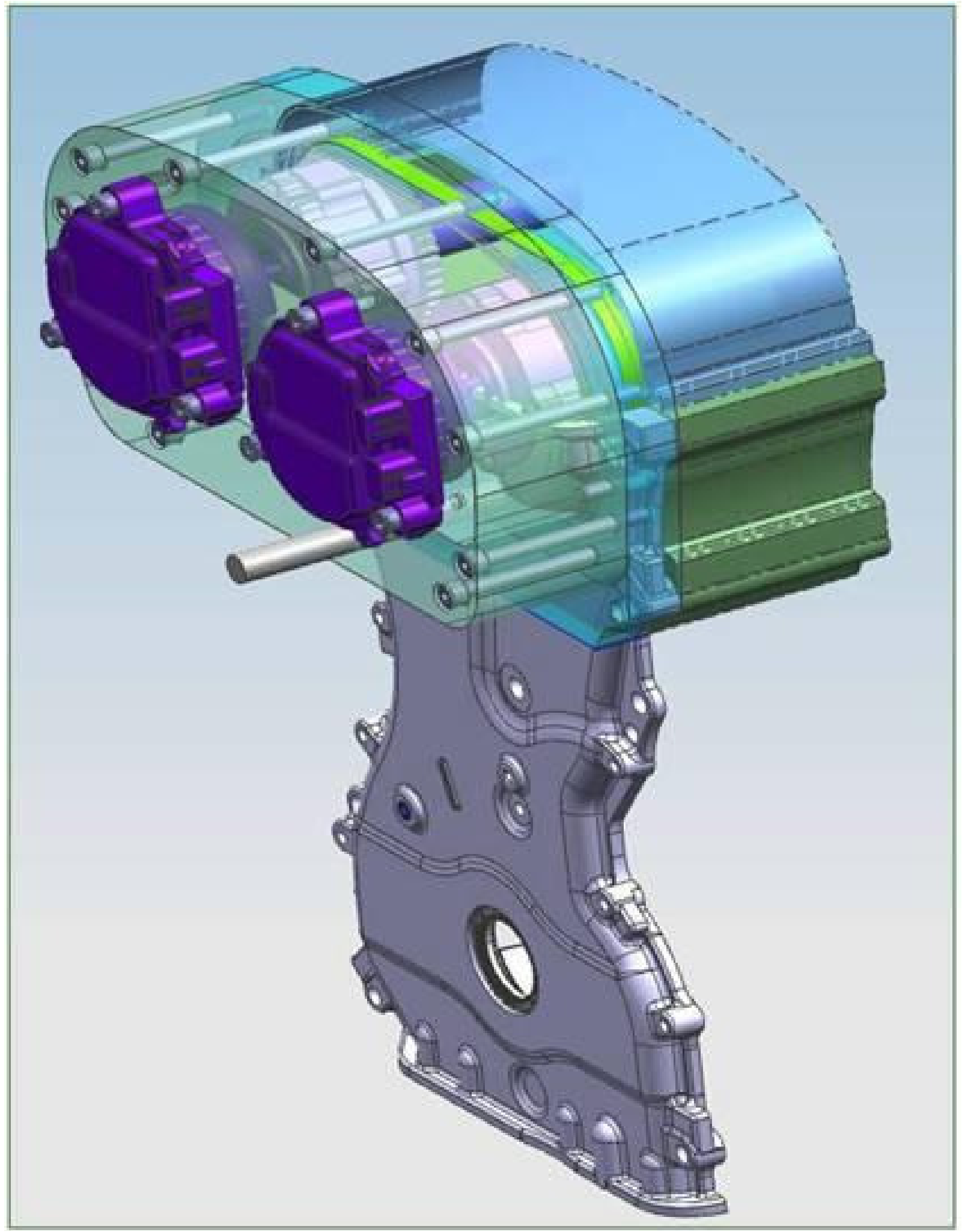

Figure 94: Electrical cam phasing system and timing belt design 
In order to warm up the engine quickly without running engine for a long time, an engine coolant heating system, see the right side of Figure 95 was developed using an external coolant pump and heating system to circulate the heated coolant when the engine is not running. After the engine start running and reaches its target speed, the external coolant pump stops and the engine water pump will take over.

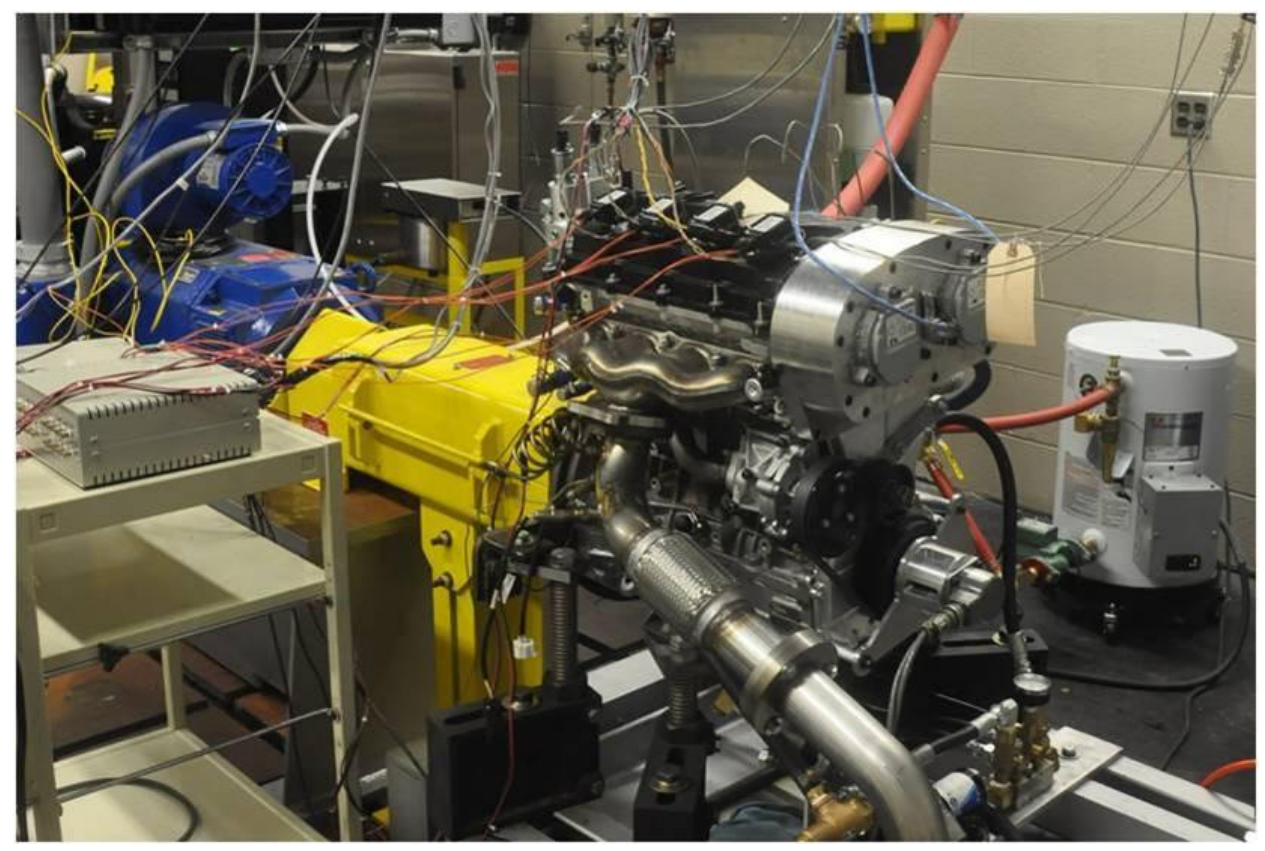

Figure 95: Target metal installed in MSU dynamometer room

Note that the engine was built using a used engine block with a modified head since a new engine was not available; and the used engine block caused certain performance issue later during the performance tests.

\subsubsection{Dynamometer setup for engine tests}

To enable closed-loop combustion control, the developed single cylinder engine prototype controller (Opal-RT based) has expanded to four cylinder operation both from hardware and software point of view. The right side of Figure 96 shows the four cylinder prototype engine controller and its I/O box, where the laptop is the engine controller host machine using to input the control command for the engine controller.

The Opal-RT based engine controller utilizes Simulink based control software. The graphic interface shown in Figure 97 is the Simulink control diagram running in real-time so that the engine control parameters can be adjusted instantly. 


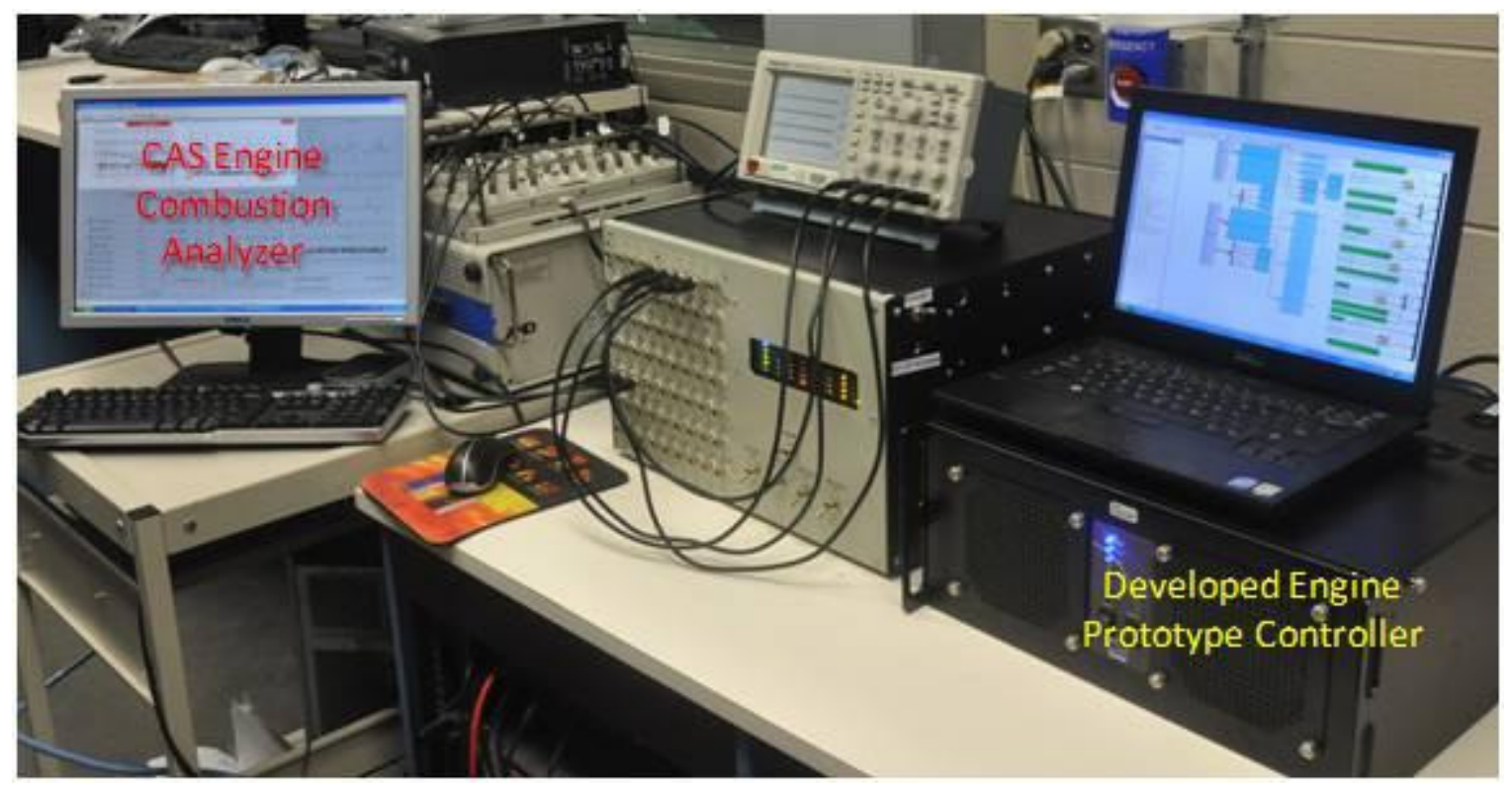

Figure 96: Engine prototype controller and dynamometer combustion analysis system

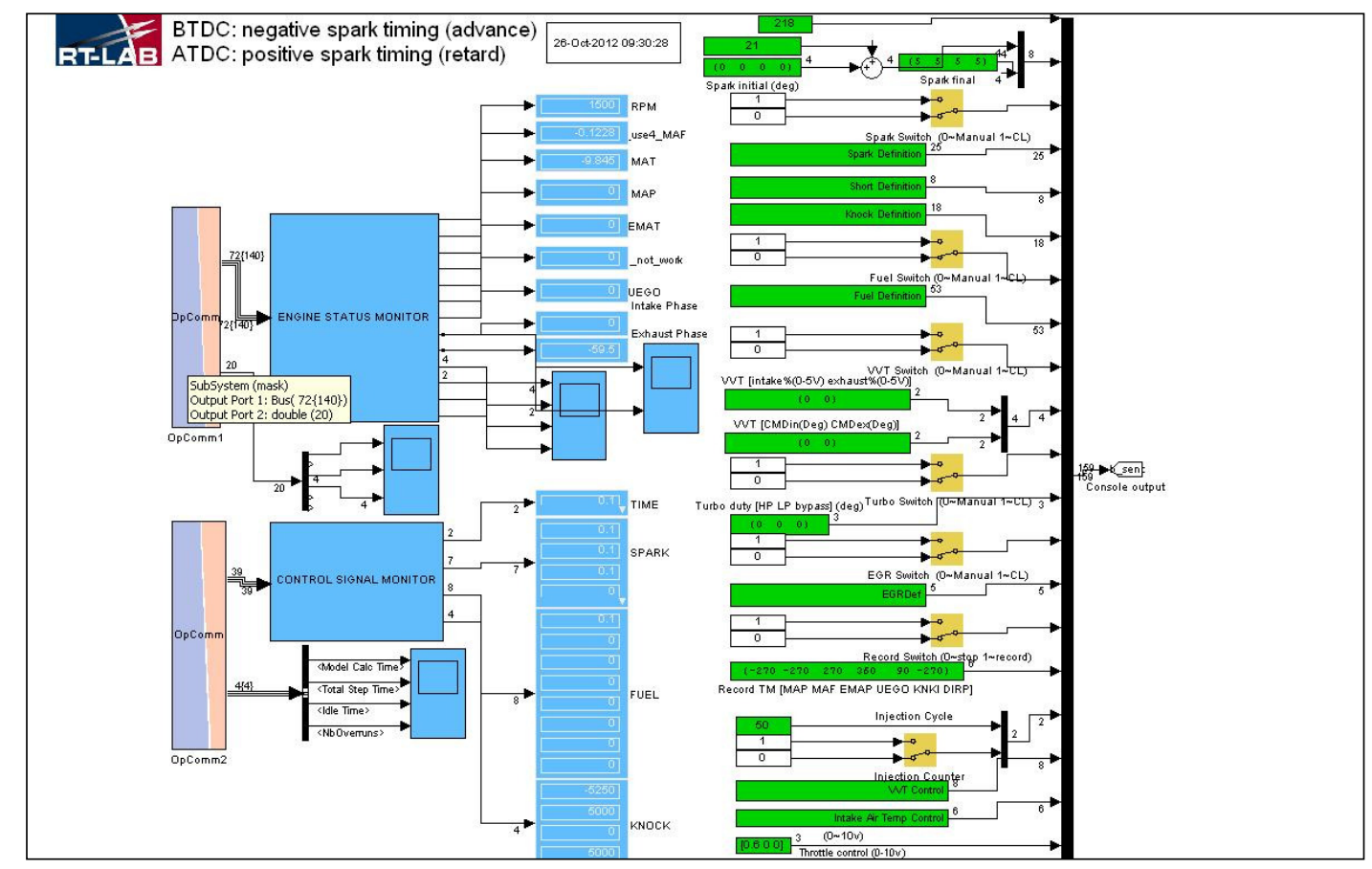

Figure 97: Engine prototype controller graphic interface

An A\&D combustion analyzer (CAS - combustion analysis system) is used for monitoring the engine combustion process in real-time using the Kistler in-cylinder pressure sensors so that engine load (IMEP - indicated mead effective pressure) can be calculated in real-time and the engine combustion process can be visualized through displayed in-cylinder pressure signals. Figure 98 shows an initial graphic interface for the A\&D CAS. 


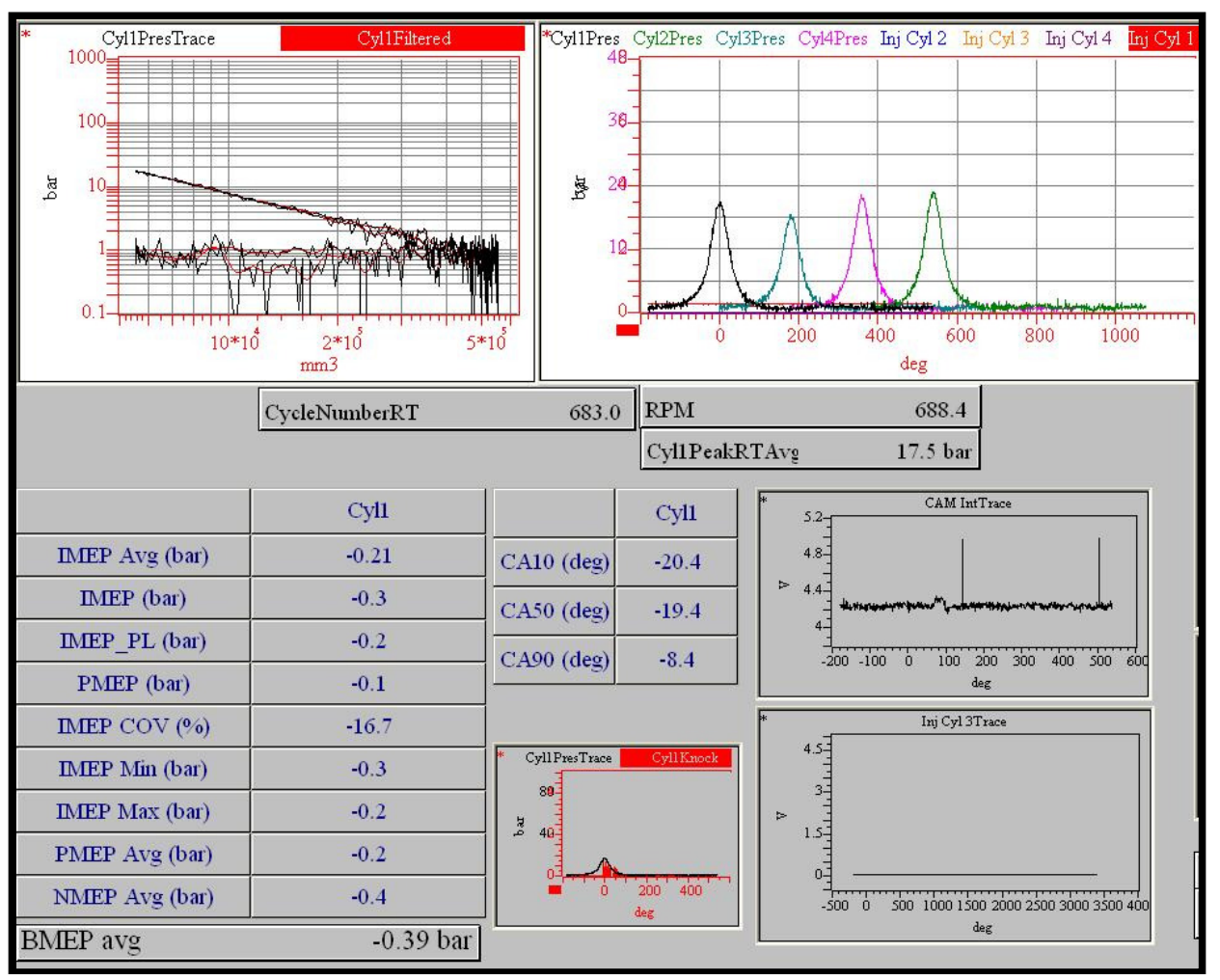

Figure 98: Combustion analyzer graphic interface

\subsubsection{Target metal engine dynamometer validation tests}

After the engine system integration was complted, the engine was installed onto the MSU research dyno, and motoring and SI combustion tests were conducted. It was found that cylinder \#3 has fairly high IMEP COV due to high blow-by. The engine was disassembled and reassembled with new pistons and piston ringes, and combustion tests indicated that the IMEP $\mathrm{COV}$ is close to normal for cylinder \#3 but the blow-by is still high. It was decided to run-in the engine ring pack while validating the other functionalities.

\section{Two-step valve lift validation:}

Firing capabilities were demonstrated under both high and low valve lift conditions at both 1500 and 2000 RPM; see Table 18.

Table 18: Firing test results for each cylinder

\begin{tabular}{|c|c|c|c|c|c|c|c|c|c|c|c|}
\hline \multirow[b]{2}{*}{ RPM } & \multirow{2}{*}{$\begin{array}{l}\text { MAP } \\
{[\mathrm{kPa}]}\end{array}$} & \multirow[b]{2}{*}{ Lambda } & \multirow[b]{2}{*}{ Valve Lift } & \multicolumn{4}{|c|}{ IMEP [bar] } & \multicolumn{4}{|l|}{$\mathrm{COV}$} \\
\hline & & & & Cyl 1 & Cyl 2 & Cyl 3 & Cyl4 & Cyl 1 & Cyl 2 & Cyl 3 & Cyl4 \\
\hline 1500 & 45.5 & 1.03 & low & 3.53 & 4.59 & 2.61 & 4.10 & 20.2 & 2.7 & 12.1 & 2.5 \\
\hline 1500 & 54.7 & 1.00 & high & 3.28 & 4.29 & 2.36 & 3.52 & 13.1 & 3.9 & 23.6 & 4.6 \\
\hline 2000 & 52.1 & 0.97 & high & 3.69 & 4.44 & 2.94 & 3.75 & 3.9 & 1.9 & 8.8 & 1.4 \\
\hline
\end{tabular}




\section{Cylinder-to-cylinder performance study:}

Data taken with the original pistons showed that high blow-by in cylinder 3 resulted in very poor combustion quality: the combustion was late and slow, leading to high COV, low peak incylinder pressure and IMEP. Adjusting the spark timing and fueling quantity to cylinder 3 had little to no effect on improving the poor combustion performance, nor did swapping hardware (injectors, spark plugs, pressure transducers, etc). Cylinder 2 performed well with low COV and the highest IMEP, while cylinder 1 was inconsistent with decent IMEP but sometimes with high COV. Cylinder 4 showed a somewhat low IMEP but good COV. A P-V diagram (Figure 99), comparing the part load 1500 RPM performance of cylinders 2 and 3, demonstrates the blow-by issue clearly. Note that the pressure trace of cylinder 3 deviates from that of cylinder 3 during compression and then results in a very weak combustion. The crank-based pressure traces of all cylinders at 2000 RPM are shown in Figure 100.

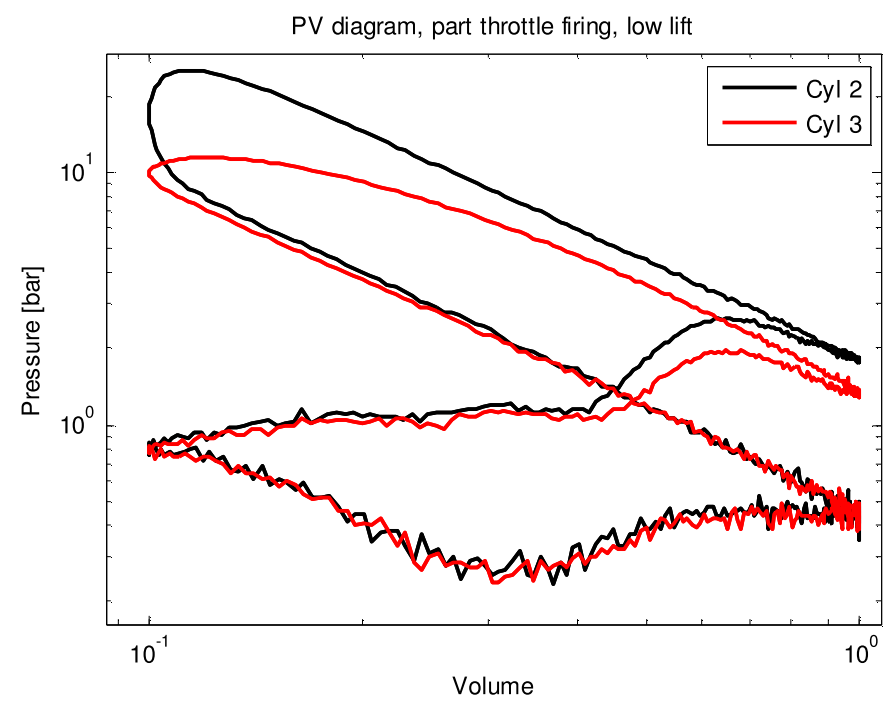

Figure 99: P-V diagram of cylinders 2 and 3

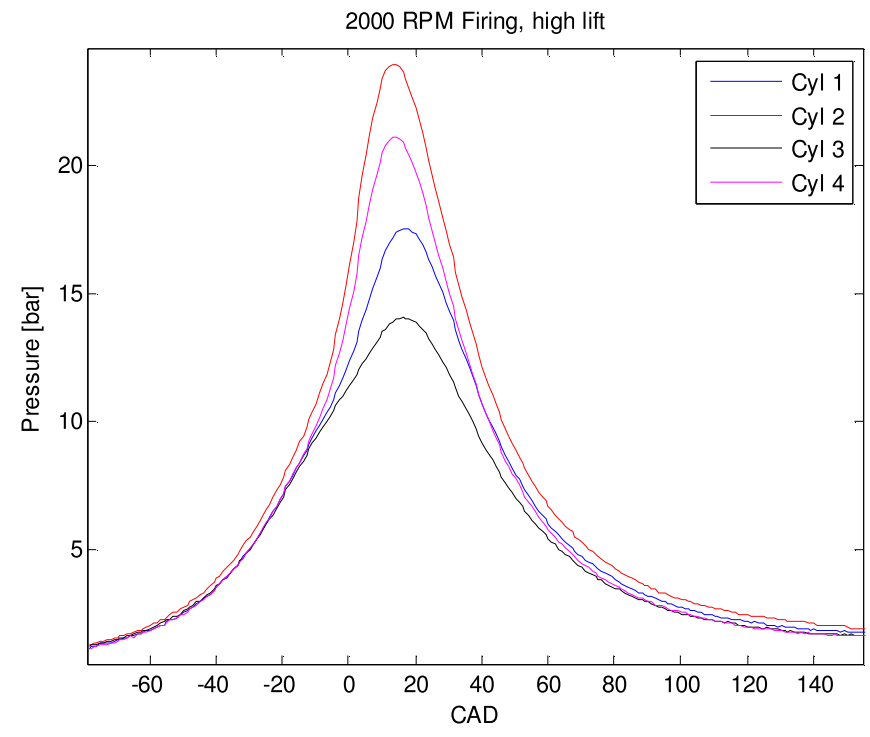

Figure 100: Crank based in-cylinder pressure signals 
However, regardless the high blow-by of cylinder 3, performance of cylinder 2 was found to be very satisfactory. Control systems for fueling, ignition, valve lift and phasing were verified to be working correctly, and the data from a test point at a given engine operation condition matches the previous test data provided by Chrysler very well with consideration of the slightly different cam profiles due to the 2-step valve-train modifications, see Table 19. It was hoped that solving the blow-by issues would result in each cylinder approaching to this performance level.

Table 19: Performance comparison of cylinder \#2

\begin{tabular}{|l|c|c|c|c|c|c|c|c|}
\hline & $\begin{array}{c}\text { Speed } \\
{[\mathrm{RPM}]}\end{array}$ & $\begin{array}{c}\text { MAP } \\
{[\mathrm{kPa}]}\end{array}$ & $\begin{array}{c}\text { IMEP } \\
{[\mathrm{bar}]}\end{array}$ & $\begin{array}{c}\text { NMEP } \\
{[\mathrm{bar}]}\end{array}$ & $\begin{array}{c}\text { CA50 } \\
\text { [CAD } \\
\text { ATDC] }\end{array}$ & $\begin{array}{c}\text { Peak } \\
\text { Pressure } \\
\text { [bar] }\end{array}$ & $\begin{array}{c}\text { Injection } \\
\text { Timing } \\
\text { [CAD } \\
\text { BTDC] }\end{array}$ & $\begin{array}{c}\text { Spark } \\
\text { Timing } \\
\text { [CAD } \\
\text { BTDC] }\end{array}$ \\
\hline $\begin{array}{l}\text { Chrysler Data } \\
\text { Sample A }\end{array}$ & 1856 & 53.1 & 4.48 & 3.99 & 5.1 & 26.6 & 299 & 22.5 \\
\hline $\begin{array}{l}\text { Chrysler Data } \\
\text { Sample B }\end{array}$ & 2112 & 51 & 4.48 & 3.95 & 5.9 & 26.9 & 304 & 22.5 \\
\hline $\begin{array}{l}\text { MSU Cylinder } \\
\# 2\end{array}$ & 2000 & 52.1 & 4.44 & 3.98 & 8.8 & 24.2 & 300 & 31 \\
\hline
\end{tabular}

\section{Blow-by and compression problem:}

Motoring traces showed the same blow-by and low compression issues in cylinder 3 that were found during the firing tests. P-V diagrams show significant leakage during the compression stroke, and this leads to low peak motoring pressures. Numerous methods were utilized to confirm and diagnose the cause of this problem.

The obtained motoring traces shows a low peak pressure in cylinder 3 compared to other cylinders, especially cylinder 2 , see Table 20. Log-scale P-V diagrams of cylinders 2 and 3 also show a significant amount of blow-by for cylinder 3, see Figure 101. Though the peak pressure in cylinder 3 is close to those of cylinders 1 and 4 in some motoring tests, P-V plots show significant blow-by for cylinder 3, whereas the other cylinders seem to ingest less intake air and display less blow-by. A close look at a P-V diagram comparing cylinders 2 and 3 shows that at the start of the compression stroke, in-cylinder pressures are nearly identical, but the blow-by in cylinder 3 causes low pressure at the end of the compression and expansion strokes, see Figure 101.

Table 20: Motoring pressure data

\begin{tabular}{|c|c|c|c|c|c|c|c|}
\hline \multirow{2}{*}{$\begin{array}{l}\text { Speed } \\
{[\text { RPM] }}\end{array}$} & \multirow[b]{2}{*}{ MAP $[\mathrm{kPa}]$} & \multirow[b]{2}{*}{ Valve Lift } & \multicolumn{4}{|c|}{ Peak Motoring Pressures [bar] } & \multirow{2}{*}{$\begin{array}{c}\text { Engine } \\
\text { temp }\end{array}$} \\
\hline & & & Cyl1 & Cyl2 & Cyl3 & Cyl4 & \\
\hline 1500 & 76.9 & Low & 17.8 & 18.6 & 16.2 & 17.0 & Cold \\
\hline 2000 & 82.1 & Low & 19.7 & 20.1 & 19.2 & 19.3 & Hot \\
\hline 2000 & 50.7 & High & 11.7 & 12.5 & 11.4 & 11.5 & Hot \\
\hline
\end{tabular}




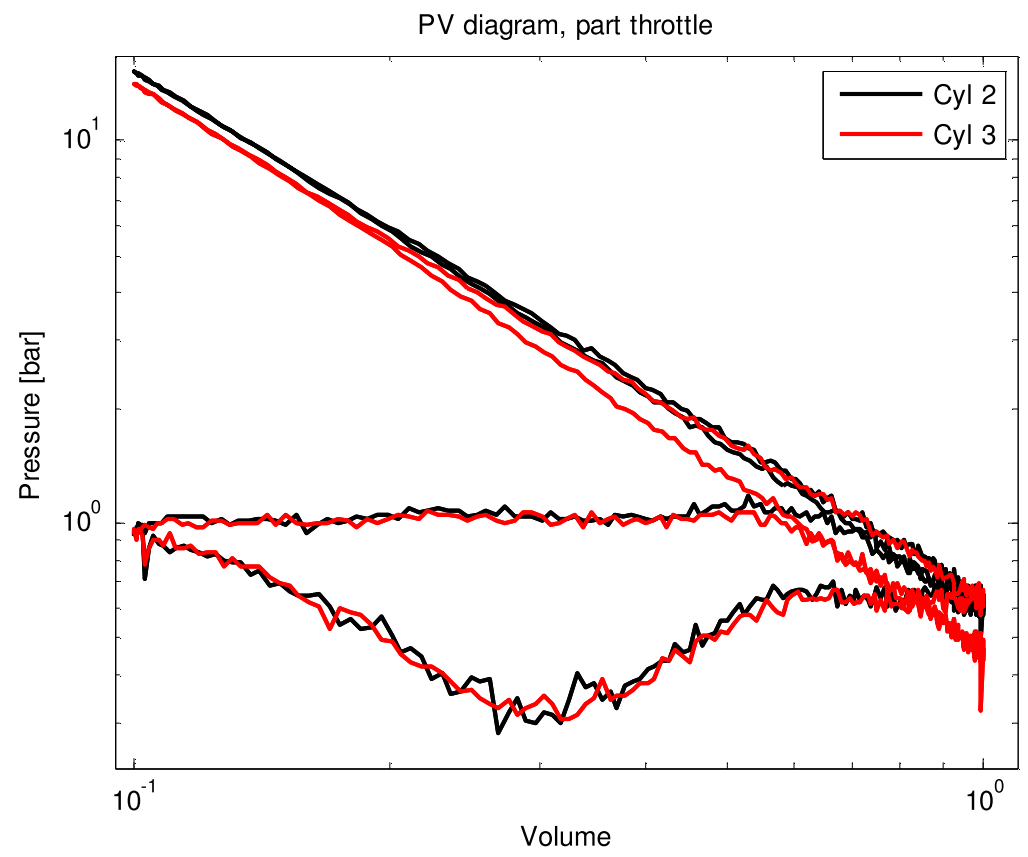

Figure 101: Motoring P-V diagrams of cylinders 2 and 3

Compression tests were performed with a Mitvac Professional Compression Test Kit. With this device the engine was motored at a low speed while a pressure gauge mounted to a cylinder through the spark plug hole; as the engine motors, the gauge reaches a maximum pressure. Cylinder 3 showed the lowest pressure at 195 psi, compared to a high of 240 psi for cylinder 2.

A second test was developed to determine if the leakage in each cylinder was indeed caused by air blowing through the piston rings and not the head gasket or valves. For this leak test, the cylinder was filled with compressed air at a constant pressure of 95 psi through a fitting located at the spark plug hole. All ventilation passages from the crankcase were blocked off except for one, which was outfitted with an airflow meter that could measure the flow rate of the compressed air that was leaking through the piston rings in standard cubic feet per hour (SCFH). This test was performed for each piston at both TDC and BDC. Cylinder 3 allowed the largest amount of leakage, indicating that it has more ring blow-by than the other cylinders.

The results from both of these tests are summarized below in Table 21. Both of these tests helped confirming the suspicions from the motoring and firing pressure data.

Table 21: Compression and leak test results

\begin{tabular}{|l|c|c|c|c|}
\hline Cylinder number: & 1 & 2 & 3 & 4 \\
\hline Compression test pressure (psi): & 225 & 240 & 195 & 210 \\
\hline Leak test flow rate at TDC (SCFH): & 11 & 11 & 16.5 & 9.5 \\
\hline Leak test flow rate at BDC (SCFH): & 15 & 13 & 19 & 12 \\
\hline
\end{tabular}

The engine was then disassembled, and the block, pistons, and rings were inspected for signs of abnormal wear that could lead to the blow-by problem. With simple bore measurement techniques, there was no obvious issue observed in cylinder 3, though further investigation using precise instruments was not available at that time. 
The new high compression pistons were installed upon the rebuild of the engine, and further motoring tests were performed. Again, low peak pressures were observed in cylinder 3, see Table 22. It is noteworthy that the blow-by seems to decrease with increasing speed and manifold pressure. The P-V diagrams shown in Figure 102 and Figure 103 demonstrate less blow-by at the 2000 RPM with WOT than that at partial load. At high in-cylinder pressure, the piston ring may be forced to seat correctly, while at low in-cylinder pressure, it may not seat well, thus causing high blow-by.

Table 22: Motoring with high CR pistons

\begin{tabular}{|r|r|r|r|r|r|}
\hline & & \multicolumn{4}{|c|}{ Peak Pressure [bar] } \\
\cline { 3 - 6 } RPM & MAP [kPa] & Cyl 1 & Cyl 2 & Cyl 3 & Cyl 4 \\
\hline 1000 & 67.9 & 18.5 & 19.3 & 16.8 & 17.3 \\
\hline 1000 & 84.8 & 23.8 & 24.2 & 21.3 & 22.5 \\
\hline 1500 & 55.6 & 15.6 & 16.2 & 14.5 & 14.9 \\
\hline 1500 & 84.8 & 24.6 & 24.8 & 23.1 & 23.6 \\
\hline 2000 & 46.2 & 12.9 & 13.5 & 12.5 & 12.5 \\
\hline 2000 & 84.7 & 25.1 & 25.3 & 24 & 24.4 \\
\hline
\end{tabular}

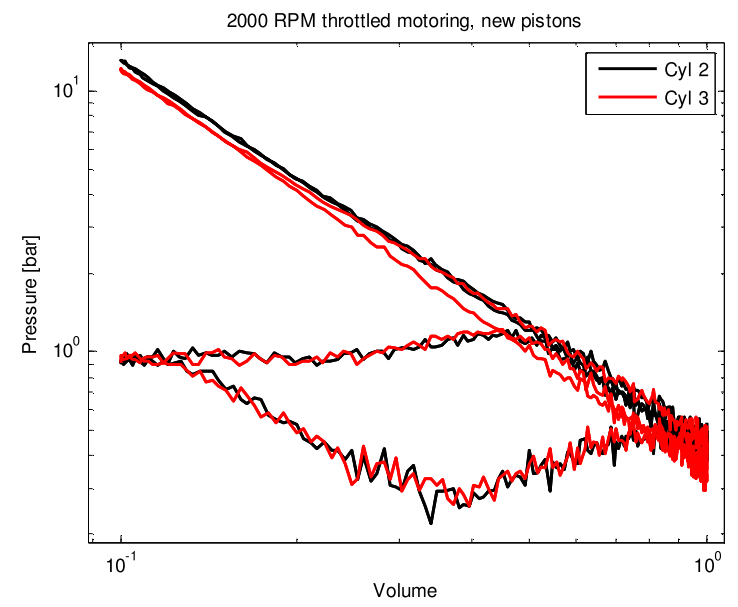

Figure 102: Throttled motoring P-V diagrams of cylinders 2 and 3 with new pistons

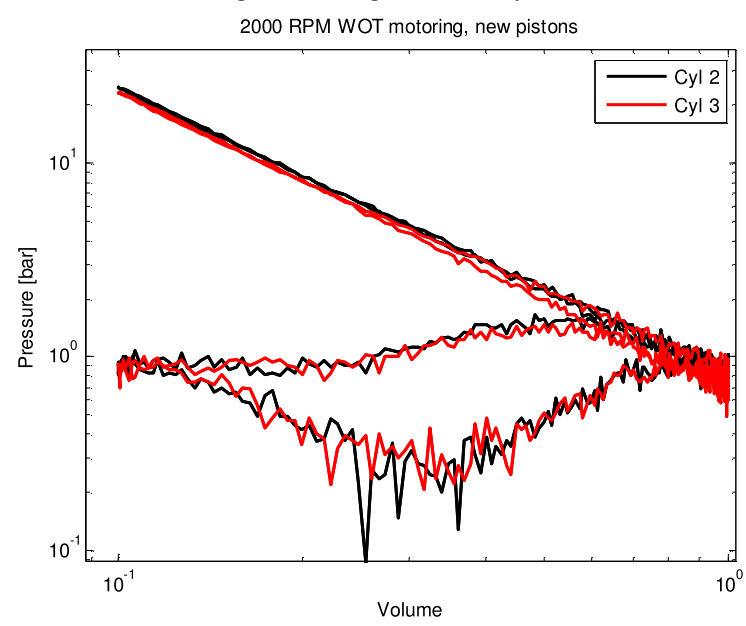

Figure 103: Un-throttled motoring P-V diagrams of cylinders 2 and 3 with new pistons 
In an attempt to eliminate the possibility that the intake and exhaust valves were not seating fully (e.g., the lifter may pressurizes the valve stem slightly even when the valve is supposed to be closed), the engine was motored without camshafts in place. These tests were inconclusive. With no air entering through the intake valves, it appeared that random ring dynamics took over as the primary factor in peak motoring pressure as the peak pressures wandered significantly in each cylinder each time this test was attempted.

With all diagnostics completed, the SI combustion tests were conducted again. The combustion stability of cylinder 3 was improved significantly but its blow-by is still higher than other cylinders. With the input of Chrysler piston engineers, it was decided to run-in the engine ring pack. The engine run for more that 100 hours to reduce the engine blow-by of cylinder \#3 with the target compression ratio (12.5:1) pistons installed. Test results show that the performance of the high blow-by cylinder (\#3) was improved but the cylinder is still very weaker comparing to the rest of the cylinders. It was decided to focus on the cylinder with the best performance; that is cylinder \#2.

\section{Load and speed benchmarking with high compression ratio pistons and high lift:}

It was decided that focusing on the performance of cylinder 2, the best performing cylinder, was a reasonable option for moving forward.

The performance gain from the increased compression ratio of approximately 12.5:1 was demonstrated by comparing the old and new pistons with tests at constant manifold pressure. With the same air flow through the engine, stoichiometric fueling, and spark timing adjusted to keep combustion the same phase, the new pistons yielded a 5.2 bar IMEP verses 4.4 bar IMEP with the old pistons (Table 23).

Table 23: Firing comparison of with low and high compression ratio pistons, constant MAP

\begin{tabular}{|l|c|c|c|c|c|c|c|c|c|c|}
\hline & \multicolumn{2}{|l|}{ IMEP [bar] } & \multicolumn{2}{|c|}{ COV } & \multicolumn{2}{c|}{ PeakP [bar] } & \multicolumn{2}{c|}{$\begin{array}{c}\text { CA50 } \\
{\left[{ }^{\circ} \text { ATDC] }\right.}\end{array}$} & \multicolumn{2}{|c|}{$\begin{array}{c}\text { Spark Timing } \\
{\left[{ }^{\circ} \text { BTDC] }\right.}\end{array}$} \\
\hline Piston: & New & Old & New & Old & New & Old & New & Old & New & Old \\
\hline & 5.2 & 4.4 & 1.7 & 1.9 & 29.4 & 24.2 & 8.7 & 8.8 & 25 & 30 \\
\hline
\end{tabular}

Three test speeds (1500, 2000, and 2500 RPM) and three load points (4.5, 6.0, and 7.5 bar IMEP) were chosen for doing some benchmarking data with the high-lift cams in spark ignition mode (see Table 24). Optimal cam timings and injection timings in these tests were based on the previous test data provided by Chrysler. Spark timing was adjusted to keep CA50 timing around $8^{\circ}$ ATDC for the best IMEP and low COV. These test points not only give an understanding of how the engine responds to a broader window of operating conditions, but also help to establish parameters for the SI conditions needed during an SI-HCCI mode transition in the future. Sample P-V and Mass Fraction Burned (MFB) plots of the low, middle, and high load points from the 2000 RPM tests are shown below (Figure 104); these figures effectively show the reduction in PMEP as load increases, and they show the consistent combustion phasing. A careful look at the MFB plot shows the slightly reduced $10-90 \%$ burn duration as the load increases. This will be useful for comparing to mode transition and HCCI combustion data. 
Table 24: Preliminary SI test point benchmarking, high-lift cams, cylinder 2

\begin{tabular}{|c|c|c|c|c|c|c|c|c|}
\hline Speed & $\begin{array}{l}\text { MAP } \\
{[\mathrm{kPa}]}\end{array}$ & $\begin{array}{l}\text { IMEP } \\
\text { [bar] }\end{array}$ & $\begin{array}{c}\text { PMEP } \\
\text { [bar] }\end{array}$ & $\begin{array}{c}\text { Spark } \\
\text { [CAD } \\
\text { BTDC] }\end{array}$ & $\begin{array}{c}\text { Injection } \\
\text { Pulse [ms] }\end{array}$ & $\begin{array}{c}\text { CA50 } \\
\text { [CAD } \\
\text { ATDC] }\end{array}$ & $\begin{array}{l}\text { B1090 } \\
\text { [CAD] }\end{array}$ & COV of IMEP \\
\hline \multirow[t]{3}{*}{1500} & 47.7 & 4.54 & 0.50 & 24 & 1.90 & 8 & 23 & 2.2 \\
\hline & 57.7 & 5.84 & 0.42 & 22 & 2.20 & 8 & 22 & 1.5 \\
\hline & 66.1 & 7.51 & 0.37 & 19 & 2.60 & 8 & 18 & 1.4 \\
\hline \multirow[t]{3}{*}{2000} & 46.0 & 4.62 & 0.53 & 27 & 1.85 & 7 & 23 & 1.2 \\
\hline & 56.4 & 5.98 & 0.44 & 23 & 2.20 & 9 & 21 & 1.7 \\
\hline & 65.4 & 7.52 & 0.35 & 20 & 2.55 & 7 & 19 & 1.2 \\
\hline \multirow[t]{3}{*}{2500} & 42.0 & 4.51 & 0.59 & 25 & 1.80 & 8 & 24 & 1.3 \\
\hline & 52.8 & 6.05 & 0.50 & 22 & 2.20 & 8 & 22 & 1.8 \\
\hline & 62.9 & 7.77 & 0.44 & 19 & 2.67 & 9 & 22 & 1.3 \\
\hline
\end{tabular}
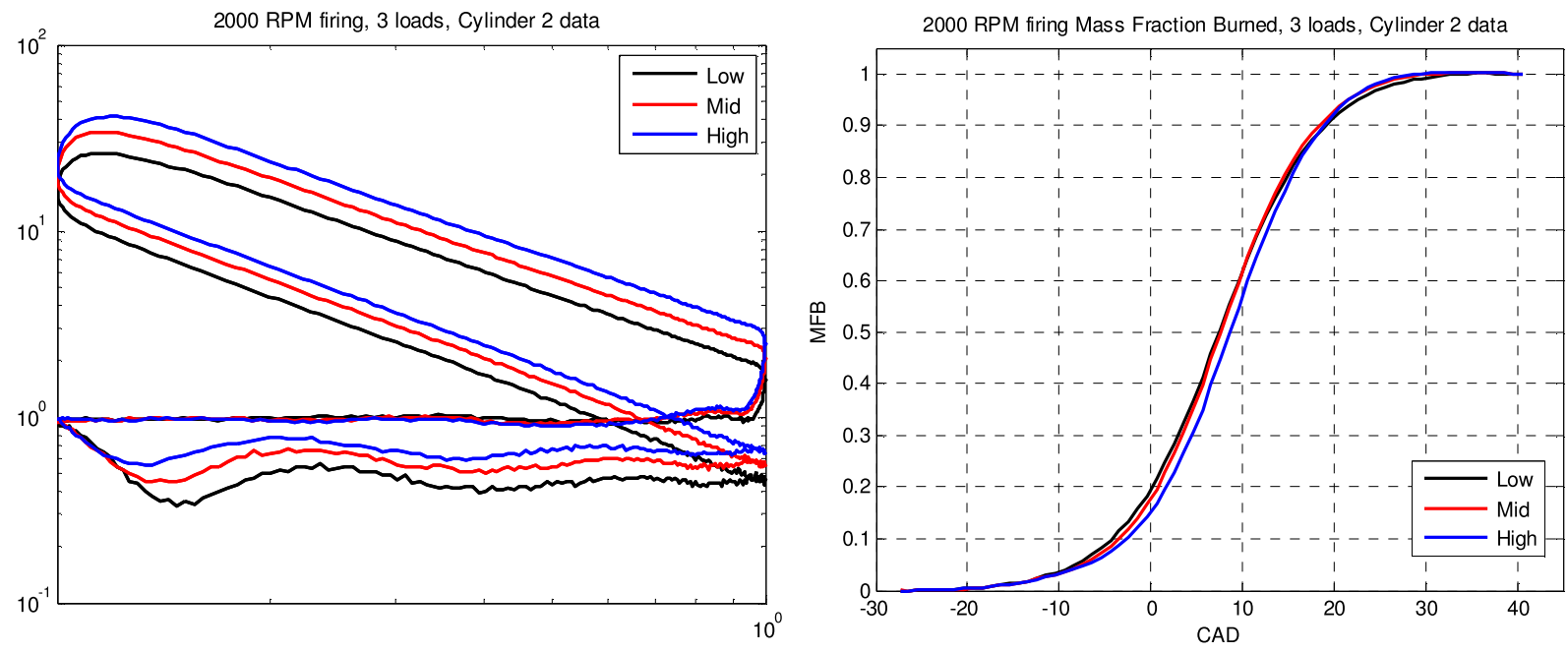

Figure 104: P-V and mass fraction burned plots of low, mid, and high load at 2000 RPM

Multi-cylinder benchmarking tests with the electrical cam phasing (variable valve timing) system were continued with an investigation of throttling through early intake valve closing (IVC); this is a typical way to use a low-lift cam in SI operation to reduce pumping losses. This technique uses the high-lift exhaust cam and allows for an easier low-pressure exhaust stroke since the high-lift cam allows for a less restrictive opening and a longer time period for pushing exhaust gas out. Advancing the low lift intake cam timing helps to limit the amount of fresh intake air that gets into the cylinder, while also to allow for an increased throttle opening and higher manifold pressure which reduce pumping work. Pressure-volume (P-V) plots can be useful to demonstrate this pumping reduction as the pumping loop becomes visible smaller. 
The P-V plots from firing cases run with a low-lift intake cam sweep are shown below (Figure 105), along with a table of the data from these cases with the key to the test number labels in the plot (Table 25). Exhaust advance timing was fixed at $6^{\circ}$, the same as what was used for high-lift benchmarking tests at this load. Though the over-laid curves make it difficult to observe, the main point from this figure is the reduction of pressure during the intake stroke due to reduced throttling with the increased intake valve timing advancement. With the intake cam advanced $60^{\circ}$ from the default position, PMEP had been reduced from 0.52 bar (high-lift) down to 0.31 bar. A run with the intake advanced $70^{\circ}$ was attempted, but cylinder 1 began knocking and misfiring badly, so operation was stopped.

Pumping efficiency, which is calculated as NMEP/IMEP, describes how efficiently the engine uses the gross power generated during the compression and expansion. The baseline with highlift cams had a pumping efficiency of 89\%; and it is improved to $93 \%$ with the use of early IVC, indicating that only $7 \%$ of the gross power made is being used for pumping air. The goal for the HCCI combustion operation is to further reduce the pumping loss.

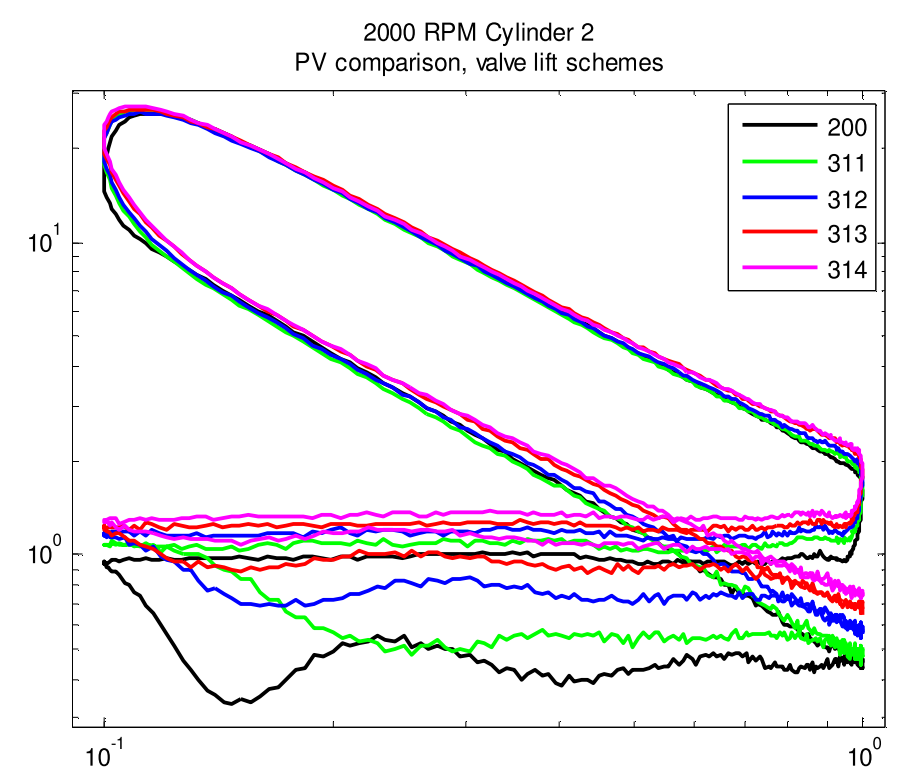

Figure 105: Low-lift intake cam sweep with decreasing pumping loop 
Table 25: Low lift intake cam sweep data

\begin{tabular}{|cccccccccc|}
\hline Test & Intake & Exhaust & IMEP & CA50 & B1090 & [bar] & $\begin{array}{c}\text { Pump } \\
\text { eff }\end{array}$ & $\begin{array}{c}\text { MAP } \\
{[\mathrm{kPa}]}\end{array}$ & Spark \\
\hline 200 & 43 high & 6 high & 4.58 & 7.6 & 23.6 & 0.52 & $89 \%$ & 45.7 & 27 \\
310 & 0 low & 6 high & 4.52 & 8.9 & 28.8 & 0.55 & $88 \%$ & 43.9 & 34 \\
311 & 20 low & 6 high & 4.57 & 8.6 & 31.8 & 0.52 & $89 \%$ & 48.1 & 42 \\
312 & 40 low & 6 high & 4.51 & 9.5 & 34.5 & 0.44 & $90 \%$ & 57.1 & 51 \\
313 & 50 low & 6 high & 4.57 & 10.0 & 36.6 & 0.36 & $92 \%$ & 66.9 & 57 \\
314 & 60 low & 6 high & 4.49 & 8.3 & 35.9 & 0.31 & $93 \%$ & 75.4 & 58 \\
\hline
\end{tabular}

A simplified figure with just the two best case SI P-V curves shows the original high-lift case (Test200) and the $60^{\circ}$ advance low-lift case (Test 314) together (Figure 106). This figure shows closely matching power loops with drastically different pumping loops.

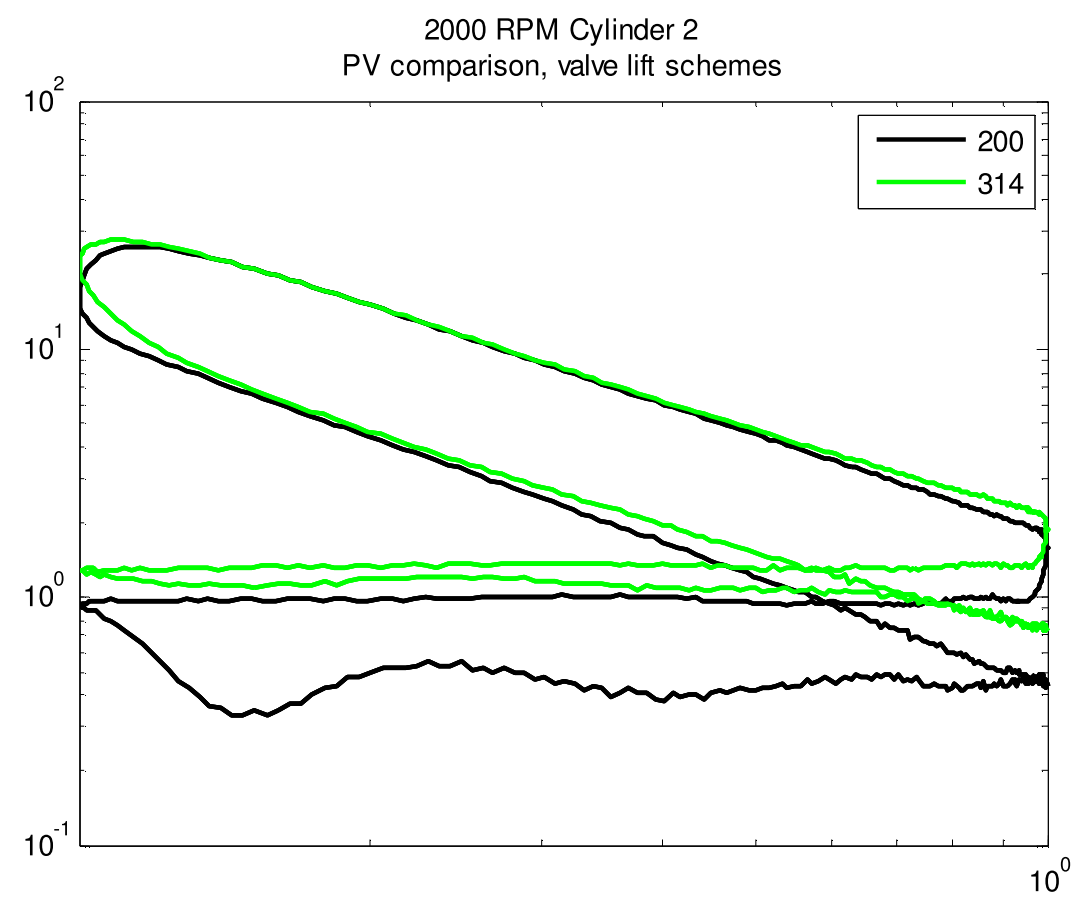

Figure 106: High and low lift intake cam comparison at constant IMEP

The multi-cylinder metal engine tests continued and the high blow-by of cylinder \#3 was not reduced over time and it was determined that high cylinder-to-cylinder variation of the multicylinder metal engine would make the SI-HCCI mode transition study impossible. With the limited time left for the project, it was decided to convert the engine into a single cylinder engine using cylinder \#2 so that the HCCI combustion and SI-HCCI mode transition study could go forward. The following is the detailed multi-cylinder test results and the single cylinder engine setup. 


\subsubsection{Switch to single cylinder operation}

Due to the ongoing blow-by problems in different cylinders (particularly cylinder 3 ) in the multicylinder engine, the decision was made to modify it into only fire the best cylinder (number 2) during further testing. A diagram of this setup is shown below in Figure 107. New intake and exhaust manifolds were fabricated with the ports to cylinders 1,3 , and 4 blocked off so no gas mixture can flow through them. While this is not the ideal way to conduct tests originally planned for a multi-cylinder engine, it eliminates some of the difficulties of trying to run with essentially four cylinders operated at quite different conditions.

A problem with attempting the HCCI combustion with poorly performing cylinders is the potential for partial-burn and misfires. While cylinder 2 might perform well, cylinder 3 might not, and this could cause difficulty in understanding the air-to-fuel ratio since the $\mathrm{O}_{2}$ sensor would observe a mixture of burned and unburned gases from all cylinders. Running the engine with one cylinder, while still allowing the others to pump air through, would lead a similar difficulty with the $\mathrm{O}_{2}$ sensor reading a mixture of exhaust and fresh charge. Furthermore, under certain conditions, such as the low-lift intake valve throttle case discussed above, cylinder 1 displayed very erratic behavior with knocking and misfiring. Cylinders 3 and 4 did not always respond as expected to changes in spark timing or valve timing either. Thus, the switch to single-cylinder operation simplifies the process of isolating the control parameters for SI and HCCI mode transition control.

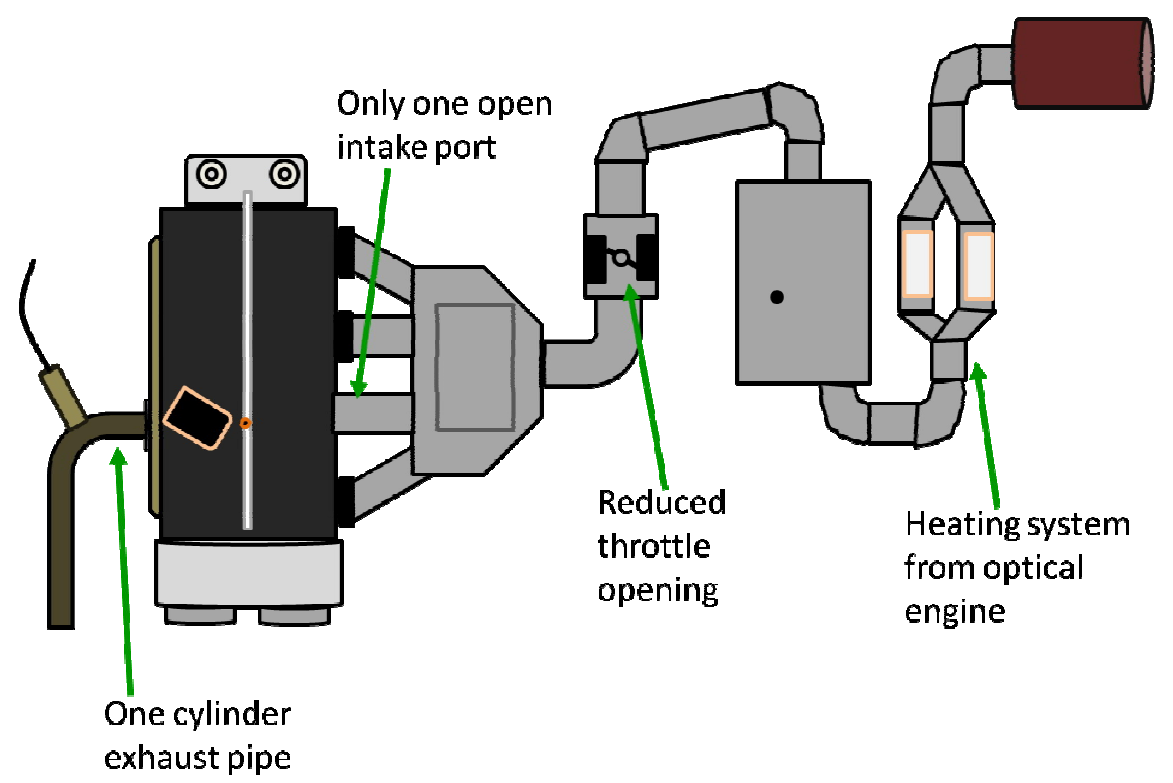

Figure 107: Diagram of single-cylinder operation on multi-cylinder engine

With the lower airflow demands of the single-cylinder setup, a new more restrictive throttle was designed. The old throttle plate was replaced with a much smaller diameter plate, and the surrounding space was press-fitted with a donut-shaped piece of aluminum. This design allows the precise control of the manifold pressure for low-load SI operations. Figure 108 shows the engine diagram for single cylinder operation. 


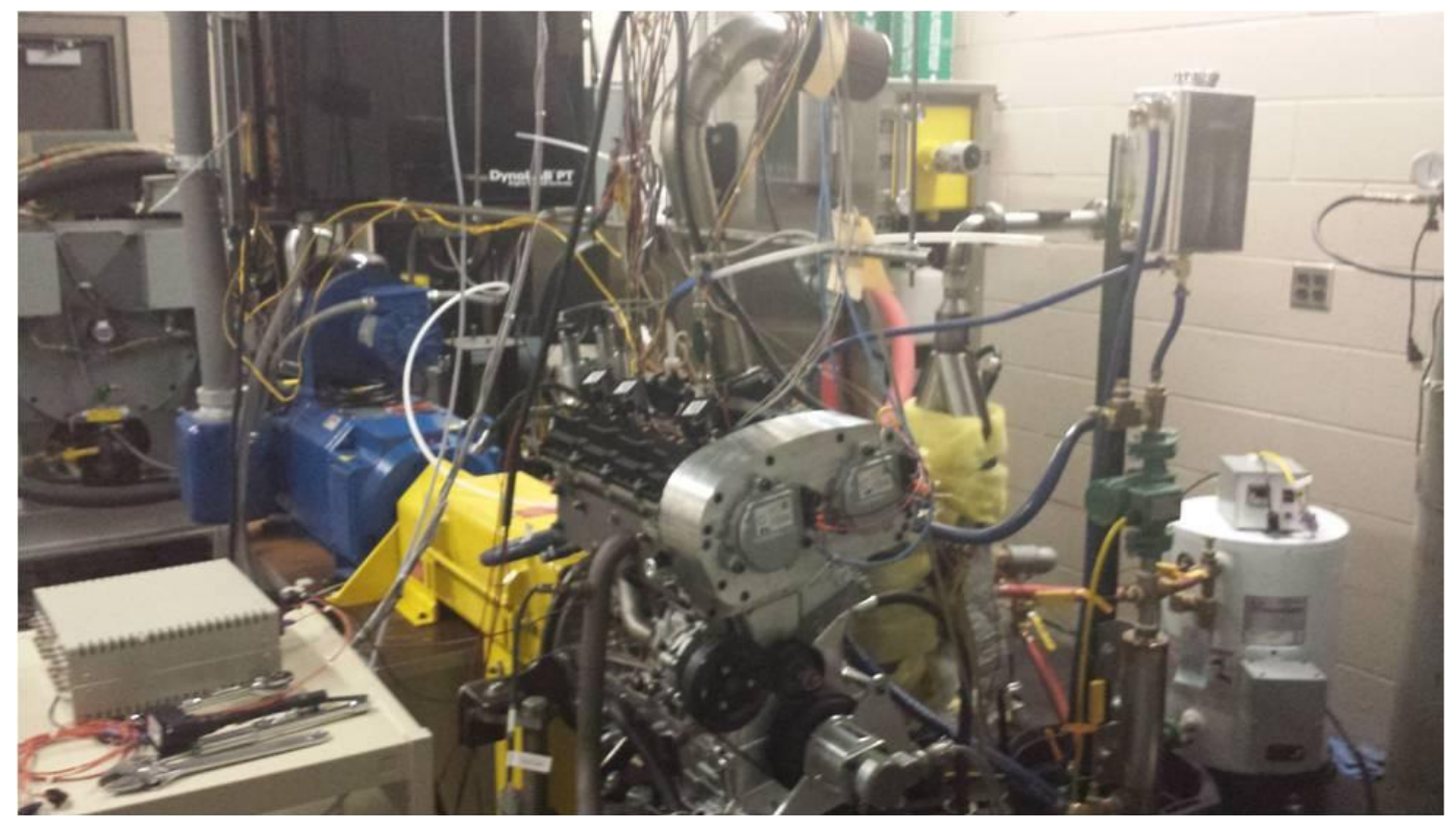

Figure 108: Single-cylinder operation on multi-cylinder engine

\subsection{HCCI and combustion mode transition control}

In this subsection, the application of the SI-HCCI combustion to the mode transition between SI and HCCI combustion was studied based on the developed control-oriented engine model in an HIL simulation environment. The main results have been published in IEEE Transaction on Control System Technology [44] (see Subsection 9.5), in 2012 American Control Conference [45] (see Subsection 9.8), and in 2011 ASME Dynamic System Control Conference [32] (see Subsection 9.9).

\subsubsection{HCCI Capable SI engine model implemented in an HIL simulator}

To demonstrate the effectiveness of using the SI-HCCI hybrid combustion mode for mode transition, the developed hybrid combustion model was implemented into a dSPACE based HIL simulation environment, and an SI to HCCI mode transition process was simulated. The implemented engine model was based on a four cylinder SI engine shown in Figure 109, where the engine parameters are listed in Table 26.

The SI engine was reconfigured to enable both SI and HCCI combustion as follows.

- The engine valvetrain was modified to enable dual lifts for both intake and exhaust valves, where the high lift is used for SI combustion and the low lift for HCCI combustion with the NVO (negative valve overlap or recompression) operation.

- External cooled EGR was added to enable high dilution charge, resulting in low charge mixture temperature. 
- The ranges of both intake and exhaust valve timings were extended to \pm 40 crank degrees to improve the controllability of the internal EGR rate, effective compression ratio, and engine volumetric efficiency during the HCCI operating condition.

- It is assumed that the engine throttle was electronically controlled to obtain the desired engine charge in both SI and unthrottled HCCI operations.

Table 26: Combustion related engine specifications

\begin{tabular}{|l|l|}
\hline PARAMETER & Model value \\
\hline bore/stroke/con-rod length & $86 \mathrm{~mm} / 86 \mathrm{~mm} / 143.6 \mathrm{~mm}$ \\
\hline compression ratio & $9.8: 1$ \\
\hline Intake /exhaust valve lifts of high stage & $9 \mathrm{~mm} / 9 \mathrm{~mm}$ \\
\hline Intake /exhaust valve lifts of low stage & $5 \mathrm{~mm} / 5 \mathrm{~mm}$ \\
\hline Intake /exhaust valve timing range (low lifts only) & $\pm 40 \mathrm{deg} / 40 \mathrm{deg}$ \\
\hline Intake /exhaust valve lifts lash & $0.2 \mathrm{~mm} / 0.25 \mathrm{~mm}$ \\
\hline
\end{tabular}

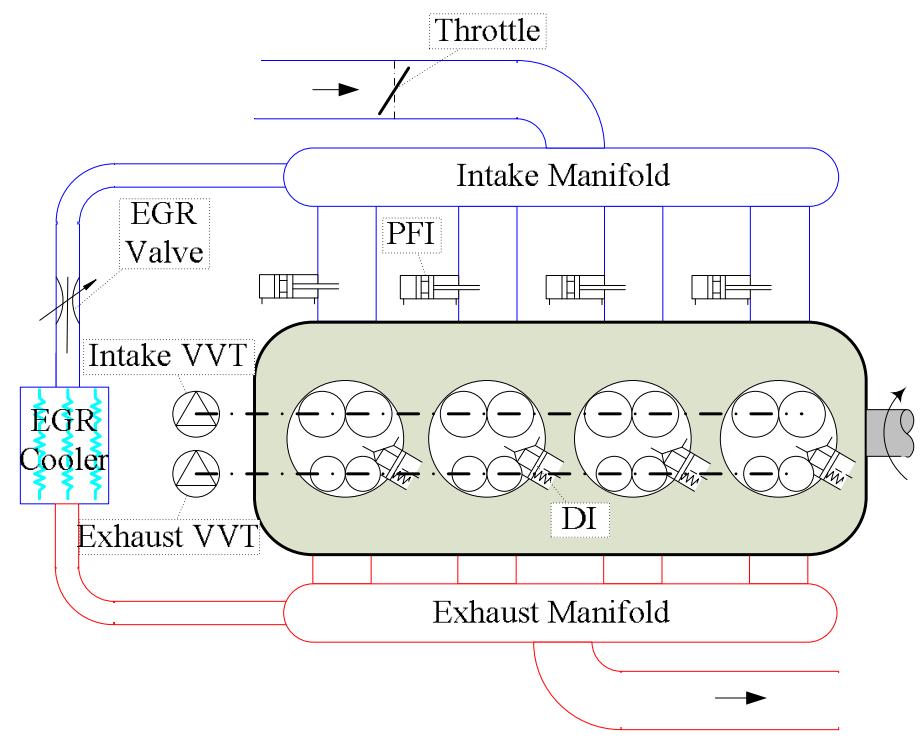

Figure 109: HCCI capable SI engine configuration

The crank-based SI-HCCI hybrid combustion model presented in [10] was used for engine combustion process. In this model, the engine air-handling subsystems, such as the external EGR system, the intake and exhaust manifolds, were modeled by mean-value time-resolved models. It has been shown that the combustion mode transition from SI to $\mathrm{HCCI}$ is more challenging than that from the HCCI to SI. For this reason the case of utilizing the hybrid combustion mode for combustion mode transition from SI to HCCI combustion was simulated under the influence of the multi-cylinder air handling system.

\subsubsection{Engine setup for SI to HCCI combustion mode transition}

Table 27 lists the engine actuator parameters for steady state SI and HCCI combustion modes at the same engine speed and load conditions. These parameters were optimized for steady state 
engine performance with the best fuel economy that satisfies engine knock limit. That is, the maximum pressure rise $(\max d p / d \theta)$ is less than or equal to 3 bar per crank degree, as shown in Figure 114. It can be seen in Table 27 that the engine operation parameters are quite different for the SI and HCCI combustion, where AGTDC and BGTDC are after and before gas exchange TDC, respectively. And it is almost impossible to adjust these engine control parameters in one engine cycle since the valve timing and throttle position cannot be changed instantly.

Table 27: Engine actuator parameters at 2000 RPM with 4.5bar IMEP

\begin{tabular}{|c|c|c|c|c|c|c|c|}
\hline & $\begin{array}{c}\text { ST } \\
\left({ }^{\circ} \text { ACTDC }\right)\end{array}$ & $\begin{array}{c}\text { EGR valve } \\
(\%)\end{array}$ & $\begin{array}{c}\text { Throttle } \\
(\%)\end{array}$ & $\begin{array}{c}\text { Fuel pulse } \\
(\mathrm{ms} / \text { cycle })\end{array}$ & $\begin{array}{c}\text { VVT_IN } \\
\left({ }^{\circ} \text { AGTDC }\right)\end{array}$ & $\begin{array}{c}\text { VVT_EX } \\
\left({ }^{\circ} \text { BGTDC }\right)\end{array}$ & Valve lift \\
\hline SI & -36 & 3 & 16.6 & 2.06 & 70 & 100 & high \\
\hline HCCI & N/A & 26 & 100 & 1.6 & 95 & 132 & low \\
\hline
\end{tabular}

The goal of the mode transition is to switch the engine operation mode from the SI to HCCI combustion without detectable engine torque fluctuation, or in other words to maintain the engine IMEP during the mode transition. However there are two main obstacles that make it very difficult to complete the smooth mode transition in one step using the steady state engine control parameters listed in Table 27. The first is the response time in the engine actuators such as throttle, VVT (Variable Valve Timing) actuators and the cooled EGR valve. In this study, the actuator response $Y(s)$ to the control input $U(s)$ is approximated as the first order system in the engine model as

$$
\frac{Y(s)}{U(s)}=\frac{1}{1+\tau s}
$$

where $s$ is the Laplace operator and $\tau$ is the associated time constant. For this simulation, $\tau$ equals to $0.15,0.12$ and 0.1 second for throttle, VVT and EGR valve, respectively.

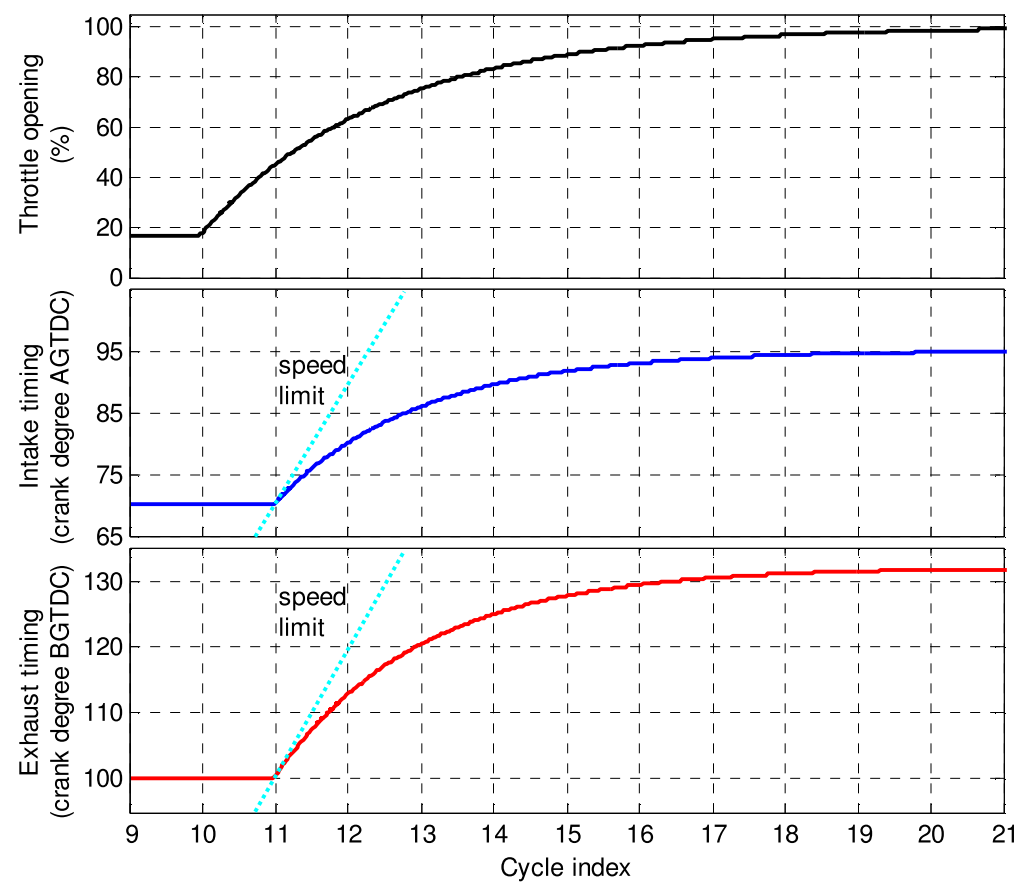

Figure 110: Transient responses of engine actuators during SI to HCCI mode transition 
Figure 110 shows the step responses of engine throttle and intake/exhaust VVT actuators from SI to HCCI operations, where the dotted lines are the response rate limit of the VVT actuators. One can see that it takes a few engine cycles for these actuators to move from the SI positions to the desired HCCI positions. The second obstacle is the gas mixture filling dynamics of the engine air charge system. Note that the gas mixture filling dynamics is coupled with the first order engine actuator dynamics. This leads to high order dynamics of the engine MAP and slows down the intake EGR response. The transient responses of the engine MAP and the intake EGR rate are shown in Figure 111. These two obstacles suggest that a few engine cycles might be required to alter the engine conditions to be suitable for the HCCI combustion during the mode transition from SI to HCCI combustion. Therefore, one cycle combustion mode transition could lead to partial combustion or misfire with large engine IMEP fluctuation. Also note that the cooled EGR rate, throttle position, intake/exhaust valve timings and lifts cannot be controlled for individual cylinder.

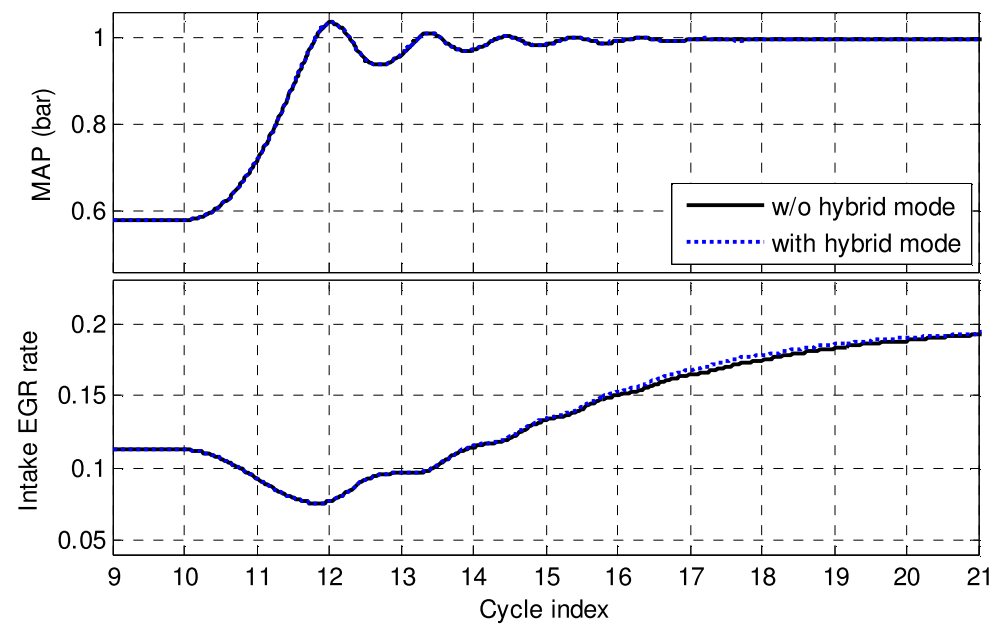

Figure 111: Transient responses of MAP and EGR rate during SI to HCCI mode transition

To compare the mode transition performance, two different mode transition strategies were studied: one utilizes the hybrid SI-HCCI combustion mode by increasing the percentage of the HCCI combustion within a few engine cycles and the other switches the combustion mode in one engine cycle (also called one-step transition). During the simulations, the engine coolant temperature was kept at $363^{\circ} \mathrm{K}$ and engine speed was set at $2000 \mathrm{RPM}$ throughout the entire simulation. In the first 9 engine cycles, the engine was operated in the SI combustion mode and all engine control parameters used are listed in Table 27 for the SI operation. At cycle 10, the engine throttle started opening to the WOT (Wide Open Throttle) position; at cycle 11, the reference of EGR valve and intake/exhaust valve timings were adjusted to the target values for steady state HCCI combustion, and the lifts of intake/exhaust valves were switched to low stages at the same time. Note that the mode transition starts at cycle 11 and between cycles 1 and 11 all the engine control parameters are the same for both strategies. At cycle 12 the spark was eliminated for the strategy without the hybrid combustion mode (or one-step transition) and the engine was forced into the HCCI combustion mode; while for the strategy using the hybrid combustion mode, spark was maintained for two more consecutive cycles and was cut at cycle 14. The injected fuel quantity is a control variable for the hybrid combustion mode, and is calculated based upon a multidimensional map as follows 


$$
M_{f u e l}=f\left(\begin{array}{llllll}
P_{I M} & \theta_{I V C} & \theta_{E V C} & \theta_{S I} & x_{E G R} & I M E P_{r e f}
\end{array}\right)
$$

where $x_{E G R}$ is the intake EGR rate; IMEP ref is the desired IMEP; $f$ represents steady state mapping of the measureable engine signals to the fuel quantity. This map was generated through intensive simulations. The calculation was updated for each cylinder due to the fact that the incylinder charge mixture is quite different from cylinder to cylinder during the mode transition.

\subsubsection{Open loop simulation results and discussion}

The combustion mode transition process was simulated for both mode transition strategies and the associated key engine parameters during the transition are plotted in Figure 112 to Figure 27. One can see that the engine parameters change significantly during the mode transition and vary greatly cylinder-by-cylinder. For instance the simulated volumetric efficiencies of cylinders 4 and 2 have small drops in cycle 11, which lead to rich combustion for cylinders 4 and 2 (see the volumetric efficiency and normalized air to fuel ratio in Figure 112). This is mainly due to the lift switch of intake and exhaust valves from high to low at cycle 11. The step change of valve lifts in one engine cycle dramatically reduced gas charged into the cylinders. To compensate this impact, engine throttle was scheduled to open toward the WOT position at cycle 10, one cycle before the valve lift change. The advanced throttle opening increases intake manifold pressure and it ensures each cylinder has enough fresh air charge to maintain the desired IMEP at cycle 11. Figure 112 also shows that it takes almost 10 engine cycles for inertial gas fraction (IGF), defined as the total burned gas (from both external and internal EGR) fraction, to reach its steady state. Note that the slow response of IGF ensures relative low dilution rate of the charge mixture at cycles 12 and 13, which makes it possible to initiate SI combustion. This is necessary for the hybrid combustion mode.

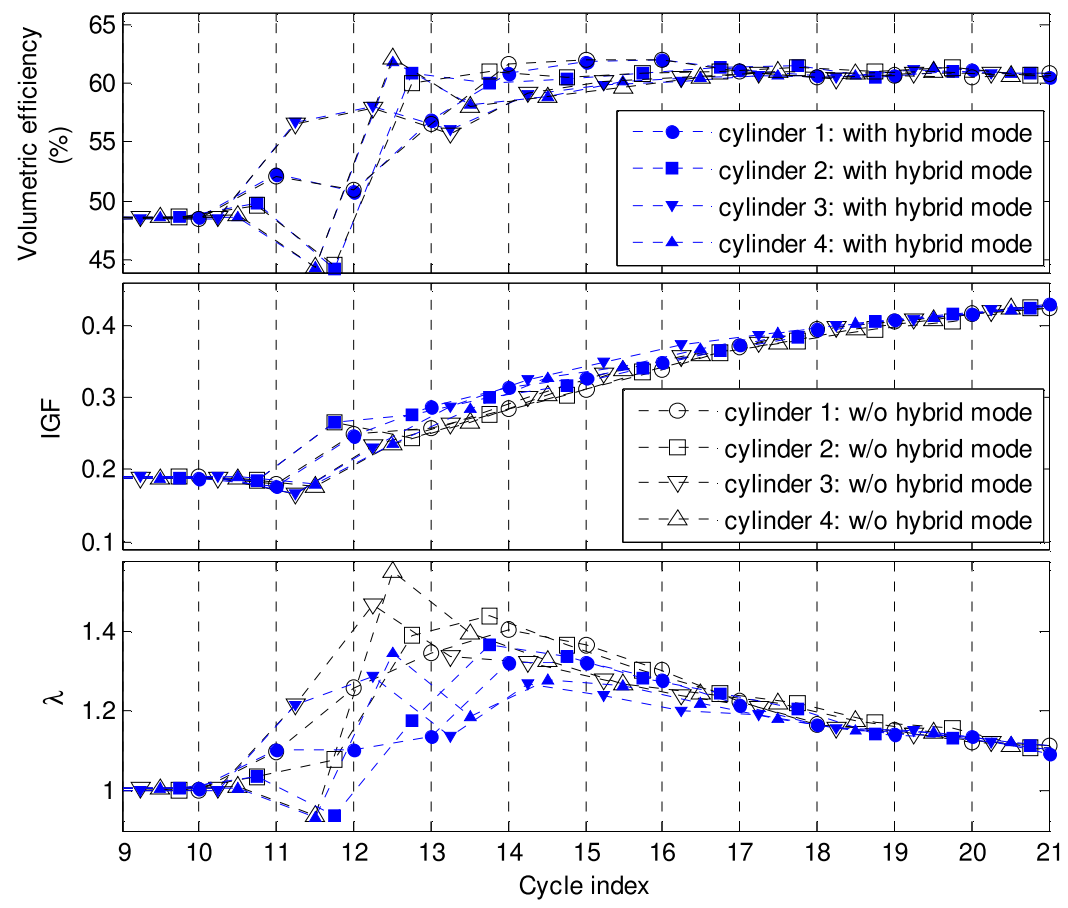

Figure 112: Volumetric efficiency, IGF and $\lambda$ during SI to HCCI mode transition 
The in-cylinder gas temperature at IVC $\left(T_{I V C}\right)$ is an important parameter that affects the HCCI combustion timing. Figure 29 shows that $T_{I V C}$ in SI combustion mode is much lower than that in HCCI combustion mode, and it takes several engine cycles to increase $T_{I V C}$ to the level required for steady state HCCI combustion. At cycle $12 T_{I V C}$ of each cylinder is still far below the target temperature. Under this condition, the SOHCCI timings of all cylinders are around 20 crank degrees ACTDC without using the hybrid combustion mode. As a result, the engine IMEP drops significantly, see Figure 114. Note that late SOHCCI timing is associated with large variation of IMEP and leads to partial combustion. Heavily retard SOHCCI timing could result in misfire at the succeeding engine cycle due to undesired $T_{I V C}$. However, with the hybrid combustion mode, the SOHCCI timings of each cylinder at cycles 11 and 12 were advanced because of the increased unburned zone gas temperature due to additional work of SI combustion occurred in the burned zone as discussed in Section 3. Note that during the hybrid combustion mode part of the gas-fuel mixture is burned in SI combustion before the HCCI combustion starts. As a result the IMEP variation during the combustion mode transition is not significant. In Figure 29 the MFB at SOHCCI is used to represent the fraction of SI combustion during the hybrid combustion. It decreases during the combustion mode transition.

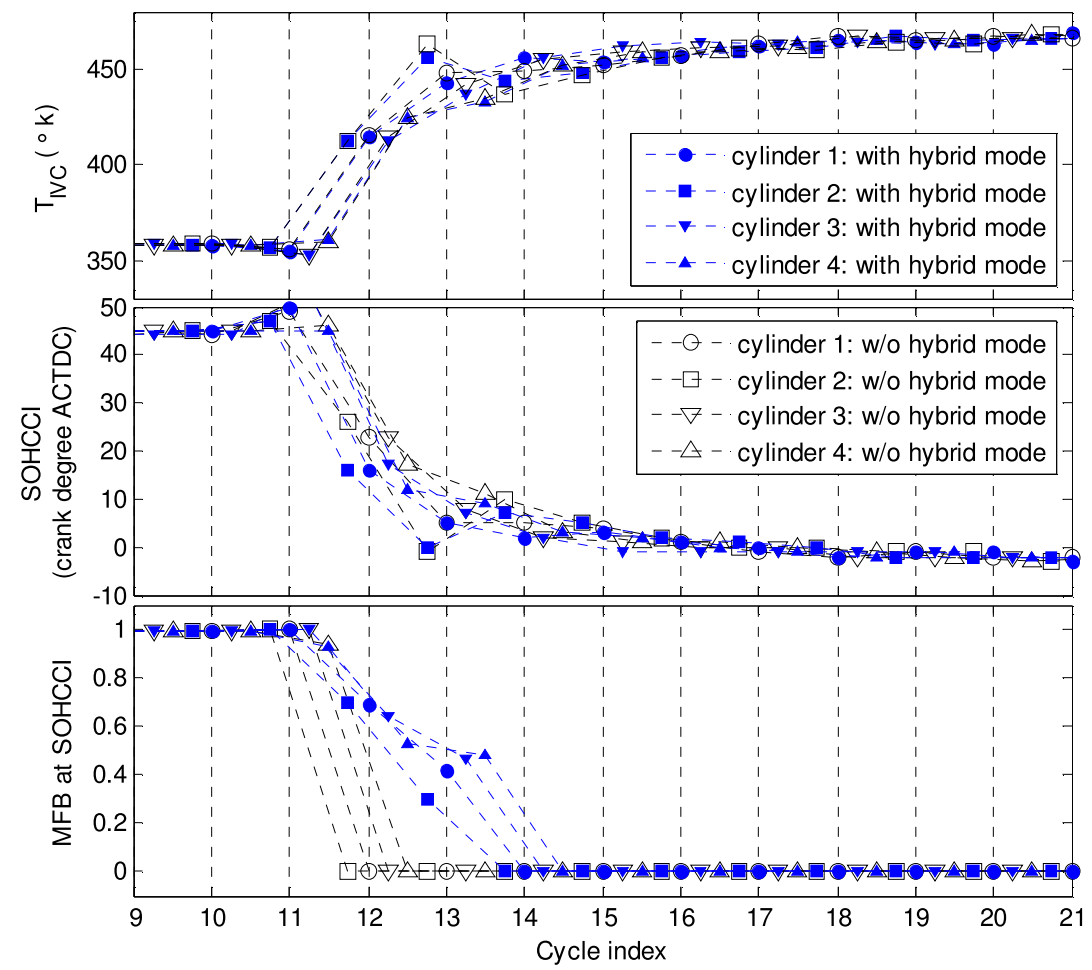

Figure 113: $T_{I V C}$, SOHCCI and SI combustion fraction during SI to HCCI mode transition

Even with the hybrid combustion mode, the engine IMEP shown in Figure 114 fluctuates around the equilibriums with about $12 \%$ error between cycle 11 and 13. Similar fluctuations can also be observed for $T_{I V C}$ and SOHCCI shown in Figure 29 for both strategies. This is mainly due to the cycle-to-cycle dynamics of the combustion process. From the engine charge dynamics one can see that the in-cylinder gas temperature at the current engine cycle is computed based upon the fresh charge mass and temperature of current engine cycle, the trapped residue gas mass and temperature from last engine cycle. Retard SOHCCI timing of the previous engine cycle leads to higher residue gas temperature, which results in high gas temperature of current cycle and 
advanced SOHCCI timing of the current cycle. Note that the fluctuations due to the residue gas dynamics are different from cylinder to cylinder. From Figure 29 and Figure 114 one can see that cylinder 2 has the largest oscillations of the simulated engine parameters. At cycle 12 the $\max$ $(d p / d \theta)$ of cylinder 2 is close to the knock limitation of 3 bar per crank degree. The influence of the residue gas dynamics can be compensated by controlling spark timing during hybrid combustion mode and adjusting the fuel quantity during HCCI combustion. This will be the subject of future research. Table 28 compares the mode transition performance of both one-step and hybrid combustion strategies. With the help of the hybrid combustion mode, the IMEP error decreases from $31 \%$ (one-step) to $11.7 \%$ (hybrid) and engine torque from $41.2 \%$ (one-step) to $15.2 \%$ (hybrid). The maximal pressure rise is also reduced from 2.96 to 2.57 due to the smooth mode transition.

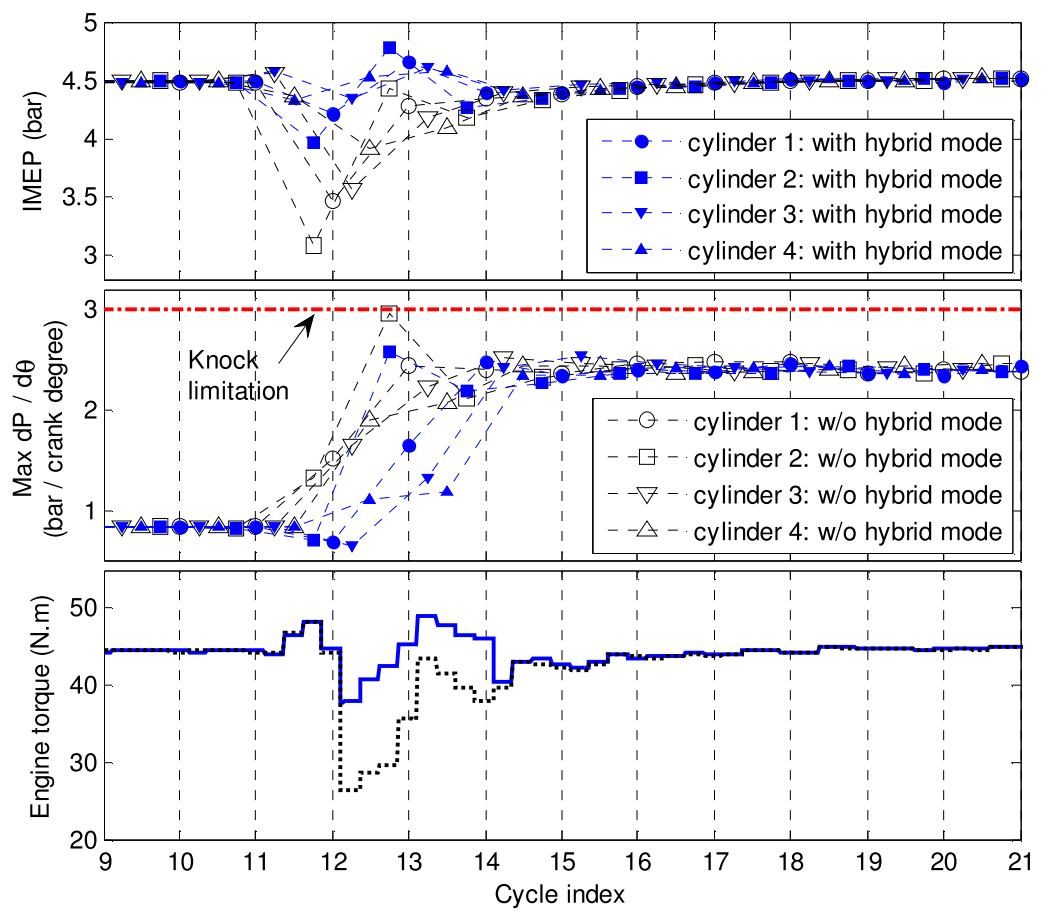

Figure 114: IMEP, $\max d p / d \theta$ and engine torque during SI to HCCI mode transition

Table 28: Performance comparison during mode transition

\begin{tabular}{|c|c|c|c|}
\hline & $\begin{array}{c}\text { IMEP error } \\
(\%)\end{array}$ & $\begin{array}{c}\text { Max } d p / d \theta \\
(\text { bar } / \text { crank } \\
\text { degree })\end{array}$ & $\begin{array}{c}\text { Torque error } \\
(\%)\end{array}$ \\
\hline $\begin{array}{c}\text { One-step } \\
\text { transition }\end{array}$ & 31 & 2.96 & 41.2 \\
\hline $\begin{array}{c}\text { Hybrid } \\
\text { transition }\end{array}$ & 11.7 & 2.57 & 15.2 \\
\hline
\end{tabular}

Figure 115 shows the in-cylinder pressure and temperature traces of cylinder 1 for both strategies. All signals vary significantly from cycle to cycle during the mode transition. One can see that the HCCI combustion lead to high peak in-cylinder pressure and low combustion 
temperature comparing with those of SI combustion. Without hybrid combustion mode the peak cylinder pressure has significantly large variation during the mode transition comparing with those using hybrid combustion mode.

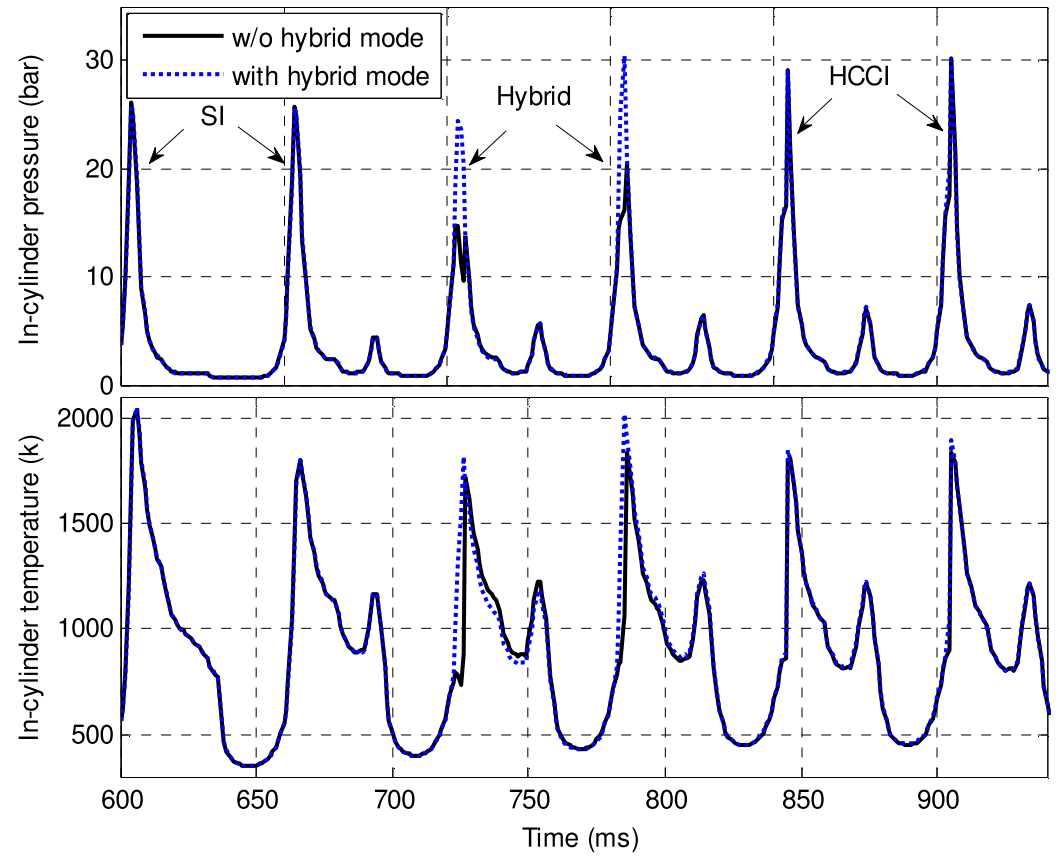

Figure 115: In-cylinder pressure traces during SI-HCCI mode transition

Multi-cycle combustion mode transition using the hybrid combustion mode was studied in this quarter and its performance was compared with these under traditional one-cycle mode transition process. The simulation results show that utilizing the SI-HCCI hybrid combustion is capable of reducing engine IMEP and torque fluctuations significantly during the mode transition between the SI and HCCI combustion.

\subsubsection{Mode Transition Strategy Development}

In Subsection 4.2.3, the one-step combustion mode transition was investigated. The control references of all engine parameters were directly switched from the SI mode to the HCCI mode, as listed in Subsection 4.2.3, in one engine cycle. The simulation results showed that misfired combustion occur during the one-step mode transition, and significant torque fluctuation was discovered. Thereby, a multistep mode transition strategy was proposed in Subsection 4.2.3 by inserting a few hybrid combustion cycles between the SI and HCCI combustion; see Figure 116. The control strategy proposed in this paper is based on this multistep strategy 


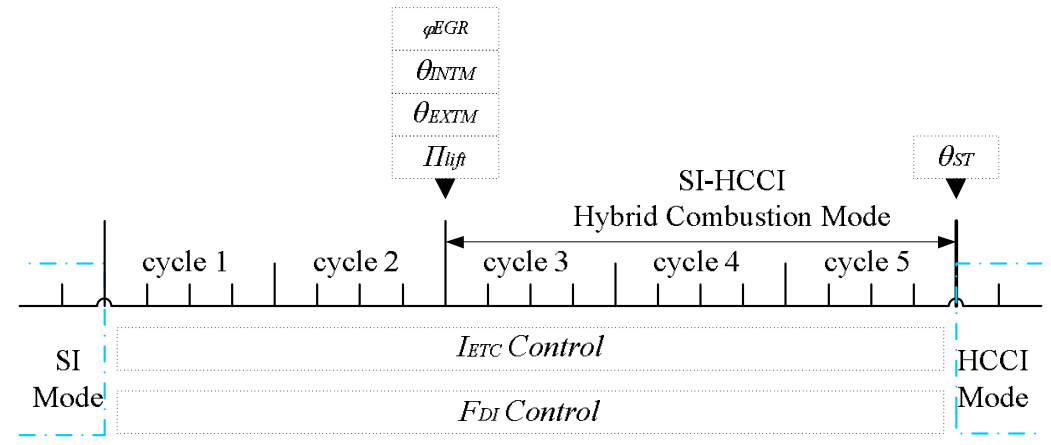

Figure 116: Multistep SI to HCCI combustion mode transition control schedule

As illustrated in Figure 116, five engine cycles are used during the SI to HCCI mode transition. During the transitional cycles some engine parameters are adjusted in an open loop according to the schedule shown in Figure 116. Cycles 1 and 2 are used for engine throttle control. They provide enough time for the engine MAP to increase to compensate the $\Pi_{\text {lift }}$ switch. At the end of cycle 2 , the intake/exhaust valve lift $\Pi_{\text {lift }}$ switches from high lift to low lift, and the control references of $\phi_{E G R}, \theta_{I N T M}$ and $\theta_{E X T M}$ are set to those of the steady state HCCI combustion mode as listed in Subsection 4.2.3. Spark timing $\theta_{S T}$ of each cylinder was kept constant during the transitional cycles and was eliminated at the end of cycle 5. Throughout the transitional cycles, the engine control parameters $I_{E T C}$ and $F_{D I}$ are regulated by time-based (1 millisecond sampling rate) and cycle-based controls, respectively.

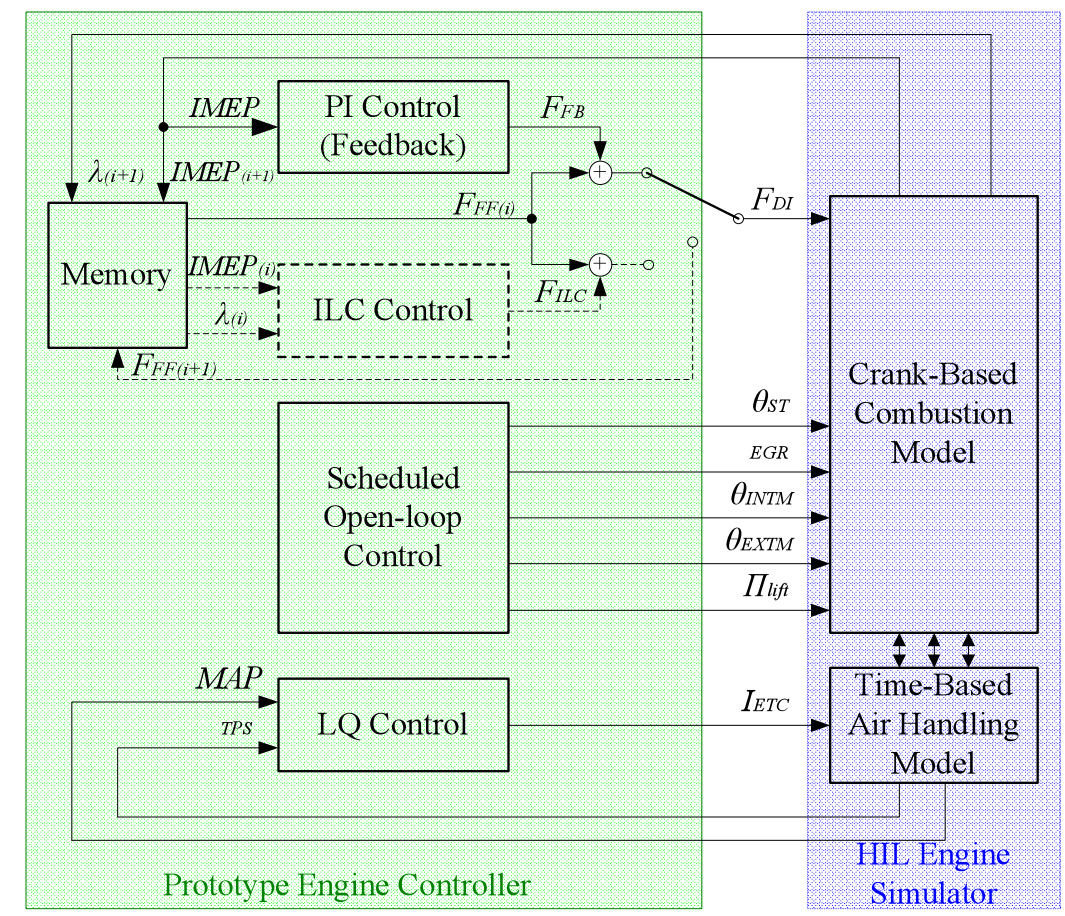

Figure 117: SI to HCCI combustion mode transition control diagram

Under the regulations of the engine parameters, the combustion characteristics during the transitional engine cycles are different from those of the typical SI and HCCI combustion modes. 
The combustion at cycles 1 and 2 are still in SI combustion mode, but the air-to-fuel ratio is no longer stoichiometric and most likely is lean due to the throttle $\left(I_{E T C}\right)$ control. During cycles 3 to 5 , engine charge temperature $T_{I V C}$ is higher than that of SI mode and lower than the desired temperature of HCCI mode. Under such condition, the engine is operated in the SI-HCCI hybrid combustion mode for cycles 3 to 5 , with the help of the maintained engine spark. The main motivation of operating the engine at the SI-HCCI hybrid combustion mode is to use the SI combustion to increase the temperature of the unburned gas mixture, such that the HCCI combustion can be achieved in the same engine cycle.

Figure 117 shows the control diagram of the multistep combustion mode transition controller and its interface with the HIL engine simulator that will be introduced later. The multistep combustion mode transition is activated as engine IMEP switches from above $I M E P_{\text {ref }}\left(I M E P_{\text {ref }}\right.$ $=4.5 \mathrm{bar}$ in the case) to below $I M E P_{\text {ref. }}$. Once the transition process is initiated, the engine parameters are controlled in different ways. The engine IMEP (or torque) is directly controlled by regulating $F_{D I}$ of individual engine cylinder; the engine air-to-fuel ratio is controlled by regulating $I_{E T C}$ through an LQ optimal MAP tracking control strategy; spark timing $\left(\theta_{S T}\right)$, EGR valve opening $\left(\phi_{E G R}\right)$, Intake/exhaust valve timings $\left(\theta_{I N T M}\right.$ and $\left.\theta_{\text {EXTM }}\right)$ and lifts $\left(\Pi_{\text {lift }}\right)$ are controlled by the open loop control schedule.

\section{Iterative control of individual cylinder fueling}

It can be observed from Figure 118 that the engine IMEP is highly correlated to $F_{D I}$ with the lean in-cylinder gas-fuel mixture for the hybrid combustion mode during the mode transition. Accordingly, it is possible to control the engine IMEP of each cylinder by regulating the corresponding $F_{D I}$.

The engine IMEP control can be operated in two modes, the learning and transient modes, as shown in Figure 117:, where the learning mode is represented by the dashed lines and the transient mode in the solid lines. For both control modes, $F_{D I}$ is adjusted every engine cycle for each cylinder. The learning mode is enabled when the engine is operated at steady state and close to the transition load. Therefore, this mode can only be operated during the vehicle cruise condition or during the engine calibration process. The transient mode is used during engine transient operations, such as the engine tip-out and tip-in operations.

When the IMEP control is operated at the learning mode, $F_{D I}$ is regulated through the following control law

$$
F_{D I}(i+1)=F_{F F}(i)+F_{I L C}(i)=F_{F F}(i)+K_{I L C}\left[I M E P_{r e f}-I M E P(i)\right]
$$

where the iterative learning control $F_{I L C}$ is calculated by a " $P$ " type (proportional) self learning algorithm as described in [32] and [33], and the iterative learning gain $K_{I L C}$ satisfies the IMEP sensitivity (shown in Figure 118) constraint, and guarantees the stability of the iterative learning.

$$
K_{L C}<\frac{\Delta F_{D I}}{\Delta M E P}
$$

The feedforward term $F_{F F}$ is the learned control variable from the last step that is stored in the memory of the controller. After each learning step $F_{F F}$ is updated by 


$$
F_{F F}(i)=F_{D I}(i)
$$

The updated feedforward term $F_{F F}$ will be used for next learning iteration or the transient mode control. When IMEP control is switched to the transient mode, the iterative learning control is deactivated and the feedback control is activated, as illustrated in Figure $117 ; F_{D I}$ is controlled by the combination of the feedforward and feedback controls, see below

$$
F_{D I}=F_{F F}+F_{F B}
$$

where the feedforward term $F_{F F}$ is the last learned control variable derived in [33]. The feedback term $F_{F B}$ is the output of a PI (proportional and integral) controller. The learned feedforward control $F_{F F}$ reduces the cylinder-to-cylinder IMEP variance, and the feedback control $F_{F B}$ provides the load tracking capability during the transient operation.

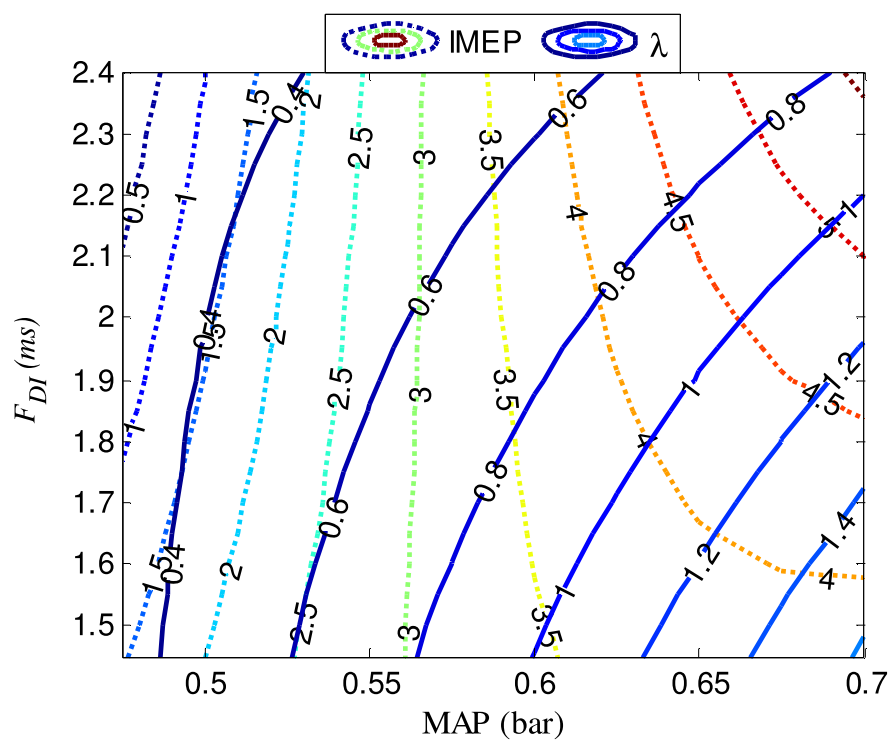

Figure 118: IMEP sensitivity analysis of the SI-HCCI hybrid combustion mode

\section{LQ optimal tracking control}

In the last section, the $F_{D I}$ controller is used to control the individual cylinder IMEP. To maintain the controllability of the DI fuel control $\left(F_{D I}\right)$, lean gas-fuel mixture is required during the mode transition. However, the combustion could become unstable if the mixture becomes extreme lean since the engine spark might not be able to ignite the gas mixture during the transitional cycles. For this study the desired normalize air-to-fuel ratio is set between $\lambda_{\min }(0.97)$ and $\lambda_{\max }$ (1.3). In [1], a step throttle pre-opening approach was proposed to prevent rich combustion at cycle 3 , but it leads to very lean combustion at the following engine cycles. In this section, an LQ tracking control strategy is developed to regulate the air-to-fuel ratio around the desired level.

As discussed above, the normalized air-to-fuel ratio needs to be maintained within the optimal range $\left(\lambda_{\min } \leq \lambda \leq \lambda_{\max }\right)$ during the SI to HCCI combustion mode transition. This control target is difficult to achieve through the air-to-fuel ratio feedback control due to transportation delay and short mode transition period. It is proposed to use the LQ tracking approach to regulate the airto-fuel mixture to the desired level. To implement this control strategy, the optimal operational range of $\lambda$ is translated into the operational range of the engine MAP shown in Figure 119, where 
the upper limit is corresponding to $\lambda_{\max }$ and lower limit is corresponding to $\lambda_{\min }$. An engine MAP tracking reference shown in Figure 119 was generated for the engine Map to stay within the desired range. The reference signal is represented by

$$
z(k)=\left\{\begin{array}{ccc}
Z_{S I} & \text { if } & k_{B}<k \leq k_{1} \\
Z_{S I}+\left(Z-Z_{S I}\right) \frac{k-k_{1}}{k_{2}-k_{1}} & \text { if } & k_{1}<k \leq k_{2} \\
Z+\left(Z_{H C C I}-Z\right) \frac{k-k_{1}}{k_{2}-k_{1}} & \text { if } & k_{2}<k \leq k_{E}
\end{array}\right.
$$

where $k$ is the sampling index; $k_{B}$ and $k_{E}$ represent the beginning and ending indices of the mode transition and they were set to 600 and 900, respectively, as shown in Figure 119; $k_{1}$ and $k_{2}$ are switch indices and they equal 670 and 720, respectively; $Z_{S I}$ and $Z_{H C C I}$ are the desired MAP of SI and HCCI modes, respectively; $Z$ is the desired MAP at $k_{2}$.

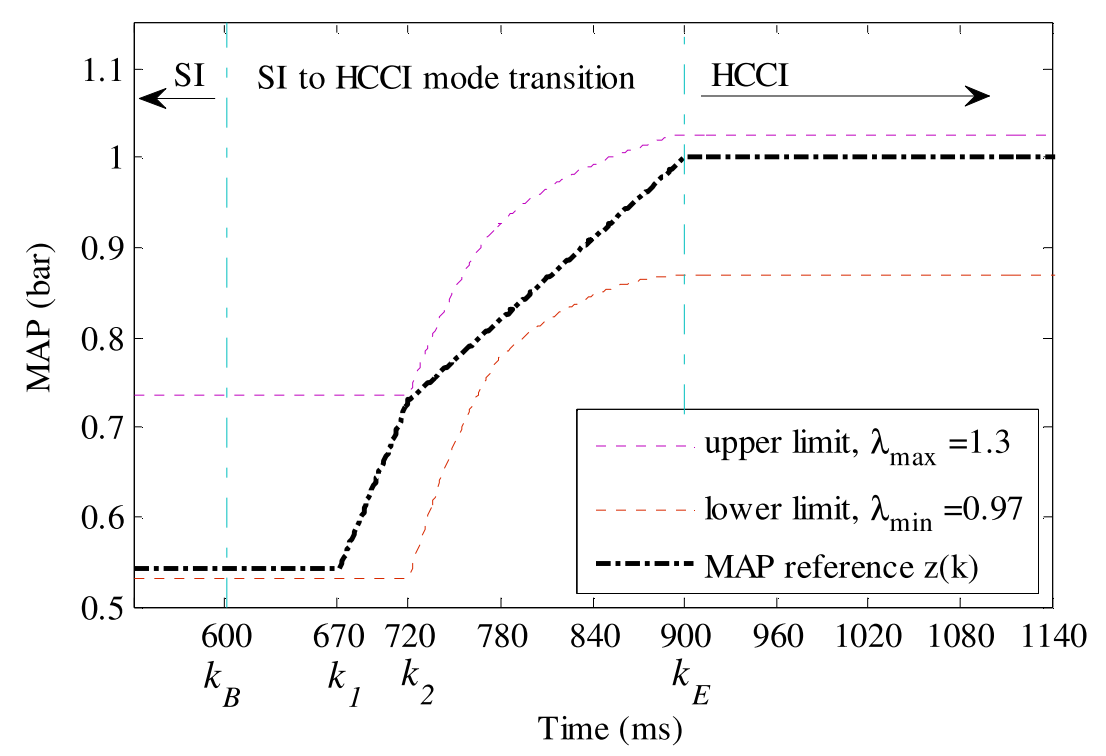

Figure 119: The target MAP operational range and MAP tracking reference

To develop the proposed LQ tracking control strategy, a simplified engine MAP model is required to represent the relationship between the control input $\left(I_{E T C}\right)$ and the system output (MAP). The simplified dynamics is represented by a second order dynamics due to the gas filling dynamics (first order) of the engine intake manifold and the first order response delay of the engine throttle. The governing equation of gas filling dynamics is represented by

$$
\frac{d M A P}{d t}=-\eta \frac{V_{d} N_{e}}{120 V_{m}} M A P+\varphi \frac{R T_{\text {amb }} C_{D} \pi r^{2} P_{\text {amb }}}{V_{m} \sqrt{2 R T_{\text {amb }}}} \phi_{T P S}
$$

and the dynamics of the throttle response is approximated by

$$
\frac{d \phi_{T P S}}{d t}=-\frac{k_{E T C}}{b_{E T C}} \phi_{T P S}+\frac{c_{E T C}}{b_{E T C}} I_{E T C}
$$

Equations (51) and (52) can be combined, discretized and represented by the following discrete state space model

$$
\begin{aligned}
& x(k+1)=A x(k)+B u(k) \\
& y(k)=C x(k)+D u(k)
\end{aligned}
$$

where 


$$
u=I_{E T C} \quad ; \quad x=\left[\begin{array}{l}
x_{1} \\
x_{2}
\end{array}\right]=\left[\begin{array}{c}
M A P \\
\phi_{T P S}
\end{array}\right] ; \quad y=M A P
$$

are the system input, state and output respectively. The system matrices are

$$
\begin{array}{ll}
A=\left[\begin{array}{cc}
1-\frac{\eta(k) V_{d} N_{e}}{120 V_{m}} \Delta T & \frac{\varphi(k) R_{a} T_{a} C_{D} \pi r^{2} P_{a}}{V_{m} \sqrt{2 R_{a} T_{a}}} \Delta T \\
0 & 1-\frac{k_{E T C}}{b_{E T C}} \Delta T
\end{array}\right], \quad B=\left[\begin{array}{c}
0 \\
\frac{k_{E T C}}{b_{E T C}} \Delta T
\end{array}\right] \\
C=\left[\begin{array}{cc}
1 & 0
\end{array}\right], & D=0
\end{array}
$$

where $\Delta T$ is the sample period. State space model (53) is linear time-variant since the volumetric efficiency $\eta$ and multiplier $\varphi$ in equations (51) and (55) are functions of the engine operating condition. Moreover, the sampling time $\Delta T$ in (55) equals 1 millisecond, and sample time index $k$ is the same as that in equation (50).

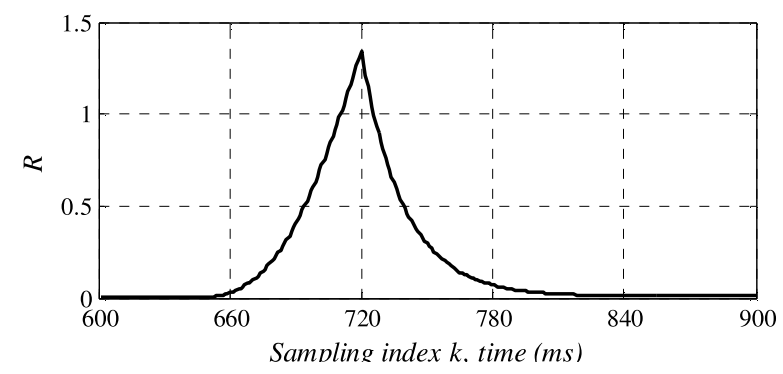

Figure 120: Weighting matrix, R, selection

Based on the control-oriented engine MAP model, a finite horizon LQ optimal tracking controller was designed to follow the reference $z(k)$. More specifically, the control objective is to minimize the tracking error $e(k)$ defined in (56) with the feasible control effort $I_{E T C}$. The tracking error $e(k)$ is defined as

$$
e(k)=y(k)-z(k)=C x(k)-z(k)
$$

and the constraint on $I_{E T C}$ is $-5 \mathrm{~A}<I_{E T C}<5 \mathrm{~A}$. The cost function of the LQ optimal controller is defined as

$$
\begin{aligned}
J= & \frac{1}{2}\left[C x\left(k_{f}\right)-z\left(k_{f}\right)\right]^{T} F\left[C x\left(k_{f}\right)-z\left(k_{f}\right)\right] \\
& +\frac{1}{2} \sum_{k=k_{i}}^{k=k_{f}-1}\left\{[C x(k)-z(k)]^{T} Q[C x(k)-z(k)]+u^{T}(k) R u(k)\right\}
\end{aligned}
$$

where $F$ and $Q$ are positive semi-definite and $R$ is positive definite. For this paper, $F$ and $Q$ are constant matrices defined in (58) and $R$ is a function of sample index and tuned to optimize the tracking error with feasible throttle control effort, see Figure 120.

$$
F=10^{-8}, \quad Q=4 \times 10^{-7}, \quad R=R(k)
$$

Based on the cost function the corresponding Hamiltonian is as follows

$$
\begin{aligned}
H= & \frac{1}{2}[C x(k)-z(k)]^{T} Q[C x(k)-z(k)]+\frac{1}{2} u^{T}(k) R u(k) \\
& +p^{T}(k+1)[A x(k)+B u(k)]
\end{aligned}
$$

According to [34], the necessary conditions for the extremum in terms of the Hamiltonian are represented as

$$
\frac{\partial H}{\partial p^{*}(k+1)}=x^{*}(k+1) \Rightarrow x^{*}(k+1)=A x^{*}(k)+B u^{*}(k)
$$




$$
\begin{gathered}
\frac{\partial H}{\partial x^{*}(k)}=p^{*}(k) \Rightarrow p^{*}(k)=A^{T} p^{*}(k+1)+C^{T} Q C x^{*}(k)-C^{T} Q z(k) \\
\frac{\partial H}{\partial u^{*}(k)}=0 \Rightarrow 0=B^{T} p^{*}(k+1)+R u^{*}(k)
\end{gathered}
$$

Note that the superscript "*" denotes the optimal trajectories of the corresponding vectors. The augmented system of (60) and (61) becomes

$$
\left[\begin{array}{c}
x^{*}(k+1) \\
p^{*}(k)
\end{array}\right]=\left[\begin{array}{cc}
A & -B R^{-1} B^{T} \\
C^{T} Q C & A^{T}
\end{array}\right]\left[\begin{array}{c}
x^{*}(k) \\
p^{*}(k+1)
\end{array}\right]+\left[\begin{array}{c}
0 \\
-C^{T} Q
\end{array}\right] z(k)
$$

Based on (62) the optimal control is in the form of

$$
u^{*}(k)=-R^{-1} B^{T}\left[P(k) x^{*}(k)-g(k)\right]
$$

Matrix $P(k)$ can be computed by solving the difference Riccati equation backwards

$$
P(k)=A^{T} P(k+1)[I+E P(k+1)]^{-1} A+C^{T} Q C
$$

with the terminal condition

$$
P\left(k_{f}\right)=C^{T} F C
$$

and vector $g(k)$ can be computed by solving the vector difference equation

$$
g(k)=A^{T}\left\{I-\left[P^{-1}(k+1)+E\right]^{-1} E\right\} g(k+1)+C^{T} Q z(k)
$$

with the terminal condition

$$
g\left(k_{f}\right)=C^{T} F z\left(k_{f}\right)
$$

The optimal control in (64) can be written into the following form

$$
u^{*}(k)=-L_{F B}(k) x^{*}(k)+L_{F F}(k) g(k+1)
$$

where the feedforward gain $L_{F F}$ is computed by

$$
L_{F F}(k)=\left[R+B^{T} P(k+1) B\right]^{-1} B^{T}
$$

and the feedback gain $L_{F B}$ is computed by

$$
L_{F B}(k)=\left[R+B^{T} P(k+1) B\right]^{-1} B^{T} P(k+1) A
$$

Note that in equation (69) the state $x^{*}$ used in the feedback control is computed exactly from the closed-loop system model defined below

$$
x^{*}(k+1)=\left[A-B L_{F B}(k)\right] x^{*}(k)+B L_{F F}(k) g(k+1)
$$

However, when the control is implemented into the HIL simulation environment or the actual engine control system, the feedback states are replaced by the actual signals (MAP and $\phi_{T P S}$ ) measured by the on-board engine sensors. In these cases the LQ controller is represented by the online form as

$$
u(k)=-L_{F B}(k) x(k)+L_{F F}(k) g(k+1)
$$

where $x$ represents the sampled states. Note that both of the states, MAP and $\phi_{T P S}$ can be measured in the HIL simulator or in the engine system

\subsubsection{Model-based control simulation results and discussions}

The multistep combustion mode transition control strategy is composed of the DI fuel quantity control and LQ optimal MAP tracking control with the SI-HCCI hybrid combustion during the mode transition. In this section, an HIL simulation environment is introduced; the HIL simulation results are presented for the control strategy validation. 


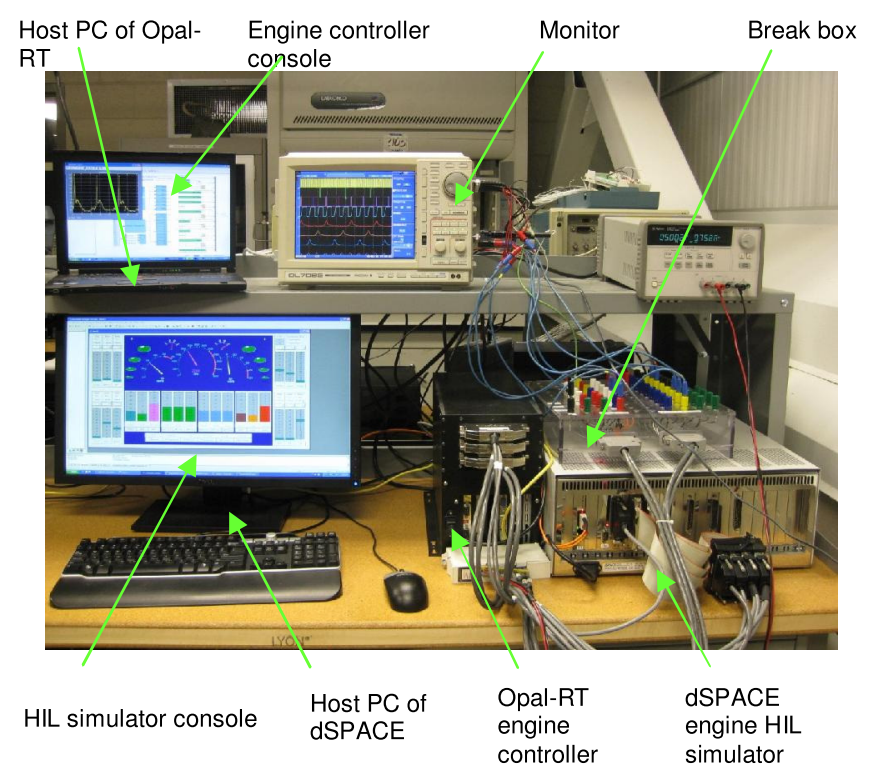

Figure 121: HIL simulation station

For the purpose of initial control strategy development and validation, an HIL simulation station, shown in Figure 121, was used. By implementing the control-oriented engine model described in [9] and [35], the dSPACE based engine HIL simulator provided all measureable engine signals (such as in-cylinder pressure and MAP signals) in real-time. These signals were directly fed into the Opal-RT based real-time prototype engine controller. Some unmeasurable engine states (such as $T_{I V C}$ ) were also available to help with the control performance analysis. On the other hand, the control signals generated by the prototype engine controller were also fed back into the engine simulator. These signals were recorded and analyzed for control strategy development and validation.

\section{Simulation results of using SI-HCCI hybrid combustion mode}

As mentioned above, the engine is operated in the SI-HCCI hybrid combustion mode between cycles 3 to 5 (see Figure 116) in the multistep mode transition strategy. To disclose the significance of using this hybrid combustion mode, the key engine variables, such as $T_{I V C}, x_{H C C I}$, and IMEP, are analyzed and plotted in Figure 122:, for the cases with and without the SI-HCCI hybrid combustion mode. Note that the simulation time from $600^{\text {th }}$ to $900^{\text {th }}$ millisecond is corresponding to the five transitional engine cycles illustrated in Figure 116.

As shown in Figure 122, charge mixture temperature at intake valve closing $\left(T_{I V C}\right)$ has response delay with or without the hybrid combustion mode due to the dynamics of the valvetrain system and the NVO operation. Under such thermo condition, if the engine was switched into the HCCI combustion mode directly, start of combustion timing would be greatly retarded (misfire could happen), causing large IMEP variance [36]. The large fluctuation of IMEP cannot be improved through combustion control due to the lack of available controllability in HCCI mode. However by using the hybrid combustion mode during the mode transition, percentage of HCCI 
combustion $x_{H C C I}$ is gradually increased as $T_{I V C}$ approaches the desired temperature. Also note that due to the LQ optimal tracking of the desired air-to-fuel ratio, the engine IMEP can be regulated by controlling $F_{D I}$. As a result, smooth IMEP can be achieved during the mode transition. Note that for the simulation results shown in Figure 122:, the proposed air-to-fuel ratio and IMEP controls are used during the mode transition with the hybrid combustion.

Therefore, using the SI-HCCI hybrid combustion during the mode transition is a key control technique for the smooth combustion mode transition.

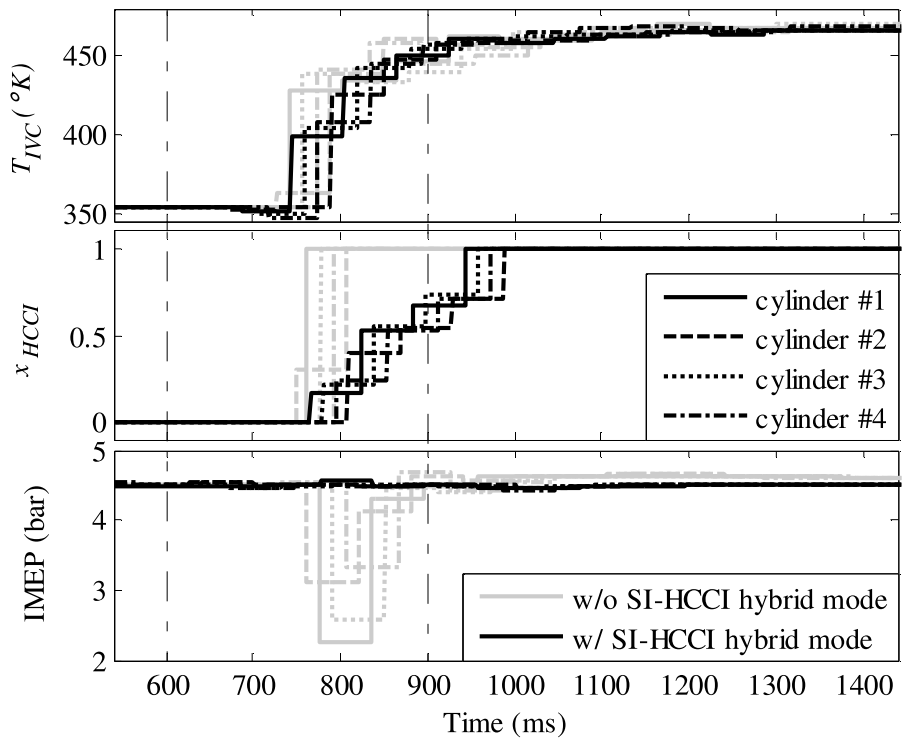

Figure 122: Combustion performances with and without SI-HCCI hybrid combustion mode

\section{$\underline{\text { Simulation results of air-to-fuel ratio control }}$}

The developed LQ optimal MAP tracking control was implemented in the prototype engine controller and validated through the HIL engine simulations. The simulated control input $I_{E T C}$, the system states MAP and $\phi_{T P S}$, and $\lambda$ are plotted in Figure 123:. For comparison purpose, the simulated responses of these variables with a step $I_{E T C}$ control approach are also shown in Figure 123:, in which $I_{E T C}$ is set to the target level before the adjustment of $\Pi_{\text {lift }}$ (happens at $720^{\text {th }} \mathrm{ms}$ ), as a result, the engine throttle is gradually opened to the wide open throttle (WOT) position and the MAP is increased before the valve lift switch. The increased MAP ensures enough fresh charge to each cylinder when the valve lift switches to the low lift. However the step $I_{E T C}$ control leads to a rapid increment of the engine MAP or excessive fresh air charge, leading to extreme lean air-to-fuel ratio $\lambda$ in the following engine cycles.

Using the proposed LQ MAP tracking control strategy, throttle current $I_{E T C}$ is regulated in a nonmonotonic increasing pattern. Note that to maintain $I_{E T C}$ in the feasible range $\left(-5 \mathrm{~A}<I_{E T C}<5 \mathrm{~A}\right)$ the weighting matrix $R$ in the cost function (57) is adjusted as illustrated in Figure 120:. The similar pattern can also be found for $\phi_{T P S}$ with a small phase lag. As a result, the engine MAP tracks the reference $z(k)$ after the intake valve lift $\Pi_{\text {lift }}$ switches to the low lift, and $\lambda$ of each cylinder is successfully maintained within the desired range. Therefore, with the help of the LQ 
optimal tracking control, the in-cylinder air-to-fuel ratio is maintained within the desired range, leading to stable combustion.

Slight oscillations in the MAP responses are found with both control approaches, which are due to the flow dynamics of the engine air-handling system and the engine MAP modeling error. It is difficult to eliminate them. Moreover, the MAP oscillation associated with the LQ optimal tracking control is within the desired MAP range.

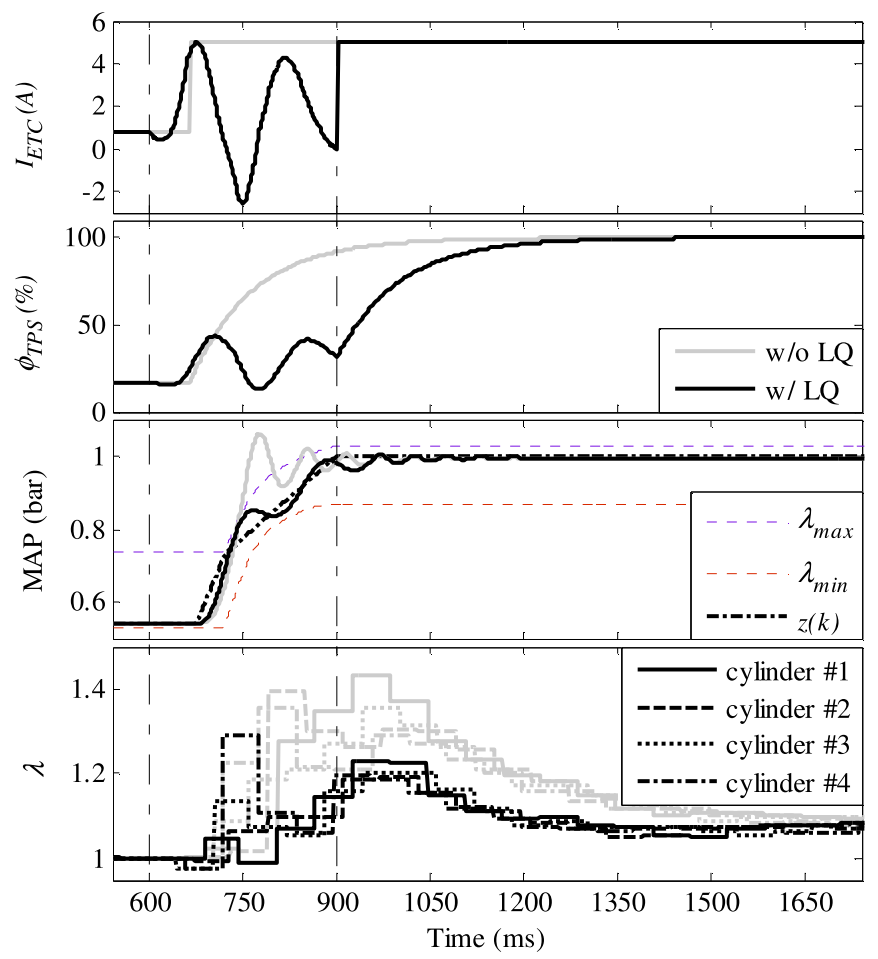

Figure 123: Engine performances of the optimal MAP tracking control

\section{Simulation results of IMEP control in learning mode}

When air-to-fuel ratio is maintained within the desired range, the iterative learning of $F_{D I}$ was conducted in the learning mode. Figure 124 shows that $F_{D I}$ of each cylinder converges after a few iterations of learning. $F_{D I}$ in cycle 2 was adjusted significantly due to the large variation of the engine MAP. The first engine cycle (cycle 3) after the intake/exhaust valve lift switch demonstrated the largest improvement, leading to significant correction of $F_{D I}$. The cylinder-tocylinder adjustment of $F_{D I}$ after iterative learning compensates for the intake charge variations due to the MAP variations, and leads to smooth IMEP of individual cylinder as shown in Figure 125. Therefore, it can be concluded that smooth SI to HCCI combustion mode transition is achievable through the iterative learning control of the DI fuel quantity. 


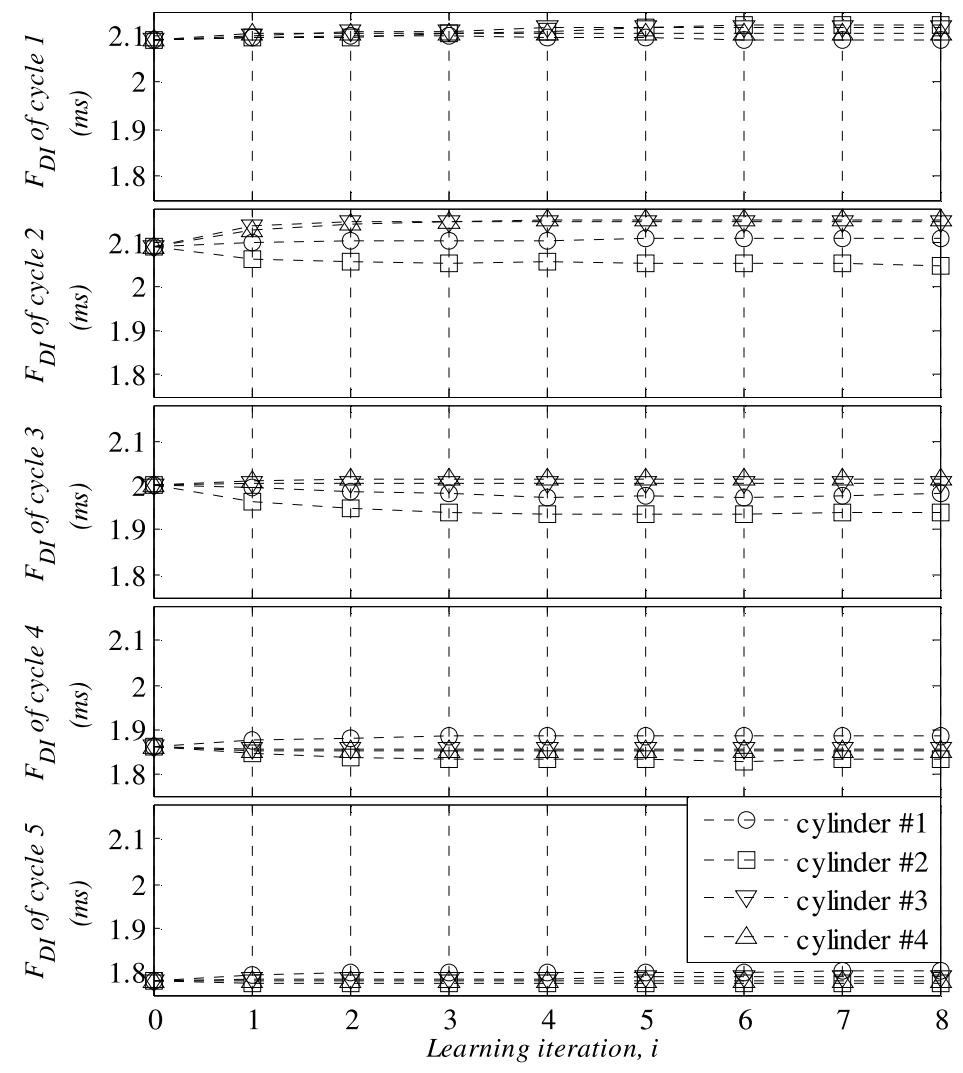

Figure 124: Correction of DI fuel quantity after iterative learning

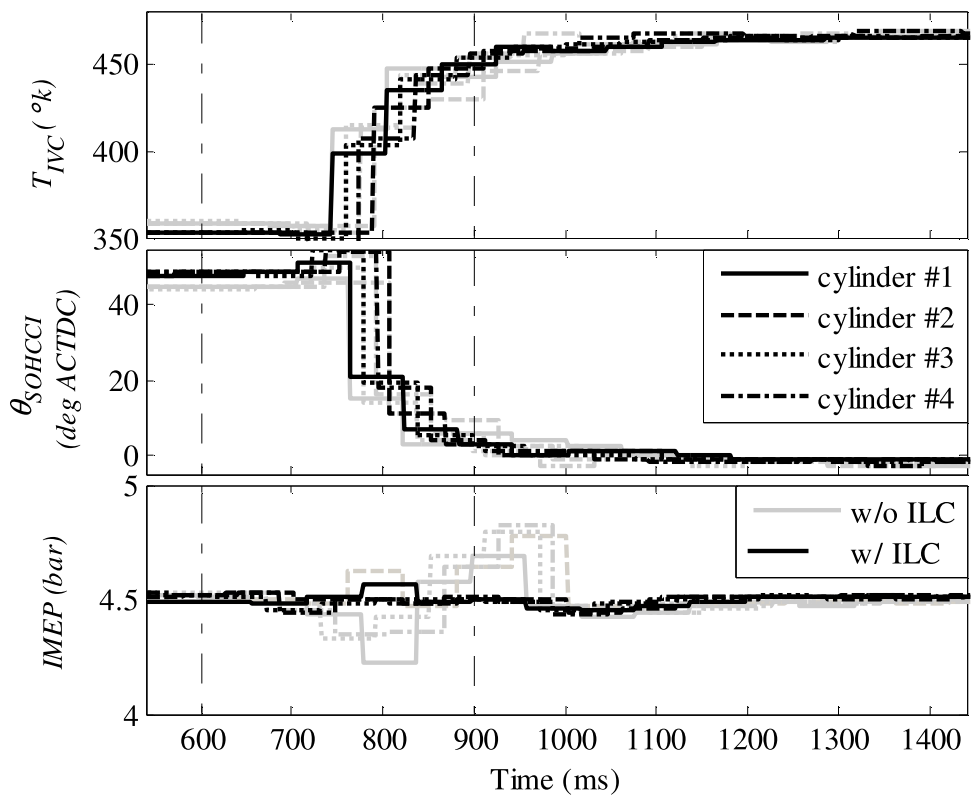

Figure 125: Combustion performances with optimal MAP tracking and DI fuel iterative learning control strategies 


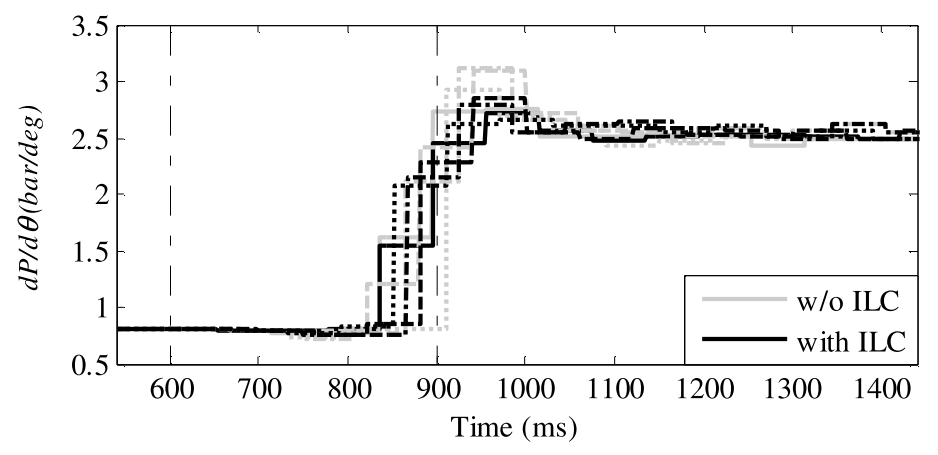

Figure 126: Engine knock index during combustion mode transition

In Figure 125, without ILC slight IMEP oscillations can be observed for $T_{I V C}$ and $\theta_{S O H C C I}$, however, the oscillations disappears after the ILC was applied. These oscillations indicate unstable combustion and are due to the cycle-to-cycle residue gas dynamics of HCCI combustion (including the SI-HCCI hybrid combustion), which has been discussed in great details in [36] and [37]. Due to the residue gas dynamics, retarded (or advanced) $\theta_{\text {SOHCCI }}$ of current engine cycle leads to advanced (or retarded) $\theta_{S O H C C I}$ for the next engine cycle. As a result, combustion oscillations occur. During the combustion mode transition, the impact of adjusting the valve lift $\Pi_{\text {lift }}$ to the individual in-cylinder combustion is quite different. Cylinder 1 experiences the most significant impact since the valve lift switch happens during its exhaust stroke. Both residue gas quantity and fresh charge quantity were affected. Therefore the IMEP fluctuations of cylinder 1 are dominated as shown in Figure 125. By using ILC of DI fuel quantity, $\theta_{S O H C C I}$ was regulated to the appropriate timing as well as the IMEP was regulated to the control reference. Accordingly the oscillations of $T_{I V C}$ and $\theta_{S O H C C I}$ were suppressed, and combustion stability during the mode transition was improved by the iterative learning control.

As a result of suppressing the oscillations of $T_{I V C}$ and $\theta_{S O H C C I}$, engine knock index was also reduced during the combustion mode transition, see Figure 126. Note that $d P / d \theta$ is a good indicator of engine knock.

\section{Engine torque performance comparison}

Engine torque responses during the combustion mode transitions using the different control strategies are plotted in Figure 127. Their statistics are listed in TABLE 29 for comparison. The largest engine torque fluctuation is produced by the one-step approach. The fluctuation of engine torque is reduced when multistep strategy is used. It is further improved as the SI-HCCI hybrid combustion mode is implemented. At last, smooth combustion mode transition is realized in multi-steps when both the air-to-fuel ratio (LQ tracking control) and the DI fuel quantity (ILC) controls are applied. The combined control of LQ tracking and ILC DI fueling with SI-HCCI hybrid combustion leads to the lowest torque fluctuations $(2.2 \%)$ when the engine operational conditions transit from the steady state SI mode to the HCCI mode. The in-cylinder gas pressure profiles are plotted in Figure 128. One can see significant improvement of combustion quality during the transitional cycles. 


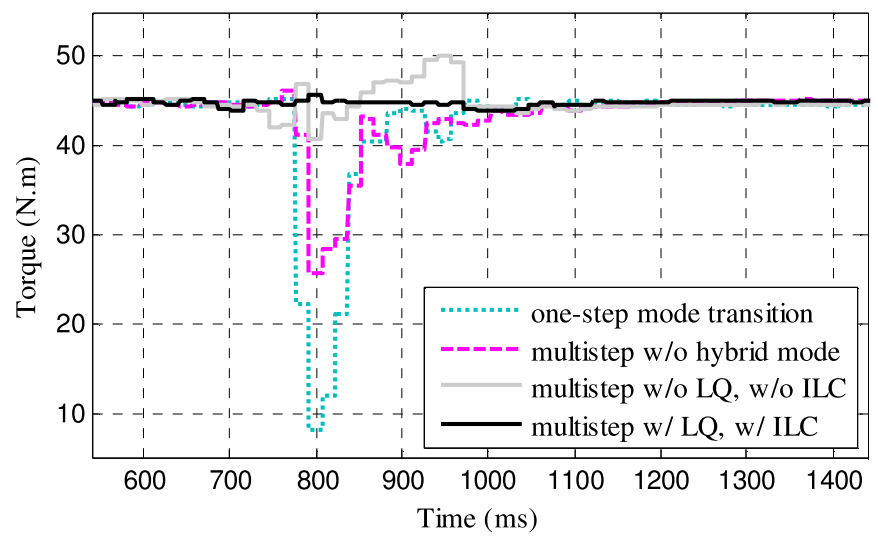

Figure 127: Engine torque performances of different control strategies

TABLE 29: Engine Performance Statistics for Different Control Strategies

\begin{tabular}{cccc}
\hline \hline & $\begin{array}{c}\text { IMEP } \\
\text { error } \\
(\%)\end{array}$ & $\begin{array}{c}\text { Max } \\
d P / d \theta \\
(\mathrm{bar} / \mathrm{deg})\end{array}$ & $\begin{array}{c}\text { Torque } \\
\text { error } \\
(\%)\end{array}$ \\
\hline $\begin{array}{c}\text { One-step mode } \\
\text { transition }\end{array}$ & 51.6 & $\mathrm{~N} / \mathrm{A}$ & 81.8 \\
\hline $\begin{array}{c}\text { Multistep w/o hybrid } \\
\text { mode }\end{array}$ & 26.9 & $\mathrm{~N} / \mathrm{A}$ & 43.1 \\
\hline $\begin{array}{c}\text { Multistep w/o LQ, w/o } \\
\text { ILC }\end{array}$ & 7.1 & 3.13 & 11.2 \\
\hline $\begin{array}{c}\text { Multistep w/ LQ, w/ } \\
\text { ILC }\end{array}$ & 1.6 & 2.82 & 2.2 \\
\hline \hline
\end{tabular}

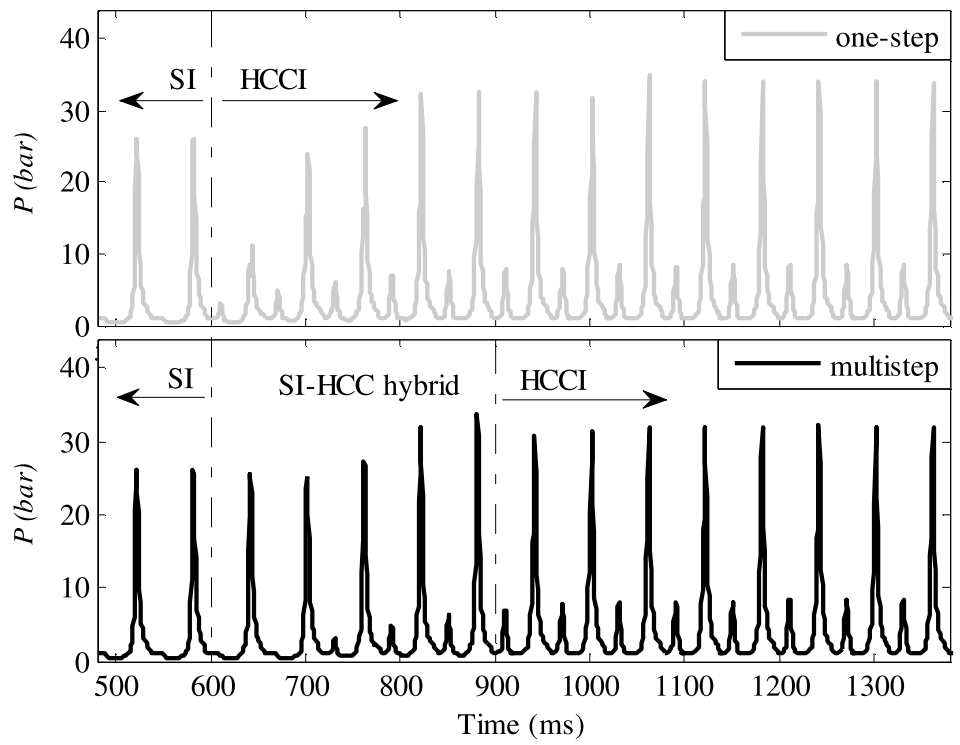

Figure 128: In-cylinder gas pressure profile of cylinder 1 during SI to HCCI combustion mode transition 


\section{Simulation results of IMEP control under mode transition}

In order to study the mode transition under the transient operation, an HIL simulation was conducted to simulate the engine throttle tip out operation, where the engine was initially operated in the SI mode. As a step input was applied to the acceleration pedal, the pedal position decreased from $40 \%$ to $15 \%$. As a result, the engine IMEP reduced from 8.1 bar to 4 bar, which crosses the combustion mode transition threshold of 4.5 bar. Accordingly the combustion mode transition was triggered. Two mode transition strategies were simulated. Both of them were multistep strategies with the hybrid combustion. One was with the optimal air-to-fuel ratio control (LQ) and the DI fuel quantity control, the other was not. Note that the DI fuel quantity control was switched to the transient mode during the engine tip out operation, and the feedforward term $F_{F F}$ used in the transient mode control was the last learned value by the ILC. The transient responses of engine variables are plotted in Figure 129.

In Figure 129, after the step input of the acceleration pedal, both $\phi_{T P S}$ and MAP are decreased at first, and then increased due to the combustion mode transition to unthrottled HCCI mode. Slightly rich combustion can be observed during the early stage of the tip out operation since the throttle opening is decreased in the SI mode. Once the mode transition started, the in-cylinder gas-fuel mixture becomes lean for both strategies. However, with the LQ MAP tracking control, the normalized air-to-fuel ratio $\lambda$ is maintained within the desired range throughout the engine tip out operation. Furthermore, with the proposed DI fuel quantity control smooth IMEP responses were achieved for all cylinders comparing with those without the iterative fuel control. Similar improvement in engine torque output can also be found in Figure 130.

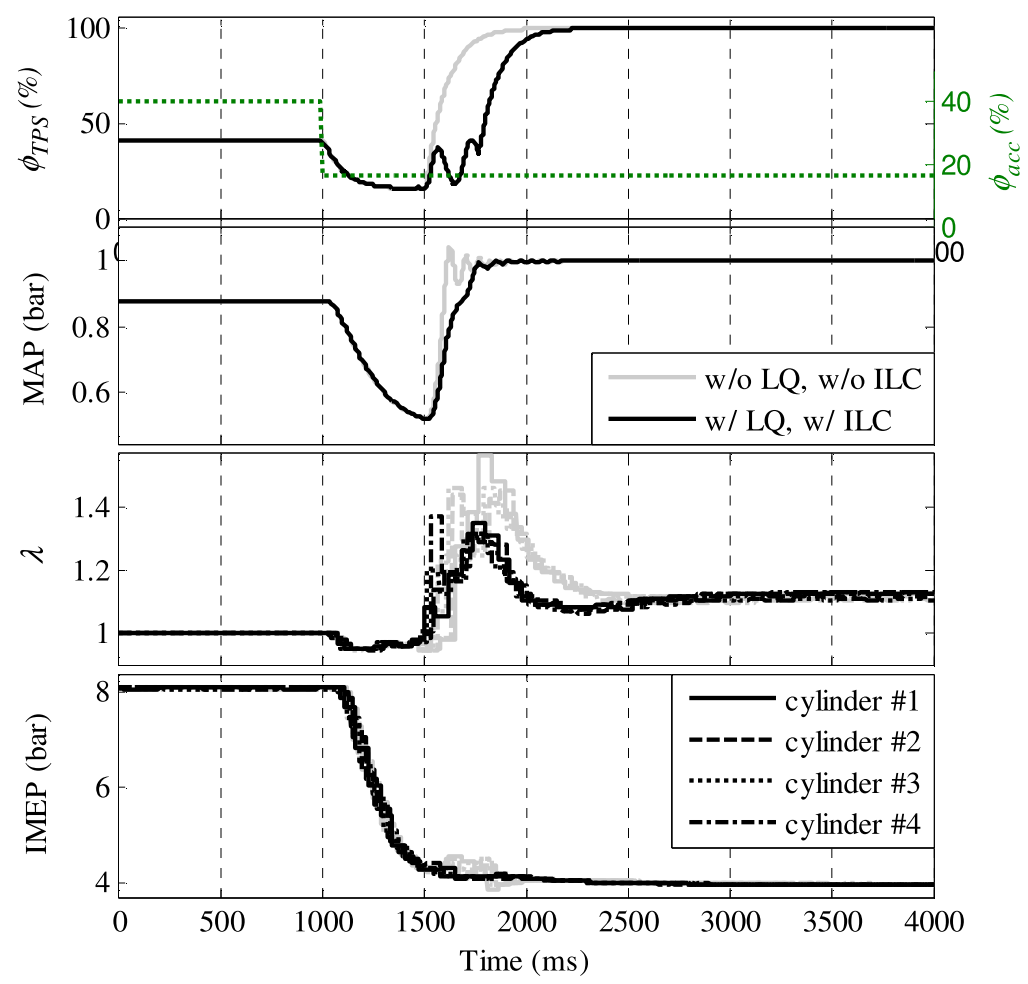

Figure 129: Engine responses during Tip out operation 


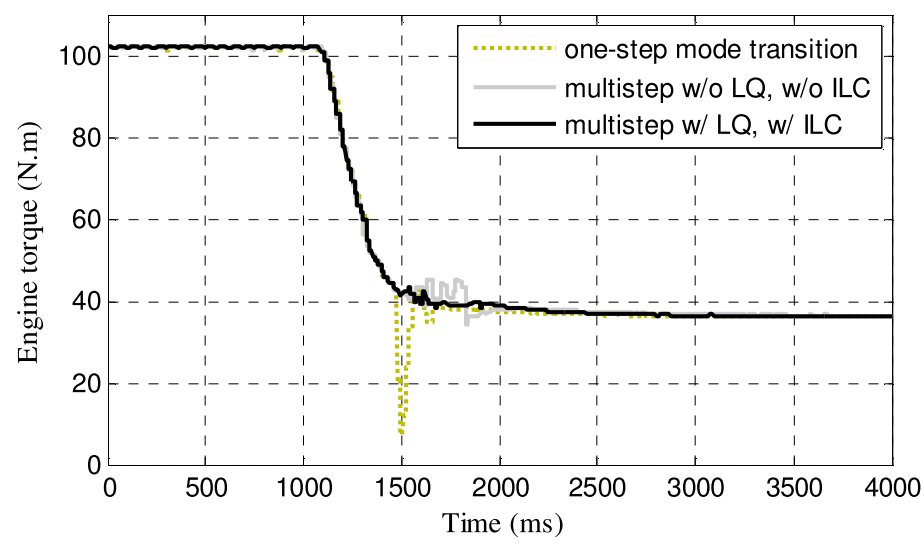

Figure 130: Engine torque response during tip out operation

Base on the simulation results shown in Figure 129 and Figure 130, it can be concluded that the proposed LQ optimal MAP tracking control is capable of maintaining the air-to-fuel ratio during engine transient operations, and the iterative DI fuel quantity control is also effective in transient mode.

\subsubsection{Control strategy implementation}

To prepare for the SI and HCCI mode transition control, the mode transition algorithms were implemented into the Opal-RT real-time controller. Mode transition from SI to HCCI was designed to be accomplished within 5 engine cycles. During these 5 cycles, intake and exhaust valves timings and lifts, injection timing and duration, spark timing, and throttle position transit from SI (or HCCI) to HCCI (or SI) targets during the mode transition to achieve a smooth transition process. The high level mode transition control block diagram is shown in Figure 131.

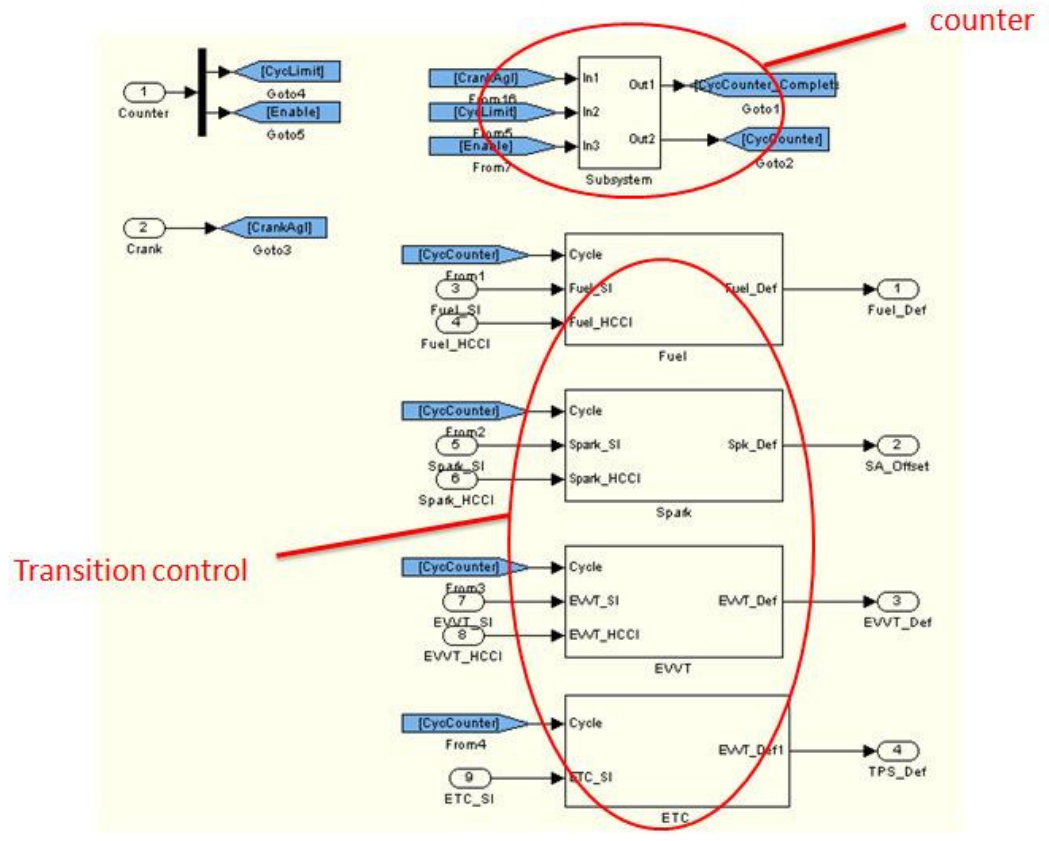

Figure 131: High level control mode transition algorithm 
The counter subsystem calculates the mode transition cycle number and other parameters based upon the internal crank angle signal, and the detailed logic can be found in Figure 132. The transition switch input is used to initiate the combustion mode transition and the number of cycles required for the combustion mode transition can be specified, too. Due to the actuator delay, it may require more than 5 cycles to complete the mode transition.

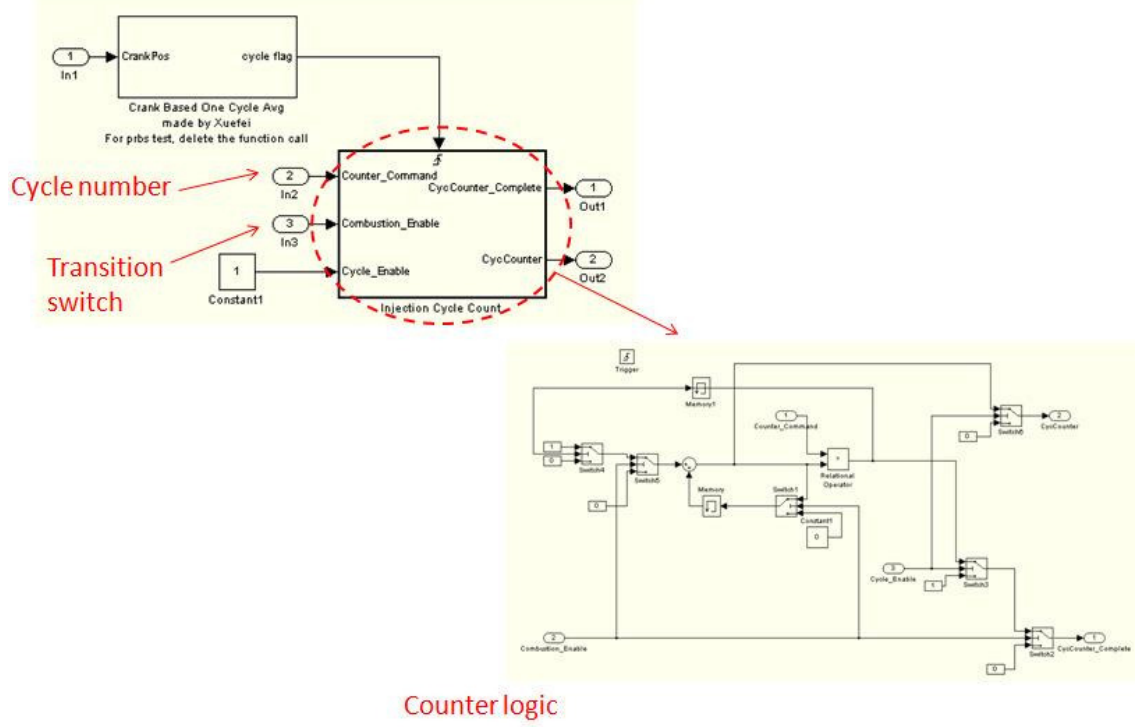

Figure 132: Transition enabling logic

Both valve lift and phasing actuators are compensated with the additional advances to accommodate the additional actuation delays. For the electronic throttle, during the mode transition from SI to HCCI, the throttle will be gradually opened to achieve desired manifold pressure; and the throttle is widely opened in the HCCI mode. The fuel and spark control is similar to the throttle control.

\subsection{HCCI combustion dynamometer tests}

This subsection provides the low lift combustion test and two HCCI test results.

\subsubsection{Low lift combustion characteristic study}

Further studies were completed to understand the performance using the low-lift cams since this is important for stable HCCI combustion. A first set of tests involved an injection timing sweep with the low-lift cams in their default retarded position. It was found that the same $300^{\circ} \mathrm{BTDC}$ injection timing used in the high-lift tests was optimal based on IMEP and COV (Figure 133).

The main interest to the low-lift cam operation was the influence of the exhaust cam timing on EGR levels and the effect on combustion and breathing efficiency. Two sets of exhaust timing sweeps were performed by advancing the exhaust valve timing over a $70^{\circ}$ window. Firstly, all parameters were kept constant to see the effect of the increased EGR on the rate of combustion, AFR (lambda), and engine load; secondly, spark and fuel were tuned to keep AFR (lambda) and CA50 (crank location when 50\% fuel is burned) constant. It is clear that exhaust cam advancement slows combustion significantly, but spark and fuel adjustments can yield stable performance with favorable combustion phasing over the entire $70^{\circ}$ window of exhaust cam 
timing even as EGR levels increase significantly (Figure 134). Note that the PMEP reduces as the exhaust valve timing is advanced with the slightly faster combustion (Table 30).

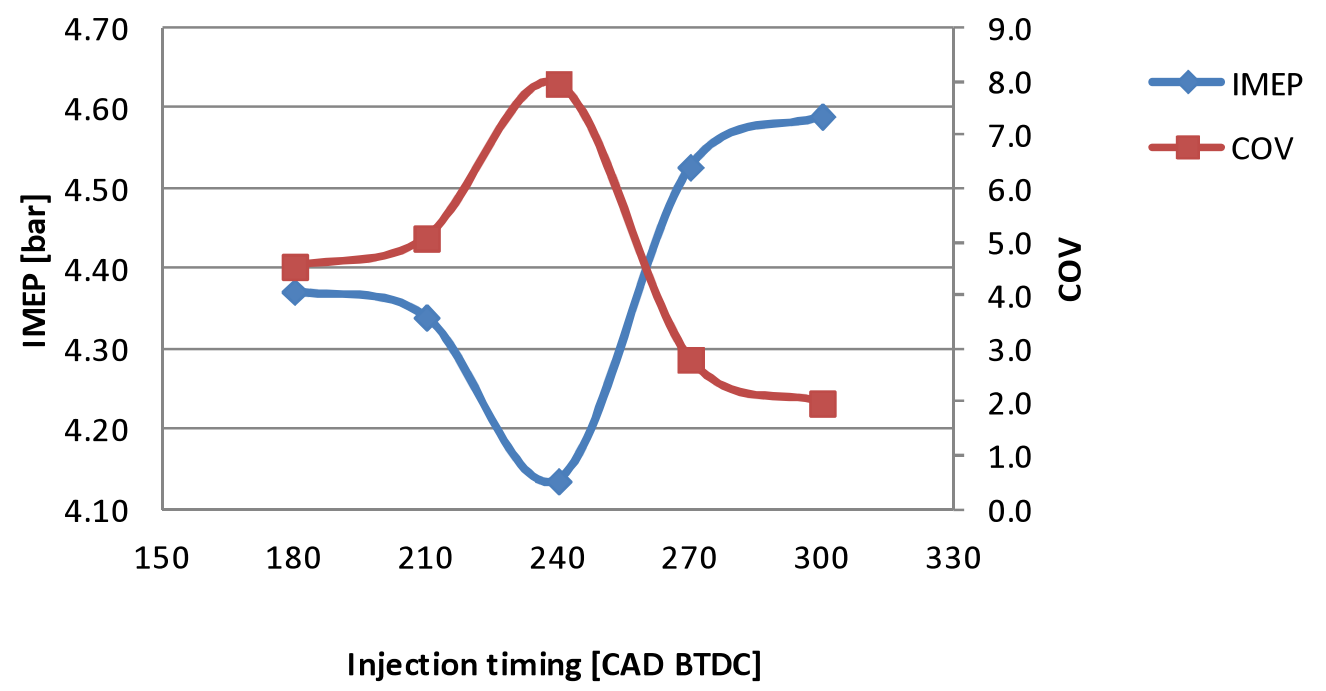

Figure 133: IMEP and COV of injection timing sweep, low lift cams

Table 30: Low-lift exhaust cam timing sweep data, $\lambda \approx 1$, CA50 $\approx 8.5$

\begin{tabular}{|c|ccccccccc|}
\hline $\begin{array}{c}\text { Exhaust } \\
\text { Advance }\end{array}$ & $\begin{array}{c}\text { IMEP } \\
{[\text { [bar] }}\end{array}$ & $\begin{array}{c}\text { NMEP PMEP } \\
{[\text { [bar] }}\end{array}$ & $\begin{array}{c}\text { [bar] } \\
\text { COV [ }\end{array}$ CATDC] & Lambda & $\begin{array}{c}\text { MAP } \\
\text { [kPa] }\end{array}$ & $\begin{array}{c}\text { Spark } \\
\text { timing } \\
{\left[{ }^{\circ} \text { BTDC] }\right.}\end{array}$ \\
\hline $\mathbf{0}^{\circ}$ & 4.54 & 3.09 & 1.45 & 1.5 & 8.1 & 0.98 & 39.8 & 33 \\
\hline $\mathbf{2 0}^{\circ}$ & 4.52 & 3.41 & 1.11 & 1.3 & 8.6 & 0.99 & 39.0 & 31 \\
\hline $\mathbf{4 0}^{\circ}$ & 4.51 & 3.67 & 0.84 & 1.4 & 9.0 & 1.01 & 41.4 & 36 \\
\hline $\mathbf{6 0}^{\circ}$ & 4.44 & 3.84 & 0.59 & 1.5 & 8.3 & 1.01 & 47.9 & 42 \\
\hline $\mathbf{7 0}^{\circ}$ & 4.42 & 3.91 & 0.51 & 1.8 & 8.9 & 0.98 & 52.6 & 47 \\
\hline
\end{tabular}

Table 31 shows a comparison of high-lift and low-lift performance with 4.5 bar IMEP at 2000 RPM. The low-lift setup slows combustion and requires a much larger spark advance, especially at extreme exhaust cam position that causes high levels of EGR. At this load, the high-lift cam is superior with a greater IMEP from the same fuel pulse and a much lower PMEP. Advancing the low lift cam $70^{\circ}$ reduces the PMEP to the level comparable to the high-lift cam, but the rate of combustion decreases significantly, and IMEP is reduced despite the increased fueling. 

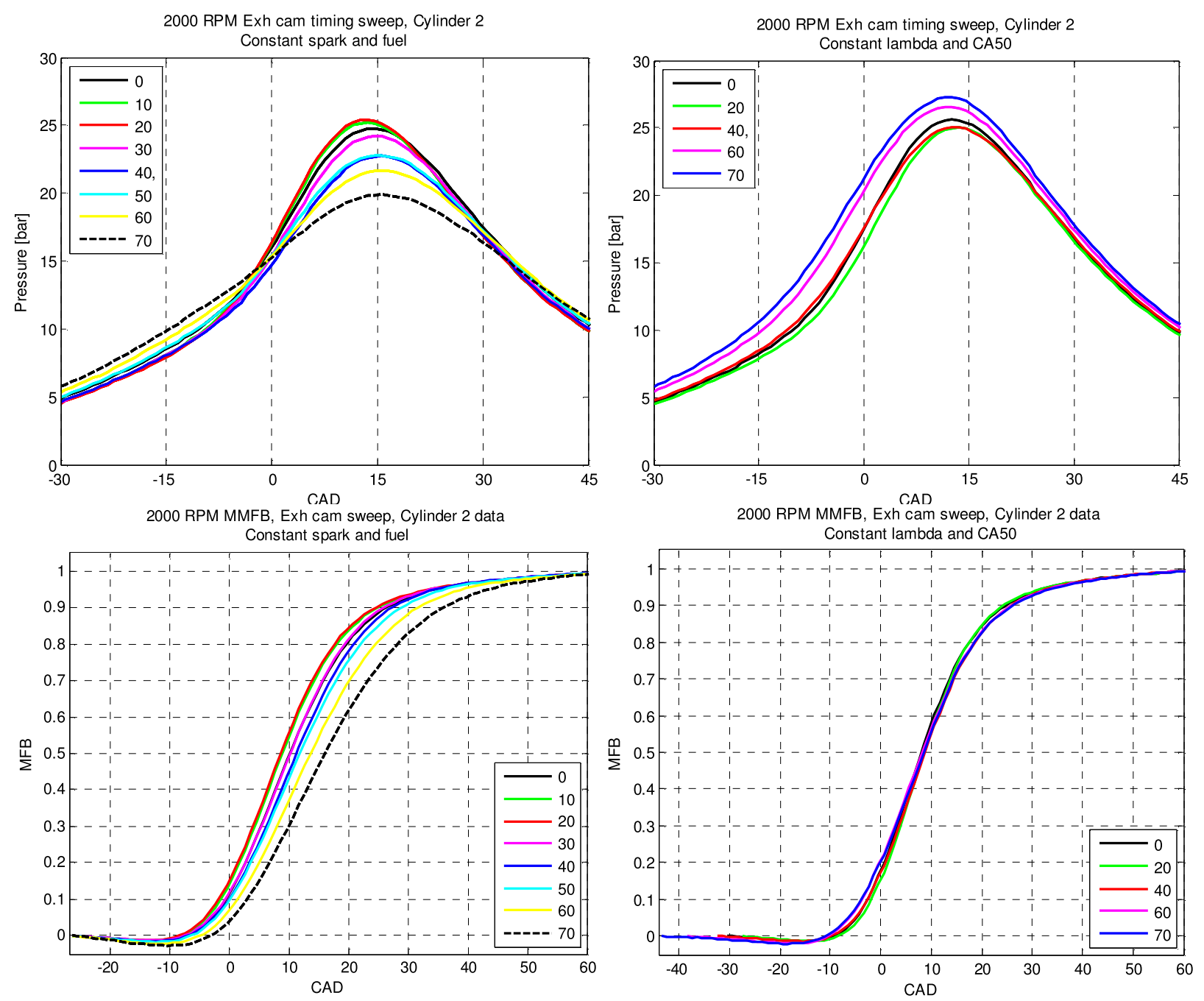

Figure 134: Pressure traces and mass fraction burned for low-lift exhaust cam sweeps

Table 31: High and Low Lift Cam Combustion Comparison

\begin{tabular}{|ccccccccccc|}
\hline Speed & $\begin{array}{c}\text { Cam } \\
\text { lift }\end{array}$ & $\begin{array}{c}\text { Exhaust } \\
\text { Advance } \\
\text { [CAD] }\end{array}$ & $\begin{array}{c}\text { MAP } \\
\text { [kPa] }\end{array}$ & $\begin{array}{c}\text { IMEP } \\
\text { [bar] }\end{array}$ & $\begin{array}{c}\text { PMEP } \\
\text { [bar] }\end{array}$ & $\begin{array}{c}\text { Ignition } \\
{\left[{ }^{\circ} \text { BTDC] }\right.}\end{array}$ & $\begin{array}{c}\text { Injection } \\
\text { Pulse } \\
\text { [ms] }\end{array}$ & $\begin{array}{c}\text { CA50 } \\
{\left[{ }^{\circ} \text { ATDC] }\right.}\end{array}$ & $\begin{array}{c}\text { B1090 } \\
\text { [CAD] }\end{array}$ & $\begin{array}{c}\text { COV } \\
\text { of } \\
\text { IMEP }\end{array}$ \\
\hline 2000 & high & 6 & 46.0 & 4.62 & 0.52 & 27 & 1.85 & 7.3 & 23.2 & 1.2 \\
2000 & low & 20 & 39.0 & 4.52 & 1.11 & 31 & 1.85 & 8.6 & 26.6 & 1.3 \\
2000 & low & 70 & 52.6 & 4.42 & 0.51 & 47 & 1.90 & 8.9 & 30.9 & 1.8 \\
\hline
\end{tabular}

Also of interest from the exhaust cam sweep is the amount of recompression (Figure 135) from negative valve overlap during the pumping loop, which becomes a key component of HCCI combustion. Recompression at TDC from early EVC begins to appear around $40^{\circ}$ of exhaust advance. By $70^{\circ}$, there is over 5 bar of recompression; this will increase significantly under WOT operation for HCCI. 


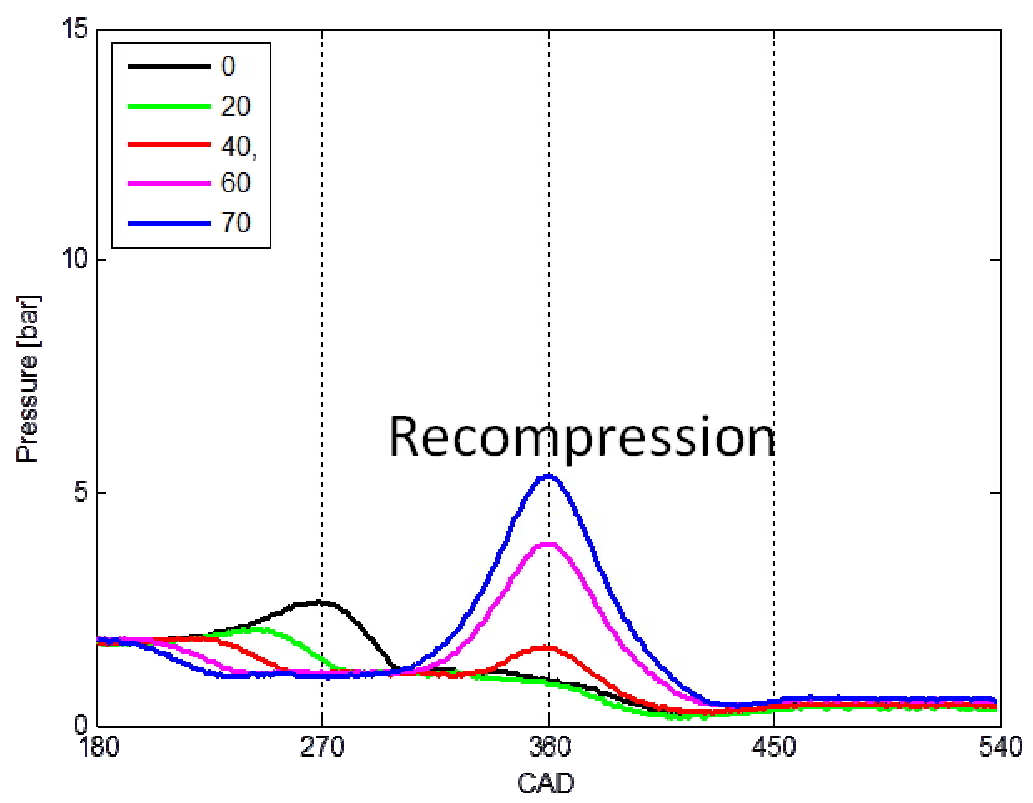

Figure 135: Negative valve overlap recompression at varying exhaust cam advances

After the multi-cylinder engine was converted into the single cylinder setup, to study the HCCI combustion, the slow SI-HCCI combustion mode transition was investigated. The preliminary HCCI combustion tests shows unstable combustion and analysis shows combustion stability can be improved by increasing recompression. The engine default cam timing was under adjustment to increase recompression capability.

\subsubsection{Preliminary HCCI combustion tests}

The mode transition strategy from above was tested with the engine firing. Following the steady SI cycles, the hybrid transition cycles consisted of misfires and late-partial burns before the first HCCI cycle occurred (Figure 136). With the control system cycle-to-cycle repeatability verified in the motoring tests and in this firing test, minimization of these misfires by tuning the spark timing and injection mass will be the focus of the transition tests. 

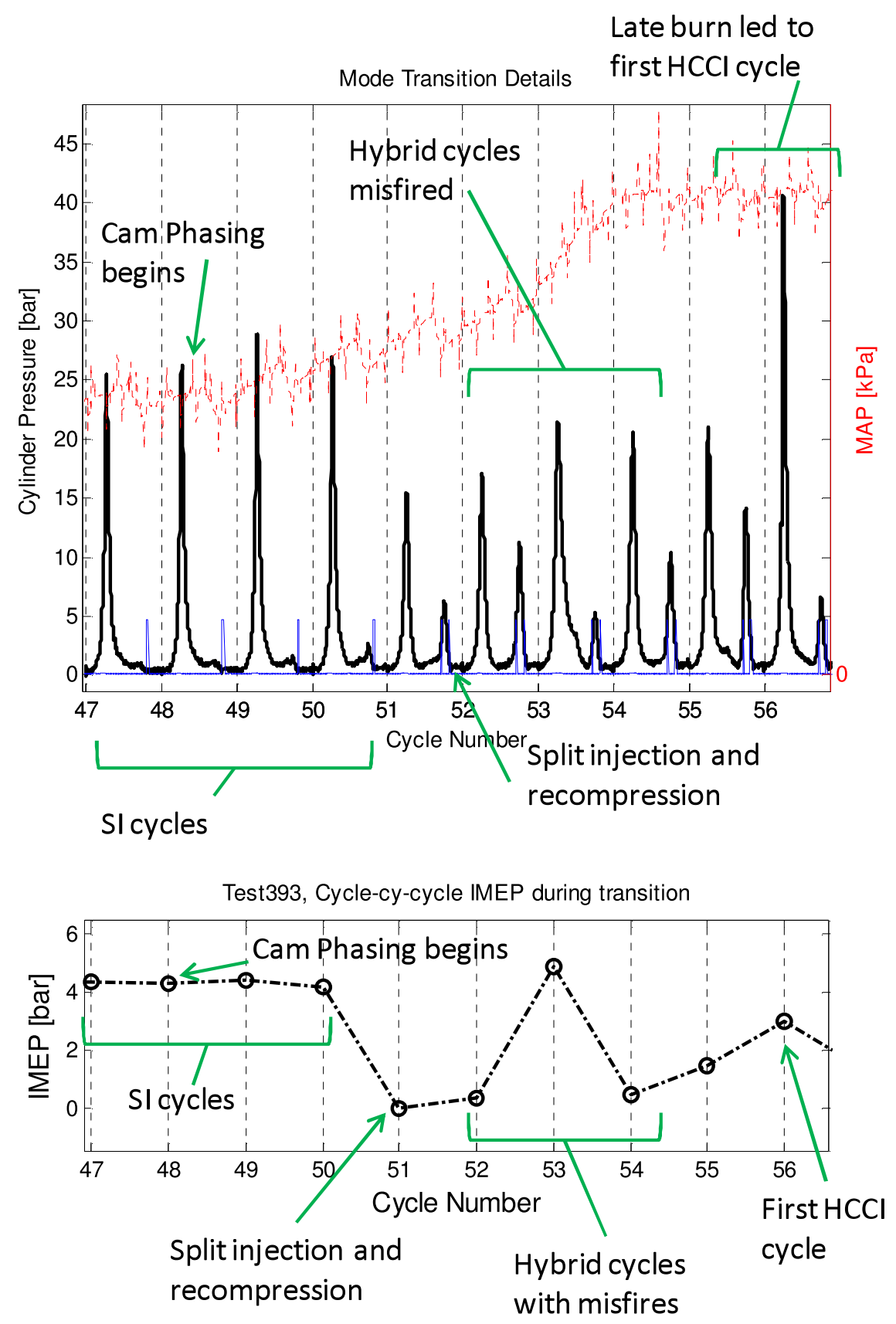

Figure 136: Detail of pressure trace, MAP, injection pulse, and IMEP during firing mode transition

Following the transition period, HCCI operation was achieved without the aid of the intake heating or boost, though it was unsteady and accompanied by numerous misfires. The misfires and late-partial burn cycles led to a small heat release during recompression, and this led to autoignition in the following compression stroke. Plots of the averaged HCCI cycle along with the averaged cycle that preceded an HCCI cycle clearly show the difference in recompression pressure (Figure 137). Note the obvious late burn and recompression heat release present in the pre-HCCI cycles in the PV diagram (Figure 138). 


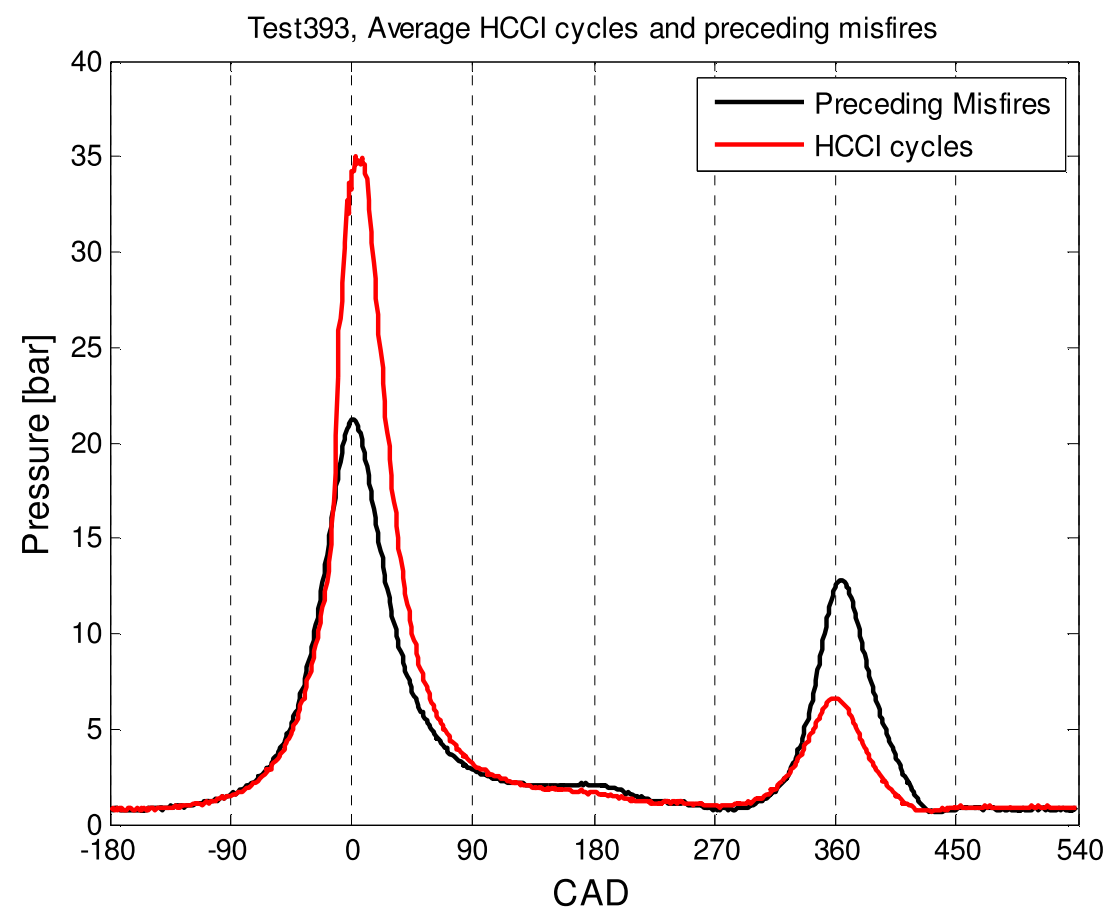

Figure 137: Average successful HCCI cycle and average pre-HCCI misfire.

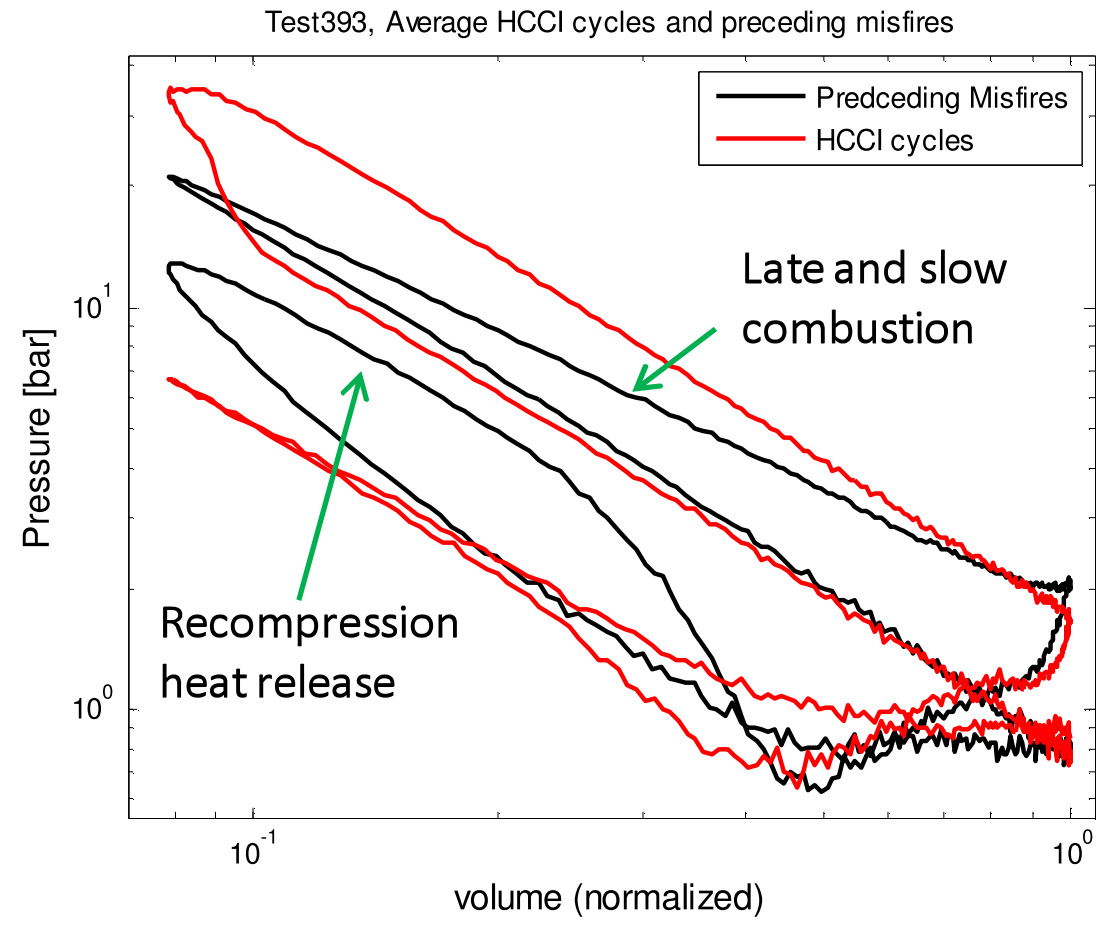

Figure 138: PV plots of average HCCI and pre-HCCI cycles.

Combustion timing in many of the HCCI cycles was earlier than desired (Figure 139), which limited the IMEP of the auto-ignition cycles despite their high peak pressures. The average IMEP for HCCI cycles was 3.4 bar, compared to 4.6 bar during SI operation with roughly the same fuel mass. For steady HCCI operation, key focuses will be on slowing down the HCCI 
combustion and on finding ways to retain more exhaust gas following HCCI cycles to bring recompression pressures up high enough to initiate combustion on the following cycle.

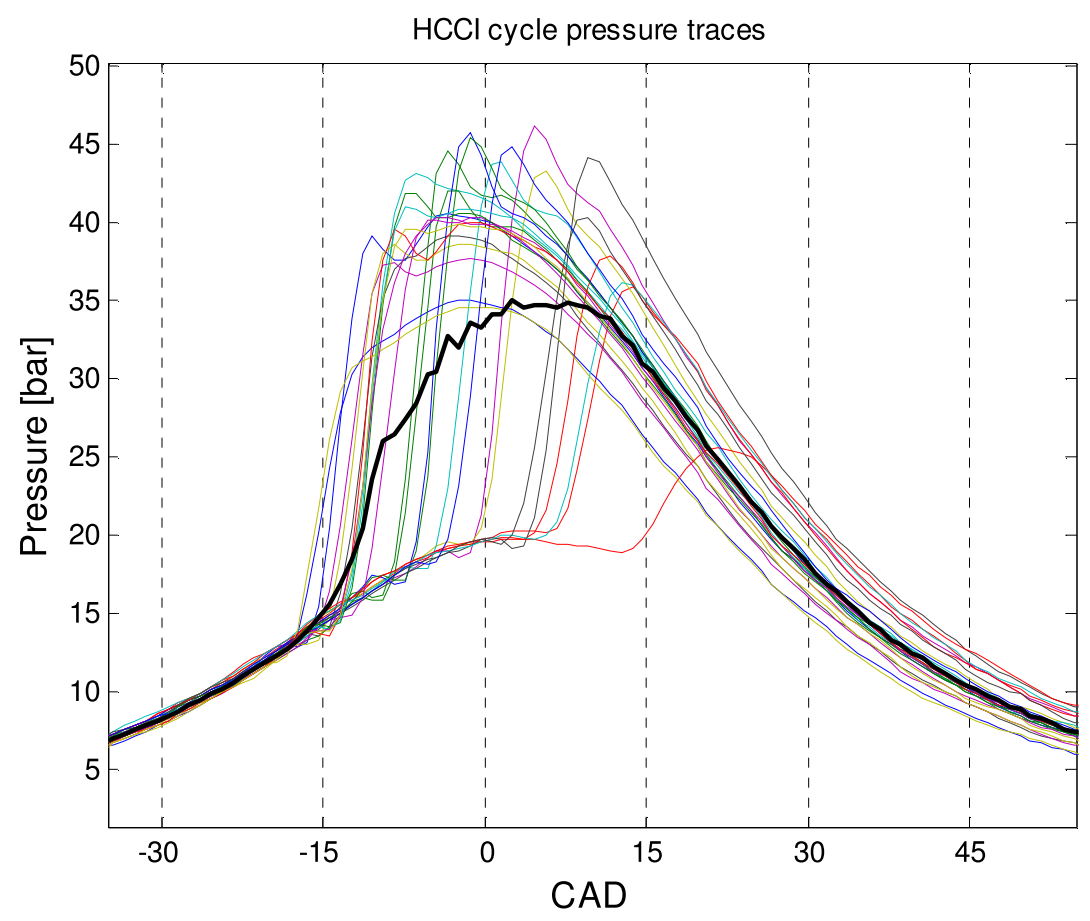

Figure 139: Detail of each HCCI cycle pressure trace.

\subsubsection{Stable HCCI combustion tests}

Following the difficulty of achieving stable HCCI operation in previous tests, efforts were made to increase the temperature of the trapped in-cylinder air-to-fuel mixture to induce consistent auto-ignition by making two significant modifications to the engine setup. First, a coolantsupplied heat exchanged has been placed in the intake manifold that preheats the intake charge to approximately $175^{\circ} \mathrm{F}$. Second, the cam shafts were repositioned to provide a longer NVO (negative valve overlap) period to trap more exhaust gas and to increase the in-cylinder pressure and temperature.

Previous test results indicate that a small amount of heat, released during recompression, is the key to achieving consistent auto-ignition in the following compression stroke. Using a pilot fuel injection and spark during the extended NVO recompression operation leads to consistently control this small heat release, leading to consistent fuel reform during the recompression. This could improve auto-ignition stability under the HCCI operation.

The new and old valve profiles of the low lift cams are shown in the Figure 140. The effective NVO period was increased from 135 CAD to 189 CAD with the cam repositioning. 

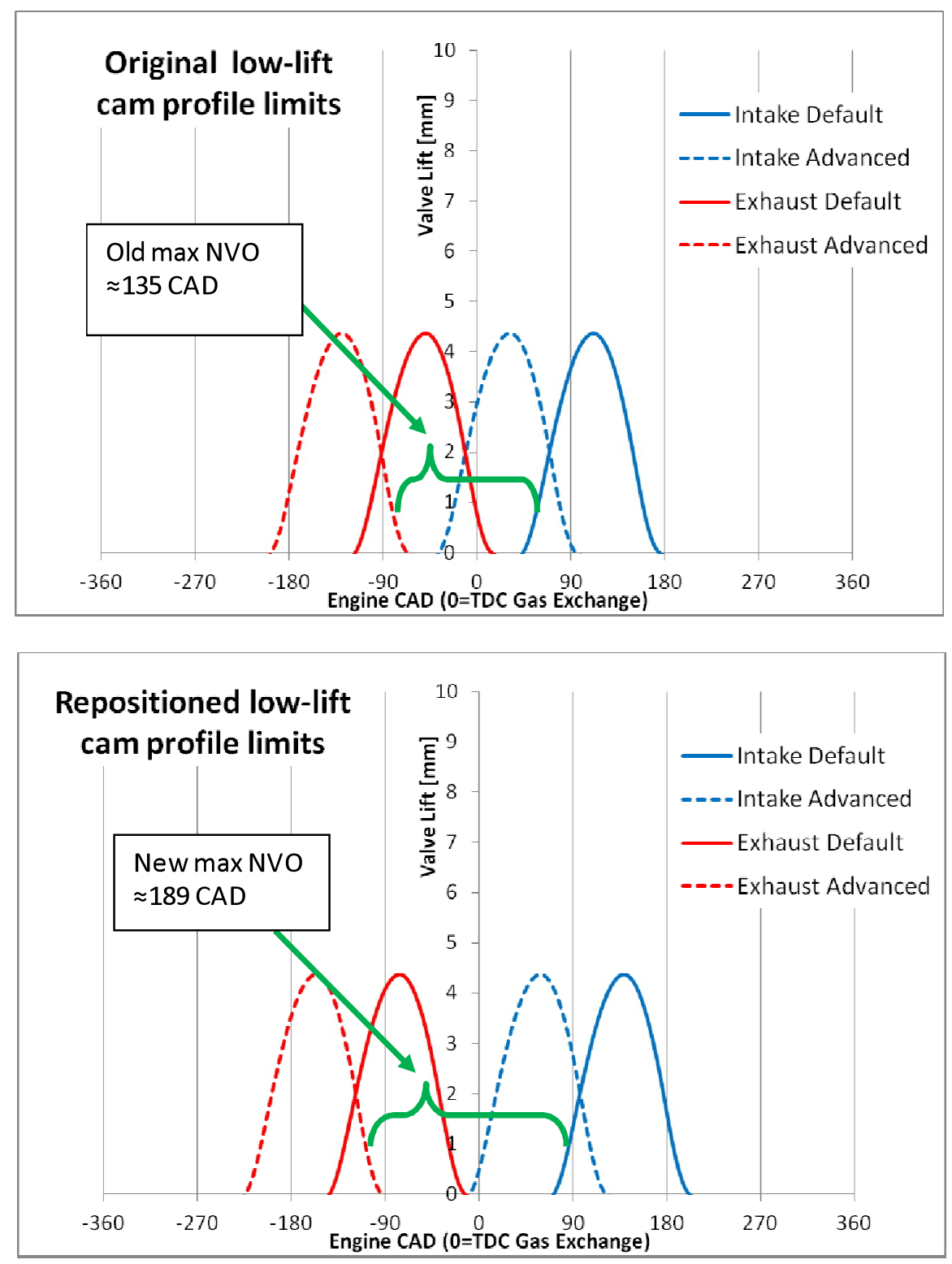

Figure 140: Low-lift cam profile limits, before and after cam repositioning.

To explore the conditions that make steady state HCCI combustion possible, the engine was slowly transitioned into HCCI combustion from an initial low-lift spark-ignition test point by adjusting appropriate parameters in small increments until stable auto-ignition was observed. This process was essentially a slow manually-controlled version of the desired mode transition strategy that will be discussed in the next section. The cam shafts were slowly phased a few degrees at a time to gradually increase NVO until a split injection could be applied; as more exhaust gas was trapped, the throttle was gradually opened. With a significant NVO period and a sufficiently opened throttle, spark timing was retarded toward TDC (top dead center) in small increments until the first signs of auto-ignition occurred. At this point, the cams were phased to their limits (most advanced for exhaust, most retarded for intake), the throttle was opened fully, and the spark timing was retarded back to just before TDC. The resulting point showed consistent fast-burning auto-ignition combustion with the acceptable COV, high IMEP, and very low PMEP. 
An average pressure trace of a 200-cycle sample of this steady HCCI point is shown in Figure 141 along with the injection and ignition strategies. The injection strategy shows a short pilot injection pulse ending at $25^{\circ}$ BTDCGE and a longer main pulse ending at $300^{\circ} \mathrm{BTDCF}$, the same main injection timing used in single-injection SI tests. The spark, which fires at the falling edge of the control signal shown, is $10^{\circ} \mathrm{BTDC}$ during both recompression and main compression events. The P-V diagram of Figure 142 is useful for showing the small positive heat release that comes as a result of the pilot injection and spark.

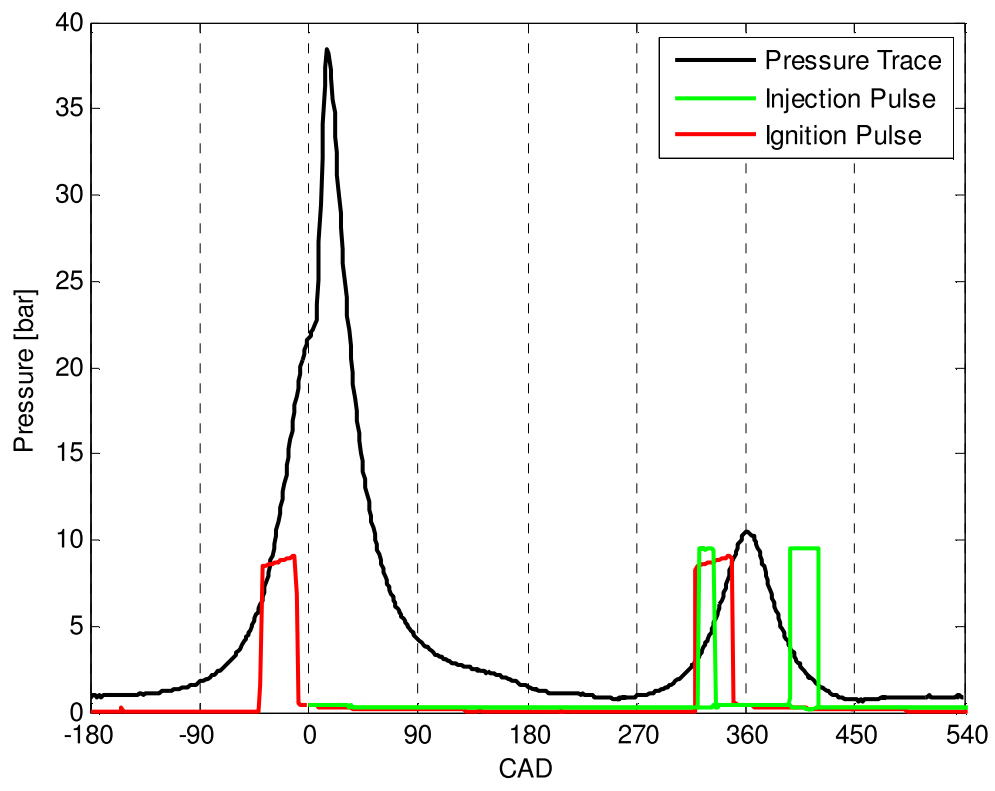

Figure 141: Average HCCI pressure trace

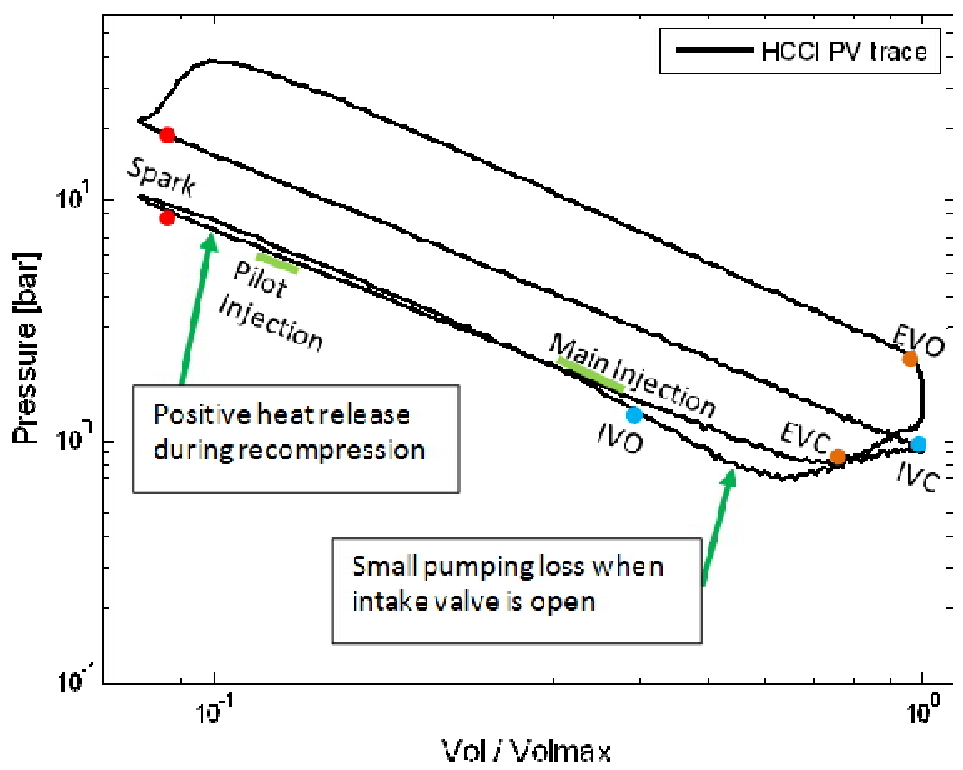

Figure 142: Average HCCI P-V diagram showing positive heat release during recompression 
A detailed overlay of all 200 cycles sampled (see Figure 143) shows some variation in peak pressure and peak pressure location, but overall a consistent trend of fast combustion initiating just after TDC. While the peak pressure varies a fair amount due to the timing of combustion, the engine IMEP fluctuations are quite small (Figure 144), with the COV of IMEP at just $2.1 \%$ over this 200 cycle sample. Also of note in this figure is the virtually nonexistent PMEP.

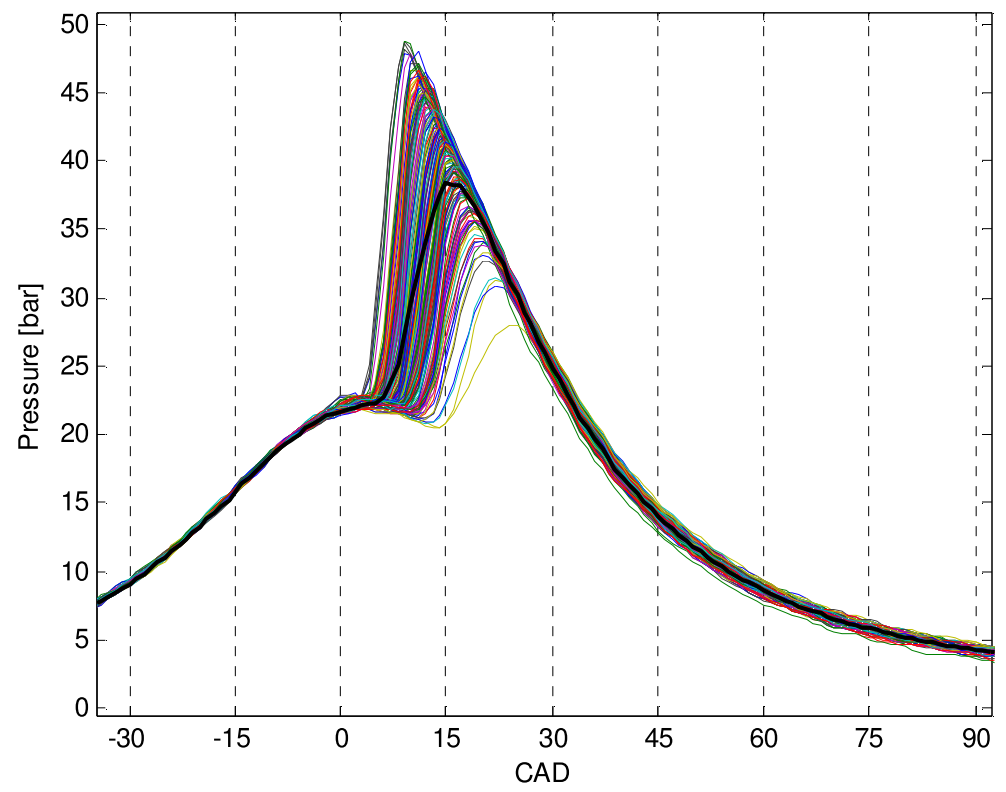

Figure 143: Overlay of 200 cycles of the in-cylinder pressure signals

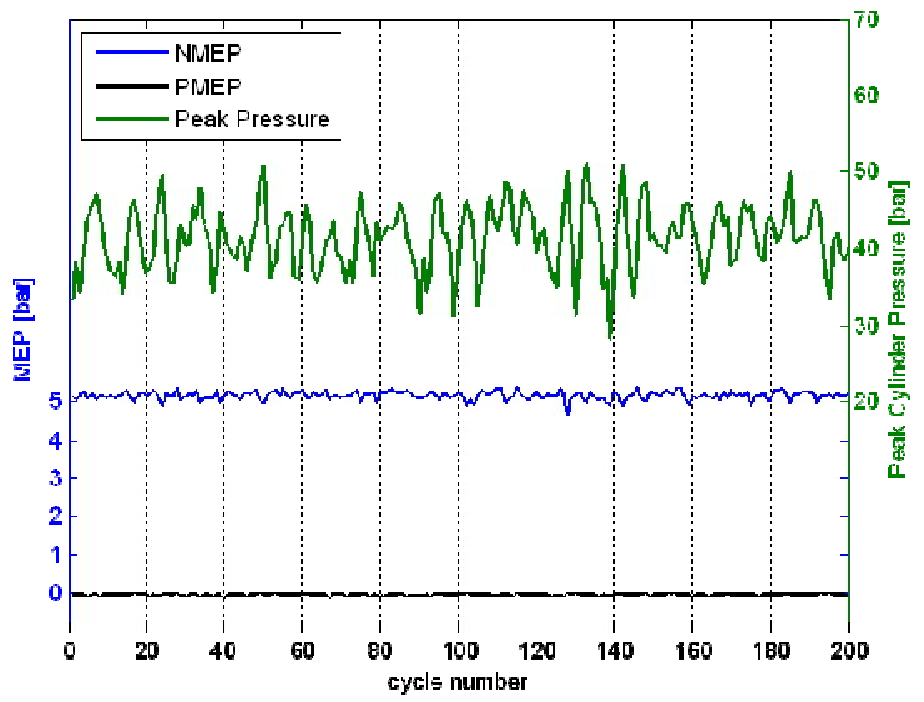

Figure 144: Peak cylinder pressure, IMEP, and PMEP of stable HCCI combustion

In an attempt to run this HCCI operational point with the same amount of fuel as an SI comparison point, a standard SI benchmark point at 4 bar NMEP was first run with a single injection; this same point was then run with a split injection by injecting approximately $15 \%$ of the total fuel in the second injection pulse and decreasing the main pulse by the appropriate amount to keep the same load and AFR (lambda) conditions. These two fuel pulse widths were 
then used for the HCCI combustion case. The results of these three cases are shown in Table 32 below. Not only were pumping losses reduced significantly, but also the engine IMEP increased significantly as well.

Table 32: SI and HCCI test point comparison

\begin{tabular}{|r|c|c|c|}
\cline { 2 - 4 } \multicolumn{1}{c|}{} & SI (single pulse) & SI (dual pulse) & HCCI (dual pulse) \\
\hline Main Inj Pulse [ms] & 1.93 & 1.90 & 1.90 \\
\hline Pilot Inj Pulse [ms] & ---- & 1.12 & 1.12 \\
\hline IMEP [bar] & 4.60 & 4.54 & 5.21 \\
\hline PMEP [bar] & -0.59 & -0.59 & -0.04 \\
\hline NMEP [bar] & 4.01 & 3.96 & 5.17 \\
\hline COV of IMEP & $0.9 \%$ & $0.9 \%$ & $2.1 \%$ \\
\hline$\lambda$ & 0.99 & 1.00 & 1.16 \\
\hline MAP [kPa] & 50 & 50 & 96 \\
\hline CA50 & 8.2 & 8.3 & 12.5 \\
\hline B1090 & 25.2 & 25.1 & 12.1 \\
\hline PRRmax [bar/CAD] & 1.4 & 1.4 & 4.1 \\
\hline Peak Pressure [bar] & 27.3 & 27.0 & 41.4 \\
\hline Peak Ploc [ ${ }^{\circ}$ ATDC] & 13.1 & 13.1 & 15.0 \\
\hline
\end{tabular}
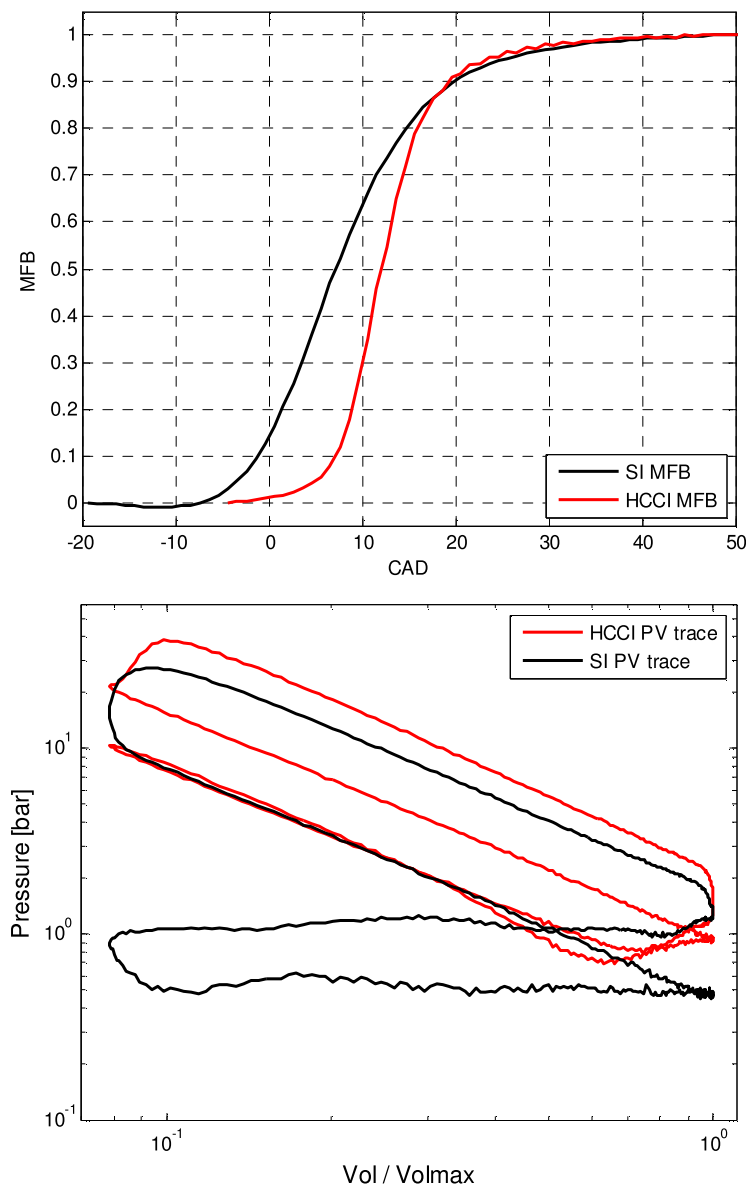

Figure 145: P-V diagram and MFB curve comparison of SI and HCCI (same fuel pulses) 
The P-V diagram and MFB curves of Figure 145 demonstrate the differences between the SI and $\mathrm{HCCI}$ combustion visually. The difference in pumping work is immediately apparent in the P-V diagram, as are the higher peak pressure, later initial burn, and faster overall burn rate. The MFB curves of both SI and HCCI combustion modes also demonstrate the difference in burn rate.

Lower load points closer to the 4 bar NMEP point of the SI combustion tests have been explored by altering the fueling amounts and pilot injection timings. These lower loads have shown greater variability, some of which can be improved upon with changes to the fuel split ratio between the main and pilot pulses.

\subsection{Summary}

The Chrysler 2.0L I4 DI engine was modified to fit the two-step vale with electrical variable valve timing actuating system. A used prototype engine was used as the base engine and the cylinder head was modified for the two-step valve with electrical VVT actuating system. Engine validation tests indicated that cylinder \#3 has very high blow-by and it cannot be reduced with new pistons and rings. Due to the time constraint, it was decided to convert the four-cylinder engine into a single cylinder engine by blocking both intake and exhaust ports of the unused cylinders. The model-based combustion mode transition control algorithm was developed in the MSU HIL simulation environment and the Simulink based control strategy was implemented into the target engine controller. With both single-cylinder metal engine and control strategy ready, stable HCCI combustion was achieved with COV of $2.1 \%$. 


\section{Phase IV: Final Performance Evaluation}

This maturation phase includes dynamometer test validation of closed-loop combustion control for SI and HCCI mode transition and final determination of the benefits of the developed dualmode closed-loop combustion strategies.

\subsection{Motoring tests of SI-to-HCCI transition}

The purpose of the motoring tests for SI-to-HCCI mode transition is to validate the operation of all actuators including cam phasing, lift control, throttle, spark, and so on. The transition involves performing simultaneous cam phasing, cam lift change, throttle opening, and ignition and injection timing changes in order to move from SI operating conditions to HCCI operating conditions in just a few engine cycles. Figure 146 details the events happening during a motoring test of this transition by showing the changes to the cylinder pressure (black) and manifold pressure (red) over each cycle. The initial dip in peak cylinder pressure denotes the start of retarding the intake cam and advancing the exhaust cam; the peak in-cylinder pressure drops while the engine MAP (manifold air pressure) increases slightly due to later IVC which allows some air to be pushed back out of the cylinder into the intake manifold; in this region, the engine would still be running with normal spark ignition (though it may be ideal to open the throttle slightly here to allow cylinder pressure to remain the same). The jump from the high-lift to low-lift cam is evident from the recompression that appears at cycle 46, and this coincides with the large jump in MAP that shows the start of throttle opening; this is where the mode transition to HCCI operation starts. By cycle 50, total HCCI operation is present.

With this setup, the whole transition takes about 10 cycles to complete, with the limiting factor being the speed at which the cam phasers can advance the exhaust valve. However, only the last 5 cycles (when the low-lift cam kicks in and the throttle is opened) will make up the true mode transition from SI to HCCI combustion.

The backward transition works much the same. Figure 147 shows the cam phasing beginning to return to the original position to eliminate recompression as the throttle closes so that the engine can return to SI combustion. 


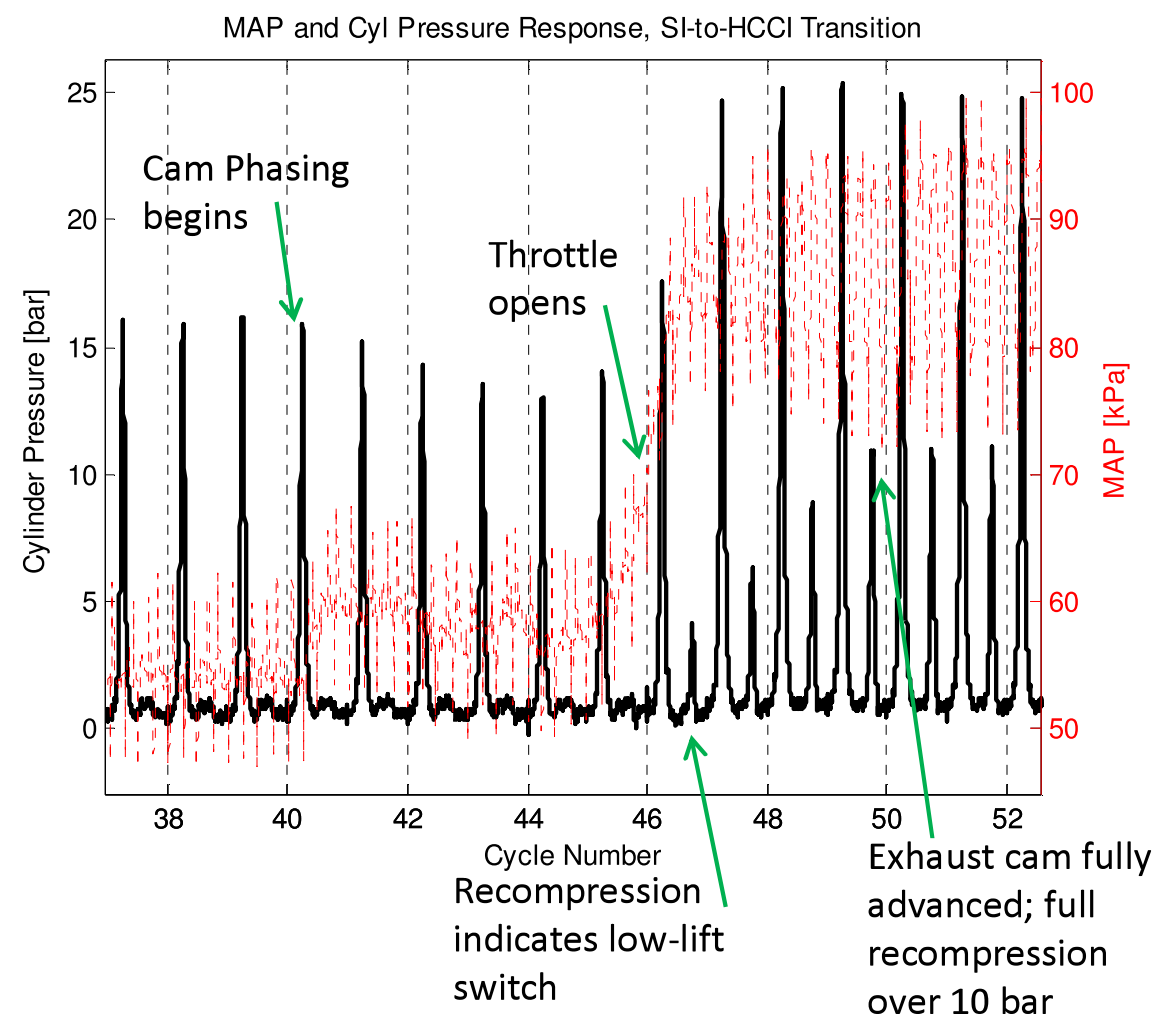

Figure 146: SI-to-HCCI mode transition motoring test details

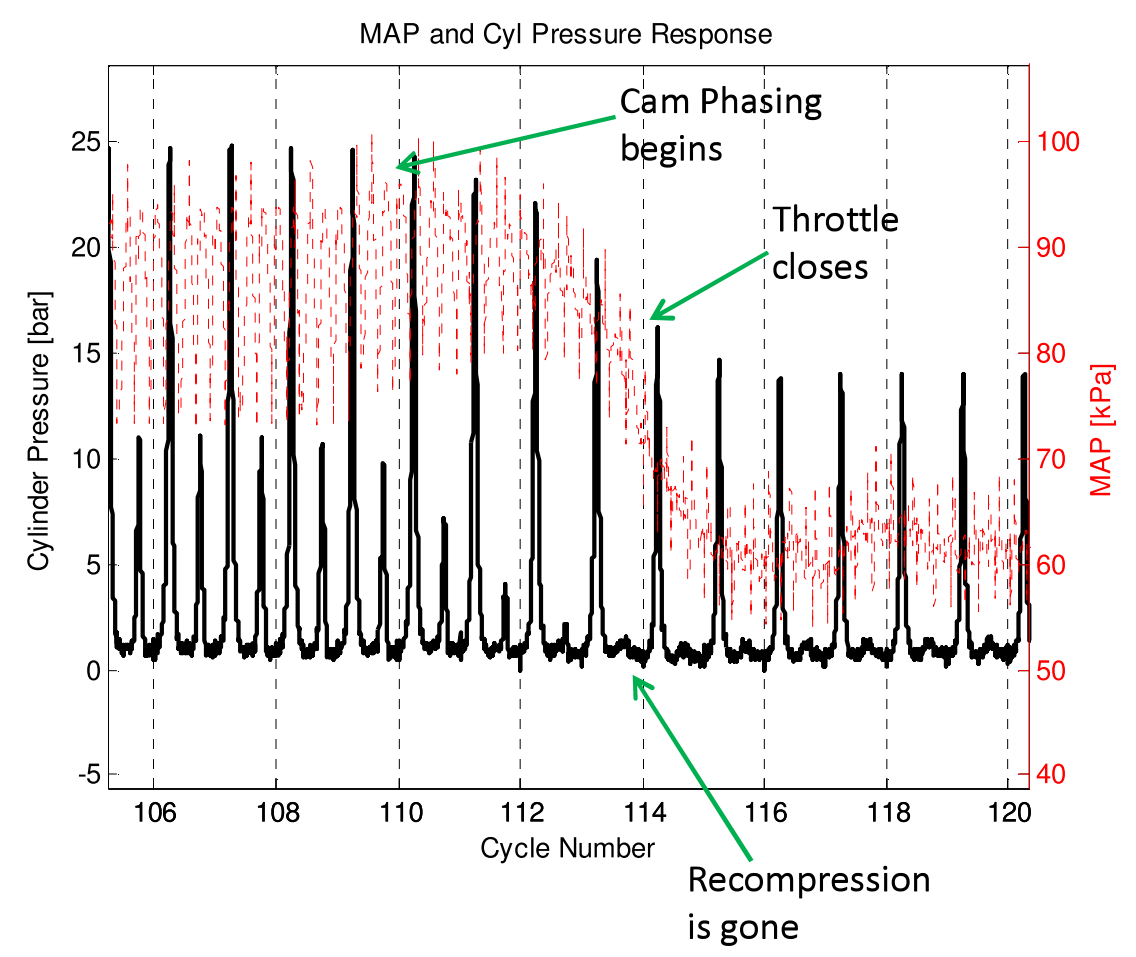

Figure 147: HCCI-to-SI mode transition motoring test details 
Improvements were also made with improved throttle response, faster cam phaser response due to the improved power supply and the addition of a split injection pulse. The split injection pulse introduces a short pilot injection during NVO recompression in order to reform some of the fuel during this recompression to help achieve auto-ignition during the next compression stroke.

While the original ideal transition plan calls for a 5-cycle total transition period, the current setup uses 8 cycles. The main reason for this extended period is slow cam phaser response. There are two cycles between the phasing command and the start of phasing, and 7 cycles needed to fully advance the exhaust valve. However, current strategy utilizes the same 3-cycle hybrid combustion transition phase following the high-to-low cam lift switch as proposed in the original plan. In Figure 148 shown below, these would be cycles 46 through 48; cycle 45 is an SI cycle, and cycle 49 should be a full HCCI cycle.

In order to determine the pulse widths needed for the two injection pulses, fuel flow rate tests were performed by firing the engine and monitoring a mass air flow sensor and $\mathrm{O}_{2}$ sensor. By adjusting fuel pulses and recording air flow and AFR (lambda), fuel flow rates for different length pulses were calculated. This allows the fuel mass to be split into any ratio desired between the pilot (during recompression) and main pulses while keeping the overall fuel mass consistent. The current setup injects $20 \%$ of the total fuel during the pilot recompression injection.

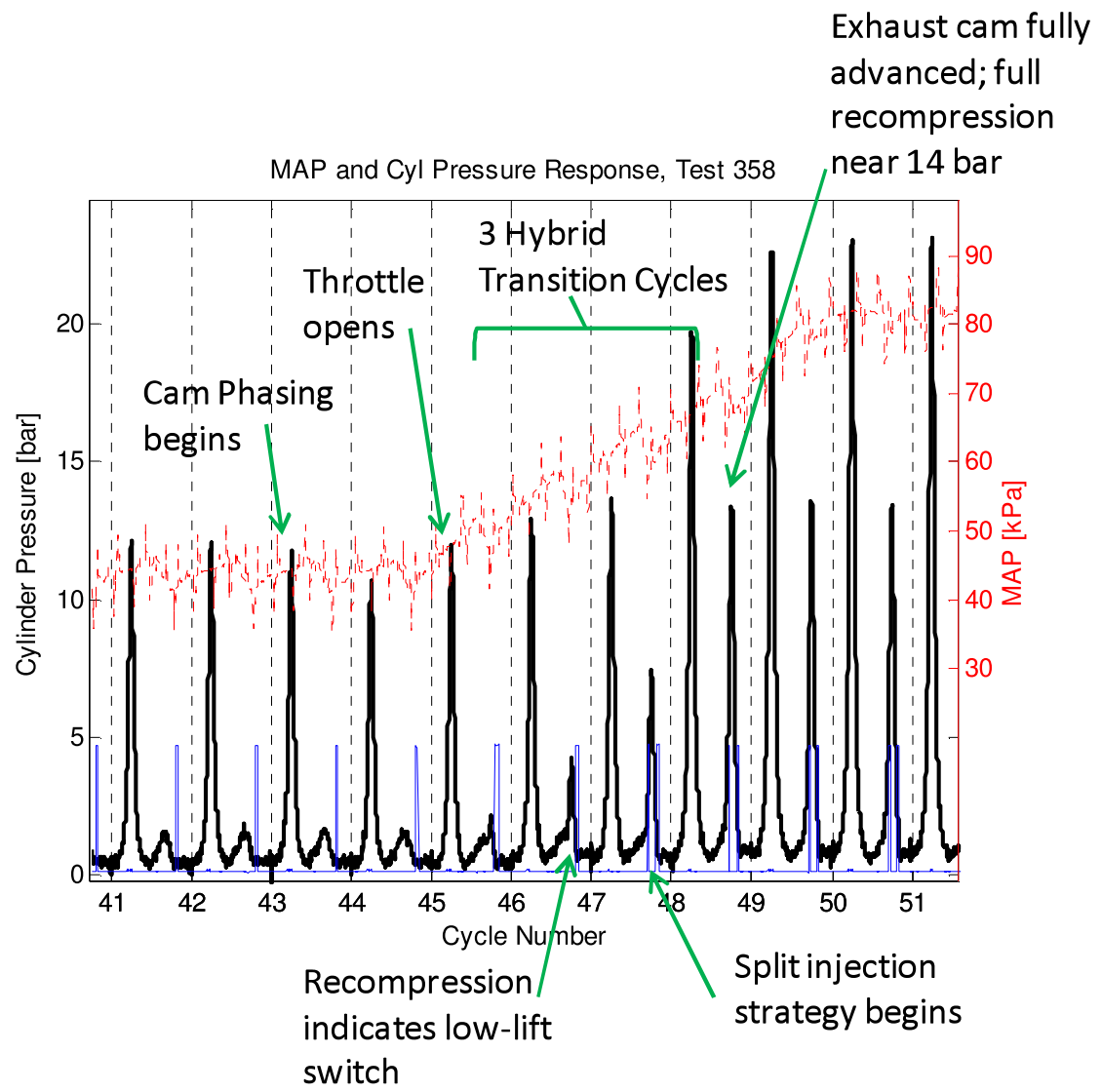

Figure 148: Detail of motoring mode transition test 


\subsection{Hybrid combustion mode during SI-to-HCCI mode transition}

In the process to obtain stable HCCI combustion by manually transiting from SI into HCCI combustion described in Subsection 4.3, combustion mode transitional points were captured that demonstrate the hybrid combustion proposed by the original mode transition concept. As the cam phasing and throttle position are changed, it is predicted that there exist a few hybrid combustion cycles which display the combustion characteristics that starts with SI combustion and ends with auto-ignition (HCCI) combustion, where combustion is initiated by a spark at normal SI timing, but after a certain amount of flame propagation, fast auto-ignition takes over for the rest of the combustion process.

Figure 149 shows a 200-cycle sample of the hybrid combustion process captured during the manual transition to HCCI. This condition was achieved with the intake cam positioned at its default fully retarded $0^{\circ}$ advance position and the exhaust cam advanced $55^{\circ}$ from its default position. This cam timing creates an asymmetrical $148^{\circ} \mathrm{NVO}$ (negative valve overlap) period, with the longer portion of recompression actually occurring on the intake side at $80^{\circ}$ compared to $68^{\circ}$ on the exhaust side. Throttle opening was larger than that for the SI combustion but not yet reaches WOT (wide open throttle), which results in a manifold pressure of $85 \mathrm{kPa}$. The air-fuel mixture was slightly at the lean side (with lambda equal to 1.02). Spark was operated at the $30^{\circ}$ BTDC (same as the standard SI spark).

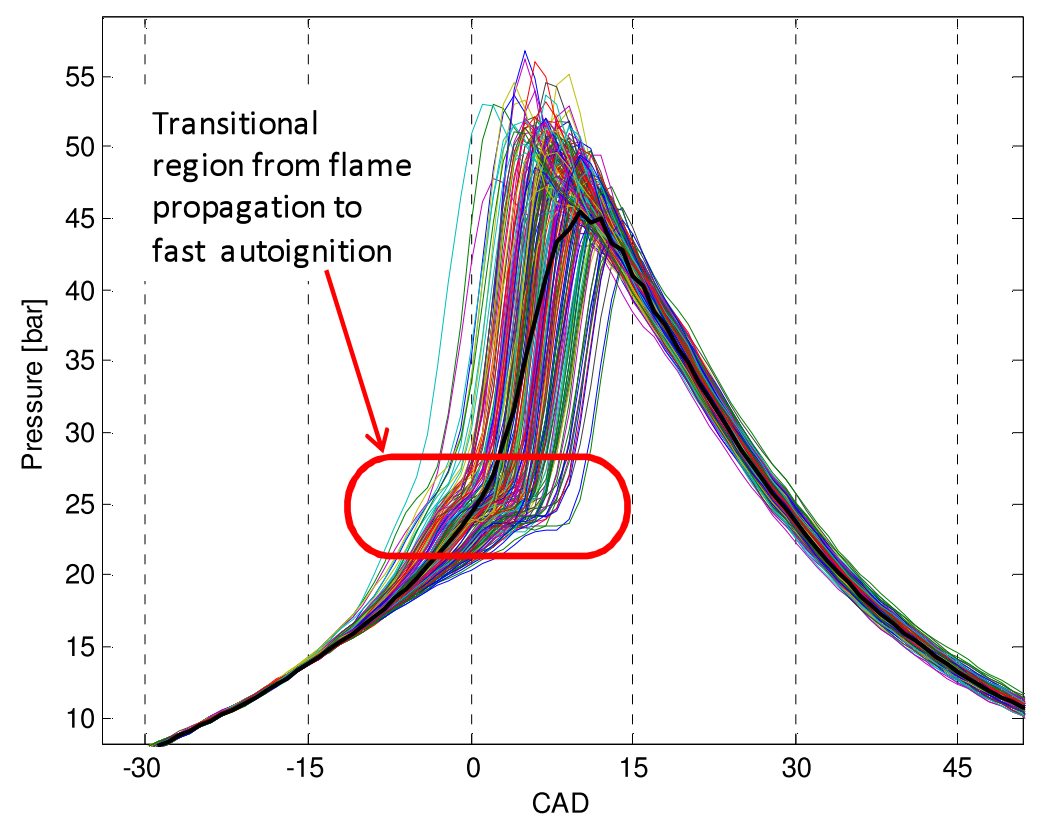

Figure 149: 200 cycles of the hybrid combustion

Other similar points were also discovered during the manual transition process with varying degrees of stability and auto-ignition phase as a function of the cam phasing, fueling, and throttle position. The auto-ignition phase represents the percentage of the HCCI combustion occurred during the hybrid combustion. Knowledge from these points was helpful when implementing a 
fast transition strategy, particularly when dealing with how to sequence cam phasing and throttle opening together to create conditions for these hybrid cycles during the transition.

\subsection{Successful fast SI-to-HCCI mode transition}

With the knowledge gained from the steady state tests and the manual transition tests from SI to HCCI combustion in Subsection 4.3.3, it was possible to calibrate the mode transition strategy to successfully move the engine in and out of HCCI mode consistently with minimal misfire or knock cycles, based upon the strategy developed in Section 4. Unfortunately, these tests were only able to be designed for a transition with the cams already in low-lift mode due to a malfunction in the exhaust cam lift mechanism.

The first successful mode transition strategy involved 15 total cycles from the start to end of cam phasing. The plots in Figure 150 show the cycle-by-cycle strategy for MAP (manifold air pressure), spark timing, cam timing, and injection pulses during the transition window. Cycleby-cycle pressure traces of these cycles are shown in Figure 151. In these figures, the process begins when cam phasing starts at cycle 50. Split injection is initiated at cycle 56 once significant recompression has built up. Note that the initial pilot injection pulse of $0.5 \mathrm{~ms}$ is below the threshold pulse and does not inject any fuel. The throttle begins to open around cycles 55 and 56, and spark timing begins to retard shortly after the combustion starts moving into hybrid mode. By cycle 63, the spark settles at $-5^{\circ}$, the throttle has moved to WOT (wide open throttle), and the exhaust cam is nearly fully advanced to allow for full HCCI combustion. The entire transition took place without partial-burn or misfire, with the lowest single-cycle IMEP stays well over 4 bar (Figure 152). AFR (lambda) rose from stoichiometric in SI mode to approximately 1.12 after the transition, with a slight drop into rich combustion for a few cycles during the transition, due to cam phasing reducing incoming intake air before the throttle began to open; this is essentially the same situation that was seen in the SI low-lift cam phasing tests summarized previously in Subsection 5.1. 

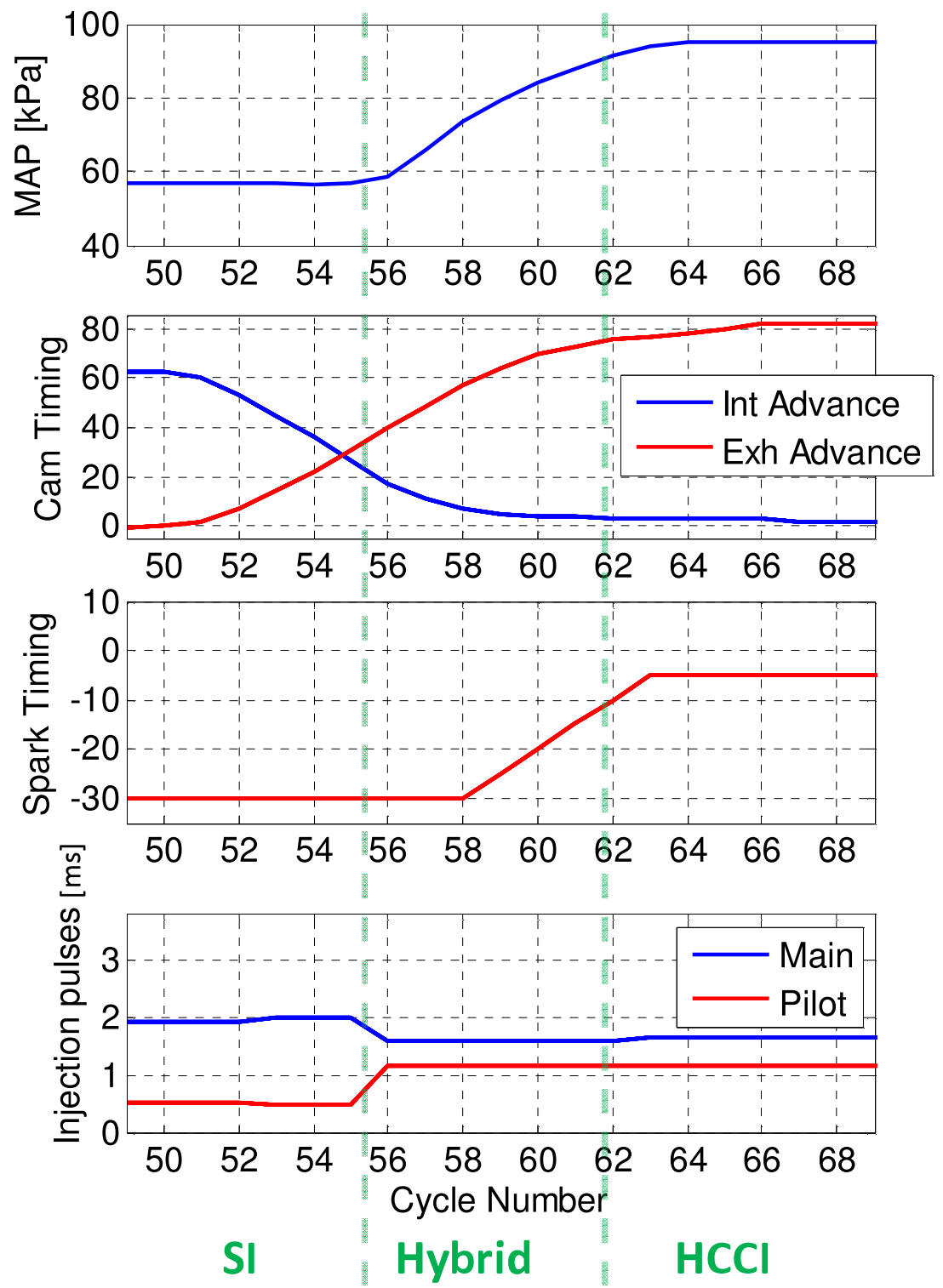

Figure 150: Control parameters for the 15-cycle mode transition 


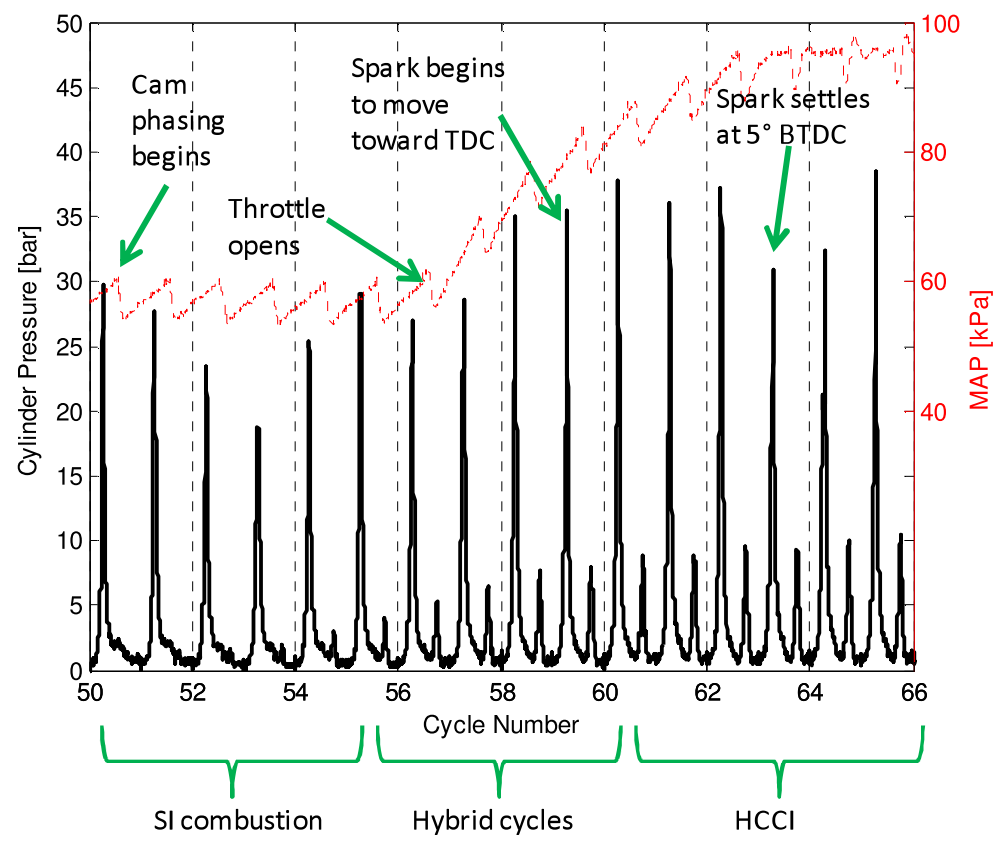

Figure 151: Cycle-by-cycle pressure trace of the 15-cycle mode transition

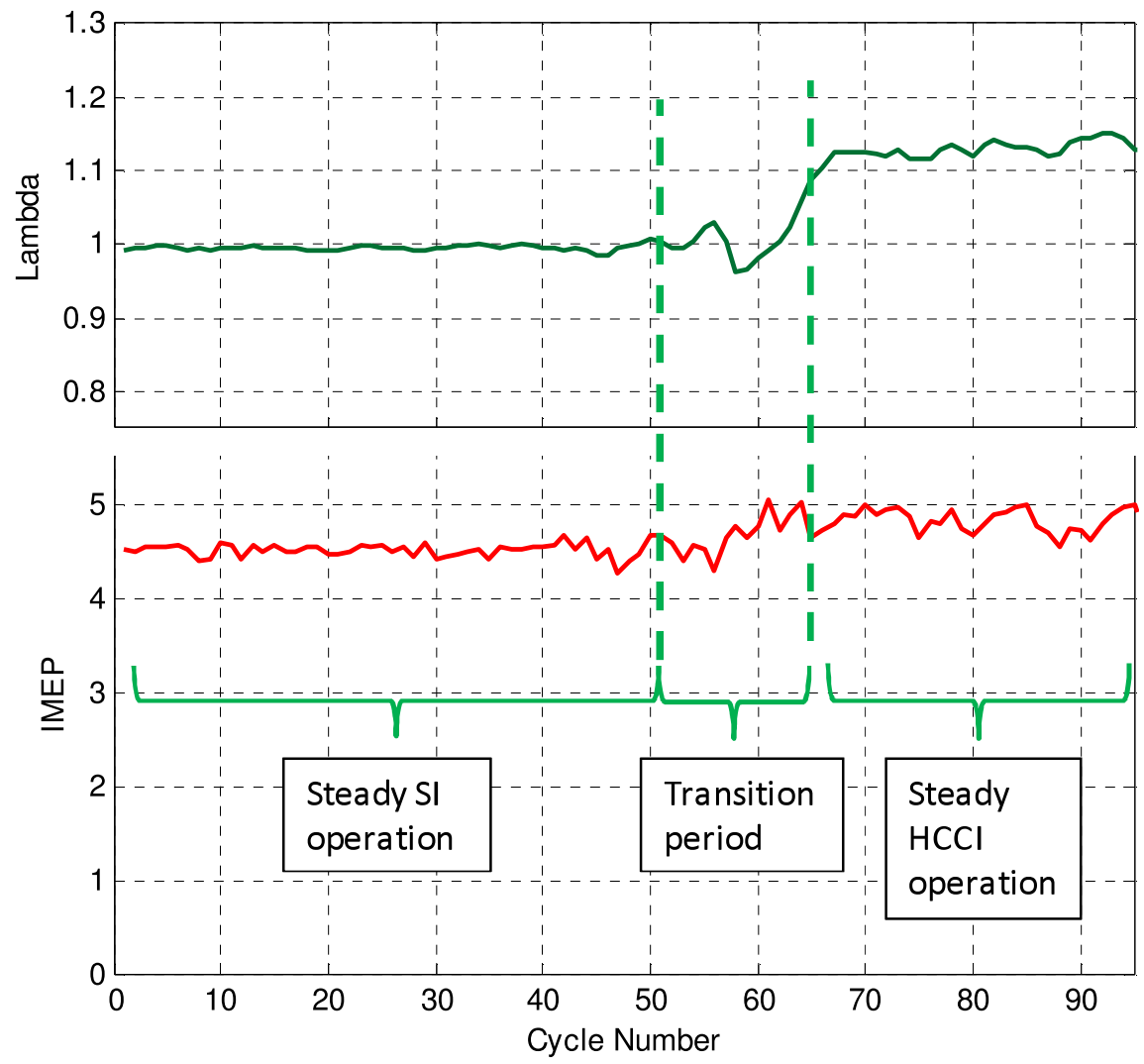

Figure 152: Lambda and IMEP traces during the 15-cycle transition 
Examining the individual pressure traces and MFB curves through the entire transition provides details of what happens as the control parameters change. Figure 153 shows the initial 5 cycles of the transition, starting at cycle 50 just as the cams begin to move. The first cycles demonstrate typical SI combustion characteristics with a CA50 phase of about $7^{\circ}$ ATDC. In cycles 52-54, the increasing NVO period starts to trap more exhaust gas, and CA50 retards as peak pressure drops and variability increases.
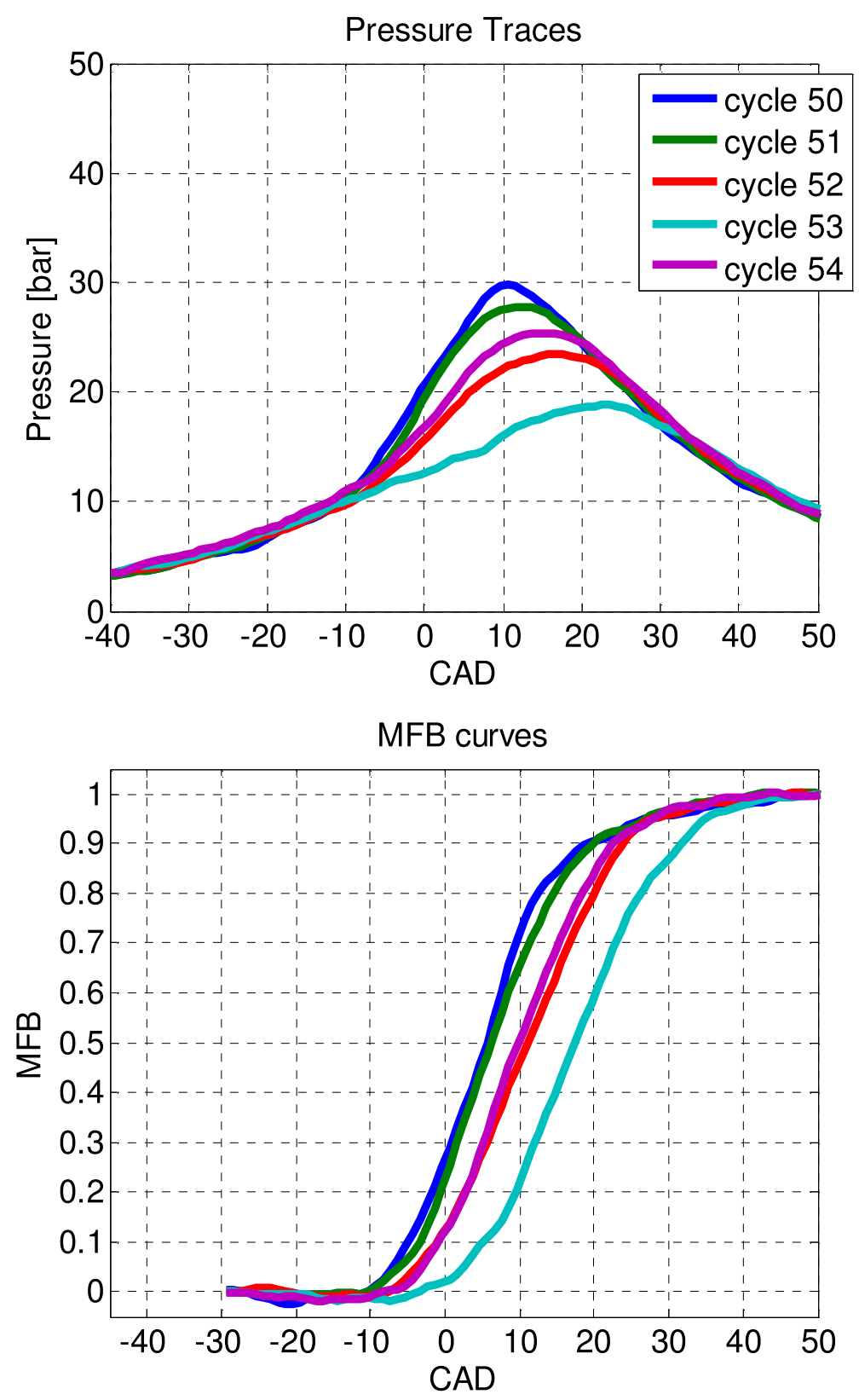

Figure 153: Pressure and MFB traces of the first five cycles of the 15-cycle transition

The next 5-cycle pressure and MFB curves are provided in Figure 154 and it is very clear that the hybrid combustion appears in cycle 55. Peak in-cylinder pressures increase from the relatively low preceding SI combustion pressures as a small amount of auto-ignition (HCCI) appears at the end of the MFB curves. As the throttle opens and the spark begins to retard, the balance between 
SI and auto-ignition shifts more towards auto-ignition, or in other words, the percentage of the HCCI combustion increases. Over these five cycles, the percentage of the SI combustion with flame propagation decreases fairly steadily from approximately $65 \%$ in cycle 55 to around $10 \%$ by cycle 59. In each of these cycles, the transition to auto-ignition (HCCI) takes place at around $10^{\circ}$ ATDC.
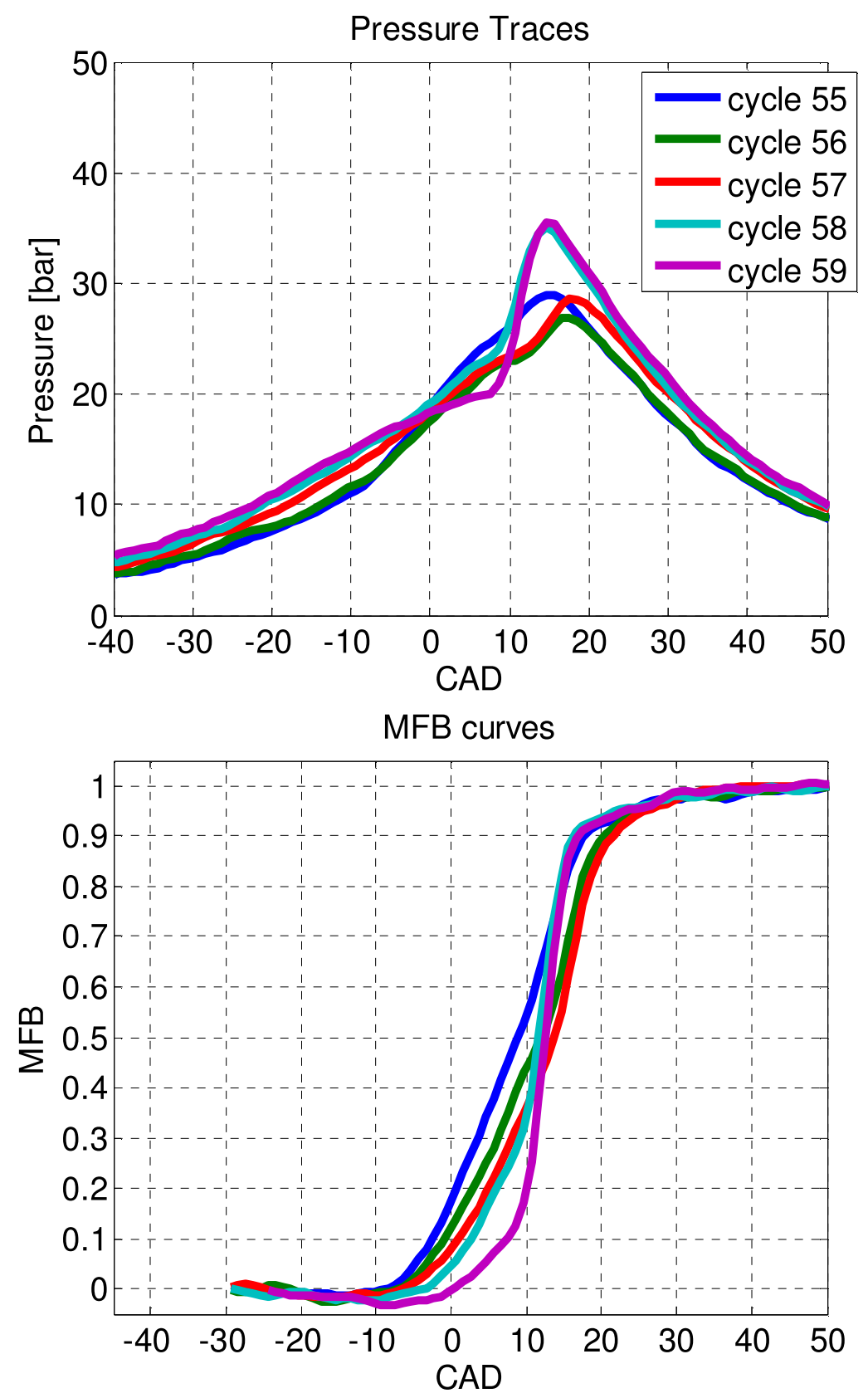

Figure 154: Pressure and MFB traces of the middle five cycles of the 15-cycle transition 
By cycle 60, the cam positions and manifold pressure are at the levels such that a consistent autoignition (HCCI combustion) is present. By cycle 63, spark has been retarded to $5^{\circ}$ BTDC, cams are fully phased for maximum NVO, and manifold pressure has reached its maximum. Following this transition, the engine was operated at the HCCI combustion mode with 5.00 bar IMEP, 2.9\% IMEP COV, and -0.15 bar PMEP.
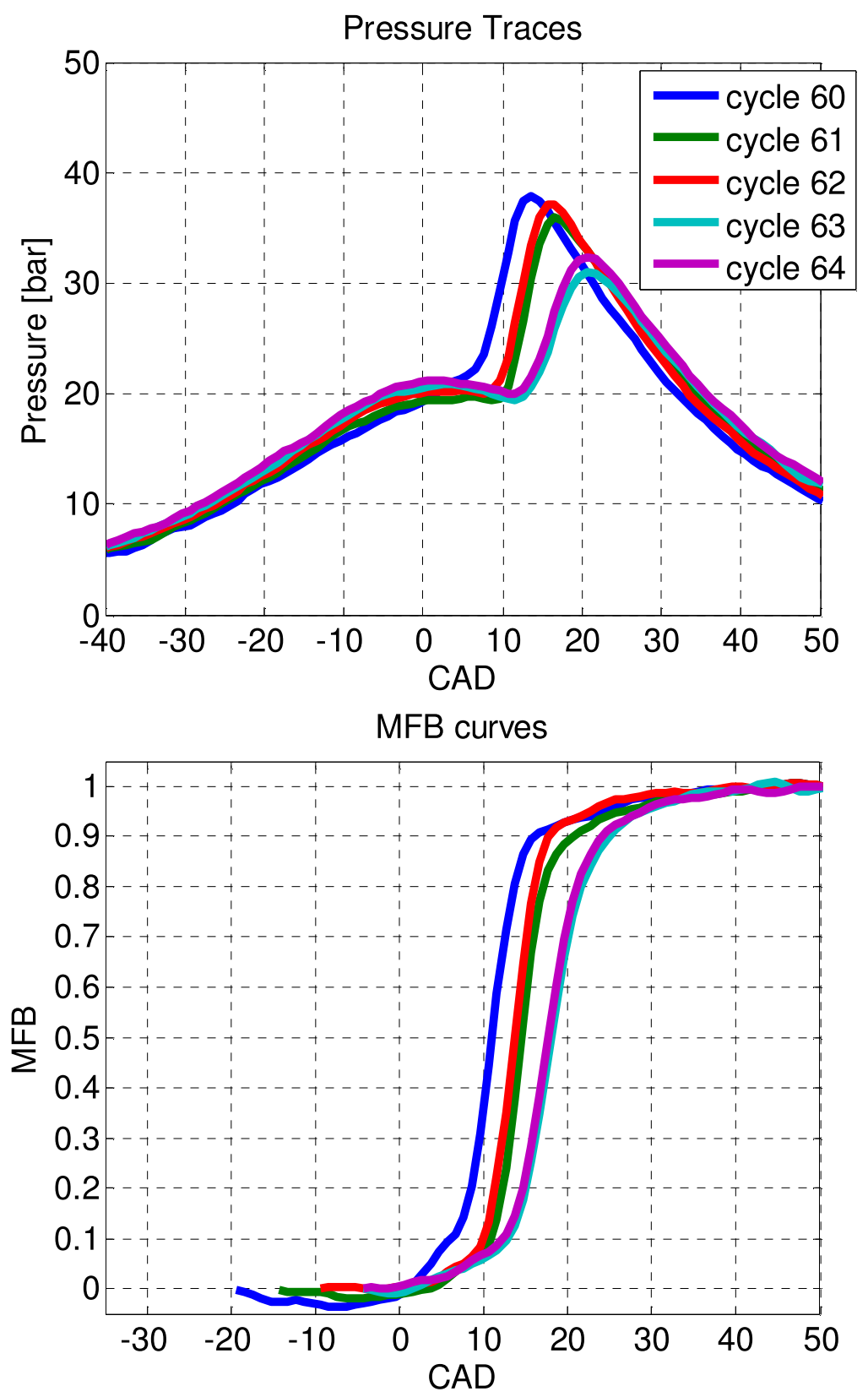

Figure 155: Pressure and MFB traces of the last five cycles of the 15-cycle transition

With the original goal of a five-cycle mode transition, further attempts were made to shorten the transition duration. Due to slow settling time of the exhaust cam phasing, total phasing time could only be reduced to 11 cycles to fully settle. However, it was possible to achieve full autoignition (HCCI combustion) at the $8^{\text {th }}$ cycle of the transition with the exhaust cam advanced to $75^{\circ}$ of its $80^{\circ}$ total phasing capability and spark settling at its final location of $-5^{\circ}$. The transition 
can effectively be called an 8-cycle transition with extra cycles for cam phaser settling time. This 8-cycle mode transition consisted of three initial SI cycles during early cam phasing followed by four to five hybrid mode combustion cycles that led to full auto-ignition (HCCI combustion).
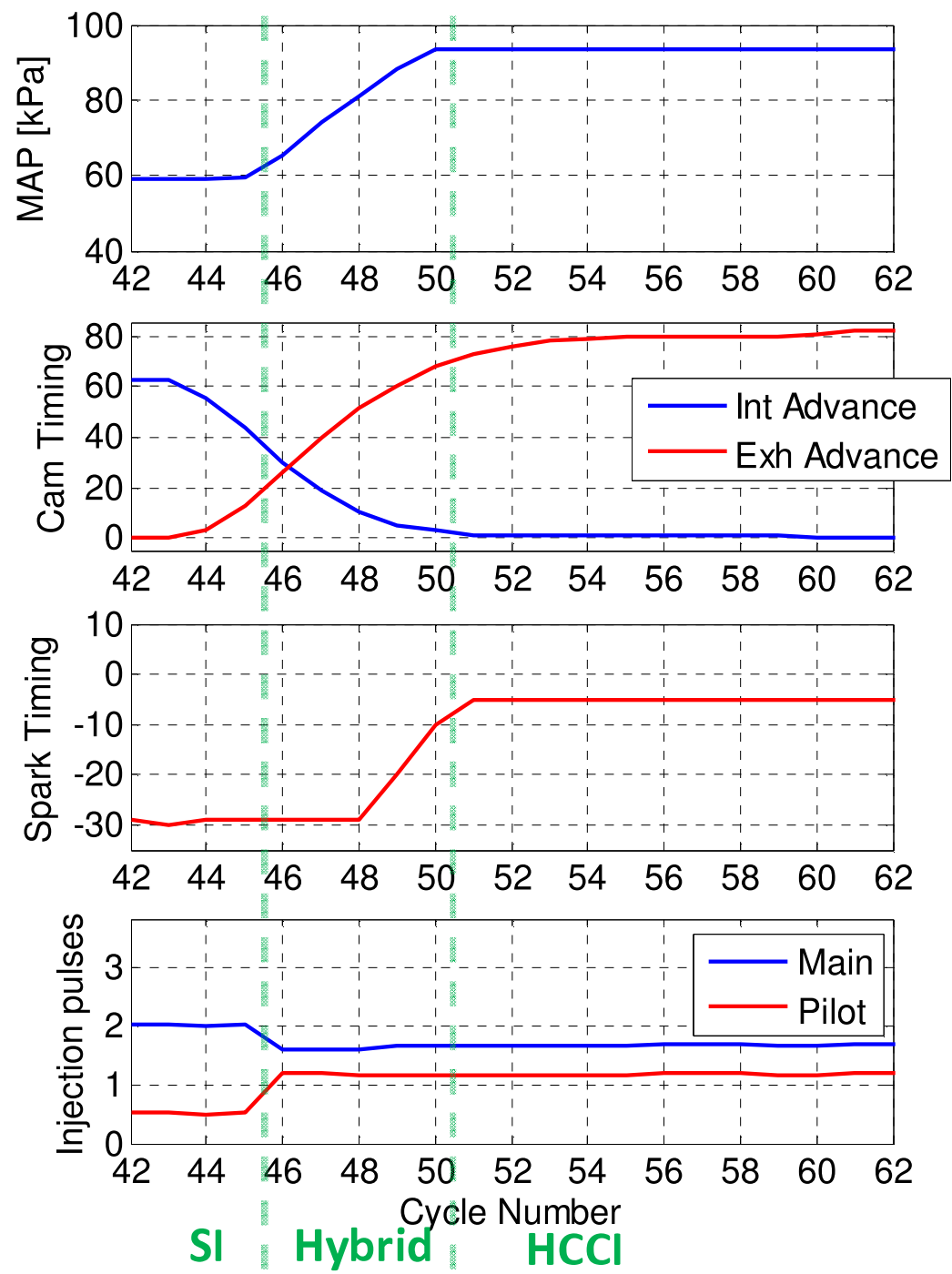

Figure 156: Control parameters of the 8-cycle transition

The control strategy for the 8-cycle transition is shown in Figure 158. Besides everything happening slightly faster, the only major differences of this transition, compared to the 15-cycle one are the timings of the throttle opening and the switch to a split injection.

The throttle was opened slightly earlier to maintain proper air-to-fuel ratio and guarantee healthy SI combustion before the hybrid combustion cycles. As the cams phase, higher levels of exhaust gas are trapped in the cylinder. These exhaust gases slow flame propagation and inhibit the intake of fresh air slightly, which may have been causing the short rich dip in the lambda trace. By opening the throttle slightly earlier, it was believed that this dip in AFR (lambda) could be 
eliminated, and the additional fresh charge could improve the combustion performance with high levels of exhaust gas.

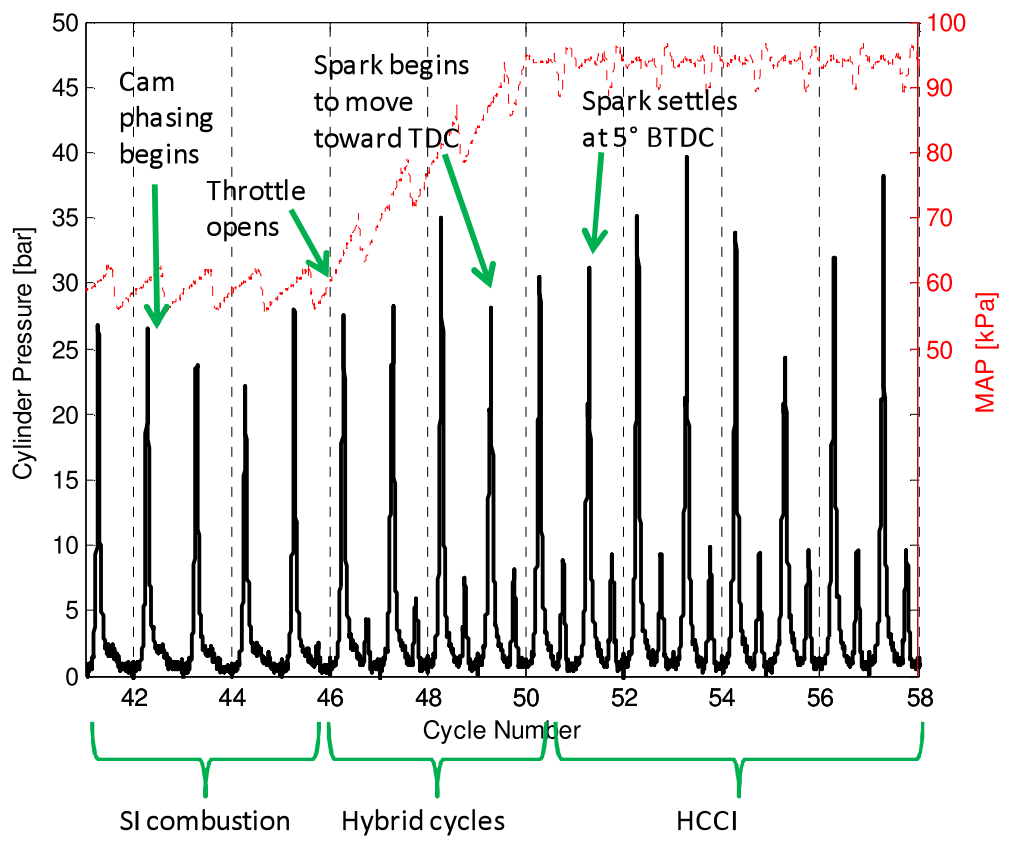

Figure 157: Cycle-by-cycle pressure trace of the 8-cycle transition

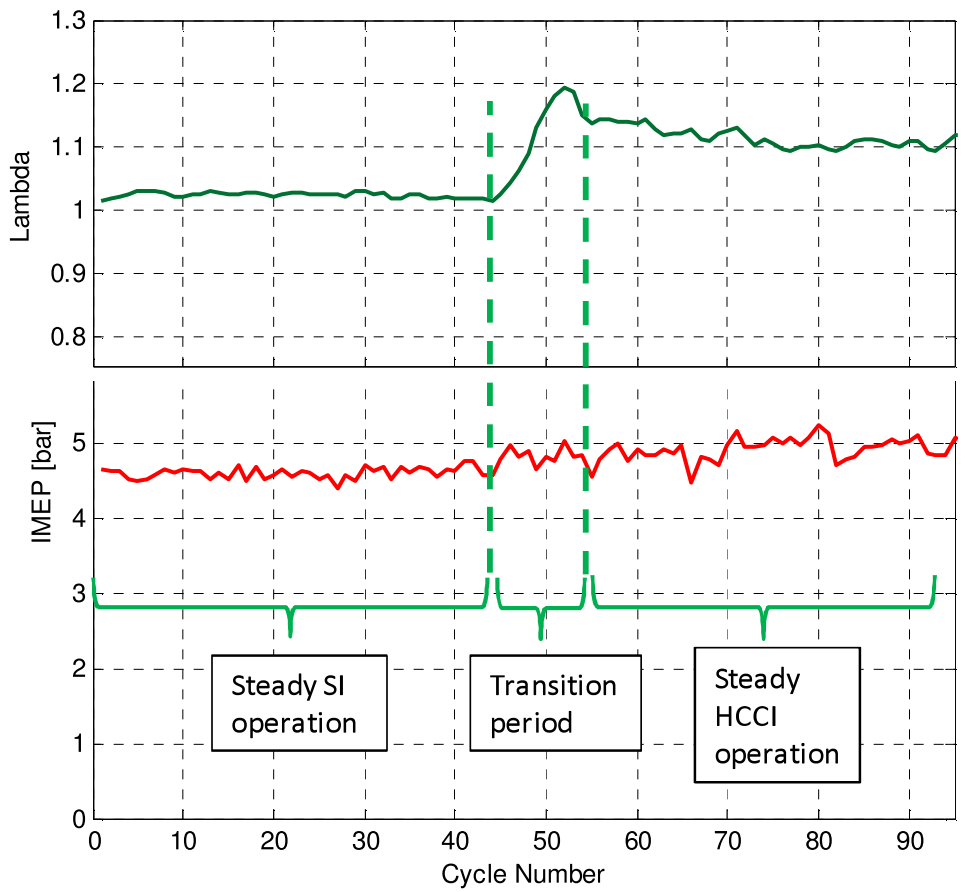

Figure 158: Lambda and IMEP traces during the 8-cycle transition

Note that the split injection for the 8-cycle transition was moved one cycle earlier relative to cam position to move the hybrid combustion ahead in the transition before SI combustion became slow and unstable. In the 15-cycle transition, the split injection was introduced when the intake and exhaust cams advanced to between $20^{\circ}$ and $40^{\circ}$ in cycle 56 (see Figure 150). However, 
slight signs of hybrid combustion appeared one cycle prior to that switch. Thus, in the 8-cycle transition, the split injection was moved forward at near the exhaust cam timing of $30^{\circ}$ (cycle 46 in Figure 156) to take advantage the hybrid combustion. This, in combination with the earlier throttle opening, can help start hybrid combustion before a weak SI cycle occurs.
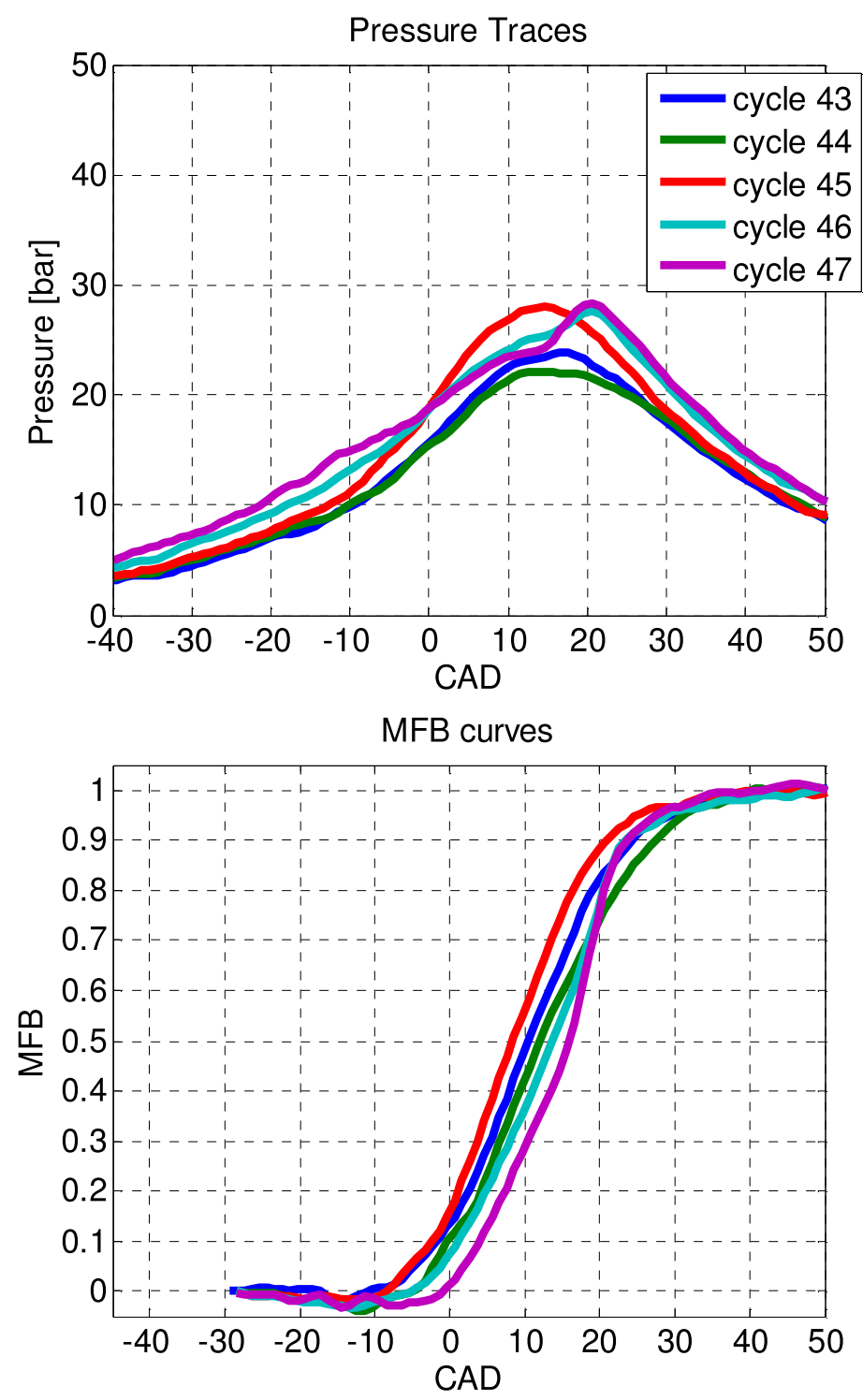

Figure 159: Pressure and MFB traces of the initial cycles of the 8-cycle transition

The cycle-by-cycle pressure traces (Figure 157) of this transition strategy and the IMEP and AFR (lambda) traces (Figure 158) show the smooth combustion mode transition. The rich air-tofuel ratio during the transition was eliminated, and the IMEP trace through the transition region is comparable with these of the stable SI and HCCI combustion. The strategy to induce earlier hybrid combustion was also successful, as shown in the cycle-by-cycle pressure trace and MFB curve detail of Figure 159. After cycle 43 (the last cycle before starting cam phasing), it only took two SI cycles before the first hybrid combustion cycle occurs in cycle 46. 
Hybrid combustion continues in cycles 48 and 49 until the percentage of SI combustion reduces down to zero. By cycle 50 , the $-10^{\circ}$ spark timing does not affect the SI combustion process, and the full auto-ignition (HCCI) combustion was achieved. The engine was operated at a steady state stable HCCI combustion with 4.90 bar IMEP, $2.4 \%$ COV, and -0.15 bar PMEP. The average maximal pressure rise rate is 3.8 bar per CAD.
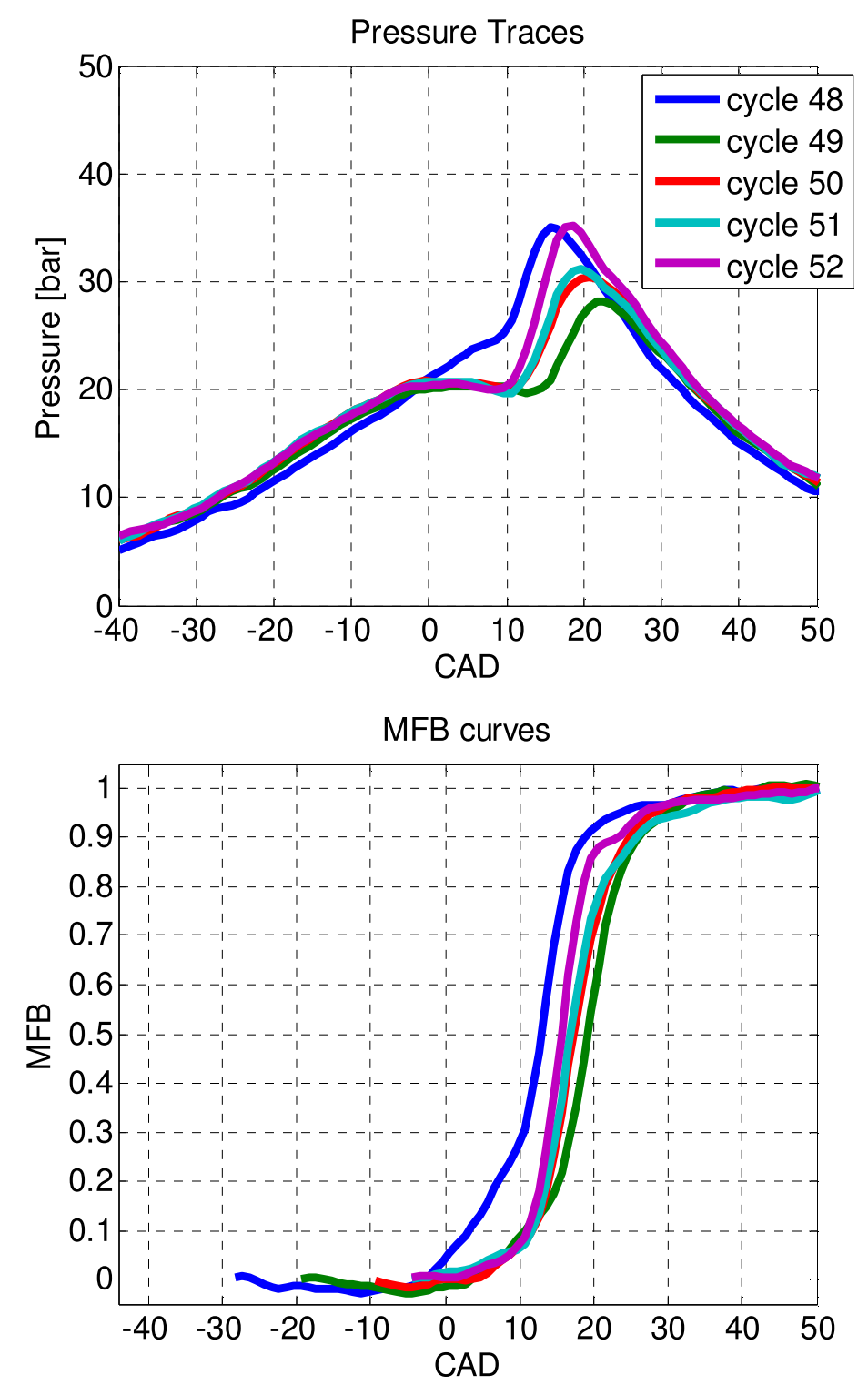

Figure 160: Pressure and MFB traces of the final cycles of the 8-cycle transition 
Figure 161 shows the MFB curves of the hybrid and HCCI combustion cycles from the 8-cycle transition. From this arrangement, it is very clear how the onset of auto-ignition advances smoothly cycle-by-cycle through the transition. The ability to traverse through this combined mode combustion quickly without a surge or loss in engine output torque is the ultimately successful achievement of this HCCI mode switch study.

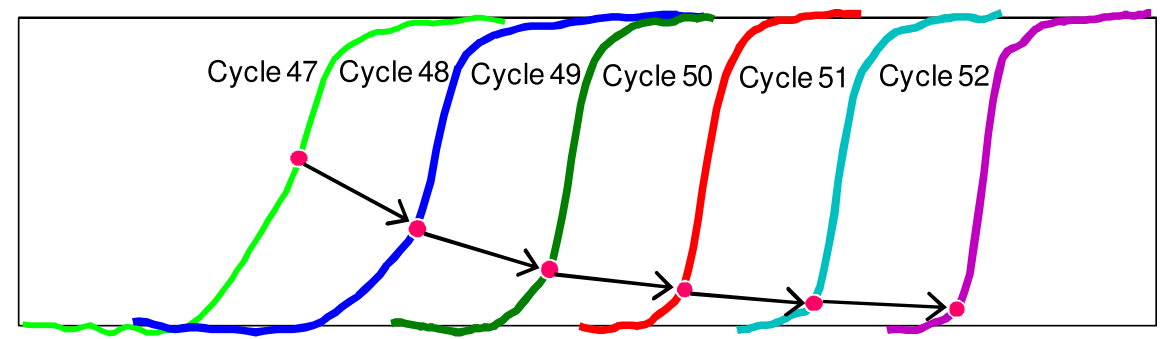

Figure 161: Progression of the hybrid combustion during the mode transition

\subsection{Temperature Sensitivity of Mode Transition}

Success of the mode transition was found to be heavily dependent on the operating temperature of the engine and charge air. Figure 162 shows the IMEP traces of two different mode transition attempts operated at slightly different engine coolant temperatures. With the coolant at $197^{\circ} \mathrm{F}$ and the charge air at $172^{\circ} \mathrm{F}$, the transition resulted in a stable HCCI condition. However, with the coolant only slightly cooler at $190^{\circ} \mathrm{F}$ and the intake air at $167^{\circ} \mathrm{F}$, the transition was not successful. Instead, after the transition, the HCCI combustion became unsustainable, leading to partial burn and misfire.

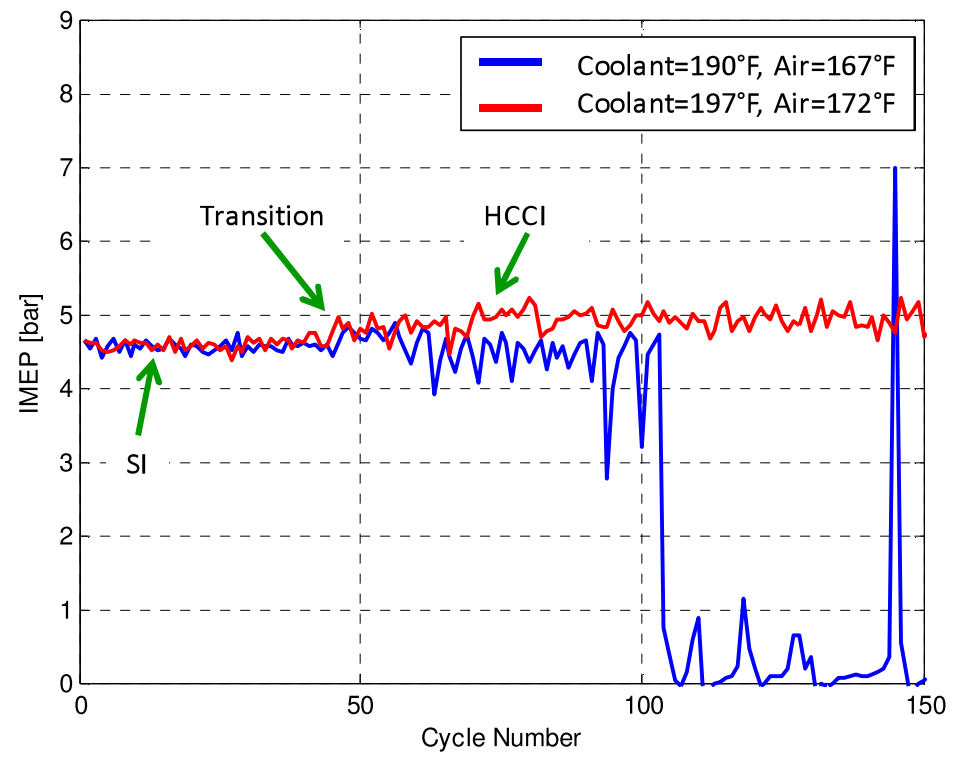

Figure 162: Engine temperature effects on mode transition stability 
Whether the coolant temperature or the intake air temperature had a greater effect on performance is an open question.

\subsection{Summary}

Motoring tests were conducted to validate the actuator transient operations including valve lift, electrical variable valve timing, electronic throttle, multiple spark and injection controls. After the actuator operations were confirmed, 15-cycle smooth combustion mode transition from SI to HCCI combustion was achieved; and fast 8-cycle smooth combustion mode transition followed. With a fast electrical variable valve timing actuator, the number of engine cycles required for mode transition can be reduced down to five. It was also found that the combustion mode transition is sensitive to the charge air and engine coolant temperatures and regulating the corresponding temperature to the target levels during the combustion mode transition is the key for a smooth combustion mode transition. 


\section{Conclusions and Recommendations}

\subsection{Conclusions}

The central objective of the proposed work is to demonstrate an HCCI capable SI engine that is capable of fast and smooth mode transition between SI and HCCI combustion modes. The model-based control technique was used to develop and validate the proposed control strategy for the fast and smooth combustion mode transition based upon the developed control-oriented engine; and an HCCI capable SI engine was designed and constructed using production ready two-step valve-train with electrical variable valve timing actuating system. Finally, smooth combustion mode transition was demonstrated on a metal engine within eight engine cycles.

- It is feasible to develop a control-oriented engine model that simulates the in-cylinder pressure and temperature in real-time.

- Model-based control for stable HCCI combustion and smooth combustion mode transition between SI and HCCI combustion is necessary since the steady state mapping parameters cannot be used for combustion mode transition control due to the transient thermal and fluid dynamics.

- Smooth combustion mode transition can be achieved for a DI engine equipped with two-step valve and electrical variable valve timing actuating system.

- It is feasible to implement the control-oriented engine model into the HIL (hardware-in-theloop) simulation environment with multiple simulation tasks.

- The electrical variable valve timing (VVT) actuating system is sensitive to the engine oil viscosity and low viscosity engine oil could improve the actuating system response time.

- It is very efficient to develop and validate the model-based control strategy in the hardwarein-the-loop simulation environment using Simulink based software such that the developed Simulink based control strategy can be implemented into Simulink based engine prototype controller directly.

- Stable HCCI combustion was achieved with COV at $2.1 \%$.

- Fast 8-cycle smooth combustion mode transition from SI to HCCI combustion was achieved; and with a fast electrical variable valve timing actuator, the number of engine cycles, required for mode transition, can be reduced down to five.

- It was also found that the combustion mode transition is sensitive to the charge air and engine coolant temperatures and regulating the corresponding temperatures to the target levels is the key for a smooth combustion mode transition.

\subsection{Recommendations}

- Studying combustion mode transition over the entire HCCI combustion operation range.

- Studying the sensitivity of the combustion mode transition to all control parameters.

- Improving the response time performance of the electrical variable valve timing actuating system to have even fast model transition. 


\section{Reference}

[1] D. J. Rausen, et al, "A mean value model for control of homogeneous charge compression ignition (HCCI) engines," ASME Journal of Dynamics, Measurement, and Control, Vol. 127, September, 2005, pp. 355-362.

[2] G. M. Shaver, et al, "Dynamic modeling of residual-affected homogeneous charge compression ignition engines with Variable Valve Actuation," ASME Journal of Dynamics, Measurement, and Control, Vol. 127, September, 2005, pp. 374-381.

[3] G. M. Shaver, "Physics based modeling and control of residual-affected HCCI engines using Variable Valve Actuation," PhD thesis, Stanford University, September, 2005.

[4] Zhang, Y., H. Xie, N. Zhou, T. Chen, and H. Zhao, "Study of SI-HCCI-SI transition on a port fuel injection engine equipped with 4VVAS," SAE Technical Paper 2007-01-0199, 2007.

[5] A-F M. Mahrous, M. L. Wyszynski, H. Xu, A. Tsolakis, and J. Qiao, “Effect of intake valves timings on in-cylinder charge characteristics in a DI engine cylinder with negative valve overlapping," SAE Technical Paper 2008-01-1347, 2008.

[6] A-F. M. Mahrous, A. Potrzebowski, M. L. Wyszynski, H. M. Xu, A. Tsolakis, and P. Luszcz, "A modeling study into the effects of variable valve timing on the gas exchange process and performance of a 4-valve DI homogeneous charge compression ignition (HCCI) engine," Energy Conversion and Management, Vol. 50, 2009, pp. 393-398.

[7] M. McCuen, Z. Sun, and G. Zhu, "Control-oriented mixing model for homogeneous charge compression ignition engine," 2010 American Control Conference, Baltimore, MD, June, 2010.

[8] X. Yang and G. Zhu, "A control oriented SI and HCCI hybrid combustion model for internal combustion engines," 2010 ASME Dynamic Systems and Control Conference, Cambridge, MA, September, 2010.

[9] X. Yang and G. Zhu, "A two-zone control oriented SI-HCCI hybrid combustion model for the HIL simulation," 2011 American Control Conference, San Francisco, CA, June, 2011.

[10] X. Yang and G. Zhu, "A control oriented hybrid combustion model of an HCCI capable SI engine," Proceedings of the Institution of Mechanical Engineers, Part D, Journal of Automobile Engineering, 226(10), 2012, pp. 1380-1395 (DOI: 10.1177/0954407012443334).

[11] C. J. Chiang and A. G. Stefanopoulou, "Stability analysis in homogeneous charge compression ignition (HCCI) engines with high dilution," IEEE Transactions on Control System Technology, Vol. 15, No. 2, March, 2007.

[12] Y. Moriya, A.Watanabe, H. Uda, H. Kawamura, M. Yoshiuka, and M. Adachi, "A newly developed intelligent variable valve timing system - continuously controlled cam phasing as applied to new 3 liter inline 6 engine", SAE Technical Paper 960579, 1996.

[13] P. H. Dugdale, R. J. Rademacher, B. R. Price, J. W. Subhedar, and R. L. Duguay, "Ecotec 2.4L VVT: a variant of GM's global 4-cylinder engine”, SAE Technical Paper 2005-011941,2005 
[14] M. Hattori, T. Inoue, Z. Mashiki, A. Takenaka, H. Urushihata, S. Morino, and T. Inohara, "Devalopment of variable valve timing system controlled by electric motor", $S A E$ Technical Paper 2008-01-1358, 2008

[15] M. Theobald, B. Lequesns, and R. Henry, "Control of engine load via electromagnetic valve actuators," SAE Technical Paper 940816, 1994

[16] R. J. Pierik and J. O. Wilson, "Engine timing drive with fixed and variable phasing", U.S. Patent 5,327,859, 1994

[17] H. Urushihata and H. Iida, "Variable valve timing control device of internal combustion engine", U.S. Patent 7,363,896 B2, 2008

[18] D. Law, D. Kemp, J. Allen, G. Kirkpatrick, and T. Copland, "Controlled combustion in an IC-engine with a fully variable valve train”, SAE Technical Paper 2001-01-0251, 2001

[19] N. Milovanovic, R. Chen, and J. Turner, "Influence of the variable valve timing strategy on the control of a homogeneous charge compression (HCCI) engine", SAE Technical Paper 2004-01-1899, 2004

[20] F. Agrell, H. Angstrom, B. Eriksson, J. Wikander, and J. Linderyd, "Integrated simulation and engine test of closed-loop HCCI control by aid of variable valve timings", $S A E$ Technical Paper 2003-01-0748, 2003

[21] A. Cairns and H. Blaxill, "The effects of two-stage cam profile switching and external EGR on SI-CAI combustion transitions," SAE Technical Paper, 2007-01-0187, 2007.

[22] G. Zhu and R. E. Skelton, "Integrated modeling and control for the large spacecraft laboratory experiment facility", Journal of Guidance, Control and Dynamics, Vol. 17, No. 3, pp. 442-450, 1994

[23] G. Zhu, K. M. Grigoriadis, and R. E. Skelton, "Covariance control design for Hubble Space Telescope", Journal of Guidance, Control and Dynamics, Vol. 18, No. 2, pp. 230236, 1995

[24] G. Zhu, M. A. Rotea, and R. Skelton, “A convergent algorithm for the output covariance constraint control problem”, SIAM J. Control Optim., Vol. 35, No.1, pp. 341-361, 1997.

[25] J. E. Shigley and C. R. Mischke, Mechanical Engineering Design, $6^{\text {th }}$ Edition, McGrawHill, 2001

[26] C. L. Phillips and R. D. Harbor, Feedback Control System, $4^{\text {th }}$ Edition, Prentice Hall, 2000.

[27] Z. Ren and G. Zhu, "Pseudo-random binary sequence closed-loop system identification error with integration control", Journal Proceedings of the Institution of Mechanical Engineers, Part I: Journal of Systems and Control Engineering, Vol. 233, pp877-884, 2009.

[28] B. Codrons, B. D. O. Anderson, and M.Gevers, "Closed-loop identification with an unstable or non-minimum phase controller," Automatica 38, pp. 2127-2137, 2002.

[29] W. Peterson, Error Correcting Coding, MIT Technical Press, Cambridge, Massachusetts, USA, 1961.

[30] G. Zhu, R. E. Skelton, and P. Li, "Q-Markov Cover identification using pseudo-random binary signals," International Journal of Control, Vol. 62, No. 1, 1995, pp. 1273-1290. 
[31] A. M. King, U. B. Desai, and R. E. Skelton, “A generalized approach to $q$-Markov covariance equivalent realization for discrete systems", Automatica, Vol. 24, No. 4, pp. 507-515, 1988.

[32] X. Yang and G. Zhu, "SI and HCCI combustion mode transition control of a multicylinder HCCI capable SI engine via iterative learning," 2011 ASME Dynamic Systems and Control Conference, Arlington, VA, November, 2011.

[33] P. R. Ouyang, B. A. Petz and F. F. Xi, "Iterative learning control with switching gain feedback for nonlinear systems," Journal of Computational and Nonlinear Dynamics, Vol. $6,2011$.

[34] D. Naidu, Optimal Control Systems, CRC Press LLC, 2003, pp. 232-239.

[35] X. Yang and G. Zhu, "A mixed mean-value and crank-based model of a dual-stage turbocharged SI engine for hardware-in-the-loop simulation," Proceedings of 2010 American Control Conference, Baltimore, MD, 2010.

[36] J. Kang, C. Chang, and T. Kuo, "Sufficient Condition on Valve Timing for Robust Load Transients in HCCI Engines," SAE Technical Paper 2010-01-1243, 2010.

[37] N. Ravi, M. J. Roelle, et al, "Model-based control of HCCI engines using exhaust recompression," IEEE Transactions on Control Systems Technology, Vol. 18, No. 6, November 2010, pp. 1289-1302.

[38] S. Zhang, G. Zhu, Y. Yoon, and Z. Sun, "A control oriented charge mixing and HCCI combustion model for internal combustion engines," 2012 ASME Dynamic System Control Conference, Ft. Lauderdale, FL, October, 2012.

[39] Z. Ren and G. Zhu, "Modeling and control of an electrical variable valve timing system for SI and HCCI combustion mode transition," 2011 American Control Conference, San Francisco, CA, June, 2011.

[40] Z. Ren and G. Zhu, "Modeling and control of an electrical variable valve timing actuator," ASME Journal of Dynamic Systems, Measurement and Control (Accepted in October, 2013).

[41] S. Zhang, G. Zhu, and Z. Sun, "A control-oriented charge mixing and two-zone HCCI combustion model," IEEE Transactions on Vehicular Technology (Accepted in September, 2013), DOI: 10.1109/TVT.2013.2285074.

[42] S. Zhang, A. White, J. Yang, and G. Zhu, "LPV control of an electronic throttle," 2013 ASME Dynamic System and Control Conference, Polo Alto, CA, October, 2013.

[43] S. Zhang, J. Yang, and G. Zhu, "LPV Gain-scheduling Control of an Electronic Throttle with Experimental Validation," Submitted to 2014 American Control Conference, Portland, OR, June, 2014.

[44] X. Yang and G. Zhu, "SI and HCCI combustion mode transition control of a multi-cylinder HCCI capable SI engine," IEEE Transaction on Control System Technology, Vol. 21, Issue 5, 2013, pp. 1558-1569 (DOI: 10.1109/TCST.2012.2201719.

[45] X. Yang and G. Zhu, "Air-to-fuel ratio regulation during SI to HCCI combustion mode transition using the LQ tracking control," Proceedings of 2012 American Control Conference, Montreal, Canada, June, 2012. 


\section{Appendix B - Publication List Associated with This Award}

1. S. Zhang, J. Yang, and G. Zhu, "LPV Gain-scheduling Control of an Electronic Throttle with Experimental Validation," Submitted to 2014 American Control Conference, Portland, OR, June, 2014.

2. S. Zhang, G. Zhu, and Z. Sun, "A control-oriented charge mixing and two-zone HCCI combustion model," IEEE Transactions on Vehicular Technology (Accepted in September, 2013), DOI: 10.1109/TVT.2013.2285074.

3. S. Zhang, A. White, J. Yang, and G. Zhu, "LPV control of an electronic throttle," 2013 ASME Dynamic System and Control Conference, Polo Alto, CA, October, 2013.

4. Z. Ren and G. Zhu, "Modeling and control of an electrical variable valve timing actuator," ASME Journal of Dynamic Systems, Measurement and Control (Accepted in October, 2013).

5. X. Yang and G. Zhu, "SI and HCCI combustion mode transition control of a multi-cylinder HCCI capable SI engine," IEEE Transaction on Control System Technology, Vol. 21, Issue 5, 2013, pp. 1558-1569 (DOI: 10.1109/TCST.2012.2201719).

6. X. Yang and G. Zhu, "A control oriented hybrid combustion model of an HCCI capable SI engine," Proceedings of the Institution of Mechanical Engineers, Part D, Journal of Automobile Engineering, 226(10), 2012, pp. 1380-1395 (DOI: 10.1177/0954407012443334).

7. S. Zhang, G. Zhu, Y. Yoon, and Z. Sun, "A control oriented charge mixing and HCCI combustion model for internal combustion engines," 2012 ASME Dynamic System Control Conference, Ft. Lauderdale, FL, October, 2012.

8. X. Yang and G. Zhu, "Air-to-fuel ratio regulation during SI to HCCI combustion mode transition using the LQ tracking control," Proceedings of 2012 American Control Conference, Montreal, Canada, June, 2012.

9. X. Yang and G. Zhu, "SI and HCCI combustion mode transition control of a multi-cylinder HCCI capable SI engine via iterative learning," 2011 ASME Dynamic Systems and Control Conference, Arlington, VA, November, 2011.

10. X. Yang and G. Zhu, "A two-zone control oriented SI-HCCI hybrid combustion model for the HIL simulation," 2011 American Control Conference, San Francisco, CA, June, 2011.

11. Z. Ren and G. Zhu, "Modeling and control of an electrical variable valve timing system for SI and HCCI combustion mode transition," 2011 American Control Conference, San Francisco, CA, June, 2011.

12. X. Yang and G. Zhu, "A control oriented SI and HCCI hybrid combustion model for internal combustion engines," 2010 ASME Dynamic Systems and Control Conference, Cambridge, MA, September, 2010. 
9 Appendix C - Published Journal and Conference (JC) Papers 


\subsection{Published JC Paper \#1}

LPV Gain-Scheduling Control of an Electronic Throttle with Experimental Validation 


\title{
LPV Gain-scheduling Control of an Electronic Throttle with Experimental Validation
}

\author{
Shupeng Zhang, Jie J. Yang, and Guoming G. Zhu
}

\begin{abstract}
Engine electronic throttle control is challenging due to its high nonlinearities. In this paper, a discrete-time electronic throttle system was modeled as an LPV (linear parameter varying) system, where the vehicle battery voltage, the nonlinear friction, and spring are the measurable time-varying parameters. Gain-scheduling $\mathrm{H}_{2}$ controller was designed for the LPV throttle control system using the linear matrix inequality (LMI) convex optimization approach. The designed controller was experimentally validated and compared with the baseline fixed gain PI controller and showed significant performance improvement.
\end{abstract}

\section{INTRODUCTION}

Electronic throttle replaces the mechanical link between the vehicle acceleration pedal and engine intake throttle valve plate by accurately regulating the throttle plate angle using either a DC motor or step motor [1] for internal combustion (IC) engines. This process is called electronic throttle control (ETC). The traditional engine throttle with the mechanical link adjusts the engine charge air directly and engine fuel quantity tracks the charge air to provide the desired air-to-fuel ratio that is critical for engine emission regulation. The advantage of using the ETC for IC engines is that the engine charge air and fuel can be regulated simultaneously, providing accurate air-fuel-ratio control, especially under the transient engine operations. The ETC is also a key enabler for torque based engine control [2], where the acceleration pedal provides desired torque and the engine control system determines the desired engine charge air and fuel to provide the desired torque. The torque based control is especially important for hybrid powertrains ([3] and [4]), where the IC engine, electric motor(s) and generator(s) are managed by their torque outputs or loads.

A conventional electric throttle consists of a DC (or step) motor, a set of speed reduction gears, throttle plate, and the limp-home (LH) spring set that keeps the valve plate at its default position. The electronic throttle system is highly nonlinear due to the rotational static and dynamic friction and the highly nonlinear LH spring set; the vehicle battery voltage fluctuation due to the vehicle electrical load variation introduces another degree of variation; in addition, the torque load introduced by the intake air flow [5] brings another uncertainty to the electronic throttle system. In this paper, these uncertainties are model as the measurable LPV (linear parameter varying) parameters.

Zhang is with the Department of Mechanical Engineering at the Michigan State University (MSU), E. Lansing, MI 48824, USA (zhangs30@msu.edu).

J. Yang is with Shanghai Jiaotong University, Shanghai, China. This work was completed while he was visiting MSU (jieyang@msu.edu)

G. Zhu is with the Department of Mechanical Engineering and Electrical/Computer Engineering at MSU, East Lansing, MI 48824, USA (phone: 517-884-1552; fax: 517-432-3341; zhug@egr.msu.edu).
Proportional-integral-derivative (PID) control is widely used in powertrain control system due to its simplicity ([6] and [7]). However, the high spring nonlinearity and charge air flow exogenous disturbances could limit the PID control to achieve the desired performance under wide operation conditions, especially in these operational conditions that require low change air flow rate. The other commonly used approach for the electronic throttle control is sliding mode control, where the nonlinearities of friction and spring force can be considered as the parameter uncertainty and bounded external disturbance ([8]-[10]); however, the robust performance cannot be guaranteed. In reference [11], a discrete-time piecewise affine approach was used to accurately model the throttle nonlinearity, and the constrained optimal control was used to design a controller satisfies all imposed constrains. However, the uncertainties, especially the battery voltage change was not considered, which could lead to poor transient performance. In [12] an LPV throttle model was established to convert the nonlinear system into a linear LPV system, and an $\mathrm{H}_{2}$ static output feedback control was designed to guarantee the robust stability and performance using the tracking error as the performance output.

In this paper, the nonlinear electronic throttle system was model as an LPV system, where the friction torque and battery voltage are the varying parameters; and the disturbance torque, induced by the air flow and other sources are modeled as exogenous input. A virtual state was added to denote the integration of the tracking error for set-point control, leading to zero steady-state error. A feed-forward control is also used to compensate the preload spring torque. An LPV gain-scheduling controller is designed for the developed LPV model utilizing the LMI (linear matrix equality) convex optimization scheme to guarantee the system stability and performance. All the performance weighting matrices were well-tuned during the control design and simulation validation process, and a sequence of controllers with control effort from low to high was designed and experimentally validated to study the trade-off between control effort and system performance under the modeling error. This provides a systematic approach for LPV control gain tuning.

The paper is organized as follows. Section II presents a discrete-time electric throttle model that is linearized into an LPV form; and Sections III and IV describe the LMI (linear matrix inequality) based convex optimization design for the LPV ETC control system and the associated experimental validation results, respectively. The last section adds some conclusions.

\section{System Modeling}

An electronic throttle system can be described by the block diagram shown below in Figure 1. A DC motor is driven by a controlled voltage $V_{m}$, which is powered by the battery. 
$V_{m}=V_{b} u$ is regulated by a PWM (pulse width modulated) duty cycle signal $u$, where the duty cycle is the output of the electronic throttle controller and $V_{b}$ is the battery voltage. An $\mathrm{H}$-bridge drive module is used to make it possible to apply the voltage in both directions. $R$ and $L$ denote the resistance and inductance of the DC motor, respectively. The motor is connected to the throttle plate shaft by a set of gears, and a pair of return springs that keeps the throttle plate resting at the default position (limp-home) when the control input is zero.

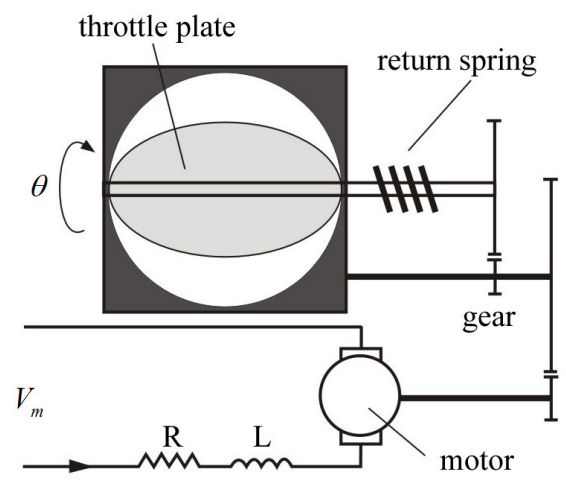

Figure 1. An electronic throttle system

Figure 2 shows the nonlinear characteristic of the return spring. The magnitude of spring torque at limp-home position $\theta_{0}$ is $T_{s}$, and the direction of the torque could switch around this position. Since the developed model in this paper is linear, the parameter denoting the throttle angle position discussed later is the position relative to $\theta_{0}$.

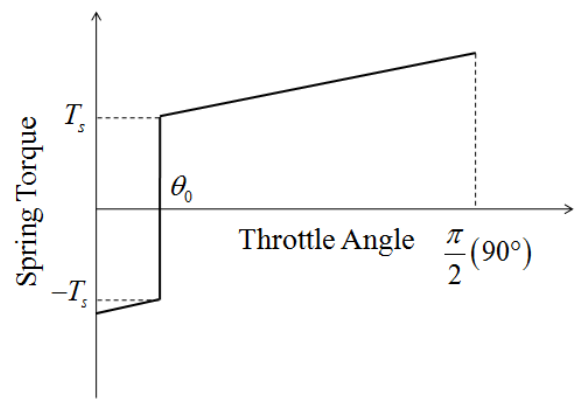

Figure 2. Nonlinear property of return spring

\section{A. Electronic Throttle Modeling}

The electronic throttle (a mechatronic system) dynamics can be expressed using the following differential equations

$$
V_{m}=i R+L \frac{d i}{d t}+E_{a}, \quad J \frac{d^{2} \theta}{d t^{2}}=T_{m}-\bar{T}_{s}-\bar{T}_{f}-T_{d}
$$

where $V_{m}, i, L$ and $R$ are the motor voltage, current, inductance and resistance, respectively; $E_{a}=K_{a} \dot{\theta}$ is the motor back EMF (electro-magnetic field) voltage; $J$ and $\theta$ are motor throttle assembly inertia and throttle angle; and $T_{m}, \bar{T}_{s}, \bar{T}_{f}$ and $T_{d}$ represent the motor output torque, nonlinear spring torque, friction torque and other exogenous input torque such as the disturbance torque due to the unbalanced force, caused by the charge air flow, applied on the throttle plate.
Since the inductance $L$ is relatively small for a DC motor, the inductance dynamics can be ignored and the motor current can be approximated by setting $L=0$. That is

$$
i=\frac{1}{R}\left(V_{m}-K_{a} \dot{\theta}\right)
$$

and the motor output torque satisfies

$$
T_{m}=K_{m} i=\frac{K_{m}}{R}\left(V_{m}-K_{a} \dot{\theta}\right)
$$

Finally the throttle system dynamics can be described by the following nonlinear differential equation.

$$
J \ddot{\theta}=\frac{K_{m}}{R}\left(V_{b} \tilde{u}-K_{a} \dot{\theta}\right)-K_{s} \theta-T_{s} \operatorname{sgn}(\theta)-K_{B} \dot{\theta}-T_{f} \operatorname{sgn}(\dot{\theta})-T_{d}
$$

where $K_{m}, K_{a}, K_{B}$ and $K_{s}$ represent the motor torque, back EMF, viscous friction, and spring stiffness coefficients; $T_{s}$ (where $\bar{T}_{s}=K_{s} \theta+T_{s} \operatorname{sgn}(\theta) \quad$ ) and $T_{f}$ (where $\left.\bar{T}_{f}=K_{B} \dot{\theta}-T_{f} \operatorname{sgn}(\dot{\theta})\right)$ represent the spring preload torque used to hold the throttle at limp-home position, and Coulomb friction torque. The differential equation can be represented by the following continuous-time state space model

$$
\dot{x}=A x+B u+\Gamma, y=C x
$$

where

$$
\begin{gathered}
A=\left[\begin{array}{cc}
0 & 1 \\
-\frac{K_{s}}{J} & -\frac{1}{J}\left(\frac{K_{m} K_{a}}{R}+K_{B}\right)
\end{array}\right], B=\left[\begin{array}{c}
0 \\
\frac{K_{m} V_{b}}{J R}
\end{array}\right], x=\left[\begin{array}{c}
x_{1} \\
x_{2}
\end{array}\right]=\left[\begin{array}{c}
\theta \\
\dot{\theta}
\end{array}\right], \\
\Gamma=\left[\begin{array}{c}
0 \\
-\frac{1}{J}\left(T_{s} \operatorname{sgn}(\theta)+T_{f} \operatorname{sgn}(\dot{\theta})+T_{d}\right)
\end{array}\right], C=\left[\begin{array}{cc}
1 & 0 \\
0 & 1
\end{array}\right]
\end{gathered}
$$

Since the system is very nonlinear due to term $\Gamma$, it will have quite different dynamic behaviors (or transfer functions) under different operation conditions. Furthermore, system parameters are coupled in the state space matrices; it will be almost impossible to use the experimental throttle step and sinusoid response data to determine all system parameters. The alternative method was adopted in this case, where the spring preload torque and stiffness were obtained by measuring the torque at different stationary points; the Coulomb friction was obtained approximately by moving the throttle plate from the same initial position to opposite directions through electronic control; the system inertia and viscous friction were obtained together by releasing the plate freely with and without electronic control. The values of the parameters are shown in Table 1.

TABLE I.

ELECTRONIC THROTTLE PARAMETERS

\begin{tabular}{c|c|c|c}
\hline \hline Parameter & Value & Parameter & Value \\
\hline$R$ & 2.07 & $J$ & 0.0058 \\
\hline$K_{m}$ & 0.537 & $K_{a}$ & 0.5589 \\
\hline$K_{B}$ & 0.005 & $K_{s}$ & 0.0914 \\
\hline$T_{f}$ & 0.137 & $T_{s}$ & 0.3193 \\
\hline
\end{tabular}

\section{B. Discrete-time LPV System}

To design an LPV gain-scheduling controller, the nonlinear system (6) needs to be converted into an LPV one. The entries in matrix $\Gamma$ were treated in different ways. 
Firstly, the spring preload torque $T_{s} \operatorname{sgn}(\theta)$ can be compensated by a control signal $u_{0}(\theta)$ as function of $\theta$ where

$$
u_{0}=\frac{J R T_{s}}{K_{m} V_{b}} \operatorname{sgn}(\theta)
$$

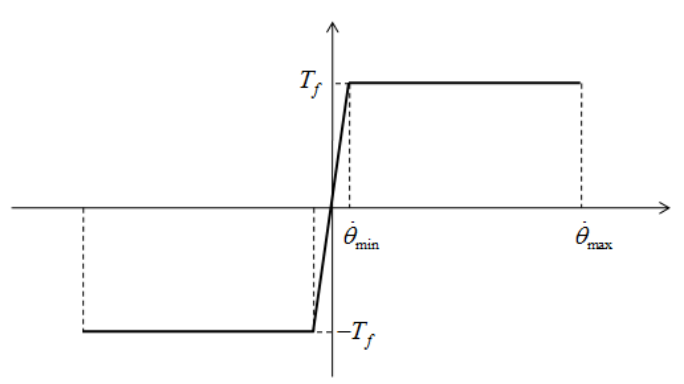

Figure 3. Approximation of sign function

Secondly, as shown in Figure $3, T_{f} \operatorname{sgn}(\dot{\theta})$ can be expressed by

$$
T_{f} \operatorname{sgn}(\dot{\theta})=\left|T_{f} / \dot{\theta}\right| \dot{\theta}+\Delta T_{f}=F_{0} \gamma_{1} \dot{\theta}+\Delta T_{f}
$$

where $\left|T_{f} / \dot{\theta}\right|$ is treated as a term containing varying parameter $\gamma_{1}$, and $F_{0}$ is a constant chosen to normalize $\gamma_{1} \cdot \dot{\theta}_{\max }$ can be experimental determined, by setting extreme operation condition; $\dot{\theta}_{\min }$ is chosen to be small enough to reduce approximation error.

Finally, $T_{d}$, along with uncertainty $\Delta T_{f}$, forms the system exogenous input $w$, where

$$
w=\frac{1}{J}\left(\Delta T_{f}+T_{d}\right)
$$

Since the battery voltage varies during the operation, $V_{b}$ can be expressed as

$$
V_{b}=\bar{V}_{b}\left(1+\gamma_{2}\right)
$$

where $\gamma_{2}$ is the second varying parameter that is measurable. The following equation defines the range of $\gamma_{1}$ and $\gamma_{2}$.

$$
\gamma_{1}(t) \in[0.000115,0.8], \quad \gamma_{2}(t) \in[-0.417,0.167]
$$

Then the continuous-time state space model can be converted into the following LPV system

$$
\begin{aligned}
& \dot{x}=\left(A_{c}+A_{\gamma} \gamma_{1}\right) x+B_{c}\left(1+\gamma_{2}\right)\left(u+u_{0}\right)+B_{w} w \\
& y=C_{c} x
\end{aligned}
$$

where the system matrices in (5) can be expressed as a sum of nominal state space matrices $A_{c}, B_{c}, B_{w}, C_{c}$, and varying parameter depended matrices $1 A_{\gamma} \gamma_{1}$ and $B_{c} \gamma_{2}$.

The system is then discretized in to a discrete-time state space model using the first order approximation system matrices as follows:

$$
A_{P}=e^{A_{c} T_{s}}+e^{A_{c} T_{s}} A_{\gamma} \gamma_{1} t_{s}, B_{P}=e^{A c T_{s}} B_{c}\left(1+\gamma_{2}\right) t_{s}, C_{P}=C_{c}
$$

where $t_{s}$ is the sampling time of the discrete-time system. For convenience, $\gamma_{1}$ and $\gamma_{2}$ are supposed to lie in the compact structure $\Theta=\operatorname{diag}\left\{\gamma_{1}, \gamma_{2}\right\}$, and

$$
\Theta \in \Omega=\left\{\operatorname{diag}\left(\gamma_{1}, \gamma_{2}\right): 0.000115 \leq \gamma_{1} \leq 0.8,-0.417 \leq \gamma_{2} \leq 0.167\right\}
$$

Then the discrete-time state-space LPV system is in the following form of

$$
\begin{aligned}
& x_{P}(k+1)=A_{P}(\Theta) x_{P}(k)+B_{P}(\Theta) u(k)+B_{w} w(k) \\
& y_{P}(k)=C_{P} x_{P}(k)
\end{aligned}
$$

Figure 4 shows the closed-loop discrete-time system architecture, where plant $P$ is the resulting discrete time state space model (15) with the third state $x_{3}$ added for the set-point control purpose that will be clarified in Section III; $K$ is the closed loop LPV controller to be designed. There are two inputs for the system plant and they are the reference signal $r$ and the summation of feedback control $u$ (from LPV controller $K$ ) and the feed-forward control $u_{0}$ (used to compensate the spring preload torque). Since $u_{0}$ can be obtained directly by (7) with known battery voltage, and will only change sign when crossing the limp-home position, it will be ignored during the control design process and will be added back during the simulations. For the LPV control design, since the system is linear, the reference signal can be set to zero, and $r$ denotes the input noise (pedal sensor error). The weighting function $W_{r}$ is selected as a low pass filter

$$
W_{r}=\frac{1}{z-0.9}
$$

which reflects the frequency character of the input noise. The weighting function $W_{u}$ is selected as a high pass filter

$$
W_{u}=\frac{a(z-0.967)}{z-0.6703}
$$

Note that the high pass filter is selected to limit the high frequency component in the control signal due to limited bandwidth of the ETC system. However, the filtering frequency was selected in such a way that the control system will have the bandwidth high enough to have satisfactory transient response (e.g., step response). Coefficient $a$ in the weighting function $W_{u}$ and the weighting functions $W_{e}$ and $W_{I}$ are selected as design constants and will be tuned during the control design and experimental validation process for the best performance possible.

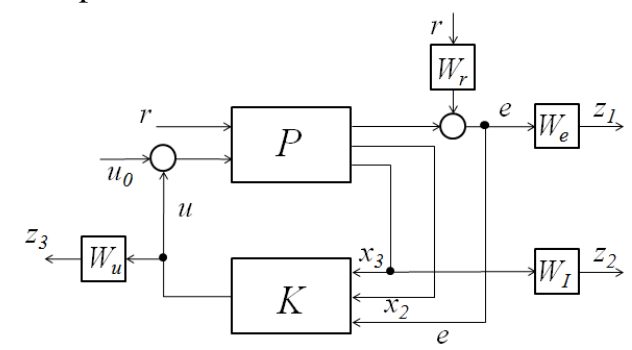

Figure 4. Closed loop system block diagram

\section{LPV CONTROL DESIGN}

\section{A. Set-point Control}

In order to achieve zero steady-state regulation error, a third state was added into the electric throttle system in the continuous-time form:

$$
\dot{x}_{I}=r-x_{1}
$$

Since the throttle position can be measured, $x_{I}$ can be obtained by integrating the tracking error online, it is available 
for the state feedback control. Then in the discrete-time system it becomes

$$
x_{I}(k+1)=x_{I}(k)+t_{s} r(k)-t_{s} x_{1}(k)
$$

\section{B. Augmented LPV System}

For LPV gain-scheduling control design, a state space model of the entire system, shown in Figure 4, is augmented with the set-point control (17) and weighting functions. The augmented state space model is in the form of

$$
\left[\begin{array}{c}
x(k+1) \\
z(k) \\
e(k)
\end{array}\right]=\left[\begin{array}{ccc}
A(\Theta) & B_{1}(\Theta) & B_{2}(\Theta) \\
C_{1} & D_{1} & D_{2} \\
C_{2} & 0 & 0
\end{array}\right]\left[\begin{array}{c}
x(k) \\
\tilde{w}(k) \\
u(k)
\end{array}\right]
$$

where $x(k)=\left[x_{P}(k) x_{I}(k) x_{r}(k) x_{W}(k)\right]^{\mathrm{T}} \in \mathbb{R}^{5}\left(x_{W}\right.$ is due to the weighting function), the exogenous input $\tilde{w}(k)=[r(k) w(k)]^{\mathrm{T}} \in \mathbb{R}^{2} \quad, \quad$ the performance output $z(k)=\left[z_{1}(k) z_{2}(k) z_{3}(k)\right]^{\mathrm{T}} \in \mathbb{R}^{3}$ and the measurement for control $y(k)=\left[y_{1}(k), y_{2}(k), y_{3}(k)\right]^{\mathrm{T}} \in \mathbb{R}^{3}$. The state space matrices are

$$
\begin{aligned}
& A(\Theta)=\left[\begin{array}{ccccc}
A_{P} & 0 & 0 & 0 \\
-t_{s} & 0 & 1 & 0.1 t_{s} & 0 \\
0 & 0 & 0 & 0.9 & 0 \\
0 & 0 & 0 & 0 & 0.6703
\end{array}\right], \\
& B_{1}(\Theta)=\left[\begin{array}{cc}
0 & B_{W} \\
0 & 0 \\
1 & 0 \\
0 & 0
\end{array}\right], B_{2}(\Theta)=\left[\begin{array}{c}
B_{P} \\
0 \\
0 \\
1
\end{array}\right] \text {, } \\
& C_{1}=\left[\begin{array}{ccccc}
-W_{e} & 0 & 0 & W_{e} & 0 \\
0 & 0 & W_{I} & 0 & 0 \\
0 & 0 & 0 & 0 & -0.2967 a
\end{array}\right], D_{1}=\left[\begin{array}{ll}
0 & 0 \\
0 & 0 \\
0 & 0
\end{array}\right], \\
& C_{2}=\left[\begin{array}{ccccc}
-1 & 0 & 0 & 1 & 0 \\
0 & 1 & 0 & 0 & 0 \\
0 & 0 & 1 & 0 & 0
\end{array}\right], D_{2}=\left[\begin{array}{lll}
0 & 0 & a
\end{array}\right]^{T} .
\end{aligned}
$$

\section{LPV Control Synthesis}

For the augmented system in (19), the $\mathrm{H}_{2}$ gain-scheduling controller $K(\Theta)$ will be synthesized to minimize the upper bound of the closed loop $\mathrm{H}_{2}$ norm from $\tilde{w}(k)$ to $z(k)$. The goal is to design a static output feedback gain-scheduling control $u(k)=K(\Theta) e(k)$ that stabilizes the closed-loop system and minimizes the upper bound for the $\mathrm{H}_{2}$ norm of the closed-loop LPV system (18) over any trajectories of $\Theta(k) \in \Theta$.

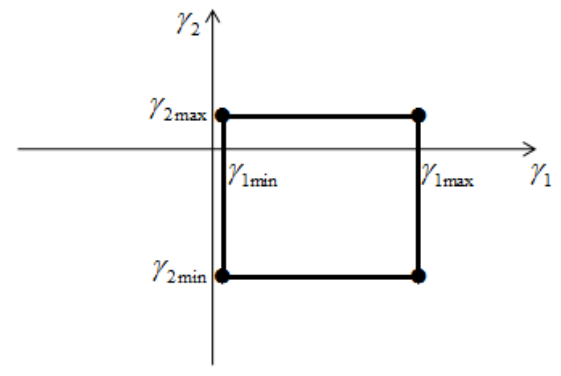

Figure 5. Parameter space polytope
The state space model (18) is converted to a discrete-time polytopic time-varying system by solving state space matrices containing varying parameters $(A(\Theta)$ and $B(\Theta)$ ) at the vertices of the parameter space polytope, as shown in Figure 5 . Any system inside the polytope is represented by a convex combination of the vertex systems as weighted by the vector $\lambda(k)$ of the barycentric coordinates, and formula for computing the barycentric coordinates is provided by [13]. The discrete-time polytopic time-varying system is given by

$$
\left[\begin{array}{c}
x(k+1) \\
z(k) \\
e(k)
\end{array}\right]=\left[\begin{array}{ccc}
\tilde{A}(\lambda(k)) & \tilde{B}_{1}(\lambda(k)) & \tilde{B}_{2}(\lambda(k)) \\
\tilde{C}_{1} & \tilde{D}_{1} & \tilde{D}_{2} \\
\tilde{C}_{2} & 0 & 0
\end{array}\right]\left[\begin{array}{c}
x(k) \\
\tilde{w}(k) \\
u(k)
\end{array}\right]
$$

where the system matrices $\tilde{A}(\lambda(k)) \in \mathbb{R}^{5 \times 5}, \tilde{B}_{1}(\lambda(k)) \in \mathbb{R}^{5 \times 2}$ and $\tilde{B}_{2}(\lambda(k)) \in \mathbb{R}^{5 \times 1}$ belong to the following polytope

$$
\begin{aligned}
\mathcal{D}=\left\{\left(\tilde{A}, \tilde{B}_{1}, \tilde{B}_{2}\right)(\lambda(k)):\right. & \\
& \left.\left(\tilde{A}, \tilde{B}_{1}, \tilde{B}_{2}\right)(\lambda(k))=\sum_{i=1}^{N} \lambda_{i}(k)\left(A, B_{1}, B_{2}\right)_{i}, \lambda(k) \in \Lambda\right\}
\end{aligned}
$$

where $A_{i}, B_{1 i}$ and $B_{2 i}$ are vertices of the polytope with $N=4$ in this case, and $\lambda(k)$ is the vector in the barycentric coordinates which belongs to the unit simplex

$$
\Lambda=\left\{\zeta \in \mathbb{R}^{N}: \sum_{i=1}^{N} \zeta_{i}=1, \zeta_{i} \geq 0, i=1, \cdots, N\right\}
$$

The rate of variation of $\lambda(k)$ can be defined as

$$
\Delta \lambda_{i}(k)=\lambda_{i+1}(k)-\lambda_{i}(k), i=1, \cdots, N
$$

which is bounded by

$$
-b \leq \Delta \lambda_{i}(k) \leq b, i=1, \cdots, N
$$

where $b \in[0,1]$.

The LMI convex optimization approach will be used to solve the LPV gain-scheduling control problem. Based on Theorem 9 of reference [14], if there exists, for $i=1, \ldots, N$, matrices $G_{i, 1} \in \mathbb{R}^{3 \times 3}, W_{i} \in \mathbb{R}^{2 \times 2}, G_{j, 2} \in \mathbb{R}^{2 \times 3}, Z_{i, 1} \in \mathbb{R}^{1 \times 3}$ and symmetric positive-definite matrices $P_{i} \in \mathbb{R}^{5 \times 5}$ and $G_{j, 3} \in \mathbb{R}^{2 \times 2}$ such that the following LMIs hold

$$
\left[\begin{array}{ccc}
(1-b) P_{i}+b P_{l} & * & * \\
G_{i}^{\mathrm{T}} \tilde{A}_{i}^{\mathrm{T}}+Z_{i}^{\mathrm{T}} \tilde{B}_{2, i}^{\mathrm{T}} & G_{i}+G_{i}^{\mathrm{T}}-P_{i} & * \\
\tilde{B}_{1, i}^{T} & 0 & I
\end{array}\right]>0
$$

for $i=1, \ldots, N$, and $l=1, \ldots, N$,

$$
\left[\begin{array}{ccc}
(1-b) P_{i}+(1-b) P_{j}+2 b P_{l} & * & * \\
G_{j}^{\mathrm{T}} \tilde{A}_{i}^{\mathrm{T}}+G_{i}^{\mathrm{T}} \tilde{A}_{j}^{\mathrm{T}}+Z_{j}^{\mathrm{T}} \tilde{B}_{2, i}^{\mathrm{T}}+Z_{i}^{\mathrm{T}} \tilde{B}_{2, j}^{\mathrm{T}} & f(i, j) & * \\
\tilde{B}_{1, i}^{T}+\tilde{B}_{1, j}^{T} & 0 & 2 I
\end{array}\right]>0
$$

where

$$
\begin{array}{r}
f(i, j)=G_{i}+G_{i}^{\mathrm{T}}+G_{j}+G_{j}^{\mathrm{T}}-P_{i}-P_{j} \\
\text { for } l=1, \ldots, N, i=1, \ldots, N-1, \text { and } j=\mathrm{i}+1, \ldots, N \\
{\left[\begin{array}{cc}
W_{i} & * \\
G_{i}^{T} \tilde{C}_{1, i}^{T}+Z_{i}^{T} \tilde{D}_{2, i}^{T} & G_{i}+G_{i}^{T}-P_{i}
\end{array}\right]>0}
\end{array}
$$

for $i=1, \ldots, N$, 


$$
\left[\begin{array}{cc}
W_{i}+W_{j} & * \\
G_{j}^{\mathrm{T}} \tilde{C}_{1, i}^{\mathrm{T}}+G_{i}^{\mathrm{T}} \tilde{C}_{1, j}^{\mathrm{T}}+Z_{j}^{\mathrm{T}} \tilde{D}_{2, i}^{\mathrm{T}}+Z_{i}^{\mathrm{T}} \tilde{D}_{2, j}^{\mathrm{T}} & f(i, j)
\end{array}\right]>0
$$

for $i=1, \ldots, N-1$, and $j=\mathrm{i}+1, \ldots, N$, then the robust static output feedback controller

$$
K(\lambda(k))=\hat{Z}(\lambda(k)) \hat{G}(\lambda(k))^{-1}
$$

where

$$
\begin{aligned}
& \hat{G}(\lambda(k))=\sum_{i=1}^{N} \lambda_{i}(k) G_{i, 1} \\
& \hat{Z}(\lambda(k))=\sum_{i=1}^{N} \lambda_{i}(k) Z_{i, 1}
\end{aligned}
$$

stabilizes the system with a guaranteed $H_{2}$ performance $v$ given by

$$
v^{2}=\min _{P_{i}, G_{i, 1}, G_{i, 2}, G_{i, 3}, Z_{i, 1}, W_{i}} \max _{i} \operatorname{Tr}\left(W_{i}\right)
$$

\section{EXPERIMENTAL VALIDATION}

The designed gain-scheduling controllers were validated in simulations using the ETC model described in Section II. Due to the page limitation, the simulation results are not included in this paper. In reality, the model and physical system are always different. For example, the friction model may not match with the actual system friction due to its nonlinearity. Therefore, the simulated performance of the designed LPV controllers will not match with the experimental one. As the control gain (effort) increases from low to high, the performance of the actual system could deviate from the expected performance, obtained through simulation validation, significantly and eventually the closed loop system may become unstable as the control gain is increased to certain level; see [15] for detail.

Therefore, in order to obtain the best performance possible for the actual physical system (ETC), a sequence of controllers with different control gains was designed by selecting different values for weighting matrices $W_{u}$ (parameter $a$ ), $W_{e}$ and $W_{l}$. Table 2 shows the selected weighting matrices to achieve control gains from low to high. The designed controllers were implemented into the electronic throttle control system and evaluated experimentally.

TABLE II. Weighting MATRICES TUNING

\begin{tabular}{c|c|c|c}
\hline \hline Controller & $\boldsymbol{W}_{\boldsymbol{e}}$ & $\boldsymbol{W}_{\boldsymbol{I}}$ & $\boldsymbol{a}\left(\boldsymbol{W}_{\boldsymbol{u}}\right)$ \\
\hline 1 & 2.5 & 2.10 & 0.25 \\
\hline 2 & 2.5 & 2.25 & 0.22 \\
\hline 3 & 2.5 & 2.50 & 0.20 \\
\hline 4 & 2.5 & 2.50 & 0.10 \\
\hline 5 & 2.5 & 1.50 & 0.05 \\
\hline
\end{tabular}

By fixing weighting matrix $W_{e}$, as the $a$ (gain of weighting matrix $W_{u}$ ) increases, the penalty to control effort $\left(z_{3}\right)$ goes up, which leads to the decreased control effort (gain). As a result, the closed loop system response becomes slow. At the same time, weighting matrix $W_{I}$ is also tuned to achieve the smallest tracking error integration $\left(z_{2}\right)$ in each case. During the simulation evaluation, all five controllers showed a trend of tracking performance improvement as the weighting coefficient $a$ decreases. Then this sequence of controllers was implemented into the prototype electronic throttle controller and validated experimentally.

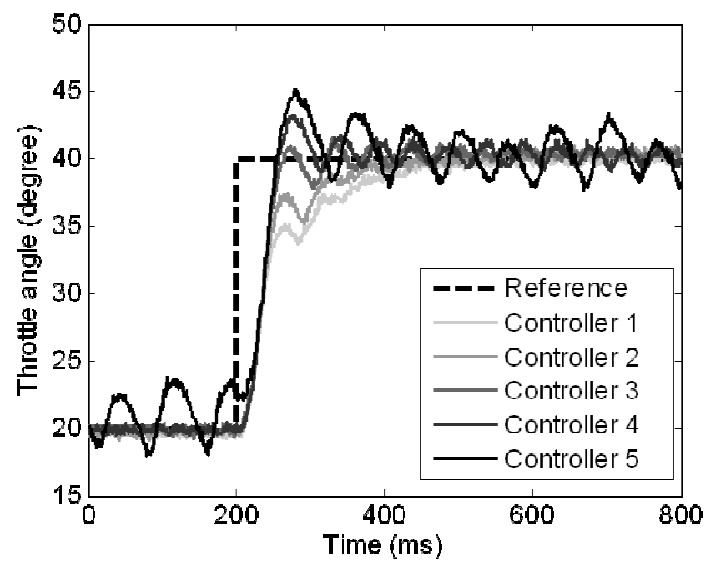

Figure 6. Step response comparison

Figure 6 shows the experimental validation results of step responses from 20 to 40 degree opening. Note that the control effort (gain) increases from controllers 1 to 5. From experimental results shown in Figure 6 it can be observed that the closed loop system with controller 5 is almost unstable and the step responses associated with controllers 1 and 2 have no overshoot with relatively slow responses. Among the five step responses, the step response associated with controller 3 provides the best performance. This can also be observed by the integration of the absolute tracking error ( $z_{2}$ performance) shown in Figure 7, while the simulation results show a uniform improvement as the control gain increases.

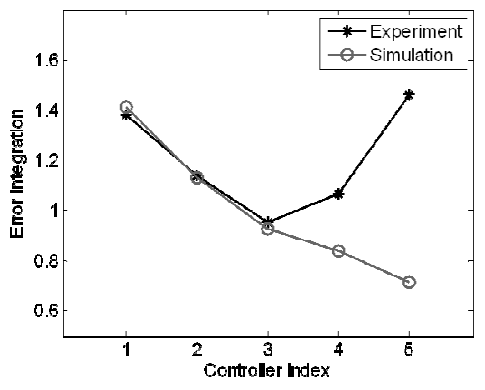

Figure 7. Performance comparison

Next, the robust performance of the throttle operation under battery voltage disturbance is studied. It is assumed that a stabilized throttle position is interrupted with a sudden vehicle battery voltage change. This could happen during the engine crank start-up process. The performance of LPV controller 3 is compared with that of the baseline well-tuned fixed gain PI controller. Both battery voltage dropping and recovery cases are studied. Figure 8 shows the system responses when the battery voltage drops from normal value $12 \mathrm{~V}$ to a low level of $7.5 \mathrm{~V}$ within $100 \mathrm{~ms}$ and remains at that level. It can be seen that the response of the LPV controller is much more robust than that of the PI controller. When battery voltage drops, throttle opening under LPV control has a tracking error less than 0.3 degree and it recovers within $600 \mathrm{~ms}$; and the tracking error of the PI control is about 0.8 degree and it recovers within 1000ms. Figure 9 shows the case 
when the battery voltage recovers to its normal level. The tracking error for the LPV controller remains unchanged at 0.3 degree and the corresponding PI one is 1.1 degrees. This is due to two main reasons. Firstly, the LPV control has a battery voltage depended $u_{0}$ (see Figure 4 ) to compensate the spring preload torque; while the PI control compensate the battery voltage change to the spring preload torque based upon the tracking error. Secondly, the LPV gain-scheduling controller adjusts its control gains as a function of the battery voltage change since battery voltage is one of the varying parameters. In this case, the control gains increase as the battery voltage drops. Combining both effects, the LPV controller provide much better performance than the PI controller.
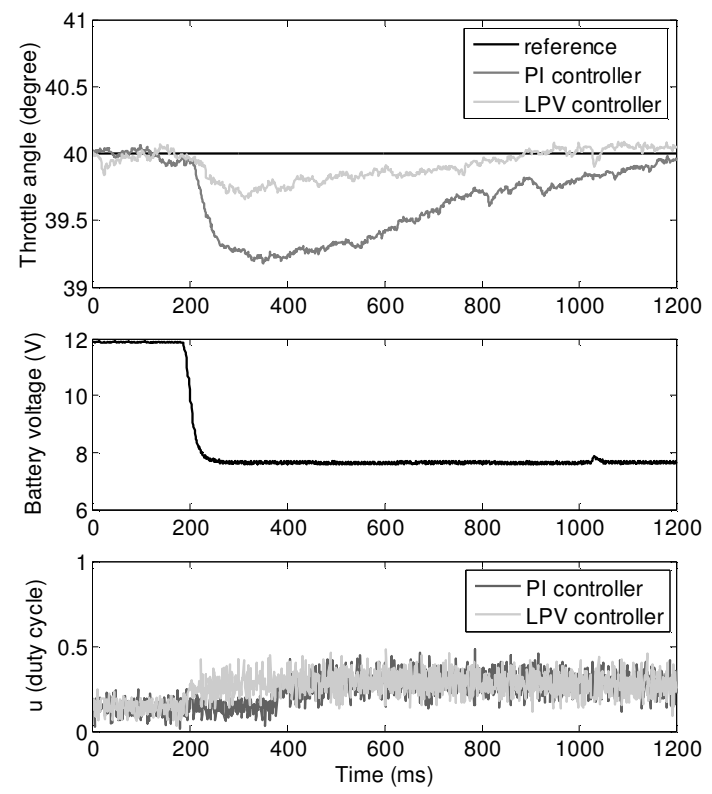

Figure 8. Battery voltage drop case
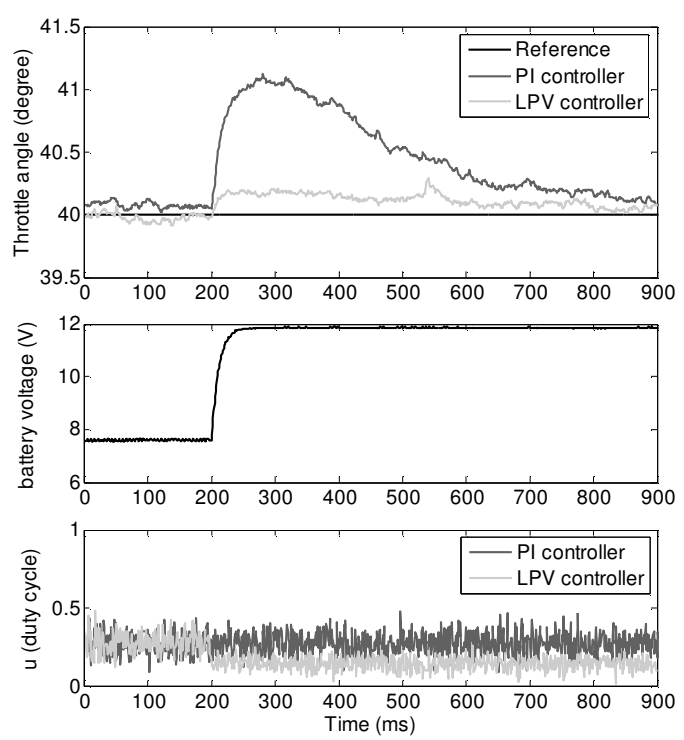

Figure 9. Battery voltage recovery case

\section{CONCLUSIONS}

In this paper a discrete-time gain-scheduling $\mathrm{H}_{2}$ controller is designed for an electronic throttle system based upon the LMI (linear matrix equality) convex optimization scheme. To enable model-based control gain tuning, a sequence of the LPV controllers were designed with control effort from low to high and evaluated through simulations and experiments. The controller associated with the best performance was chosen through experimental validation and used for performance comparison with the conventional fixed gain PI (proportional-integral) controller under battery voltage variation. The experimental results show significant tracking performance improvement of the LPV controller over the PI one. The future work is to reduce the modeling error and extend the $H_{2}$ LPV control design to mixed $H_{2}$ and $H_{\infty}$ LPV control design.

\section{REFERENCES}

[1] C. Rossi, A. Tilli, and A. Tonielli, "Robust Control of a Throttle Body for Drive by Wire Operation of Automotive Engines," IEEE Transactions on control systems technology, Vol. 8, No.6, November, 2000.

[2] S. Satou, S. Nakagawa, etc, "An Accurate Torque-based Engine Control by Learning Correlation between Torque and Throttle Position," SAE Technical Paper Series, 2008-01-1015, 2008.

[3] H. Lee and S. Sul, "Fuzzy-Logic-Based Torque Control Strategy for Parallel-Type Hybrid Electric Vehicle," IEEE Transactions on Industrial Electronics, Vol. 45, No. 4, August, 1998.

[4] V. H. Johnson, K. B. Wipke, and D. J. Rausen, "HEV Control Strategy for Real-Time Optimization of Fuel Economy and Emissions," SAE Technical Paper Series, 2000-01-1543, 2000.

[5] C. C. de Witt, I. Kolmanovsky, and J. Sun, "Adaptive Pulse Control of Electronic Throttle," 2001 American Control Conference, Arlington, VA, June, 2001.

[6] Z. Quyang and W. Junli, "Nonlinear PID Control of Electronic Throttle Valve," 2011 IEEE Conference on Electronic and Control Engineering, September, 2011.

[7] C. Lifeng and C. Ran, "A Fuzzy Immune PID Controller for Electronic Throttle," 2009 Second International Symposium on Computational Intelligence and Design, 2009.

[8] K. Nakano, U. Sawut, etc. "Modeling and Observer-based Sliding Mode Control of Electronic Throttle Systems," ECTI transactions on electrical eng., Vol.4, No.1 February, 2006.

[9] U. Ozguner, H. Sulgi, and Y. Pan, "Discrete-time Sliding Mode Control of Electronic Throttle Valve," 2001 IEEE Conference on Decision and Control, Orlando, FL, December, 2011.

[10] M. Horn, A. Hofer, and M. Reichhartinger, "Control of an electronic throttle valve based on concepts of sliding-mode control," 2008 IEEE International Conference on Control Applications, September, 2008.

[11] M. Vasak, etc. "Constrained Optimal Control of an Electronic Throttle," International Journal of Control, Vol. 79, No. 5, May, 2006, pp. 465-478.

[12] S. Zhang, A. P. White, J. Yang, and G. Zhu, "LPV Control of an Electronic Throttle," 2013 ASME Dynamic Systems and Control Conference, San Francisco, CA, October, 2013.

[13] J. Warrem, S. Schaefer, etc. "Barycentric Coordinates for Convex Sets," Advances in Computational Mathematics, Vol. 27, No. 3, 2007, pp. 319-338.

[14] J. D. Caigny, J. F. Camino, R. Oliveira, P. Peres, and J. Swevers. "Gain-scheduled $\mathrm{H}_{2}$ and $H_{\infty}$ Control of Dicrete-time Polytopic Time-varying Systems," IET Control Theory and Applications, 4: 362-380, 2010.

[15] G. Zhu, R. E. Skelton. "Integrated Modeling and Control for the Large Spacecraft Control Laboratory Experiment Facility," Journal of Guidance, Control, and Dynamics, Vol. 17, No. 3, May-June 1994. 
9.2 Published JC Paper \#2

A Control-Oriented Charge Mixing and Two-Zone HCCI Combustion Model 


\title{
A Control-Oriented Charge Mixing and Two-Zone HCCI Combustion Model
}

\author{
Shupeng Zhang, Guoming Zhu, and Zongxuan Sun
}

\begin{abstract}
Control-oriented engine model is often used in HIL (hardware-in-the-loop) simulation environment for developing and validating the corresponding control strategies. For the HCCI (homogeneous charge compression ignition) combustion, mixture heterogeneity should be considered in the control-oriented model to provide accurate combustion phase prediction. This paper describes a two-zone HCCI combustion model, where the in-cylinder charge is divided into the well-mixed and unmixed zones as the result of charge mixing. Simplified fluid dynamics is used to predict the residual gas fraction before the combustion phase starts, which defines the mass of the unmixed zone, during real-time simulations. The unmixed zone size not only determines how well the in-cylinder charge is mixed, which affects the start of HCCI combustion, but also the resulting peak in-cylinder pressure and temperature during the combustion process. The developed model was validated in the HIL simulation environment. The HIL simulation results show that the proposed charge mixing and HCCI combustion model provides better agreement with the corresponding GT-Power simulation results than the previously developed one-zone model.
\end{abstract}

Index Terms-internal combustion engine, charge mixing, two-zone combustion model.

\section{INTRODUCTION}

$I^{N}$ $\mathrm{N}$ recent years the HCCI (homogeneous charge compression ignition) capable SI (spark ignition) engines have been widely investigated, as an alternative combustion technology, to obtain the benefit of high efficiency of compression ignition (CI) engines and the benefit of low emissions of SI engines, to meet the increasingly strict emissions legislation. The major advantage of HCCI engines is realized by eliminating the formation of flames, which results in a much lower combustion temperature. As the consequence of the low flame temperature,

Manuscript received Jan. 30, 2013. This work was supported in part by the U.S. Department of Energy under Grant DE-EE000211 and in part by US National Science Foundation under Grants CMMI-1030360/1030380.

Copyright (c) 2013 IEEE. Personal use of this material is permitted. However, permission to use this material for any other purposes must be obtained from the IEEE by sending a request to pubs-permissions@ieee.org.

S. Zhang is with the Department of Mechanical Engineering at Michigan State University, East Lansing, MI 48824, USA (email: zhangs30@msu.edu).

G. Zhu is with the Department of Mechanical Engineering at Michigan State University, East Lansing, MI 48824, USA (phone: 517-884-1552; fax: 517-432-3341; e-mail: zhug@egr.msu.edu ).

Z. Sun is with the department of Mechanical Engineering at University of Minnesota, Minneapolis, MN 55455, USA. (e-mail:zsun@umn.edu). the formation of NOx (nitrogen oxides) is greatly reduced. The lean burn nature of the HCCI engine also enables un-throttled operation to improve vehicle fuel economy [1], [2].

However, one challenge for the HCCI combustion control is to predict the start of combustion precisely. Moreover, HCCI combustion mode can only operate in a certain range of engine conditions and is limited at high engine load due to knock and low load due to misfire. Several approaches have been demonstrated to achieve auto-ignition combustion for an SI engine, such as intake charge heating, increasing the compression ratio, exhaust gas recirculation, and residual gas trapping that is achieved by negative valve overlap (NVO) [1], along with corresponding control strategies. Nowadays for the purpose of reducing research cost and shortening the development duration, one of the most efficient approaches is to develop and validate the control strategy using an HIL (hardware-in-the-loop) simulation environment, where the real engine is replaced by a control-oriented real-time engine model.

To accurately control the HCCI combustion process, a precise charge mixing and combustion model is a necessity. Widely used high fidelity engine models, such as GT-Power and WAVE, provide fairly accurate engine charge mixing and combustion models. However, they can only be used for off-line simulations and cannot be used for model-based control, where real-time HIL simulations are required. Multi-zone models based on chemical kinetics, that divide the cylinder into adiabatic core zones and thermal boundary layers, are capable of simulating more realistic HCCI combustion phenomena [3], [4]. Unfortunately, these models are not fast enough for real-time simulations. The mean-value single-zone method was used in [5] to model the averaged chemical kinetics and thermodynamic properties and a control-oriented modeling approach was used for multi-mode HCCI engine in [6].

In [7] a control-oriented one-zone HCCI combustion model was constructed based on the assumption that the in-cylinder fuel, air, and residual gas charges are uniformly premixed at the intake valve closing (IVC). However, during the engine intake process, some of the residual gas is not mixed with the fresh intake gas, which remains at certain position of the cylinder. This unmixed portion is so-called unmixed residual gas fraction. Ignoring the unmixed residual gas fraction will result in modeling errors for the peak in-cylinder pressure and temperature since the unmixed residual gas may have a quite 
different temperature compared with the well mixed zone. Also the volume and air-to-fuel ratio (AFR) of the mixed zone will be affected by the unmixed portion in the cylinder. During the gas exchange process described in [1], a first order transfer function was used to approximate the in-cylinder pressure, which led to large errors compared with the actual pressure. In [8] and [9] a two-zone HCCI model were established taking into consideration of unmixed zone during combustion phase. Reference [8] developed a two-zone model based on thermochemistry and chemical kinetics, which ensures better combustion results, but the charge mixing process is not modeled. As a result, the size of the unmixed zone cannot be determined in real-time. In [9] the unmixed zone was assumed to remain at the bottom of the cylinder with a columnar intake flow jet, and the mass transfer rate from unmixed zone to mixed zone is proportional to the kinetic energy of the intake flow, which denoted that the charge mixing only occurs during the intake phase; however, this simplified model ignored the turbulent phenomenon caused by both of the bifurcated intake flow shear and high speed moving piston, which would last for the entire intake phase and compression phase.

In this article, to make the real-time simulation possible, mass fraction burned Wiebe function, along with energy conservation principle, was used to model the combustion process to guarantee the accuracy of thermodynamics characteristics such as in-cylinder pressure and temperature. One dimensional flow dynamics equations are used to model the detailed gas exchange dynamic process. During the intake phase, the in-cylinder charge is divided into two zones, mixed and unmixed zones, and modeled based upon the turbulent flow analysis approach.

The main contribution of this article is to provide a control-oriented HCCI engine model, capable of real-time HIL simulations and model-based engine combustion control, with simulation accuracy comparable with that of the high fidelity off-line engine model. It is true that the accuracy of the developed control oriented model is not compatible with high fidelity 3-D model due to the simplification of the engine charge mixing and combustion models. However, the developed control oriented engine model is able to provide crank-based combustion information while the traditional mean-value engine model cannot.

The rest of this paper is organized as follows. In section II the engine description and modeling framework are discussed. In section III detailed modeling approach is described for each combustion phase and simulation results are compared with GT-Power simulation results and experimental data in section IV. Section V addresses the conclusions.

\section{ENGINE DESCRIPTION AND MODELING FRAMEWORK}

The engine used for the modeling work is a $2.0 \mathrm{~L}$ four-cylinder equipped with two-step valve lift and electrical cam phaser. The engine is capable of SI and HCCI combustion. Figure 1 shows the architecture of the entire engine model.
Compared with the one-zone model in [7], it has the similar overall framework. The engine model receives inputs from the engine controller, including spark signal, injection signal, throttle signal, etc. The outputs of the engine model include mean-value based crank shaft speed, exhaust pressure, crank based in-cylinder pressure, temperature, etc. Also, the engine crank, cam and gate signals are generated to synchronize with engine control unit for HIL simulations. The engine model consists of five subsystem models: combustion model, throttle and manifold model, EGR (exhaust gas recirculation) model, piston/crank dynamics and dyno model. Crank-based combustion related variables are updated every crank degree; and mean-value engine variables, including throttle, manifold model, crank and dynamometer models are updated with a fixed sample time at $1 \mathrm{~ms}$.

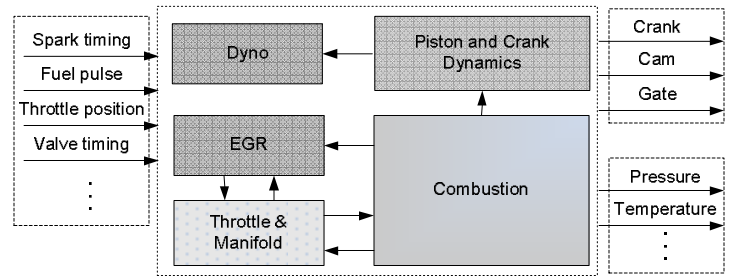

Fig. 1. Two-zone model architecture.

For the charge mixing process, the in-cylinder charge mixture is divided into two zones, mixed and unmixed, before the combustion starts. In order to predict the size of the unmixed zone, it is essential to model the flow dynamics during the entire intake process. A compressible flow dynamics method is used to predict the flow rate in real-time. When combined with turbulent diffusion analysis, the transfer rate between the two zones can finally be obtained. During the intake phase, due to the NVO recompression occurred before the intake phase, the in-cylinder pressure is usually higher than the intake manifold pressure at the start of the intake phase. Therefore, at the beginning of the intake process, the trapped in-cylinder gas flows from cylinder to manifold and then flow back to the cylinder. This intake process is considered in the model.
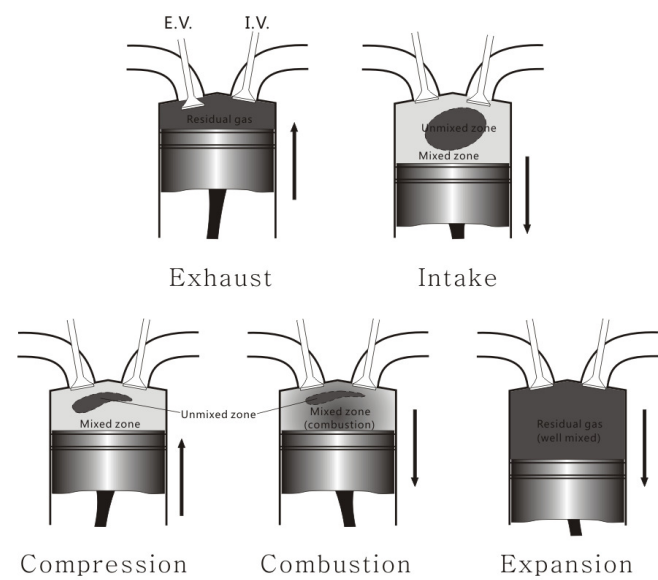

Fig. 2. Two-zone charge mixing and HCCI combustion model.

Figure 2 shows a diagram of the two-zone mixing process over an entire engine cycle. The unmixed zone is assumed to be 
located in the inner part of the cylinder, and its size is shrinking due to the gas diffusion to the mixed zone. Once auto-ignition occurs, it is assumed that the mass of unmixed zone does not change and a polytropic compression is assumed. When combustion ends, the two-zone assumption is not essential to in-cylinder analysis, hence it is assumed that the in-cylinder residual gas distributes homogeneously. Since the calculated temperature and volume of the unmixed zone can be updated each crank degree under this assumption, it is easy to analyze the combustion process in the mixed zone. In this article, it is also assumed that there is no heat exchange between the two zones, but the mixed zone exchanges heat with the cylinder wall.

\section{Two-ZOne Charge MiXing AND COMBustion Model}

The thermodynamic characteristics of the in-cylinder gas, such as in-cylinder pressure and temperature, are of great interest in the SI and HCCI combustion modeling research. This is especially important at certain critical combustion phases such as the IVC (intake valve closing).

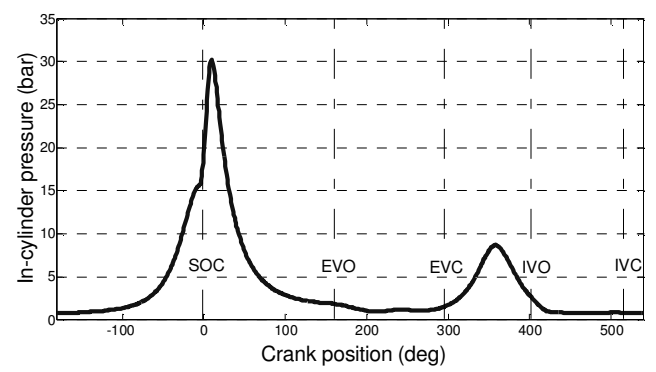

Fig. 3. Combustion phases within an engine cycle.

Figure 3 shows five key combustion phases of an HCCI combustion process within one engine cycle. They are: intake valve closing; start of HCCI combustion (SOC); exhaust valve opening (EVO); exhaust valve closing (EVC); and intake valve opening (IVO). For each combustion phase, the in-cylinder combustion variables, such as pressure and temperature are modeled using thermodynamic governing partial differential equations that are discretized every crank degree and solved analytically. For the HCCI combustion process, Wiebe based mass fraction burned (MFB) function, calibrated using experimental data, was used to approximate the complicated chemical reaction process to make the real-time simulation possible with required simulation accuracy; for the gas exchange process, the discretized governing equations (6) and (8) were solved iteratively, along with 1-D flow dynamics equations, with the guaranteed convergence for in-cylinder thermodynamics characteristics. In the rest of this section, crank resolved model of each combustion phase are presented.

\section{A. Intake Phase}

During this phase, the fresh charge enters the cylinder and mixes gradually with the residual gas. As a result, the total mass of the unmixed zone reduces. The goal is to predict the size of the unmixed zone at IVC; hence, it is essential to model the flow dynamics during the entire intake phase. Calculation of the intake flow rate is based on the one-dimensional compressible flow equations [10]:

a) When the flow is not choked $\left(P_{T} / P_{0}>[2 /(\gamma+1)]^{\gamma /(\gamma-1)}\right)$,

$$
\dot{m}_{v}=\frac{C_{D} A_{v} P_{0}}{\sqrt{R T_{0}}}\left(\frac{P_{T}}{P_{0}}\right)^{1 / \gamma}\left\{\frac{2 \gamma}{\gamma-1}\left[1-\left(\frac{P_{T}}{P_{0}}\right)^{(\gamma-1) / \gamma}\right]\right\}^{1 / 2}
$$

b) When the flow is choked $\left(P_{T} / P_{0} \leq[2 /(\gamma+1)]^{\gamma /(\gamma-1)}\right)$,

$$
\dot{m}_{v}=\frac{C_{D} A_{v} P_{0}}{\sqrt{R T_{0}}} \gamma^{1 / 2}\left(\frac{2}{\gamma+1}\right)^{(\gamma+1) / 2(\gamma-1)}
$$

where $C_{D}$ is the discharge coefficient and experimentally determined, $P_{0}$ and $T_{0}$ are the upstream stagnation pressure and temperature, $P_{T}$ is the downstream pressure, $A_{v}$ is the intake valve reference area, and $\gamma$ is the specific heat ratio.

For an HCCI combustion engine with the NVO strategy, most often, the in-cylinder pressure is higher than the intake manifold pressure at the IVO due to the residual gas recompression and early exhaust valve closing. Hence, a certain portion of the residual gas will escape into the manifold after IVO. This is called backflow. In order to simplify the modeling process, the entire intake phase is divided into three stages under certain assumptions:

Firstly, right after the IVO, the trapped residual gas flows out of the cylinder through the intake valve. In this case, $P_{0}$ and $T_{0}$ in (1) and (2) are in-cylinder pressure and temperature, respectively, $P_{T}$ is the manifold pressure, and $\dot{m}$ is treated as a negative value in the model for calculation convenience. During this stage, as the in-cylinder total mass is decreasing while the cylinder volume is increasing, the in-cylinder pressure and temperature both drop significantly.

Secondly, once the in-cylinder pressure becomes lower than the intake manifold pressure, the flow direction reverses. It is assumed that the escaped residual gas in the first stage was not mixed with the fresh charge in the manifold. Since the GT-Power and CFD (computational fluid dynamics) simulation results show that the back flow occurred during the first stage will be charged back into the cylinder completely. Therefore, this assumption will not lead to large modeling error at the end of the charge mixing process. For equations (1) and (2), in this case, $P_{0}$ is the manifold pressure, $P_{T}$ is the in-cylinder pressure, and $T_{0}$ is the residual gas temperature reduced by a factor governed by heat transfer and expansion.

Finally, after all the escaped residual gas flows back into the cylinder, the actual fresh charge process begins. During this stage, $P_{0}$ and $T_{0}$ are the intake manifold pressure and temperature, respectively, and $P_{T}$ is the in-cylinder pressure. This stage has the longest duration among the three stages. Mixing occurs in this stage.

The calculation of in-cylinder pressure and temperature is 
based upon the first law of thermodynamics. Since there is only one-direction flow path at one time due to the NVO operation, the energy conservation equation can be written as

$$
\frac{d(m u)}{d t}=\dot{Q}_{w}-\dot{W}+\dot{m} h
$$

where $\dot{W}$ is the rate of the transferred work, which equals $p \dot{V}$. $\dot{Q}_{w}$ is the total heat-transfer rate to the cylinder walls, which can be obtained using the Woschni correlation model [11], [12]:

$$
\dot{Q}_{w}=-A_{c} h_{c}\left(T-T_{w}\right) / N_{e}
$$

where $A_{c}$ is the contact area between gas and cylinder wall, $T_{w}$ is average temperature of cylinder wall, $N_{e}$ is the engine speed, and $h_{c}$ is the instantaneous convection coefficient that can be calculated by

$$
h_{c}=\alpha B^{-0.2} P^{0.8} T^{-0.55}\left[C_{1} \bar{S}_{p}+C_{2} \frac{V_{d} T_{r}}{p_{r} V_{r}}\left(P-P_{m o t}\right)\right]^{0.8}
$$

where $B$ is the bore; $P$ is the in-cylinder pressure; $\bar{S}_{p}$ is the mean piston speed; $V_{d}$ is the displaced volume; $T_{r}, p_{r}$, and $V_{r}$ are the in-cylinder temperature, pressure and volume at some reference state, such as intake valve closing; and $P_{m o t}$ is the motored in-cylinder pressure at the current crank position. $\alpha$, $C_{1}$ and $C_{2}$ are the scaling factors used as model calibration parameters. To simplify calculation, it is assumed that $h_{c}$ is constant within the calculation step but variable step-by-step.

In the developed model equation (3) is discretized and solved analytically. Note that $C_{p}$ and $C_{v}$ do change as a function of temperature and species. However, within one computational step (one crank degree) the variations of $C_{p}$ and $C_{v}$ are fairly small. To simplify calculation, it is assumed that $C_{p}$ and $C_{v}$ are constant within the calculation step but variable step-by-step. It is also assumed that the pressures in mixed and unmixed zones are identical. Then the in-cylinder temperature can be determined at every crank degree by the following equation

$T\left(\theta_{i}\right)=\frac{Q_{w}-P\left(\theta_{i-1}\right)\left[V\left(\theta_{i}\right)-V\left(\theta_{i-1}\right)\right]+\dot{m} \Delta t C_{p} T_{0}+m\left(\theta_{i-1}\right) C_{v} T\left(\theta_{i-1}\right)}{\left[m\left(\theta_{i-1}\right)+\dot{m} \Delta t\right] C_{v}}$

where $\theta$ is crank angle; $T_{0}$ is the intake flow temperature; $\Delta t$ is the time interval for each crank degree; and $Q_{w}$ is the heat transfer to the cylinder wall during the time interval, which can be obtained by

$$
Q_{w}=\dot{Q}_{w}\left(\theta_{i-1}\right) \Delta t
$$

based on the assumption that the heat transfer rate remains unchanged within one calculation step.

Since the mixture can be considered as an ideal gas, in-cylinder pressure can be obtained by

$$
P\left(\theta_{i}\right)=\frac{m\left(\theta_{i}\right) R T\left(\theta_{i}\right)}{V\left(\theta_{i}\right)}
$$

Equations (1) and (2) are discretized at each crank step and solved numerically, where the solution is obtained using iterative approach for a given step, along with (6) and (8).
In-cylinder pressure, temperature and intake flow rate are updated at each iteration. During the third phase of the process, the residual gas mixes with the fresh charge gradually in a fairly complicated dynamic process. The main task of the modeling work is to describe the mass transfer rate from the unmixed zone to the mixed zone using a simple approach, which is solvable in real-time for HIL simulations.

Due to the high intake flow velocity and piston motion, there is significant in-cylinder turbulent motion, combined with tumble and swirl. The flow field changes significantly as manifold shape, combustion chamber geometry and valve timing vary. CFD models were widely used to provide the relatively accurate estimation of in-cylinder gas motion [13], [14]. Figure 4 shows a side view of a simulated charge mixing process with NVO. The entire process can be considered as a turbulent diffusion process. Figure 4 (a) shows that at the beginning of the third stage, as discussed previously, the fresh charge comes in, and bifurcates into two jets; the right jet flows along the cylinder wall towards the piston, and the left jet flows along the cylinder head and past the exhaust valve. As the valve lift increases, additional incoming fresh charge leads to faster in-cylinder flow velocity and turbulent intensity, and forms two main vortices: the left-top vortex caused by the shear between the left jet and cylinder wall, and the right-bottom vortex caused by the interaction between the right jet and piston, as shown in Figure 4 (b). The mass transfer from residual gas to well-mixed gas, resulting from the species gradient between fresh charge and residual gas, is augmented by this in-cylinder turbulence. Once the intake valve is about to close and the piston is approaching to BDC (bottom dead center), the in-cylinder average flow velocity drops, but turbulence keeps the process going albeit at a decreasing rate, as shown in Figure 4 (c). When the piston moves up towards the TDC (top dead center), it pushes the residual gas to the upper location of the cylinder, and a newly formed vortex is conducive to charge mixing, as shown in Figure 4 (d).

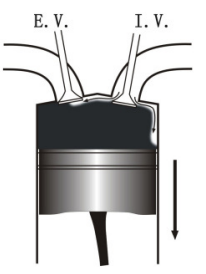

ล.

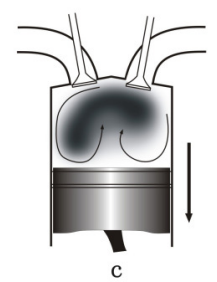

Fig. 4. Charge mixing process.
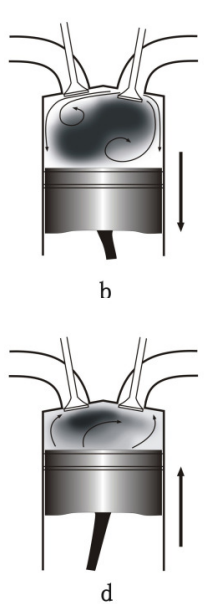

Based on the CFD simulation results [13], [14], although different valve timing strategies will lead to different flow fields and residual gas shapes, the shape of the unmixed zone 
formed by the residual gas is similar. In most of the cases, the residual gas is surrounded by the mixed charge due to continuous shear flow. Hence a simplified model is proposed in Figure 5.

Fig. 5. A simplified charge mixing model

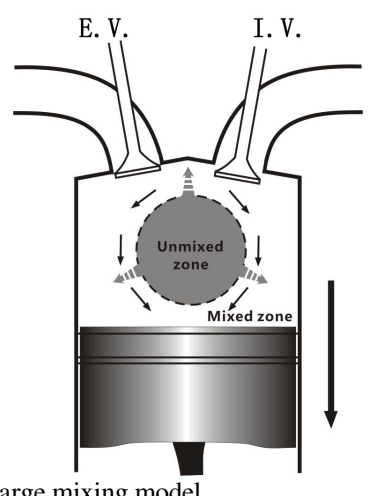

It is assumed that during the charge mixing process the unmixed zone (formed by residual gas) remains in the center of the cylinder, and the shape is assumed to be spherical. The fresh charge surrounds the unmixed zone with velocity tangent to the sphere and mixes with the residual gas gradually, which forms the mixed zone. The mass transport from the unmixed zone to mixed zone is caused by gas diffusion, which consists of both molecular diffusion and turbulent diffusion. Since the turbulent diffusion rate is much higher than that of molecular diffusion, the latter is ignored. Under this assumption Fick's first law of diffusion will be applied [15]:

$$
j_{t}=-\rho D_{t} \frac{d \omega}{d r}
$$

where $j_{t}$ is the turbulent mass flux of the residual gas; $\rho$ is the density of the residual gas; $\mathscr{D}_{t}$ is the turbulent diffusivity and the last term describes the mass fraction of the residual gas distribution in the mixed zone in the radius direction. This mass flux denotes the mass flow rate from unmixed zone to mixed zone per unit interaction area per unit time. To simplify the problem, it is assumed that at any time the residual gas in the mixed zone has a constant distribution gradient, hence the last term can be rewritten as $-\omega_{0} / l$, where $\omega_{0}$ is the concentration of residual gas at the interaction surface and $l$ is the thickness of mixed zone. The relationship between turbulent diffusivity and turbulent viscosity leads to the following equation that can be used to solve $\mathscr{D}_{t}$ :

$$
S c_{t}=\frac{v_{t}}{\mathscr{D}_{t}}
$$

Here, $S c_{t}$ is a dimensionless constant known as the turbulent Schmidt number. The turbulent viscosity can be obtained by:

$$
v_{t}=c_{1} \bar{v} l
$$

where $\bar{v}$ is the average velocity in the mixed zone, which is approximated by mean value of intake flow velocity and piston velocity; $l$ is the thickness of the mixed zone, the same as in residual gas distribution gradient calculation; and $c_{1}$ is a constant to be calibrated. Finally the mass transfer rate can be expressed as

$$
\dot{m}_{t r}\left(\theta_{i}\right)=\rho\left(\theta_{i}\right) c_{1} \bar{v}\left(\theta_{i}\right) \omega_{0} c_{2} A\left(\theta_{i}\right) / S c_{t}
$$

where $A$ is the surface area of the unmixed zone, which can be easily calculated under the "sphere" assumption; and $c_{2}$ is a calibration constant due to the assumptions made. Notice that at the beginning of the charge mixing process, the amount of the incoming fresh charge is not enough to surround the residual gas, and in this case $A$ should be the surface area of the mixed zone and calculated by assuming that the thickness of the mixed zone is identical to the intake valve lift.

Notice that during the entire intake phase temperature gradient exists between two zones, resulting in heat transfer from the unmixed zone to the mixed zone. Hence a polytropic process can be approximated in the unmixed zone, and the polytropic exponent $n$ was calibrated as a function of temperature to match the GT-Power simulation results over a wide engine operation range. Then the temperature in both zones can be obtained sequentially, assuming that the pressures of the mixed and unmixed zones are identical:

$$
\begin{gathered}
T\left(\theta_{i}\right)_{\text {unmixed }}=T\left(\theta_{I V O}\right) \cdot\left(\frac{P\left(\theta_{I V O}\right)}{P\left(\theta_{i}\right)}\right)^{n-1 / n} \\
T\left(\theta_{i}\right)_{\text {mixed }}=\frac{m\left(\theta_{i}\right) T\left(\theta_{i}\right)-m_{\text {unmixed }}\left(\theta_{i}\right) T\left(\theta_{i}\right)_{\text {unmixed }}}{m_{\text {mixed }}\left(\theta_{i}\right)}
\end{gathered}
$$

The volume of both zones can be determined by ideal gas law.

Since the developed model is capable of SI and HCCI combustion modes, the modeling methodology described above can be also used for the positive valve overlap (PVO) case. Similar analysis can be used during the intake valve opening and exhaust valve closing; however, during the valve overlap stage, complicated in-cylinder mixing process exists. Due to the strong in-cylinder motion (combination of turbulence and tumble) after intake valve opening, it is assumed that intake fresh charge will not get out through the exhaust valve, that is, there is no fresh charge flow into the exhaust port. This assumption is due to the fact that under most engine operational conditions there is no fresh charge escaped through the exhaust valve. Hence the intake charge mixing process and residual gas exhaust process can be calculated separately. Note that in some case the backflow phenomenon does exist, including flow from cylinder to intake manifold, and flow from exhaust manifold to cylinder. Similar criteria and method can be applied to this case as in the first two stages mentioned above.

\section{B. Compression phase}

Since at the end of compression phase the in-cylinder temperature might be very high, the heat transfer portion cannot be neglected for achieving an accurate prediction of the start of HCCI combustion. Here an iteration method is used to determine the in-cylinder pressure and temperature for each zone separately. First (14) is used to approximate the average in-cylinder temperature, based on an isentropic process with 
heat transfer assumption for the entire cylinder.

$$
\begin{aligned}
& T\left(\theta_{i}\right)_{\text {unmixed }}=\frac{m_{\text {unmixed }}\left(\theta_{i-1}\right) C_{v} T_{\text {unmixed }}\left(\theta_{i-1}\right)-P\left(\theta_{i-1}\right) \Delta \bar{V}\left(\theta_{i}\right)-F\left(\theta_{i}\right)}{\left[m_{\text {unmixed }}\left(\theta_{i-1}\right)-\dot{m}_{t r}\left(\theta_{i}\right) \Delta t\right] C_{v}} \\
& T\left(\theta_{i}\right)_{\text {mixed }}=\frac{Q_{\text {mixed }}-P\left(\theta_{i-1}\right) \Delta \hat{V}\left(\theta_{i}\right)+m_{\text {mixed }}\left(\theta_{i-1}\right) C_{v} T_{\text {mixed }}\left(\theta_{i-1}\right)+F\left(\theta_{i}\right)}{\left[m_{\text {mixed }}\left(\theta_{i-1}\right)+\dot{m}_{t r}\left(\theta_{i}\right) \Delta t\right] C_{v}}
\end{aligned}
$$

where

$$
\begin{aligned}
\Delta \bar{V}\left(\theta_{i}\right) & =\left[V_{\text {unmixed }}\left(\theta_{i}\right)-V_{\text {unmixed }}\left(\theta_{i-1}\right)\right], \Delta \hat{V}\left(\theta_{i}\right)=V_{\text {mixed }}\left(\theta_{i}\right)-V_{\text {mixed }}\left(\theta_{i-1}\right) \\
F\left(\theta_{i}\right) & =\dot{m}_{t r}\left(\theta_{i}\right) \Delta t C_{p} T\left(\theta_{i}\right)_{\text {unmixed }}
\end{aligned}
$$

Note that $Q_{\text {mixed }}$ is heat transfer to the cylinder walls from the mixed zone; and $\dot{m}_{t r}$ is the mass transfer rate from unmixed zone to mixed zone that can be obtained by (12). Heat transfer between mixed zone and unmixed zone is neglected here. Then, the average in-cylinder temperature can be obtained by

$$
T\left(\theta_{i}\right)=\frac{m_{\text {mixed }} T\left(\theta_{i}\right)_{\text {mixed }}+m_{\text {unmixed }} T\left(\theta_{i}\right)_{\text {unmixed }}}{m_{I V C}}
$$

The compression phase ends when the HCCI combustion starts. A commonly used criterion for the start of combustion (SOC) timing is the Arrhenius integral [16], [17], that depends on the oxygen and fuel concentration, which is described as

$$
A R I=\int_{\theta_{I V C}}^{\theta_{i}} A p^{a}\left[\mathrm{O}_{2}\right]^{b}[F u e l]^{c} e^{-\frac{E_{a}}{R \cdot T_{\text {mixed }}(\theta)}} d \theta
$$

where $E_{a}$ is the activation energy for the auto ignition reaction and is chosen to be a constant; and $A$ is a scaling factor related to fuel composition. Since for NVO strategy EGR rate has a strong effect on ignition delay [18], $A$ is also a function of EGR, and a lookup table can be used to calibrate $A$.

The SOC crank position is defined as the crank angle for $1 \%$ fuel burned under HCCI combustion. During this phase, the Arrhenius integral continues its integration. As the in-cylinder temperature and pressure increase gradually due to compression, the Arrhenius integral increases as well. Once the $A R I$ reaches criteria of the SOC $(A R I \geq 1)$, it shows that the HCCI combustion phase starts. At this moment, the temperature of mixed zone will be recorded as $T\left(\theta_{\text {SOC }}\right)$ and volume of unmixed zone as $V_{\text {unmixed }}\left(\theta_{S O C}\right)$ to be used for calculation in the next phase.

\section{Combustion and expansion phase}

In the HCCI combustion phase, the following two assumptions are made:

1) There is no mass exchange between the mixed (burned) and the unmixed zones due to the weak in-cylinder gas motion during the combustion phase (near TDC).

2) There is no heat transfer between the two zones but each zone has heat transfer to the cylinder wall.

Under the two assumptions, thermodynamic activity in both zones can be solved separately. In order to simplify the coupled equations, in-cylinder pressure in the last crank degree is used to calculate the volume of the current unmixed zone.

$$
V_{\text {unmixed }}\left(\theta_{i}\right)=V_{\text {unmixed }}\left(\theta_{\text {SOC }}\right)\left[\frac{P\left(\theta_{\text {SOC }}\right)}{P\left(\theta_{i-1}\right)}\right]^{1 / \gamma}
$$

In the mixed zone, the fuel MFB is modeled based upon the Wiebe MFB function and associated heat release rate function. Experimental results show that the heat release rate curve for HCCI combustion varies significantly and is heavily dependent on the fuel types and engine operational conditions. Typically the combustion process can be divided into two stages, cool-flame reactions and main combustion [19], [20]. Under certain lean combustion cases, especially with very high EGR rate (combined with both NVO trapped residual mass and external EGR), or highly diluted mixture case (high $\mathrm{N}_{2}$ concentration or low fuel concentration), the main HCCI combustion stage can be further divided into two stages: fast reaction (heat release) rate stage and slow reaction rate stage [21], [22], as shown in Figure 6. In order to develop a generic combustion model that can be applied to all types of four-stroke SI and CI engines, a generalized formula for MFB curve, that covers most possible combustion processes, is modeled by a combination of three functions

$$
x(\theta)=\alpha x_{1}(\theta)+\beta x_{2}(\theta)+(1-\alpha-\beta) x_{3}(\theta)
$$

and each of these three functions is modeled by the Wiebe function [10] with ( $\alpha \geq 0, \beta \geq 0, \alpha+\beta \leq 1$ )

$$
x_{i}(\theta)=1-e^{-a_{i}\left(\frac{\theta-\theta_{0 i}}{\Delta \theta_{i}}\right)^{m_{i}+1}}, i=1,2,3
$$

where coefficients $a_{i}, m_{i}$, factors $\alpha, \beta$, and predicted burn duration $\Delta \theta_{i}$ are calibration parameters of engine speed and load, and coolant temperature; $\theta_{0 i}$ represents the start of combustion for the stage $i$; and $\theta_{01}=\theta_{S O C}$ is given by the Arrhenius criterion in (18). All these parameters were calibrated within certain engine operation condition range and lookup tables were used as functions of engine speed and air-to-fuel ratio. For the combustion with relatively low EGR rate and not extremely lean combustion the third combustion stage does not exist and $\alpha+\beta$ can be set to one; and for gasoline type fuels combustion where the cool-flame reaction is not evident $\alpha$ can be set to one and $\beta$ to zero
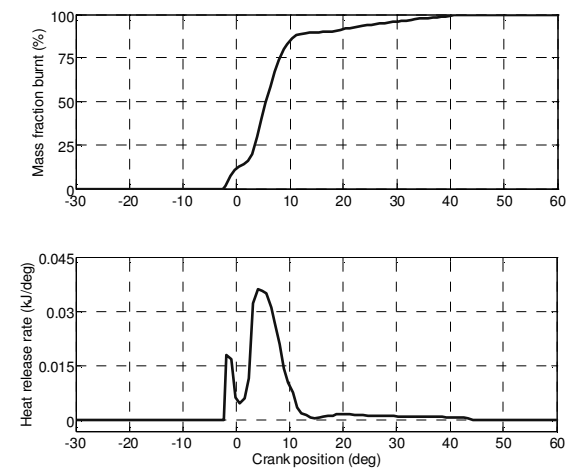

Fig.6. HCCI combustion mass fraction burnt and heat release rate.

The energy conservation equation applied to the mixed zone has the form

$$
m_{\text {mixed }} \frac{d u}{d \theta}+p \frac{d V}{d \theta}+Q_{w}=\eta_{H C C I} m_{f u e l} Q_{L H V} \frac{d x}{d \theta}
$$


where the combustion efficiency $\eta_{H C C I}$ is a calibration parameter used to match the simulated IMEP (indicated mean effective pressure) provided by GT-Power model, and $Q_{L H V}$ is the low heating value of the fuel. Considering the time interval for each crank degree is fairly small, the temperature calculation was simplified into two steps shown in (23) to make real-time simulation possible. They are a polytropic volume change process without heat exchange and a heat exchange process without volume change, where in the numerical simulations parameter $n$ is equal to $\gamma$. Then the temperature, pressure and volume of mixed zone can be solved by

$$
\begin{gathered}
T_{\text {mixed }}\left(\theta_{i}\right)=T_{\text {mixed }}\left(\theta_{i-1}\right) \cdot\left(\frac{V_{\text {mixed }}\left(\theta_{i-1}\right)}{V_{\text {mixed }}\left(\theta_{i}\right)}\right)^{n-1}+\frac{\eta_{H C C} m_{\text {fuel }} Q_{L H V}\left[x\left(\theta_{i}\right)-x\left(\theta_{i-1}\right)\right]-Q\left(\theta_{i}\right)}{m_{\text {mixed }} C_{v}} \\
P\left(\theta_{i}\right)=P\left(\theta_{i-1}\right) \cdot \frac{V_{\text {mixed }}\left(\theta_{i-1}\right)}{V_{\text {mixed }}\left(\theta_{i}\right)} \cdot \frac{T_{\text {mixed }}\left(\theta_{i}\right)}{T_{\text {mixed }}\left(\theta_{i-1}\right)}
\end{gathered}
$$

and

$$
V_{\text {mixed }}\left(\theta_{i}\right)=V\left(\theta_{i}\right)-V_{\text {unmixed }}\left(\theta_{i}\right)
$$

Temperature in the unmixed zone can be obtained by (15).

After the HCCI combustion phase, the two-zone analysis is no longer essential for in-cylinder combustion behavior, and the two zones are assumed to be well mixed instantaneously. The in-cylinder average temperature can be obtained by (13) with the initial condition:

$$
T\left(\theta_{e}\right)=\frac{m_{\text {mixed }} T\left(\theta_{e}\right)+m_{\text {unmixed }} T_{\text {unmixed }}\left(\theta_{e}\right)}{m_{I V C}}
$$

where the index $e$ denotes the crank position when combustion terminates.

\section{Exhaust phase}

The exhaust process is similar to the intake phase. Equations (1), (2), (6) and (7) are used for calculating the exhaust flow rate, in-cylinder temperature and pressure. Note that during this phase the in-cylinder pressure is higher than the exhaust manifold pressure in most of time, however, the situation can be reversed. Therefore, the backflow occurring is also considered. At the exhaust valve closed (EVC), the trapped mass can be calculated by

$$
m_{E V C}=\frac{P_{E V C} V_{E V C}}{R T_{E V C}}
$$

\section{E. NVO phase}

The NVO phase is called as engine recompression. During this phase the trapped in-cylinder gas is polytropically compressed or expanded in a closed system with heat transfer to the cylinder wall, so (28) and (8) are used to calculate both temperature and pressure with $m\left(\theta_{i}\right)$ replaced by $m_{E V C}$ in (8).

$$
T\left(\theta_{i}\right)=T\left(\theta_{i-1}\right) \cdot\left(\frac{V\left(\theta_{i-1}\right)}{V\left(\theta_{i}\right)}\right)^{n-1}
$$

\section{F. Throttle and manifold model}

Throttle and intake manifold models provide the manifold pressure required for intake flow rate calculation in real-time. The fresh air flow rate through the throttle plate can be calculated using the one-dimensional compressible flow equation similar to the one used to solve intake valve case:

$$
\dot{m}_{t}=\frac{C_{d} A_{t} P_{0} \sqrt{2 \gamma /(\gamma-1)}}{\sqrt{R T_{0}}} \Psi\left(p_{r}\right)
$$

where $C_{d}$ is the discharge coefficient; $P_{0}$ and $T_{0}$ are the atmosphere pressure and temperature; $A_{t}$ is the throttle reference area; and $p_{r}=p_{\text {man }} / p_{0}$ where $p_{\text {man }}$ denotes the manifold pressure. The function $\Psi$ is given by

$$
\Psi\left(p_{r}\right)= \begin{cases}\sqrt{p_{r}^{\frac{2}{\gamma}}-p_{r}^{\frac{\gamma+1}{\gamma}}} & , \text { if } p_{r} \geq\left(\frac{2}{\gamma+1}\right)^{\frac{\gamma}{\gamma-1}} \\ \sqrt{\left(\frac{\gamma-1}{2 \gamma}\right)\left[\frac{2}{\gamma+1}\right]^{\frac{\gamma+1}{\gamma-1}}}, & \text { if } p_{r}<\left(\frac{2}{\gamma+1}\right)^{\frac{\gamma}{\gamma-1}}\end{cases}
$$

Considering the throttle geometry, $A_{t}$ can be approximated by

$$
A_{t}=\frac{\pi}{4} D^{2}\left[1-\cos \left(\alpha-\alpha_{0}\right)\right]
$$

where $D$ is the diameter of the throttle plate, and $\alpha_{0}$ is the closed throttle angle.

Actual flow dynamics and thermodynamics in the manifold are quite complicated. In order to simplify the problem, a uniform condition assumption for the manifold is made, which assumes that there is no pressure gradient or temperature gradient in spatial distribution. By choosing the entire manifold as the control volume and applying the mass conservation, the mass change in the manifold is the difference between inlet mass flow (combined with air mass flow past the throttle and external EGR flow) and outlet mass flow (flow enter the cylinder through the valves). Then the equation for manifold mass change rate can be expressed as

$$
\dot{m}_{\text {man }}=\dot{m}_{t}+\dot{m}_{E G R}-\dot{m}_{v}
$$

Applying the ideal gas law in the manifold volume $V$, the pressure differential equation can be written as

$$
\dot{p}_{\text {man }}=\frac{\dot{m}_{\text {man }} R T_{\text {man }}}{V}
$$

Neglect the different gas thermodynamic properties between fresh air and EGR, the difference equation for manifold temperature is

$$
\begin{aligned}
& {\left[T_{\text {man }}\left(\theta_{i}\right) m_{\text {man }}\left(\theta_{i}\right)-T_{\text {man }}\left(\theta_{i-1}\right) m_{\text {man }}\left(\theta_{i-1}\right)\right] C_{v, \text { man }}=} \\
& \quad\left[T_{0} \dot{m}_{t}\left(\theta_{i}\right) C_{v 0}+T_{E G R} \dot{m}_{E G R}\left(\theta_{i}\right) C_{v, E G R}-T_{\text {man }}\left(\theta_{i-1}\right) \dot{m}_{v}\left(\theta_{i}\right) C_{v, \text { man }}\right] \Delta t
\end{aligned}
$$

where $\Delta t$ is the time interval for each crank degree. Then equations (32) and (33) can be discretized, along with (29) and (34), $p_{\text {man }}$ and $T_{\text {man }}$ can be solved online iteratively, which is similar to solving equations $(1)-(8)$.

\section{Simulation Results}

The two-zone HCCI combustion model was validated in the HIL simulation environment (see Figure 7 for the HIL system 
architecture). On top of the figure a host computer is used for running the Opal-RT based engine controller; the lower host computer is used for running the dSPACE based real-time engine simulator which was introduced in this paper. The two host computers can communicate with each other to get all the data updated each crank angel to achieve the real-time simulation. An oscilloscope can be applied to display the simulation results.

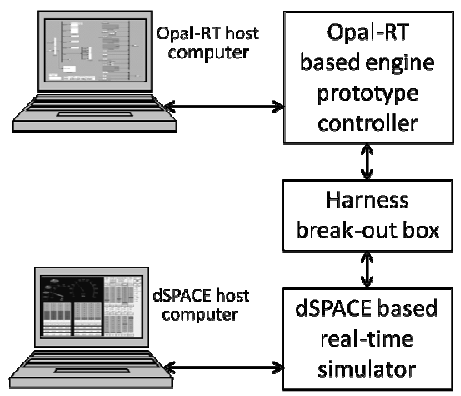

Fig.7. HIL simulation environment.

TABLE I

ENGINE SPECIFICATIONS

\begin{tabular}{ll}
\hline \hline \multicolumn{1}{c}{ Parameter } & \multicolumn{1}{c}{ Model value } \\
\hline bore/stroke/con-rod length & $86 \mathrm{~mm} / 86 \mathrm{~mm} / 143.6 \mathrm{~mm}$ \\
compression ratio & $9.8: 1$ \\
intake valve opening duration & 148 crank degree \\
exhaust valve opening duration & 148 crank degree \\
Intake/exhaust valve lifts & $5 \mathrm{~mm}$ \\
\hline \hline
\end{tabular}

The engine parameters are given in Table 1, and Table 2 shows the model calibration parameters and how they are calibrated.

TABLE 2

CALIBRATION PARAMETERS

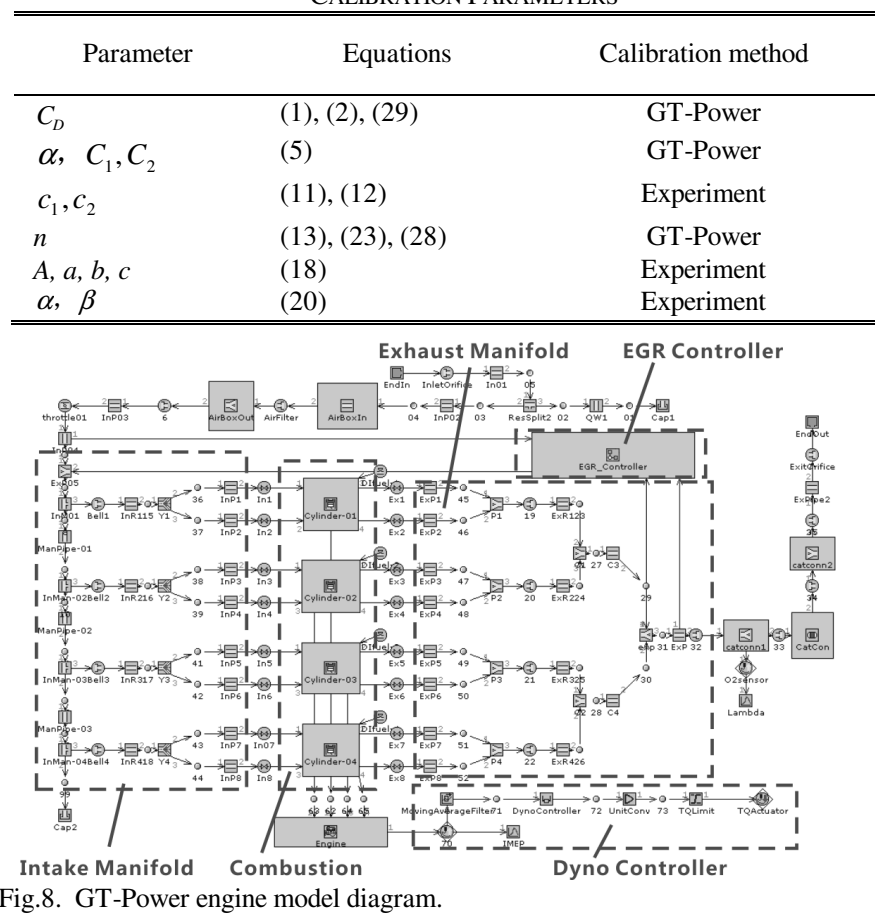

The two-zone model was validated for the engine operation at $2000 \mathrm{rpm}$ with various loads. The simulation results at 4.2 bar IMEP are presented below. The associated valve timing for EVO, EVC, IVO and IVC are $156^{\circ}, 304^{\circ}, 382^{\circ}$, and $530^{\circ}$ after TDC (top dead center). A four-cylinder GT-Power model is also developed and used to provide baseline simulation results, and Figure 8 shows the architecture of the model. For the purpose of validation, the proposed two-zone charge mixing and combustion model was compared with the one-zone model in [7], along with the improved one-zone model where the gas exchange flow dynamics was included but with the assumption of homogeneous mixing during the entire gas exchange process.

Figures 9 and 10 show the in-cylinder pressures and temperatures over an engine cycle. Note that the two-zone charge mixing model can provide an accurate simulation results that match with GT-Power simulation results quite well.

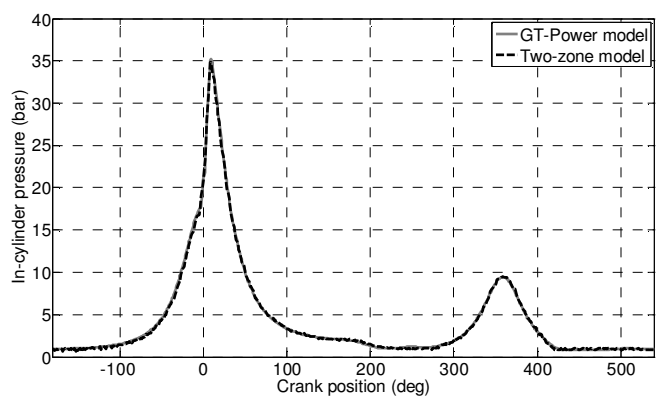

Fig.9. In-cylinder pressure comparison.

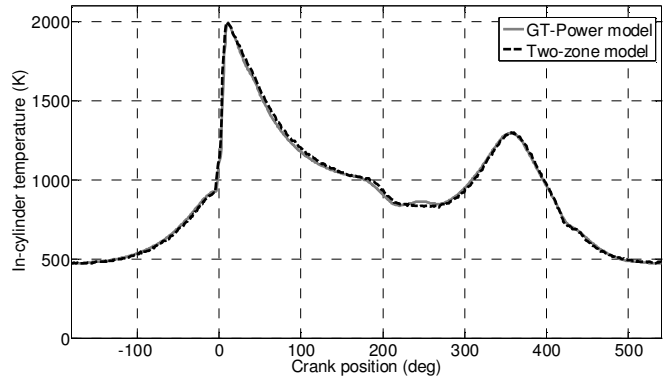

Fig.10. In-cylinder temperature comparison.

Figures 11 and 12 show the in-cylinder pressures and temperatures during the gas exchange process. Compared with one-zone model without fluid dynamics, two-zone charge mixing model shows a significant improvement, and the simulated pressure and temperature responses match with these provided by GT-Power simulations quite well. On the other hand, the in-cylinder pressure of one-zone model has a large error with respect to GT-Power simulation, especially at the beginning of valve opening, but at the end of the intake process it converges to the accurate value due to the effect of the first-order approximation; the in-cylinder temperature of one-zone model has significant error during the entire gas exchange process since it was obtained based upon the in-cylinder pressure, assuming an isentropic process at each time interval. Moreover, at IVC the temperature does not converge to a reasonable value, leading to inaccurate trapped mass calculation and SOC prediction. 


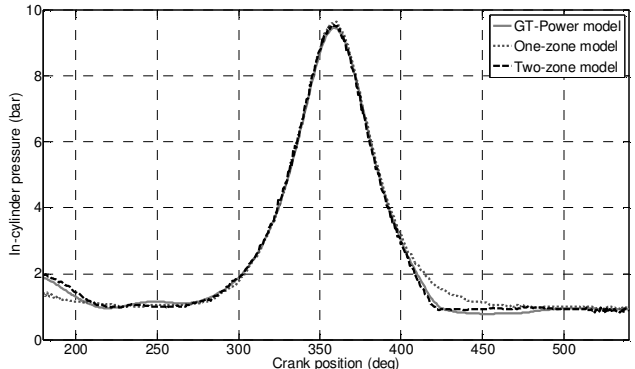

Fig.11. In-cylinder pressure during the gas exchange process.

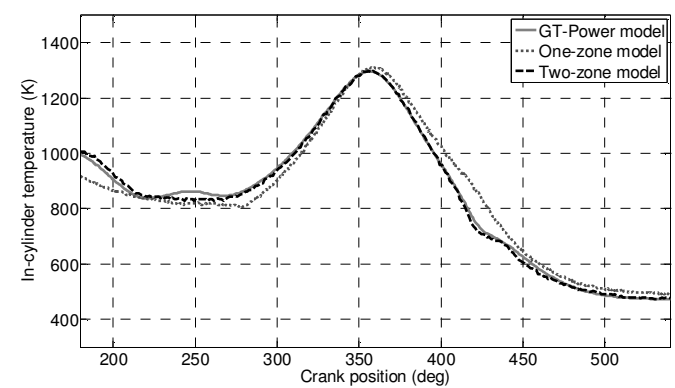

Fig.12. In-cylinder temperature during the gas exchange process.

Table 3 compares the simulation results between two-zone and one-zone models under the same engine operation condition, where GT-Power simulation results are used as the baseline. Since the temperature obtained in one-zone model is always higher than the unmixed zone temperature in two-zone model, the SOC prediction is much earlier than that of the two-zone model. The IMEP is also higher due to the error in intake phase and combustion phase.

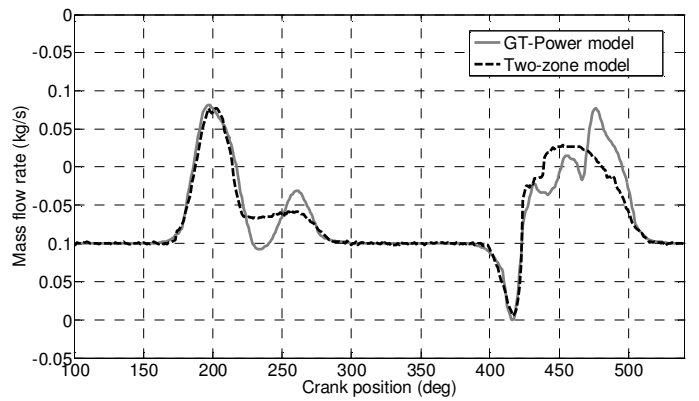

Fig.13. Mass flow rate comparison.

TABLE 3

SIMULATION RESULTS COMPARISON

\begin{tabular}{llll}
\hline \hline Model & Fuel (mg) & SOC & IMEP \\
\hline GT-Power & 13.2 & 2 & 4.2 \\
Two-zone model & 13.2 & 2 & 4.22 \\
One-zone model (w/ flow dynamics) & 13.2 & 4 & 4.23 \\
One-zone model (w/o flow dynamics) & 13.2 & 8 & 4.36 \\
\hline \hline
\end{tabular}

In Figure 13 both of the simulated exhaust and intake flow rates obtained from the two-zone model were compared with these of GT-Power simulations. Again they matched quite well; on the other hand, the simulation result of one-zone model is not shown here since a first order transfer function was used to approximate the in-cylinder pressure, the flow rate calculation is trivial. This indicates the benefit of the proposed charge mixing model. Note that the error between GT-Power and two-zone model responses are due to the un-modeled pressure wave in both of the intake and exhaust manifolds; and this dynamics is fairly difficult to model using a simplified modeling approach for real-time simulations. However, the proposed charge mixing model does provide good charge flow estimation without pressure wave.

Figure 14 shows the charge mixing process during the intake phase. At the beginning of intake valve open, the total in-cylinder trapped mass is reducing due to the backflow in the first stage; afterward, the backflow is sucked back to the cylinder and the trapped mass returns to the level before IVO. Then the third stage begins, fresh charge flows into the cylinder, which causes the mixed zone expanding and unmixed zone shrinking. At first the mass transfer rate is relatively slow since the unmixed zone is not been surrounded by the fresh charge thoroughly; then the transfer rate increases due to the increased intake flow and piston movement; and after IVC, the unmixed zone continues shrinking tardily, which indicates the effect of piston movement on mass diffusion is relatively weaker than that of the intake flow.

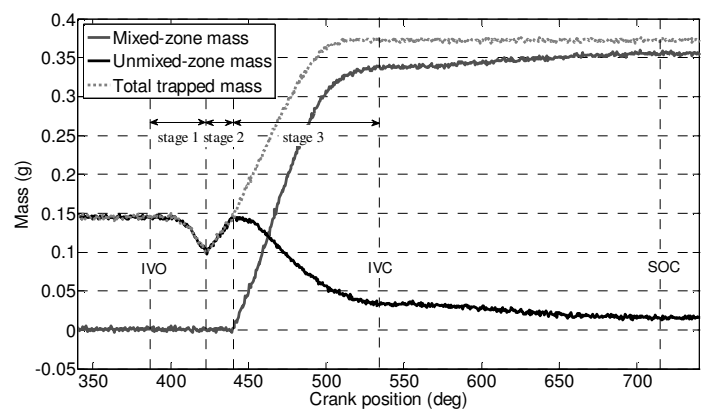

Fig.14. Size change of each zone.

Figure 15 shows the in-cylinder average temperature, along with temperature in both of mixed zone and unmixed zone. Obviously, before combustion starts, since the unmixed zone consists of residual gas, its temperature is higher than that of the mixed zone; hence the average in-cylinder temperature is a little higher than that of the mixed zone, since the mixed zone occupies most portion of the cylinder volume. After combustion, the temperature in the mixed zone rises significantly, but there is no combustion occurs in the unmixed zone, which results in higher temperature in the mixed zone than the average temperature.

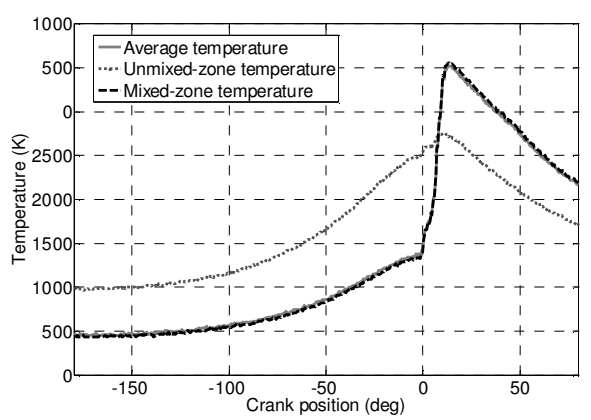

Fig.15. Temperature of each zone. 
Figure 16 compares in-cylinder pressure of the two-zone model with that of the improved one-zone model during compression and combustion phases. This improved one-zone model contains the flow dynamics during the gas exchange phase. Since the in-cylinder pressure is assumed to be uniformly distributed at any time, the averaged in-cylinder pressure of the two-zone model is much closed to these of the improved one-zone model. It can be seen that in the two-zone model the prediction of the SOC in the two-zone model is later than the one-zone model, and also the peak in-cylinder pressure is lower. This is due to the difference in estimated in-cylinder temperatures since the one-zone model uses the averaged temperature of two zones to estimate the SOC while the two-zone model uses the mixed zone temperature that is lower than the unmixed zone. It can be seen in Figure 17 that the Arrhenius integral increases slower for the two-zone model than that for the one-zone model.
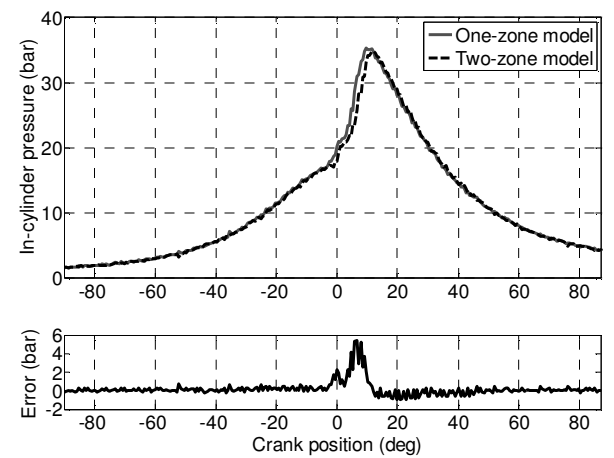

Fig.16. In-cylinder pressure of two-zone and one-zone models.

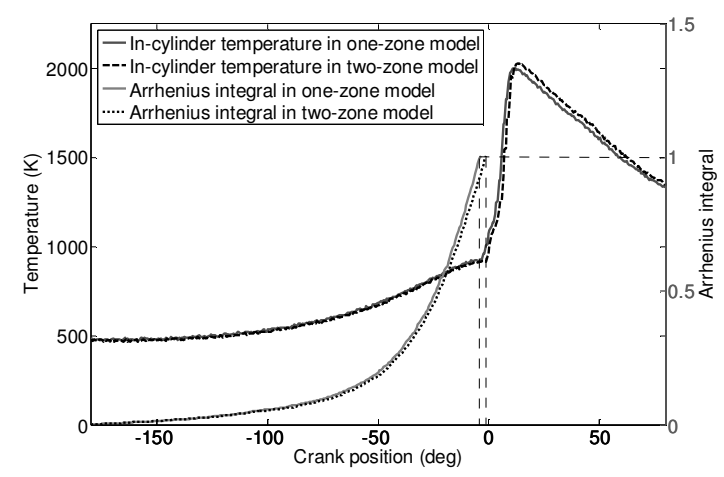

Fig.17. In-cylinder temperature of two-zone and one-zone models.

To validate the charge mixing two-zone model over an operating range, additional simulations were conducted with nine different load conditions at $2000 \mathrm{rpm}$, where the injected fuel mass varies between $13.2 \mathrm{mg}$ and $10.8 \mathrm{mg}$; exhaust valve timing EVO and EVC at $156^{\circ}$ and $304^{\circ}$, respectively; and intake valve timing IVO and IVC at $382^{\circ}$ and $530^{\circ}$, respectively. Figure 18 compares the IMEP and SOC of the two-zone model with those of GT-Power model, and shows that two-zone model has good agreement with GT-Power model.

The two-zone HCCI combustion model was then validated using experimental data. The engine was equipped with intake air heater and without external EGR, and the engine parameters are listed in Table 1 . The associated valve timing for EVO, EVC, IVO and IVC are $146^{\circ}, 294^{\circ}, 392^{\circ}$, and $540^{\circ}$ after TDC, respectively. Intake air temperature is $330 \mathrm{~K}$. Figure 19 shows the in-cylinder pressure and MFB of the two-zone model and experimental results during combustion phase. Note that the MFB was calibrated based upon the two-piece Wiebe function (a special case of the three-piece one) using experimental data. The parameters in equations (20) and (21) are: $\alpha=0.79, \beta=0.21, a_{1}=a_{2}=0.61, \theta_{02}=-6, m_{1}=2$, $m_{2}=1.5, \Delta \theta_{1}=8$, and $\Delta \theta_{2}=22$. It shows that the two-zone model matches experimental result fairly well during combustion process.
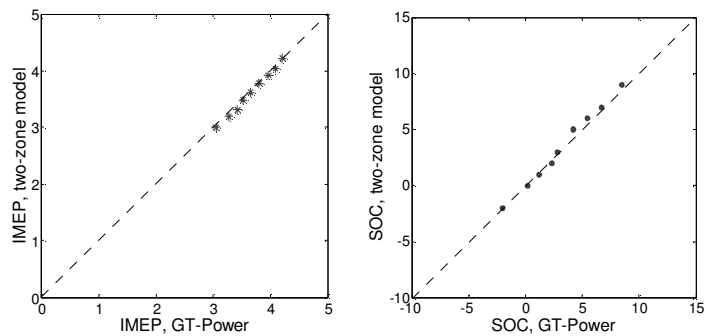

Fig.18. IMEP and SOC of two-zone model and GT-Power model.

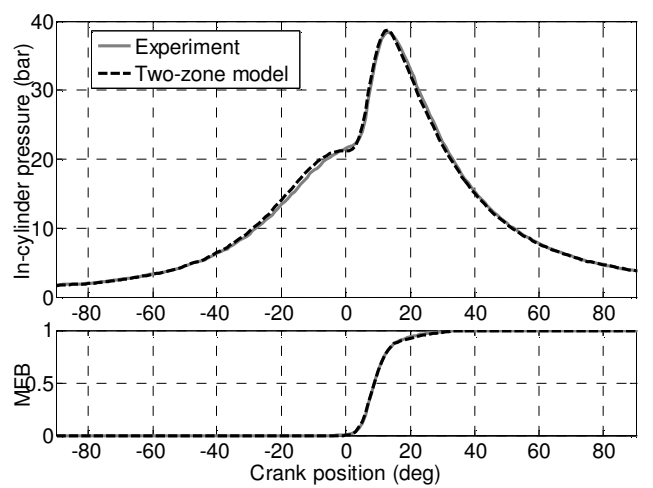

Fig.19. Experimental comparison of In-cylinder pressure and MFB.

In summary, the charge mixing two-zone model is capable of achieving much more accurate simulation results than that of the one-zone model due to utilizing the fluid dynamics analysis. The two-zone charge mixing and combustion model provides the simulation results that are comparable with these of GT-Power model and experimental results.

\section{CONCLUSION}

A two-zone charge mixing and HCCI (homogeneous charge compression ignition) combustion model is proposed in this paper based upon the simplified fluid dynamics. The developed model was implemented into the hardware-in-the-loop simulation environment for model validation. The simulation results of the proposed model match with the GT-Power simulation and experimental data well, and it is also demonstrated that the discretized fluid dynamics approach provides a satisfactory simulation results compared with GT-Power model. This indicates that it is feasible to develop a real-time control-oriented engine model that provides comparable simulation results to these provided by high fidelity 
model such as GT-Power. The simulation results also show that the unmixed zone plays an important role in predicting the start of combustion, in-cylinder pressure and temperature during the combustion process. It is believed that the two-zone charge mixing and HCCI combustion model provides an improved simulation platform for developing the real-time HCCI control strategy. The future work is to further validate and calibrate the developed model using experimental data.

\section{ACKNOWLEDGEMENT}

Authors would like to thank Professor Indrek Wichman of Michigan State University for helping reviewing this paper and provide valuable inputs.

\section{REFERENCES}

[1] H. Zhao, HCCI and CAI Engines for the Automotive Industry. Cambridge, UK: Woodhead Publishing, 2007, pp. 6-36.

[2] R. H. Stanglmaier and C. E. Roberts, "Homogeneous Charge Compression Ignition (HCCI): Benefits, Compromises, and Future Engine Applications," SAE Technical Paper, SAE 1999-01-3682, 1999.

[3] P. Kongsereeparp, et al, "A Stand-along Multi-zone Model for Combustion in HCCI Engines," ASME Internal Combustion Engine Division 2005 Fall Technical Conference, Ottawa, ON, Canada, September, 2005, pp. 265-274.

[4] N. P. Komninos, "Modeling HCCI combustion: Modification of a multi-zone model and comparison to experimental results at varying boost pressure," Applied Energy, 86 (2009), pp. 2141-2151.

[5] D. J. Rausen and A. G. Stefanopoulou, "A Mean-Value Model for Control of Homogeneous Charge Compression Ignition (HCCI) Engines," ASME Journal of Dynamics, Measurement, and Control, vol. 127, Sep 2005.

[6] G. M. Shaver, M. J. Roelle, and J. C. Gerdes, "Modeling Cycle-to-cycle Dynamics and Mode Transition in HCCI Engines with Variable Valve Actuation," Control Engineering Practice, 14 (2006), pp 213 - 222.

[7] X. Yang and G. Zhu, "A Control-oriented Hybrid Combustion Model of a Homogeneous Charge Compression Ignition Capable Spark Ignition Engine," Journal of Automobile Engineering, San Francisco, CA, June, 2011.

[8] V. Tandra and N. Srivastava, "A Two Zone Model of a Single Cylinder HCCI Engine for Control Applications," 2008 ASME Dynamics and Control Conference, Ann Arbor, Michigan, October, 2008.

[9] S. Zhang, G. Zhu, Y. Yoon, and Z. Sun, "A Control Oriented Charge Mixing and HCCI Combustion Model for Internal Combustion Engines ," 2012 ASME Dynamic Systems and Control Conference, 2012 vol. 226 pp.1380-1395.

[10] J. B. Heywood, Internal Combustion Engine Fundamentals. New York: McGraw-Hill, Inc., 1988.

[11] G. Woschni, "A Universally Applicable Equation for Instantaneous heat Transfer Coefficient in the Internal Combustion Engine," SAE Technical Paper, SAE 670931, 1967.

[12] J. Chang and O. Guralp, "New Heat Transfer Correlation for an HCCI Engine Derived from Measurements of Instantaneous Surface Heat," SAE Technical Paper, SAE 2004-01-2996, 2004.

[13] A. M. Mahrous, et al, "Effect of Intake Valves Timings on In-Cylinder Charge Characteristics in a DI Engine Cylinder with Negative Valve Overlapping," SAE Technical Paper, SAE 2008-01-1347, 2008.

[14] T. Lucchini, G. D'Errico, and M. Fiocco, "Multi-Dimensional Modeling of Gas Exchange and Fuel-Air Mixing Process in a Direct-Injection, Gas Fueled Engine," SAE Int., SAE 2011-24-0036, 2011.

[15] R. B. Bird, W. E. Stewart, E. N. Lightfoot, Transport Phenomena. John Wiley \& Sons, Inc., 2007, pp. 659.

[16] M. Hillion, J. Chauvin, and N. Petit, "Controlling the Start of Combustion on an HCCI Diesel Engine," 2008 American Control Conference, Seattle, Washington, June, 2008.

[17] J. Bengtsson, M. Gafvert, and P. Strandh, "Modeling of HCCI Engine Combustion for Control Analysis," 43rd IEEE Conference on Decision and Control, Atlantis, Paradise Island, Bahamas, December, 2004.
[18] S. Tanaka, F. Ayala, J. C. Keck, and B. Heywood, "Two-stage ignition in HCCI combustion and HCCI control by fuels and additives," Combustion and Flame, 132 (2003), pp. 219-239.

[19] A. Iqbal, et al, "Ignition Delay Correlation for Predicting Autoignition of a Toluene Reference Fuel Blend in Spark Ignition Engines," SAE Int., SAE 2011-01-0338.

[20] M. Canova, et al, "A Control-Oriented Model of Combustion Process in a HCCI Diesel Engine," 2005 American Control Conference, Portland, OR, June, 2005.

[21] D. Kawano, et al, "Effect of fuel properties on combustion and exhaust emissions of homogeneous charge compression ignition (HCCI) engine," SAE Technical Paper, SAE 2004-01-1966.

[22] K. Kuwahara, et al, "Chemical Kinetics Study on Two-stage Main Heat Release in Ignition Process of Highly Diluted Mixtures," SAE Int., SAE 2013-01-1657.

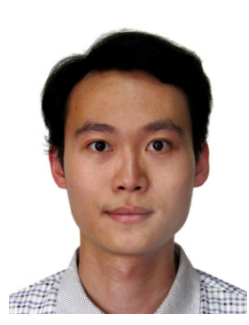

Shupeng Zhang received the B.S. and M.S. degrees in Automotive Engineering from Tsinghua University, Beijing, China, in 2006 and 2009, respectively. He is currently a Ph.D. candidate in the Department of Mechanical Engineering, Michigan State University, East Lansing, MI.

His research interests include engine modeling, combustion and mode transition control of HCCI engine, LPV (linear parameter varying) control with applications to the automotive powertrain and engine systems.

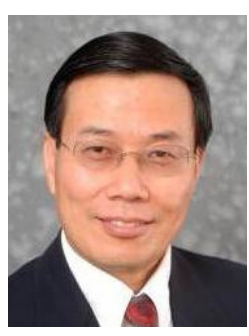

Guoming (George) Zhu received the B.S. and M.S. degrees from Beijing University of Aeronautics and Astronautics, Beijing, China, in 1982 and 1984, respectively, and the Ph.D. degree in aerospace engineering from Purdue University, West Lafayette, IN, in 1992.

He is currently a professor in the Department of Mechanical Engineering (ME), and Electrical and Computer Engineering (ECE) at Michigan State University, East Lansing, MI. Prior to joining the ME and ECE Departments, he was a Technical Fellow in advanced powertrain systems at Visteon Corporation. He also worked for Cummins Engine Co., Ltd as a technical advisor. His teaching interests focus on control classes at both undergraduate and graduate levels; and his current research interests include closed-loop combustion control of internal combustion (IC) engines, engine system modeling and identification, hybrid powertrain control and optimization, etc. He has more than 25 years of experience related to control theory, engine diagnostics, and combustion control. He has authored or coauthored more than 140 refereed technical papers and received 40 U.S. patents.

Dr. Zhu was an Associate Editor for the ASME Journal of Dynamic Systems, Measurement, and Control and is a member of editorial board for the International Journal of Powertrain. He is a fellow of SAE and ASME.

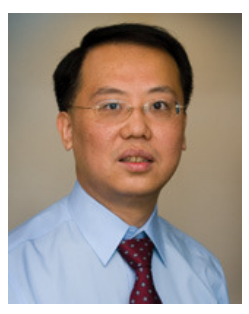

Zongxuan Sun (M'01) received the M.S. and Ph.D. degrees in Mechanical Engineering from the University of Illinois at Urbana-Champaign in 1998 and 2000 respectively, and the B.S. degree in Automatic Control from Southeast University, Nanjing, China, in 1995.

$\mathrm{He}$ is currently an Associate Professor of Mechanical Engineering at the University of Minnesota. He was a staff researcher (2006-2007) and a senior researcher (2000-2006) at General Motors Research and Development Center in Warren, MI. His research interests include controls and mechatronics with applications to the automotive propulsion systems. Dr. Sun has published over eighty referred technical papers and received nineteen US patents. He served on the conference editorial board of the ASME DSCD division.

Dr. Sun is a recipient of the George W. Taylor Career Development Award from College of Science and Engineering, University of Minnesota, NSF CAREER Award, SAE Ralph R. Teetor Educational Award, Best Paper Award from 2012 International Conference on Advanced Vehicle Technologies and Integration, Inventor Milestone Award, Spark Plug Award, and Charles L. McCuen Special Achievement Award from GM R\&D. 
9.3 Published JC Paper \#3

LPV Control of an Electronic Throttle 


\title{
LPV CONTROL OF AN ELECTRONIC THROTTLE
}

\author{
Shupeng Zhang, Andrew P. White, Jie J. Yang, and Guoming G. Zhu \\ Department of Mechanical Engineering \\ Michigan State University \\ East Lansing, MI 48824 \\ (zhug@egr.msu.edu)
}

\begin{abstract}
In this paper, a discrete-time electronic throttle model was developed based upon the parameters obtained through system identification. To design gain-scheduling controllers using $L P V$ (linear parameter varying) scheme, the throttle was modeled as an LPV system, where the vehicle battery voltage and the non-linear friction coefficient are the measurable time-varying parameters. Gain-scheduling $\mathrm{H}_{2}$ controller was designed for the LPV throttle system using the linear matrix inequality (LMI) convex optimization approach. The designed controller is validated through simulations and show that the proposed controller provides improved performance over the baseline fixed gain controller.
\end{abstract}

\section{INTRODUCTION}

Electronic throttle of internal combustion (IC) engines replaces the mechanical link between the vehicle acceleration pedal and engine intake throttle valve plate by accurately regulating the throttle plate angle using either a DC motor or step motor [1]. This process is called electronic throttle control (ETC). The traditional engine throttle with the mechanical link controls the engine charge air directly and engine fueling tracks the charge air to provide the desired air-to-fuel ratio, which is critical for engine emission regulation. The advantage of using the ETC for IC engines is that the engine charge air and fuel can be regulated simultaneously, providing accurate air-fuel-ratio control, especially during the transient engine operations. The ETC is also a key enabler for torque based engine control [2], where the acceleration pedal provides desired torque and the engine control system determines the desired engine charge air and fuel to provide the desired torque. The torque based control is especially important for hybrid powertrains ([3] and [4]), where the IC engine, electrical motor(s)/generator(s) are managed by their torque output or load.
A conventional electric throttle consists of a DC (or step) motor, a set of speed reduction gears, throttle plate, and springs that keep the valve plate at its default position. The electronic throttle system is highly nonlinear due to the rotational static and dynamic friction and the highly nonlinear limp-home (LH) spring set; the vehicle battery voltage fluctuation due to the vehicle electrical load variation introduces another degree of variations; in addition, the torque load introduced by the intake air flow [5] brings another uncertainty to the electronic throttle system. In this paper, these uncertainties are model as the measurable LPV parameters.

Proportional-integral-derivative (PID) control is widely used in powertrain control system due to its simplicity. However, the high spring nonlinearity and charge air flow exogenous disturbances could limit the PID control to achieve the desired performance under wide operation conditions, especially in these operational conditions that require low change air flow rate. In [6] a non-linear gain-scheduling PID controller with PID control parameters as a function of regulation error was developed to achieve fast response. In [7] a fuzzy immune adaptive PID controller was presented which is capable of providing improved robustness. The other common used approach for the electronic throttle control is sliding mode control, where the nonlinearities of friction and spring force can be considered as the parameter uncertainty and bounded external disturbance ([8]-[10]); however, the robust performance cannot be guaranteed. In reference [11], a discrete-time piecewise affine approach was used to accurately model the throttle nonlinearity, and the constrained optimal control was used to design a controller satisfies all imposed constrains. However, the uncertainties, especially the battery voltage change was not considered, which could lead to poor transient performance. 
In this paper, the nonlinear electronic throttle system was model as a linear parameter varying (LPV) system, where the friction torque and battery voltage are the varying parameters; and the disturbance torque, induced by the air flow and other sources are modeled as exogenous input. An inverse dynamic feedforward control is also used to compensate the preload spring torque. An LPV gain-scheduling controller is designed for the LPV model utilizing the LMI (linear matrix equality) convex optimization scheme to guarantee the system stability and performance.

The paper is organized as follows. The next section presents a discrete-time LPV electric throttle model that is calibrated using system identification in the LFT (linear fraction transformation) form; and the following two sections describe the process of the LPV control design based upon the LMI convex optimization and the simulation validation results. The last section adds some conclusions.

\section{ETC SYSTEM MODELING}

An electronic throttle system can be described by the block diagram shown below in Figure 1.

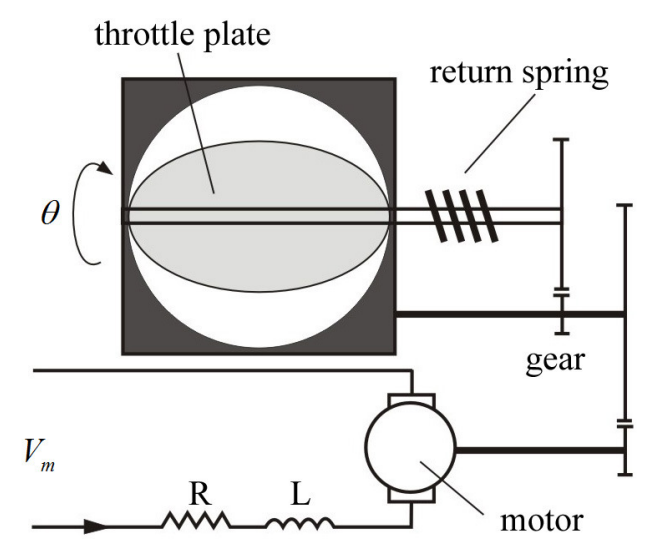

\section{FIGURE 1: AN ELECTRONIC THROTTLE SYSTEM}

A DC motor is driven by a controlled voltage $V_{m}$, which is powered by the battery. $V_{m}=V_{b} u$ is regulated by a PWM (pulse width modulated) duty cycle signal $u$, where the duty cycle is the output of the electronic throttle controller and $V_{b}$ is the battery voltage. An H-bridge drive module is used to make it possible to apply the voltage in both directions. $R$ and $L$ denote the resistance and inductance of the DC motor, respectively. The motor is connected to the throttle plate shaft by a set of gears, and a pair of returning springs that keeps the throttle plate resting at the default position (limp-home) when the control input is zero. Figure 2 shows the nonlinear characteristic of the return spring. The magnitude of spring torque at limp-home position $\theta_{0}$ is $T_{s}$, and the direction of the torque could switch around this position. Since the developed model in this paper is linear, the parameter denoting the throttle angle position discussed later is the position relative to $\theta_{0}$.

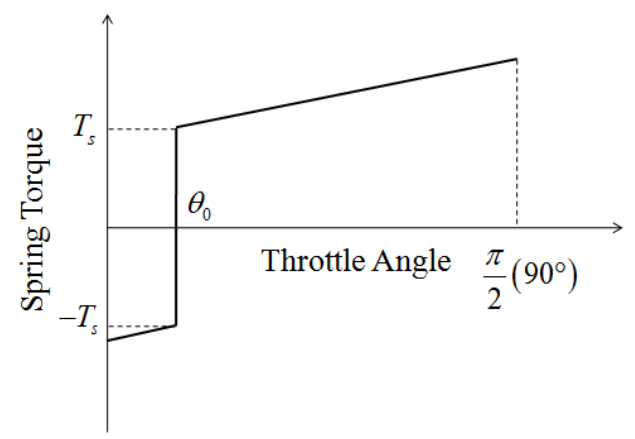

FIGURE 2: NONLINEAR PROPERTY OF RETURN SPRING

\section{Electronic Throttle Modeling}

The electronic throttle (a mechatronic system) dynamics can be expressed using the following differential equation

$$
\begin{gathered}
V_{m}=i R+L \frac{d i}{d t}+E_{a} \\
J \frac{d^{2} \theta}{d t^{2}}=T_{m}-\bar{T}_{s}-\bar{T}_{f}-T_{d}
\end{gathered}
$$

where $V_{m}, i, L$ and $R$ are the motor voltage, current, inductance and resistance, respectively; $E_{a}=K_{a} \dot{\theta}$ is the motor back EMF (electro-magnetic field) voltage; $J$ and $\theta$ are motor throttle assembly inertia and throttle angle; and $T_{m}, \bar{T}_{s}, \bar{T}_{f}$ and $T_{d}$ represent the motor output torque, nonlinear spring torque, friction torque and other exogenous input torque such as the disturbance torque due to the unbalanced force, caused by the charge air flow, applied the throttle plate.

Since the inductance $L$ is relatively small for a DC motor, the inductance dynamics can be ignored and the motor current can be approximated by setting $L=0$. That is

$$
i=\frac{1}{R}\left(V_{m}-K_{a} \dot{\theta}\right)
$$

and the motor output torque satisfies

$$
T_{m}=K_{m} i=\frac{K_{m}}{R}\left(V_{m}-K_{a} \dot{\theta}\right)
$$

Finally the throttle system dynamics can be described by the following nonlinear differential equation.

$$
J \ddot{\theta}=\frac{K_{m}}{R}\left(V_{b} \tilde{u}-K_{a} \dot{\theta}\right)-K_{s} \theta-T_{s} \operatorname{sgn}(\theta)-K_{B} \dot{\theta}-T_{f} \operatorname{sgn}(\dot{\theta})-T_{d}
$$

where $K_{m}, K_{a}, K_{B}$ and $K_{s}$ represent the motor torque, back EMF, viscous friction, and spring stiffness coefficients; $T_{s} \quad$ (where $\quad \bar{T}_{s}=K_{s} \theta+T_{s} \operatorname{sgn}(\theta) \quad$ ) and $T_{f} \quad$ (where 
$\left.\bar{T}_{f}=K_{B} \dot{\theta}+T_{f} \operatorname{sgn}(\dot{\theta})\right)$ represent the spring preload torque used to hold the throttle at limp-home position, Coulomb friction torque. The differential equation can be represented by the following continuous-time state space model

$$
\dot{x}=A x+B u+\Gamma, \quad y=C x
$$

where

$$
\begin{gathered}
A=\left[\begin{array}{cc}
0 & 1 \\
-\frac{K_{s}}{J} & -\frac{1}{J}\left(\frac{K_{m} K_{a}}{R}+K_{B}\right)
\end{array}\right], \quad B=\left[\begin{array}{c}
0 \\
\frac{K_{m} V_{b}}{J R}
\end{array}\right], x=\left[\begin{array}{c}
x_{1} \\
x_{2}
\end{array}\right]=\left[\begin{array}{c}
\theta \\
\dot{\theta}
\end{array}\right], \\
\Gamma=\left[\begin{array}{c}
0 \\
-\frac{1}{J}\left(T_{s} \operatorname{sgn}(\theta)+T_{f} \operatorname{sgn}(\dot{\theta})+T_{d}\right)
\end{array}\right], C=\left[\begin{array}{ll}
1 & 0
\end{array}\right]
\end{gathered}
$$

The system modeling parameters was calibrated based upon the experimental throttle step and sinusoid response data, along with the measured spring stiffness. The values of the parameters are shown in Table 1.

\section{TABLE 1: ELECTRONIC THROTTLE PARAMETERS}

\begin{tabular}{c|c|c|c}
\hline \hline Parameter & Value & Parameter & Value \\
\hline$R$ & 2 & $J$ & 0.008 \\
\hline$K_{m}$ & 1.08 & $K_{a}$ & 0.2 \\
\hline$K_{B}$ & 0.1 & $K_{s}$ & 0.18 \\
\hline$T_{f}$ & 0.09 & $T_{s}$ & 1.02 \\
\hline
\end{tabular}

\section{Discrete-time LPV System}

To design an LPV gain-scheduling controller, the nonlinear system (6) needs to be converted into an LPV one. The items in matrix $\Gamma$ were treated in different ways.

Firstly, the spring preload torque $T_{s} \operatorname{sgn}(\theta)$ can be compensated by a control signal $u_{0}(\theta)$ as function of $\theta$ where

$$
u_{0}=\frac{R T_{s}}{K_{m} V_{b}} \operatorname{sgn}(\theta)
$$

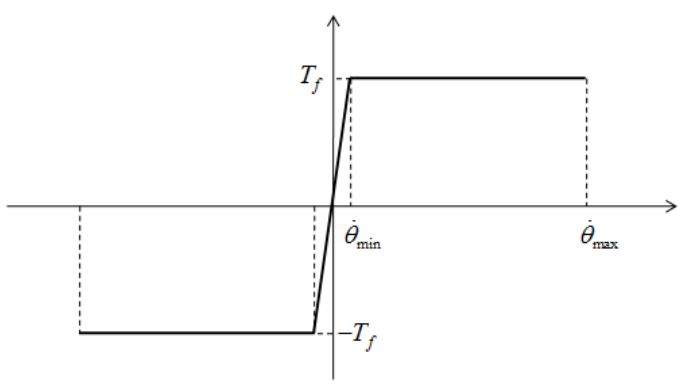

\section{FIGURE 3: APPROXIMARION OF SIGN FUNCTION}

Secondly, as shown in Figure 3, $T_{f} \operatorname{sgn}(\dot{\theta})$ can be expressed by

$$
T_{f} \operatorname{sgn}(\dot{\theta})=\left|T_{f} / \dot{\theta}\right| \dot{\theta}+\Delta T_{f}=F_{0} \gamma_{1} \dot{\theta}+\Delta T_{f}
$$

where $\left|T_{f} / \hat{\theta}\right|$ is treated as a term containing varying parameter $\gamma_{1}$, and $F_{0}$ is a constant chosen to normalize $\gamma_{1} \cdot \dot{\theta}_{\max }$ can be experimental determined, by setting extreme operation condition; $\dot{\theta}_{\min }$ is chosen to be small enough to reduce approximation error.

Finally, $T_{d}$, along with uncertainty $\Delta T_{f}$, forms the system exogenous input $w$, where

$$
w=\frac{1}{J}\left(\Delta T_{f}+T_{d}\right)
$$

Since the battery voltage varies during the operation, $V_{b}$ can be expressed as

$$
V_{b}=\bar{V}_{b}\left(1+\gamma_{2}\right)
$$

where $\gamma_{2}$ is the second varying parameter that is measurable. The following equation defines the range of $\gamma_{1}$ and $\gamma_{2}$.

$$
\gamma_{1}(t) \in[0.02,0.8], \quad \gamma_{2}(t) \in[-0.417,0.167]
$$

Then continuous-time state space model can be put into the format of an LPV system as follows

$$
\begin{aligned}
& \dot{x}=\left(A_{c}+A_{\gamma} \gamma_{1}\right) x+B_{c}\left(1+\gamma_{2}\right)\left(u+u_{0}\right)+B_{w} w \\
& y=C_{c} x
\end{aligned}
$$

where the system matrices in (5) can be expressed as a sum of nominal state space matrices $A_{c}, B_{c}, B_{w}, C_{c}$ and varying parameter depended matrices $A_{\gamma} \gamma_{1}$ and $B_{c} \gamma_{2}$.

The system is then discretized in to a discrete-time state space model using the first order approximation system matrices as follows:

$$
\begin{aligned}
& A_{P}=e^{A_{c} T_{s}}+e^{A_{c} T_{s}} A_{\gamma} \gamma_{1} t_{s} \\
& B_{P}=e^{A c T_{s}} B_{c}\left(1+\gamma_{2}\right) t_{s} \\
& C_{P}=C_{c}
\end{aligned}
$$

where $t_{s}$ is the sampling time of the discrete-time system. For convenience, $\gamma_{1}$ and $\gamma_{2}$ are supposed to lie in the compact structure $\Theta=\operatorname{diag}\left\{\gamma_{1}, \gamma_{2}\right\}$, and

$$
\Theta \in \Omega=\left\{\operatorname{diag}\left(\gamma_{1}, \gamma_{2}\right): 0.02 \leq \gamma_{1} \leq 0.8,-0.417 \leq \gamma_{2} \leq 0.167\right\}
$$

Then the discrete-time state-space LPV system is in the form of 


$$
\begin{aligned}
& x_{P}(k+1)=A_{P}(\Theta) x_{P}(k)+B_{P}(\Theta) u(k)+B_{w} w(k) \\
& y_{P}(k)=C_{P} x_{P}(k)
\end{aligned}
$$

Figure 4 shows the closed-loop discrete-time system architecture, where plant $P$ is the resulting discrete time state space model (15); a feed-forward controller $G$ is designed to provide fast transient response; and $K$ is the closed loop LPV controller to be designed. There are three inputs for the system plant and they are the feedback control $u$ from LPV controller $K$, the feed-forward control $u_{f f}$ from controller $G$, and $u_{0}$ used to compensate the spring preload torque. Since $u_{0}$ can be obtained directly by (7) with known battery voltage, and will only changes sign when crossing the limp-home position, it will be ignored during the control design process and will be added back during the simulations. Integrator $I$ is used to eliminate the steady-state error between the throttle angle and the reference command $r$. For the LPV control design, since the system is in a linear structure, the reference signal can be set to zero, and $r$ denotes the input noise (pedal sensor error). The weighting function $W_{r}$ is selected as a low pass filter

$$
W_{r}=\frac{1}{z-0.9}
$$

Note that the low pass filter is used to ensure that the closed loop system is able to provide good tracking performance. The weighting functions $W_{z}, W_{e}$ and $W_{u}$ are selected as constants and will be tuned during the control design process for the best performance.

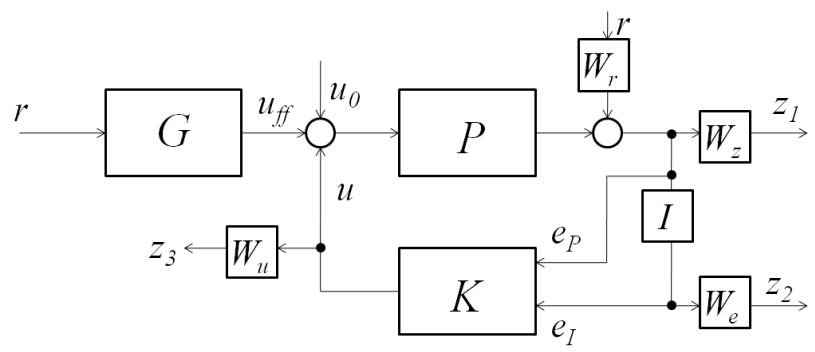

FIGURE 4: BLOCK DIAGRAM OF CLOSED-LOOP SYSTEM

\section{LPV CONTROLLER DESIGN}

\section{Feed-Forward Controller}

The objective of using a feed-forward controller is to provide fast transient response, especially in the case of sudden change in the input command. Ideally a feed-forward controller can be designed using the inverse plant dynamics, the output of feed-forward control shall provide accurate tracking to the command signal. However, in reality, the exact inverse system dynamics is impossible to obtain, and feedback control is used to achieve improved performance with zero steady-state error.
Since the electronic throttle system is nonlinear, through the system identification, a linearized system transfer function was obtained. The resulting second-order discrete-time transfer function from input $u$ (motor voltage) to output $y$ (throttle position) is

$$
\frac{y(z)}{u(z)}=\frac{0.0007776 z-0.00007893}{z^{2}-1.068 z+0.068}
$$

To make the feed-forward controller casual, the inverse transfer function is connected in series with a unite-gain firstorder low pass filter

$$
G(z)=\frac{u_{f f}(z)}{r(z)}=\frac{z^{2}-1.068 z+0.068}{0.0007776 z-0.00007893} \cdot \frac{0.05}{z-0.95}
$$

where the low pass filter is used to make the feedforward control casual and to eliminate the noise generated by the inverse dynamics. Then a state space realization for this feedforward controller can be obtained.

$$
\begin{aligned}
& x_{G}(k+1)=A_{G} x_{G}(k)+B_{G} r(k) \\
& u_{f f}(k)=C_{G} x_{G}(k)+D_{G} r(k)
\end{aligned}
$$

where $A_{G}, B_{G}, C_{G}$ and $D_{G}$ are the state space matrices of the feed-forward controller.

\section{Augmented LPV System}

For LPV gain-scheduling control design, a state space model of the entire system, shown in Figure 4, is augmented with the feed-forward control (18), the integrator and weighting functions. The augmented state space model is in the form of

$$
\left[\begin{array}{c}
x(k+1) \\
z(k) \\
e(k)
\end{array}\right]=\left[\begin{array}{ccc}
A(\Theta) & B_{1}(\Theta) & B_{2}(\Theta) \\
C_{1} & D_{1} & D_{2} \\
C_{2} & 0 & 0
\end{array}\right]\left[\begin{array}{c}
x(k) \\
\tilde{w}(k) \\
u(k)
\end{array}\right]
$$

where $\quad x(k)=\left[x_{G}(k) x_{P}(k) x_{r}(k) x_{I}(k)\right]^{\mathrm{T}} \in \mathbb{R}^{6}$. Note that $x_{r}(k)$ is the state with respect to the weighting function $W_{r}$, and $x_{I}(k)$ is the state with respect to the integrator $I$. The exogenous input $\tilde{w}(k)=[r(k) w(k)]^{\mathrm{T}} \in \mathbb{R}^{2}$, the performance output $z(k)=\left[z_{1}(k) z_{2}(k)\right]^{\mathrm{T}} \in \mathbb{R}^{2}$ and the measurement for control $e(k)=\left[e_{P}(k), e_{I}(k)\right]^{\mathrm{T}} \in \mathbb{R}^{2}$. The state space matrices are 


$$
\begin{aligned}
A(\Theta)= & {\left[\begin{array}{cccc}
A_{G} & 0 & 0 & 0 \\
B_{P}(\Theta) C_{G} & A_{P}(\Theta) & 0 & 0 \\
0 & -C_{P} T_{s} & 1 & 0.1 T_{s} \\
0 & 0 & 0 & 0.9
\end{array}\right], } \\
B_{1}(\Theta)= & {\left[\begin{array}{cc}
B_{G} & 0 \\
B_{P}(\Theta) D_{G} & B_{w} \\
0 & 0 \\
1 & 0
\end{array}\right], B_{2}(\Theta)=\left[\begin{array}{c}
0 \\
B_{P}(\Theta) \\
0 \\
0
\end{array}\right], } \\
C_{1}= & {\left[\begin{array}{cccc}
0 & -W_{z} C_{P} & 0 & W_{z} \\
0 & 0 & W_{e} & 0 \\
0 & 0 & 0 & 0
\end{array}\right], D_{1}=\left[\begin{array}{ll}
0 & 0 \\
0 & 0 \\
0 & 0
\end{array}\right], } \\
C_{2}= & {\left[\begin{array}{cccc}
0 & -C_{P} & 0 & 0.1 \\
0 & 0 & 1 & 0
\end{array}\right], D_{2}=\left[\begin{array}{lll}
0 & 0 & W_{u}
\end{array}\right]^{T} . }
\end{aligned}
$$

\section{LPV Control Synthesis}

For the augmented system in (19), the $H_{2}$ gainscheduling controller $K(\Theta)$ will be synthesized to minimize the upper bound of the closed loop $H_{2}$ norm from $\tilde{w}(k)$ to $z(k)$. To simplify the design process, during the design process, reference input to the feedforward control is ignored. That is, during the design the feedforward dynamics is not considered by setting $B_{G}=0$ and $D_{G}=0$. The goal is to design a static output feedback gain-scheduling control $u(k)=K(\Theta) e(k)$, a proportional and integral controller, that stabilizes the closed-loop system and minimizes the upper bound $\mathrm{H}_{2}$ norm of the closed-loop LPV system (18) over any trajectories of $\Theta(k) \in \Theta$.

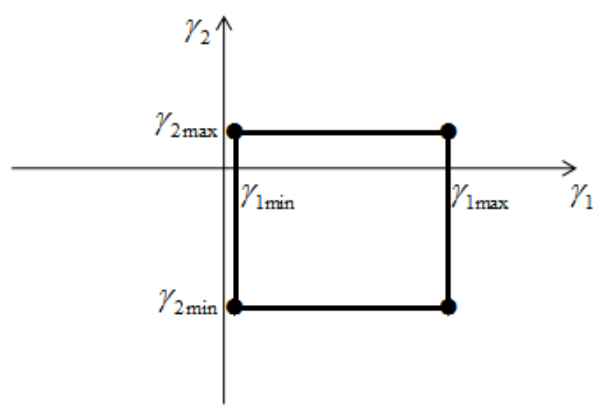

FIGURE 5: PARAMETER SPACE POLYTOPE

The state space model (18) is converted to a discrete-time polytopic time-varying system by solving state space matrices containing varying parameters $(A(\Theta)$ and $B(\Theta))$ at the vertices of the parameter space polytope, as shown in Figure 5. Any system inside the polytope is represented by a convex combination of the vertex systems as weighted by the vector $\lambda(k)$ of the barycentric coordinates, and formula for computing the barycentric coordinates is provided by [12]. The discrete-time polytopic time-varying system is given by

$$
\left[\begin{array}{c}
x(k+1) \\
z(k) \\
e(k)
\end{array}\right]=\left[\begin{array}{ccc}
\tilde{A}(\lambda(k)) & \tilde{B}_{1}(\lambda(k)) & \tilde{B}_{2}(\lambda(k)) \\
\tilde{C}_{1} & \tilde{D}_{1} & \tilde{D}_{2} \\
\tilde{C}_{2} & 0 & 0
\end{array}\right]\left[\begin{array}{c}
x(k) \\
\tilde{w}(k) \\
u(k)
\end{array}\right]
$$

where the system matrices $\tilde{A}(\lambda(k)) \in \mathbb{R}^{6 \times 6}, \quad \tilde{B}_{1}(\lambda(k)) \in \mathbb{R}^{6 \times 2}$ and $\tilde{B}_{2}(\lambda(k)) \in \mathbb{R}^{6 \times 1}$ belong to the polytope

$$
\begin{aligned}
\mathcal{D}=\left\{\left(\tilde{A}, \tilde{B}_{1}, \tilde{B}_{2}\right)(\lambda(k)):\right. & \\
& \left.\left(\tilde{A}, \tilde{B}_{1}, \tilde{B}_{2}\right)(\lambda(k))=\sum_{i=1}^{N} \lambda_{i}(k)\left(A, B_{1}, B_{2}\right)_{i}, \lambda(k) \in \Lambda\right\}
\end{aligned}
$$

where $A_{i}, B_{1 i}$ and $B_{2 i}$ are vertices of the polytope with $N=4$ in this case, and $\lambda(k)$ is the vector in the barycentric coordinates which belongs to the unit simplex

$$
\Lambda=\left\{\zeta \in \mathbb{R}^{N}: \sum_{i=1}^{N} \zeta_{i}=1, \zeta_{i} \geq 0, i=1, \cdots, N\right\}
$$

The rate of variation of $\lambda(k)$ can be defined as

$$
\Delta \lambda_{i}(k)=\lambda_{i+1}(k)-\lambda_{i}(k), i=1, \cdots, N
$$

which is bounded by

$$
-b \leq \Delta \lambda_{i}(k) \leq b, i=1, \cdots, N
$$

where $b \in[0,1]$.

Linear matrix inequality (LMI) approach will be used to solve the LPV gain-scheduling control problem. Based on Theorem 9 of reference [13], if there exists, for $i=1, \ldots, N$, matrices $G_{i, 1} \in \mathbb{R}^{2 \times 2}, \quad W_{i} \in \mathbb{R}^{2 \times 2}, \quad G_{j, 2} \in \mathbb{R}^{4 \times 2}, Z_{i, 1} \in \mathbb{R}^{1 \times 2}$ and symmetric positive-definite matrices $P_{i} \in \mathbb{R}^{6 \times 6}$ and $G_{j, 3} \in \mathbb{R}^{4 \times 4}$ such that the following LMIs hold

$$
\left[\begin{array}{ccc}
(1-b) P_{i}+b P_{l} & * & * \\
G_{i}^{\mathrm{T}} \tilde{A}_{i}^{\mathrm{T}}+Z_{i}^{\mathrm{T}} \tilde{B}_{2, i}^{\mathrm{T}} & G_{i}+G_{i}^{\mathrm{T}}-P_{i} & * \\
\tilde{B}_{1, i}^{T} & 0 & I
\end{array}\right]>0
$$

for $i=1, \ldots, N$, and $l=1, \ldots, N$,

$$
\left[\begin{array}{ccc}
(1-b) P_{i}+(1-b) P_{j}+2 b P_{l} & * & * \\
G_{j}^{\mathrm{T}} \tilde{A}_{i}^{\mathrm{T}}+G_{i}^{\mathrm{T}} \tilde{A}_{j}^{\mathrm{T}}+Z_{j}^{\mathrm{T}} \tilde{B}_{2, i}^{\mathrm{T}}+Z_{i}^{\mathrm{T}} \tilde{B}_{2, j}^{\mathrm{T}} & G_{i}+G_{i}^{\mathrm{T}}+G_{j}+G_{j}^{\mathrm{T}}-P_{i}-P_{j} & * \\
\tilde{B}_{1, i}^{\mathrm{T}}+\tilde{B}_{1, j}^{\mathrm{T}} & 0 & 2 I
\end{array}\right]>0
$$

for $l=1, \ldots, N, i=1, \ldots, N-1$, and $j=\mathrm{i}+1, \ldots, N$,

$$
\left[\begin{array}{cc}
W_{i} & * \\
G_{i}^{T} \tilde{C}_{1, i}^{T}+Z_{i}^{T} \tilde{D}_{2, i}^{T} & G_{i}+G_{i}^{T}-P_{i}
\end{array}\right]>0
$$


for $i=1, \ldots, N$,

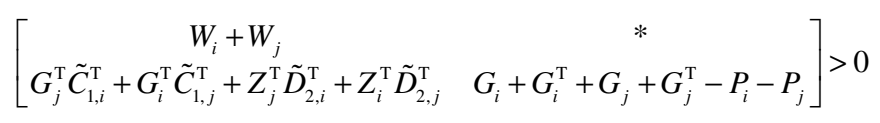

for $i=1, \ldots, N-1$, and $j=\mathrm{i}+1, \ldots, N$, then the robust static output feedback controller

$$
K(\lambda(k))=\hat{Z}(\lambda(k)) \hat{G}(\lambda(k))^{-1}
$$

where

$$
\begin{aligned}
& \hat{G}(\lambda(k))=\sum_{i=1}^{N} \lambda_{i}(k) G_{i, 1} \\
& \hat{Z}(\lambda(k))=\sum_{i=1}^{N} \lambda_{i}(k) Z_{i, 1}
\end{aligned}
$$

stabilizes the system with a guaranteed $H_{2}$ performance $v$ given by

$$
v^{2}=\min _{P_{i}, G_{i, 1}, G_{i, 2}, G_{i, 3}, Z_{i, 1}, W_{i}} \max _{i} \operatorname{Tr}\left(W_{i}\right)
$$

\section{SIMULATION VALIDATION}

The proposed gain-scheduling controller is validated in simulation and compared to a conventional PID controller with fixed gain. The first simulation was set up to represent different electronic throttle operating conditions without parameter variation. Figure 6 shows the response of the ETC system for the first simulation, where the input is a multi-step signal filtered by a low pass filter with a constant battery voltage and no external disturbance torque. The simulation results show that both of the LPV gain-scheduling and PI controls are able to provide a good tracking of the reference signal since there is no parameter variations.

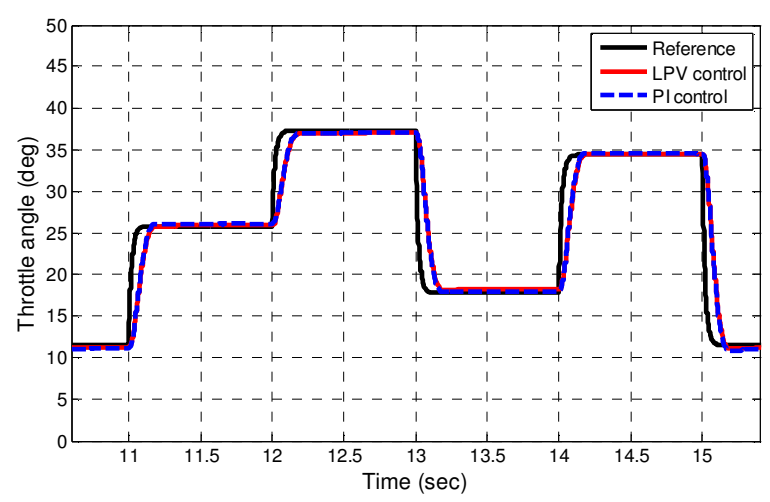

FIGURE 6: RECATNGULAR SIHGNAL RESPONSE

In the second simulation, it is assumed that a stabilized throttle position is interrupted with a vehicle battery voltage drop due to the additional vehicle electrical load, for instance, during engine cranking. Figure 7 shows throttle responses in the top graph, associated control duty cycle in the middle graph, and the vehicle battery variation in the bottom graph. From Figure 7, it can be observed that the LPV control provides much more stable response than that of fixed gain PID one. This is due to two reasons. Firstly, in LPV control design, a battery voltage depended $u_{0}$ is adjusted online to compensate the spring preload torque, in contrast with which a significant drop of throttle opening in PI control design reflects that the spring preload torque overcomes the control effort. Secondly, the LPV gain-scheduling controller adjusts its gain as battery voltage changes since battery voltage is one of the varying parameters. In this case, the control gains increases as the voltage drops. This can be seen from the middle graph of Figure 7 . When the battery voltage drops, the increased control gain leads an increased control duty cycle to compensate it.
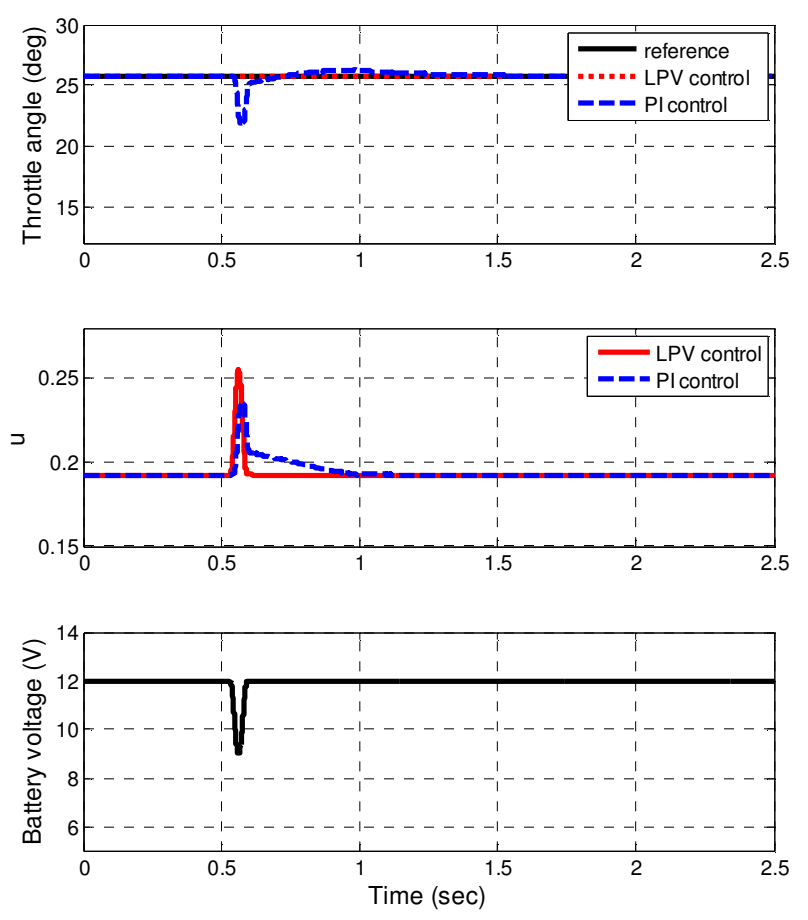

FIGURE 7: BATTERY VOLTAGE VARYING CASE 

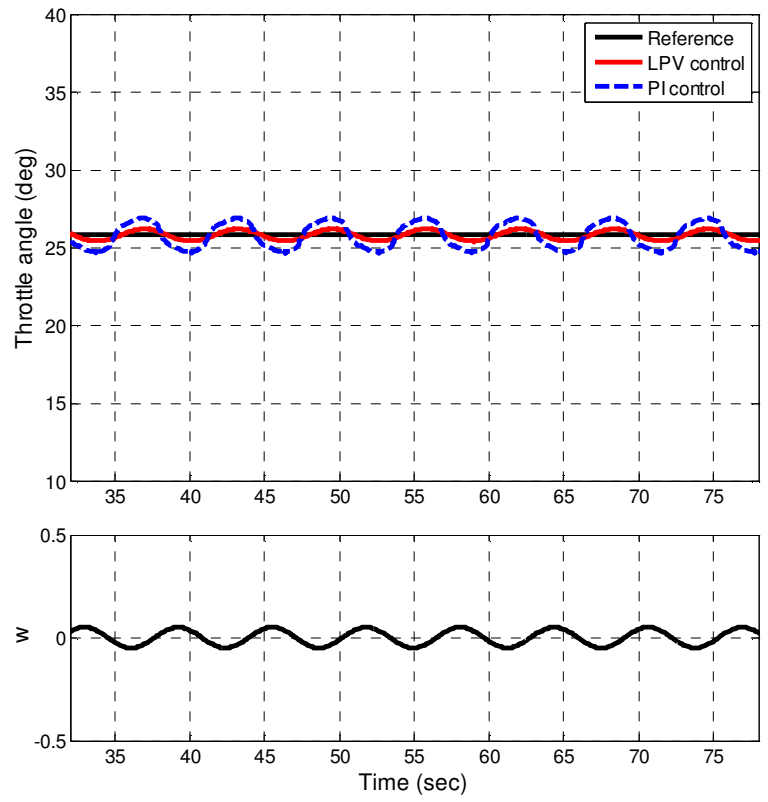

FIGURE 8: EFFECT OF EXTURNAL DISTURBANCE

In the third simulation, a sinusoidal disturbance is imposed on the plant model, which simulates the uncertain disturbance torque caused by the intake air flow wave dynamics. Figure 7 shows both the uncertain torque load and throttle positions, where the lower graph is sinusoid torque disturbance; and upper graph shows the throttle position responses of both conventional and LPV gain-scheduling PI controllers, along with the reference signal. The simulation results show that the LPV system have much smaller steadystate error, since it is designed to minimize the closed loop $\mathrm{H}_{2}$ norm from external disturbance to the throttle position output.

\section{CONCLUSION}

In this paper a discrete-time gain-scheduling controller is designed for an electronic throttle system based upon the LMI (linear matrix equality) convex optimization scheme. The designed LPV gain-scheduling controller was validated in simulations against the conventional PID (proportionalintegral-derivative) controller, and demonstrates the robustness to the parameter variation and external disturbance. The future work is to extend the $\mathrm{H}_{2}$ LPV control design to mixed $\mathrm{H}_{2}$ and $H_{\infty}$ LPV control design and validate the design LPV gain-scheduling control on an actual throttle body.

\section{REFERENCES}

[1] C. Rossi, A. Tilli, and A. Tonielli, "Robust Control of a Throttle Body for Drive by Wire Operation of Automotive
Engines," IEEE Transactions on control systems technology, Vol. 8, No.6, November, 2000.

[2] S. Satou, S. Nakagawa, etc, "An Accurate Torque-based Engine Control by Learning Correlation between Torque and Throttle Position," SAE Technical Paper Series, 2008-01-1015, 2008.

[3] H. Lee and S. Sul, "Fuzzy-Logic-Based Torque Control Strategy for Parallel-Type Hybrid Electric Vehicle," IEEE Transactions on Industrial Electronics, Vol. 45, No. 4, August, 1998.

[4] V. H. Johnson, K. B. Wipke, and D. J. Rausen, "HEV Control Strategy for Real-Time Optimization of Fuel Economy and Emissions," SAE Technical Paper Series, 2000-01-1543, 2000.

[5] C. C. de Witt, I. Kolmanovsky, and J. Sun, "Adaptive Pulse Control of Electronic Throttle," 2001 American Control Conference, Arlington, VA, June, 2001.

[6] Z. Quyang and W. Junli, "Nonlinear PID Control of Electronic Throttle Valve," 2011 IEEE Conference on Electronic and Control Engineering, September, 2011.

[7] C. Lifeng and C. Ran, "A Fuzzy Immune PID Controller for Electronic Throttle," 2009 Second International Symposium on Computational Intelligence and Design, 2009.

[8] K. Nakano, U. Sawut, etc. "Modeling and Observerbased Sliding Mode Control of Electronic Throttle Systems," ECTI transactions on electrical eng., Vol.4, No.1 February, 2006

[9] U. Ozguner, H. Sulgi, and Y. Pan, "Discrete-time Sliding Mode Control of Electronic Throttle Valve," 2001 IEEE Conference on Decision and Control, Orlando, FL, December, 2011.

[10] M. Horn, A. Hofer, and M. Reichhartinger, "Control of an electronic throttle valve based on concepts of slidingmode control," 2008 IEEE International Conference on Control Applications, September, 2008.

[11] M. Vasak, etc. "Constrained Optimal Control of an Electronic Throttle," International Journal of Control, Vol. 79, No. 5, May, 2006, pp. 465-478.

[12] J. Warrem, S. Schaefer, etc. "Barycentric Coordinates for Convex Sets," Advances in Computational Mathematics, Vol. 27, No. 3, 2007, pp. 319-338.

[13] J. D. Caigny, J. F. Camino, R. Oliveira, P. Peres, and J. Swevers. "Gain-scheduled $H_{2}$ and $H_{\infty}$ Control of Dicrete-time Polytopic Time-varying Systems," IET Control Theory and Applications, 4: 362-380, 2010. 


\subsection{Published JC Paper \#4}

Modeling and Control of an Electrical Variable Valve Timing System 


\title{
Modeling and Control of an Electric Variable Valve Timing System
}

\author{
Zhen $\operatorname{Ren}^{1}\left(1^{\text {st }}\right.$ author $)$ \\ Michigan State University \\ Mechanical Engineering, East Lansing, MI 48824 \\ e-mail: renzhen@msu.edu \\ ASME Membership (if applicable) \\ Guoming G. Zhu ${ }^{2}$ ( $^{\text {nd }}$ author - Corresponding Author) \\ Michigan State University \\ 1497 Engineering Research Court, Room E148, East Lansing, MI 48824 \\ e-mail: zhug@egr.msu.edu \\ ASME Membership (000005818646)
}

\begin{abstract}
This paper presents a model of an electric variable valve timing (EVVT) system and its closed-loop control design with experimental validation. The studied EVVT uses a planetary gear system to control the engine cam timing. The main motivation of utilizing the EVVT system is its fast response time and the accurate timing control capability. This is critical for the combustion mode transition control between the spark ignition and homogeneous charge compression ignition combustion, where the engine cam timing needs to follow a desired trajectory to accurately control the engine charge and recompression process. A physics-based model was developed to study the characteristics of the EVVT system, and a control oriented EVVT model, with the same structure as the physics-based one, was obtained using closed-loop system identification. The closed-loop control strategies were developed to control the EVVT to follow a desired trajectory. Both simulation and bench test results are included.
\end{abstract}

\section{INTRODUCTION}

Variable valve timing (VVT) systems, used in internal combustion engines, were developed in the nineties [1] and have since been widely used due to the growing fuel economy demands and emission regulations. VVT systems improve fuel economy and reduce emissions at low engine speed, and improve engine power and torque at high engine speed. Conventional electronic-hydraulic VVT ([1] and [2]), also called hydraulic VVT, is the most widely used in the automotive engines today. The hydraulic VVT systems require minor changes when applied to

\footnotetext{
${ }^{1}$ Zhen Ren was with Mechanical Engineering Department, Michigan State University, East Lansing, MI 48824 USA. He is now with Delphi Powertrain Systems, Auburn Hills, MI 48326 USA (e-mail: zhen.ren@ delphi.com).

${ }^{2}$ Guoming G. Zhu is with the Department of Mechanical Engineering and the Department of Electrical and Computer Engineering at Michigan State University, East Lansing, MI 48824 USA (e-mail: zhug@egr.msu.edu, phone: 517-884-1552).
} 
traditional non-VVT valve-trains [1], which makes design and manufacturing relatively easy. However due to its mechanism, the hydraulic VVT system also has its limitations [3].

The performance of hydraulic VVT systems is significantly affected by the engine operational conditions such as engine oil temperature and pressure. For instance, at low engine temperature, the hydraulic VVT system cannot be activated and has to remain at its default lock position so that the cold start performance and emissions cannot be improved [3]. This leads to the study of other variable valve-train systems, such as electromagnetic [4], hydraulic [5], electro-pneumatic [6], and electric motor-driven planetary gear systems ([7] and [8]). Comparing to hydraulic VVT, the electric motor-driven VVT (or electric VVT) is that its performance is less dependent of engine oil temperature and pressure [3]; and it has wider operational range, and hence, it improves engine performance over a wider operational range. Especially, since the electric VVT (EVVT) system is actuated by an electric motor, its performance, such as response time, is independent of the engine oil pressure and can be greatly improved by selecting a proper electric motor.

The major advantage of homogeneous charge compression ignition $(\mathrm{HCCl})$ combustion is realized by eliminating the formation of flames. That results in much lower combustion temperature. As a result, the formation of NOx (nitrogen oxides) is greatly reduced. The lean burn nature of the $\mathrm{HCCl}$ combustion also enables un-throttled operation to improve engine fuel economy. Unfortunately, $\mathrm{HCCl}$ combustion is feasible only over a limited engine operational range due to engine knock at high load and misfire at light load. Achieving $\mathrm{HCCl}$ in an automotive internal combustion engine requires it to operate in the spark ignition (SI) combustion mode at high load and in the $\mathrm{HCCl}$ combustion mode at low and medium load ([9] and [10]). This makes it necessary to have a smooth transition between $\mathrm{SI}$ and $\mathrm{HCCl}$ combustion modes.

Achieving $\mathrm{HCCl}$ combustion and controlling the mode transition between $\mathrm{SI}$ and $\mathrm{HCCl}$ combustion in a practical engine require a few enabling actuating and sensing technologies. They are used to control the thermodynamic and gas mixture conditions in the combustion chamber at the intake valve closing. The engine actuating subsystems under consideration includes variable valve actuation (cam-based or camless), dual fuel systems (port and direct fuel injection with multiple fuel injections), supercharger and/or turbocharger, exhaust energy recuperation and fast thermal conditioning of the intake charge mixture, spark-assist $\mathrm{HCCl}$ combustion, etc. Variable valve actuation can be used to control the effective compression ratio (via the intake valve closing time), the internal residual fraction via the negative valve overlap (also called recompression); see [11] and [12], or secondary opening of the exhaust valve (residual re-induction); see [11] and [12]. Although hydraulic VVT systems played critical roles in accomplishing smooth mode transitions from $\mathrm{SI}$ to $\mathrm{HCCl}$ and vice versa, see [13], [14], and [15], in addition to providing the basic control of the $\mathrm{HCCl}$ combustion (i.e., ignition timing and burn rate or duration), its slow response time limits the performance of mode transition and extends the transition duration. A typical EVVT system is more than five times fast than the traditional VVT one and is capable of accurately tracking the desired cam phase. The EVVT system studied in this paper was used to control the engine valve timings at either $\mathrm{SI}$ or $\mathrm{HCCl}$ combustion mode, and to track the desired cam phase trajectory during the combustion mode transition for optimized performance.

A feedback controller was introduced in [8] to control the electric planetary VVT system. The 
closed-loop EVVT system needs to meet both steady-state regulation and dynamic tracking performance requirements, due to the steady-state and transient requirements of the $\mathrm{HCCl}$ combustion. Therefore, a feedback control combined with the feedforward control was developed for the simulation study. The cam phase is the integration of speed difference between the EVVT motor and engine camshaft in an EVVT system. This leads to using the camshaft reference speed as the feedforward control for the EVVT motor. An output covariance constraint (OCC) controller ([16], [17], and [18]), an $\mathrm{H}_{2}$ controller with specially selected output weight [18], was used for feedback control to reduce both steady state and tracking errors. Performance of the OCC controller was compared to well-tuned proportional-derivative (PD) controllers, and the OCC controller with feedforward provides improved cam phase tracking control over the PD control. Different cam phase sample rates were also studied and results show that four samples per engine cycle are sufficient for the OCC feedback control.

The physical based model and simulation results provide a modeling and control framework for the EVVT bench. The EVVT system with a local speed controller was mounted on an engine head. An electric motor was used to simulate the rotating engine crankshaft. The EVVT pulley was connected to the motor through a timing belt. Because of the complexity of the actual system and unknown motor local speed control parameters, the developed physical-based model was difficult to calibrate. As a result, closed-loop system identification [19] was used to obtain the model calibrations. The $q$-Markov COVariance Equivalent Realization ( $q$-Markov Cover) system identification method ([20], [21], and [22]) using PRBS (pseudo-random binary signal [22]) was used to obtain the closed-loop system models. The EVVT open-loop system model was calculated based upon the identified closed-loop model. The $q$-Markov Cover theory was originally developed for model reduction. It guarantees that the reduced order system model preserves the first $q$-Markov parameters of the original system. The realization of all $q$ Markov Covers using input and output data of a discrete time system is capable of system identification. Q-Markov Cover for system identification uses pulse, white noise, or PRBS as input excitations. It can be used to obtain the linearized model with matching input/output sequence for nonlinear systems [22]. It was also been extended to identify multi-rate discretetime systems when input and output sampling rates are different [23]. An OCC controller [18] was designed based upon the identified model.

This paper is organized as following. Section II describes the EVVT model and system architecture. Section III introduces the feedforward control and the closed-loop OCC control design. Section IV provides the simulation results. EVVT test bench setup is discussed in Section V. System identification frameworks are discussed in Section VI; and the experimental results are shown in Section VII, along with the study of performance impact of the engine oil viscosity. Conclusions are drawn in Section VIII.

\section{EVVT MODELING}

\section{A. Planetary EVVT Components}

The planetary gear EVVT system studied in this paper consists of four major components (see Figure 1). Ring gear, serves as VVT pulley, is driven directly by a crankshaft through a timing belt at half crankshaft speed. Planet gear carrier is driven by an electric motor. Planet gears engage both ring and sun gears. Sun gear is connected to the camshaft. The sun and planet gears are 
passive components that obtain kinetic energy from carrier and ring gears. Comparing to other components, the inertia of engine fly wheel and crankshaft is fairly large. As a result, dynamics of the ring gear is ignored in the model. All other components have known mechanical properties and their dynamics are considered in the modeling.

\section{B. Planetary Gear System Kinematics}

In a planetary gear system [24] shown in Figure 1, angular velocities of components are determined by

$$
\frac{\omega_{s}(t)-\omega_{c}(t)}{\omega_{r}(t)-\omega_{c}(t)}=-\frac{n_{r}}{n_{s}}
$$

where $\omega_{s}, \omega_{c}$ and $\omega_{r}$ are angular velocities of the sun, carrier, and ring gears, respectively. $n_{r}$ and $n_{s}$ are the teeth numbers of ring and sun gears. Laplace transformation [25] of (1) can be expressed as

$$
\Omega_{s}(s)=-\frac{n_{r}}{n_{s}} \Omega_{r}(s)+\frac{n_{r}}{n_{s}} \Omega_{c}(s)+\Omega_{c}(s)
$$

The cam phase angle $\phi$ is the integration of the double difference between camshaft and crankshaft speeds. That is,

$$
\phi=2 \int_{0}^{t}\left[\omega_{s}(\tau)-\omega_{r}(\tau)\right] d \tau
$$

and its corresponding Laplace transformation is

$$
\Phi(s)=\frac{2}{s}\left[\Omega_{s}(s)-\Omega_{r}(s)\right]
$$

Substituting (2) into (4) yields,

$$
\Phi=\frac{2}{s}\left(\frac{n_{s}+n_{r}}{n_{s}}\right)\left(\Omega_{c}-\Omega_{r}\right)
$$

Equation (5) shows that the cam phase is an integral function of speed difference between carrier and ring gears. By controlling the EVVT motor speed with respect to the engine crankshaft speed, cam phase can be adjusted. When the carrier speed is equal to the ring speed, cam phase is held; when the carrier speed is greater than the ring speed, cam phase is advancing; and when the carrier speed is slower than the ring speed, cam phase is retarding. Notice that equation (5) contains an integrator, and target cam phase reference cannot be used as feedforward control directly.

\section{Planetary Gear System Dynamics}

Planetary gear system dynamics with an electric motor are modeled in this section. For simplicity, the gear system friction is ignored in this modeling study. Figure 2 shows free body diagrams of planetary gear components.

Without loss of generality comparing with the system in Figure 1, the system is treated as having only one planet gear (Figure 2a). Assuming that all the gears are properly engaged, the following equation is derived: 


$$
\frac{n_{s}}{r_{s}}=\frac{n_{p}}{r_{p}}=\frac{n_{r}}{r_{r}}, \quad r_{r}=2 r_{p}+r_{s}
$$

where $n_{p}$ is planet gear number of teeth. $r_{s}, r_{p}$, and $r_{r}$ are pitch circle radius of sun, planet, and ring gears, respectively. In this study, the gears use a standard pressure angle $\theta$ of 20 degrees. Since the ring has a very large inertia comparing to other components, angular velocity of the ring $\omega_{r}$ is assumed to be constant during the phase shifting. From (2):

$$
\dot{\omega}_{s}=\frac{n_{r}+n_{s}}{n_{s}} \dot{\omega}_{c}
$$

There are two torques applied to the sun gear (Figure $2 \mathrm{~b}$ ). They are camshaft load $T_{\text {cam }}$ and torque from tooth force $F_{34}$.

$$
F_{34} \cdot r_{s} \cdot \cos \theta-T_{\text {cam }}=J_{s} \dot{\omega}_{s}
$$

where $J_{s}$ is sun gear's moment of inertia with respect to its center of gravity.

Two tooth forces $\left(F_{43}\right.$ and $\left.F_{13}\right)$ and one bearing force from carrier $F_{23}$ are applied to planet gear (see Figure2c) that rotates around the bearing on the carrier at $\omega_{p}$ :

$$
\frac{\omega_{p}(t)-\omega_{c}(t)}{\omega_{s}(t)-\omega_{c}(t)}=-\frac{n_{s}}{n_{p}}
$$

and from torque balance with respect to bearing point:

$$
\left(F_{43}+F_{13}\right) \cdot r_{p} \cdot \cos \theta=J_{p} \dot{\omega}_{p}
$$

where $J_{p}$ is planet gear moment of inertia with respect to its center of gravity. The planet gear also rotates about the center of sun gear. Therefore,

$$
\left[F_{13}\left(2 r_{r}\right)-F_{43} r_{s}\right] \cos \theta+F_{23}\left(r_{p}+r_{s}\right) \cos \alpha=J_{p s} \dot{\omega}_{c}
$$

where the direction and magnitude of bearing force $F_{23}$ are unknown. The planet gear moment of inertia with respect to the center of sun gear $J_{p s}$ can be calculated by

$$
J_{p s}=J_{p}\left[1+m_{p}\left(r_{s}+r_{p}\right)^{2}\right]
$$

Since the carrier is driven directly by the motor shaft, the carrier inertia is also considered as part of motor shaft inertia, and modeled in the next sub-section. Torque balance of carrier is

$$
F_{32} \cos \alpha\left(r_{p}+r_{s}\right)=T_{\text {load }}
$$

where $T_{\text {load }}$ is the mechanical load to the motor shaft and $F_{32}$ is the bearing force from planet gear.

Equations (6) to (13) can be simplified as follows.

$$
T_{\text {load }}=J_{\text {gears }} \dot{\omega}_{c}+k T_{\text {cam }}
$$

where constant $J_{\text {gears }}$ is an equivalent inertia of the planetary gear system, and $k$ is a factor of gear ratio.

\section{EVVT Motor Dynamics}

An electric motor is used to drive carrier in the planetary system. A local closed-loop speed 
governor is used to control both the motor speed and direction. The input to the local motor controller is the reference speed and direction. The motor and its controller are treated as an actuator in this study (Figure 3). It is modeled with two inputs of motor velocity command and cam load, and one output of motor shaft speed.

The mechanical load of the motor can be modeled [25] as

$$
J_{c} \dot{\omega}_{c}=\tau-B \omega_{c}-T_{\text {load }}
$$

where $J_{c}$ is the moment of inertia of motor shaft and carrier, $B$ is the friction coefficient, and $\tau$ is the motor torque. Substituting (14) into (15) leads to

$$
\left(J_{c}+J_{\text {gears }}\right) \dot{\omega}_{c}+B \omega_{c}=\tau-k T_{\text {cam }}
$$

and the associated transfer function can be written as

$$
\Omega_{c}(s)=\frac{1}{\left(J_{c}+J_{\text {gears }}\right) s+B}\left[T(s)-k T_{\text {cam }}(s)\right]
$$

Modeling procedure of the electrical portion can be found in [25]. Let $J=J_{c}+J_{\text {gears }}$, the electric motor with planetary gear load (Figure 3) can then be represented by

$$
\Omega_{c}(s)=G_{e}(s) E_{a}(s)+G_{m}(s) T_{\text {cam }}(s)
$$

where the voltage input transfer function is

$$
G_{e}=\frac{K_{\tau}}{\left(L_{m} s+R_{m}\right)\left(J_{s}+B\right)+K_{\tau} K_{m}} \cong \frac{K_{\tau}}{R_{m}\left(J_{s}+B\right)+K_{\tau} K_{m}}
$$

and the mechanical input transfer function is

$$
G_{m}=\frac{-\left(L_{m} s+R_{m}\right)}{\left(L_{m} s+R_{m}\right)(J s+B)+K_{\tau} K_{m}} \cong \frac{-R_{m} k}{R_{m}(J s+B)+K_{\tau} K_{m}}
$$

and $K_{\tau}, K_{m}, L_{m}$, and $R_{m}$ are the motor parameters representing motor torque constant, back EMF (electric magnetic field) constant, armature inertia and resistance, respectively.

Note that the model described by equations (18) to (20) were used to determine the order of the identified system model described in Section $\mathrm{VI}$ and used for simulation validation and evaluation of the closed-loop system performance.

\section{FEEDFORWARD AND CLOSED-LOOP CONTROL DESIGN}

\section{A. Output Covariance Constraint (OCC)}

Consider the following state-space linear time-invariant system

$$
\begin{aligned}
\mathbf{x}_{\mathbf{p}}(k+1) & =\mathbf{A}_{\mathbf{p}} \mathbf{x}_{\mathbf{p}}(k)+\mathbf{B}_{\mathbf{p}} \mathbf{u}(k)+\mathbf{D}_{\mathbf{p}} \mathbf{w}_{\mathbf{p}}(k) \\
\mathbf{y}_{\mathbf{p}}(k) & =\mathbf{C}_{\mathbf{p}} \mathbf{x}_{\mathbf{p}}(k) \\
\mathbf{z}(k) & =\mathbf{M}_{\mathbf{p}} \mathbf{x}_{\mathbf{p}}(k)+\mathbf{v}(k)
\end{aligned}
$$

where $\mathbf{x}_{p}$ is the state; $\mathbf{u}$ is the control input; $\mathbf{w}_{\mathbf{p}}$ represents process noise; and $\mathbf{v}$ is the measurement noise. The vector $\mathbf{y}_{\mathbf{p}}$ contains all variables whose dynamic responses are of interest. Vector $\mathbf{z}$ is the noisy measurements. Suppose that a strictly proper output feedback stabilizing control law, expressed in state-space form below, is employed for plant (21). 


$$
\begin{aligned}
\mathbf{x}_{\mathbf{c}}(k+1) & =\mathbf{A}_{\mathbf{c}} \mathbf{x}_{\mathbf{c}}(k)+\mathbf{F z}(k) \\
\mathbf{u}(k) & =\mathbf{G x}_{\mathbf{c}}(k)
\end{aligned}
$$

where $\mathbf{x}_{c}$ is the controller state; $\mathbf{u}$ is the control output; and $\mathbf{z}$ is the noisy measurement input. Note that matrices $\mathbf{F}, \mathbf{A}_{\mathbf{c}}$, and $\mathbf{G}$ are control design parameters. Then the resulting closed-loop system is

$$
\begin{aligned}
& \mathbf{x}(k+1)=\mathbf{A} \mathbf{x}(k)+\mathbf{D} \mathbf{w}(k) \\
& \mathbf{y}(k)=\left[\begin{array}{c}
\mathbf{y}_{\mathbf{p}}(k) \\
\mathbf{u}(k)
\end{array}\right]=\left[\begin{array}{c}
\mathbf{C}_{\mathbf{y}} \\
\mathbf{C}_{\mathbf{u}}
\end{array}\right] \mathbf{x}(k)=\mathbf{C x}(k)
\end{aligned}
$$

where $\mathbf{x}=\left[\begin{array}{ll}\mathbf{x}_{\mathbf{p}}^{\mathbf{T}} & \mathbf{x}_{\mathbf{c}}^{\mathbf{T}}\end{array}\right]^{\mathbf{T}}$ and $\mathbf{w}=\left[\begin{array}{ll}\mathbf{w}_{\mathbf{p}}^{\mathbf{T}} & \mathbf{v}^{\mathbf{T}}\end{array}\right]^{\mathbf{T}}$. Formulas for the closed-loop system matrices $\mathbf{A}, \mathbf{C}$, and $\mathbf{D}$ can be obtained based upon (21) and (22).

Consider the closed-loop system (23). Let $\mathbf{W}_{\mathbf{p}}$ and $\mathbf{V}$ denote positive definite symmetric matrices with dimensions compatible to the process noise $\mathbf{w}_{\mathbf{p}}$ and measurement vector $\mathbf{z}$, respectively. Define $\mathbf{W}=$ block $\operatorname{diag}\left[\begin{array}{ll}\mathbf{W}_{\mathbf{p}} & \mathbf{V}\end{array}\right]$ and let $\mathbf{X}$ denote the closed-loop controllability Gramian from the input $\mathbf{W}^{-\frac{1}{2}} \mathbf{w}$. Since $\mathbf{A}$ is stable, $\mathbf{X}$ satisfies

$$
\mathbf{X}=\mathbf{A X A}^{\mathbf{T}}+\mathbf{D W D}^{\mathbf{T}}
$$

The goal is to find controllers of form (12) that minimize the (weighted) control energy $\operatorname{trace}\left(\mathbf{R C}_{\mathbf{u}} \mathbf{X C}_{\mathbf{u}}^{\mathbf{T}}\right)$ with $\mathbf{R}>0$ subject to the following constraint

$$
\mathbf{Y}=\mathbf{C X C}^{\mathbf{T}} \leq \overline{\mathbf{Y}}
$$

where $\overline{\mathbf{Y}}>0$ is given and $\mathbf{X}$ solves (24). This problem, called the OCC problem [18], is defined as finding a full-order dynamic output feedback controller (22) for system (21) that minimizes the following cost

$$
J_{\text {occ }}=\operatorname{trace}\left(\mathbf{R C}_{\mathbf{u}} \mathbf{X C}_{\mathbf{u}}^{\mathbf{T}}\right), \quad \mathbf{R}>0
$$

subject to constraints (24) and (25).

The OCC problem has several interesting interpretations. For instance, assume first that $\mathbf{w}_{\mathbf{p}}$ and $\mathbf{v}$ are uncorrelated zero-mean white noises with intensity matrices $\mathbf{W}_{\mathbf{p}}>0$ and $\mathbf{v}>0$. Let $E$ be an expectation operator, and

$$
\begin{aligned}
E\left[\mathbf{w}_{\mathbf{p}}(k)\right] & =0 ; E\left[\mathbf{w}_{\mathbf{p}}(k) \mathbf{w}_{\mathbf{p}}^{\mathbf{T}}(k-n)\right]=\mathbf{W}_{\mathbf{p}} \delta(n) \\
E[\mathbf{v}(k)] & =0 ; E\left[\mathbf{v}(k) \mathbf{v}^{\mathbf{T}}(k-n)\right]=\mathbf{V} \delta(n)
\end{aligned}
$$

Define $E_{\infty}[\cdot]:=\lim _{k \rightarrow \infty} E[\cdot]$, it is easy to see that OCC is the problem of minimizing $E_{\infty} \mathbf{u}^{\mathbf{T}} \mathbf{R u}$ subject to the OCC constraint $\mathbf{Y}:=E_{\infty} \mathbf{y}(k) \mathbf{y}^{\mathbf{T}}(k) \leq \overline{\mathbf{Y}}$. It is well known that this constraint may be interpreted as constraint on the variance of the performance variables or lower bounds on the residence time (in a given ball around the origin of the output space) of the performance variables; see [26] for details.

The OCC problem can also be interpreted from a deterministic point of view. To see this, define the $\ell_{\infty}$ and $\ell_{2}$ norms as follows 


$$
\begin{aligned}
& \|\mathbf{y}\|_{\infty}^{2}:=\sup _{k \geq 0} \mathbf{y}^{\mathbf{T}}(k) \mathbf{y}(k) \\
& \|\mathbf{w}\|_{2}^{2}:=\sum_{k=0}^{\infty} \mathbf{w}^{\mathbf{T}}(k) \mathbf{w}(k)
\end{aligned}
$$

and define the (weighted) $\ell_{2}$ disturbance set

$$
\mathscr{W}:=\left\{\mathbf{w}: \mathbf{R} \rightarrow \mathbf{R}^{n_{w}} \text { and }\left\|\mathbf{W}^{-1 / 2} \mathbf{w}\right\|_{2}^{2} \leq 1\right\}
$$

where $\mathbf{W}>0$ is a real symmetric matrix. Then, for any $\mathbf{w} \in \mathscr{W}$, the following is true (see [27] and [28] for details)

$$
\|\mathbf{y}\|_{\infty}^{2} \leq \bar{\sigma}[\mathbf{Y}] \text {, and }\left\|\mathbf{u}_{i}\right\|_{\infty}^{2} \leq\left[\mathbf{C}_{\mathbf{u}} \mathbf{X} \mathbf{C}_{\mathbf{u}}^{\mathbf{T}}\right]_{i i}, i=1,2, \ldots n_{u}
$$

where $n_{u}$ is the dimension of $\mathbf{u} ; \bar{\sigma}[\cdot]$ denotes the maximum singular value; and $[\cdot]_{i i}$ is the $i^{\text {th }}$ diagonal entry. Moreover, references [27] and [28] show that the bounds in (30) are the least upper bounds for any signal $\mathbf{w} \in \mathscr{W}$.

Thus, using $\overline{\mathbf{Y}}=I \epsilon^{2}$ in (25) and $\mathbf{R}=\operatorname{diag}\left[r_{1}, r_{2}, \ldots, r_{n_{u}}\right]$ in (26), the OCC is the problem of minimizing the weighted sum of worst-case peak values on the control signals (related to actuator sizing) given by

$$
J_{o c c}=\sum_{i=1}^{n_{u}} r_{i}\left\{\sup _{\mathbf{w} \in \mathscr{W}}\left\|\mathbf{u}_{i}\right\|_{\infty}^{2}\right\}
$$

subject to the constraint on the worst-case peak value of the performance variables of the form

$$
\sup _{\mathbf{w} \in \mathscr{W}}\|\mathbf{y}\|_{\infty}^{2} \leq \epsilon^{2}
$$

This interpretation is important in applications where hard constraints on responses or actuator signals cannot be ignored such as space telescope pointing error and machine tool control problems. Detailed proof can be found in [16]. The controller matrices $\mathbf{A}_{\mathbf{c}}, \mathbf{F}$, and G can be calculated using an iterative algorithm introduced in [16] and [18].

\section{B. Control Design Parameters}

The EVVT system model includes the VVT controller, the local motor controller, motor/planetary dynamics, and planetary kinematics. Figure 4 shows an overview of the control system architecture. The system parameters are listed as following and the controllers were designed based on these parameters.

The voltage input transfer function is

$$
G_{e}=\frac{45}{0.2 s+1}
$$

the mechanical input transfer function is

$$
G_{m}=\frac{-5}{0.2 s+1}
$$

and the motor has a local PID controller defined by 


$$
K_{\text {motor }}=1+\frac{0.1}{s}+0.05\left(\frac{s}{0.01 s+1}\right)
$$

Teeth numbers of the gear-train are listed in Table 1 . Substituting these values into (5) results the planetary kinematics

$$
\Phi=\frac{6}{s}\left(\Omega_{c}-\Omega_{r}\right)
$$

Figure 5 describes the cam torque load of each cylinder over an engine cycle. It consists of three portions: constant friction load, sinusoidal load representing cam profile, and steps represent the valve spring pre-load. In the study, a four cylinder engine is simulated. The total load is a combination of four single cylinder loads with 180 degree phase shift for each cylinder, assuming the cam drives both intake and exhaust valves.

\section{Feedforward Control}

A feedforward controller was employed to improve the system response. The reference cam phase was not used directly as feedforward control due to the physical characteristics of the EVVT system stated in the previous section; instead, the derivative of the cam phase reference signal was used due to the planetary kinematics shown in (36). The feedforward gain was determined by the ratio between desired cam phase slope and the motor speed. Using (36), feedforward gain $K_{F F}$ can be determined as:

$$
u_{F F}=K_{F F} \dot{\theta}_{r e f}+\omega_{r}=\frac{1}{6} \dot{\theta}_{r e f}+\omega_{r}
$$

where $u_{F F}$ is the feedforward control effort. $\dot{\theta}_{r e f}$ is the filtered derivative of the reference signal $\theta_{\text {ref }}$

$$
\dot{\theta}_{\text {ref }}=\frac{s}{0.05 s+1} \theta_{\text {ref }}
$$

\section{Baseline Control}

Since the electrical cam phase actuator plant contains an integrator, PD controllers were used as our baseline ones. Two baseline feedback controllers were tuned for performance comparison, where $P D_{W F}$ was tuned without feedforward and $P D_{W O F}$ was tuned with feedforward, and they are

$$
\begin{gathered}
P D_{W F}(s)=7+0.03 s \\
P D_{W O F}(s)=1+0.005 s
\end{gathered}
$$

\section{E. OCC Feedback Control Design}

The OCC control design considers the mechanical cam load as a disturbance, the EVVT controller input as a plant input, and the cam phase as an output, the electrical portion system matrices of the EVVT system (Figure 4) can then be written as 


$$
\begin{aligned}
& \mathbf{A}=\left[\begin{array}{cccc}
-1455 & -23023 & -2250 & 0 \\
1 & 0 & 0 & 0 \\
0 & 1 & 0 & 0 \\
0 & 0 & 1 & 0
\end{array}\right], \quad \mathbf{B}=\left[\begin{array}{l}
1 \\
0 \\
0 \\
0
\end{array}\right] \\
& \mathbf{C}=\left[\begin{array}{llll}
0 & 8100 & 135130 & 13500
\end{array}\right], \quad \mathbf{D} \cong 0
\end{aligned}
$$

The control design parameters were chosen as

$$
\mathbf{W}_{\mathbf{p}}=2, \quad \mathbf{V}=0.01, \quad \mathbf{R}=1
$$

Using the control design algorithm introduced in [18], the resulting OCC controller is

$$
K=\frac{113.6 s^{3}+1.65 \times 10^{5} s^{2}+2.6 \times 10^{6} s+2.6 \times 10^{5}}{s^{4}+1541 s^{3}+1.5 \times 10^{5} s^{2}+2.12 \times 10^{6} s+2.11 \times 10^{5}}
$$

The system transfer function (42) and its state-space realization (40) provide a system model for control design and simulation study conducted in the next section; and it also provide the system order selection information for system identification conducted in Section VI.

\section{SIMULATION STUDY}

The EVVT reference signal was selected as a forty crank degree phase retard that completes in three engine cycles to simulate the EVVT operation under $\mathrm{SI}$ and $\mathrm{HCCl}$ combustion transition. The transition reference signal is divided into three stages with a constant slope within one engine cycle for simplicity. The retard phase is 50\% (20 degrees) for the first engine cycle, $33.3 \%$ (40/3 degrees) for the second cycle, and $16.7 \%$ (20/3 degrees) for the third. The phase control signal was sampled every $5 \mathrm{~ms}$ and the feedback signal is updated four times per engine cycle. For instance, at $1500 \mathrm{rpm}$ the cam phase is sampled every 20ms. The closed-loop system performance at two engine speeds, $1500 \mathrm{rpm}$ and $2000 \mathrm{rpm}$, was studied.

Figure 6 compares the cam phase responses among three controllers, OCC, $P D_{W F}$ and $P D_{W O F}$, at $1500 \mathrm{rpm}$. It shows that the initial response of the PD controller with feedforward $\left(P D_{W F}\right)$ is much faster than the PD controller without feedforward $\left(P D_{W O F}\right)$. However, due to the relatively low gain of the PD controller with feedforward, after the second cycle, it has a larger overshoot with longer settling time than the PD controller without feedforward. However, the OCC controller demonstrates fast response time with low overshoot. Table 2 shows cam phase angles at the end of each engine cycle after the $\mathrm{SI}$ and $\mathrm{HCCl}$ transition starts. The OCC controller with feedforward has the lowest overall tracking errors. Figure 7 provides the same time responses as Figure 6 at $2000 \mathrm{rpm}$. It is noticed that the performance is quite different at engine speeds of 1500 and $2000 \mathrm{rpm}$ (see Figures 6 and 7). Table 2 compares the cam phase error at the end of each engine cycle numerically and shows that the tracking error increases at $2000 \mathrm{rpm}$. This could be due to the increased rate change of the reference cam phase signal in time domain as the engine speed increases from 1500 to $2000 \mathrm{rpm}$ since the engine cycle period reduced from $80 \mathrm{~ms}$ at $1500 \mathrm{rpm}$ to $60 \mathrm{~ms}$ at $2000 \mathrm{rpm}$.

The tracking errors with increased feedback sampling rates were also studied and the results are shown in Table 3. The simulation data shows that the tracking error reduces when the 
number of samples increased from four samples per engine cycle to eight. However, further increment of number of samples does not reduce the tracking error significantly. Especially, with the OCC design, the tracking error is fairly small with four samples per cycle. Therefore, considering the limited tracking error reduction and increased computational requirement with high sample rate, it is determined that using four samples per engine cycle is proper for this application.

As a summary, simulation results show that the OCC controller has a lower overshoot than a well-tuned proportional controller, while with comparable response time, and four samples per engine cycle for the cam position is suitable for the EVVT control application.

\section{THE EVVT TEST BENCH SETUP}

A test bench was constructed for system identification and control validation of the studied EVVT actuator system. The test setup was very similar to the hydraulic VVT system bench [19], except the hydraulic VVT was replaced by an EVVT system. The EVVT system has a phasing range of 45 degrees and is controlled by an AC motor with its own PID speed governor. An Opal-RT (Hardware-in-the-loop test equipment manufacture, located in Montréal, Québec, Canada) real-time prototype controller controls the cam phase by adjusting the reference speed pulse-width modulation (PWM) signal sent to the motor speed governor. The PWM signal frequency is directly proportional to the command speed and the local motor controller has its own cut-off frequencies at both high and low motor speeds.

A Ford 5.4L V8 engine head was used for the test bench. An electric motor was used to simulate the motion of the engine crank shaft. An encoder was installed on the motor shaft that generates crank angle and gate (360 degrees per pulse) signals. A hall-effect cam position sensor was installed with a four-slot cam disc, which generates four pulses per engine cycle. Therefore, the cam position is updated four times every engine cycle. Figure 8 shows the diagram of the EVVT test bench with key system components and Figure 9 is a picture of the associated test bench. The cam position sensing system has a theoretical resolution of 1/64 crank degree. An electric oil pump was used to supply oil for the valve-train system and the EVVT planetary gear system, and the EVVT bench was running at room temperature $\left(25^{\circ} \mathrm{C}\right)$. This setup enables the system identification and control experimental work.

\section{SYSTEM IDENTIFICATION FRAMEWORK}

Consider a general form of linear time-invariant closed-loop system shown in Figure 10, where $r$ is the reference signal; $n$ is the measurement noise; $u$ and $y$ are system input and output, respectively. As discussed in the Introduction section, there are many approaches for the closed-loop system identification, which can be categorized as direct, indirect, and joint input-output approaches. In this paper, the controller knowledge is used to calculate the openloop plant model from identified closed-loop system model, which is called indirect approach. To ensure the accuracy of the identified plant, the closed-loop controller in this paper was selected to be proportional ([29] and [30]).

The input and output relationship of the generalized closed-loop system, shown in Figure 10, can be expressed below: 


$$
\mathbf{y}=\mathbf{H} \cdot \mathbf{r}=\mathbf{G K}(\mathbf{I}+\mathbf{G K})^{-1} \mathbf{r}
$$

Let $\hat{\mathbf{H}}$ be identified closed-loop transfer functions from $r$ to $y$. The open-loop system model $\mathbf{G}_{I D}$ can be calculated using identified $\hat{\mathbf{H}}$, assuming that $(\mathbf{I}-\hat{\mathbf{H}})$ is invertible. The controller transfer function is used to solve for the open-loop system models. Then, the following can be derived:

$$
\mathbf{G}_{I D}=\hat{\mathbf{H}}(\mathbf{I}-\hat{\mathbf{H}})^{-1} \mathbf{K}^{-1}
$$

PRBS signal is used as an input signal for identifying the closed-loop system model. The most commonly used PRBSs are based on maximum length sequences (called $m$-sequences) [31] for which the length of the PRBS is $m=2^{n}-1$, where $n$ is an integer (order of PRBS). Let $z^{-1}$ represent a delay operator, and define $\hat{p}\left(z^{-1}\right)$ and $p\left(z^{-1}\right)$ to be polynomials

$$
p\left(z^{-1}\right)=a_{n} z^{-n+1} \oplus \cdots \oplus a_{2} z^{-1} \oplus a_{1}=\hat{p}\left(z^{-1}\right) z^{-1} \oplus 1
$$

where $a_{i}$ is either 1 or 0 , and $\oplus$ obeys binary addition, i.e.,

$$
1 \oplus 1=0=0 \oplus 0 \text { and } 0 \oplus 1=1=1 \oplus 0
$$

and the non-zero coefficients $a_{\mathrm{i}}$ of the polynomial are defined in the following (Table 4) and also in [31].

Then the PRBS can be generated by the following formula

$$
\hat{u}(k+1)=\hat{p}\left(z^{-1}\right) \hat{u}(k), k=0,1,2, \ldots
$$

where $\hat{u}(0)=1$ and $\hat{u}(-1)=\hat{u}(-2)=\cdots=\hat{u}(-n)=0$. Defined the following sequence

$$
s(k)= \begin{cases}a ; & \text { If } k \text { is even; } \\ -a ; & \text { If } k \text { is odd }\end{cases}
$$

Then, the signal

$$
u(k)=s(k) \otimes[-a+2 a \hat{u}(k)]
$$

is called the inverse PRBS, where $\otimes$ obeys

$$
\mathrm{a} \otimes \mathrm{a}=-\mathrm{a}=-\mathrm{a} \otimes-\mathrm{a} \quad \& \quad \mathrm{a} \otimes-\mathrm{a}=\mathrm{a}=-\mathrm{a} \otimes \mathrm{a}
$$

It is clear after some analysis that $u$ has a period $2 m$ and $u(k)=-u(k+m)$. The mean of the inverse PRBS is

$$
m_{u}=E_{2 m} u(k) \equiv \frac{1}{2 m} \sum_{k=0}^{2 m-1} u(k)=0
$$

and the autocorrelation $\left(R_{u u}(\tau)=E_{2 m} u(k+\tau) u(k)\right)$ of $u$ is

$$
R_{u u}(\tau) \equiv \frac{1}{2 m} \sum_{k=0}^{2 m-1} u(k+\tau) u(k)= \begin{cases}a^{2}, & \tau=0 ; \\ -a^{2}, & \tau=m ; \\ -a^{2} / m, & \tau \text { even; } \\ a^{2} / m, & \tau \text { odd }\end{cases}
$$

Note that the first and second order information of the inverse PRBS signal is very close to these of white noise for a large enough $m$. The inverse PRBS is used in the $q$-Markov Cover 
identification. For convenience, in the rest of this paper, the term "PRBS" is used to represent the inverse PRBS.

Consider an unknown (presumed nonlinear) system:

$$
\begin{aligned}
\mathbf{x}(k+1) & =f(\mathbf{x}(k), \mathbf{w}(k)) \\
\mathbf{y}(k) & =g(\mathbf{x}(k), \mathbf{w}(k))
\end{aligned}
$$

subjected to an input sequence $\{w(0), w(1), w(2), \ldots\}$ generating the output sequence $\{y(0), y(1), y(2), \ldots\}$. The unknown system is $q$-identifiable, if there exists a linear model of the form:

$$
\begin{aligned}
\mathbf{x}(k+1) & =\mathbf{A x}(k)+\mathbf{D} \mathbf{w}(k) \\
\mathbf{y}(k) & =\mathbf{C} \mathbf{x}(k)+\mathbf{H} \mathbf{w}(k)
\end{aligned}
$$

that can reproduced the same output sequence $\{y(0), y(1), y(2), \ldots, y(q-1)\}$, subject to the same input sequence $\{w(0), w(1), w(2), \ldots, w(q-1)\}$. In case that the system is not $q$-identifiable, it is possible for $q$-Markov Cover to construct the least squares fit using linear model for the inputoutput sequence (see [32] and [33]). The above q-Markov Cover system identification framework was used in the next section to obtain the linearized EVVT model for control design.

\section{EVVT CONTROL SYSTEM BENCH TESTS}

This section discusses the bench system identification and control design based upon the identified EVVT model. The closed-loop control is presented at the end of the section.

\section{A. Closed-loop Identification of EVVT System}

The engine feedforward speed (half engine speed) was used during the system identification. The main reason is that the EVVT model uses the speed difference between the motor and half engine speed as the input; see equation (5). Since the engine speed changes much slower than the EVVT phasing speed, it is considered as a constant during the cam phasing. The resulting identified model has the following form:

$$
\Phi=G_{\text {evvt }}(s)\left(u-\frac{1}{2} \omega_{\text {eng }}\right)
$$

where $\Phi$ is the cam phase; $G_{\text {evvt }}(s)$ is the identified EVVT model; $u$ is the speed command from the controller; and the constant $\frac{1}{2} \omega_{\text {eng }}$ is half engine speed. The PRBS was used as the reference signal for the closed-loop identification and its amplitude was selected to be 10 degrees centered at 20 degree advance from the most retarded position. A proportional controller with a gain of 0.07 was used for the system identification.

The EVVT system was identified at 1000 and $1500 \mathrm{rpm}$ due to the speed limitation on the test bench. Parameters used in the identification are listed in Table 5. The order of the identified EVVT model was chosen to be four that is the same as the physical model (40). A nominal system model was obtained as

$$
G_{\text {evvt }}(s)=\frac{-9.7 s^{3}+139 s^{2}+5760 s+5785}{s^{4}+12.2 s^{3}+159 s^{2}-62 s+11}\left(\frac{\text { degree }}{1000 \mathrm{rpm}}\right)
$$




\section{B. Control Design for EVVT System Test Bench}

An OCC controller was designed for the EVVT bench system. The system plant matrices of the nominal model were obtained from equation (56)

$$
\begin{aligned}
& \mathbf{A p}=\mathbf{A}=\left[\begin{array}{cccc}
-12.2 & -159 & 62 & -11 \\
1 & 0 & 0 & 0 \\
0 & 1 & 0 & 0 \\
0 & 0 & 1 & 0
\end{array}\right], \mathbf{B p}=\mathbf{B}=\left[\begin{array}{l}
1 \\
0 \\
0 \\
0
\end{array}\right] \\
& \mathbf{C p}=\mathbf{M p}=\mathbf{C}=\left[\begin{array}{llll}
-9.6 & 139.9 & 5756 \quad 5783
\end{array}\right], \mathbf{D p} \cong \mathbf{0}
\end{aligned}
$$

Controller design parameters were selected as

$$
\mathbf{W}_{\mathbf{p}}=1, \quad \mathbf{V}=0.01, \quad \mathbf{R}=1
$$

Using the OCC iterative control design algorithm in [18], an OCC controller was obtained

$$
K_{O C C}(s)=\frac{60 s^{3}+8.5 \times 10^{2} s^{2}+1 \times 10^{4} s+6.7 \times 10^{3}}{s^{4}+148 s^{3}+6937 s^{2}+8.6 \times 10^{4} s+8.2 \times 10^{4}}\left(\frac{1000 \mathrm{rpm}}{\text { degree }}\right)
$$

A proportional controller (60) was tuned for performance comparison. Note that the DC gains of both controllers are fairly close. The proportional controller was tuned to achieve fast response time with low overshoot at both 1000 and $1500 \mathrm{rpm}$ for a square wave reference signal (see Figure 11). Note that the same OCC controller was used at both engine speeds.

$$
K_{P}(s)=0.08(1000 \mathrm{rpm} / \text { degree })
$$

\section{Control Performance Evaluation}

The controllers were tested at 1000 and $1500 \mathrm{rpm}$. Both proportional and OCC controllers have feedforward portions from engine speed as shown in (38). Different reference signals were used to validate the trajectory tracking performance of the EVVT bench system.

The reference cam phase is a 20 crank degree phase advance or retard signal with a 10 degree linear phase change for the first engine cycle, 6.67 degrees for the second, and 3.33 degrees for the third, respectively. This signal was also used as the reference signal in simulations (Figure 12). From Figure 12, it is shown that the OCC controller has lower overshoot than the proportional controller at $1000 \mathrm{rpm}$. At $1500 \mathrm{rpm}$, overshoots for both controllers are very low. The OCC controller has a faster response time than the proportional controller in both engine speeds. Settling times for the two controllers are also very close to each other.

A series of sinusoidal waves was used to test the frequency response of the closed-loop systems to further investigate trajectory tracking performance of the EVVT controllers. The amplitude of the sinusoidal signal was set to be 10 degrees and centered at 20 degree advance from the most retarded position, and the frequencies of the signals vary from 0.01 to $2.00 \mathrm{~Hz}$. The test results show that both controllers have very good tracking performance at low frequency (see Figure 13 and Table 6). As the excitation frequency increases, the phase responses associated with both controllers show increased phase leg with decreased magnitudes. The two controllers have almost identical gains at different frequencies, but the OCC has less phase delay than the proportional controller. 
It was observed that when the test bench engine was running at $1000 \mathrm{rpm}$, the closed-loop EVVT system has overshoots when the excitation frequency is closed to $1.0 \mathrm{~Hz}$. After $1.5 \mathrm{~Hz}$, the system gain decays quickly. However, the frequency response of the identified fourth order nominal model in (56) does not show this behavior at $1.0 \mathrm{~Hz}$. To study this phenomena, a seventh order model was obtained using closed-loop system identification at $1000 \mathrm{rpm}$ and its frequency response (predicted performance) was compared with the measured data, obtained from magnitude and phase responses of the sinusoidal input at different frequency, in Figure 14. A peak near $1.0 \mathrm{~Hz}$ can be found in the magnitude of the measured and predicted frequency responses. The overshoot of the closed-loop system can be duplicated under simulation environment using the identified seventh order model at $1000 \mathrm{rpm}$. However the physical dynamics behind the phenomenon are still not clear and it is believed that it could be caused by the timing belt vibration.

\section{Performance Variation due to the Engine Oil Viscosity}

Nonlinear friction in the hydraulic system [34] could also affect the performance of the EVVT. Two different types of engine oil, SAE 5W20 and SAE 30, were used during the bench tests to study this effect. The engine was running at $1500 \mathrm{rpm}$ at room temperature $\left(25^{\circ} \mathrm{C}\right)$, and OCC controller (59) was used in both cases. The reference signal was a 20 degree advance step. The 10 to 90 percent rising time was 0.3 second for SAE 5W20, and 0.48 second for SAE 30 engine oil. The response time difference is due to the viscous friction in the planetary gear system caused by the engine oil viscosity. Figure 15 shows the system step response and normalized speed difference between the EVVT motor and half engine speed (750 rpm). The EVVT motor speed was saturated at 30 units above $750 \mathrm{rpm}$ with SAE 30 and at about 50 units above 750 rpm with SAE 5W20. The system response slows down as the difference between the EVVT motor and half of the engine speed increases. Notice that the EVVT motor speed is measured by calculating PWM frequency from the EVVT local speed controller.

The test result also suggests that the frictionless assumption during the simulation study is not true. However, on an actual engine, the nominal operating temperature is much higher than the room temperature and the engine oil viscosity will have much less effect for a warm engine than a cold engine.

As a summary, it was observed from the EVVT bench tests that the engine oil viscosity has a large impact on EVVT performance. Since the engine oil is used to lubricate the planetary gear system, the hydraulic friction introduced by the engine oil adds additional load to the electric motor and limits the peak motor speed. As a result the maximum phasing speed is reduced when high viscosity engine oil was used. Two kinds of engine oil with different viscosities were used on the test bench. The results show that at room temperature, the EVVT system response is 1.6 times slower using SAE 30 than using SAE 5W20 engine oil.

\section{CONCLUSIONS}

An electric variable valve timing (EVVT) system with planetary gear-train was modeled based upon individual system component kinematics and dynamics. A closed-loop OCC (output covariance constraint) controller with feedforward was designed to reduce the cam phase tracking error during the $\mathrm{SI}$ and $\mathrm{HCCl}$ combustion mode transition, where the filtered derivative 
of the cam reference phase is used as the feedforward control. Compared to the well-tuned PD controllers, simulation results show that the OCC controller provides fast response with low overshot and low tracking error.

An EVVT system was installed on an engine head and bench tests were conducted. The EVVT system plant model was obtained by using closed-loop system identification. The model has fairly similar responses to the physical system. An OCC controller was developed based on the identified model. Different signals were used as reference to test the controller performance. The experimental results showed that the OCC controller provides a faster response time than that of a well-tuned proportional controller. The OCC controller performance also has less phase delay than the proportional controller under high frequency sinusoidal reference input. The bench test results show that the OCC has a better overall performance and is suitable for an $\mathrm{HCCl}$ capable SI engine.

The impact of engine oil viscosity was also investigated. The test results showed that the EVVT response slows down as the engine oil viscosity increases. It suggested that it is necessary to use low viscosity engine oil to achieve the maximum performance. This could mean either operating at a high oil temperature or using low viscosity engine oil.

\section{REFERENCES}

[1] Moriya, Y., Watanabe, A., Uda, H., Kawamura, H., Yoshiuka, M., and Adachi, M., 1996, “A Newly Developed Intelligent Variable Valve Timing System - Continuously Controlled Cam Phasing as Applied to New 3 Liter Inline 6 Engine", SAE Technical Paper, 960579.

[2] Dugdale, P. H., Rademacher, R. J., Price, B. R., Subhedar, J. W., and Duguay, R. L., 2005, "Ecotec 2.4L VVT: A Variant of GM's Global 4-Cylinder Engine", SAE Technical Paper, 200501-1941.

[3] Hattori, M., Inoue, T., Mashiki, Z., Takenaka, A., Urushihata, H., Morino, S., and Inohara, T., 2008, "Devalopment of Variable Valve Timing System Controlled by Electric Motor", SAE Technical Paper, 2008-01-1358.

[4] Theobald, M., Lequesns, B., and Henry, R., 1994, "Control of Engine Load via Electromagnetic Valve Actuators," SAE Technical Paper, 940816.

[5] Sun, Z. and Kuo, T., 2010, "Transient Control of Electro-Hydraulic Fully Flexible Engine Valve Actuation System", IEEE Transactions on Control Systems Technology, 18(3), pp. 613-621.

[6] Ma, J., Zhu, G., and Schock, H., 2010, "A dynamic model of an electro-pneumatic valve actuator for internal combustion engines," ASME Journal of Dynamic Systems, Measurement and Control, 132(2), doi: 10.1115/1.4000816.

[7] Pierik, R. J. and Wilson, J. O., 1994, "Engine Timing Drive with Fixed and Variable Phasing", U.S. Patent 5,327,859.

[8] Urushihata, H. and Lida, H., 2008, "Variable Valve Timing Control Device of Internal Combustion Engine", U.S. Patent 7,363,896.

[9] Zhang, Y., Xie, H., Zhou, N., Chen, T., and Zhao, H., 2007, "Study of SI-HCCI-SI Transition on a Port Fuel Injection Engine Equipped with 4VVAS," SAE Technical Paper, 2007-01-0199.

[10] Cairns, A. and Blaxill, H., 2007, "The Effects of Two-Stage Cam Profile Switching and External EGR on SI-CAI Combustion Transitions," SAE Technical Paper, 2007-01-0187. 
[11] Shaver, G. M., et al, 2005, "Dynamic modeling of residual-affected homogeneous charge compression ignition engines with Variable Valve Actuation," ASME Journal of Dynamics, Measurement, and Control, 127(5), pp. 374-381.

[12] Shaver, G. M., 2005, Physics based modeling and control of residual-affected $\mathrm{HCCl}$ engines using Variable Valve Actuation, PhD thesis, Stanford University.

[13] Law, D., Kemp, D., Allen, J., Kirkpatrick, G., and Copland, T., 2001, "Controlled Combustion in an IC-Engine with a Fully Variable Valve Train", SAE Technical Paper, 2001-01-0251.

[14] Milovanovic, N., Chen, R., and Turner, J., 2004, "Influence of the Variable Valve Timing Strategy on the Control of a Homogeneous Charge Compression ( $\mathrm{HCCl}$ ) Engine", SAE Technical Paper, 2004-01-1899.

[15] Agrell, F., Angstrom, H., Eriksson, B., Wikander, J., and Linderyd, J., 2003, "Integrated Simulation and Engine Test of Closed Loop HCCl Control by Aid of Variable Valve Timings", SAE Technical Paper, 2003-01-0748.

[16] Zhu, G. and Skelton, R. E., 1994, "Integrated Modeling and Control for the Large Spacecraft Laboratory Experiment Facility", Journal of Guidance, Control and Dynamics, 17(3), pp. 442-450.

[17] Zhu, G., Grigoriadis, K. M., and Skelton, R. E., 1995, "Covariance Control Design for Hubble Space Telescope", Journal of Guidance, Control and Dynamics, 18(2), pp. 230-236.

[18] Zhu, G., Rotea, M. A., and Skelton, R., 1997, "A Convergent Algorithm for the Output Covariance Constraint Control Problem", SIAM J. Control Optim., 35(1), pp. 341-361.

[19] Ren, Z. and Zhu, G., 2011, "Integrated System ID and Control Design for an IC Engine Variable Valve Timing System", J. Dyn. Sys., Meas., Control, 133(2), 021012, doi: 10.1115/1.4003263.

[20] Skelton, R. E. and Anderson, B. D. O., 1986, “Q-Markov covariance equivalent realization," International Journal of Control, 53(1).

[21] Liu, K. and Skelton, R. E., 1991, "Identification and control of NASA's ACES structure," Proceedings of American Control Conference, Boston, Massachusetts, USA.

[22] Zhu, G., Skelton, R. E., and Li, P., 1995, "Q-Markov Cover identification using pseudorandom binary signals," International Journal of Control, 62(1), pp. 1273-1290.

[23] Zhu, G., 2000, "Weighted multirate q-Markov Cover identification using PRBS - an application to engine systems," Mathematical Problems in Engineering, 6, pp. 201-224.

[24] Shigley, J. E. and Mischke, C. R., 2001, Mechanical Engineering Design, 6th Edition, McGraw-Hill.

[25] Phillips, C. L. and Harbor, R. D., 2000, Feedback Control System, 4th Edition, Prentice Hall.

[26] Meerkov, S. and Runolfsson, T., 1989, "Output residence time control," IEEE Trans. Automat. Control, 34, pp. 1171-1176.

[27] Wilson, D. A., 1989, "Convolution and Hankel operator norms for linear systems," IEEE Trans. Automat. Control, 34, pp. 94-97.

[28] Zhu, G., Corless, M., and Skelton, R., 1989, "Robustness properties of covariance controllers," in Proceedings of Allerton Conf., Monticello, IL.

[29] Ren, Z. and Zhu, G., 2009, "Pseudo-random binary sequence closed-loop system identification error with integration control", Journal Proceedings of the Institution of Mechanical Engineers, Part I: Journal of Systems and Control Engineering, 233, pp. 877884. 
[30] Codrons, B., Anderson, B. D. O., and Gevers, M., 2002, "Closed-loop identification with an unstable or nonminimum phase controller," Automatica, 38, pp. 2127-2137.

[31] Peterson, W.W., 1961, Error Correcting Coding, MIT Technical Press, Cambridge, Massachusetts, USA.

[32] Anderson, B. D. O. and Skelton, R. E., 1988, "The Generation of all q-Markov Covers", IEEE Transactions on Circuits and Systems, 35(4), pp. 375-384.

[33] King, A. M., Desai, U. B., and Skelton, R. E., 1988, "A Generalized Approach to q-Markov Covariance Equivalent Realization for Discrete Systems," Automatica, 24(4), pp. 507-515.

[34] Shi, Y. and Burton, R., 2011, "Modeling and robust discrete-time sliding-mode control design for a fluid power electrohydraulic actuator (EHA) system," IEEE/ASME Transaction on Mechatronics, doi: 10.1109/TMECH.2011.2160959.

TABLE 1 PLANETARY SYSTEM PARAMETERS

\begin{tabular}{|c|c|c|c|}
\hline Component & Sun & Ring & Planet \\
\hline Number of teeth & 30 & 60 & 15 \\
\hline
\end{tabular}

TABLE 2: OUTPUT COMPARISON AT END OF EACH CYCLE

\begin{tabular}{|c|c|c|c|c|}
\hline \multirow{2}{*}{$\begin{array}{c}\text { Engine } \\
\text { speed }\end{array}$} & $\begin{array}{c}\text { Cycle } \\
\text { Number }\end{array}$ & \multicolumn{3}{|c|}{ Error (Deg) } \\
\cline { 2 - 5 } & 1 & +2.6 & +0.1 & +0.3 \\
\hline \multirow{4}{*}{ 1500rpm } & 2 & +1.8 & -1.0 & -0.6 \\
\cline { 2 - 5 } & 3 & +0.8 & -1.3 & -0.8 \\
\cline { 2 - 5 } & 4 & +0.0 & -0.8 & -0.5 \\
\hline \multirow{4}{*}{ 2000rpm } & 1 & +2.5 & +0.1 & +0.4 \\
\cline { 2 - 5 } & 2 & +1.7 & -1.2 & -0.7 \\
\cline { 2 - 5 } & 3 & +1.0 & -1.1 & -0.6 \\
\cline { 2 - 5 } & 4 & -0.2 & -1.3 & -0.9 \\
\hline
\end{tabular}

TABLE 3: OUTPUT COMPARISON AT 2000RPM WITH DIFFERENT SAMPLE RATE

\begin{tabular}{|c|c|c|c|c|}
\hline Sample & Cycle & \multicolumn{3}{|c|}{ Error (Deg) } \\
\cline { 3 - 5 } Rate & Number & PD & PD w/ff & OCC w/ ff \\
\hline \multirow{4}{*}{ 8/ cycle } & 1 & +2.2 & +0.4 & +0.7 \\
\cline { 2 - 5 } & 2 & +1.5 & -0.5 & +0.0 \\
\cline { 2 - 5 } & 3 & +0.8 & -1.0 & -0.5 \\
\cline { 2 - 5 } & 4 & +0.2 & -0.6 & -0.4 \\
\hline \multirow{3}{*}{$\begin{array}{c}\text { 16/ } \\
\text { cycle }\end{array}$} & 1 & +2.2 & +0.6 & +0.9 \\
\cline { 2 - 5 } & 2 & +1.5 & -0.2 & +0.4 \\
\cline { 2 - 5 } & 3 & +0.7 & -0.6 & -0.2 \\
\cline { 2 - 5 } & 4 & +0.0 & -0.5 & -0.1 \\
\hline
\end{tabular}


TABle 4: Nonzero CoefFicientS OF PRBS Polynomial

\begin{tabular}{|c|c|c|}
\hline Polynomial order $(n)$ & $\begin{array}{c}\text { Period of sequence } \\
(m)\end{array}$ & Non-zero Coefficient \\
\hline 6 & 63 & $\mathrm{a}_{5}, \mathrm{a}_{6}$ \\
\hline 7 & 127 & $\mathrm{a}_{4}, \mathrm{a}_{7}$ \\
\hline 8 & 255 & $\mathrm{a}_{2}, \mathrm{a}_{3}, \mathrm{a}_{4}, \mathrm{a}_{8}$ \\
\hline 9 & 511 & $\mathrm{a}_{5}, \mathrm{a}_{9}$ \\
\hline 10 & 1023 & $\mathrm{a}_{7}, \mathrm{a}_{10}$ \\
\hline 11 & 2047 & $\mathrm{a}_{9}, \mathrm{a}_{11}$ \\
\hline
\end{tabular}

TABLE 5: CLOSED-LOOP IDENTIFICATION PARAMETERS FOR THE EVVT BENCH

\begin{tabular}{|c|c|c|}
\hline Engine Speed (rpm) & 1000 & 1500 \\
\hline Input Sample Rate (ms) & 5 & 5 \\
\hline Output Sample Rate (ms) & 30 & 20 \\
\hline Output/Input Sample Ratio & 0.167 & 0.25 \\
\hline PRBS order & 13 & 13 \\
\hline Signal length (s) & 81.88 & 81.88 \\
\hline Markov parameter. \# & 100 & 100 \\
\hline ID open-loop model order & 4 & 4 \\
\hline
\end{tabular}

TABLE 6: FREQUENCY RESPONSES OF THE CLOSED-LOOP EVVT SYSTEM

\begin{tabular}{|c||c|c|c|c||c|c|c|c|}
\hline \multicolumn{1}{|c||}{$\begin{array}{c}\text { Input } \\
\text { Freq. } \\
(\mathrm{Hz})\end{array}$} & \multicolumn{4}{c||}{$1000 \mathrm{rpm}$} & \multicolumn{4}{c|}{$1500 \mathrm{rpm}$} \\
\cline { 2 - 9 } & Magnitude & \multicolumn{1}{c|}{ Phase (deg) } & Magnitude & \multicolumn{2}{c|}{ Phase (deg) } \\
\hline \hline \multirow{2}{*}{0.01} & 1 & 1 & 6 & 5 & 1 & 1 & 4 & 4 \\
\hline 0.1 & 0.98 & 0.98 & 21 & 16 & 0.97 & 0.97 & 21 & 9 \\
\hline 0.2 & 0.97 & 0.96 & 30 & 19 & 0.95 & 0.96 & 33 & 19 \\
\hline 0.4 & 0.95 & 0.97 & 46 & 30 & 0.93 & 0.95 & 50 & 21 \\
\hline 0.8 & 1.18 & 1.18 & 74 & 49 & 0.96 & 1.02 & 66 & 46 \\
\hline 1 & 1.33 & 1.35 & 94 & 58 & 0.95 & 0.98 & 87 & 58 \\
\hline 1.5 & 1.05 & 1.04 & 161 & 108 & 0.82 & 0.78 & 119 & 91 \\
\hline 2 & 0.70 & 0.72 & 202 & 137 & 0.60 & 0.61 & 144 & 115 \\
\hline
\end{tabular}




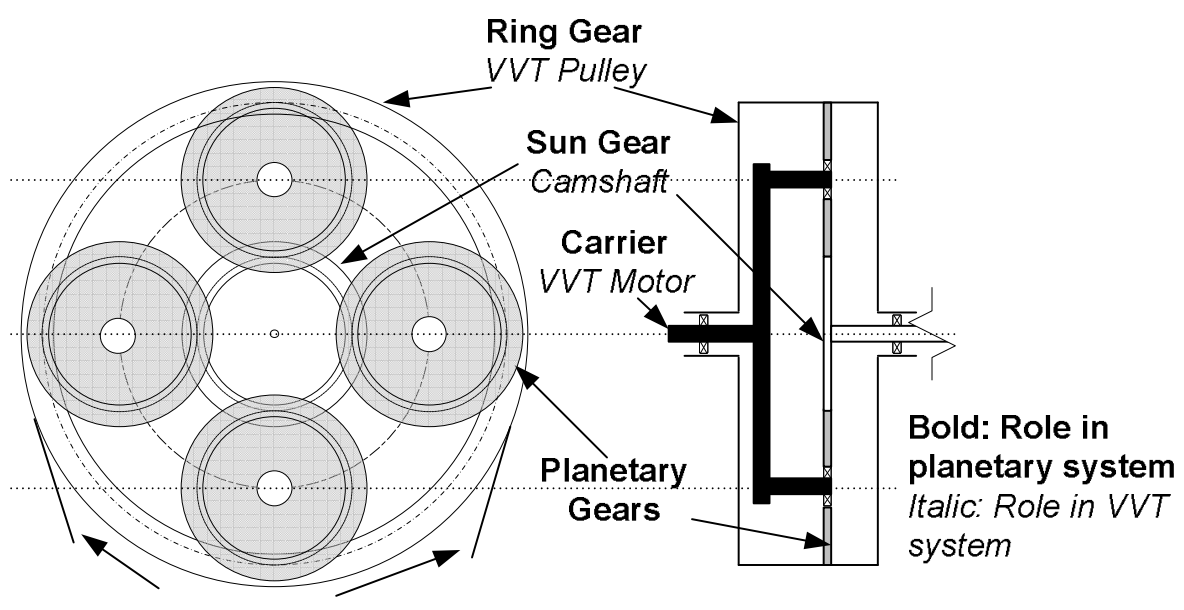

Figure 1: Electric planetary gear VVT system

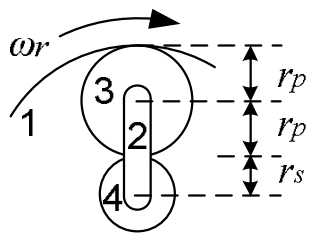

a) Planetary System

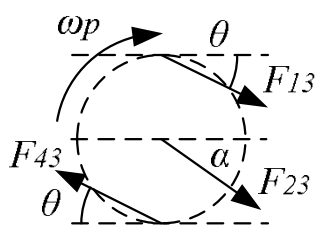

c) Planet Gear 3

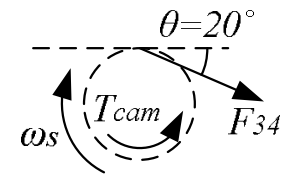

b) Sun Gear 4

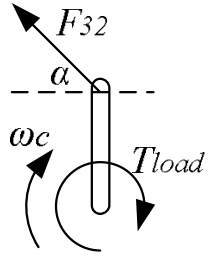

d) Carrier 2

Figure 2: Free body diagrams of planetary gear components

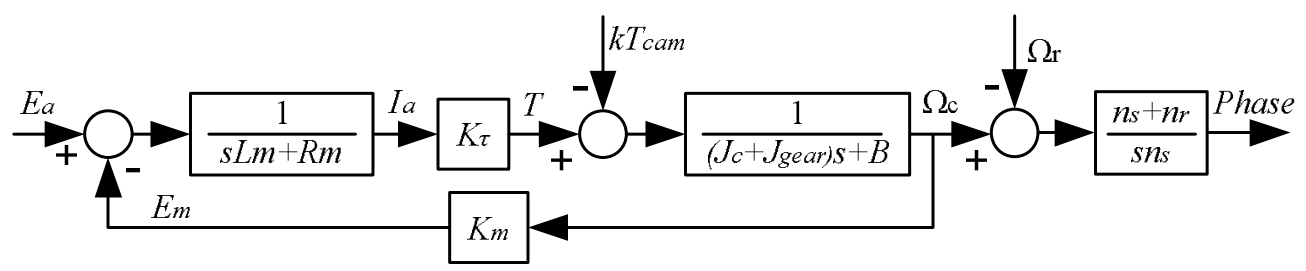

Figure 3: Block diagram of an electric motor with the planetary gear system 


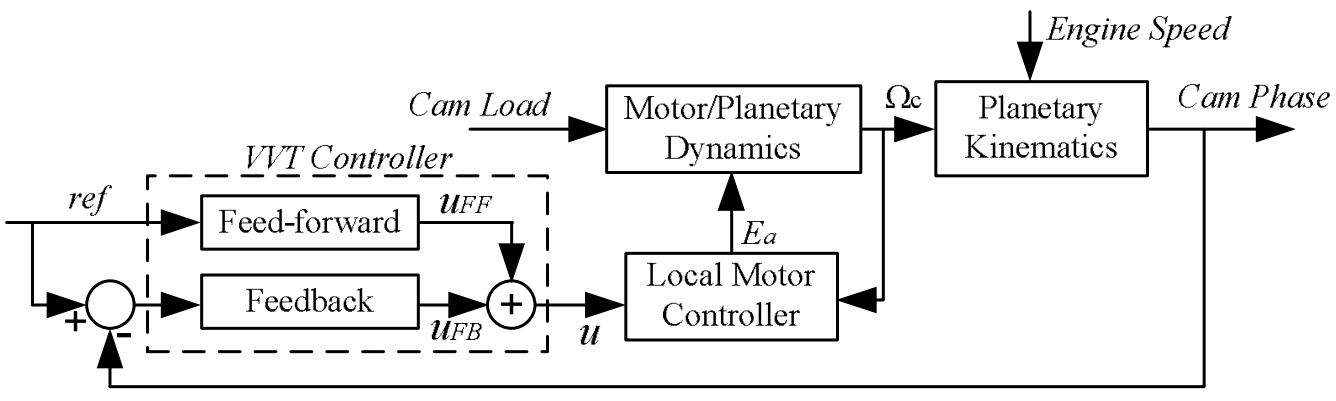

Figure 4: EVVT control system architecture

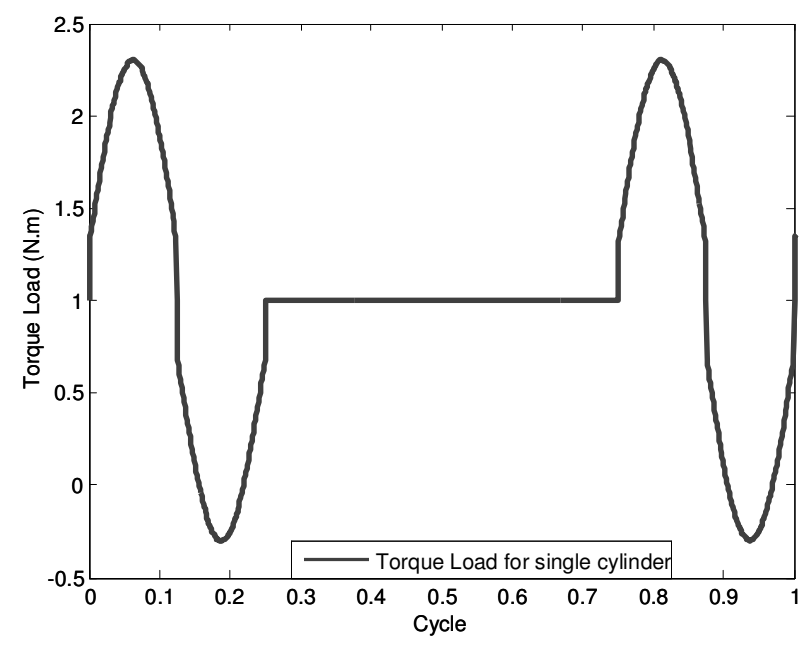

Figure 5: Torque load for single cylinder

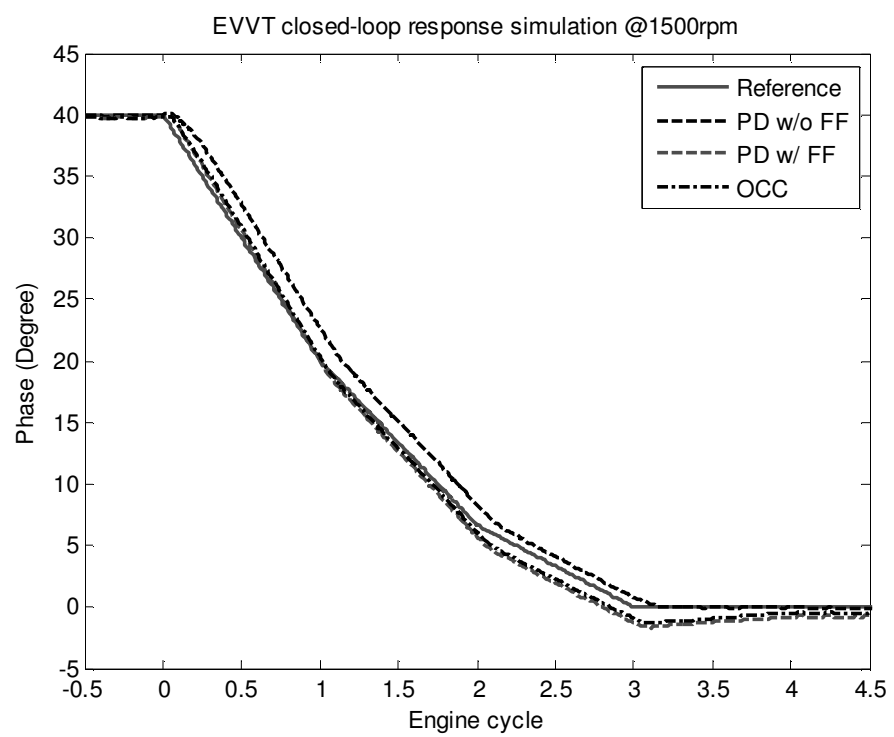

Figure 6: Simulated cam phase responses at $1500 \mathrm{rpm}$ 


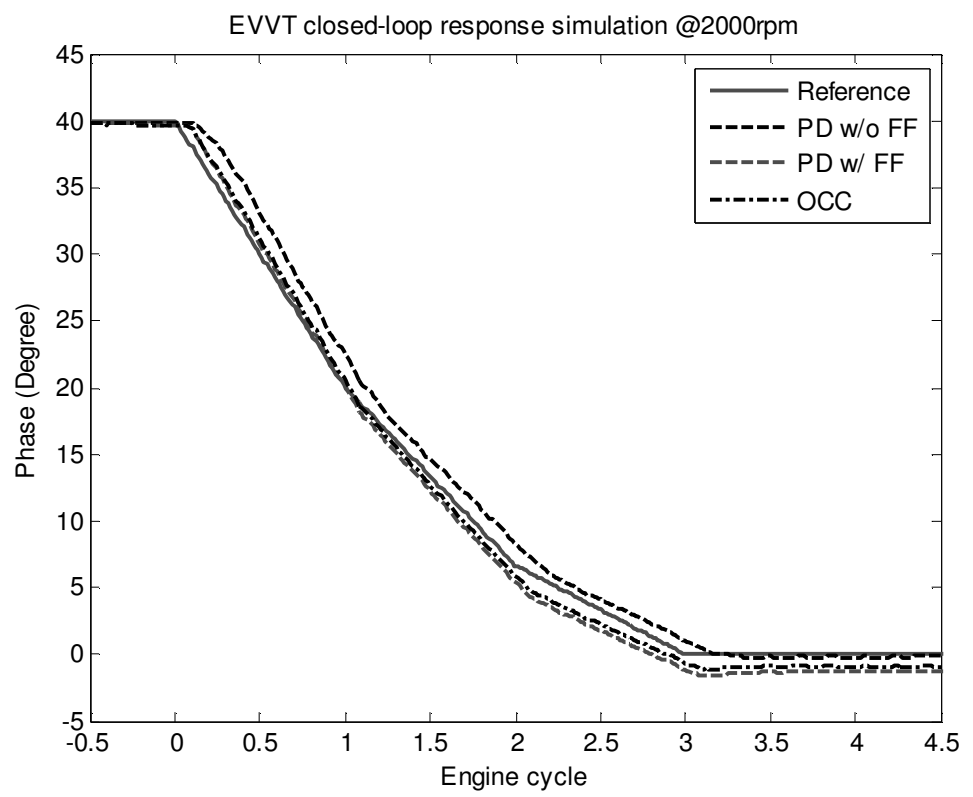

Figure 7: Cam phase output comparison at 2000 rpm

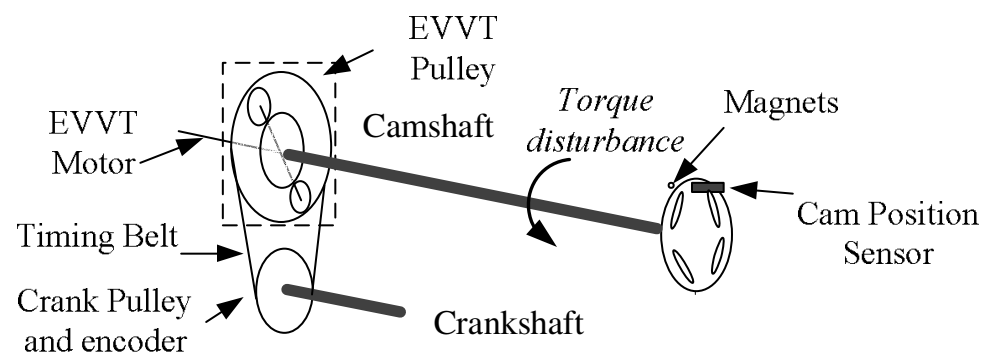

Figure 8: EVVT system test bench diagram

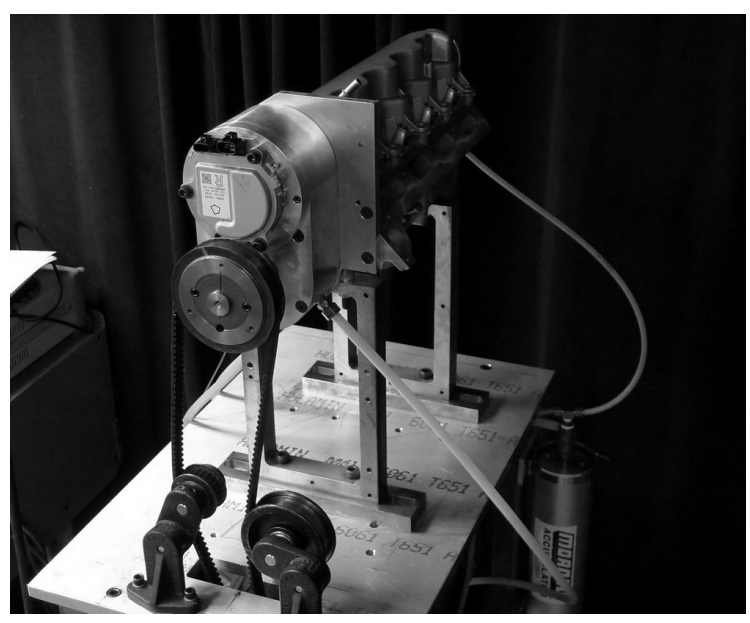

Figure 9: EVVT test bench 


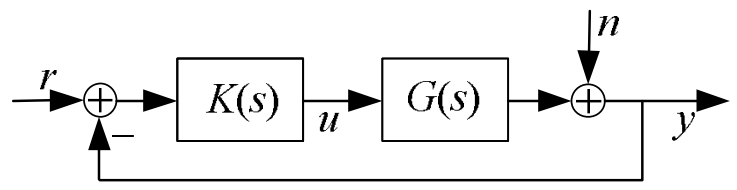

Figure 10: Closed-loop identification framework
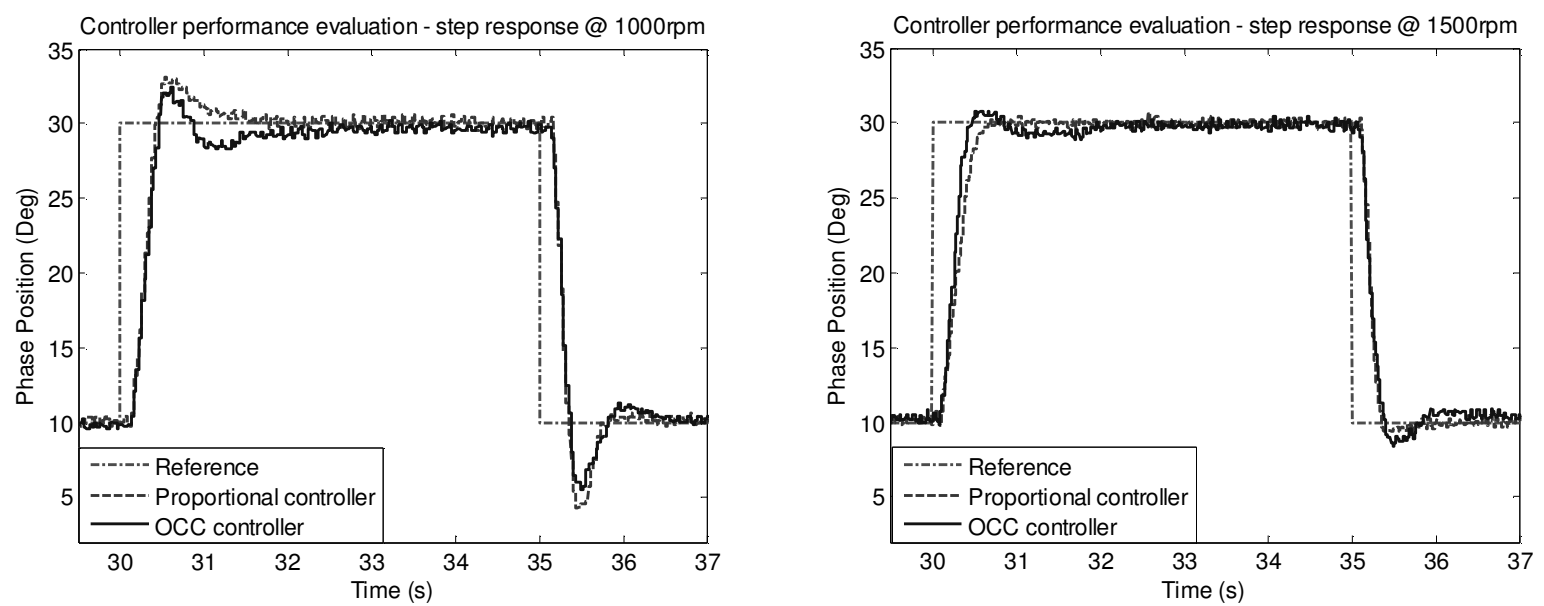

Figure 11: EVVT bench step response comparison
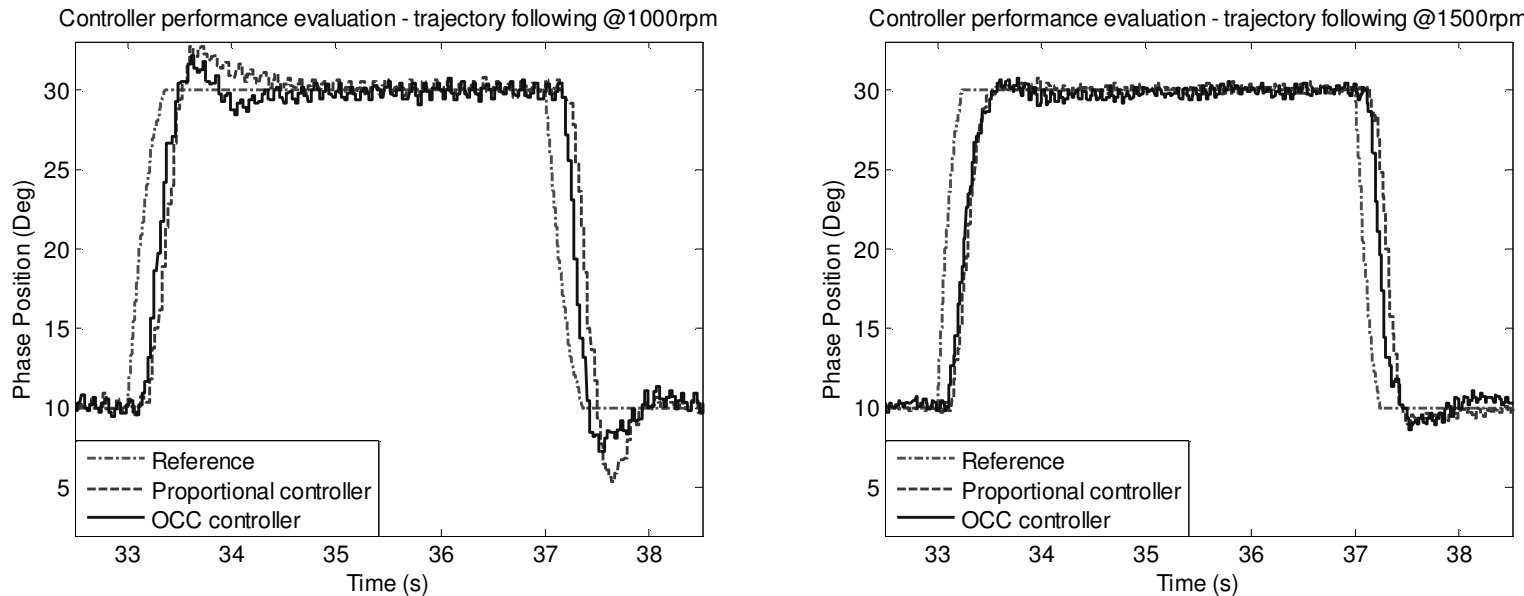

Figure 12: EVVT phase tracking comparison 

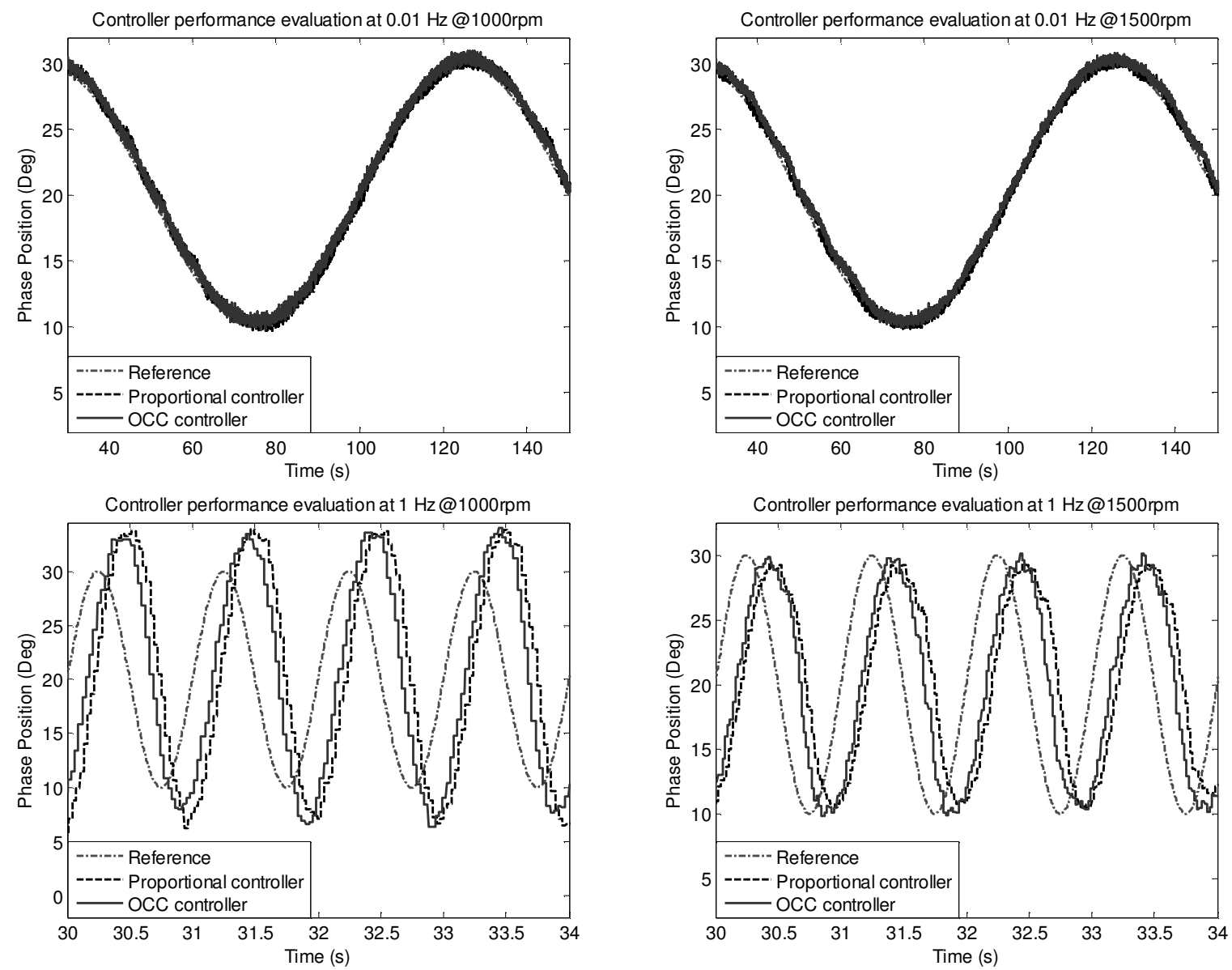

Figure 13: Sinusoidal responses of the closed-loop EVVT system at 0.01 and $1.00 \mathrm{~Hz}$

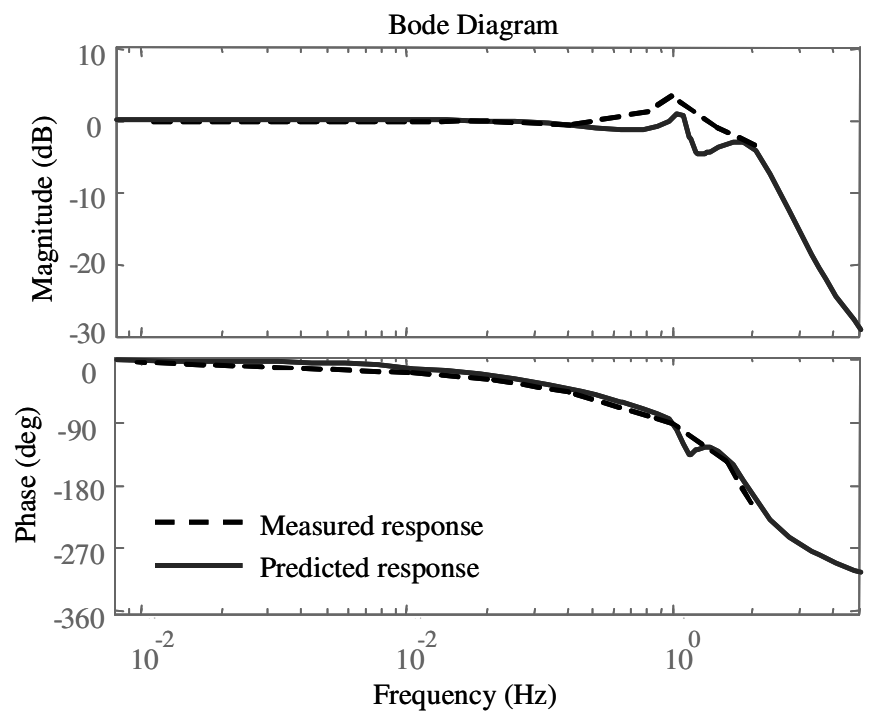

Figure 14: Measured and identified EVVT frequency responses at $1000 \mathrm{rpm}$ 
ASME Journal of Dynamic Systems, Measurement, and Control (DOI: 10.1115/1.4025914)
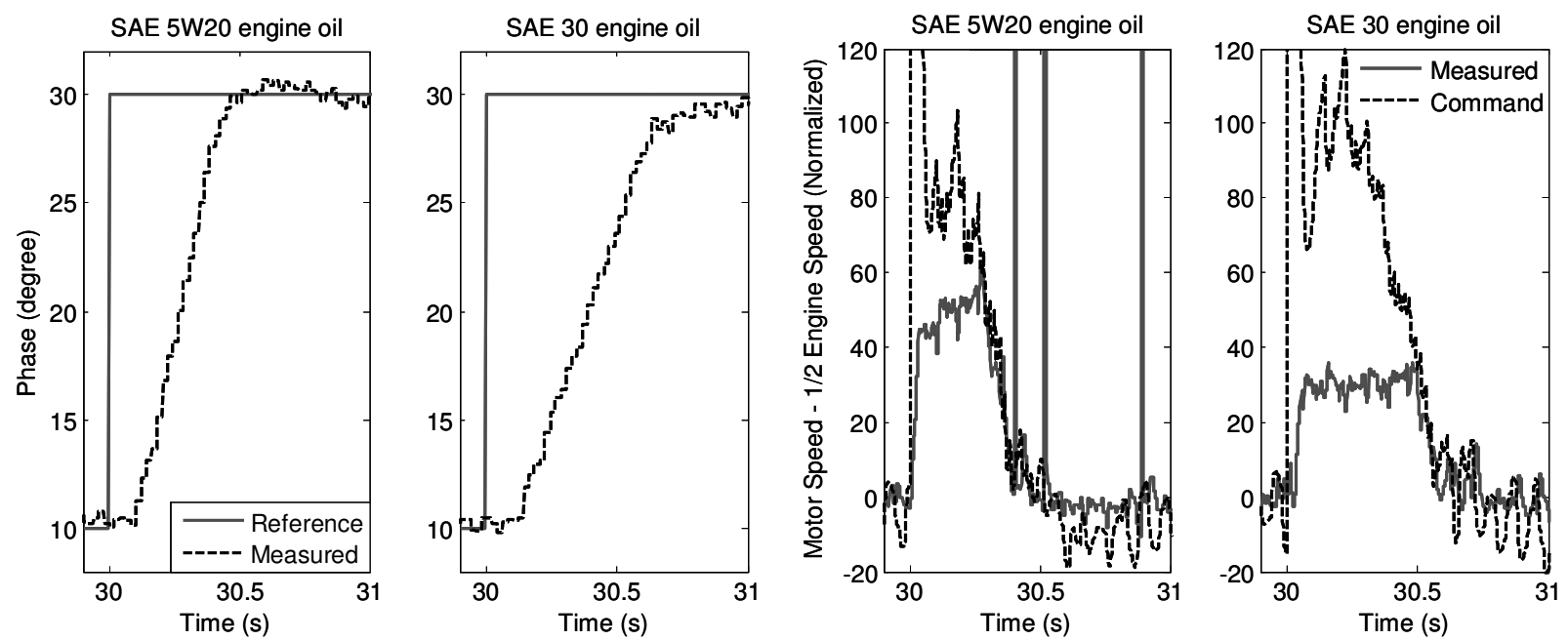

Figure 15: Impact of engine oil viscosity on EVVT response 
9.5 Published JC Paper \#5

SI and HCCI Combustion Mode Transition Control of an HCCI Capable SI Engine 


\title{
SI and HCCI Combustion Mode Transition Control of an HCCI Capable SI Engine
}

\author{
Xiaojian Yang and Guoming Zhu
}

\begin{abstract}
The combustion mode transition between spark ignition (SI) and homogeneous charge compression ignition (HCCI) combustion of an internal combustion (IC) engine is challenging due to the distinct engine operating parameters over the two combustion modes and the cycle-to-cycle residue gas dynamics during the mode transition. The control problem becomes even more complicated for a multicylinder engine without camless variable valve actuators. This paper studies the combustion mode transition problem of a multicylinder IC engine equipped with dual-stage valve lift and electrical variable valve timing (VVT) systems. Hardware-in-the-loop (HIL) simulations were used as a tool to develop and validate the proposed control strategies. Based on the HIL simulation results, this paper shows that smooth combustion mode transition can be realized in a few engine cycles. During the mode transition, a model-based linear quadratic tracking strategy was used to track the desired engine manifold pressure through the engine throttle control to maintain the engine AFR in a desired range; the fuel quantity of individual cylinder was controlled via the iterative learning; and engine spark was maintained for the SI-HCCI (or spark assistant) hybrid combustions during the combustion mode transition. The HIL simulations demonstrated the effectiveness of the developed control strategies under both steady state and transient engine operating conditions. As a result, it is feasible to have a smooth combustion mode transition for an HCCI capable SI engine equipped with dual-stage valve lift and electrical VVT systems.
\end{abstract}

Index Terms-Closed-loop systems, control synthesis, numerical simulation, optimal control.

\section{NOMENCLATURE}

$\lambda \quad$ Normalized air-to-fuel ratio.

$P \quad$ In-cylinder gas pressure (bar).

$\theta \quad$ Crank angle position (degree ACTDC).

$\theta_{\text {ST }} \quad$ Spark timing (degree ACTDC).

$\theta_{\text {SOHCCI }}$ Start of HCCI combustion timing (degree ACTDC).

IETC Actuator current of ETC system (A).

$F_{\mathrm{DT}} \quad$ DI fueling pulse duration (ms/cycle).

$F_{\mathrm{FB}}, F_{\mathrm{FF}} \quad$ Feedback/feedforward portions of $F_{\mathrm{DT}}$ (ms/cycle).

$F_{\text {ILC }} \quad$ ILC portion of DI pulse duration (ms/cycle).

Manuscript received June 25, 2011; accepted October 18, 2011. Manuscript received in final form May 22, 2012. This work was supported in part by the U.S. Department of Energy under Grant DE-EE0000211 and in part by the National Science Foundation under Grant CMMI 1030360. Recommended by Associate Editor G. E. Stewart.

The authors are with the Mechanical Engineering Department, Michigan State University, East Lansing, MI 48824 USA (e-mail: yangxia2@egr.msu.edu; zhug@egr.msu.edu).

Color versions of one or more of the figures in this paper are available online at http://ieeexplore.iee.org.

Digital Object Identifier 10.1109/TCST.2012.2201719

\author{
$x_{\mathrm{EGR}}$ \\ $\phi_{\text {TPS }}$ \\ $\phi_{\text {acc }}$ \\ $\phi_{\mathrm{EGR}}$ \\ $\Pi_{\text {lift }}$ \\ $T_{\text {IVC }}$ \\ $M_{\mathrm{EVC}}$ \\ $\theta_{\mathrm{INTM}}$ \\ $\theta_{\text {EXTM }}$ \\ $V_{d}$ \\ $V_{m}$ \\ $N_{e}$ \\ $R_{a}$ \\ $P_{a}$ \\ $T_{a}$ \\ $r$ \\ $k_{\mathrm{ETC}}$ \\ $b_{\text {ETC }}$ \\ $c_{\text {ETC }}$ \\ $i$ \\ j \\ k \\ MAP \\ IMEP \\ IMEP $_{\text {ref }}$ \\ ACTDC \\ AGTDC \\ BGTDC \\ DI \\ EGR \\ ETC \\ EVC \\ HCCI \\ HIL \\ ILC \\ IVC \\ LQ \\ MFB \\ NVO \\ SI \\ TDC \\ UEGO \\ VVT \\ EGR gas fraction in engine intake manifold. \\ Engine throttle position (\%). \\ Acceleration pedal position (\%). \\ External EGR valve opening (\%). \\ Peak lift of intake/exhaust valve ( $\mathrm{mm})$. \\ In-cylinder gas temperature at $\operatorname{IVC}\left({ }^{\circ} \mathrm{K}\right)$. \\ Residue gas mass at EVC (mg). \\ Intake valve timing at peak lift (degree \\ AGTDC). \\ Exhaust valve timing at peak lift (degree \\ BGTDC). \\ Engine displacement $\left(\mathrm{m}^{3}\right)$. \\ Volume of intake manifold $\left(\mathrm{m}^{3}\right)$. \\ Engine speed (rpm). \\ Gas constant (J/gK). \\ Ambient pressure (bar). \\ Ambient temperature (K). \\ Radius of throttle plate $(\mathrm{m})$. \\ Spring constant of throttle plate. \\ Damping ratio of throttle plate. \\ Torque constant of throttle drive motor \\ $(\mathrm{N} \cdot \mathrm{m} / \mathrm{A})$. \\ Index of ILC iteration. \\ Engine cycle index. \\ Index of sampling time for time based control \\ logic. \\ Intake manifold absolute pressure (bar). \\ Indicated mean effective pressure (bar). \\ IMEP learning reference used in ILC (bar). \\ After combustion TDC. \\ After gas exchange TDC. \\ Before gas exchange TDC. \\ Direct injection. \\ Exhaust gas recirculation. \\ Electric throttle control. \\ Exhaust valve closing. \\ Homogeneous charge compression ignition. \\ Hardware-in-the-loop. \\ Iterative learning control. \\ Intake valve closing. \\ Linear quadratic. \\ Mass fraction burned. \\ Negative valve overlap. \\ Spark ignition. \\ Top dead center. \\ universal exhaust gas oxygen. \\ Variable valve timing.
}


VVA Variable valve actuation.

WOT Wide open throttle.

\section{INTRODUCTION}

$\mathbf{H}$ OMOGENEOUS charge compression ignition (HCCI) combustion has the potential for internal combustion (IC) engines to meet the increasingly stringent emission regulations with improved fuel economy [1]. The flameless nature of the HCCI combustion and its high dilution operation capability lead to low combustion temperature. As a result, the formation of NOx (nitrogen oxides) can be significantly reduced [2]. Furthermore, HCCI engine is capable of unthrottled operation that greatly reduces pumping loss and improves fuel economy [3], [4].

On the other hand, HCCI combustion has its own limitations. It is limited at high engine load due to the audible knock; and at low load due to engine misfire caused by the lack of sufficient thermal energy to initiate the auto-ignition of the gas-fuel mixture during the compression stroke [5]. In fact, HCCI combustion can be regarded as a type of engine operating mode rather than a type of engine [6]. In order to take advantage of the HCCI combustion mode in an spark ignition (SI) engine, the traditional SI combustion mode, is required at high load, at high speed, at ultralow load (such as idle), and at certain operating conditions, such as cold start.

It is fairly challenging to operate the engine in two distinct combustion modes, and it is even more difficult to have the smooth combustion mode transition between the SI and HCCI combustions, because the favorable thermo conditions for one combustion mode are always adverse to the other [7]. For example, high intake charge temperature is required in the HCCI mode to initiate the combustion, while in the SI mode it leads to reduced volumetric efficiency and increased knock tendency. For this reason, engine control parameters, such as intake and exhaust valve timings and lifts, throttle position and exhaust gas recirculation (EGR) valve opening, are controlled differently between these two combustion modes. During the combustion mode transition, these engine parameters need to be adjusted rapidly. However, the physical actuator limitations on response time prevent them from completing their transitions within the required duration, specifically, within one engine cycle. The multicylinder operation makes it challenging [8]. And this problem becomes more difficult when two-stage valve lift and electrical variable valve timing (VVT) systems are adopted. Accordingly the combustion performance during the transition cannot be maintained unless proper control strategy is applied.

The control of the HCCI combustion process has been widely studied in past decades. Robust HCCI combustions can be achieved through model-based control as described in [9]-[11]. To make the HCCI combustion feasible in a practical SI engine, the challenge of the combustion mode transition is inevitable. In recent years, more and more attentions have been paid to the mode transition control between the SI and HCCI combustions. In [12] and [13], smooth mode transitions between the SI and HCCI combustions are realized for a single cylinder engine equipped with the camless variable valve actuation (VVA) system. However, high cost prevents the implementation of the camless VVA system in production engines. In [14], a VVT system with dual-stage valve lift is used on a multicylinder engine for the study of the mode transition. Experimental results show the potential of achieving smooth mode transition by controlling the step throttle opening timing and the direct injection (DI) fuel quantity. However, satisfactory mode transition has not been accomplished due to the lack of the robust mode transition control strategy. Reference [15] uses a hybrid robust control of air-path for dual mode diesel engines with conventional and low temperature combustions, and the control of the thermoacoustic instability is studied in [16].

In this paper, a control strategy of the combustion mode transition was developed. The studied four-cylinder engine is equipped with external cooled EGR, dual-stage valve lift and electrical VVT systems. Hardware-in-the-loop (HIL) simulations were used for control development and validation. For this paper, the real-time HIL engine model used in the HIL simulation was validated by the well-known GT-Power model [5] that has been calibrated using experimental data. It is also worth mentioning that the GT-Power modeling tool is widely used in automotive industry to predict the engine performance. Also the control strategies, discussed in this paper, mainly deal with the engine intake charge and air-to-fuel ratio (AFR) dynamics that the GT-Power-based model (1-D flow dynamic model) is capable of predicting them accurately and the HIL model (also a 1-D flow dynamic model) matches with the GT-Power simulations closely. Therefore, it is believed that the proposed strategies can be applied to an actual HCCI capable SI engine; however, the control algorithm might need to be recalibrated to compensate for the model calibration error between the model used for control design and the physical engine system. Also, the HIL simulation model used for the mode transition simulations did not include emission models and the emission performance was not evaluated.

The HIL simulation results demonstrated that unstable combustions during the transition can be eliminated by using the multistep strategy as discussed in [5]. In addition, the linear quadratic (LQ) optimal MAP tracking control strategy was developed to maintain the AFR in the desired range. Under the optimal MAP control, smooth combustion mode transition was achieved with the help of the iterative learning control (ILC) of the DI fuel quantity of individual cylinder. The entire control strategy was validated in the HIL engine simulation environment [17], and satisfactory engine performance was achieved during the combustion mode transition for both steady state and transient operating conditions. It is also worth mentioning that our control algorithm assumes that the incylinder pressure sensor is available for feedback control. Since both price and reliability of the pressure sensors are continued improving and we believe that it will be feasible for production engines in near future. The other option is to use the in-cylinder ionization sensing.

This paper is organized as follows. Section II discusses the control performance target of the combustion mode transition and the associated engine configuration. Section III introduces the SI-HCCI hybrid combustion mode and the associated 


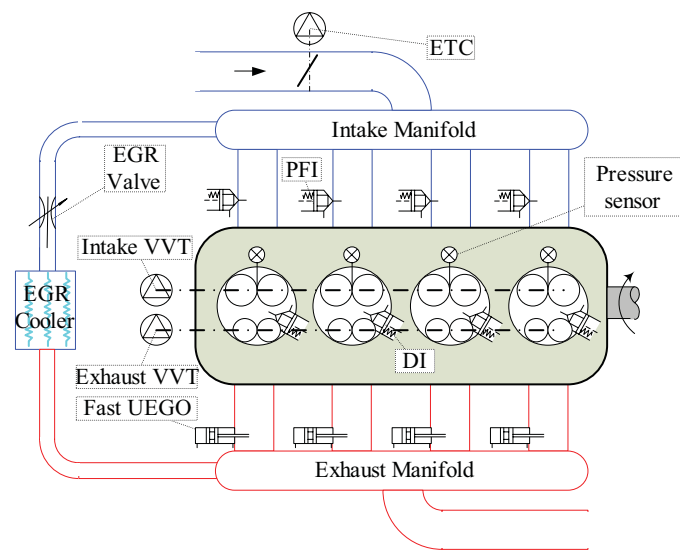

Fig. 1. Configuration of the HCCI capable SI engine.

TABLE I

HCCI CAPABLE SI ENGINE SPECIFICATIONS

\begin{tabular}{ll}
\hline Engine parameter & Model value \\
\hline Bore/stroke/con-rod length & $86 \mathrm{~mm} / 86 \mathrm{~mm} / 143.6 \mathrm{~mm}$ \\
Compression ratio & $9.8: 1$ \\
Intake/exhaust valve lifts of high stage & $9 \mathrm{~mm} / 9 \mathrm{~mm}$ \\
Intake/exhaust valve lifts of low stage & $5 \mathrm{~mm} / 5 \mathrm{~mm}$ \\
Intake/exhaust valve timing range & $\pm 40^{\circ} / \pm 40^{\circ}$ \\
Intake/exhaust valve lifts lash & $0.2 \mathrm{~mm} / 0.25 \mathrm{~mm}$ \\
Intake manifold volume & $3.2 \mathrm{~L}$ \\
Throttle diameter & $42 \mathrm{~mm}$ \\
\hline
\end{tabular}

control oriented model. The multistep combustion mode transition strategy is proposed in Section IV, and the following two sections present the IMEP control and the AFR tracking control strategies. In Section VII, the real-time HIL engine simulation system is described, and the simulation results are presented for validating the control strategies. Conclusions are finally drawn in Section VIII.

\section{Combustion Mode Transition Control Problem}

The purpose of the combustion mode transition control is to minimize the engine output torque fluctuation during the mode transition process.

\section{A. Target Engine Configuration}

Fig. 1 shows the configuration of the target HCCI capable SI engine, and the engine specifications are listed in Table I. The key feature of this engine is its valve train system. It has two-stage lift for both intake and exhaust valves. The high lift is $9 \mathrm{~mm}$ for the SI combustion mode, and the low lift is $5 \mathrm{~mm}$ for the HCCI combustion mode. The ranges of both intake and exhaust valve timings are extended to \pm 40 crank degrees to improve the controllability of the internal EGR fraction, the effective compression ratio, and the engine volumetric efficiency during the combustion mode transition and HCCI operations.

In addition to the modification of the engine valvetrain, external cooled EGR is used to enable high dilution charge with low charge mixture temperature. The engine throttle can be electronically controlled to obtain the desired engine charge
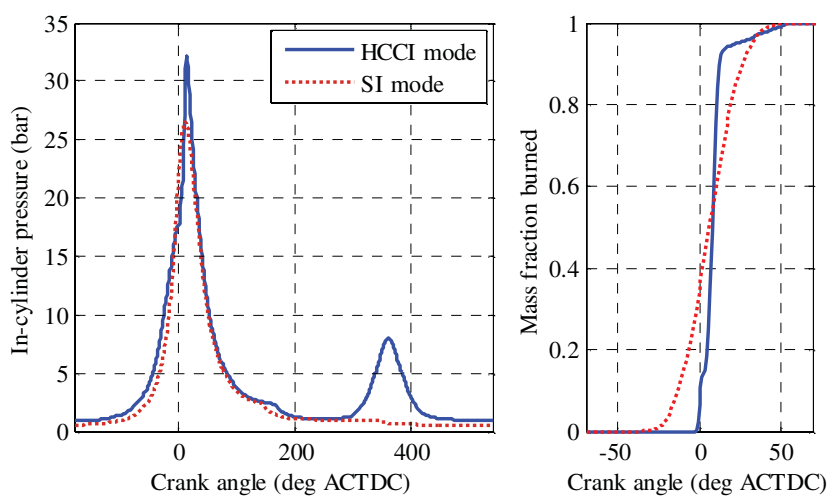

Fig. 2. Steady state combustion characteristics of SI and HCCI modes.

TABLE II

ENGINE CONTROL PARAMETERS FOR SI AND HCCI COMBUSTION MODES

\begin{tabular}{lcc}
\hline Engine control parameter & SI & HCCI \\
\hline$\theta_{\text {ST }}($ deg ACTDC) & -36 & None \\
$\varphi_{\text {EGR }}(\%)$ & 3 & 26 \\
$I_{\text {ETC }}(\mathrm{A})$ & 0.84 & 5 \\
$F_{\text {DT }}(\mathrm{ms} /$ cycle $)$ & 2.06 & 1.6 \\
$\theta_{\text {INTM }}($ deg AGTDC) & 70 & 95 \\
$\theta_{\text {EXTM }}($ deg BGTDC) & 100 & 132 \\
$\Pi_{\text {lift }}(\mathrm{mm})$ & 9 & 5 \\
\hline
\end{tabular}

in both SI and unthrottled HCCI operations. Each cylinder of the test engine is equipped with piezoelectric pressure transducer. Four fast response universal exhaust gas oxygen (UEGO) sensors are installed at the exhaust ports for feedback control.

\section{B. SI and HCCI Steady-State Operational Parameters}

For this paper, the combustion mode transition was studied for the engine operated at $2000 \mathrm{rpm}$ with 4.5 bar indicated mean effective pressure (IMEP). Table II lists the engine parameters associated with the SI and HCCI combustions. These parameters were optimized for the steady state engine operations with the best fuel economy that satisfies the engine knock limit requirement. That is, the maximum pressure rise rate $(\mathrm{d} P / \mathrm{d} \theta)$ is less than or equal to 3 bar per crank degree. It can be seen in Table II that the optimized engine control parameters are quite different between the SI and HCCI combustion modes. Some of these parameters can be adjusted within one engine cycle, such as spark timing $\theta_{\mathrm{ST}}$, ETC drive current $I_{\mathrm{ETC}}$, DI fuel quantity $F_{\mathrm{DT}}$, and valve lift $\Pi_{\text {lift }}$; the others cannot due to actuator dynamics.

The combustion characteristics are also quite different between these two combustion modes as illustrated in Fig. 2. For example, the HCCI combustion has higher peak incylinder pressure comparing with that of the SI combustion due to the faster fuel burn rate. Most likely, it also has a recompression phase (see the second peak of the solid line in Fig. 2) due to negative valve overlap (NVO) operation, while the SI combustion does not. The goal of the combustion mode transition is to switch the combustion mode without 


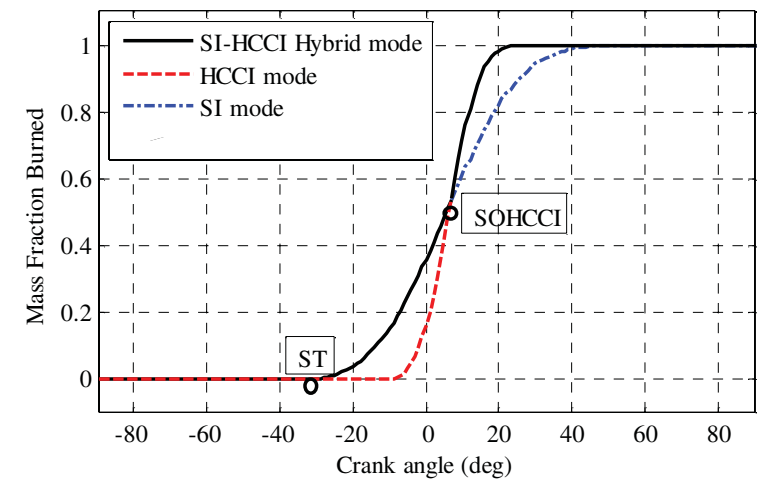

Fig. 3. MFB trace of SI-HCCI hybrid combustion mode.

detectable engine torque fluctuation by regulating the engine control parameters, or in other words, to maintain the engine IMEP during the combustion mode transition.

\section{SI-HCCI HybRid COMbustion Mode}

The earlier work in [18] demonstrated that the engine charge temperature $\left(T_{\mathrm{IVC}}\right)$ has response delay during the combustion mode transition, mainly caused by the response delays of the engine intake/exhaust valve timings. Under such transient conditions, if the engine was forced to switch to the HCCI combustion mode the engine IMEP could not be maintained with cycle-by-cycle fuel control $F_{\mathrm{DT}}$. Also the increased charge cooling effect caused by the increment of $F_{\mathrm{DT}}$ reduces the charge temperature and leads to degraded HCCI combustion. However, the transitional thermo condition is suitable for the SI-HCCI hybrid combustion mode proposed in [5] and [17].

By maintaining the engine spark, combustion during the mode transition could start in the SI combustion mode with a relatively low heat release rate, and once the thermo and chemical conditions of the unburned gas satisfy the start of HCCI (SOHCCI) combustion criteria, the combustion continues in HCCI combustion mode, which is illustrated by the solid curve of mass fraction burned (MFB) shown in Fig. 3. During an ideal SI to HCCI combustion transition process, the HCCI combustion percentage (the vertical distance from SOHCCI to MFB $=1$ ) increases gradually along with the increment of charge temperature $\left(T_{\mathrm{IVC}}\right)$. For the HCCI to SI combustion transition, the process is reversed. More importantly, during the SI-HCCI hybrid combustion, engine IMEP can be controlled by regulating the DI fuel quantity. This is the other motivation of utilizing the hybrid combustion mode during the combustion mode transition.

In [5] and [17], a crank-based SI-HCCI hybrid combustion model was developed for real-time control strategy development. It models the SI combustion phase under two-zone assumptions and the HCCI combustion phase under one-zone assumptions. The SI and HCCI combustion modes are actually special cases of the SI-HCCI hybrid combustion mode in the model, since the SI combustion occurs when the HCCI combustion percentage is zero, and the HCCI combustion occurs when the percentage is hundred. Accordingly this combustion model is applicable for all combustion modes

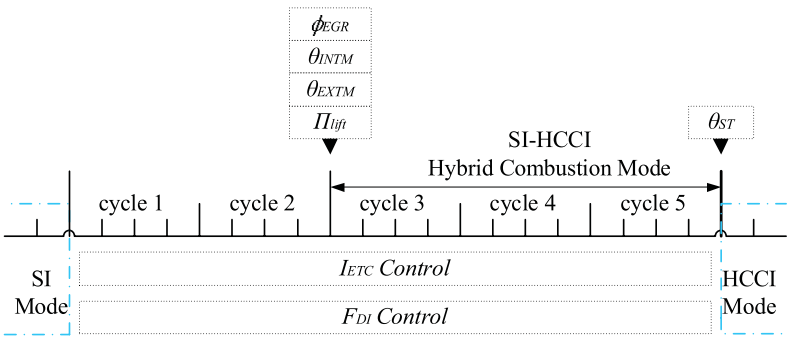

Fig. 4. Multistep SI to HCCI combustion mode transition control schedule.

during the mode transition. In the engine model, the cycleto-cycle dynamics of engine combustion can be expressed in a nonlinear state space form as follows:

$$
\begin{aligned}
x(j+1) & =f(x(j), u(j)) \\
y(j) & =h(x(j), u(j))
\end{aligned}
$$

where $x(j), u(j)$, and $y(j)$ are states, inputs, and outputs, respectively. They are defined by

$$
\begin{aligned}
& x(j)=\left[M_{\mathrm{EVC}}(j) T_{\mathrm{IVC}}(j)\right]^{T} \\
& u(j)=\left[\theta_{\mathrm{ST}} F_{\mathrm{DT}} \theta_{\mathrm{INTM}} \theta_{\mathrm{EXTM}} x_{\mathrm{EGR}} \operatorname{MAP}_{\text {lift }}\right]^{T}(j) \\
& y(j)=\operatorname{IMEP}(j) .
\end{aligned}
$$

The state and output functions, $f$ and $h$, in (1) are composed by the governing equations of the engine combustion process. The details of the model are described in [5] and [17].

\section{Multistep Mode Transition Control Strategy}

In [18], the one-step combustion mode transition was investigated. The control references of all engine parameters were directly switched from the SI to HCCI mode, as listed in Table II, in one engine cycle. The simulation results showed that misfired combustions occur during the one-step mode transition, and significant torque fluctuation was discovered. Thereby, a multistep mode transition strategy was proposed in [18] by inserting a few hybrid combustion cycles between the SI and HCCI combustions, see Fig. 4. The control strategy proposed in this paper is based on this multistep strategy. Ideally, we would like to have the lowest number of engine cycles for the mode transition. For this specific engine architecture, since it takes three cycles for the engine valve timing to transit to its target timing, we picked three transition cycles. Due to the pre-throttle opening requirement to avoid rich combustions, two more engine cycles were added, which results a five-cycle transition process. For the engine operated at $1500 \mathrm{rpm}$ it takes $0.4 \mathrm{~s}$.

As illustrated in Fig. 4, five engine cycles are used during the SI to HCCI mode transition. During the transitional cycles some engine parameters are adjusted in an open loop manner according to the schedule shown in Fig. 4. Cycles 1 and 2 are used for engine throttle preopening control. They provide enough time for the engine MAP to rise to compensate the $\Pi_{\text {lift }}$ switch. At the end of cycle 2, the intake/exhaust valve lift $\Pi_{\text {lift }}$ switches from high to low lift, and the control references of $\varphi_{\mathrm{EGR}}, \theta_{\mathrm{INTM}}$, and $\theta_{\mathrm{EXTM}}$ are set to those of the steady state HCCI combustion mode as listed in Table II. Spark timing 


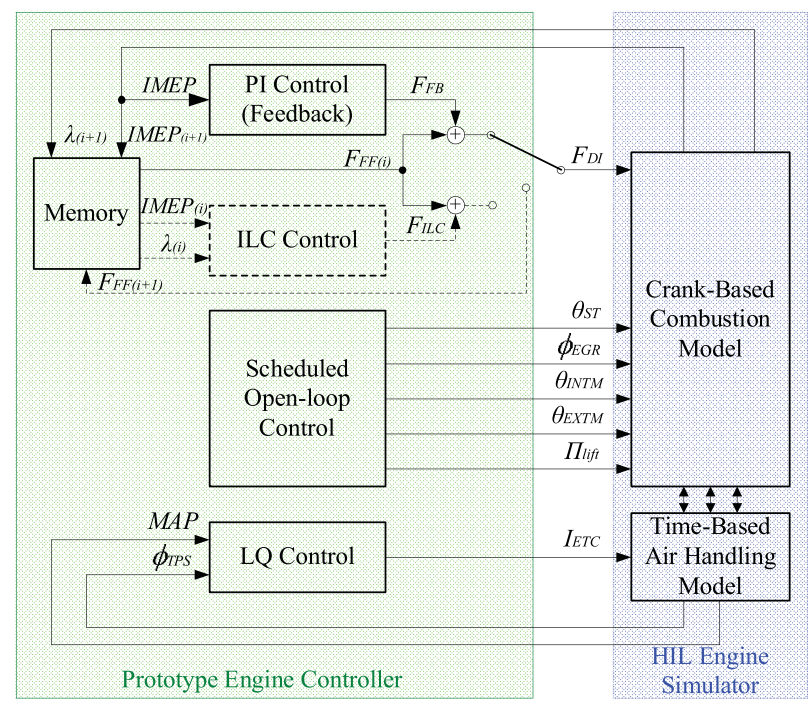

Fig. 5. SI to HCCI combustion mode transition control diagram.

$\theta_{\text {ST }}$ of each cylinder was kept constant during the transitional cycles and was eliminated at the end of cycle 5. Throughout the transitional cycles, the engine control parameters $I_{\text {ETC }}$ and $F_{\mathrm{DT}}$ are regulated by time-based $(1 \mathrm{~ms}$ sampling rate) and cycle-based controls, respectively. The corresponding control algorithm will be described in the next two sections. Since the mode transition between the SI and HCCI combustions occurs over a relatively small speed and load range, about five to six transition points will be used to form lookup tables for each "scheduled open-loop control" parameters $\left(\theta_{\mathrm{ST}}, \phi_{\mathrm{EGR}}, \theta_{\mathrm{INTM}}\right.$, $\theta_{\text {EXTM }}$, and $\left.\Pi_{\text {lift }}\right)$ shown in Fig. 5.

Under the regulations of the engine parameters, the combustion characteristics during the transitional engine cycles are different from those of the typical SI and HCCI combustion modes. The combustion at cycles 1 and 2 are still in SI combustion mode, but the AFR is no longer stoichiometric and most likely is lean due to the throttle $\left(I_{\mathrm{ETC}}\right)$ preopening. From cycles 3 to 5 , engine charge temperature $T_{\text {IVC }}$ is higher than that of the SI mode and lower than the desired temperature for the HCCI mode. Under such condition, the engine is operated in the SI-HCCI hybrid combustion mode from cycles 3 to 5 due to the maintained engine spark. The main motivation of operating the engine at the SI-HCCI hybrid combustion mode is to use the SI combustion to increase the temperature of the unburned gas mixture so that the HCCI combustion can be achieved later in the same engine cycle.

Fig. 8 shows the diagram of the multistep combustion mode transition controller and its interface with the HIL engine simulator that will be introduced in Section VII. The multistep combustion mode transition is activated as engine IMEP switches from above IMEP ref $\left(\mathrm{IMEP}_{\text {ref }}=4.5 \mathrm{bar}\right.$ in

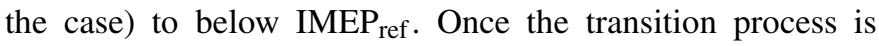
initiated, the engine parameters are controlled in different ways. The engine IMEP (or torque) is directly controlled by regulating $F_{\mathrm{DT}}$ of individual engine cylinder; the engine AFR is regulated by $I_{\mathrm{ETC}}$ through an LQ optimal MAP tracking control strategy; spark timing $\left(\theta_{\mathrm{ST}}\right)$, EGR valve opening

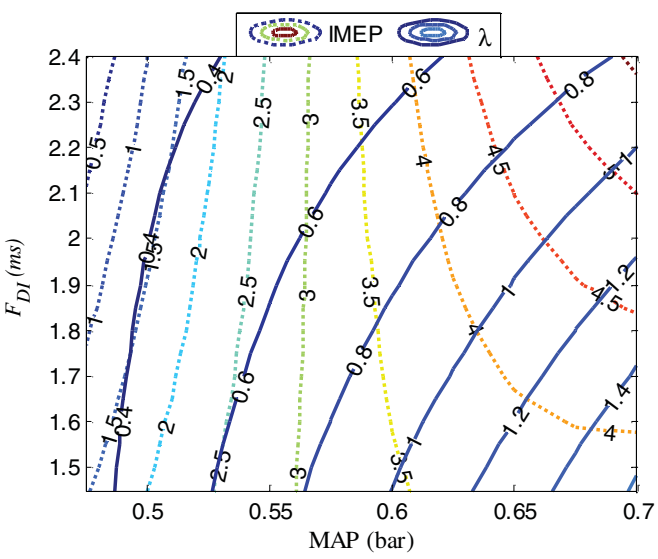

Fig. 6. IMEP sensitivity analysis of the SI-HCCI hybrid combustion mode.

$\left(\phi_{\text {EGR }}\right)$, Intake/exhaust valve timings $\left(\theta_{\text {INTM }}\right.$ and $\left.\theta_{\text {EXTM }}\right)$ and lifts $\left(\Pi_{\text {lift }}\right)$ are controlled by the open loop scheduled control.

In the next two sections, the engine IMEP control and AFR control will be described.

\section{INDIVIDUAL CYLINDER IMEP CONTROL}

As shown in the right side of Fig. 6, IMEP is sensitive to $F_{\mathrm{DT}}$ input at the relatively high MAP or lean combustion condition for the hybrid combustion mode during the mode transition. On the other hand, IMEP is sensitive to MAP at the low MAP or rich condition; see the left side of Fig. 6. This is why the AFR control is essential to the ILC of $F_{\mathrm{DT}}$. Accordingly, it is possible to control the engine IMEP of each cylinder by regulating the corresponding $F_{\mathrm{DT}}$.

The engine IMEP control can be operated in two modes, the learning and transient modes, as shown in Fig. 8, where the learning mode is represented by the dashed lines and the transient mode in the solid lines. Note that the injection pulse width unit is millisecond in Fig. 8 and it can be converted into $\mathrm{mg}$ by multiplying a factor of approximately $8 \mathrm{mg} / \mathrm{ms}$. For both control modes, $F_{\mathrm{DT}}$ is adjusted every engine cycle for each cylinder. The learning mode is enabled when the engine is operated at steady state and close to the transition load. Therefore, this mode can only be operated during the vehicle cruise condition or during the engine calibration process. The transient mode is used during engine transient operations, such as the engine tip-out and tip-in operations.

When the IMEP control is operated at the learning mode, $F_{\mathrm{DT}}$ is regulated through the following control law:

$$
\begin{aligned}
F_{\mathrm{DT}}(i+1) & =F_{\mathrm{FF}}(i)+F_{\mathrm{ILC}}(i) \\
& =F_{\mathrm{FF}}(i)+K_{\mathrm{ILC}}\left[\operatorname{IMEP}_{\text {ref }}-\operatorname{IMEP}(i)\right]
\end{aligned}
$$

where the ILC $F_{\text {ILC }}$ is calculated by a "P" type (proportional) self learning algorithm as described in [19] and [19], and the iterative learning gain $K_{\text {ILC }}$ satisfies the IMEP sensitivity (shown in Fig. 6) constraint, and guarantees the stability of the iterative learning

$$
K_{\mathrm{ILC}}<\frac{\Delta F_{\mathrm{DT}}}{\Delta \mathrm{IMEP}} .
$$




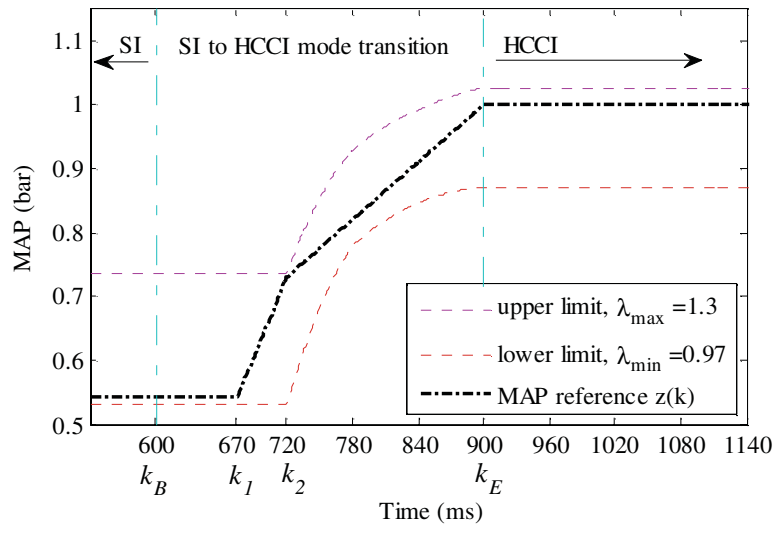

Fig. 7. Target MAP operational range and MAP tracking reference.

The feedforward term $F_{\mathrm{FF}}$ is the learned control variable from the last step that is stored in the memory of the controller. After each learning step $F_{\mathrm{FF}}$ is updated by

$$
F_{\mathrm{FF}}(i)=F_{\mathrm{DT}}(i) \text {. }
$$

The updated feedforward term $F_{\mathrm{FF}}$ will be used for next learning iteration or the transient mode control.

When IMEP control is switched to the transient mode, the ILC is deactivated and the feedback control is activated, as illustrated in Fig. $8, F_{\mathrm{DT}}$ is controlled by the combination of the feedforward and feedback controls, as shown below

$$
F_{\mathrm{DT}}=F_{\mathrm{FF}}+F_{\mathrm{FB}}
$$

where the feedforward term $F_{\mathrm{FF}}$ is the last learned control variable derived in (5). The feedback term $F_{\mathrm{FB}}$ is the output of a proportional and integral (PI) controller. The learned feedforward control $F_{\mathrm{FF}}$ reduces the cylinder-to-cylinder IMEP variance, and the feedback control $F_{\mathrm{FB}}$ provides the load tracking capability during the transient operation.

\section{ENGINE AFR CONTROL}

In the last section, the $F_{\mathrm{DT}}$ controller is used to control the individual cylinder IMEP. To maintain the controllability of the DI fuel control $\left(F_{\mathrm{DT}}\right)$ to the IMEP, lean gas-fuel mixture is required during the mode transition. However, the combustion could become unstable if the mixture becomes extreme lean since the engine spark might not be able to ignite the gas mixture during the transitional cycles. For this paper, the desired normalized AFR is set between $\lambda_{\min }(0.97)$ and $\lambda_{\max }$ (1.3). In [19], a step throttle pre-opening approach was proposed to prevent rich combustions at cycle 3 , but it leads to very lean combustion at the following engine cycles. In this section, an LQ MAP tracking control strategy is developed to regulate the AFR at the desired range.

Since during the combustion mode transition period the engine fueling quantity is adjusted to maintain desired IMEP (or torque), the AFR control during the mode transition is accomplished by regulating the in-cylinder charge air. Assuming that the in-cylinder pressure equals to the MAP at intake valve closing (IVC), the quantity of the charge air can be calculated from the MAP and IVC position. Therefore, the AFR

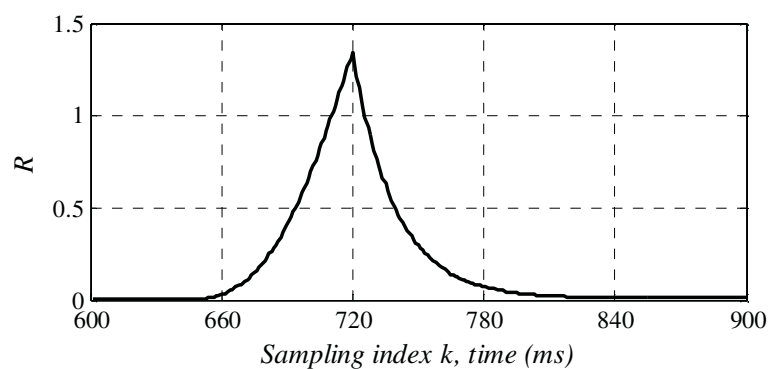

Fig. 8. Adjustment of weighting matrix R.

control can be achieved by regulating the engine MAP and it is naturally to convert the AFR control problem to a MAP regulation problem. Fig. 6 illustrates the relationship between the engine MAP and AFR, and Fig. 7 shows how the AFR operational boundary is translated into MAP constraints for the studied engine operational conditions during the combustion mode transition.

\section{A. MAP Tracking Reference}

As discussed above, the normalized AFR needs to be maintained within the optimal range $\left(\lambda_{\min } \leq \lambda \leq \lambda_{\max }\right)$ during the SI to HCCI combustion mode transition. This control target is difficult to achieve through the AFR feedback control due to the transportation delay and short mode transition period. It is proposed to use the LQ tracking approach to regulate the air-to-fuel mixture to the desired range by controlling the engine MAP. To implement this control strategy, the desired operational range of $\lambda$ is translated into the operational range of the engine MAP shown in Fig. 7, where the upper limit is corresponding to $\lambda_{\max }$ and lower limit is corresponding to $\lambda_{\min }$. An engine MAP tracking reference shown in Fig. 7 was generated for the engine MAP to stay within the desired range. The reference signal is represented by the following function:

$$
z(k)= \begin{cases}Z_{\mathrm{SI}}, & \text { if } k_{B}<k \leq k_{1} \\ Z_{\mathrm{SI}}+\left(Z-Z_{\mathrm{SI}}\right) \frac{k-k_{1}}{k_{2}-k_{1}}, & \text { if } k_{1}<k \leq k_{2} \\ Z+\left(Z_{\mathrm{HCCI}}-Z\right) \frac{k-k_{1}}{k_{2}-k_{1}}, & \text { if } k_{2}<k \leq k_{E}\end{cases}
$$

where $k$ is the sampling index; $k_{B}$ and $k_{E}$ represent the beginning and ending indices of the mode transition and they were set to 600 and 900, respectively, as shown in Fig. 7; $k_{1}$ and $k_{2}$ are switch indices and they equal 670 and 720 , respectively; $Z_{\mathrm{SI}}$ and $Z_{\mathrm{HCCI}}$ are the desired MAP of SI and HCCI modes, respectively; $Z$ is the desired MAP at $k_{2}$.

The MAP tracking reference is divided into three stages due to the natures of the three different combustion stages during the mode transition. They are the SI, SI-HCCI hybrid, and HCCI combustion stages. In the SI stage the MAP reference is divided into two straight lines. The first piece is a horizontal line used to compensate the intake air transportation delay from throttle to manifold and the second one is a straight line with positive slop to increase the MAP quickly to avoid the rich combustion after the valve lift is switched from high to low. In the SI-HCCI stage, a straight line is used to bridge between the SI and HCCI target MAP to allow the MAP to transit to the HCCI stage MAP smoothly. In the HCCI stage 
the MAP reference is actually a constant equal to the ambient air pressure due to the un-throttled operation of the HCCI combustion. It shall be noticed that during the combustion mode transition selecting the MAP tracking reference is very important. Using a piecewise linear reference signal may not be optimal and it could be optimized in the future. The focus of this paper is to show that the combustion mode transition performance can be improved by accurately controlling the engine MAP during the mode transition to regulate the engine AFR within the desired range.

As discussed in Section II-B, during the combustion mode transition all engine control parameters listed in Table II are changing, including the EGR valve opening $\left(\phi_{\mathrm{EGR}}\right)$ and exhaust valve timing. As a result, the overall inertia gas fraction due to both internal and external EGR gas is increased from $19 \%$ at steady-state SI combustion to $44 \%$ at steady-state HCCI combustion. However, due to the long transportation delay of the cooled EGR loop and the dynamics of the VVT actuators, the variation of the overall inertia gas fraction was less significant. For the studied operational condition, it dropped from $19 \%$ down to $16 \%$ and then increased to $24 \%$ during the five transitional combustion cycles. Therefore, the constant inertia gas rate is assumed when the upper and lower MAP bounds, shown in Fig. 7, are derived. Also note that the AFR is concerned only during the five transitional cycles when the engine spark is required to initiate the hybrid combustions. This is why the MAP tracking is critical during the five transitional cycles.

\section{B. Simplified Engine MAP Model}

To develop the proposed LQ tracking control strategy, a simplified engine MAP model is required to represent the relationship between the control input $\left(I_{\mathrm{ETC}}\right)$ and the system output (MAP). The simplified dynamics is represented by a second-order dynamics due to the gas filling dynamics (first order) of the engine intake manifold and the first-order response delay of the engine throttle. Note that the HIL real-time model uses the 1-D nonlinear flow dynamics. The governing equation of gas

$$
\frac{d \mathrm{MAP}}{d t}=-\eta \frac{V_{d} N_{e}}{120 V_{m}} \mathrm{MAP}+\phi \frac{R T_{\mathrm{amb}} C_{D} \pi r^{2} P_{\mathrm{amb}}}{V_{m} \sqrt{2 R T_{\mathrm{amb}}}} \varphi_{\mathrm{TPS}}
$$

and the dynamics of the throttle response is approximated by

$$
\frac{d \varphi_{\mathrm{TPS}}}{d t}=-\frac{k_{\mathrm{ETC}}}{b_{\mathrm{ETC}}} \varphi_{\mathrm{TPS}}+\frac{c_{\mathrm{ETC}}}{b_{\mathrm{ETC}}} I_{\mathrm{ETC}} .
$$

Equations (8) and (9) can be combined, discretized, and represented by the following discrete state-space model:

$$
\begin{aligned}
x(k+1) & =A x(k)+B u(k) \\
y(k) & =C x(k)+D u(k)
\end{aligned}
$$

where

$$
u=I_{\mathrm{ETC}} ; x=\left[\begin{array}{c}
x_{1} \\
x_{2}
\end{array}\right]=\left[\begin{array}{c}
\mathrm{MAP} \\
\varphi_{\mathrm{TPS}}
\end{array}\right] ; y=\mathrm{MAP}
$$

are the system input, state and output, respectively. The system matrices are

$$
\begin{aligned}
& A=\left[\begin{array}{cc}
1-\frac{\eta(k) V_{d} N_{e}}{120 V_{m}} \Delta T & \frac{\phi(k) R_{a} T_{a} C_{D} \pi r^{2} P_{a}}{V_{m} \sqrt{2 R_{a} T_{a}}} \Delta T \\
0 & 1-\frac{k_{\mathrm{ETC}}}{b_{\mathrm{ETC}}} \Delta T
\end{array}\right] \\
& B=\left[\begin{array}{c}
0 \\
\frac{k_{\mathrm{ETC}}}{b_{\mathrm{ETC}}} \Delta T
\end{array}\right] \\
& C=\left[\begin{array}{ll}
1 & 0
\end{array}\right] \\
& D=0
\end{aligned}
$$

where $\Delta T$ is the sample period. State space model (10) is linear time-variant since the volumetric efficiency $\eta$ and multiplier $\phi$ in (8) and (12) are functions of the engine operating condition. Moreover, the sampling time $\Delta T$ in (12) equals $1 \mathrm{~ms}$, and sample time index $k$ is the same as that in (7).

\section{LQ Optimal Tracking Control Synthesis}

Based on the control oriented engine MAP model, a finite horizon LQ optimal tracking controller was designed to follow the reference $z(k)$. More specifically, the control objective is to minimize the tracking error $e(k)$ defined in (13) with the feasible control effort $I_{\mathrm{ETC}}$. The tracking error $e(k)$ is defined as

$$
e(k)=y(k)-z(k)=C x(k)-z(k)
$$

and the constraint on $I_{\text {ETC }}$ is $-5 \mathrm{~A}<I_{\mathrm{ETC}}<5 \mathrm{~A}$. The cost function of the LQ optimal controller is defined as

$$
\begin{gathered}
J=\frac{1}{2}\left[C x\left(k_{f}\right)-z\left(k_{f}\right)\right]^{T} F\left[C x\left(k_{f}\right)-z\left(k_{f}\right)\right] \\
+\frac{1}{2} \sum_{k=k_{1}}^{k_{f}-1}\left\{[C x(k)-z(k)]^{T} Q[C x(k)-z(k)]\right. \\
\left.+u^{T}(k) R u(k)\right\}
\end{gathered}
$$

where $F$ and $Q$ are positive semi-definite and $R$ is positive definite. Note that the LQ MAP tracking control cannot guarantee that the controlled AFR stays within desired range. The LQ design weight matrices, $F, Q$, and $R$, were tuned during validation simulations to make the MAP stay within the desired range. As a result, $F$ and $Q$ are constant matrices defined in (16) and $R$ is a function of sample index and tuned to optimize the tracking error with feasible throttle control effort, see Fig. 8

$$
F=10^{-8}, Q=4 \times 10^{-7}, R=R(k)
$$

Based on the cost function the corresponding Hamiltonian is as follows:

$$
\begin{aligned}
H= & \frac{1}{2}[C x(k)-z(k)]^{T} Q[C x(k)-z(k)]+\frac{1}{2} u^{T}(k) \\
& \times R u(k)+p^{T}(k+1)[A x(k)+B u(k)] .
\end{aligned}
$$


According to [21], the necessary conditions for the extremum in terms of the Hamiltonian are represented as

$$
\begin{aligned}
\frac{\partial H}{\partial p^{*}(k+1)}= & x^{*}(k+1) \Rightarrow x^{*}(k+1)=A x^{*}(k) \\
& +B u^{*}(k) \\
\frac{\partial H}{\partial x^{*}(k)}= & p^{*}(k) \Rightarrow p^{*}(k)=A^{T} p^{*}(k+1) \\
& +C^{T} Q C x^{*}(k)-C^{T} Q z(k) \\
\frac{\partial H}{\partial u^{*}(k)}= & 0 \Rightarrow 0=B^{T} p^{*}(k+1)+R u^{*}(k) .
\end{aligned}
$$

Note that the superscript “*” denotes the optimal trajectories of the corresponding vectors. The augmented system of (19) and (20) becomes

$$
\begin{aligned}
{\left[\begin{array}{c}
x^{*}(k+1) \\
p^{*}(k)
\end{array}\right]=} & {\left[\begin{array}{cc}
A & -B R^{-1} B^{T} \\
C^{T} Q C & A^{T}
\end{array}\right]\left[\begin{array}{c}
x^{*}(k) \\
p^{*}(k+1)
\end{array}\right] } \\
& +\left[\begin{array}{c}
0 \\
-C^{T} Q
\end{array}\right] z(k) .
\end{aligned}
$$

Based on (21) the optimal control is in the form of

$$
u^{*}(k)=-R^{-1} B^{T}\left[P(k) x^{*}(k)-g(k)\right]
$$

where matrix $P(k)$ can be computed by solving the difference Riccati equation backwards

$$
P(k)=A^{T} P(k+1)[I+E P(k+1)]^{-1} A+C^{T} Q C
$$

with the terminal condition

$$
P\left(k_{f}\right)=C^{T} F C
$$

and vector $g(k)$ can be computed by solving the vector difference equation

$$
\begin{aligned}
g(k)= & A^{T}\left\{I-\left[P^{-1}(k+1)+E\right]^{-1} E\right\} \times g(k+1) \\
& +C^{T} Q z(k)
\end{aligned}
$$

with the terminal condition

$$
g\left(k_{f}\right)=C^{T} F z\left(k_{f}\right) .
$$

The optimal control in (23) can be written into the following form:

$$
u^{*}(k)=-L_{\mathrm{FB}}(k) x^{*}(k)+L_{\mathrm{FF}}(k) g(k+1)
$$

where the feedforward gain $L_{\mathrm{FF}}$ is computed by

$$
L_{\mathrm{FF}}(k)=\left[R+B^{T} P(k+1) B\right]^{-1} B^{T}
$$

and the feedback gain $L_{\mathrm{FB}}$ is computed by

$$
L_{\mathrm{FB}}(k)=\left[R+B^{T} P(k+1) B\right]^{-1} B^{T} P(k+1) A .
$$

Note that in (28) the state $x^{*}$ used in the feedback control is computed exactly from the closed loop system model defined below

$$
x^{*}(k+1)=\left[A-B L_{\mathrm{FB}}(k)\right] x^{*}(k)+B L_{\mathrm{FF}}(k) g(k+1) .
$$

However, when the control is implemented into the HIL simulation environment or the actual engine control system, the feedback states are replaced by the actual signals (MAP

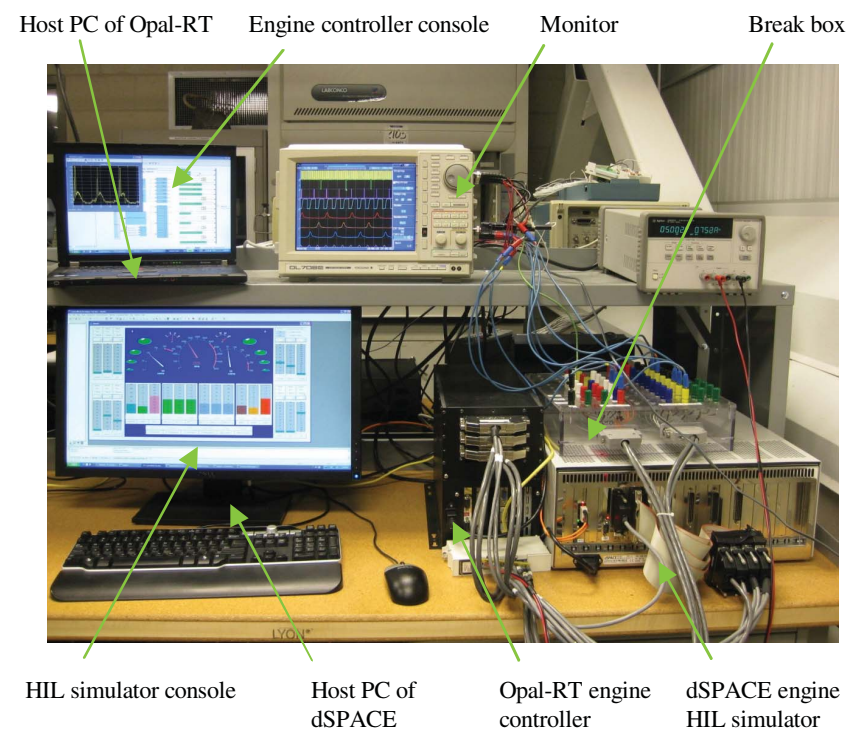

Fig. 9. HIL simulation station.

and $\left.\phi_{\text {TPS }}\right)$ measured by the physical engine sensors. In these cases the LQ controller is represented by the online form as

$$
u(k)=-L_{\mathrm{FB}}(k) x(k)+L_{\mathrm{FF}}(k) g(k+1)
$$

where $x$ represents the sampled states. Note that both states, MAP and $\phi_{\text {TPS }}$, can be measured in the HIL simulator or in the engine system.

\section{HIL SimUlation VALIDATION}

The multistep combustion mode transition control strategy is composed of the DI fuel quantity control and LQ optimal MAP tracking control with the SI-HCCI hybrid combustions during the mode transition. In this section, an HIL simulation environment is introduced; the HIL simulation results are presented for the control strategy validation.

\section{A. HIL Simulation Station}

For the purpose of initial control strategy development and validation, an HIL simulation station, shown in Fig. 9, was used. By implementing the control oriented engine model described in [5] and [17], the dSPACE-based engine HIL simulator provided all measureable engine signals (such as incylinder pressure and MAP signals) in real-time. These signals were directly fed into the Opal-RT-based real-time prototype engine controller. Some unmeasurable engine states (such as $T_{\text {IVC) }}$ ) were also available to help with the control performance analysis. On the other hand, the control signals generated by the prototype engine controller were also fed back into the engine simulator. These signals were recorded and analyzed for control strategy development and validation.

\section{B. Simulation Results of Using SI-HCCI Hybrid Mode}

As mentioned above, the engine is operated in the SI-HCCI hybrid combustion mode between cycles 3 and 5 (see Fig. 4) for the multistep mode transition strategy. To disclose the significance of using this hybrid combustion mode, the key 


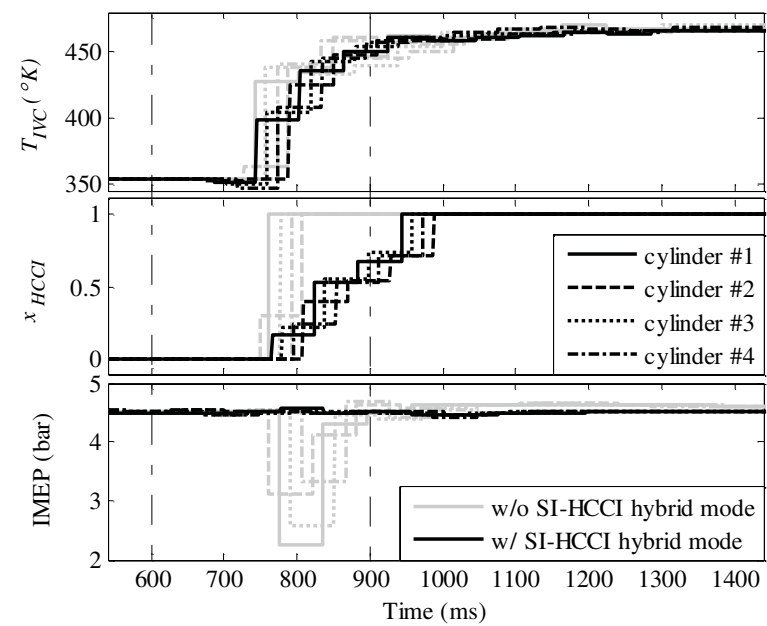

Fig. 10. Combustion performances with and without SI-HCCI hybrid combustion mode.

engine variables, such as $T_{\mathrm{IVC}}, x_{\mathrm{HCCI}}$, and IMEP, are analyzed and plotted in Fig. 10, for the cases with and without the SIHCCI hybrid combustion mode. Note that the simulation time from 600th to 900th $\mathrm{ms}$ is corresponding to the five transitional engine cycles illustrated in Fig. 4.

As shown in Fig. 10, charge mixture temperature at intake valve closing $\left(T_{\mathrm{IVC}}\right)$ has response delay with or without the hybrid combustion mode due to the dynamics of the valvetrain system and the NVO operation. Under such thermal condition, if the engine was switched into the HCCI combustion mode directly, start of combustion timing would be greatly retarded (misfire could happen), causing large IMEP variance [20]. The large fluctuation of IMEP cannot be improved through combustion control due to the lack of available controllability in the HCCI mode as discussed in Section III. However, by using the hybrid combustion mode during the mode transition, percentage of $\mathrm{HCCI}$ combustion $x_{\mathrm{HCCI}}$ is gradually increased as the in-cylinder temperature at the IVC $T_{\text {IVC }}$ approaches the desired level. Also note that due to the LQ optimal tracking of the desired MAP, the engine IMEP can be regulated by controlling $F_{\mathrm{DT}}$. As a result, smooth IMEP can be achieved during the mode transition. Note that for the simulation results shown in Fig. 10, the proposed AFR and IMEP controls are used during the mode transition with the hybrid combustions.

Therefore, using the SI-HCCI hybrid combustions during the mode transition is a key control technique for the smooth combustion mode transition.

\section{Simulation Results of AFR Control}

The developed LQ optimal MAP tracking control was implemented in the prototype engine controller and validated through the HIL engine simulations. The simulated control input $I_{\mathrm{ETC}}$, the system states MAP and $\phi_{\mathrm{TPS}}$, and $\lambda$ are plotted in Fig. 11. For comparison purpose, the simulated responses of these variables with a step $I_{\text {ETC }}$ control approach are also shown in Fig. 11, in which $I_{\text {ETC }}$ is set to the target level before the adjustment of $\Pi_{\text {lift }}$ (happens at 720 th ms), as a result, the engine throttle is gradually opened to the wide open throttle (WOT) position and the MAP is increased before the valve lift

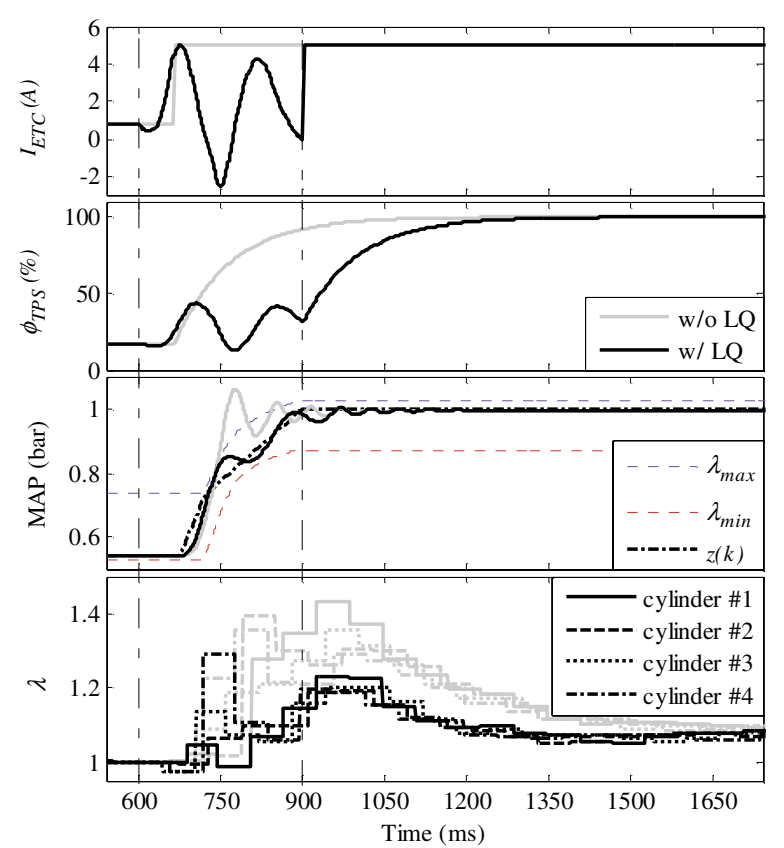

Fig. 11. Engine performances of the optimal MAP tracking control.

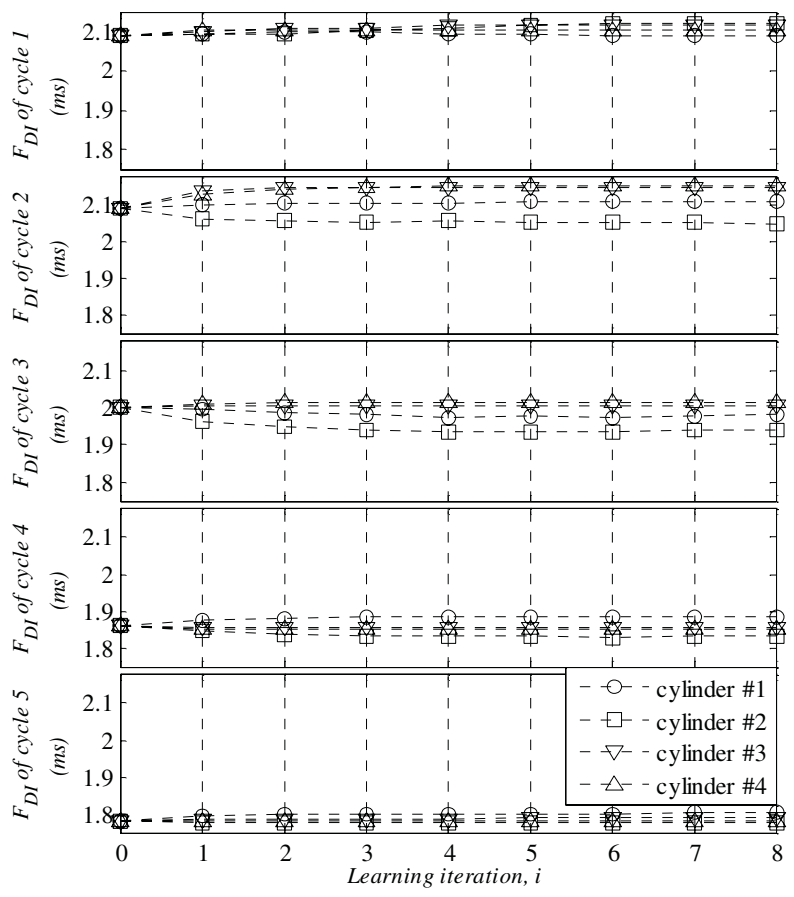

Fig. 12. Correction of DI fuel quantity after iterative learning.

switches. The increased MAP ensures enough fresh air charge to each cylinder when the valve lift switches to the low lift. However, the step $I_{\text {ETC }}$ control leads to a rapid increment of the engine MAP or excessive fresh air charge, leading to extreme lean AFR $\lambda$ in the following engine cycles.

Using the proposed LQ MAP tracking control strategy, throttle current $I_{\text {ETC }}$ is regulated in a nonmonotonic increasing pattern. Note that to maintain $I_{\mathrm{ETC}}$ in the feasible range ( $-5 \mathrm{~A}$ $<I_{\mathrm{ETC}}<5 \mathrm{~A}$ ) the weighting matrix $R$ in the cost function (15) is adjusted as illustrated in Fig. 8. The similar pattern 


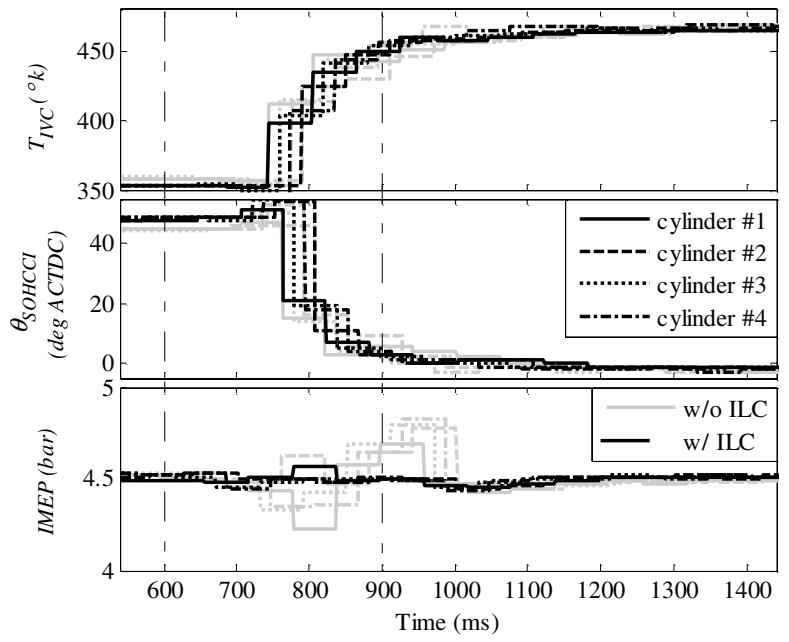

Fig. 13. Combustion performances of using optimal MAP tracking and DI fuel ILC strategies.

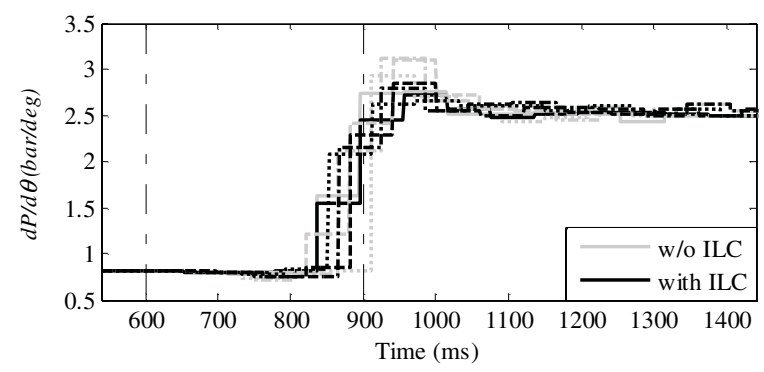

Fig. 14. Engine knock index during combustion mode transition.

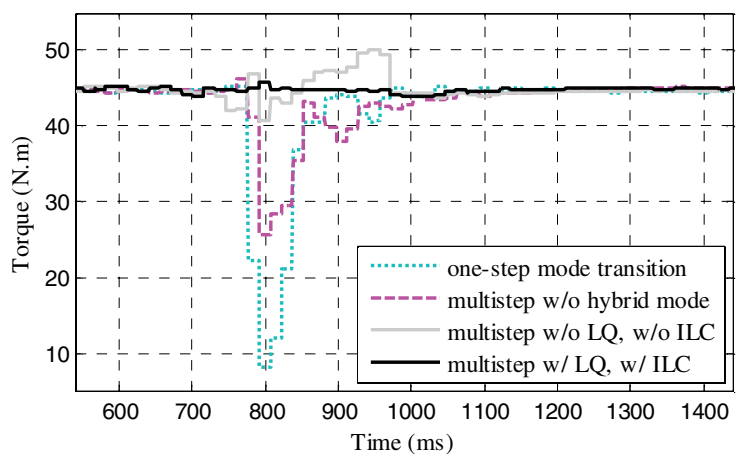

Fig. 15. Engine torque performances of different control strategies.

can also be found for $\phi_{\text {TPS }}$ with a small phase lag. As a result, the engine MAP tracks the reference $z(k)$ after the intake valve lift $\Pi_{\text {lift }}$ switches to the low lift, and $\lambda$ of each cylinder is successfully maintained within the desired range. Therefore, with the help of the LQ optimal tracking control, the in-cylinder AFR is maintained within the desired range, leading to stable combustions since the lean mixture can be ignited by engine spark.

Slight oscillations in the MAP responses are found with both control approaches, which are due to the flow dynamics of the engine air-handling system and the engine MAP modeling error. It is difficult to eliminate them. Moreover, the MAP oscillation associated with the LQ optimal tracking control is within the desired MAP range.

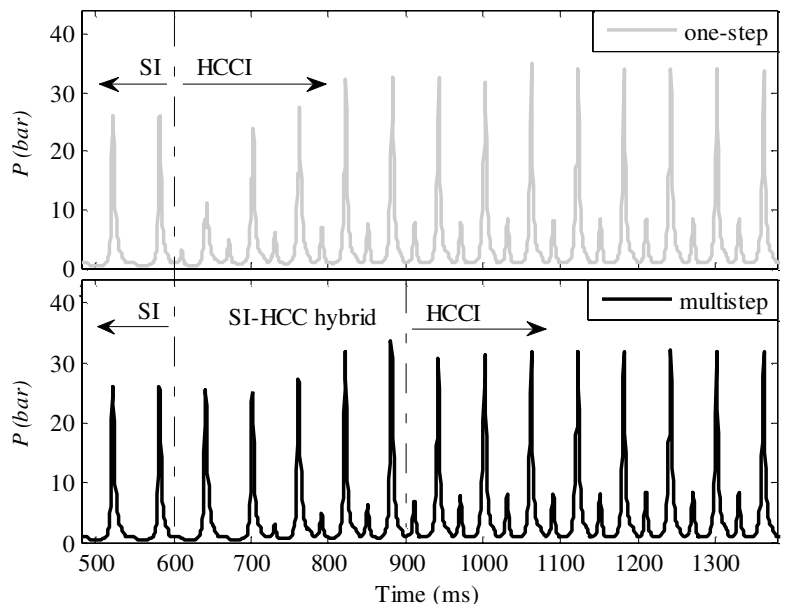

Fig. 16. In-cylinder gas pressure profile of cylinder 1 during SI to HCCI combustion mode transition.

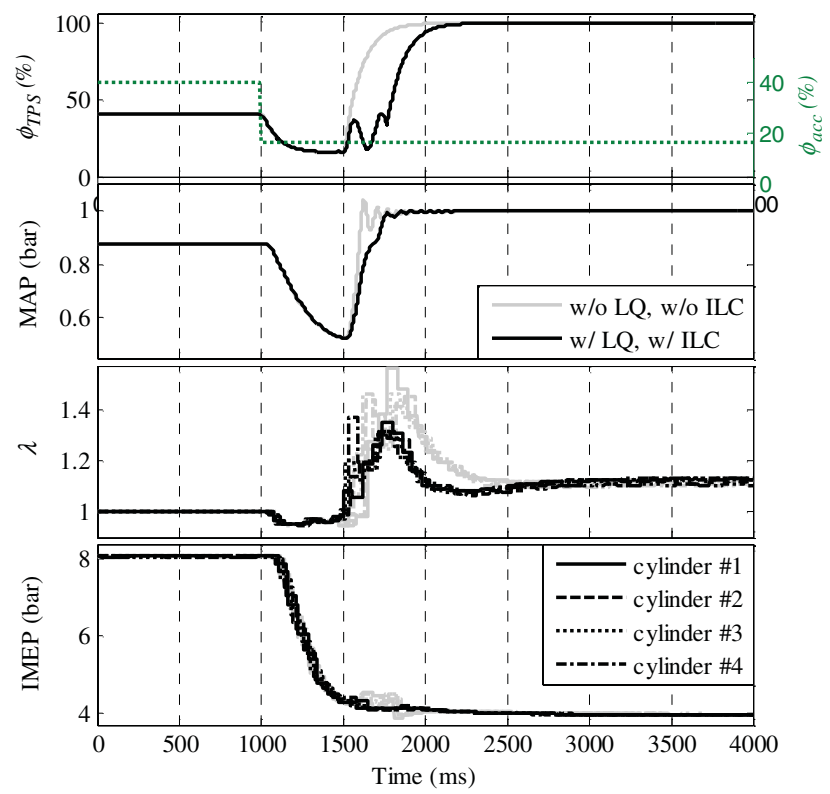

Fig. 17. Engine responses during tip-out operation.

\section{Simulation Results of IMEP Control in Learning Mode}

When the AFR is maintained within the desired range, the iterative learning of $F_{\mathrm{DT}}$ was conducted in the learning mode. Fig. 12 shows that $F_{\mathrm{DT}}$ of each cylinder converges after a few learning iterations. $F_{\mathrm{DT}}$ in cycle 2 was adjusted significantly due to the large variation of the engine MAP. The first SI-HCCI engine cycle (cycle 3) after the intake/exhaust valve lift switch demonstrated the largest improvement, leading to significant correction of $F_{\mathrm{DT}}$. The cylinder-to-cylinder adjustment of $F_{\mathrm{DT}}$ after iterative learning compensates for the intake charge variations due to the MAP variations, and leads to smooth IMEP of individual cylinder as shown in Fig. 13. Therefore, it can be concluded that smooth SI to HCCI combustion transition is achievable through the ILC of the DI fuel quantity when the AFR is regulated within the desired range by the LQ MAP tracking control.

In Fig. 13, without the ILC, slight IMEP oscillations can be observed for $T_{\mathrm{IVC}}$ and $\theta_{\mathrm{SOHCCI}}$, however, the oscillations 


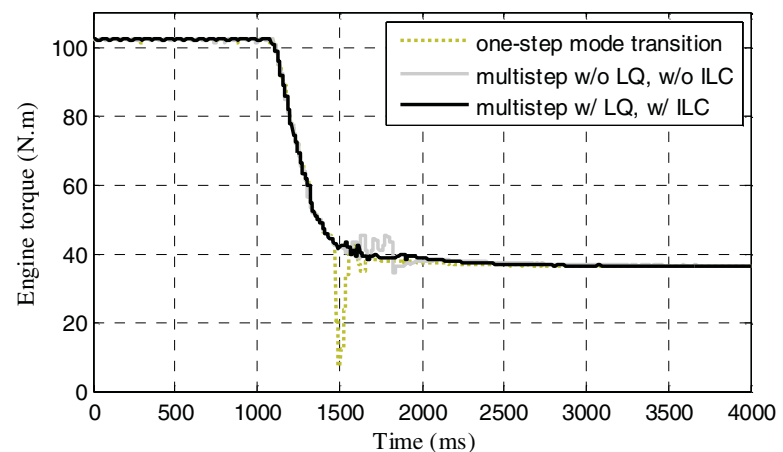

Fig. 18. Engine torque response during tip-out operation.

disappear after the ILC was applied. These oscillations indicate unstable combustions and are due to the cycle-to-cycle residue gas dynamics of HCCI combustions (including the SI-HCCI hybrid combustions), which has been discussed in great details in [9], [10], and [16]. Due to the residue gas dynamics, retarded (or advanced) $\theta_{\text {SOHCCI }}$ of current engine cycle leads to advanced (or retarded) $\theta_{\text {SOHCCI }}$ for the next engine cycle. As a result, combustion oscillations occur. During the combustion mode transition, the impact of adjusting the valve lift $\Pi_{\text {lift }}$ to the individual in-cylinder combustion is quite different. Cylinder 1 experiences the most significant impact since the valve lift switch happens during its exhaust stroke. Both residue gas quantity and fresh charge quantity were affected. Therefore the IMEP fluctuations of cylinder 1 are dominated as shown in Fig. 13. By using the ILC of DI fuel quantity, $\theta_{\text {SOHCCI was regulated to the appropriate timing as well as }}$ the IMEP was regulated to the control target. Accordingly the oscillations of $T_{\mathrm{IVC}}$ and $\theta_{\mathrm{SOHCCI}}$ were suppressed, and combustion stability during the mode transition was improved by the ILC.

As a result of suppressing the oscillations of $T_{\mathrm{IVC}}$ and $\theta_{\text {SOHCCI}}$, engine knock index was also reduced during the combustion mode transition, see Fig. 14 . Note that $\mathrm{d} P / \mathrm{d} \theta$ is a good indicator of engine knock [22].

\section{E. Engine Torque Performance Comparison}

Engine torque responses during the combustion mode transitions using the different control strategies are plotted in Fig. 15. Their statistics are listed in Table III for comparison. The largest engine torque fluctuation is produced by the onestep approach. The fluctuation of engine torque is reduced when multistep strategy is used. It is further improved as the SI-HCCI hybrid combustion mode is implemented. At last, smooth combustion mode transition is realized in multi-steps when both the MAP (LQ tracking control) and the DI fuel quantity (ILC) controls are applied. The combined control of LQ MAP tracking and ILC DI fueling with SI-HCCI hybrid combustions leads to the lowest torque fluctuations $(2.2 \%)$ when the engine operational conditions transit from the steady state SI mode to the HCCI mode. The in-cylinder gas pressure profiles are plotted in Fig. 16. One can see significant improvement of combustion quality during the transitional cycles.
TABLE III

Engine Performance Statistics For DifFERENT CONTROL STRATEgIES

\begin{tabular}{lccc}
\hline & $\begin{array}{c}\text { IMEP } \\
\text { error }(\%)\end{array}$ & $\begin{array}{c}\text { Max d } P / \mathrm{d} \theta \\
(\mathrm{bar} / \mathrm{deg})\end{array}$ & $\begin{array}{c}\text { Torque } \\
\text { error } \\
(\%)\end{array}$ \\
\hline One-step mode transition & 51.6 & N/A & 81.8 \\
Multistep w/o hybrid mode & 26.9 & N/A & 43.1 \\
Multistep w/o LQ, w/o ILC & 7.1 & 3.13 & 11.2 \\
Multistep w/ LQ, w/ ILC & 1.6 & 2.82 & 2.2 \\
\hline
\end{tabular}

\section{F. Simulation Results of IMEP Control in Transient Mode}

In order to study, the mode transition under the transient operation, an HIL simulation was conducted to simulate the engine throttle tip-out operation, where the engine was initially operated in the SI mode. As a step input was applied to the acceleration pedal, the pedal position decreased from $40 \%$ to $15 \%$. As a result, the engine IMEP reduced from 8.1 to 4 bar, which crosses the combustion mode transition threshold of 4.5 bar. Accordingly the combustion mode transition was triggered. Two-mode transition strategies were simulated. Both of them were multistep strategies with the hybrid combustions. One was with the optimal MAP tracking control (LQ) and the DI fuel quantity control, the other was not. Note that the DI fuel quantity control was switched to the transient mode during the engine tip-out operation, and the feedforward term $F_{\mathrm{FF}}$ used in the transient mode control was the last learned value by the ILC. The transient responses of engine variables are plotted in Fig. 17.

In Fig. 17, after the step input of the acceleration pedal, both $\phi_{\text {TPS }}$ and MAP are decreased at first, and then increased due to the combustion mode transition to the unthrottled HCCI mode. Slightly rich combustions can be observed during the early stage of the tip-out operation since the throttle opening is decreased in the SI mode. Once the mode transition started, the in-cylinder gas-fuel mixture becomes lean for both strategies. However, with the LQ MAP tracking control, the normalized AFR $\lambda$ is maintained within the desired range throughout the engine tip-out operation. Furthermore, with the proposed DI fuel quantity control smooth IMEP responses were achieved for all cylinders comparing with those without the ILC fuel control. Similar improvement in engine torque output can also be found in Fig. 18.

Based on the simulation results shown in Figs. 17 and 18, it can be concluded that the proposed LQ optimal MAP tracking control is capable of regulating the AFR within the desired range during engine transient operations, and the ILC DI fuel quantity control is also effective in transient mode.

\section{CONCLUSION}

The combustion mode transition between the SI and HCCI combustion is challenging but necessary to implement the promising HCCI combustion technology to production SI engines. As demonstrated in this paper, smooth combustion mode transition can be realized in a multicylinder HCCI capable SI engine equipped with the dual-valve lift and electrical VVT systems. The mode transition was accomplished 
within five engine cycles with the help of the LQ MAP tracking control and the ILC of DI fuel quantity. Based on the HIL simulation results presented in this paper, the LQ control tracks the engine MAP to the desired target with small oscillations; and as a result, the normalized AFR is maintained within the desired range. This makes it feasible to control the individual cylinder IMEP by regulating the corresponding DI fuel quantity. With the help of the ILC DI fuel control, the cylinder-to-cylinder variations of the engine IMEP can be reduced significantly, and the engine IMEP tracks the control target. Furthermore, the ILC is able to compensate the influence of the cycle-to-cycle residue gas dynamics, and accordingly reduces the fluctuations of the engine knock index during the mode transition. In addition, combining the learned feedforward control with the PI feedback control, smooth SI to HCCI combustion mode transition can also be realized during transient engine operations.

\section{REFERENCES}

[1] F. Zhao, T. Asmus, D. Assanis, J. E. Dec, J. A. Eng, and P. M. Najt, "Homogeneous charge compression ignition (HCCI) engines key research and development issues," SAE, Warrendale, PA, Tech. Rep. PT-94, 2003.

[2] R. M. Wagner and K. D. Edwards, "Hybrid SI-HCCI combustion modes for low emissions in stationary power applications," in Proc. 3rd Annu. Adv. Stationary Reciprocat. Engines Meeting Argonne Nat. Lab., Argonne, IL Jun. 2006.

[3] N. J. Killingsworth, S. M. Aceves, D. L. Flowers, F. Espinosa-Loza, and M. Krstic, "HCCI engine combustion-timing control: Optimizing gains and fuel consumption via extremum seeking," IEEE Trans. Control Syst. Technol., vol. 17, no. 6, pp. 1350-1361, Nov. 2009.

[4] C. J. Chiang and A. G. Stefanopoulou, "Stability analysis in homogeneous charge compression ignition (HCCI) engines with high dilution," IEEE Trans. Control Syst. Technol., vol. 15, no. 2, pp. 209-219, Mar. 2007.

[5] X. Yang and G. Zhu, "A two-zone control oriented SI-HCCI hybrid combustion model for the HIL engine simulation," in Proc. Amer. Control Conf., San Francisco, CA, 2011, pp. 973-978.

[6] S. C. Kong and R. D. Reitz, "Application of detailed chemistry and CFD for predicting direct injection HCCI engine combustion and emission," Proc. Combustion Inst., vol. 29, no. 1, pp. 663-669, 2002.

[7] X. Yang, G. Zhu, and Z. Sun, "A control oriented SI and HCCI hybrid combustion model for internal combustion engines," in Proc. ASME Dynamic Syst. Control Conf., Cambridge, MA, 2010, pp. 973-978.

[8] N. Kalian, H. Zhao, and J. Qiao, "Investigation of transition between spark ignition and controlled auto-ignition combustion in a V6 directinjection engine with cam profile switching," in Proc. IMechE, vol. 222. 2008, pp. 1911-1926.

[9] N. Ravi, M. J. Roelle, H.-H. Liao, A. F. Jungkunz, C.-F. Chang, S. Park, and J. C. Gerdes, "Model-based control of HCCI engines using exhaust recompression," IEEE Trans. Control Syst. Technol., vol. 18, no. 6, pp. 1289-1302, Nov. 2010.

[10] J. Kang, C. Chang, and T. Kuo, "Sufficient condition on valve timing for robust load transients in HCCI engines," SAE International, Warrendale, PA, Tech. Rep. 2010-01-1243, 2010.

[11] G. M. Shaver, "Physics based modeling and control of residual-affected HCCI engines using variable valve actuation," Ph.D. thesis, Dept. Mech. Eng., Stanford University, Stanford, CA, Sep. 2005.

[12] M. J. Roelle, G. M. Shaver, and J. C. Gerdes, "Tackling the transition: A multi-mode combustion model of SI and HCCI for mode transition control," in Proc. Int. Mech. Eng. Conf. Exposit., Anaheim, CA, Nov. 2004, pp. 329-336.

[13] Y. Zhang, H. Xie, N. Zhou, and T. Chen, "Study of SI-HCCI-SI transition on a port fuel injection engine equipped with 4VVAS," SAE, Warrendale, PA, Tech. Rep. 2007-01-0199, 2007.

[14] H. Wu, N. Collings, S. Regitz, J. Etheridge, and M. Kraft, "Experimental investigation of a control method for SI-HCCI-SI transition in a multicylinder gasoline engine," SAE International, Warrendale, PA, Tech. Rep. 2010-01-1245, 2010.
[15] J. Wang, "Hybrid robust air-path control for diesel engines operating conventional and low temperature combustion modes," IEEE Trans. Control Syst. Technol., vol. 16, no. 6, pp. 1138-1151, Nov. 2008.

[16] A. M. Annaswamy, M. Fleifil, J. W. Rumsey, R. Prasanth, J. P. Hathout, and A. F. Ghoniem, "Thermoacoustic instability: Model-based optimal control designs and experimental validation," IEEE Trans. Control Syst. Technol., vol. 8, no. 6, pp. 905-918, Nov. 2000.

[17] X. Yang and G. Zhu, "A mixed mean-value and crank-based model of a dual-stage turbocharged SI engine for hardware-in-the-loop simulation," in Proc. Amer. Control Conf., Baltimore, MD, 2010, pp. 3791-3796.

[18] X. Yang and G. Zhu, "SI and HCCI combustion mode transition control of a multi-cylinder HCCI capable SI engine via iterative learning," in Proc. 4th Annu. Dynamic Syst. Control Conf., Arlington, VA, Oct.-Nov. 2011, pp. 663-670.

[19] P. R. Ouyang, B. A. Petz, and F. F. Xi, "Iterative learning control with switching gain feedback for nonlinear systems," J. Comput. Nonlinear Dynamics, vol. 6, p. 011020, Jan. 2011.

[20] H. H. Liao and N. Ravi, "Representing recompression HCCI dynamics with a switching linear model," in Proc. Amer. Control Conf., Baltimore, MD, Jul. 2010, pp. 3803-3808.

[21] D. Naidu, Optimal Control Systems. Boca Raton, FL: CRC Press, 2003, pp. 232-239.

[22] W. K. Cheng, T. Kenney, and J. Yang, "On HCCI engine knock," SAE, Warrendale, PA, Tech. Rep. 2007-01-1858, 2007.

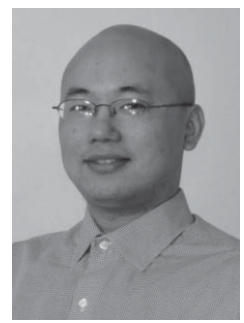

Xiaojian Yang received the B.S. and M.S. degrees in vehicle engineering from the Hefei University of Technology, Hefei, China, in 2000 and 2003, respectively, and the Ph.D. degree in mechanical engineering from Michigan State University (MSU), East Lansing, in 2011.

$\mathrm{He}$ is currently a Senior Control Engineer with the Advanced Powertrain Group, Delphi Automotive PLC, Auburn Hill, MI. Before joining Delphi, he conducted research in modeling and control of homogeneous-charge-compression-ignition engine systems with the Energy and Automotive Research Laboratory, MSU. His current research interests include modeling and control of advanced internal combustion engine systems.

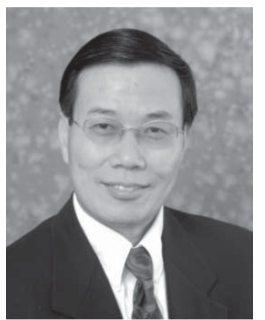

Guoming Zhu received the B.S. and M.S. degrees from the Beijing University of Aeronautics and Astronautics, Beijing, China, in 1982 and 1984, respectively, and the $\mathrm{Ph} . \mathrm{D}$. degree in aerospace engineering from Purdue University, West Lafayette, IN, in 1992.

$\mathrm{He}$ is an Associate Professor with the Department of Mechanical Engineering (ME) and the Department of Electrical and Computer Engineering (ECE), Michigan State University, East Lansing. Prior to joining the ME and ECE Departments, he was a Technical Fellow in advanced powertrain systems with the Visteon Corporation, Van Buren Township, MI. He was also a Technical Advisor with Cummins Engine Company, Columbus, IN. His teaching interests focus on control classes at both the undergraduate and graduate levels. He has over 24 years of experience related to control theory, engine diagnostics, and combustion control. He has authored or co-authored over 100 refereed technical papers and holds 40 U.S. patents. His current research interests include closed-loop combustion control of internal combustion engines, engine system modeling and identification, hybrid powertrain control and optimization, and linear parameter varying control with application to automotive systems.

Dr. Zhu was an Associate Editor of the ASME Journal of Dynamic Systems, Measurement, and Control. He is currently an Editorial Board Member of the International Journal of Powertrains. He is an ASME fellow. 
9.6 Published JC Paper \#6

A Control-Oriented Hybrid Combustion Model of an HCCI Capable SI Engine 


\title{
A control-oriented hybrid combustion model of a homogeneous charge compression ignition capable spark ignition engine
}

Proc IMechE Part D:

J Automobile Engineering

226(10) 1380-1395

(c) IMechE 2012

Reprints and permissions:

sagepub.co.uk/journalsPermissions.nav DOI: I0.I I 77/09544070I2443334

pid.sagepub.com

(SAGE

\author{
Xiaojian Yang and Guoming G Zhu
}

\begin{abstract}
To implement the homogeneous charge compression ignition combustion mode in a spark ignition engine, it is necessary to have smooth mode transition between the spark ignition and homogeneous charge compression ignition combustions. The spark ignition-homogeneous charge compression ignition hybrid combustion mode modeled in this paper describes the combustion mode that starts with the spark ignition combustion and ends with the homogeneous charge compression ignition combustion. The main motivation of studying the hybrid combustion mode is that the percentage of the homogeneous charge compression ignition combustion is a good parameter for combustion mode transition control when the hybrid combustion mode is used during the transition. This paper presents a control oriented model of the spark ignition-homogeneous charge compression ignition hybrid combustion mode, where the spark ignition combustion phase is modeled under the two-zone assumption and the homogeneous charge compression ignition combustion phase under the one-zone assumption. Note that the spark ignition and homogeneous charge compression ignition combustions are special cases in this combustion model. The developed model is capable of simulating engine combustion over the entire operating range, and it was implemented in a real-time hardware-in-the-loop simulation environment. The simulation results were compared with those of the corresponding GT-Power model, and good correlations were found for both spark ignition and homogeneous charge compression ignition combustions.
\end{abstract}

\section{Keywords}

Spark ignition combustion, homogeneous charge compression ignition combustion, combustion modeling, combustion control

Date received: 20 July 20I I; accepted: 2I February 2012

\section{Introduction}

The continuing pursuit of improving fuel economy and the increasingly stringent emission regulations has rekindled the research interest in the homogeneous charge compression ignition (HCCI) combustion in recent years. The flameless nature of the HCCI combustion and its high dilution operation capability lead to low combustion temperature. As a result, the formation of $\mathrm{NO}_{x}$ (nitrogen oxides) can be significantly reduced. Furthermore, an HCCI capable engine is capable of unthrottled operation that greatly reduces pumping loss and improves fuel economy. ${ }^{1}$

On the other hand, HCCI combustion has its own limitations. The HCCI combustion is limited at high engine load due to the audible engine knock, ${ }^{2}$ and at low load due to misfire caused by the lack of sufficient thermal energy to trigger the auto-ignition of the gas-fuel mixture late in the compression stroke. ${ }^{3}$ In fact, the HCCI combustion can be regarded as a type of engine operating mode rather than a type of engine. ${ }^{4}$ In order to take advantage of the HCCI combustion mode in an internal combustion engine, an other combustion mode, such as the SI (spark ignited) combustion, is required at high load, at ultra-low load (such as idle), and at certain operational conditions such as cold start, and at high engine speed. It is fairly challenging to operate the engine in two distinct combustion modes, and it is even more difficult to have smooth combustion mode

Department of Mechanical Engineering, Michigan State University, USA

\section{Corresponding author:}

Guoming G Zhu, Department of Mechanical Engineering, Michigan State University, E I 48 ERC South, East Lansing, MI 48824, USA.

Email: zhug@egr.msu.edu 
transition between SI and HCCI combustions modes, since the favorable thermo conditions for one combustion mode are always adverse to the other. Due to the significant response delay of the hydraulic and electric variable valve timing (VVT) systems, cycle-to-cycle residual gas dynamics, and the response delay in the engine air handling system, it is almost impossible to achieve desired thermo conditions for the combustion mode transition in one engine cycle. ${ }^{5,6}$ To address this issue, multi-cycle combustion mode transition strategies were proposed. ${ }^{7,8}$ In Zhang et al., ${ }^{8}$ experimental data show that a hybrid (or spark assisted) combustion mode occurred during the mode transition. It starts in the SI combustion mode with a relatively low heat release rate, and once the thermo and chemical conditions of the unburned gas satisfy the start of HCCI (SOHCCI) combustion criteria, the combustion continues in the HCCI combustion mode. The hybrid combustion process is illustrated by the dashed curves of mass fraction burned (MFB) shown in Figure 1. During an ideal SI to HCCI combustion transition process, as the charge temperature, defined as in-cylinder gas mixture temperature at intake valve closing, increases gradually cycle-aftercycle, the percentage of SI combustion, defined as the MFB percentage at SOHCCI (see Figure 1), decreases gradually over each combustion cycle while the HCCI combustion percentage, MFB between SOHCCI and the end of combustion, increases gradually. For the HCCI to SI combustion transition, the process is reversed. This hybrid combustion mode makes it feasible to use the conventional valvetrain for the HCCI capable SI engine since it allows the engine cam phase to change gradually. ${ }^{9}$ In the rest of this paper, the term hybrid combustion refers to the SI-HCCI hybrid combustion mode.

Smooth combustion mode transition can be realized by utilizing appropriate control strategies to regulate the SI-HCCI hybrid combustions properties during the mode transition, as demonstrated. ${ }^{5,7}$ Yang and $\mathrm{Zhu}^{5}$ also demonstrated the effectiveness of using spark assisted HCCI (or SI-HCCI combustion in this paper)

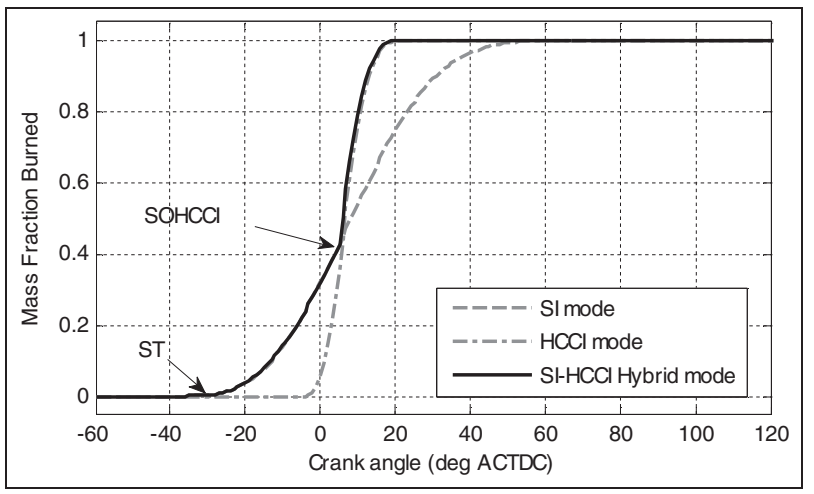

Figure I. MFB of the SI-HCCI hybrid combustion. SI: spark ignition; $\mathrm{HCCl}$ : homogeneous charge compression ignition; ST: spark timing; ACTDC: after combustion total dead center. to avoid the misfire by controlling the in-cylinder airto-fuel ratio in the proper range such that SI is possible. Also, the SI to HCCI transition mode has been known as more difficult to control than the HCCI to SI transition mode since the residual gas management or incylinder temperature control is completely different in terms of thermal energy introduction and thermal inertia aspects. To develop and validate the combustion mode transition control strategies, a precise control oriented combustion model is a necessity. It should be able to simulate the engine combustion process in realtime with enough modeling complexity for combustion feedback control. Multi-zone, three-dimensional computational fluid dynamics (CFD) models with detailed chemical kinetics can be found ${ }^{4,10,11}$ and they are capable of describing the engine thermal and flow dynamics, heat transfer, and pollutant formation phenomena of both SI and HCCI combustions with reasonable accuracy. Similar combustion models have been implemented into commercial codes such as GTPower ${ }^{12}$ and Wave. ${ }^{13}$ However, these high-fidelity models cannot be used for control strategy development and validation since they can only be operated in the offline simulation mode, but they can be used as reference models for the control-oriented combustion model presented in this paper.

In this paper, the zero-dimensional mean-value modeling method ${ }^{14,15}$ is used to model average chemical kinetics and thermodynamic properties of the combustion process, due to its low computational throughput required for real-time simulations. The modeled combustion variables, such as in-cylinder gas mixture pressure and temperature, are updated every crank degree. ${ }^{16}$ The nonlinear equations describing the chemical kinetics and thermodynamics of the combustion process are solved by discretizing the continuous nonlinear equations. Furthermore, the combustion model presented in this paper for the SI-HCCI hybrid combustion treats both SI and HCCI combustion modes as special cases of the hybrid combustion. Therefore, the SI-HCCI hybrid combustion model contains all possible combustion modes during the mode transition. In the SI-HCCI hybrid combustion model, the SI combustion phase from ST to SOHCCI (see Figure 1) is modeled under the two-zone assumption, and the thermodynamic and chemical properties of the unburned zone gas mixture are modeled to accurately estimate the start of HCCI combustion phase; whereas the HCCI combustion phase from the SOHCCI to the end of combustion is modeled under the one-zone assumption to reduce the computational throughput.

The developed SI-HCCI hybrid combustion model was implemented into a dSPACE based hardware-inthe-loop (HIL) engine simulation environment for realtime simulations and model validations. The simulation results were also compared with those obtained from the corresponding high-fidelity GT-Power model, and fairly good correlations were observed between these two combustion models. This shows that the proposed 
combustion model can be used to approximate the high-fidelity combustion model and implemented in real-time control at the same time.

The paper is organized as follows. In the second section the target engine configuration is described and the SI-HCCI hybrid combustion model architecture is presented according to the engine configuration. The third and fourth sections provide the governing equations of the combustion and gas exchange phases. The fifth section addresses the implementation of the developed combustion model into the real-time HIL simulation environment. The model calibration and validation results are presented in the sixth section. Conclusions are drawn in the seventh section

\section{SI-HCCI hybrid combustion model architecture}

This section discusses the target engine configuration capable of the SI, HCCI, and SI-HCCI hybrid combustions. The model architecture is presented to combine the three combustion modes into a single combustion model.

\section{Target engine configuration}

Figure 2 shows the configuration of the target $\mathrm{HCCI}$ capable SI engine, and the engine specifications are listed in Table 1. The key feature of the engine is its valvetrain system that has two-stage valve lifts for both intake and exhaust valves. The high lift is $9 \mathrm{~mm}$ for the SI combustion mode; and the low lift is $5 \mathrm{~mm}$ for the HCCI combustion mode. Note that the cam profiles for the high and low lift are different, which provides additional freedom to optimize both SI and HCCI combustions. Furthermore, both intake and exhaust valve timings can be adjusted within \pm 40 crank degrees to improve the controllability of the internal exhaust gas recirculation (EGR) fraction, the effective compression ratio, and the engine volumetric efficiency during the combustion mode transition and steady state SI and HCCI operations. Note that using the two-step valve system with electrical VVT for HCCI combustion has been demonstrated. ${ }^{17}$ For the engine considered, the valve lift and its profile were optimized using GTPower simulations. In addition to the modification of the engine valvetrain, external cooled EGR is used to enable high dilution charge with low charge mixture temperature, which leads to $7 \%$ and $19 \%$ external EGR rate during the SI to HCCI combustion mode transition. The engine throttle can be electronically controlled to obtain the desired engine charge in both SI and unthrottled HCCI operations. To enable the closed-loop combustion control of each engine cylinder, each engine cylinder is equipped with a piezoelectric pressure transducer and each exhaust port is fitted with a fast response UEGO (universal exhaust gas oxygen) sensor. Accordingly, the proposed control oriented

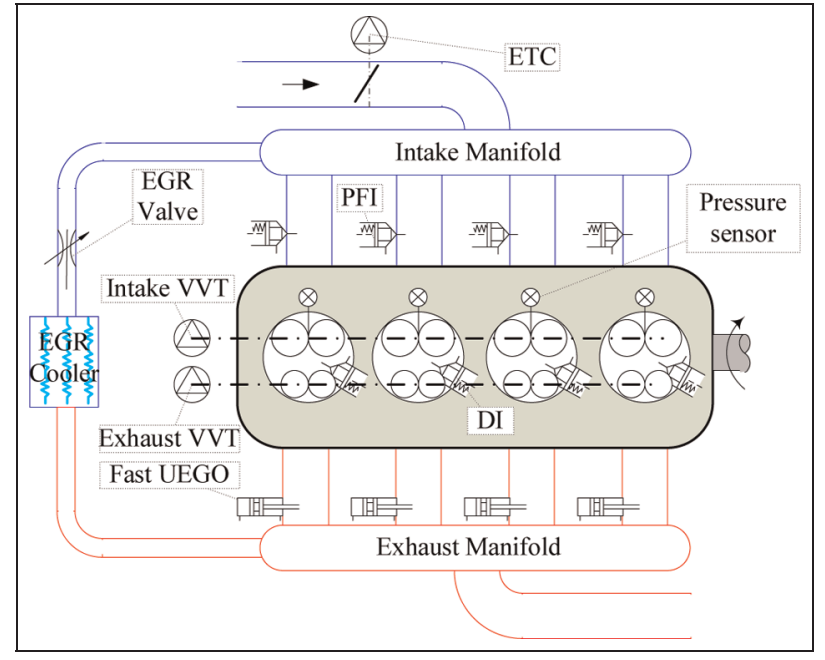

Figure 2. Configuration of the $\mathrm{HCCl}$ capable SI engine. ETC: electronic throttle control; EGR: exhaust gas recirculation; PFI: port fuel injection; VVT: variable valve timing; DI: diesel injection; UEGO: universal exhaust gas oxygen.

Table I. The $\mathrm{HCCl}$ capable SI engine specifications.

\begin{tabular}{|c|c|}
\hline Parameter & Model value \\
\hline Bore/stroke/con-rod length & $86 \mathrm{~mm} / 86 \mathrm{~mm} / \mathrm{I} 43.6 \mathrm{~mm}$ \\
\hline Compression ratio & $9.8: 1$ \\
\hline $\begin{array}{l}\text { Intake/exhaust valve lifts of high } \\
\text { stage }\end{array}$ & $9 \mathrm{~mm} / 9 \mathrm{~mm}$ \\
\hline $\begin{array}{l}\text { Intake/exhaust valve lifts of low } \\
\text { stage }\end{array}$ & $5 \mathrm{~mm} / 5 \mathrm{~mm}$ \\
\hline $\begin{array}{l}\text { Intake/exhaust valve timing } \\
\text { range (low lifts only) }\end{array}$ & $\pm 40 \mathrm{deg} / \pm 40 \mathrm{deg}$ \\
\hline Intake/exhaust valve lifts lash & $0.2 \mathrm{~mm} / 0.25 \mathrm{~mm}$ \\
\hline
\end{tabular}

combustion model is required to simulate the corresponding in-cylinder gas pressure and air-to-fuel ratio signals.

The target engine is capable of operating at the SI combustion mode with high intake and exhaust valve lifts, low EGR fraction, and with SI, at the SI-HCCI hybrid combustion mode with low intake and exhaust valve lifts, medium EGR rate and with SI, and at the HCCI combustion mode with low intake and exhaust valve lifts, high EGR rate, and without SI. During the combustion mode transition between the SI and HCCI combustions, the engine will be operated at the three combustion modes from SI to SI-HCCI to HCCI combustion. Thereby, the proposed combustion model is required to model all three combustion modes. At the same time, it also needs to have computational throughput low enough for real-time simulations, since the developed combustion model is to be used for the control strategy development and validation of the combustion mode transition.

This paper focuses mainly on the combustion model development, therefore the EGR and EGR cooler models were not presented. For the integrated engine model, the EGR valve was model based upon the valve model 
described by Fiveland and Assanis, ${ }^{18}$ and the EGR cooler was modeled based on the equation (12.2) given on the WOT website. ${ }^{17}$ Details about the integrated engine model can be found in Heywood. ${ }^{19}$ In the integrated engine model, the air-handling subsystem models, such as the exhaust manifold model and EGR loop model, are coupled with the combustion variables of individual cylinders and the cycle-to-cycle and cylinderto-cylinder dynamics are shown in these simulations.

\section{Combustion model architecture}

To model the in-cylinder gas properties, such as mass, pressure and temperature, with the accuracy over the entire engine cycle to meet the real-time HIL simulation requirements, the proposed combustion model needs to be updated every crank degree (crank-based), which is different from the modeling approaches used by Rausen et al. ${ }^{14}$ and Canova and Mohler. ${ }^{15}$ There are two additional reasons for using the crank-based modeling approach. The first is due to the fact that most combustion characteristics are usually functions of the crank angle, such as spark timing, burn duration and the crank angle of peak in-cylinder pressure location. The second is that the entire combustion process can be divided into several combustion phases as functions of crank angle. For this paper, the combustion process is divided into six phases separated by six events. They are intake valve closing (IVC), spark timing (ST), SOHCCI, exhaust valve opening (EVO), exhaust valve closing (EVC), and intake valve opening (IVO), as shown in Figure 3. The in-cylinder gas properties are modeled differently for each phase that is defined between two combustion events.

However the crank-based modeling approach has its own challenges. During the real-time simulation the entire combustion model needs to be updated within the period associated with one crank degree. This leads to fairly short computational duration at high engine speed. For example, at $6000 \mathrm{rpm}$ one crank degree corresponds to about 28 micro-seconds. In order to avoid overruns during real-time simulations, the combustion model must be as simple as possible but with satisfactory simulation accuracy. Accordingly, all in-cylinder gas properties are assumed to be in quasi-steady state during the period of each crank degree, and in-cylinder gas flow dynamics are not modeled for any of the combustion phases.

In Figure 3, events ST and SOHCCI are marked in the dashed boxes to distinguish them from other events, since the existences of these two events depend on the engine combustion mode, and the other four events always exist for any 4-stroke internal combustion engine. Note that the main difference among the SI, HCCI, and SI-HCCI combustion modes depends on the events between IVC and EVO. When the SOHCCI event does not occur, the engine is operated at the SI combustion mode; when the SOHCCI event occurs before or at the SI event, the engine is operated at the

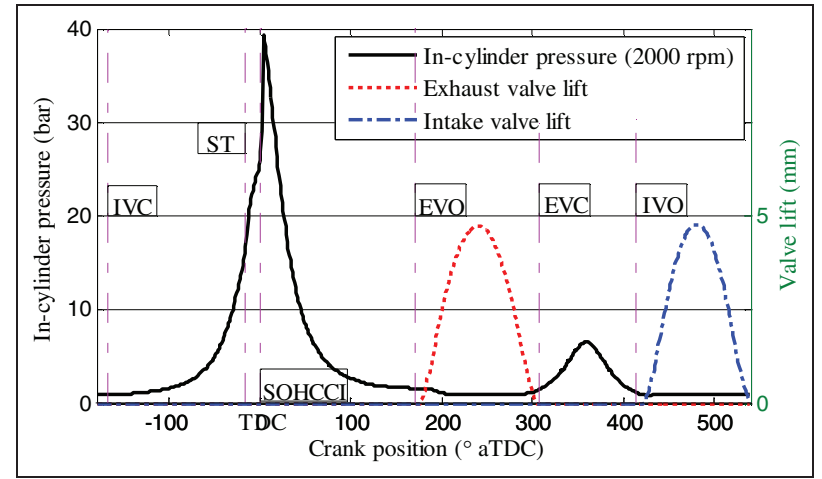

Figure 3. $\mathrm{SI}-\mathrm{HCCl}$ hybrid combustion events and phases. ATCD: after top dead center; IVC: intake valve closing; ST: spark timing; SOHCCl: start of $\mathrm{HCCl}$ combustion; EVO: exhaust valve opening; EVC: exhaust valve closing; IVO: inlet valve opening.

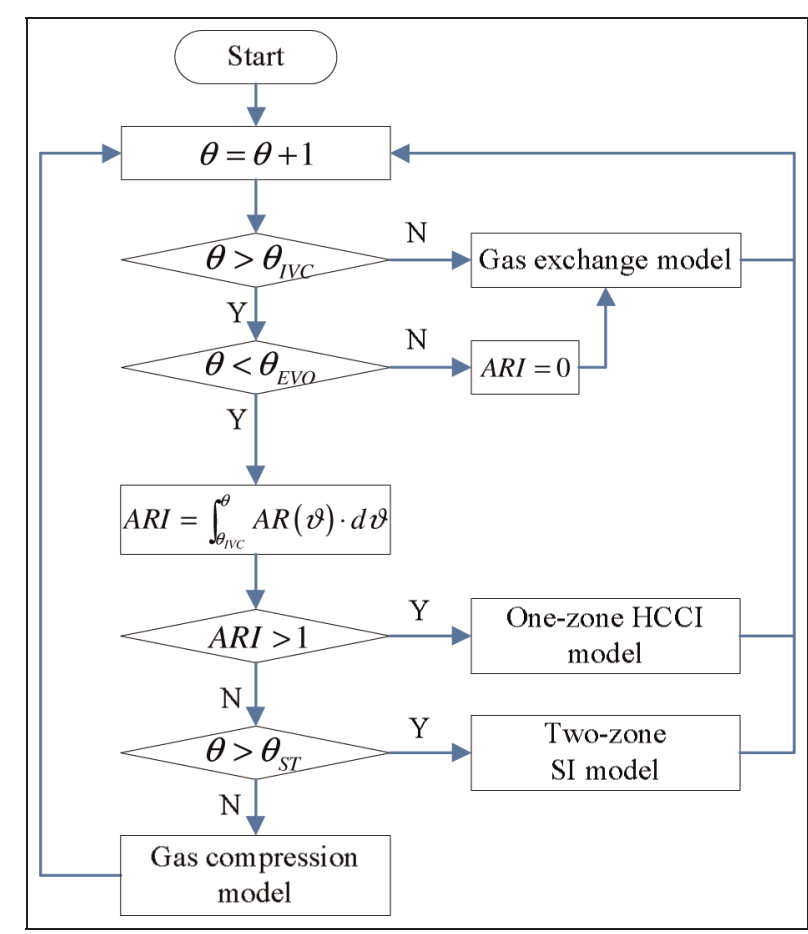

Figure 4. Diagram of the $\mathrm{SI}-\mathrm{HCCl}$ hybrid combustion model. ARI: Arrhenius integration; $\mathrm{HCCl}$ : homogeneous charge compression ignition; Sl: spark ignition.

HCCI combustion mode; while when the SOHCCI occurs after the SI combustion the engine is operated at the SI-HCCI hybrid combustion mode. Note that both SI and HCCI combustion modes are special cases of the SI-HCCI hybrid combustion mode. In fact, there also exists a fourth combustion mode, in which both SI and SOHCCI do not occur in the combustion phase, or SOHCCI happens very late without the SI combustion. The fourth mode is undesired and needs to be avoided since it means misfire or partial burn. Figure 4 illustrates the block diagram of the combustion model.

As shown in Figure 4, the Arrhenius integration (ARI) is used as the criterion of the start of HCCI combustion in the unburned zone, and it is described by ${ }^{20}$ 


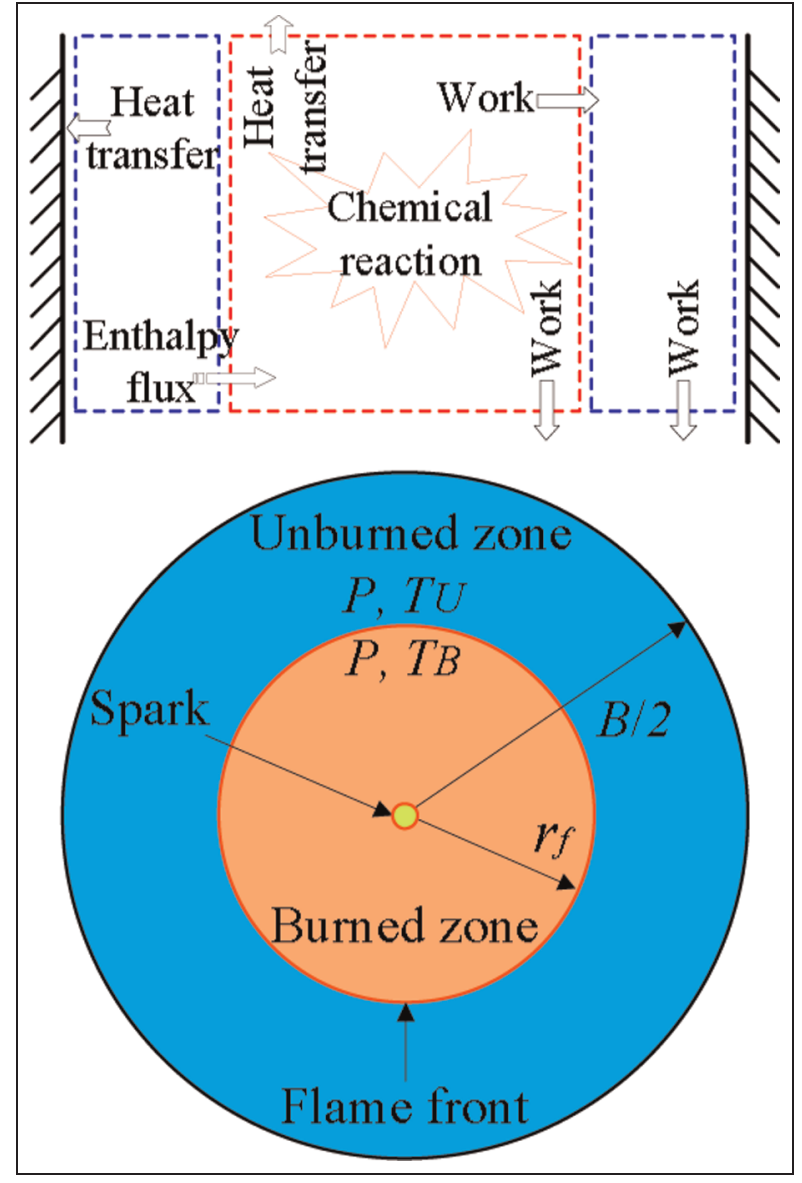

Figure 5. Two-zone SI combustion model.

$$
A R I=\int_{\theta_{I V C}}^{\theta_{i}} A x_{f}^{b} x_{o x}^{c} e^{\frac{-E_{a}}{R T}} d \vartheta
$$

where $x_{f}$ and $x_{o x}$ are unburned fuel and oxidizer concentrations; exponents $b$ and $c$ are the influence factors; $R$ is gas constant; multiplier $A$ and Arrhenius activation energy $E_{a}$ can be obtained by matching the combustion burn rate experimentally. The SOHCCI crank position $\theta_{i}$ is defined as the crank angle at which $1 \%$ of the fuel is burned under HCCI combustion. Accordingly, once $A R I$ exceeds 1 the combustion model switches to the HCCI combustion calculation. The Arrhenius integration in equation (1) starts at $\theta_{I V C}$, and is reset at $\theta_{E V O}$. During this period the integration is updated every crank degree. The variable $T$ in equation (1) is a key parameter and affects the rising rate of the $A R I$ significantly. If spark ignition never occurs, the variable $T$ in equation (1) equals the in-cylinder gas temperature, while during the SI combustion phase $T$ equals the unburned gas temperature $T_{U}$, which is calculated by the two-zone SI combustion model that is presented in the third section. This is also the main motivation of developing the two-zone SI combustion model, since the one-zone SI combustion model proposed by Yang and colleagues ${ }^{21,22}$ does not provide this required information.
In Figure 3, the phase between IVC and EVO is defined as combustion phase. It is modeled by three sub-models: the gas compression model, the one-zone HCCI combustion model, and the two-zone SI combustion model, as shown in Figure 4. The governing equations of the combustion phase are presented in the third section.

The gas exchange phase starts at EVO and ends at IVO of the next engine cycle as illustrated in Figure 3. Two valve timing strategies are modeled in the gas exchange phase of the HCCI capable SI engine. They are negative valve overlap (NVO) with low valve lift and positive valve overlap (PVO) with low valve lift. $\mathrm{PVO}$ is mainly used for SI combustion mode, in which IVO occurs before EVC. On the contrary NVO is mainly used for the HCCI and SI-HCCI hybrid combustion modes, in which EVC comes earlier than IVO. Figure 3 illustrates the NVO operation, and one can see the pressure increment of the in-cylinder gas during the NVO operation. This is due to the recompression applied to the trapped gas. The fourth section describes the governing equations for the gas exchange phase.

\section{Combustion phase models}

As illustrated in Figure 3, the combustion phase consists of three sub-phases: the compression phase between IVO and ST, the SI combustion phase between ST and SOHCCI, and the HCCI combustion phase between SOHCCI and EVO. This section discusses the mathematical models for the three phases. For this control oriented combustion model, the in-cylinder gas fuel mixture was assumed to be completely homogeneous before the start of combustion.

\section{SI combustion phase model}

During the SI combustion it is assumed that the spark ignited flame front divides the in-cylinder gas mixture into two zones: the burned and unburned zones as shown in Figure 5. To simplify the two-zone combustion model, the shape of the burned zone is assumed to be a circle centered at the cylinder (see Figure 5). For the SI combustion the temperature of the unburned zone is quite different from that of the burned zone as seen in Figure 11 and Figure 12 (see later). However, this difference is neglected in the one-zone SI combustion model discussed by Yang and $\mathrm{Zhu},{ }^{21}$ where an average temperature is used for both zones. Although the in-cylinder gas temperature stratification affects the local combustion speed and emission formation, the developed control oriented model is not capable of estimating the temperature distribution due to the realtime implementation requirement. It turns out that the simulation error of the in-cylinder pressure using the one-zone model is relatively small. However, for the SIHCCI hybrid combustion, the unburned zone temperature is an important parameter for predicting the start of HCCI combustion. The expansion of the burned 
zone during the initial SI combustion process applies work to the unburned zone. As a result it makes the unburned zone temperature rise quickly, leading to the start of HCCI combustion in the unburned zone. On the other hand, the two-zone SI combustion model approximates the SI combustion better than the onezone model. These are the main motivations for developing this two-zone SI combustion model.

To simplify the modeling process, the following assumptions were made in the two-zone SI combustion model:

- The fuel, air, and residual gas charges are uniformly premixed at IVC.

- The pressure of the in-cylinder gas mixture is assumed to be evenly distributed throughout both burned and unburned zones, while the temperatures of the two zones are different.

- At each combustion simulation step (crank degree), it is assumed that a portion of gas-fuel mixture is transferred from the unburned to burned zone, and the amount of the gas-fuel mixture is governed by the fuel burn rate that can be derived from equation (2) below. Also the flow of each zone is assumed in steady state. The heat transfer between the burned and unburned zones is neglected.

After SI, the fuel mass fraction burned is approximated by the following Wiebe function ${ }^{15}$

$$
x\left(\theta_{i}\right)=1-\exp \left[-a\left(\frac{\theta_{i}-\theta_{S T}}{\Delta \theta}\right)^{m+1}\right]
$$

where $x$ is the MFB of fuel; $\theta_{i}$ is the current crank position; the predicted burn duration $\Delta \theta$ and the Weibe exponent $m$ are calibration parameters as functions (implemented as lookup table) of engine speed, load, and coolant temperature. Coefficient $a$ depends on how burn duration $\Delta \theta$ is defined. When $\Delta \theta$ is specified as the crank angle between $10 \%$ and $90 \%$ MFB, $a$ can be calculated by

$$
a=\left\{[-\ln (1-0.9)]^{\frac{1}{m+1}}-[-\ln (1-0.1)]^{\frac{1}{m+1}}\right\}^{m+1}
$$

Within the SI combustion phase, the mass of the burned zone gas is calculated based upon the mass of burned fuel that can be calculated by (2). According to the first law of thermodynamics, ${ }^{23}$ the energy balance of the burned zone is represented by

$$
\frac{d\left(M_{B} e_{B}\right)}{d \theta}+P \frac{d V_{B}}{d \theta}+Q_{B}=\eta_{S I} h_{L H V} M_{f} \frac{d x}{d \theta}+\frac{d M_{B}}{d \theta} h_{U}
$$

where $M_{B}, V_{B}$, and $e_{B}$ are the mass, volume, and internal energy of the burned zone, respectively; $Q_{B}$ is the heat transfer from the burned zone, where $Q_{B}=x Q$ and $Q$ is given by equation (9); $M_{f}$ is the total fuel mass trapped in the cylinder for the given engine cycle; $P$ is the gas pressure of both zones; $h_{L H V}$ is the low heating value of fuel; $h_{U}$ is the specific enthalpy of the unburned zone; $\eta_{S I}$ is the combustion efficiency due to incomplete combustion.

The energy balance equation of unburned zone is

$$
\frac{d\left(M_{U} e_{U}\right)}{d \theta}+P \frac{d V_{U}}{d \theta}+Q_{U}=\frac{d M_{U}}{d \theta} h_{U}
$$

where $M_{U}, V_{U}$, and $e_{U}$ are the mass, volume, and internal energy of unburned zone, respectively; $Q_{U}$ is the heat transfer from the unburned zone and $Q_{U}=(1-x) Q$.

Moreover, the gases of both burned and unburned zones can be considered as ideal gases, ${ }^{18,24}$ and therefore ideal gas law holds for both zones. For the burned zone, we have

$$
\frac{P V_{B}}{R T_{B}}=M_{B}=x M_{t}
$$

where $M_{t}$ is the total gas mass of both zones; $T_{B}$ is the burned zone gas temperature; $R$ is gas constant.

For the unburned zone, the ideal gas law can be expressed by

$$
\frac{P V_{U}}{R T_{U}}=M_{U}=(1-x) M_{t}
$$

where $T_{U}$ is the unburned zone gas temperature.

Additionally, the cylinder geometry yields the following equation

$$
V_{B}+V_{U}=V
$$

where $V$ is the instant cylinder volume.

For an SI gasoline engine the heat transfer due to radiation is relatively small, compared to the convective heat transfer. ${ }^{19}$ Therefore only convective heat transfer $Q$ is computed in the energy balance equations. The Woschni correlation model ${ }^{19,20}$ is used to calculate the heat transfer quantity

$$
Q\left(\theta_{i}\right)=A_{c} h_{c}\left[T\left(\theta_{i-1}\right)-T_{w}\right]
$$

and

$$
h_{c}=q B^{l-1} P^{l} w^{l} T^{0.75-1.62 l} / N_{e}
$$

where $B$ is the cylinder bore; $w$ is the gas flow velocity that is a function of engine speed $N_{e} ; A_{c}$ is the contact area between gas and cylinder wall; $T_{w}$ is the average temperature of the cylinder wall; coefficient $q$ and exponent $l$ are used as model calibration parameters, where $q=0.54$ and $l=0.8$ provide the best correlation for the SI combustion mode.

Note that the gas temperature $T$ in equations (9) and (10) is the average temperature of both burned and unburned zones that can be calculated by

$$
T=\frac{x C_{v B} T_{B}+(1-x) C_{v U} T_{U}}{x C_{v B}+(1-x) C_{v U}}
$$

where $C_{v}$ is the specific heat for constant volume. 


\section{$\mathrm{HCCl}$ combustion phase model}

The HCCI combustion is modeled under the one-zone assumption due to its flameless nature. Fuel and air are assumed to be mixed homogeneously throughout the unburned gas mixture; thermodynamic characteristics such as pressure and temperature are uniformly distributed in the cylinder. Accordingly, only the mean values of in-cylinder gas pressure and temperature are modeled.

Unlike the SI combustion, there is no direct control (such as spark for SI combustion) to initiate the HCCI combustion. The fast heat release rate of the $\mathrm{HCCI}$ combustion is actually triggered by a very slow chemical reaction of the gas-fuel mixture during the compression phase between the IVC and start of HCCI combustion, where it takes certain crank degrees for one percent fuel be burned. The most commonly used approach in control oriented modeling of the HCCI combustion is to assume that the chemical reaction process is governed by a single rate Arrhenius equation ${ }^{19}$

$$
A R=A x_{f}^{b} x_{o x}^{c} e^{\frac{-E_{a}}{R T}}
$$

where $A R$ is the rate of disappearance of unburned fuel and other parameters have been discussed in equation (1). As mentioned at the beginning of the second section the integration of the Arrhenius function is used to estimate the SOHCCI timing, where the SOHCCI timing separates the slow chemical reaction from the fast one. During the fast combustion phase the fuel MFB can also be approximated by the following Wiebe function

$$
x\left(\theta_{i}\right)=1-\exp \left[-a\left(\frac{\theta_{i}-\theta_{S O H C C I}}{\Delta \theta_{H C C I}}\right)^{m+1}\right]
$$

where the coefficients $a$, the exponential $m$ and the combustion duration $\Delta \theta_{H C C I}$ are functions of engine speed, load (fuel quantity for HCCI combustion), and coolant temperature. They are different from those used in the SI combustion model (see equation (2)). Based on the calculated MFB, the in-cylinder gas pressure and temperature are calculated by

$$
\begin{aligned}
T\left(\theta_{i}\right)= & T\left(\theta_{i-1}\right)\left(\frac{V\left(\theta_{i-1}\right)}{V\left(\theta_{i}\right)}\right)^{(\kappa-1)} \\
& +\frac{\eta_{H C C I} M_{f} h_{L H V}\left[x\left(\theta_{i}\right)-x\left(\theta_{i-1}\right)\right]-Q\left(\theta_{i}\right)}{M_{t} C_{v}}
\end{aligned}
$$

and

$$
P\left(\theta_{i}\right)=P\left(\theta_{i-1}\right) \frac{V\left(\theta_{i-1}\right)}{V\left(\theta_{i}\right)} \cdot \frac{T\left(\theta_{i}\right)}{T\left(\theta_{i-1}\right)}
$$

where $\kappa$ is the average heat capacity ratio of the gas charged into the cylinder; $\eta_{H C C I}$ is a function of engine speed and fuel mass, and it is a calibrated to match the simulated Indicated mean effective pressure (IMEP) is provided by the GT-Power model. The in-cylinder gas temperature is derived based on isentropic assumption and energy balance of a closed system.
There are two terms in the right hand of equation (14). The first term represents an isentropic compressing or expanding process, and the second calculates the temperature increase due to the heat released during the combustion. Therefore, the complicated combustion thermodynamics is simplified into an isentropic volume change process without heat exchange in one crank degree period and a heat exchange process without volume change in an infinitely small time period.

\section{Compression phase model}

The governing equations of the gas compression phase between IVC and ST are the same as equations (14) and (15) with $x=0$ throughout the phase.

\section{Gas exchange phase models}

As shown in Figure 3, the gas exchange process occurs between EVO and IVC. During this process exhaust gas leaves the cylinder, and fresh charge enters the cylinder and mixes with residual gas from the last engine cycle. The gas exchange process is normally very complicated and difficult to model for real-time simulations. A CFD model is required to model it accurately, but it is too complicated to be used in the control oriented model due to the real-time simulation requirement. This paper combines both correlation and physical modeling approaches to model the gas exchange process.

\section{Gas exhaust process model}

The gas exhaust phase corresponds to the phase between EVO and IVO for PVO and the phase between EVO and EVC for NVO. In this phase, the in-cylinder gas mixture expands in the cylinder, exhaust runner, and exhaust manifold. The in-cylinder pressure drops quickly, but not instantaneously, down to the level of the exhaust manifold pressure, see Figure 6. It normally takes a few crank degrees for the in-cylinder pressure to reach the exhaust manifold pressure. It is quite difficult to model this process accurately using simple dynamic equations for real-time simulations. For simplicity, a first-order transfer function is used to approximate this process as follows

$$
P(z)=\frac{1-\tau_{E V O}}{1-\tau_{E V O} \cdot z^{-1}} P_{E M}(z)
$$

where $z$ is the unit delay operator for one crank degree; $\tau_{E V O}$ is the transition time constant as a function of engine speed and load for exhaust valve opening; $P_{E M}$ is the exhaust manifold absolute pressure.

The in-cylinder gas temperature is calculated as a function of the pressure drop. That is

$$
T\left(\theta_{i}\right)=T\left(\theta_{i-1}\right)\left[\frac{P\left(\theta_{i}\right)}{P\left(\theta_{i-1}\right)}\right]^{\frac{\kappa-1}{\kappa}}-\frac{Q\left(\theta_{i-1}\right)}{M\left(\theta_{i-1}\right) C_{v}}
$$




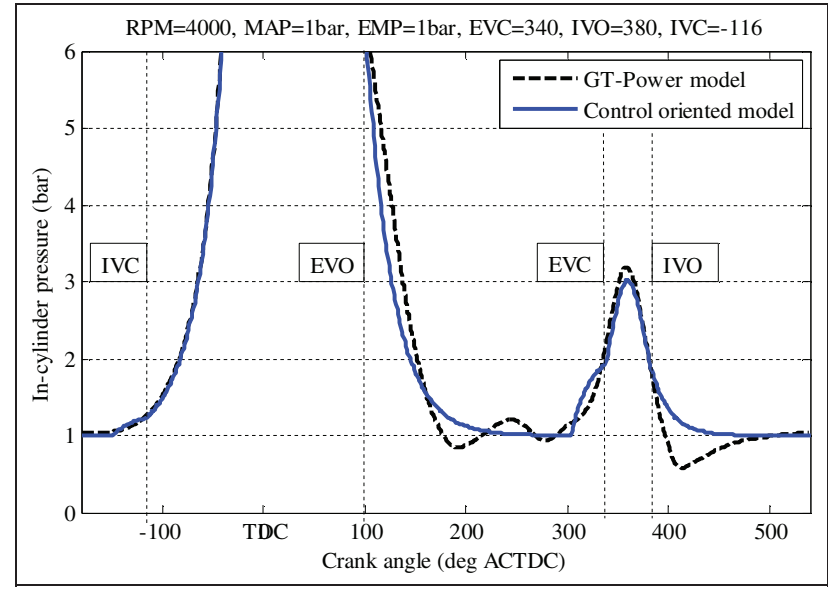

Figure 6. In-cylinder pressures in gas exchange process. RPM: revolutions per minute; MAP: intake manifold air pressure; EMP: exhaust manifold pressure; EVC: exhaust valve closing; IVO: intake valve opening; IVC: intake valve closing; ACTDC: after combustion top dead center.

By investigating the pressure signal from GT-Power simulation in Figure 6, one can find that during the late stage of the gas exhaust phase the in-cylinder pressure increases significantly, and it finally reaches 1.9 bar at EVC, which is much bigger than the exhaust manifold pressure (1.0 bar in this case). There are two possible causes. One is that the exhaust valve is almost closed during the late stage, which prevents gas from leaving the cylinder; and the other is that the EVC timing is before gas exchange TDC. This phenomenon cannot be ignored since $P\left(\theta_{E V C}\right)$ is used to calculate residual gas mass $M_{r}$, which affects HCCI combustion timing and duration significantly. The dynamics of the pressure increase can also be modeled using a first-order transfer function

$$
P(z)=\frac{1-\tau_{E V C}}{1-\tau_{E V C} \cdot z^{-1}} P_{E M}(z) \eta_{E V C}
$$

where $\eta_{E V C}$ is the predicted pressure ratio at EVC, and it is mainly affected by engine speed, EVC timing and exhaust valve lift; $\tau_{E V C}$ is the transition time constant of EVC and it is a function of engine speed and load. As shown in Figure 6, this model yields a good match between the pressure signals provided by simulation results of both GT-Power and the control oriented combustion models, especially at the EVC timing.

\section{Residual gas recompression during NVO operation}

NVO is often used to regulate the HCCI combustion properties such as combustion timing. There are two main advantages. One is to reform the trapped pilot fuel in this phase (EVC-IVO in Figure 3); ${ }^{25}$ and the other is to adjust the residual gas temperature. As a result the in-cylinder gas temperature at IVC can be optimized for the desired SOHCCI timing. The first effect is hard to model using governing equations. In Ravi et al., ${ }^{25}$ the Arrhenius threshold is correlated based on the experimental data. It is well known that pilot fuel reforming due to recompression can effectively affect HCCI combustion timing and the pilot fuel injection timing and quantity can be used as control parameters in a few HCCI control strategies. However, during the SI to HCCI mode transition of a multi-cylinder engine equipped with electrical VVT and a two-step valve system, the reforming effect is less significant than that in the steady HCCI mode due to the electrical VVT response dynamics, which reduces the effect of negative valve overlap and impairs the recompression of residue gas and fuel. Therefore, this paper ignores this effect due to the lack of the experimental data. Although the recompression has less effect on combustion timing control during the combustion mode transition than during the steady state HCCI operation, reforming the pilot fuel during the recompression could still be useful. It can be used as a quick control variable to micro-tune the combustion timing for the consequent combustion event.

The second effect can be approximated by a combination of an isentropic volume change process of ideal gas in a closed system and a heat transfer process. Temperature and pressure are calculated by

$$
T\left(\theta_{i}\right)=T\left(\theta_{i-1}\right) \cdot\left(\frac{V\left(\theta_{i-1}\right)}{V\left(\theta_{i}\right)}\right)^{(\kappa-1)}-\frac{Q\left(\theta_{i-1}\right)}{M\left(\theta_{i-1}\right) C_{v}}
$$

and

$$
P\left(\theta_{i}\right)=P\left(\theta_{i-1}\right) \cdot\left(\frac{V\left(\theta_{i-1}\right)}{V\left(\theta_{i}\right)}\right)^{\kappa}
$$

Another important parameter calculated in this phase is the residual gas mass. It is calculated based upon the ideal gas law, and updated once per engine cycle at EVC for NVO

$$
M_{r}=M\left(\theta_{E V C}\right)=\frac{P\left(\theta_{E V C}\right) \cdot V\left(\theta_{E V C}\right)}{R \cdot T\left(\theta_{E V C}\right)}
$$

\section{Gas exchange behavior during PVO}

The gas exchange behavior during the phase between IVO and EVC for the PVO is also very complicated. Since both intake and exhaust valves are partially opened in this phase, residual gas and fresh charge can flow in many ways depending on the pressure ratio across each valve. For simplification, it is assumed that gas exchange across the exhaust valve is terminated in this phase and part of the residual gas can flow to the intake port but it flows back into the cylinder completely during the remaining intake process. Based upon this assumption, the mass of residual gas for PVO can be calculated at IVO by 


$$
M_{r}=M\left(\theta_{I V O}\right)=\frac{P\left(\theta_{I V O}\right) \cdot V\left(\theta_{I V O}\right)}{R \cdot T\left(\theta_{I V O}\right)}
$$

This modeling approach may result in slight error in $M_{r}$ calculation. Fortunately, the PVO only occurs during the SI combustion process, in which the influence of residual gas to the entire engine performance is much less than that during the SI-HCCI and HCCI combustion processes that usually involve NVO.

For the cooled EGR, the EGR valve was modeled according to the valve model described in Fiveland and Assanis, ${ }^{18}$ and the EGR cooler was modeled based on the equation (12.2) of the 'WOT' website. ${ }^{17}$

\section{Air intake process model}

The air intake process from IVO to IVC is also a process of in-cylinder gas mixing. During this phase, the fresh charge air, injected fuel vapor, and residual gas are assumed to be mixed homogeneously. At the same time, the in-cylinder pressure approaches the intake manifold pressure. A first-order transfer function is also used in this model as follows

$$
P(z)=\frac{1-\tau_{I V O}}{1-\tau_{I V O} \cdot z^{-1}} \cdot P_{I M}(z)
$$

and

$$
T\left(\theta_{i}\right)=\frac{M_{f} \cdot C_{v f} \cdot T_{f}+M_{a} \cdot C_{v a} \cdot T_{I M}+M_{r} \cdot C_{v r} \cdot T_{r}}{M_{f} \cdot C_{v f}+M_{a} \cdot C_{v f}+M_{r} \cdot C_{v r}}
$$

where $\tau_{I V O}$ is the transition time constant for intake valve opening, which is a function of engine speed and load; $P_{I M}$ is the intake manifold absolute pressure; $M_{a}$ is the fresh charge mass (note that it could be the mixture of fresh air and external EGR gas); $T_{r}$ is the temperature of residual gas and it can be calculated by the approach described in equation (17).

During the late stage of the air intake phase, the incylinder pressure may also deviate from the intake manifold pressure, see Figure 6. This phenomenon is similar to the case of the exhaust phase. The same approach is used to model the transition as follows.

$$
P(z)=\frac{1-\tau_{I V C}}{1-\tau_{I V C} \cdot z^{-1}} P_{I M}(z) \eta_{I V C}
$$

where $\eta_{I V C}$ is the predicted pressure ratio at IVC, which is a function of engine speed, IVC timing and exhaust valve lift; and $\tau_{I V C}$ is the transition time constant for intake valve closing and it is a function of engine speed and load. At IVC, the total in-cylinder gas mass $M_{t}$ is calculated by

$$
M_{t}=M\left(\theta_{I V C}\right)=\frac{P\left(\theta_{I V C}\right) \cdot V\left(\theta_{I V C}\right)}{R \cdot T\left(\theta_{I V C}\right)}
$$

\section{Model implementation in HIL simulation environment}

This section describes the dSPACE based real-time HIL engine simulation environment and the implementation of the mathematical models presented in the third and fourth sections into the HIL simulation environment.

\section{Combustion model solution}

The entire engine model consists of numerous mathematic equations. Some of them are nonlinear differential equations. To solve these equations analytically in real-time is almost impossible. During the HIL simulations, these equations are solved numerically. This sub-section discusses the numeric solution of the combustion model presented in this paper.

For most of the mathematical models presented in the third and fourth sections, the combustion variables of present crank angle degree are directly calculated based upon those of the previous crank angle degree. Whereas in the two-zone SI combustion model, the governing equations (4) to (8), are nonlinear differential equations. To solve them, the equations are discretized and converted into the following format

$$
\left\{\begin{array}{l}
a_{1} V_{B}\left(\theta_{i}\right)+b_{1} T_{B}\left(\theta_{i}\right)=c_{1} \\
a_{2} V_{U}\left(\theta_{i}\right)+b_{2} T_{U}\left(\theta_{i}\right)=c_{2} \\
V_{B}\left(\theta_{i}\right) P\left(\theta_{i}\right) / T_{B}\left(\theta_{i}\right)=c_{3} \\
V_{U}\left(\theta_{i}\right) P\left(\theta_{i}\right) / T_{U}\left(\theta_{i}\right)=c_{4} \\
V_{B}\left(\theta_{i}\right)+V_{U}\left(\theta_{i}\right)=c_{5}
\end{array}\right.
$$

where

$$
\begin{aligned}
a_{1}= & P\left(\theta_{i-1}\right) \\
b_{1}= & x\left(\theta_{i}\right) M_{t} C v \\
a_{2}= & P\left(\theta_{i-1}\right) \\
b_{2}= & {\left[1-x\left(\theta_{i}\right)\right] M_{t} C v } \\
c_{1}= & \eta_{S I} H_{L H V} M_{f}\left[x\left(\theta_{i}\right)-x\left(\theta_{i-1}\right)\right]+P\left(\theta_{i-1}\right) V_{B}\left(\theta_{i-1}\right)-x\left(\theta_{i}\right) Q\left(\theta_{i}\right) \\
& +M_{t} x\left(\theta_{i-1}\right) C v T_{B}\left(\theta_{i-1}\right)+M_{t}\left[x\left(\theta_{i}\right)-x\left(\theta_{i-1}\right)\right] C p T_{U}\left(\theta_{i-1}\right) \\
c_{2}= & P\left(\theta_{i-1}\right) V_{U}\left(\theta_{i-1}\right)-M_{t}\left[x\left(\theta_{i}\right)-x\left(\theta_{i-1}\right)\right] C p T_{U}\left(\theta_{i-1}\right) \\
& +M_{t}\left[1-x\left(\theta_{i-1}\right)\right] C v T_{U}\left(\theta_{i-1}\right)-\left[1-x\left(\theta_{i}\right) Q\left(\theta_{i}\right)\right. \\
c_{3}= & x\left(\theta_{i}\right) M_{t} R \\
c_{4}= & {\left[1-x\left(\theta_{i}\right)\right] M_{t} R } \\
c_{5}= & V\left(\theta_{i}\right)
\end{aligned}
$$

By solving the algebraic equations set (27), the five unknowns $\left[V_{B}\left(\theta_{i}\right) V_{U}\left(\theta_{i}\right) T_{B}\left(\theta_{i}\right) T_{U}\left(\theta_{i}\right) P\left(\theta_{i}\right)\right]^{T}$ in (27) can be represented as functions of the information computed in the last crank angle degree $\left[V_{B}\left(\theta_{i-1}\right) V_{U}\left(\theta_{i-1}\right) T_{B}\left(\theta_{i-1}\right)\right.$ $\left.T_{U}\left(\theta_{i-1}\right) P\left(\theta_{i-1}\right) x\left(\theta_{i-1}\right)\right]^{T}$ and the MFB of current crank angle degree $x\left(\theta_{i}\right)$ is calculated by the Wiebe function (2). Equation (27) was solved analytically using Matlab Symbolic Math Toolbox, and the rational solution, see Appendix 3, was used for numerical solution of the algebraic equations set (27) in the HIL simulations.

Note that due to the nonlinearity of equations (6) and (7) the solutions of equation set (27) are not 
unique. After implementing the model into the dSPACE HIL simulation environment for real-time simulation, the two-zone engine model can be simulated in real-time at engine speeds up to $5000 \mathrm{rpm}$ without overrun. Fortunately the possible operating range of the two-zone model (SI-HCCI hybrid mode) is between $1000 \mathrm{rpm}$ and $3500 \mathrm{rpm}$.

For the SI-HCCI combustion simulations, the incylinder gas temperatures of the one-zone HCCI model and the two-zone SI model are updated every crank degree during the SI combustion. The SI unburned zone temperature is mainly used to accurately estimate the start of HCCI combustion. Once the start of HCCI combustion criterion is satisfied, updating of both burned and unburned zone temperatures stops, and the overall in-cylinder gas temperature is updated till the end of the current engine cycle due to the continued heat release of HCCI combustion and volumetric change in the cylinder. In-cylinder gas pressure is assumed to be identical over both zones for the two-zone SI model.

\section{HIL simulation system architecture}

Figure 7 shows the system architecture of the HIL simulation environment. For simplicity, not all the signals and subsystems are included in the diagram. It is only used to demonstrate the implementation of the engine model into the HIL simulation environment. In Figure 7, the ECU (engine control unit) on top of the diagram is used for implementing the combustion mode transition control strategies that are presented by Yang and $\mathrm{Zhu}^{5}$ and it is not the focus of this paper. All blocks below the ECU block are to be implemented in the HIL engine simulator. Within the combustion block, the mathematical models proposed in Sections 3 and 4 are updated every crank angle degree. The wall wetting dynamic model of PFI (port fuel injection) fueling as represented by the fuel inject block is updated every engine cycle for each injector. The wall wetting dynamics describes the PFI fuel injection dynamics where part of the injected fuel flows into the cylinder directly and part of it remains on the back of the intake valve, and can be found in Fiveland and Assanis. ${ }^{18}$ The rest of the blocks are the time-based (1 millisecond) models for crankshaft torque balance, air intake/ exhaust and EGR flow dynamics, etc. Accordingly, there are three parallel tasks executed in the engine simulator processor (dSPACE DS-1006 CPU). Note that the mathematical models for all blocks other than the combustion model block are presented by Yang and Zhu. ${ }^{21,26}$ They are well developed mean-value engine models that are widely utilized in numerous technique papers. ${ }^{14,19,24}$

During the HIL simulations, engine crank signal (720 pulses per cycle), TDC signal (2 pulses per cycle) and cam signal (1 pulse per cycle) are generated based on the modeled engine speed $N_{e}$ using the dSPACE hardware (DS-2211 APU board). These signals are sent to the ECU to synchronize the engine simulator and the ECU.

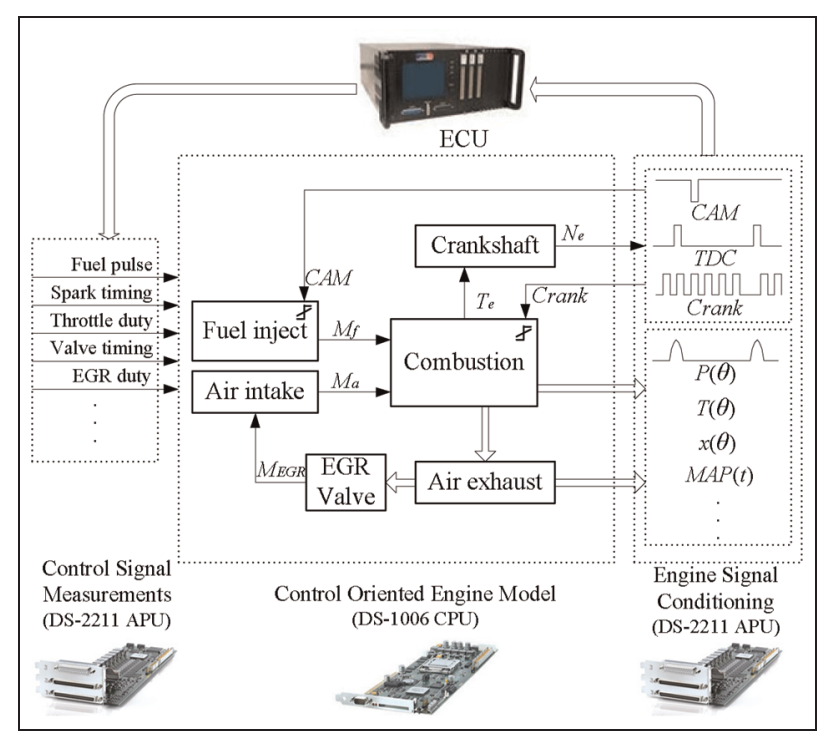

Figure 7. System architecture of the integrated HIL simulation environment.

ECU: engine control unit; CAM: cam position signal; TDC: top dead center; EGR: exhaust gas recirculation; CPU: central processing unit; APU: accelerated processing unit.

At the same time, crank and cam signals are looped back to the HIL simulation CPU board to generate interruption signals for crank and cycle based simulations, and to trigger crank based and cycle based model calculations. The interruption triggered by crank signal has the highest priority in the interruption queue, since the crank based routine needs to be executed within a specific crank angle. The cycle based interruption has the second highest priority. On the output side (right) of the engine simulation diagram, the signal conditioning block converts all computed engine variables to actual engine signals that the ECU can utilize. On the input side (left) of the engine simulation diagram, the signal preprocessing block translates all ECU control signals back to the parameters that the engine model can use.

A diagram of the entire HIL engine simulation platform is shown in Figure 8. Two host computers are used to interact with the $\mathrm{dSPACE}$ based real-time engine simulator and the Opra-RT based engine prototype controller, respectively. They are used to set the simulation parameters and to display the simulation results. An oscilloscope is also adopted to display the real-time HIL simulation results. The nonlinear equations (4) to (8) are solved using the solution provided in Appendix 3.

\section{Model calibration and validation}

To calibrate and validate the developed SI-HCCI hybrid combustion model, a GT-Power single cylinder engine model was also developed for the engine configuration shown in Figure 2 with specifications shown in Table 1. GT-Power is an engine and powertrain modeling tool widely used in the automotive industry. 


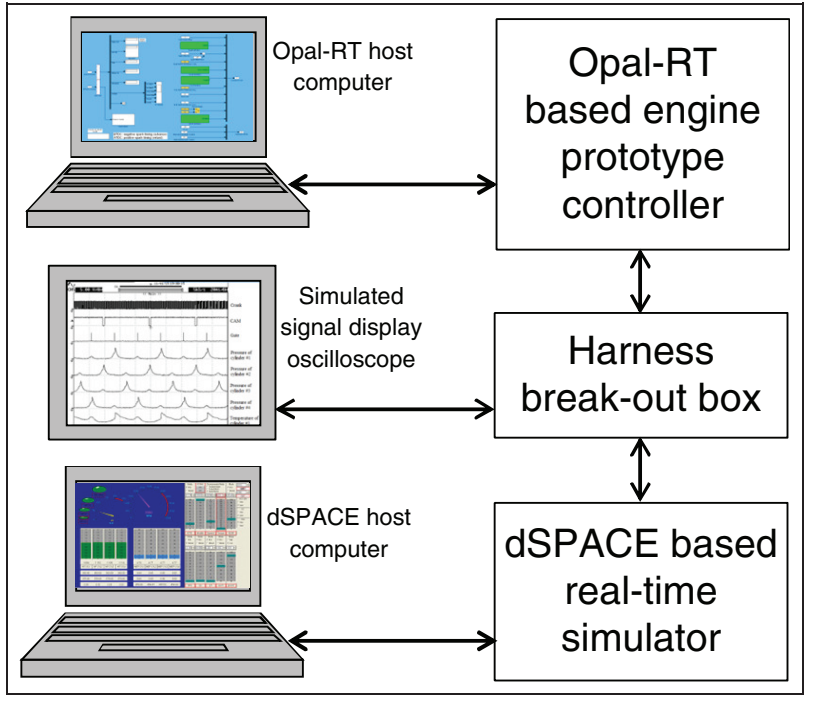

Figure 8. HIL simulation platform.

Comparing with the combustion model developed in this paper, the 1D (one dimensional) combustion model employed by the GT-Power software provides highfidelity in-cylinder combustion information such as pressure and temperature. However, its high computational throughput prevents it from being directly used for the real-time HIL simulations. In this paper, the GT-power model is used as the reference model to calibrate and validate the combustion model developed in this paper.

Model calibration data were generated by GT-Power simulations over the engine speed and load operational ranges. For each evaluation condition, GT-Power simulation results were used as the baseline. Firstly, $\eta_{E V C}$ and $\eta_{I V C}$ (look-up tables) in equations (18) and (25) were calibrated as functions of engine speed, valve timing and lift to make the in-cylinder gas pressure at EVC and IVC match with the GT-Power simulation results; secondly, coefficients such as $m$ and $\kappa$ in equations (2) and (14) were calibrated to match the MFB profiles, finally, $\eta_{S I}$ and $\eta_{H C C I}$ in equations (4) and (14) were calibrated as functions of engine speed and load to match engine IMEP.

\section{Two-zone SI combustion model validation}

The two-zone SI combustion model was validated at $3000 \mathrm{rpm}$ under three different engine load conditions with intake manifold pressure $P_{I M}=0.4,0.7$ and $1.0 \mathrm{bar}$ corresponding to low, medium, and full load conditions. The combustion durations under the three conditions are quite different, the associated spark timings were set to the MBT (Minimal advance for the Best Torque) at each load condition, and the valve strategy is PVO. Fairly good agreement of both pressure and temperature signals can be found in simulation results shown in Figure 9 and Figure 10. This demonstrates that the developed two-zone SI combustion model is capable of providing comparable simulation results to these of the

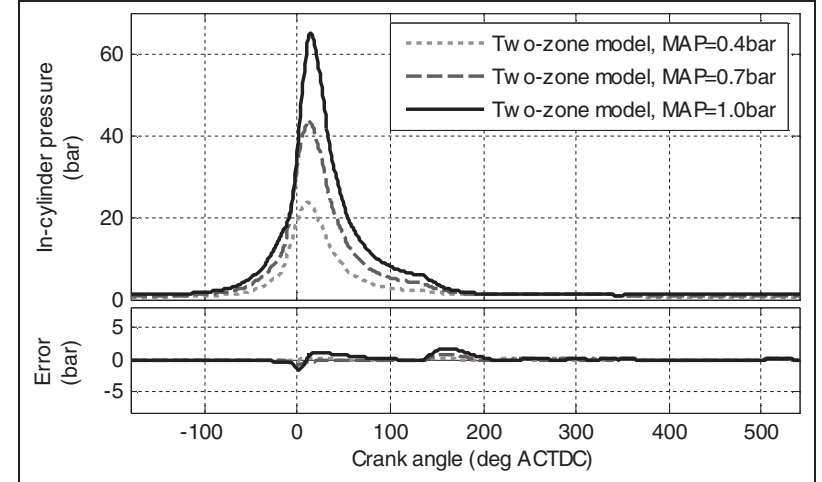

Figure 9. In-cylinder pressures of GT-Power and two-zone SI models at $3000 \mathrm{rpm}$.

MAP: intake manifold air pressure; ACTDC: after combustion top dead center.

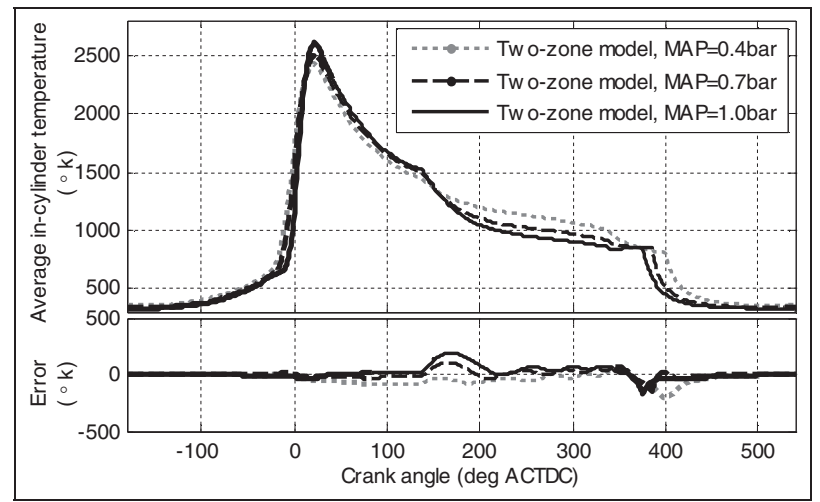

Figure 10. In-cylinder temperatures of GT-Power and twozone SI models at $3000 \mathrm{rpm}$.

MAP: intake manifold air pressure; ACTDC: after combustion top dead center.

GT-Power model. Note that the in-cylinder temperature of the two-zone SI combustion model, see Figure 10, is the averaged temperature, provided by equation (11), of both burned and unburned zones. Although the modeling error of temperature is fairly large at certain crank angles, especially around the exhaust valve opening timing, the error at the region of interest (for instance, start of combustion) is fairly small.

Figure 11 and Figure 12 present the two temperature profiles (burned and unburned zones) of the two-zone SI combustion model, where the burned zone temperature is shown in Figure 11 and unburned zone temperature in Figure 12. Note that the one-zone model can only provide the averaged temperature of the burned and unburned zones. Both temperature profiles show good agreement with the GT-Power simulation results. This confirms the effectiveness of the developed twozone model.

In Figure 12 both unburned zone temperatures are also compared with the in-cylinder gas temperature simulated without combustion. It shows that before 


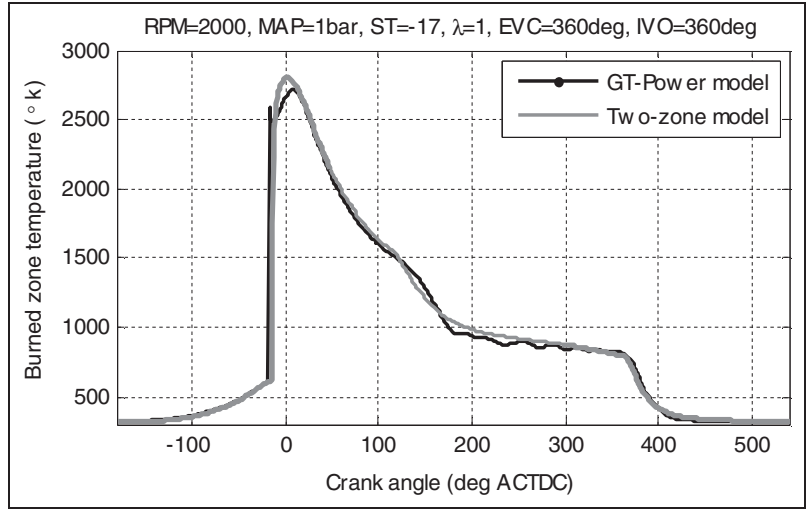

Figure I I. Burned zone gas temperatures of GT-Power and two-zone SI combustion models.

RPM: revolutions per minute; MAP: intake manifold air pressure; ST: spark timing; EVC: exhaust valve closing; IVO: intake valve opening; ACTCD: after combustion top dead center.

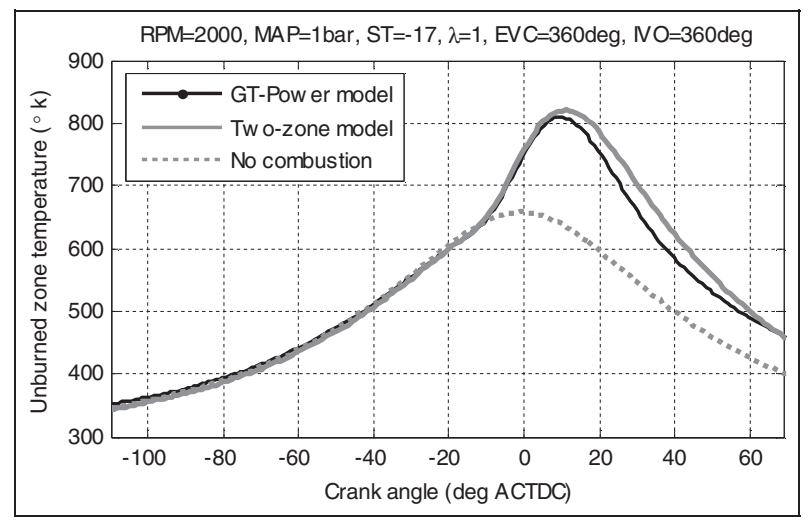

Figure 12. Unburned zone gas temperatures of GT-Power and two-zone SI combustion models.

RPM: revolutions per minute; MAP: intake manifold air pressure; ST: spark timing; EVC: exhaust valve closing; IVO: intake valve opening; ACTDC: after combustion top dead center.

spark timing ( $\mathrm{ST}=-17^{\circ}$ ACTDC in this case), the three temperatures are the same; and after the spark timing, the unburned zone temperature increases much faster than that without combustion. This is due to the work applied by the burned zone gas. The Arrhenius integration in equation (1) is more sensitive to higher unburned zone temperature, which indicates that the slow chemical reaction in the unburned zone before SOHCCI can be accelerated by the rapid increment of the unburned zone temperature. Accordingly if the unburned zone gas temperature can be controlled precisely, so can the SOHCCI timing of both HCCI and SI-HCCI hybrid combustions.

To validate the combustion model over a broad engine operational range, additional simulations were conducted at the engine speed varied between $1000 \mathrm{rpm}$ and $5000 \mathrm{rpm}$ with a $1000 \mathrm{rpm}$ interval and low, medium, and full engine loads. Both PVO and NVO valve strategies were used. The valve timings for PVO are $\mathrm{IVO}=340^{\circ} \mathrm{ACTDC}$ and $\mathrm{EVC}=380^{\circ} \mathrm{ACTDC}$ and for $\mathrm{NVO}, \mathrm{IVO}=380^{\circ} \mathrm{ACTDC}$ and $\mathrm{EVC}=340^{\circ}$ ACTDC. To simplify the presentation, only a few key engine variables were plotted in Figure 13 and Figure 14. Note that these key variables, shown in Figure 13 and Figure 14, outline the agreement of the pressure, temperatures (burned and unburned) of both GTPower and developed models.

From Figure 13 and Figure 14, one can find that all pressure related variables, such as IMEP, peak cylinder pressure $P_{\max }$ and the crank position of $P_{\max }$, have fairly good agreement with those of the GT-Power simulations, while for the temperature related variables, the errors are relatively larger. One possible reason is that the gas property parameters such as $C_{v}$ and $R$ used in the two models are different. In the GT-Power model these parameters are functions of not only the gas temperature but also the gas chemical composition, while in the developed two-zone model the chemical composition is not considered due to the simplified real-time modeling.

\section{One-zone $\mathrm{HCCl}$ combustion model validation}

Figure 15 shows the HCCI combustion simulation results using the developed one-zone and GT-Power models. For this simulation, $16 \mathrm{mg}$ of fuel was injected into each cylinder per engine cycle to generate 5.02 bar IMEP at $2000 \mathrm{rpm}$, which is close to the load limitation of the HCCI combustion. Note that for HCCI combustion, the engine load is not determined by the engine MAP but by the injected fuel quantity due to the unthrottled operation. The lift profiles of both intake and exhaust valves were switched to the low lift stages, since the low exhaust valve lift makes it possible to trap more internal residual gas with the NVO operation and the low intake valve lift enables un-throttled engine operation.

Figure 15 presents the simulation results of the incylinder pressure, temperature, and the normalized HRR (heat release rate) obtained by taking the derivative of the $\mathrm{MFB}^{27}$ (a magnified HRR plot is also provided). From Figure 15, it can be seen that there is significant difference in HRR at the start and end of the combustion. This is mainly due to the different combustion models used, where a combination of three Wiebe functions was used in the GT-Power model to describe the start, intermediate, and end of combustion and the proposed model uses only one Wiebe function. This results in a fairly large difference at the start and end of combustions. The 50\% MFB locations for the proposed and GT-Power model are 1.19 and $2.01^{\circ}$ after TDC, respectively.

The engine intake valve timing was advanced to reduce the effective compression ratio and to avoid engine knock at the upper load limit of the HCCI combustion. The HCCI combustion timing is also sensitive to both intake and exhaust valve timings. Proper valve timing selection leads to improved combustion 


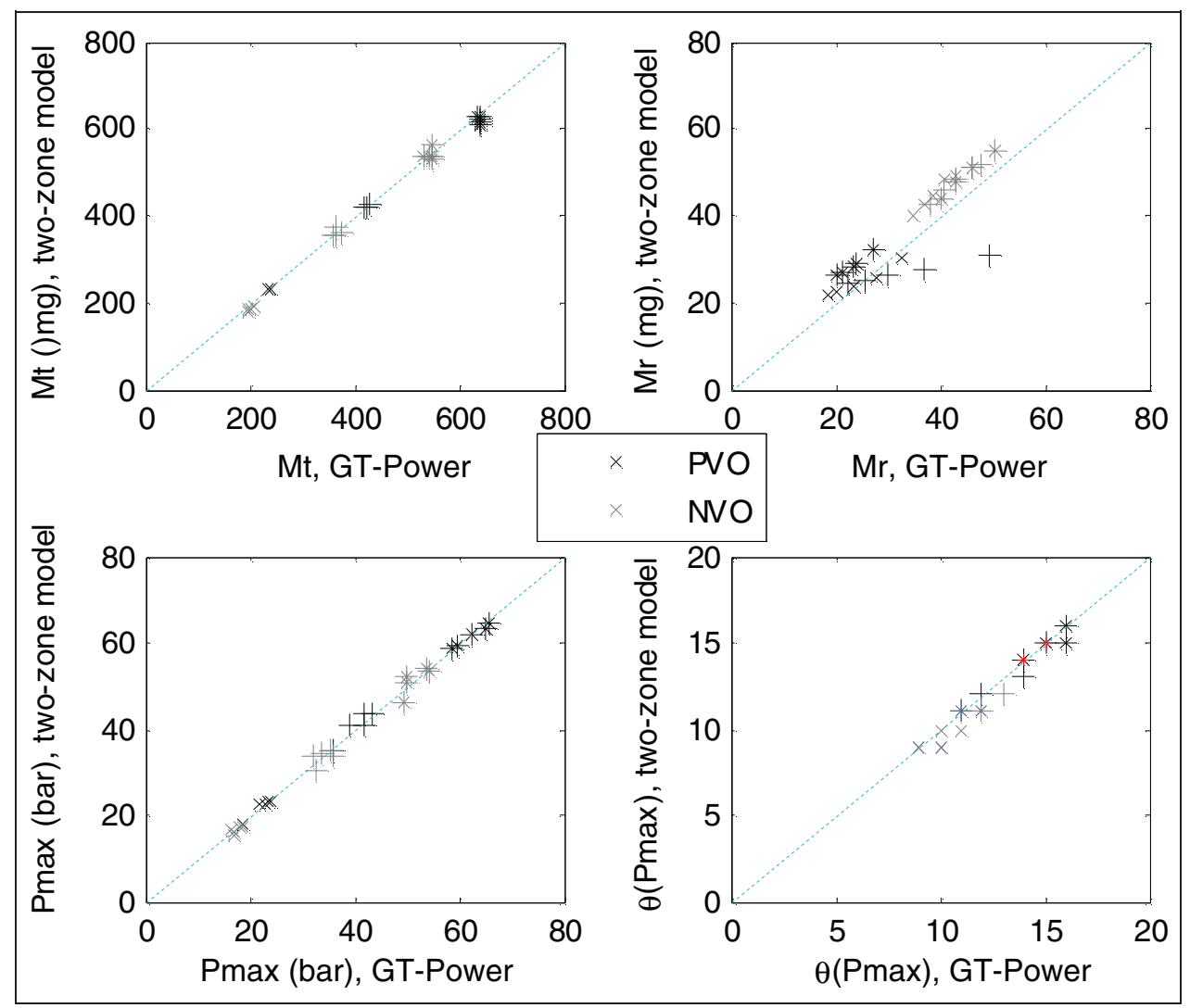

Figure 13. Correlation of the two-zone and GT-Power SI combustion model: $M_{t}, M_{r} P_{\max }$ and $P_{\max }$ location. PVO: positive valve overlap; NVO: negative valve overlap.

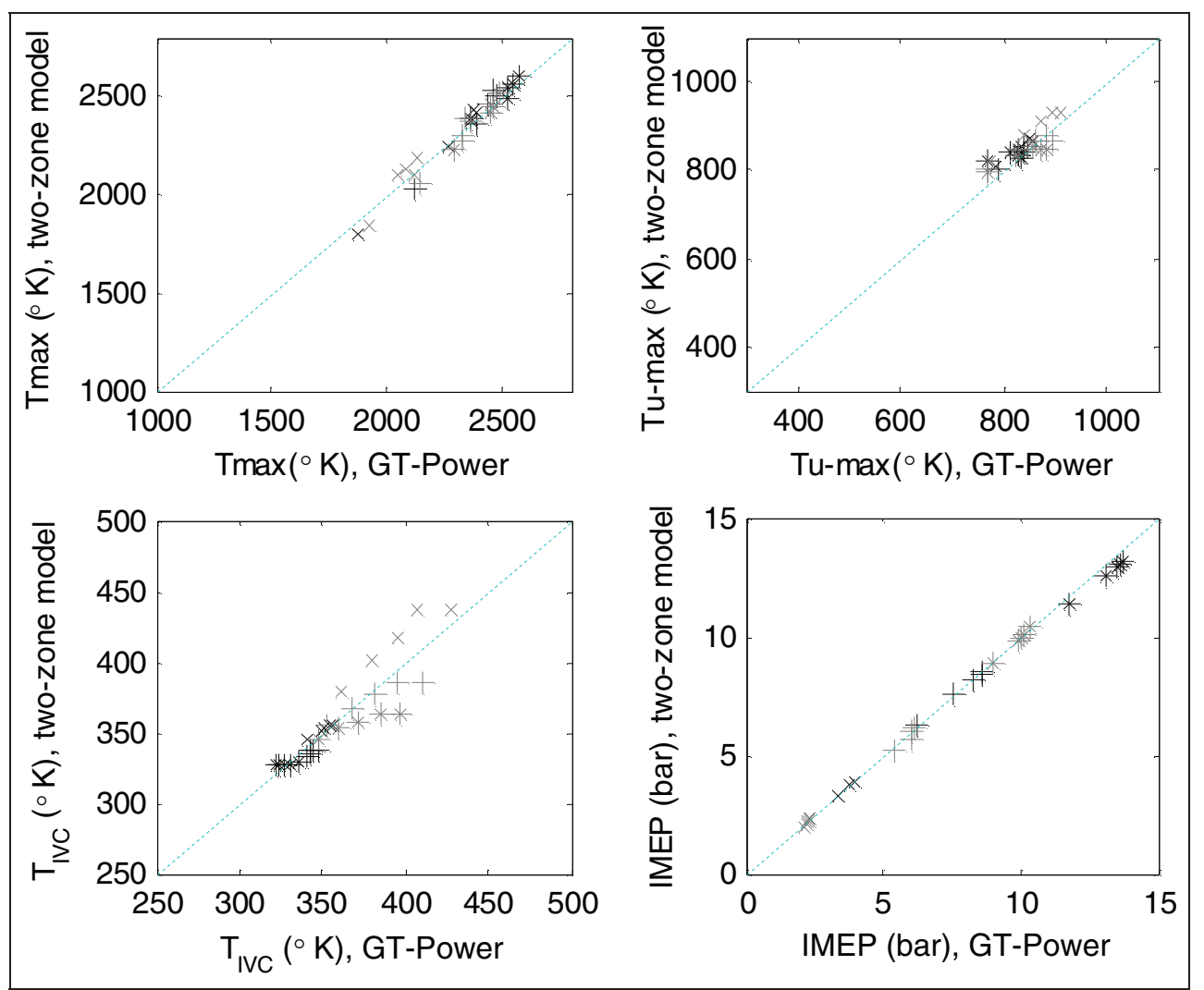

Figure 14. Correlation of the two-zone SI combustion models: $T_{\max }, T_{u-\max }, T_{I V C}$ and IMEP. 


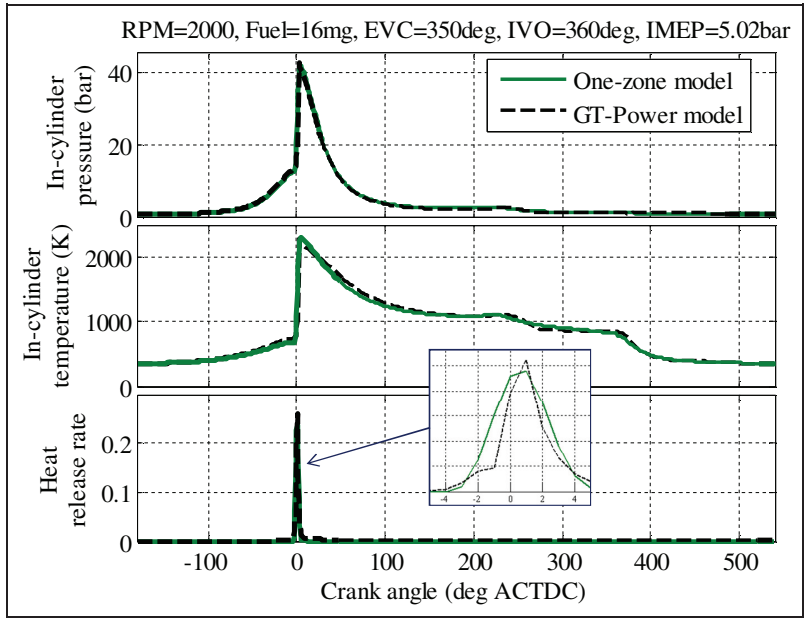

Figure 15. $\mathrm{HCCl}$ combustion in-cylinder pressure and temperature of the developed one-zone and GT-Power models. RPM: rotations per minute; EVC: exhaust valve closing; IVO: intake valve opening; IMEP: indicated man effective pressure; ACTDC: after combustion top dead center.

efficiency. For the simulation results shown in Figure 16 , the intake valve opening (IVO) timing was between 360 and $400^{\circ}$ after TDC, and the exhaust valve closing (EVC) timing was between 310 and $350^{\circ}$ after TDC. The simulation results were also compared with the corresponding GT-Power simulation results with good agreement. Note that the HCCI combustion model used in the GT-Power engine model is a predictive, single-zone model. The chemical reaction properties are defined by the GT-Power reference object "EngCylChemGas". The HCCI combustion model was not calibrated by test data for the engine configuration described in this paper since the main purpose of this paper is to demonstrate the potential of using the control oriented combustion model to match the complicated, chemical reaction based combustion model.

Due to the unavailability of the GT-Power SI-HCCI combustion model, the simulation results of the SIHCCI combustion model was not compared with that of the GT-Power model and this will be part of the future work when the experimental data are available. It is worth mentioning that the simulation results of the developed SI-HCCI combustion model have the same trend, compared to the experimental results of the spark assisted HCCI combustion shown by Zhang et al. ${ }^{8}$ The simulations of the SI-HCCI combustion mode transition control can be found in Yang. ${ }^{28}$

\section{Conclusions}

This paper presents a control oriented SI-HCCI hybrid combustion model of an HCCI (homogeneously charge compression ignition) capable SI (spark ignited) engine. The developed combustion model is capable of simulating the SI-HCCI hybrid combustion that starts with the SI combustion and ends with the HCCI combustion,

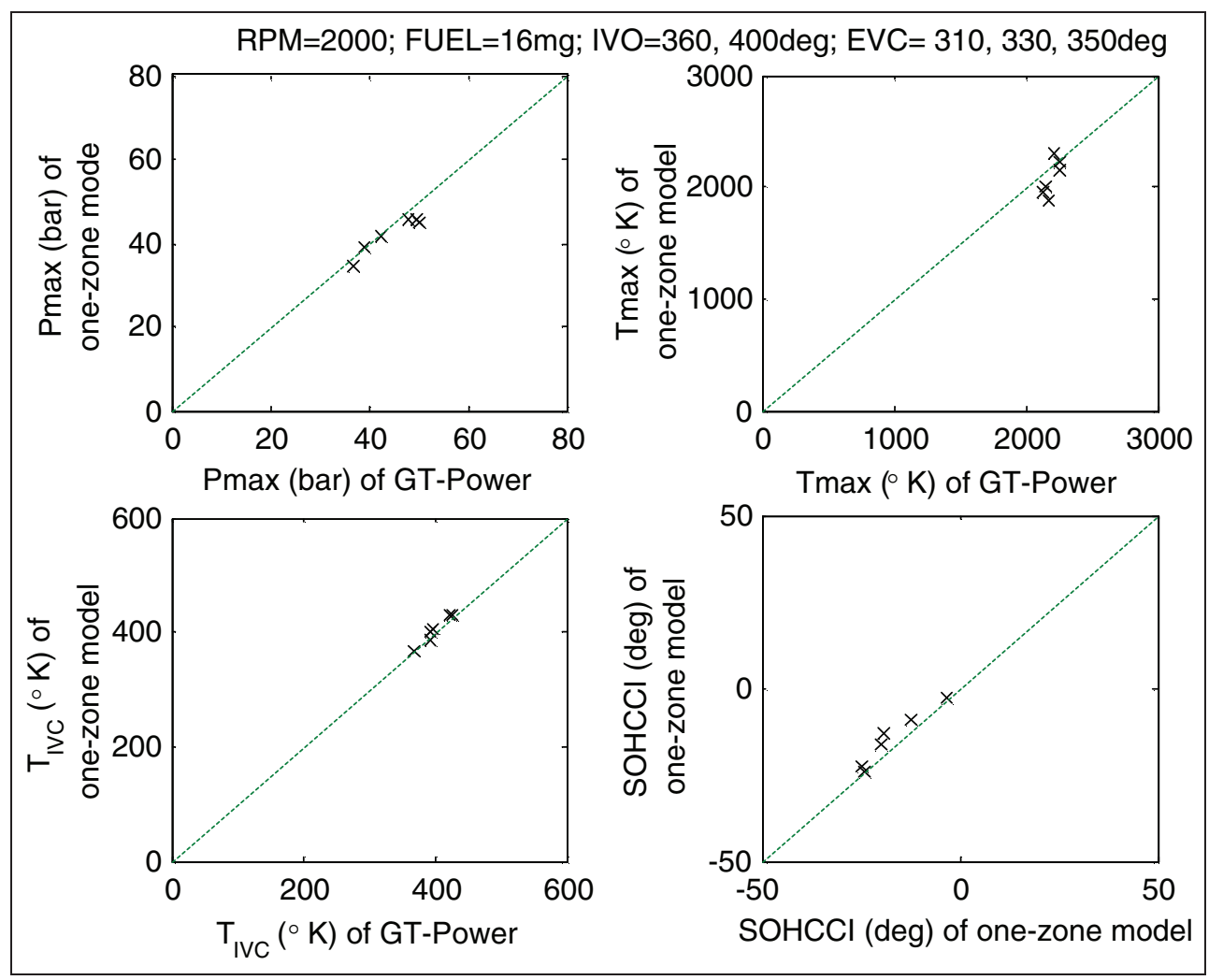

Figure 16. Comparison of the one-zone and GT-Power models with the $\mathrm{HCCl}$ combustion properties $P_{\max }, T_{\max }, T_{I V C}$ and SOHCCl timing.

RPM: revolutions per minute; IVO: intake valve opening; EVC: exhaust valve opening. 
where the SI and HCCI combustions are its special cases. The SI and HCCI combustions were modeled under the two-zone and one-zone assumptions, respectively. The developed combustion model was implemented in an HIL (hardware-in-the-loop) real-time engine simulation environment. The HIL simulation results were validated with the data generated by the corresponding GT-Power model. It is concluded that the developed SI-HCCI hybrid combustion model can be used for real-time simulation of the combustion mode transition; the accuracy of the SI and HCCI combustion model is comparable to the relatively high-fidelity GT-Power model; and it is suitable for engine control strategy development and validation.

\section{Funding}

This work was supported by the US National Science Foundation (grant no. CMMI-1030360) and the U.S. Department of Energy (grant no. DE-EE0000211).

\section{References}

1. Zhao F, Asmus T, Assanis D, et al. Homogeneous charge compression ignition (HCCI) engines key research and development issues. Warrendale, PA: Society of Automotive Engineers, 2003.

2. Cheng WK, Kenney $\mathrm{T}$ and Yang J. On HCCI engine knock. SAE paper 2007-01-1858, 2007.

3. Etheridge J, Mosbach S, Kraft $\mathrm{M}$ and $\mathrm{Wu}, \mathrm{H}$. A fast detailed-chemistry modeling approach for simulating the SI-HCCI transition. SAE paper 2010-01-1241, 2010.

4. Kong SC and Reitz RD. Application of detailed chemistry and CFD for predicting direct Injection HCCI engine combustion and emission. Proc Comb Inst 2002; 29: 663669.

5. Yang $X$ and Zhu G. SI and HCCI combustion mode transition control of a multi-cylinder HCCI capable SI engine via iterative learning. In: Proceedings of the 4th annual dynamic systems and control conference, Westin Arlington Gateway, Arlington, VA, USA, 31 October-2 November 2011.

6. $\mathrm{Wu} \mathrm{H}$ and Craft $\mathrm{M}$. Experimental investigation of a control method for SI-HCCI-SI transition in a multi-cylinder gasoline engine. SAE paper 2010-01-1245, 2010.

7. Roelle MJ, Shaver GM and Gerdes JC. Tackling the transition: a multi-mode combustion model of SI and HCCI for mode transition CONTROL. In: Proceedings of IMECE'04, 2004 international mechanical engineering conference and exposition, Anaheim, CA, USA, 13 November 2004.

8. Zhang Y, Xie H, Zhou N, Chen T, et al. Study of SIHCCI-SI transition on a port fuel injection engine equipped with 4VVAS. SAE paper 2007-01-0199, 2007.

9. Cairns A and Blaxhill H. The effects of two-stage cam profile switching and external EGR on SI-CAI combustion transitions. SAE paper 2007-01-0187, 2007.

10. Etheridge J, Mosbach S and Kraft M. A fast detailedchemistry modeling approach for simulating the SI-HCCI transition. SAE paper 2010-01-1241, 2010.

11. Aceves SM, Flowers DL, Dibble RW and Babajimopoulos A. Overview of modeling techniques and their application to $\mathrm{HCCI} / \mathrm{CAI}$ engines. In: Zhao $\mathrm{H}$ (eds) HCCI and CAI engines for the automotive industry. Cambridge: Woodhead Publishing, 2007.

12. http://www.gtisoft.com/, 2011.

13. http://www.ricardo.com/What-we-do/Software/Products/ WAVE/, 2011.

14. Rausen DJ, Stefanopoulou AG, Kang JM, et al. A mean value model for control of homogeneous charge compression ignition (HCCI) engines. ASME J Dyn Meas Contr 2005; 127(5): 355-362.

15. Canova $\mathbf{M}$ and Mohler SM. Mean value modeling and analysis of HCCI diesel engines with external mixture formation. ASME J Dyn, Meas, Contr 2009; 131(1): 011002.

16. Tandra V, Srivastava N and Soliman A. A discrete VVAbased phenomenological model of an HCCI Engine. SAE paper 2010-01-1240, 2010.

17. http://wot.motortrend.com/gm-hcci-engine-offers-lowemissions-and-diesellike-economy-732.html, 2011.

18. Fiveland SB and Assanis DN. Development of a twozone HCCI combustion model accounting for boundary layer effects. SAE paper 2001-01-1028, 2001.

19. Heywood JB. Internal combustion engine fundamentals. New York: McGraw-Hill, 1988.

20. Shaver GM, Roelle MJ and Gerdes JC. Modeling cycleto-cycle dynamics and mode transition in HCCI engines with variable valve actuation. Contr Engng Practice 2006; 14: 213-222.

21. Yang $X$ and Zhu G. A mixed mean-value and crankbased model of a dual-stage turbocharged SI engine for hardware-in-the-loop simulation. In: Proceedings of 2010 American control conference, Baltimore, MD, June, 2010.

22. Yang $\mathrm{X}, \mathrm{Zhu} \mathrm{G}$ and Sun Z. A control oriented SI and HCCI hybrid combustion model for internal combustion engines. In: Proceedings of 2010 ASME dynamic systems and control conference, Cambridge, MA, 2010.

23. Cengel YA and Boles MA. Thermodynamics: An engineering approach, 5th ed. New York: McGraw-Hill, 2006.

24. Guzzella L and Onder CH. Introduction to modeling and control of internal combustion engine systems. Springer, 2004.

25. Ravi N, Liao HH and Jungkunz AF. Modeling and control of exhaust recompression HCCI using split injection. In: Proceedings of 2010 American control conference, Baltimore, MD, June, 2010.

26. Yang $\mathrm{X}$ and Zhu G. A two-zone control oriented SIHCCI hybrid combustion model for the HIL engine simulation. In: Proceedings of 2011 American control conference, San Francisco, CA, USA, 2011.

27. Husted H, Kruger D, Fattic G, et al. Cylinder pressurebased control of pre-mixed diesel combustion. SAE paper 2007-01-0773, 2007.

28. Yang X. Modeling and control of SI and SI-HCCI hybrid combustion engines. $\mathrm{PhD}$ Thesis, Michigan State University, 2011.

\section{Appendix I}

\section{Abbreviations}

ACTDC after combustion TDC

ECU engine control unit

EGR exhaust gas recirculation

EVC exhaust valve closing

EVO exhaust valve opening 


$\begin{array}{llll}\text { HCCI } & \begin{array}{l}\text { homogeneous charge compression } \\ \text { ignition }\end{array} & \begin{array}{l}\text { PFI } \\ \text { PVO }\end{array} & \begin{array}{l}\text { port fuel injection } \\ \text { positive valve overlap } \\ \text { spark ignition }\end{array} \\ \text { HIL } & \text { hardware-in-the-loop } & \text { SI } & \text { SI and HCCI hybrid combustion } \\ \text { IMEP } & \text { indicated mean effective pressure (bar) } & \text { SI-HCCI } & \text { spark timing } \\ \text { IVC } & \text { intake valve closing } & \text { ST } & \text { start of HCCI combustion } \\ \text { IVO } & \text { intake valve opening } & \text { SOHCCI } & \text { star } \\ \text { MAP } & \text { intake manifold air pressure } & \text { TDC } & \text { top dead center } \\ \text { MFB } & \text { mass fraction burned } & \text { UEGO } & \text { universal exhaust gas oxygen } \\ \text { NVO } & \text { negative valve overlap } & \text { VVT } & \text { variable valve timing }\end{array}$

\section{Appendix 2}

List of simulation parameters

\begin{tabular}{|c|c|c|c|}
\hline Parameter & Symbol & Value (if constant) & Unit \\
\hline Arrhenius scaling coefficient & $A$ & 0.054 & \\
\hline Influence factor of fuel concentration & $b$ & 0.05 & \\
\hline Influence factor of oxidizer & c & 0.2 & \\
\hline Intake manifold air pressure & $P_{I M}$ & & bar \\
\hline Fuel concentration & $x_{f}$ & & $\mathrm{~g} / \mathrm{m}^{3}$ \\
\hline Oxidizer concentration & $x_{o x}$ & & $\mathrm{~g} / \mathrm{m}^{3}$ \\
\hline Arrhenius activation energy & $E_{a}$ & 388 & $\mathrm{~kJ} / \mathrm{kg}$ \\
\hline Peak in-cylinder pressure & $P_{\max }$ & & bar \\
\hline Predicted burn duration & $\Delta \theta$ & & $\operatorname{deg}$ \\
\hline Wiebe exponent & $m$ & 2 & \\
\hline Combustion efficiency in $\mathrm{HCCl}$ combustion mode & $\eta_{\mathrm{HCCl}}$ & & \\
\hline Combustion efficiency in SI combustion mode & $\eta_{S I}$ & & \\
\hline Scaling coefficient in equation $(10)$ & $q$ & 0.54 & \\
\hline Specific heat at constant volume & $C_{v}$ & 0.72 & $\mathrm{~kJ} / \mathrm{kg} . \mathrm{K}$ \\
\hline Gas constant & $R$ & 1.39 & $\mathrm{~kJ} / \mathrm{kg} . \mathrm{K}$ \\
\hline Influence factor in equation (10) & 1 & 0.8 & \\
\hline Lower heating value & $h_{L H V}$ & 44000 & $\mathrm{~kJ} / \mathrm{kg}$ \\
\hline
\end{tabular}

\section{Appendix 3}

\section{Solution of equation (27)}

$V_{B}\left(\theta_{i}\right)=\left(b_{2} c_{1} c_{3}-D^{0.5}+b_{1} c_{2} c_{4}+a_{1} b_{2} c_{3} c_{5}-a_{2} b_{1} c_{4} c_{5}\right) / 2 C$

$V_{U}\left(\theta_{i}\right)=-\left(b_{2} c_{1} c_{3}-D^{0.5}+b_{1} c_{2} c_{4}-a_{1} b_{2} c_{3} c_{5}+a_{2} b_{1} c_{4} c_{5}\right) / 2 C$

$T_{B}\left(\theta_{i}\right)=-\left(a_{1} c_{2} c_{4}+a_{2} c_{1} c_{4}-a_{1}\left(D^{0.5}+b_{2} c_{1} c_{3}+b_{1} c_{2} c_{4}-a_{2} b_{1} c_{3} c_{5}-a_{1} b_{2} c_{4} c_{5}\right) / 2 b_{1}-a_{1} a_{2} c_{4} c_{5}\right) / C$

$T_{U}\left(\theta_{i}\right)=\left(a_{1} c_{2} c_{3}+a_{2} c_{1} c_{3}-a_{2}\left(D^{0.5}+b_{2} c_{1} c_{3}+b_{1} c_{2} c_{4}-a_{1} b_{2} c_{3} c_{5}-a_{2} b_{1} c_{4} c_{5}\right) / 2 b_{2}-a_{1} a_{2} c_{3} c_{5}\right) / C$

$P\left(\theta_{i}\right)=\left(D^{0.5}+b_{2} c_{1} c_{3}+b_{1} c_{2} c_{4}-a_{1} b_{2} c_{3} c_{5}-a_{2} b_{1} c_{4} c_{5}\right) /\left(2 b_{1} b_{2} c_{5}\right)$

With

$$
\begin{aligned}
C= & a_{1} b_{2} c_{3}-a_{2} b_{1} c_{4} \\
D= & a_{1}^{2} b_{2}^{2} c_{3}^{2} c_{5}^{2}-2 a_{1} a_{2} b_{1} b_{2} c_{3} c_{4} c_{5}^{2}+2 a_{1} b_{1} b_{2} c_{2} c_{3} c_{4} c_{5}-2 a_{1} b_{2}^{2} c_{1} c_{3}^{2} c_{5}+a_{2}^{2} b_{1}^{2} c_{4}^{2} c_{5}^{2} \\
& -2 a_{2} b_{1}^{2} c_{2} c_{4}^{2} c_{5}+2 a_{2} b_{1} b_{2} c_{1} c_{3} c_{4} c_{5}+b_{1}^{2} c_{2}^{2} c_{4}^{2}+2 b_{1} b_{2} c_{1} c_{2} c_{3} c_{4}+b_{2}^{2} c_{1}^{2} c_{3}^{2}
\end{aligned}
$$


9.7 Published JC Paper \#7

A Control-Oriented Charge Mixing and HCCI Combustion Model for IC Engines 


\section{A CONTROL ORIENTED CHARGE MIXING AND HCCI COMBUSTION MODEL FOR INTERNAL COMBUSTION ENGINES}

\author{
Shupeng Zhang and Guoming G. Zhu \\ Mechanical Engineering \\ Michigan State University \\ East Lansing, MI 48824 \\ zhangs30@msu.edu and zhug@egr.msu.edu
}

\author{
Yongsoon Yoon and Zongxuan Sun \\ Mechanical Engineering \\ University of Minnesota \\ Minneapolis, MN 55455 \\ yoonx216@umn.edu and zsun@umn.edu
}

\begin{abstract}
This paper describes a control-oriented charge mixing and Homogeneous Charge Compression Ignition (HCCI) combustion model, where the in-cylinder charge is divided into the well-mixed and unmixed zones as the result of charge mixing. Simplified fluid dynamics is used to predict the residual gas fraction at the intake valve closing, which defines the size of the unmixed zone, during real-time simulations. The unmixed zone size not only determines how well the in-cylinder charge is mixed, which affects the start of HCCI combustion, the peak in-cylinder pressure and also the temperature during the combustion process. The developed model was validated in the HIL (hardware-in-the-loop) simulation environment. The HIL simulation results show that the proposed charge mixing and HCCI combustion model provides better agreement with these of the corresponding GT-Power than the previously developed one-zone model.
\end{abstract}

\section{INTRODUCTION}

To obtain the benefit of high efficiency of compression ignition (CI) engines and the benefit of low emissions of spark ignition engines, the homogeneous charge compression ignition (HCCI) engines have been widely investigated in recent years. The major advantage of HCCI engines is realized by eliminating the formation of flames and results in a much lower combustion temperature. As the consequence of the low flame temperature, the formation of NOx (nitrogen oxides) is greatly reduced. The lean burn nature of the HCCI engine also enables un-throttled operation to improve vehicle fuel economy ([1] and [2]).

However, one challenge in HCCI combustion control is to predict the start of combustion precisely. Moreover, HCCI combustion mode can only operate in a certain range of engine condition and is limited at high engine load due to knock, or at low load due to misfire. To accurately control the HCCI combustion process, a precise charge mixing and combustion model is a necessity. Widely used high fidelity engine models, such as GT-Power and WAVE, provides fairly accurate engine charge mixing and combustion models, however, they can only be used for off-line simulations and cannot be used for modelbased control, where real-time HIL (hardware-in-the-loop) simulations are often required. Multi-zone model based on chemical kinetics, that divides the cylinder into adiabatic core zones and thermal boundary layers, is capable of simulating more realistic HCCI combustion phenomena ([3] and [4]). Similarly, these kinds of models are not fast enough for realtime simulations. Mean-value and single-zone method was used in [5] to model averaged chemical kinetics and thermodynamic properties and simple and control-oriented modeling approach was used for multi-mode HCCI engine in [6]. In [1] a control-oriented one-zone HCCI combustion model was constructed based on the assumption that the incylinder fuel, air, and residual gas charges are uniformly premixed at the intake valve closing (IVC). However, during the engine intake process, some of the residual gas is not mixed with the fresh intake gas, which remains at the bottom of the cylinder. This unmixed portion is so-called unmixed residual gas fraction. Ignoring the unmixed residual gas fraction would result in modeling errors of the peak in-cylinder pressure and temperature since the unmixed residual gas could have quite different temperature comparing with the well mixed zone. Also the volume and air-to-fuel ratio (AFR) of the mixed zone will be affected. Furthermore, during the gas exchange process described in [1], a first order transfer function was used to approximate the in-cylinder pressure, which led to large errors compared with the actual pressure. In [7] and [8] a two-zone HCCI model was established taking into 
consideration of unmixed zone during combustion phase. Reference [7] developed a two-zone model based on thermochemistry and chemical kinetics, which ensures better combustion results, but the charge mixing process is not modeled. As a result, the size of the unmixed zone cannot be determined in real-time. In [8] a pseudo-velocity method was used to predict the unmixed zone size during intake phase; however, for the negative valve over-lap (NVO) case the simulation error is fairly large between pseudo-velocity and actual intake flow velocity.

In this paper, to meet the real-time simulation requirement, the in-cylinder charge mixture is divided into two zones, mixed and unmixed zones before the combustion starts. In order to predict the size of the unmixed zone, it is essential to model the flow dynamics during the entire intake process. A compressible flow dynamics method is used to predict the flow rate in real-time. Combined with energy conservation law, the transfer rate between the two zones can be finally obtained. During the intake phase, due to the previous NVO recompression, the cylinder-manifold circumstance is more complicated than the positive valve overlap (PVO) case, thus the change of downstream and upstream is considered. Between the IVC and start of combustion events, it is assumed that the mass of unmixed zone does not change due to weak incylinder motion. The isentropic compression is assumed with heat transfer to the cylinder wall. The heat transfer process is also used in combustion process until the exhaust valve opens. Since the temperature and volume of the unmixed zone can be updated each crank angle under this assumption, it is easy to analyze the combustion process in the mixed zone. In this paper, it is also assumed that there is no heat exchange between the two zones, but both of them have heat exchange with the cylinder wall. Pressure dynamic interactions between the two zones are also modeled during the combustion process. Figure 1 shows a diagram of the two-zone approach between the intake valve opening and exhaust valve opening.

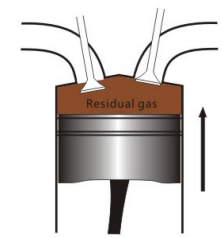

Exhaust

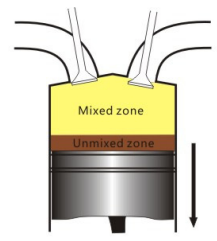

Intake

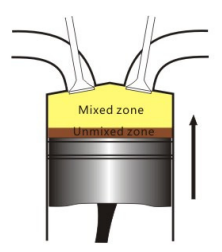

Compression

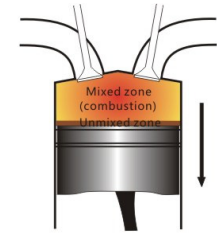

Combustion

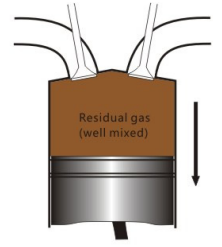

Expansion
FIGURE 1: TWO-ZONE CHARGE MIXING AND HCCI COMBUSTION MODEL

\section{TWO-ZONE CHARGE MIXING AND COMBUSTION} MODEL

The thermodynamic characteristics of in-cylinder gas, such as in-cylinder pressure and temperature, are of great interest in the SI and HCCI combustion modeling research. This is especially important at certain critical combustion phases such as the intake valve closing.

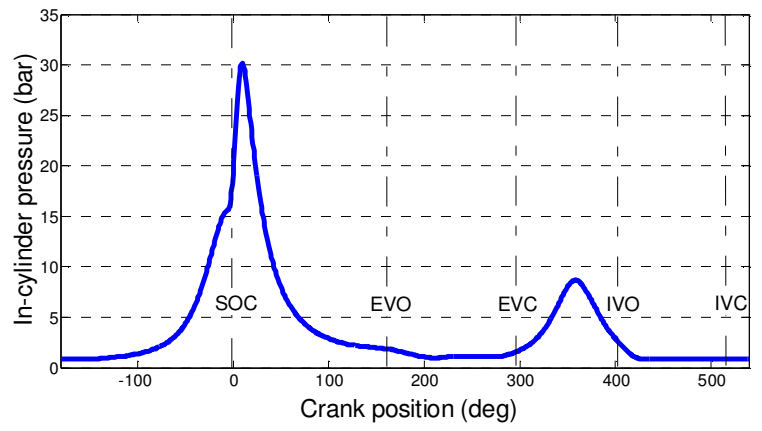

FIGURE 2: COMBUSTION PHASES WITHIN AN ENGINE
CYCLE

Figure 2 shows five key combustion phases of an HCCI combustion process within one engine cycle. We consider five key combustion phases in the SI or HCCI combustion process. They are intake valve closing (IVC), start of HCCI combustion (SOC), exhaust valve opening (EVO), exhaust valve closing (EVC), and intake valve opening (IVO). For each combustion phase, the in-cylinder behaviors (such as pressure, temperature, etc.) are modeled using thermodynamic governing equations with initial conditions derived from the last combustion phase. In the rest of this section, crank angle based model of each combustion phase is presented.

\section{Intake Phase}

During this phase, the fresh charge enters the cylinder and mixes with the residual gas gradually. As a result, the total mass of the unmixed zone reduces consequently. The goal is to predict the size of the unmixed zone at IVC; hence, it is essential to model the flow dynamics during the entire intake phase. The calculation of intake flow rate is based on the onedimensional compressible flow equation [9]:

a) When the flow is not choked $\left(P_{T} / P_{0}>[2 /(\gamma+1)]^{\gamma / \gamma-1}\right)$,

$$
\dot{m}=\frac{C_{D} A_{R} P_{0}}{\sqrt{R T_{0}}}\left(\frac{P_{T}}{P_{0}}\right)^{1 / \gamma}\left\{\frac{2 \gamma}{\gamma-1}\left[1-\left(\frac{P_{T}}{P_{0}}\right)^{(\gamma-1) / \gamma}\right]\right\}^{1 / 2}
$$

b) When the flow is choked $\left(P_{T} / P_{0} \leq[2 /(\gamma+1)]^{\gamma / \gamma-1}\right)$,

$$
\dot{m}=\frac{C_{D} A_{R} P_{0}}{\sqrt{R T_{0}}} \gamma^{1 / 2}\left(\frac{2}{\gamma+1}\right)^{(\gamma+1) / 2(\gamma-1)}
$$


where $C_{D}$ is the discharge coefficient, which is experimentally determined, $P_{0}$ and $T_{0}$ are the upstream stagnation pressure and temperature, $P_{T}$ is the downstream pressure, $A_{R}$ is the intake valve reference area, and $\gamma$ is the specific heat ratio.

For an HCCI combustion engine with the NVO strategy, most often, the in-cylinder pressure is higher than the intake manifold pressure at the IVO (intake valve opening) due to the residual gas recompression and early exhaust valve closing. Hence, certain portion of the residual gas will escape into the intake port after IVO. This is called backflow. In order to simplify the modeling process, the entire intake phase is divided into three stages under certain assumptions:

Firstly, right after the IVO, the trapped residual gas flows out of the cylinder through intake valve. $P_{0}$ and $T_{0}$ in equations (1) and (2) are in-cylinder pressure and temperature, respectively, $P_{T}$ is the manifold pressure, and $\dot{m}$ is treated as a negative value in the model for calculation convenience. During this stage, as the in-cylinder total mass is reducing while the cylinder volume is increasing, the in-cylinder pressure and temperature drops significantly.

Secondly, once the in-cylinder pressure becomes lower than the intake manifold pressure, flow direction reverses. It is assumed that the escaped residual gas in the first stage was not mixed with the fresh charge in the manifold, and during this stage all the escaped residue flows back into the cylinder, where $P_{0}$ is the manifold pressure, $P_{T}$ is the in-cylinder pressure, and $T_{0}$ is the residual gas temperature multiplied by the coefficient due to the heat release and expansion. In the model $T_{0}$ is approximated by the current in-cylinder temperature.

Finally, after all the escaped residual gas flows back into the cylinder, the actual fresh charge process begins. During this stage, $P_{0}$ and $T_{0}$ are the intake manifold pressure and temperature, respectively, $P_{T}$ is the in-cylinder pressure. This stage takes the longest duration among the three stages, and the mixing occurs within this stage.

The calculation of in-cylinder pressure and temperature is based upon the first law of thermodynamics. Since there is only one-direction flow path at one time due to the NVO operation, the energy conservation equation can be written as

$$
\frac{d(m u)}{d t}=\dot{Q}_{w}-\dot{W}+\dot{m} h
$$

where $\dot{W}$ is the rate of the transferred work, which equals $p \dot{V}$. $\dot{Q}_{w}$ is the total heat-transfer rate to the cylinder walls, which can be obtained using the Woschni correlation model ([10] and [11]):

$$
\dot{Q}_{w}=-A_{c} h_{c}\left(T-T_{w}\right)
$$

where $A_{c}$ is the contact area between gas and cylinder wall, $T_{w}$ is average temperature of cylinder wall, and $h_{c}$ is the average convection coefficient, which can be calculated by

$$
h_{c}=\alpha B^{-0.2} P^{0.8} T^{-0.73}\left(2.28 \bar{S}_{p}\right)^{0.8}
$$

where $B$ is the bore, $\bar{S}_{p}$ is the mean piston speed and $\alpha$ is the scaling factor used as model calibration parameters.

In the developed model equation (3) is discretized under the assumptions that within one calculation step, the gas thermodynamic properties $\left(C_{p}\right.$ and $\left.C_{v}\right)$ remain unchanged.

It is also assumed that the pressures in mixed and unmixed zones are identical. Then the in-cylinder temperature can be determined every crank degree by the following equation

$$
T\left(\theta_{i}\right)=\frac{Q_{w}-P\left(\theta_{i-1}\right)\left[V\left(\theta_{i}\right)-V\left(\theta_{i-1}\right)\right]+\dot{m} \Delta t C_{p} T_{0}+m\left(\theta_{i-1}\right) C_{v} T\left(\theta_{i-1}\right)}{\left[m\left(\theta_{i-1}\right)+\dot{m} \Delta t\right] C_{v}}
$$

where $\theta$ is crank position, $T_{0}$ is the intake flow temperature, and $\Delta t$ is the time interval for each crank degree.

Since the mixture can be considered as the ideal gas, incylinder pressure can be obtained by

$$
P\left(\theta_{i}\right)=\frac{m\left(\theta_{i}\right) R T\left(\theta_{i}\right)}{V\left(\theta_{i}\right)}
$$

While during the fresh charge process, the residual gas mixes with the fresh gas gradually, and the main modeling work is to describe the mass transfer rate from the unmixed zone to the mixed zone. The actual in-cylinder fluid dynamics is fairly complicated and difficult to be modeled accurately using a simple approach. As shown in Figure 3, it is assumed that the in-cylinder charge is mixed in a thermodynamically uniform state and the mixing dynamics is purely due to impinging effect of the intake fluid jet. 


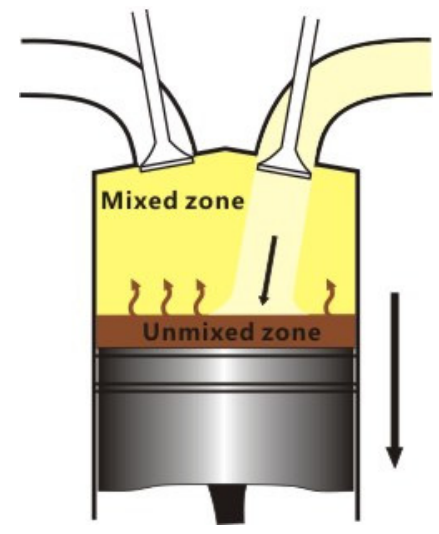

FIGURE 3: MASS TRANSFER FROM UNMIXED ZONE TO MIXED ZONE

Under these assumptions, applying conservation of energy, the mass transfer rate is assumed to be proportional to the kinetic energy of the intake flow.

$$
\dot{m}_{t r}=K \frac{\dot{m} v^{2}}{2 R T}
$$

where $K$ is the proportional coefficient to be calibrated, $\dot{m}$ is the intake flow rate which can be obtained by equations (1) and (2), and $v$ is the intake flow velocity which can be calculated from system geometry

$$
v=\frac{\dot{m}}{\rho A_{R}}
$$

At IVC, a residual gas fraction can be defined as

$$
\beta=\frac{m_{\text {unmixed }}}{m_{I V C}}
$$

where $m_{I V C}$ denotes the total trapped mass in the cylinder at IVC.

Notice that the constituent of the unmixed zone is always a portion of the entire residual gas at IVO, for there is no fresh charge entering this zone. Hence during the entire intake phase the unmixed zone can be approximated as an isentropic process, and at IVC the temperature in both zones can be obtained sequentially:

$$
\begin{gathered}
T\left(\theta_{I V C}\right)_{\text {unmixed }}=T\left(\theta_{I V O}\right) \cdot\left(\frac{P\left(\theta_{I V O}\right)}{P\left(\theta_{I V C}\right)}\right)^{\gamma-1 / \gamma} \\
T\left(\theta_{I V C}\right)_{\text {mixed }}=\frac{m_{I V C} T\left(\theta_{I V C}\right)-m_{\text {unmixed }} T\left(\theta_{I V C}\right)_{\text {unmixed }}}{m_{\text {mixed }}}
\end{gathered}
$$

under the identical pressure of mixed and unmixed zone assumption. The volume of both zones can be finally determined by ideal gas law.

\section{Compression Phase}

The compression phase can be approximated as an isentropic process with heat transfer for both zones. Since at the end of this phase the in-cylinder temperature might be very high, the heat transfer portion cannot be neglected for achieving accurate prediction of the start of HCCI combustion. Here it is assumed that there is no mass exchange between the two zones due to the weak in-cylinder motion. Thus the incylinder pressure and temperature for each zone can be determined separately. Equation (13) is used first to calculate the average in-cylinder temperature.

$$
T\left(\theta_{i}\right)=T\left(\theta_{i-1}\right) \cdot\left(\frac{V\left(\theta_{i-1}\right)}{V\left(\theta_{i}\right)}\right)^{(\kappa-1)}-\frac{Q_{w}}{m_{I V C} C_{v}}
$$

Then in-cylinder pressure can be obtained by equation (7) and temperatures of both mixed and unmixed zones can be calculated sequentially:

$$
\begin{gathered}
T\left(\theta_{i}\right)_{\text {unmixed }}=T\left(\theta_{i-1}\right) \cdot\left(\frac{P\left(\theta_{i-1}\right)}{P\left(\theta_{i}\right)}\right)^{\gamma-1 / \gamma}-\frac{Q_{\text {unmixed }}}{m_{\text {unmixed }} C_{v}} \\
T\left(\theta_{i}\right)_{\text {mixed }}=\frac{m_{I V C} T\left(\theta_{i}\right)-m_{\text {unmixed }} T\left(\theta_{i}\right)_{\text {unmixed }}}{m_{\text {mixed }}}
\end{gathered}
$$

where $Q_{\text {unmixed }}$ is heat-transfer to the cylinder walls from the unmixed zone.

The compression phase ends when the HCCI combustion starts. A commonly used criterion for the start of combustion (SOC) timing is the Arrhenius integral ([12] and [13]), which is described as

$$
A R I=\int_{\theta_{I V C}}^{\theta_{i}} \frac{A}{N_{e}} e^{-\frac{E_{a}}{R \cdot T_{\text {mixed }}(\theta)}} d \theta
$$

where $N_{e}$ is engine speed; $A$ is a scaling constant; $E_{a}$ is the activation energy for the auto ignition reaction.

The SOC crank position is defined as the crank angle for one percent fuel burned under the HCCI combustion. During this phase, the Arrhenius integral continues its integration. As the in-cylinder temperature and pressure increase gradually due to compression, the Arrhenius integral increases as well. Once the $A R I$ reaches criteria of the SOC $(A R I \geq 1)$, it shows that the HCCI combustion phase starts. At this moment, the temperature of mixed zone will be recorded as $T\left(\theta_{S O C}\right)$ and volume of unmixed zone as $V_{\text {unmixed }}\left(\theta_{S O C}\right)$ to be used for calculation in the next phase.

\section{Combustion and Expansion Phase}

In the HCCI combustion phase, the following two assumptions are made: 
1) There is no mass exchange between the mixed (burned) and the unmixed zones.

2) There is no heat transfer between the two zones but each zone has heat transfer to the cylinder wall.

Under the two assumptions, thermodynamic activity in both zones can be solved separately. In order to simplify the coupled equations, in-cylinder pressure in the last crank degree is used to calculate the volume of the current unmixed zone.

$$
V_{\text {unmixed }}\left(\theta_{i}\right)=V_{\text {unmixed }}\left(\theta_{\text {SOC }}\right)\left[\frac{P\left(\theta_{\text {SOC }}\right)}{P\left(\theta_{i-1}\right)}\right]^{1 / \gamma}
$$

In the mixed zone, the fuel mass fraction burned (MFB) are modeled by the following Wiebe function [9]

$$
x\left(\theta_{i}\right)=1-e^{-a\left(\frac{\theta_{i}-\theta_{S O C}}{\Delta \theta}\right)^{m+1}}
$$

where coefficients $a, m$, and predicted burn duration $\Delta \theta$ are calibration parameters of engine speed and load, and coolant temperature. The mixed zone temperature can be calculated by

$$
\begin{aligned}
T_{\text {mixed }}\left(\theta_{i}\right) & =T_{\text {mixed }}\left(\theta_{i-1}\right) \cdot\left(\frac{V_{\text {mixed }}\left(\theta_{i-1}\right)}{V_{\text {mixed }}\left(\theta_{i}\right)}\right)^{\gamma-1} \\
& +\frac{\eta_{H C C I} m_{\text {fuel }} Q_{L H V}\left[x\left(\theta_{i}\right)-x\left(\theta_{i-1}\right)\right]-Q\left(\theta_{i}\right)}{m_{\text {mixed }} C v} \\
P\left(\theta_{i}\right) & =P\left(\theta_{i-1}\right) \cdot \frac{V_{\text {mixed }}\left(\theta_{i-1}\right)}{V_{\text {mixed }}\left(\theta_{i}\right)} \cdot \frac{T_{\text {mixed }}\left(\theta_{i}\right)}{T_{\text {mixed }}\left(\theta_{i-1}\right)}
\end{aligned}
$$

and

$$
V_{\text {mixed }}\left(\theta_{i}\right)=V\left(\theta_{i}\right)-V_{\text {unmixed }}\left(\theta_{i}\right)
$$

where the combustion efficiency $\eta_{H C C I}$ is a calibration parameter used to match the simulated IMEP provided by GTPower model, and $Q_{L H V}$ is the low heat value of the fuel. Temperature in the unmixed zone can be obtained by equation (14).

After the HCCI combustion phase, the two-zone analysis is no longer essential for in-cylinder combustion behavior, and the two zones are supposed to be well mixed instantaneously. The in-cylinder average temperature can be obtained by equation (13) with the initial condition:

$$
T\left(\theta_{e}\right)=\frac{m_{\text {mixed }} T\left(\theta_{e}\right)+m_{\text {unmixed }} T_{\text {unmixed }}\left(\theta_{e}\right)}{m_{I V C}}
$$

where the index $e$ denotes the crank position when combustion terminates.

\section{Exhaust Phase}

The exhaust process is similar to the intake phase. Equations (1), (2), (6) and (7) are used for calculating the exhaust flow rate, in-cylinder temperature and pressure. Note that during this phase the in-cylinder pressure is higher than the exhaust manifold pressure in most of time; however, the situation can be reversed. Therefore, the backflow occurring is also considered. At the exhaust valve closed (EVC), the trapped mass can be calculated by

$$
m_{E V C}=\frac{P_{E V C} V_{E V C}}{R T_{E V C}}
$$

\section{NVO Phase}

The NVO phase is called as engine recompression. During this phase the trapped in-cylinder gas is isentropically compressed or expanded in a closed system with heat transfer to the cylinder wall, so equations (13) and (7) are used to calculate both temperature and pressure with $m\left(\theta_{i}\right)$ replaced by $m_{E V C}$ in equation (7).

\section{SIMULATION RESULTS}

The two-zone HCCI combustion model was validated in the HIL simulation environment (see Figure 4 for the HIL system architecture) with the engine parameters given in Table 1.

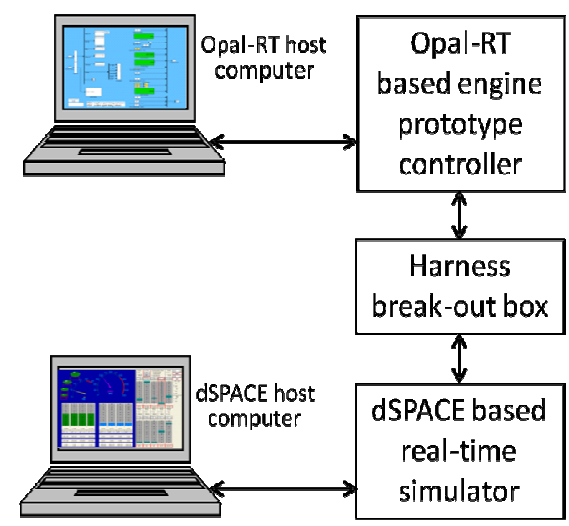

FIGURE 4: HIL SIMULATION ENVIROMENT

TABLE 1: ENGINE SPECIFICATIONS

\begin{tabular}{l|l}
\hline \hline Parameter & Model value \\
\hline bore/stroke/con-rod length & $86 \mathrm{~mm} / 86 \mathrm{~mm} / 143.6 \mathrm{~mm}$ \\
\hline compression ratio & $9.8: 1$ \\
\hline intake valve opening duration & 120 crank degree \\
\hline exhaust valve opening duration & 128 crank degree \\
\hline Intake/exhaust valve lifts & $5 \mathrm{~mm}$ \\
\hline
\end{tabular}

The two-zone model was validated for the engine operation at $2000 \mathrm{rpm}$ with 4.0 bar IMEP. The associated valve timing for EVO, EVC, IVO and IVC are 160, 288, 400, and 520 degree after TDC (top dead center). Figure 5 and Figure 6 show the in-cylinder pressure and temperature during the gas 
exchange process. A single cylinder GT-Power model is also developed and its simulation results were used as the baseline. Compared with one-zone model, two-zone charge mixing model based on fluid dynamics shows a significant improvement, and the simulated pressure and temperature responses match with these of GT-Power simulation results quite well.

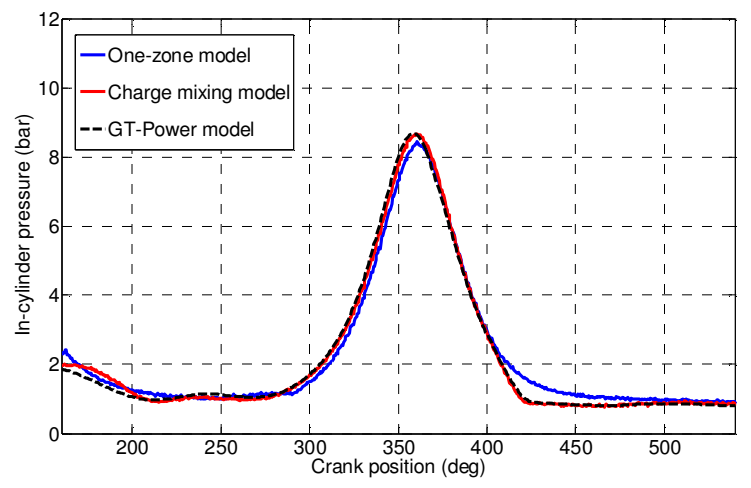

FIGURE 5: IN-CYLINDER PRESSURE

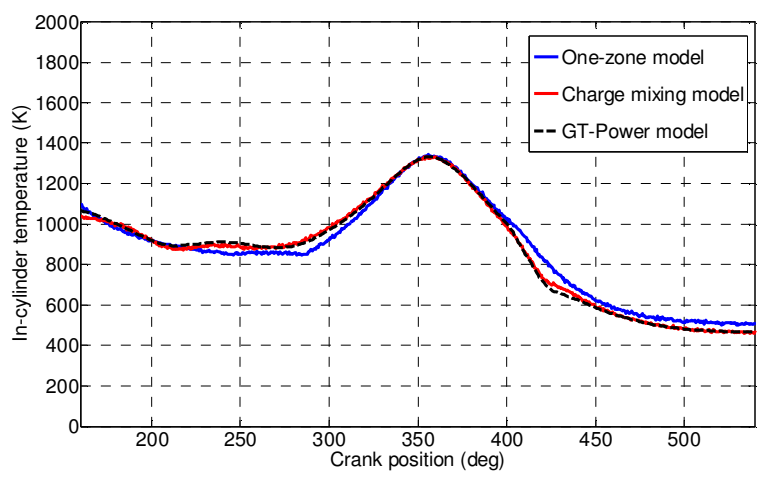

FIGURE 6: IN-CYLINDER TEMPERATURE

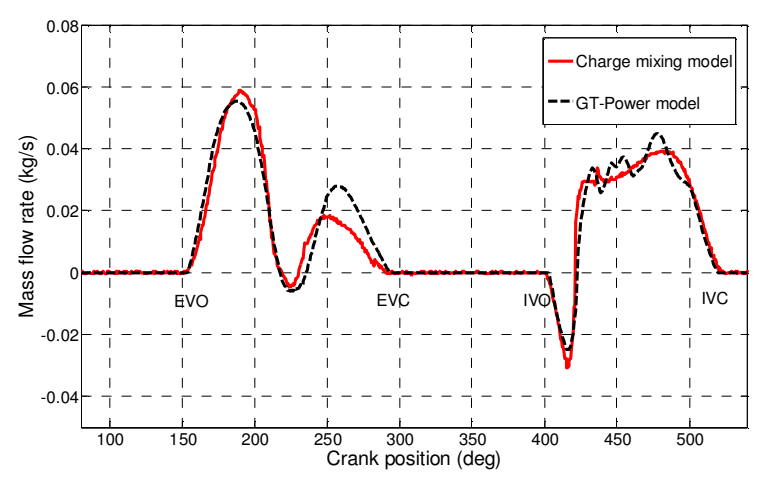

FIGURE 7: MASS FLOW RATE

In Figure 7 both of the simulated exhaust and intake flow rates obtained from the two-zone model were compared with these of GT-Power simulations. Again they matched quite well; on the other hand, the simulation result of the one-zone model is not shown here since a first order transfer function was used to approximate the in-cylinder pressure, the flow rate calculation is trivial. This indicates the benefit of the charge mixing modeling. Note that the small error between GT-Power and two-zone model responses are due to the unmodeled instantaneous pressure fluctuation in the manifold; this dynamics is fairly difficult to model using a simplified modeling approach for real-time simulations.

Figure 8 shows the expanding process of the mixed zone and shrinking process of the unmixed zone during the intake phase.

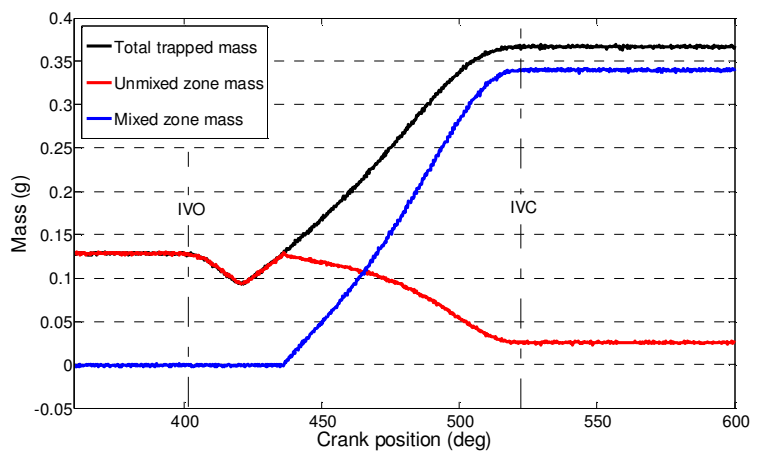

FIGURE 8: SIZE OF BOTH ZONES
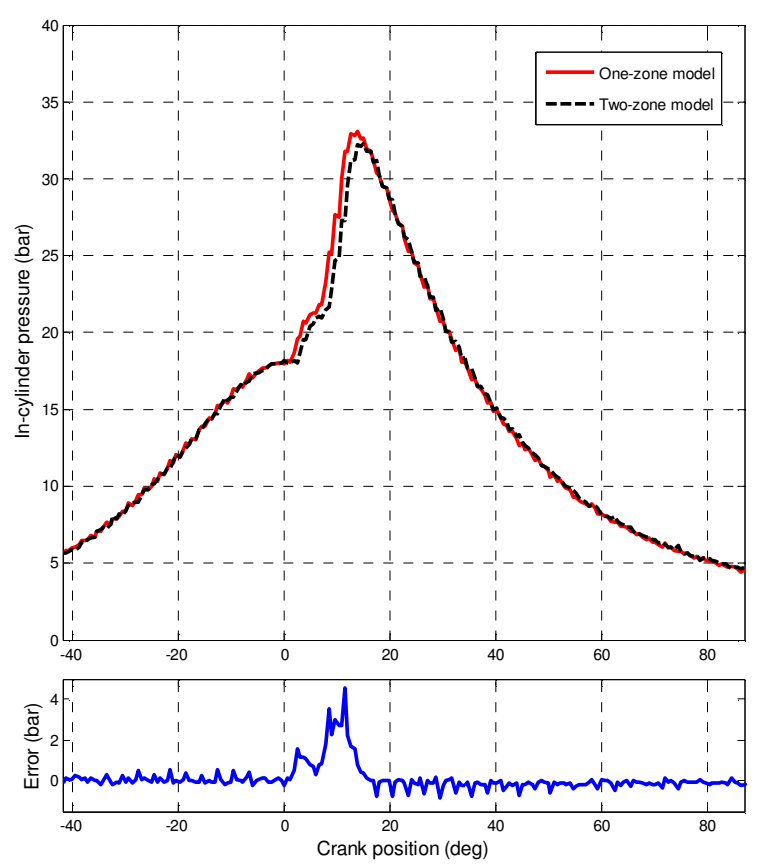

\section{FIGURE 9: IN-CYLINDER PRESSURE FOR SINGLE-ZONE AND TWO-ZONE MODELS}

Figure 9 compares the in-cylinder pressure of the twozone model with that of the one-zone model during compression and combustion phases. It can be seen that in the two-zone model the prediction of the SOC in the two-zone model is later than the one-zone model, and also the peak in- 
cylinder pressure is four bars lower than that of one-zone model. It is believed that this is due to the difference in estimated in-cylinder temperatures since the one-zone model uses the averaged temperature of two zones to estimate the SOC while the two-zone model uses the mixed zone temperature that is lower than the unmixed zone.

In summary, the charge mixing two-zone model is capable of achieving much more accurate simulation results than that of the one-zone model due to utilizing the fluid dynamics analysis. The two-zone charge mixing and combustion model provides the simulation results that are comparable with these of GT-Power model.

\section{CONCLUSION}

A two-zone charge mixing HCCI combustion model is proposed in this paper based upon the simplified fluid dynamics. The developed model was implemented into the hardware-in-the-loop simulation environment. The simulation results of the proposed model match with the GT-Power simulation well, and it is also demonstrated that the discretized fluid dynamics approach provides a satisfactory simulation results compared with the GT-Power model. Moreover, the unmixed zone plays an important role in predicting the start of combustion, in-cylinder pressure and temperature during the combustion process. It is believed that the two-zone charge mixing approach provides an improved simulation platform for developing the HCCI control strategy for real-time applications. The future work is to validate the proposed model using an optical engine.

\section{ACKNOWLEDGMENTS}

This work was supported by the US National Science Foundation under Grants CMMI-1030360 and CMMI1030380.

\section{REFERENCES}

[1] X. Yang and G. Zhu, "A two-zone control oriented SI_HCCI hybrid combustion model for the HIL simulation," 2011 American Control Conference, San Francisco, CA, June, 2011.

[2] R. H. Stanglmaier and C. E. Roberts, "Homogeneous Charge Compression Ignition (HCCI): Benefits, Compromises, and Future Engine Applications," SAE Technical Paper, SAE 1999-01-3682, 1999.
[3] P. Kongsereeparp, et al, "A Stand-along Multi-zone Model for Combustion in HCCI Engines," ASME Internal Combustion Engine Division 2005 Fall Technical Conference, Ottawa, ON, Canada, September, 2005, pp. 265-274.

[4] N. P. Komninos, "Modeling HCCI combustion: Modification of a multi-zone model and comparison to experimental results at varying boost pressure," Applied Energy, 86 (2009), pp. 2141-2151.

[5] D. J. Rausen and A. G. Stefanopoulou, "A Mean-Value Model for Control of Homogeneous Charge Compression Ignition (HCCI) Engines," ASME Journal of Dynamics, Measurement, and Control, Vol. 127, Sep, 2005.

[6] G. M. Shaver, M. J. Roelle, and J. C. Gerdes, "Modeling cycle-to-cycle dynamics and mode transition in HCCI engines with variable valve actuation," Control Engineering Practice, 14 (2006) 213 - 222

[7] V. Tandra and N. Srivastava, "A TWO ZONE MODEL OF A SINGLE CYLINDER HCCI ENGINE FOR CONTROL APPLICATIONS," 2008 ASME Dynamics and Control Conference, Ann Arbor, Michigan, October, 2008

[8] M. J. McCuen, Z. Sun, and G. Zhu, "Control-Oriented Mixing Model for Homogeneous Charge Compression Ignition Engines," 2010 American Control Conference, Marriott Waterfront, Baltimore, MD, June, 2010

[9] J. B. Heywood, Internal Combustion Engine Fundamentals, McGraw-Hill, Inc., 1988.

[10]G. Woschni, "A Universally Applicable Equation for Instantaneous heat Transfer Coefficient in the Internal Combustion Engine,” SAE Technical Paper, SAE 670931, 1967.

[11]J. Chang and O. Guralp, "New Heat Transfer Correlation for an HCCI Engine Derived from Measurements of Instantaneous Surface Heat," SAE Technical Paper, SAE 2004-01-2996, 2004.

[12]M. Hillion, J. Chauvin, and N. Petit, "Controlling the Start of Combustion on an HCCI Diesel Engine," American Control Conference, June, 2008.

[13] J. Bengtsson, M. Gafvert, and P. Strandh, "Modeling of HCCI Engine Combustion for Control Analysis," 43rd IEEE Conference on Decision and Control, December, 2004. 
9.8 Published JC Paper \#8

Air-to-Fuel Ratio Regulation during SI to HCCI Combustion Mode Transition Using the LQ Tracking Control 


\title{
Air-to-Fuel Ratio Regulation during SI to HCCI Combustion Mode Transition Using the LQ Tracking Control
}

\author{
Xiaojian Yang, Guoming G. Zhu, and Xuefei Chen
}

\begin{abstract}
The combustion mode transition between spark ignition (SI) and homogeneous charge compression ignition (HCCI) combustions of an internal combustion (IC) engine is challenging due to the distinct engine operating parameters over the two combustion modes and the cycle-to-cycle residue gas dynamics during the mode transition. The control problem becomes even more complicated for a multi-cylinder engine without camless valve actuation. This paper studies the combustion mode transition problem of a multi-cylinder IC engine with dual-stage valve lift and electrical variable valve timing (VVT) systems. Hardware-in-the-loop (HIL) simulations were used to develop and validate the proposed control strategies. The HIL simulation results show that smooth combustion mode transition can be realized utilizing the hybrid combustion mode in a few engine cycles and in-cylinder air-to-fuel ratio during the mode transition needs to be regulated to the desired level. This paper presents a model based linear quadratic tracking strategy to track the desired air-to-fuel ratio by controlling the engine throttle. The HIL simulations demonstrated the effectiveness of the developed control strategies. As a result, it is feasible to have a smooth combustion mode transition with dual-stage valve lift and electrical VVT systems.
\end{abstract}

\section{INTRODUCTION}

$\mathrm{H}$ OMOGENEOUS charge compression ignition (HCCI) combustion has the potential for internal combustion (IC) engines to meet the increasingly stringent emissions regulations with improved fuel economy [1]. The flameless nature of the HCCI combustion and its high dilution operation capability lead to low combustion temperature. As a result, the formation of NOx (nitrogen oxides) can be significantly reduced [2]. Furthermore, HCCI engine is capable of un-throttled operation that greatly reduces pumping loss and improves fuel economy ([3], [4]).

On the other hand, the HCCI combustion has its own limitations. It is limited at high engine load due to the mechanical limit; and at low load due to misfire caused by the lack of sufficient thermal energy to initiate the auto-ignition of the gas-fuel mixture during the compression stroke [5]. In fact, $\mathrm{HCCI}$ combustion can be regarded as a type of engine

Manuscript received September 23, 2011. This work is partly supported by the National Science Foundation under Grant CMMI-1030360 and partly supported by the U.S. Department of Energy under Grant DE-EE0000211.

Yang, Zhu, and Chen are with the Michigan State University, East Lansing, MI 48824, USA (yangxia2@egr.msu.edu, zhug@egr.msu.edu, chenxuef@msu.edu, respectively). operating mode rather than a type of engine [6]. That is, the engine has to be operated in dual-combustion mode to cover entire operational range.

It is fairly challenging to operate the engine in two distinct combustion modes, and it is even more difficult to have the smooth combustion mode transition between SI and HCCI combustions because the favorable thermo conditions for one combustion mode are often adverse to the other [7]. For example, high intake charge temperature is required in HCCI mode to initiate the combustion, while in SI mode it leads to reduced volumetric efficiency and increased knock tendency. For this reason, engine control parameters, such as intake and exhaust valve timings and lifts, throttle position and EGR (exhaust gas recirculation) valve opening, are controlled differently between these two combustion modes. During the combustion mode transition, these engine parameters need to be adjusted rapidly. However, the physical actuator limitations on response time prevent them from completing their transitions within the required duration, specifically, within one engine cycle. The multi-cylinder operation makes it challenging [8]. And this problem becomes more difficult when two-stage lift valve and electrical variable valve timing (VVT) systems are adopted. Accordingly the combustion performance during the transition cannot be maintained unless proper control strategy is applied.

The control of HCCI combustion has been widely studied in past decades. Robust HCCI combustions can be achieved through model based control as described in [9]-[11]. To make the HCCI combustion feasible in a practical SI engine, the challenge of the combustion mode transition is inevitable. In recent years, more and more attention has been paid to the mode transition control between SI and HCCI combustion. In [12] and [13], smooth mode transitions between SI and HCCI combustions are realized for a single cylinder engine equipped with the camless VVA (variable valve actuation) system. However, high cost prevents the implementation of the camless VVA system in production engines. In [14] a VVT system with dual-stage lifts is used on a multi-cylinder engine for the study of the mode transition. Experimental results show the potential of achieving smooth mode transition by controlling the step throttle opening timing and the DI (direct injection) fuel quantity. However, satisfactory mode transition has not been accomplished due to the lack of the robust mode transition control strategy.

The hardware-in-the-loop (HIL) simulation results 
demonstrated that unstable combustions during the transition can be eliminated by using the multi-step strategy as discussed in [5] for a four-cylinder engine equipped with external cooled EGR, dual-stage valve lift and electrical VVT system. This paper utilizes the LQ (linear quadratic) optimal MAP tracking control strategy to maintain the air-to-fuel ratio in the desired range so that the hybrid (or spark assisted) combustion is feasible. Under the optimal MAP control, smooth combustion mode transition can be achieved with the help of the iterative learning control (ILC) of the DI fuel quantity of individual cylinder. Note that the ILC is mainly used to generate transient fuel calibrations. The entire control strategy was validated in the HIL engine simulation environment [15], and satisfactory engine performance was achieved during the combustion mode transition for both steady state and transient operating conditions.

The paper is organized as follows. Section II introduces the multi-step SI to HCCI combustion mode transition control strategy. Section III discusses the air-to-fuel ratio control problem. The engine throttle and manifold dynamics modeling is presented in Section IV and the air-to-fuel ratio tracking control strategies in Section V. The control strategy is demonstrated in Section VI through simulations. Conclusions are finally drawn in Section VII.

\section{Multi-Step Combustion Mode Transition}

The configuration of the target HCCI capable SI engine and the engine specifications are listed in TABLE 1 . The key feature of this engine is its valvetrain system. It has two-stage lift for both intake and exhaust valves. The high lift has $9 \mathrm{~mm}$ maximum lift for the SI combustion mode; and the low lift has $5 \mathrm{~mm}$ maximum lift for the HCCI combustion mode. The ranges of both intake and exhaust valve timing are extended to \pm 40 crank degrees to improve the controllability of the internal EGR fraction, the effective compression ratio, and the engine volumetric efficiency during the combustion mode transition and HCCI operations. The externally cooled EGR is used to enable high dilution charge with a low charge mixture temperature.

TABLE 1 HCCI capable SI engine specifications

\begin{tabular}{ll}
\hline \hline Engine parameter & Model value \\
bore/stroke/con-rod length & $86 \mathrm{~mm} / 86 \mathrm{~mm} / 143.6 \mathrm{~mm}$ \\
compression ratio & $9.8: 1$ \\
Intake / exhaust valve lifts of high stage & $9 \mathrm{~mm} / 9 \mathrm{~mm}$ \\
Intake / exhaust valve lifts of low stage & $5 \mathrm{~mm} / 5 \mathrm{~mm}$ \\
Intake / exhaust valve timing range & $\pm 40 \mathrm{deg} / \pm 40 \mathrm{deg}$ \\
Intake / exhaust valve lifts lash & $0.2 \mathrm{~mm} / 0.25 \mathrm{~mm}$ \\
Intake manifold volume & $3.2 \mathrm{liter}$ \\
Throttle diameter & $42 \mathrm{~mm}$ \\
\hline \hline
\end{tabular}

For this paper, the combustion mode transition was studied for the engine operated at $2000 \mathrm{rpm}$ with 4.5 bar IMEP. TABLE 2 lists the engine parameters associated with the SI and HCCI combustion. These parameters were optimized for steady state engine operation with the best fuel economy that satisfies the engine knock limit requirement. It can be seen in
TABLE 2 that the optimized engine control parameters are quite different between the SI and HCCI combustion modes. Some of these parameters can be adjusted within one engine cycle, such as spark timing $\theta_{S T}$, electronic throttle control (ETC) drive current $I_{E T C}$ that is proportional to throttle motor torque, DI fuel quantity $F_{D I}$ and valve lift $\Pi_{l i f t}$; the others cannot due to actuator dynamics.

TABLE 2 Engine control parameters for SI and HCCI modes

\begin{tabular}{lll}
\hline \hline Engine control parameter & SI & HCCI \\
$\theta_{S T}($ deg ACTDC) & -36 & none \\
$\phi_{E G R}(\%)$ & 3 & 26 \\
$I_{E T C}(\mathrm{~A})$ & 0.84 & 5 \\
$F_{D I}(\mathrm{~ms} /$ cycle $)$ & 2.06 & 1.6 \\
$\theta_{I N T M}($ deg AGTDC) & 70 & 95 \\
$\theta_{E X T M}(\mathrm{deg}$ BGTDC) & 100 & 132 \\
$\Pi_{\text {lift }}(\mathrm{mm})$ & 9 & 5 \\
\hline \hline
\end{tabular}
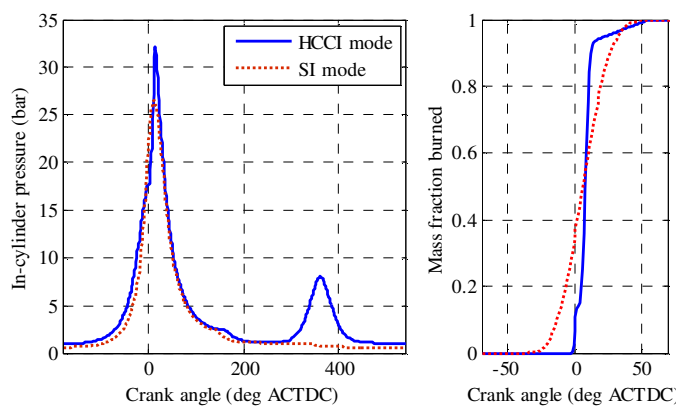

Fig. 1. Steady state combustion characteristics of SI and HCCI modes

The combustion characteristics are also quite different between these two combustion modes as illustrated in Fig. 1. For example, the HCCI combustion has higher peak in-cylinder pressure compared with that of the SI combustion due to the faster burn rate. Most likely, it also has a recompression phase (see the second peak of the solid line in Fig. 1) due to negative valve overlap (NVO) operation, while the SI combustion does not. The goal of the combustion mode transition is to switch the combustion mode without detectable engine torque fluctuation by regulating the engine control parameters, or in other words to maintain the engine IMEP during the combustion mode transition.

The earlier work in [16] demonstrated that the engine charge temperature $\left(T_{I V C}\right)$ has a response delay during the combustion mode transition, mainly caused by the response delays of the engine intake/exhaust valve timings and manifold filling dynamics. As a result, if the engine were forced to switch to the HCCI combustion mode, the engine IMEP could not be maintained with cycle-by-cycle fuel control $F_{D I}$. Also the increased cooling effect caused by the increment of $F_{D I}$ reduces the charge temperature and leads to degraded HCCI combustions. However, the transitional thermodynamic conditions are suitable for the SI-HCCI hybrid combustion mode proposed in [5] and [15].

By maintaining the engine spark (SI spark location), combustions during the mode transition could start in SI combustion mode with a relatively low heat release rate; and 
once the thermo and chemical conditions of the unburned gas satisfy the start of HCCI (SOHCCI) combustion criteria, the combustion continues in HCCI combustion mode, which is illustrated by the solid curve of mass fraction burned (MFB), shown in Fig. 2, obtained through GT-Power simulations. During an ideal SI to HCCI combustion transition process, the HCCI combustion percentage (the vertical distance from SOHCCI to MFB $=1$ ) increases gradually along with the incremental increase of charge temperature $\left(T_{I V C}\right)$. For the HCCI to SI combustion transition, the process is HCCI combustion percentage will be gradually reduced. More importantly, during the SI-HCCI hybrid combustion, engine IMEP can be controlled by regulating the DI fuel quantity that will be discussed in the next section. This is the other motivation of utilizing the hybrid combustion mode during the combustion mode transition.

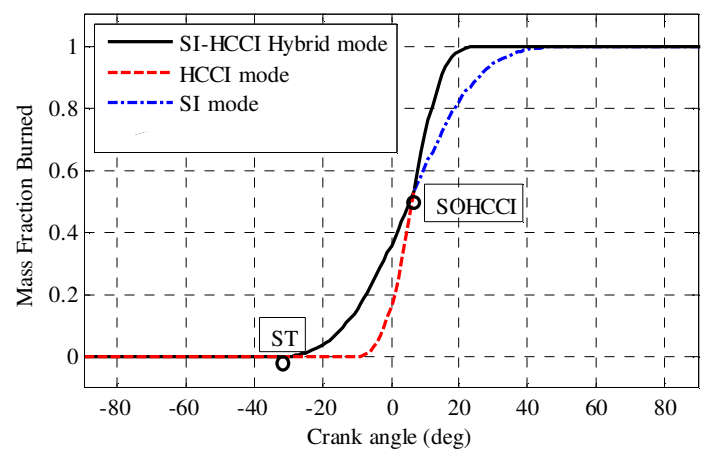

Fig. 2. MFB trace of SI-HCCI hybrid combustion mode

In [15], a crank based SI-HCCI hybrid combustion model was developed for real-time control strategy development. It models the SI combustion phase under two-zone assumptions and the HCCI combustion phase under one-zone assumptions. The SI and HCCI combustion modes are actually special cases of the SI-HCCI hybrid combustion mode in the model, since the SI combustion occurs when the HCCI combustion does not occur, and the HCCI combustion occurs when the percentage is one hundred percent. Accordingly this combustion model is applicable for all combustion modes during the mode transition.

In [16], the one-step combustion mode transition was investigated. The control references of all engine parameters were directly switched from the SI mode to the HCCI mode, as listed in TABLE 2, in one engine cycle. The simulation results showed that misfires occur during the one-step mode transition, and significant torque fluctuation was discovered. Thereby, a multistep mode transition strategy was proposed in [16] by inserting a few hybrid combustion cycles between the SI and HCCI combustions, see Fig. 3. The proposed control strategy is based on this multistep strategy.

As illustrated in Fig. 3, five engine cycles are used during the SI to HCCI mode transition. During the transitional cycles some engine parameters are adjusted in open loop according to the schedule shown in Fig. 3. Cycles 1 and 2 are used for engine throttle control. They provide enough time for the engine MAP to increase to compensate the valve lift $\left(\Pi_{l i f t}\right)$ switch. At the end of cycle 2 , the intake/exhaust valve lift $\Pi_{\text {lift }}$ switches from high lift to low lift, and the control references of EGR fraction $\phi_{E G R}$, intake valve timing $\theta_{I N T M}$ and exhaust valve timing $\theta_{\text {EXTM }}$ are set to those of the steady state HCCI combustion mode as listed in TABLE 2. Spark timing $\theta_{S T}$ of each cylinder was kept constant during the transitional cycles and was eliminated at the end of cycle 5. Throughout the transitional cycles, the engine control parameters, throttle current $I_{E T C}$ and DI fueling $F_{D I}$, are regulated at 1 millisecond sampling period and cycle-based controls, respectively. The corresponding control algorithm will be described in the next two sections.

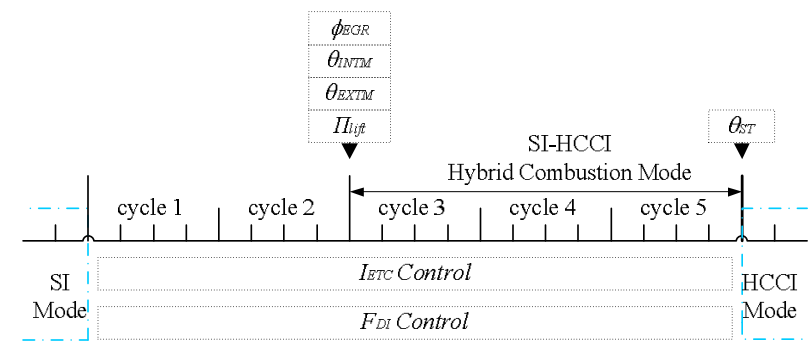

Fig. 3. Multistep SI to HCCI combustion mode transition control schedule

\section{The Air-to-Fuel Ratio Tracking Problem}

To study the feasibility of using fuel injection quantity $F_{D I}$ to regulating the engine IMEP, intensive simulations were conducted to map out the engine IMEP and air-to-fuel ratio as functions of engine fuel injection quantity $F_{D I}$ and MAP (manifold air pressure). The simulation results are shown in Fig. 4, indicating that the engine IMEP is highly correlated to $F_{D I}$ with the lean air-to-fuel mixture. As a result, it is possible to control the individual cylinder IMEP by regulating the corresponding $F_{D I}$.

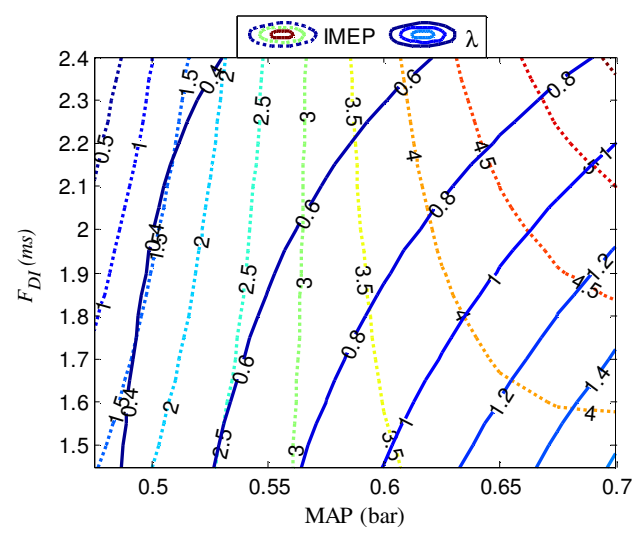

Fig. 4. IMEP sensitivity analysis of the SI-HCCI hybrid combustion mode

To maintain the controllability of the DI fueling $\left(F_{D I}\right)$, lean gas-fuel mixture is required during the mode transition. However, the combustion could become unstable if the mixture becomes extremely lean since the engine spark might 
not be able to ignite the gas mixture. For this study the desired normalize air-to-fuel ratio is set between $\lambda_{\min }(0.97)$ and $\lambda_{\max }$ (1.3). In [16], a step throttle pre-opening approach was proposed to prevent rich combustions at cycle 3, but it leads to very lean combustions at the following engine cycles. In this paper, an LQ tracking control strategy is developed to regulate the air-to-fuel ratio around the desired level.

As discussed above, the normalized air-to-fuel ratio needs to be maintained within the optimal range $\left(\lambda_{\min } \leq \lambda \leq \lambda_{\max }\right)$ during the SI to HCCI combustion mode transition. This control target is difficult to achieve through the air-to-fuel ratio feedback control due to delay and a short mode transition period. It is proposed to use the LQ optimal tracking approach to regulate the air-to-fuel mixture to the desired level. To implement this control strategy, the optimal operational range of $\lambda$ is translated into the operational range of the engine MAP shown in Fig. 5, where the upper limit is corresponding to $\lambda_{\max }$ and lower limit is corresponding to $\lambda_{\min }$. This provided an engine MAP tracking reference, shown in Fig. 5, to maintain the engine MAP to stay within the desired range. The reference signal is represented by

$$
z(k)=\left\{\begin{array}{ccc}
Z_{S I} & \text { if } & k_{B}<k \leq k_{1} \\
Z_{S I}+\left(Z-Z_{S I}\right) \frac{k-k_{1}}{k_{2}-k_{1}} & \text { if } & k_{1}<k \leq k_{2} \\
Z+\left(Z_{H C C I}-Z\right) \frac{k-k_{1}}{k_{2}-k_{1}} & \text { if } & k_{2}<k \leq k_{E}
\end{array}\right.
$$

where $k$ is the time based sampling index; $k_{B}$ and $k_{E}$ represent the beginning and ending indices of the mode transition and they were set to 600 and 900, respectively, as shown in Fig. 5; $k_{1}$ and $k_{2}$ are switch indices and they equal 670 and 720, respectively; $Z_{S I}$ and $Z_{H C C I}$ are the desired MAP of SI and $\mathrm{HCCI}$ modes, respectively; $Z$ is the desired MAP at $k_{2}$.

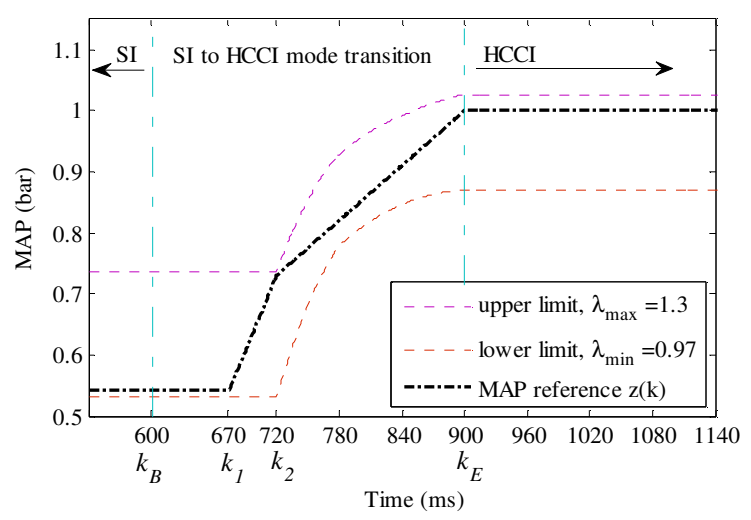

Fig. 5. The target MAP operational range and MAP tracking reference

\section{Engine Air Charge Dynamic Model}

To develop the proposed LQ tracking control strategy, a simplified engine MAP model is required to represent the relationship between the control input $\left(I_{E T C}\right)$ and the system output (MAP). The simplified dynamics are represented by second order dynamics due to the gas filling dynamics (first order) of the engine intake manifold and the first order response delay of the engine throttle. The governing equation of gas filling dynamics is represented by

$$
\frac{d M A P}{d t}=-\eta \frac{V_{d} N_{e}}{120 V_{m}} M A P+\varphi \frac{R T_{a m b} C_{D} \pi r^{2} P_{a m b}}{V_{m} \sqrt{2 R T_{a m b}}} \phi_{T P S}
$$

With filling dynamics time constant around $60 \mathrm{~ms}$. The dynamics of the throttle response is approximated by

$$
\frac{d \phi_{T P S}}{d t}=-\frac{k_{E T C}}{b_{E T C}} \phi_{T P S}+\frac{c_{E T C}}{b_{E T C}} I_{E T C}
$$

where $\eta, V_{d}, V_{m}$ and $N_{e}$ are volumetric efficiency of intake process, engine displacement, intake manifold volume, and engine speed, respectively; $R, T_{a n b}, P_{a m b}$ and $C_{D}$ are gas constant, ambient temperature, ambient pressure, and valve discharge constant, respectively; and $\phi_{T P S}, k_{E T C}, b_{E T C}$ and $c_{E T C}$ are engine throttle position, spring stiffness of the throttle plate, damping coefficient of the throttle plate, and throttle motor torque constant, respectively. The throttle time constant is around 50ms. Equations (2) and (3) can be combined, discretized and represented by the following discrete state space model

$$
\begin{aligned}
& x(k+1)=A x(k)+B u(k) \\
& y(k)=C x(k)+D u(k)
\end{aligned}
$$

where

$$
u=I_{E T C} \quad ; \quad x=\left[\begin{array}{l}
x_{1} \\
x_{2}
\end{array}\right]=\left[\begin{array}{c}
M A P \\
\phi_{T P S}
\end{array}\right] ; y=M A P
$$

are the system input, state and output respectively. The system matrices are

$$
\begin{array}{ll}
A=\left[\begin{array}{cc}
1-\frac{\eta(k) V_{d} N_{e}}{120 V_{m}} \Delta t & \frac{\varphi(k) R_{a} T_{a} C_{D} \pi r^{2} P_{a}}{V_{m} \sqrt{2 R_{a} T_{a}}} \Delta t \\
0 & 1-\frac{k_{E T C}}{b_{E T C}} \Delta t
\end{array}\right], \quad B=\left[\begin{array}{c}
0 \\
\frac{k_{E T C}}{b_{E T C}} \Delta t
\end{array}\right] \\
C=\left[\begin{array}{cc}
1 & 0
\end{array}\right], & D=0
\end{array}
$$

where $\Delta t$ is the sample period. State space model (4) is linear time-variant since the volumetric efficiency $\eta$ and multiplier $\varphi$ in equations (2) and (6) are functions of the engine operating condition. Moreover, the sampling time $\Delta T$ in (6) equals 1 millisecond, and sample time index $k$ is the same as that in equation (1).

\section{LQ TRACKIng CONTROL SYNTHESIS}

Based on the control oriented engine MAP model, a finite horizon LQ optimal tracking controller was designed to follow the reference $z(k)$. More specifically, the control objective is to minimize the tracking error $e(k)$ defined in (7) with the feasible control effort $I_{E T C}$. The tracking error $e(k)$ is defined as

$$
e(k)=y(k)-z(k)=C x(k)-z(k)
$$

and the constraint on $I_{E T C}$ is $-5 \mathrm{~A}<I_{E T C}<5 \mathrm{~A}$. The cost function of the LQ optimal controller is defined as 


$$
\begin{aligned}
J= & \frac{1}{2}\left[C x\left(k_{f}\right)-z\left(k_{f}\right)\right]^{T} F\left[C x\left(k_{f}\right)-z\left(k_{f}\right)\right] \\
& +\frac{1}{2} \sum_{k=k_{i}}^{k=k_{f}-1}\left\{[C x(k)-z(k)]^{T} Q[C x(k)-z(k)]+u^{T}(k) R u(k)\right\}
\end{aligned}
$$

where $F$ and $Q$ are positive semi-definite and $R$ is positive definite. For this paper, $F$ and $Q$ are constant matrices defined in (9) and $R$ is a function of sample index and tuned to optimize the tracking error with feasible throttle control effort, see Fig. 6.

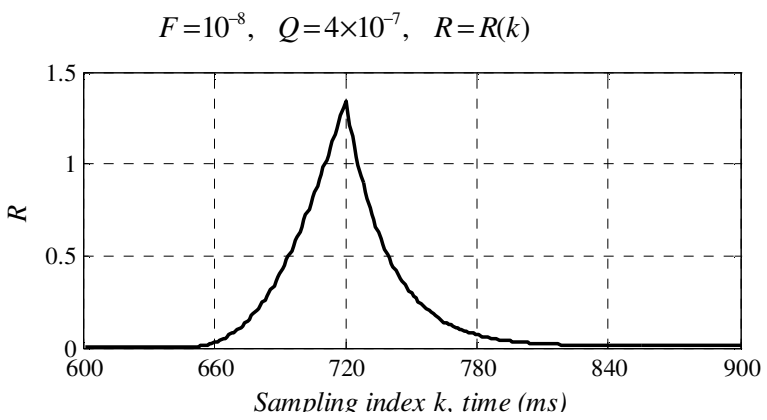

Fig. 6. Adjustment of weighting matrix R

Based on the cost function the corresponding Hamiltonian is as follows

$$
\begin{aligned}
H= & \frac{1}{2}[C x(k)-z(k)]^{T} Q[C x(k)-z(k)]+\frac{1}{2} u^{T}(k) R u(k) \\
& +p^{T}(k+1)[A x(k)+B u(k)]
\end{aligned}
$$

According to [17], the necessary conditions for the extremum in terms of the Hamiltonian are represented as

$$
\begin{gathered}
\frac{\partial H}{\partial p^{*}(k+1)}=x^{*}(k+1) \Rightarrow x^{*}(k+1)=A x^{*}(k)+B u^{*}(k) \\
\frac{\partial H}{\partial x^{*}(k)}=p^{*}(k) \Rightarrow p^{*}(k)=A^{T} p^{*}(k+1)+C^{T} Q C x^{*}(k)-C^{T} Q z(k) \\
\frac{\partial H}{\partial u^{*}(k)}=0 \Rightarrow 0=B^{T} p^{*}(k+1)+R u^{*}(k)
\end{gathered}
$$

Note that the superscript "**" denotes the optimal trajectories of the corresponding vectors. The augmented system of (11) and (12) becomes

$$
\left[\begin{array}{c}
x^{*}(k+1) \\
p^{*}(k)
\end{array}\right]=\left[\begin{array}{cc}
A & -B R^{-1} B^{T} \\
C^{T} Q C & A^{T}
\end{array}\right]\left[\begin{array}{c}
x^{*}(k) \\
p^{*}(k+1)
\end{array}\right]+\left[\begin{array}{c}
0 \\
-C^{T} Q
\end{array}\right] z(k)
$$

Based on (13) the optimal control is in the form of

$$
u^{*}(k)=-R^{-1} B^{T}\left[P(k) x^{*}(k)-g(k)\right]
$$

Matrix $P(k)$ can be computed by solving the difference Riccati equation backwards

$$
P(k)=A^{T} P(k+1)[I+E P(k+1)]^{-1} A+C^{T} Q C
$$

with the terminal condition

$$
P\left(k_{f}\right)=C^{T} F C
$$

and vector $g(k)$ can be computed by solving the vector difference equation

$$
g(k)=A^{T}\left\{I-\left[P^{-1}(k+1)+E\right]^{-1} E\right\} g(k+1)+C^{T} Q z(k)
$$

with the terminal condition

$$
g\left(k_{f}\right)=C^{T} F z\left(k_{f}\right)
$$

The optimal control in (15) can be written into the following form

$$
u^{*}(k)=-L_{F B}(k) x^{*}(k)+L_{F F}(k) g(k+1)
$$

where the feedforward gain $L_{F F}$ is computed by

$$
L_{F F}(k)=\left[R+B^{T} P(k+1) B\right]^{-1} B^{T}
$$

and the feedback gain $L_{F B}$ is computed by

$$
L_{F B}(k)=\left[R+B^{T} P(k+1) B\right]^{-1} B^{T} P(k+1) A
$$

Note that in equation (20) the state $x^{*}$ used in the feedback control is computed exactly from the closed loop system model defined below

$$
x^{*}(k+1)=\left[A-B L_{F B}(k)\right] x^{*}(k)+B L_{F F}(k) g(k+1)
$$

However, when the control is implemented into the HIL simulation environment or the actual engine control system, the feedback states are replaced by the actual signals (MAP and $\left.\phi_{T P S}\right)$ measured by the on-board engine sensors. In these cases the LQ controller is represented by the online form as

$$
u(k)=-L_{F B}(k) x(k)+L_{F F}(k) g(k+1)
$$

where $x$ represents the sampled states. Note that both of the states, MAP and $\phi_{T P S}$ can be measured in the HIL simulator or in the engine system.

\section{Simulation Results AND Discussion}

The developed LQ optimal MAP tracking control was implemented into the prototype engine controller and validated through the HIL engine simulations. The simulated control input $I_{E T C}$, the system states MAP and $\phi_{T P S}$, and $\lambda$ are plotted in Fig. 7. For comparison purpose, the simulated responses of these variables with a step $I_{E T C}$ control approach are also shown in Fig. 7, in which $I_{E T C}$ is set to the target level before the adjustment of $\Pi_{\text {lift }}$ (happens at $720^{\text {th }} \mathrm{ms}$ ), as a result, the engine throttle is gradually opened to the wide open throttle (WOT) position and the MAP is increased before the valve lift switch. The increased MAP ensures enough fresh charge to each cylinder when the valve lift switches to the low lift. However the step $I_{E T C}$ control leads to a rapid increment of the engine MAP or excessive fresh air charge, leading to extreme lean air-to-fuel ratio $\lambda$ in the following engine cycles.

Using the proposed LQ MAP tracking control strategy, throttle current $I_{E T C}$ is regulated in a non-monotonic increasing pattern. Note that to maintain $I_{E T C}$ in the feasible range $\left(-5 \mathrm{~A}<I_{E T C}<5 \mathrm{~A}\right)$ the weighting matrix $R$ in the cost function (8) is adjusted as illustrated in Fig. 6. The similar pattern can also be found for $\phi_{T P S}$ with a small phase lag. As a result, the engine MAP tracks the reference $z(k)$ after the intake valve lift $\Pi_{\text {lift }}$ switches to the low lift, and $\lambda$ of each cylinder is successfully maintained within the desired range. Therefore, with the help of the LQ optimal tracking control, the in-cylinder air-to-fuel ratio is maintained within the 
desired range, leading to stable combustions.

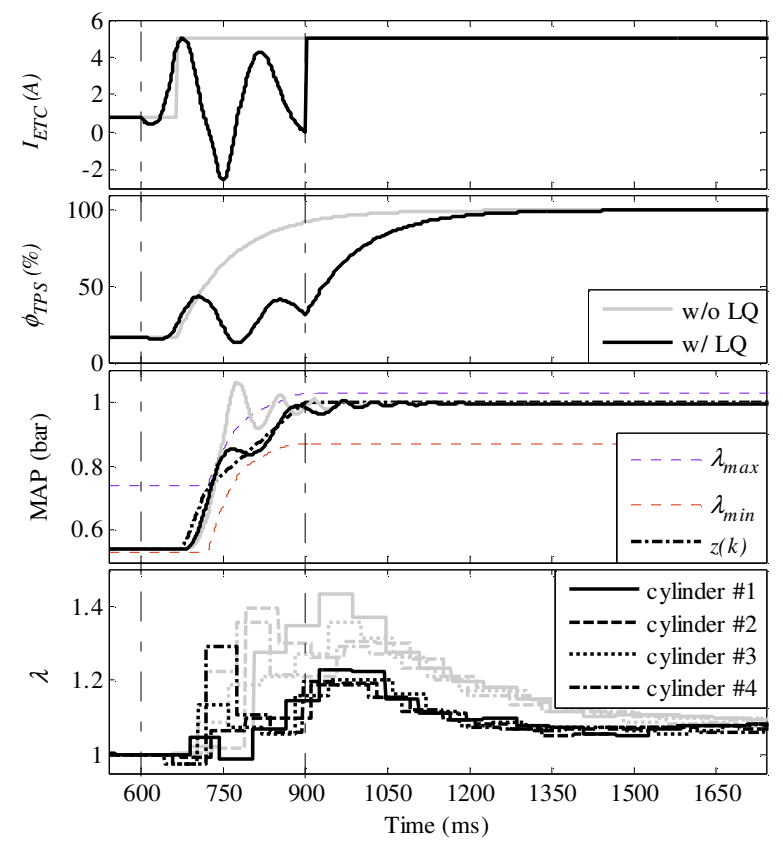

Fig. 7. Engine performances of the optimal MAP tracking control

Slight oscillations in the MAP responses are found with both control approaches, which are due to the flow dynamics of the engine air-handling system and the engine MAP modeling error. It is almost impossible to eliminate them. Moreover, the MAP oscillation associated with the LQ optimal tracking control is within the desired MAP range.

\section{CONCLUSIONS}

The combustion mode transition between the spark ignition (SI) and homogeneous charge compression ignition (HCCI) combustions is challenging but necessary to implement the promising HCCI combustion technology to production SI engines. To ensure the smooth combustion mode transition the engine air-to-fuel ratio needs to be precisely controlled. This paper shows through the hardware-in-the-loop simulations that the LQ control is capable of tracking the engine MAP to the desired target with small oscillations. As a result, the engine air-to-fuel ratio is maintained within the desired range. This makes it feasible to regulate the individual cylinder IMEP through adjusting the corresponding direct injection fuel quantity.

\section{REFERENCES}

[1] F. Zhao, T. Asmus, D. Assanis, J. E. Dec, J. A. Eng, and P. M. Najt, "Homogeneous Charge Compression Ignition (HCCI) Engines Key Research and Development Issues," 2003, Warrendale, Pennsylvania: Society of Automotive Engineers.

[2] R. M. Wagner, K. D. Edwards, et al, "Hybrid SI-HCCI Combustion Modes for Low Emissions in Stationary Power Applications," 3rd Annual Advanced Stationary Reciprocating Engines Meeting Argonne National Laboratory, Argonne, IL June 28-30, 2006.
[3] N. J. Killingsworth, S. M. Aceves, et al, "HCCI Engine Combustion-Timing Control: Optimizing Gains and Fuel Consumption Via Extremum Seeking," IEEE Transactions on Control Systems Technology, Vol. 17, No. 6, November 2009, pp. 1350-1361.

[4] C. J. Chiang and A. G. Stefanopoulou, "Stability Analysis in Homogeneous Charge Compression Ignition (HCCI) Engines With High Dilution," IEEE Transactions on Control System Technology, Vol. 15, No. 2, March 2007.

[5] X. Yang and G. Zhu, "A Two-Zone Control Oriented SI-HCCI Hybrid Combustion Model for the HIL Engine Simulation," Proceedings of 2011 American Control Conference, San Francisco, California, USA, 2011.

[6] S. C. Kong and R. D. Reitz, "Application of Detailed Chemistry and CFD for Predicting Direct Injection HCCI Engine Combustion and Emission," Proceedings of the Combustion Institute, Vol. 29, 2002, pp. 663-669.

[7] X. Yang, G. Zhu, and Z. Sun, "A Control Oriented SI and HCCI Hybrid Combustion Model for Internal Combustion Engines," Proceedings of 2010 ASME Dynamic Systems and Control Conference, Cambridge, MA, 2010.

[8] N Kalian, H Zhao, and J Qiao, "Investigation of transition between spark ignition and controlled auto-ignition combustion in a V6 direct-injection engine with cam profile switching," Proc. IMechE Vol. 222 Part D: J. Automobile Engineering, IMechE 2008.

[9] N. Ravi, M. J. Roelle, et al, "Model-Based Control of HCCI Engines Using Exhaust Recompression," IEEE Transactions on Control Systems Technology, Vol. 18, No. 6, November 2010, pp. 1289-1302.

[10] J. Kang, C. Chang, and T. Kuo, "Sufficient Condition on Valve Timing for Robust Load Transients in HCCI Engines," SAE International, 2010-01-1243.

[11] G. M. Shaver, "Physics based modeling and control of residual-affected HCCI engines using Variable Valve Actuation," PhD thesis, Stanford University, September, 2005.

[12] M. J. Roelle, G. M. Shaver, and J. C. Gerdes, "Tackling the Transition: A Multi-Mode Combustion Model of SI and HCCI for Mode Transition Control," Proceedings of IMECE 2004 International Mechanical Engineering Conference and Exposition, Anaheim, California, USA, November 13-19, 2004.

[13] Y. Zhang, H. Xie, et al, "Study of SI-HCCI-SI Transition on a Port Fuel Injection Engine Equipped with 4VVAS", SAE Paper 2007-01-0199.

[14] H. Wu, M. Kraft, et al, "Experimental Investigation of a Control Method for SI-HCCI-SI Transition in a Multi-Cylinder Gasoline Engine," SAE International, 2010-01-1245.

[15] X. Yang and G. Zhu, "A Mixed Mean-Value and Crank-based Model of a Dual-Stage Turbocharged SI Engine for Hardware-In-the-Loop Simulation", Proceedings of 2010 American Control Conference, Baltimore, MD, 2010.

[16] X. Yang and G. Zhu, "SI and HCCI Combustion Mode Transition Control of A Multi-Cylinder HCCI Capable SI Engine Via Iterative Learning," Proceedings of the 4th Annual Dynamic Systems and Control Conference, Westin Arlington Gateway, Arlington, VA, USA. Oct 31 Nov 2, 2011.

[17] D. Naidu, Optimal Control Systems, CRC Press LLC, 2003, pp. 232-239. 
9.9 Published JC Paper \#9

SI and HCCI Combustion Mode Transition Control of a Multi-Cylinder HCCI Capable SI Engine via Iterative Learning 


\section{SI AND HCCI COMBUSTION MODE TRANSITION CONTROL OF A MULTI-CYLINDER HCCI CAPABLE SI ENGINE VIA ITERATIVE LEARNING}

\author{
Xiaojian Yang \\ Mechanical Engineering \\ Michigan State University \\ East Lansing, MI 48824 \\ Yangxia2@msu.edu
}

\author{
Guoming G. Zhu \\ Mechanical Engineering \\ Michigan State University \\ East Lansing, MI 48824 \\ zhug@egr.msu.edu
}

\begin{abstract}
The combustion mode transition between spark ignition (SI) and homogeneously charged compression ignition (HCCI) combustions of an internal combustion (IC) engine is challenging due to the distinct engine operational parameters over these two combustion modes and the cycle-to-cycle residue gas dynamics of the HCCI combustion. The control problem becomes even more complicated when multi-cylinder operation is involved. This paper studies the combustion mode transition problem of a multi-cylinder IC engine with dual-stage valve lifts and electrical variable valve timing systems. A control oriented engine model was used to develop a multistep mode transition control strategy via iterative learning for combustion mode transition between SI to HCCI with minimal engine torque fluctuations. The hardware-in-the-loop (HIL) simulations demonstrated the effectiveness of the developed control strategy for the combustion mode transition under both constant load and transient engine operational conditions.
\end{abstract}

\section{NOMENCLATURE}

$\lambda \quad$ Normalized air-to-fuel ratio

$x_{E G R} \quad$ EGR gas fraction in engine intake manifold

$\theta_{S T} \quad$ Spark timing in degree after combustion TDC (top dead center)

$\theta_{\text {SOHCCI }} \quad$ Start of HCCI combustion timing

$N_{T P O} \quad$ Throttle pre-opening timing before intake/exhaust valve lift switch (quarter cycle)

$F_{D I} \quad$ Direct injection (DI) fuel pulse duration (ms/cycle)

$F_{F B}, F_{F F} \quad$ Feedback/feedforward portions of $F_{D I}(\mathrm{~ms} /$ cycle $)$

$F_{I L C} \quad$ ILC (iterative learning control) portion of DI pulse duration (ms/cycle)

$\begin{array}{ll}\phi_{\text {throttle }} & \text { Engine throttle position (\%) } \\ \phi_{a c c} & \text { Acceleration pedal position (\%) } \\ \phi_{E G R} & \text { External EGR valve opening (\%) } \\ \Pi_{\text {lift }} & \text { Peak lift of intake/exhaust valve (mm) } \\ T_{I V C} & \text { In-cylinder gas temperature at IVC }\left({ }^{\circ} \mathrm{K}\right) \\ M_{E V C} & \text { Residue gas mass at EVC (mg) } \\ \theta_{I N T M} & \begin{array}{l}\text { Intake valve timing at peak lift (degrees after gas } \\ \text { exchange TDC) }\end{array} \\ \theta_{E X T M} & \begin{array}{l}\text { Exhaust valve timing at peak lift (degrees before } \\ \text { gas exchange TDC) }\end{array} \\ M A P & \text { Intake manifold absolute pressure (bar) } \\ I M E P & \text { Indicated mean effective pressure (bar) } \\ I M E P_{\text {des }} & \text { Desired IMEP due to driver need (bar) } \\ I M E P & \text { IMEP learning reference used in ILC (bar) } \\ i, k, n & \text { Indexes of ILC iteration, engine cycle, and cylinder }\end{array}$

\section{INTRODUCTION}

Homogeneously charged compression ignition (HCCI) combustion has the potential for internal combustion (IC) engines to meet the increasingly stringent emissions regulations with improved fuel economy. The flameless nature of the HCCI combustion and its high dilution operation capability lead to low combustion temperature. As a result, the formation of $\mathrm{NO}_{\mathrm{x}}$ (Nitrogen Oxides) can be significantly reduced. Furthermore HCCI engine is capable of un-throttled operation that greatly reduces pumping loss and improves fuel economy ([1] and [2]).

On the other hand, HCCI combustion has its own limitations. It is limited at high engine load due to the audible 
knock, and at low load due to misfire caused by the lack of sufficient thermal energy to trigger the auto-ignition of the gasfuel mixture late in the compression stroke [3]. In fact, HCCI combustion can be regarded as a type of engine operating mode rather than a type of engine [4]. In order to take the advantage of the HCCI combustion mode in an IC engine, other combustion mode, such as spark ignited (SI) combustion, is needed at high load, at high speed, at ultra-low load (such as idle), and at certain operating conditions (such as cold start).

However, it is fairly challenging to operate the engine in two distinct combustion modes, and it is even more difficult to have smooth combustion mode transition between SI and HCCI combustions. Because the favorable thermo conditions for one combustion mode are always adverse to the other. For example, high intake charge temperature is required in HCCI mode to initiate the combustion, while in SI mode it leads to reduced volumetric efficiency and increased knock tendency. For this reason, engine control parameters, such as intake and exhaust valve timings and lifts, throttle position and EGR (exhaust gas recirculation) valve opening, are controlled differently between these two combustion modes. During the combustion mode transition, these engine parameters need to be changed rapidly. However, the physical actuator limitations on response time prevent them from completing their transitions within the required duration, specifically, within one engine cycle. The multi-cylinder operation makes it even more difficult. Accordingly the combustion performance during the transition cannot be maintained unless proper control strategy is applied.

The control of HCCI combustion process has been widely studied in past decades. Robust HCCI combustions can be achieved through model based control as described in [5], [6], and [7]. To implement the HCCI combustion in a practical IC engine, the challenge of the combustion mode transition is inevitable. In recent years, more and more attentions have been paid to the mode transition control between SI and HCCI combustions. In [8] and [9], smooth mode transitions between SI and HCCI combustions are realized for a single cylinder engine equipped with the camless VVA (Variable Valve Actuation) system. However, high cost prevents the implementation of the camless VVA system in production engines. In [10] a VVT (Variable Valve Timing) system with dual-stage lifts is used on a multi-cylinder engine. Mode transition is investigated. Experimental results show that smoother mode transition can be achieved by controlling the throttle opening timing and the direct injection (DI) fuel quantity. However, satisfactory mode transition is not achieved due to the lack of the robust mode transition control strategy.

In this paper, the combustion mode transition control strategy is investigated through the hardware-in-the-loop (HIL) engine simulations. The studied four-cylinder engine is equipped with external cooled EGR, dual-stage valve lifts and electrical VVT valvetrain system. The SI-HCCI hybrid combustion mode, as discussed in [1], was implemented during the multistep mode transition and iterative learning control
(ILC) strategy was used for regulating the throttle opening timing and the individual cylinder DI fuel quantity. Smooth combustion mode transitions were achieved when the engine is operated at both stead constant load and transient conditions.

\section{THE HCCI CAPABLE SI ENGINE}

Figure 1 shows the target HCCI capable SI engine configuration. It is a four-cylinder 2.0 liter engine with compression ration of 9.8. The key feature of this engine is its valvetrain system. It has two stages of lifts for both intake and exhaust valves. The high lift stage has $9 \mathrm{~mm}$ maximum lift and is used for SI combustion mode; the low lift stage has $5 \mathrm{~mm}$ maximum lift and is used for HCCI combustion mode. The ranges of both intake and exhaust valve timing were extended to \pm 40 crank degrees to improve the controllability of the internal EGR fraction, effective compression ratio, and engine volumetric efficiency during the HCCI operating condition. In addition to the modification of the valvetrain, external cooled EGR is added to the engine to enable high dilution charge, resulting in low charge mixture temperature. The engine throttle can be electronically controlled to obtain the desired engine charge in both SI and unthrottled HCCI operations. Each cylinder of the test engine will be fitted with piezoelectric pressure transducer. Four fast response UEGO (Universal Exhaust Gas Oxygen) sensors are also assumed to be connected to each exhaust pipe, corresponding to each cylinder.

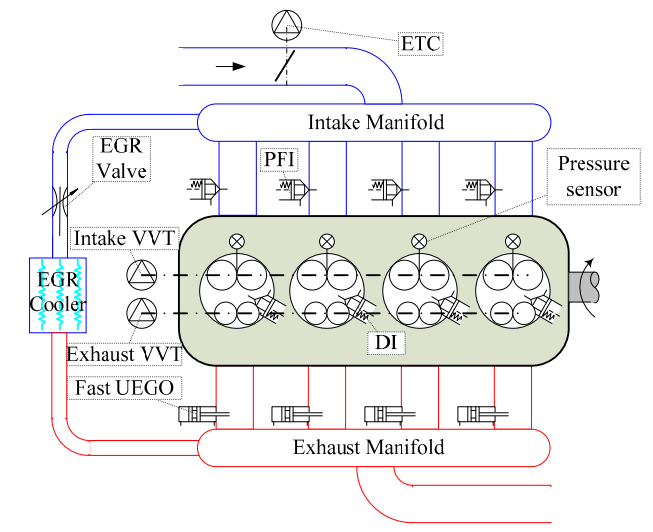

\section{FIGURE 1. HCCI CAPABLE SI ENGINE CONFIGURATION}

An HIL simulation station, shown in Fig. 2, is used for the purpose of initial control strategy development and validation. The dSPACE based engine HIL simulator outputs all measureable engine signals (such as in-cylinder pressure and MAP signals) in real-time. These signals can be directly fed into the Opal-RT based real-time prototype engine controller. Some immeasurable engine states (such as in-cylinder temperature) are also generated by the simulator to help with the control strategy development. On the other hand, the control signals generated by the prototype engine controller are also fed back into the dSPACE based engine simulator. These signals are also monitored and recorded for all the simulations that will be discussed in the rest of this paper. The proposed control strategy discussed in this paper had been validated in 
the HIL simulation environment, and will be verified against measurements obtained from engine dynamometer tests after the test engine is ready.

The HIL simulation is a useful tool for control strategy development and validation, but the effectiveness of the HIL simulation relies on the engine model that is being executed in the dSPACE simulator. A control oriented real-time engine model is required. It must reflect all dominant engine dynamics with relatively high fidelity. Next section introduces the engine model used in this study.

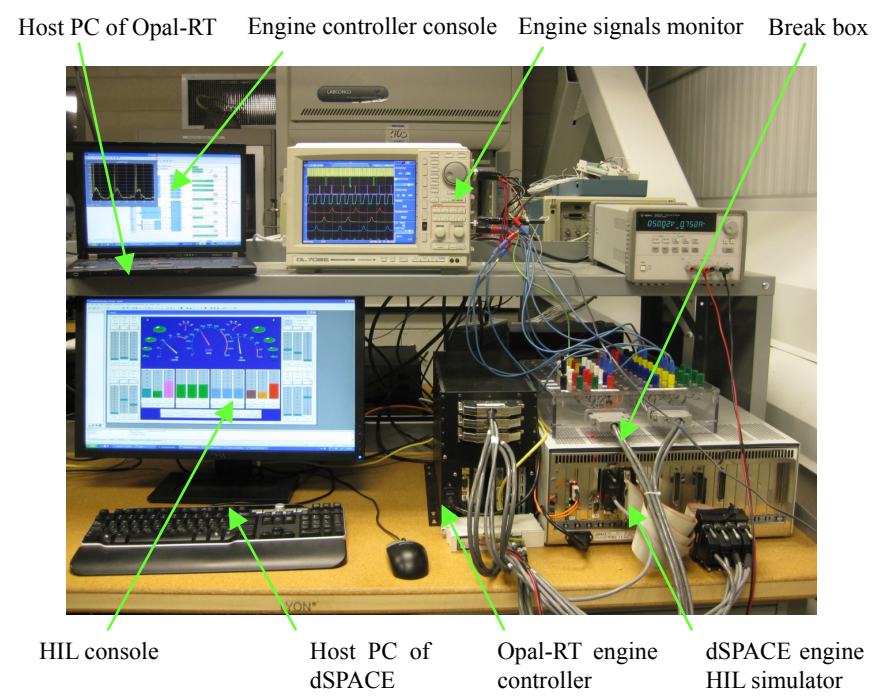

FIGURE 2. HIL SIMULATION STATION

\section{THE HCCI CAPABLE SI ENGINE MODEL}

The engine model used for the HIL simulation of this paper has been presented in previous work ([1] and [15]). It models the physical characteristics of the engine system. And it consists of two main sub-system models. One is the crank based model for combustion process; and the other is the time based models for engine air-handling system, crankshaft dynamics, and engine actuators.

The crank based combustion model has the resolution of every crank degree. It models the SI-HCCI hybrid (or spark assistant) combustion process that starts with the SI combustion and ends with the HCCI combustion, where the SI combustion phase is modeled under two-zone assumption and the HCCI combustion phase is modeled under one-zone assumption. The SI and HCCI combustion modes are actually special cases of the SI-HCCI hybrid combustion mode. Accordingly this combustion model is applicable for any operations during the combustion mode transition. The combustion model of the cycle-to-cycle dynamics can be expressed in the nonlinear state space form as

$$
\begin{array}{r}
x(k+1)=f(x(k), u(k)) \\
y(k)=h(x(k), u(k))
\end{array}
$$

where $x(k), u(k)$ and $y(k)$ are states, inputs and outputs, respectively. They are given by

$$
\begin{aligned}
& x(k)=\left[\begin{array}{ll}
M_{E V C}(k) & T_{I V C}(k)
\end{array}\right]^{T} \\
& u(k)=\left[\begin{array}{lllllll}
\theta_{S T} & F_{D I} & \theta_{I N T M} & \theta_{E X T M} & x_{E G R} & M A P & \Pi_{l i j]}
\end{array}\right]^{T}(k) \\
& y(k)=I M E P(k)
\end{aligned}
$$

The state and output functions, $f$ and $g$, in Eq. (1) are composed by the governing equations of the engine combustion process. The details of the combustion model are described in [1].

The engine crankshaft model and air handling system submodels, such as the gas filling dynamical model of intake manifold and external EGR model, are developed in time based and updated every millisecond, since the dynamics of these sub-systems are continuous. The complete description of these models is presented in [15].

In the HIL simulation of combustion mode transition, the engine control actuator dynamics, such as engine throttle, VVT actuator and EGR valve, are found to affect the combustion performance significantly. They must be reflected in the engine model. In this study, the actuator response $Y(s)$ with respect to the control input $U(s)$ is approximated as the first order system in the engine model as

$$
\frac{Y(s)}{U(s)}=\frac{1}{1+\tau s}
$$

where $s$ is the Laplace operator and $\tau$ is the associated actuator time constant. For the HIL simulations of this paper, $\tau$ equals to $0.15,0.12$ and 0.1 second for engine throttle, VVT actuator and EGR valve, respectively. The transient responses of these actuators can be found in Fig. 4, Fig. 6 and Fig. 12. Their influences to the combustion mode transition will be discussed.

\section{ONE-STEP COMBUSTION MODE TRANSITION}

The combustion mode transition was first studied for constant load engine operating condition. At $2000 \mathrm{rpm}$ engine speed the transition is assumed to occur at 4.5 bar IMEP. Table 1 lists the engine parameters (actuator positions) associated with the SI and HCCI combustions. These parameters are optimized for the steady state engine performance with the best fuel economy that satisfies the engine knock limit. That is, the maximum pressure rise $(d p / d \theta)$ is less than or equal to 3 bar per crank degree. It can be seen in Tab. 1 that the engine control parameters are quite different between the SI and HCCI combustions. Among these control parameters, some of them can be adjusted to the target positions within one engine cycle, such as $\theta_{S T}, F_{D I}$ and $\Pi_{\text {lifft }}$; but the others cannot, due to the response delays of these engine actuators.

The combustion characteristics are also quite different between these two combustion modes. For example, the HCCI combustion has higher peak in-cylinder pressure comparing with that of the SI combustion, due to the faster fuel burn rate as illustrated in Fig. 3. Most likely, it also has a recompression phase but the SI combustion does not. The goal of the 
combustion mode transition is to transfer the combustion mode without detectable engine torque fluctuation by regulating those engine control parameters, or in other words to maintain the engine IMEP during the combustion mode transition.

The control strategy development in this paper starts with one-step transition strategy. That is to directly change the control references of all engine control parameters from the SI to the HCCI engine parameters, as listed in Tab. 1, in one engine cycle. The HIL simulation of the one-step transition was conducted and key engine variables are plotted in Fig. 4.

\section{TABLE 1. STEADY STATE ENGINE PARAMETERS FOR SI} AND HCCI COMBUSTION MODES

\begin{tabular}{|l|l|l|}
\hline Engine parameter & SI & HCCI \\
\hline$\theta_{S T}(\operatorname{deg}$ ACTDC $)$ & -36 & none \\
\hline$\phi_{E G R}(\%)$ & 3 & 26 \\
\hline$\phi_{\text {throttle }}(\%)$ & 16.6 & 100 \\
\hline$F_{D I}(\mathrm{~ms} /$ cycle $)$ & 2.06 & 1.6 \\
\hline$\theta_{I N T M}(\operatorname{deg}$ AGTDC $)$ & 70 & 95 \\
\hline$\theta_{E X T M}(\operatorname{deg}$ BGTDC $)$ & 100 & 132 \\
\hline$\Pi_{\text {lift }}(\mathrm{mm})$ & 9 & 5 \\
\hline
\end{tabular}
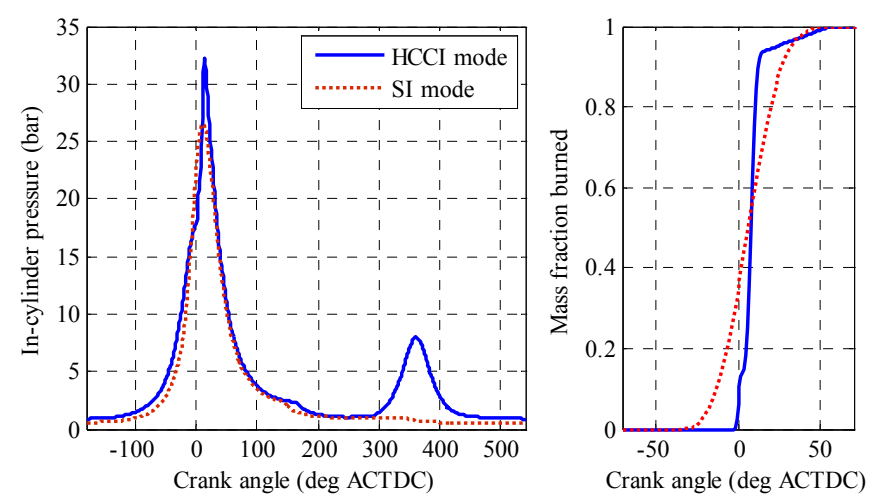

FIGURE 3. STEADY STATE COMBUSTION CHARACTERISTICS OF SI AND HCCI COMBUSTION MODES

As illustrated in Fig. 4, during the one-step mode transition, once the step references apply to the engine control actuators, some engine parameters response instantaneously, such as the intake/exhaust valve lifts. DI fuel quantity can also be adjusted to its control reference value within one engine cycle. Whereas the other engine parameters, such as the intake/exhaust valve timings and the throttle opening, are with response delays. It takes them several engine cycles to reach their reference values, as seen in Fig. 4. When the gas filling dynamics of the intake manifold is coupled with the first order dynamics of these engine actuators, higher order dynamical response can be observed on the engine MAP signal, see Fig. 4. Furthermore, the intake/exhaust valve lift switch imposes a big impact to the engine respiration process. It decreases the charge mass and increases residue gas mass. As a result, the volumetric efficiency of each cylinder for the engine cycle right after the valve lift switch drops significantly, leading to rich combustions for all four cylinders. For the succeeding engine cycles the volumetric efficiency is increased due to the increment of MAP.

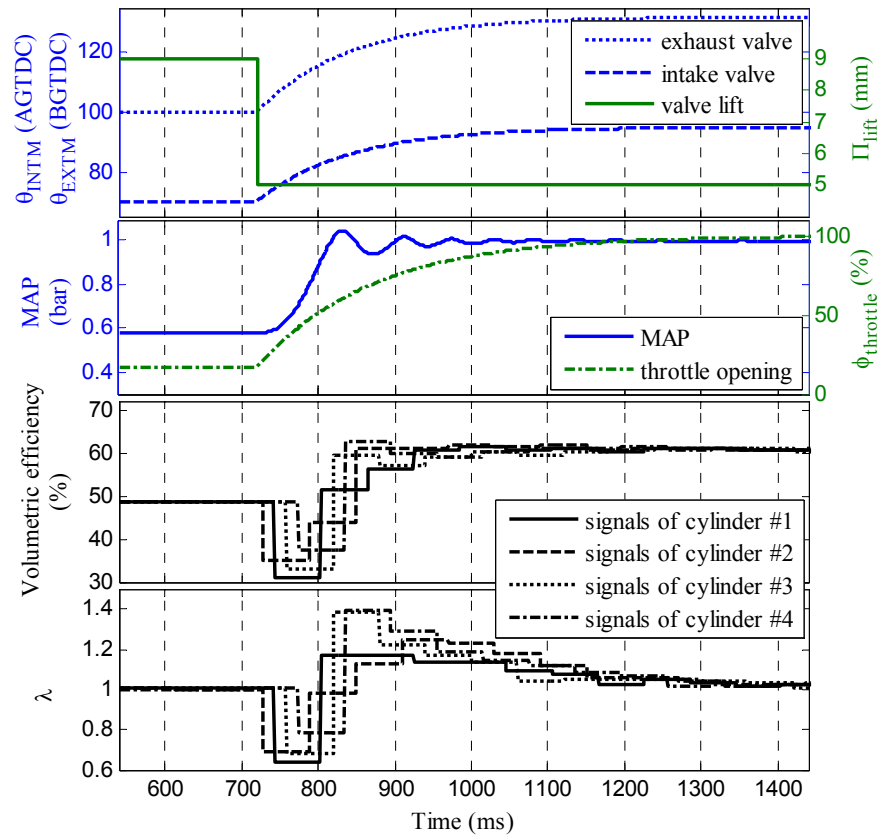

FIGURE 4. ONE-STEP TRANSITION ENGINE RESPONSES

The HIL simulation results indicate that the smooth combustion mode transition cannot be realized by the one-step transition strategy due to the response delay of the engine actuators and the flow dynamics of engine respiration process. Multiple engine cycles need to be inserted between the two distinct combustion modes. This is the main motivation of the proposed multistep combustion mode transition strategy that will be discussed in the next section.

\section{MULTISTEP COMBUSTION MODE TRANSITION}

In the one-step mode transition, rich combustions are found for the engine cycle right after the valve lift switch. This implies the lack of fresh in-cylinder charge. Without enough fresh charge the combustion will inevitably be degraded. There are two ways to increase the charge during the transient condition. One is to advance the intake valve timing and retard the exhaust valve timing; the other is to open the engine throttle before the valve lift switch. Using the first approach, both intake and exhaust valve timings need to be moved to one direction first and then to the opposite direction. This prolongs the settling time of the valve timings, and complicates the engine flow dynamics during the combustion mode transition. The approach of pre-opening the throttle does not have this issue. As illustrated in Fig. 5 two engine cycles (cycle 1 and 2) are inserted between the SI and HCCI combustion modes. Control events are scheduled at different timings during the two cycles. At the beginning of cycle 1 the engine controller computes $N_{T P O}\left(0 \leq N_{T P O} \leq 8\right)$. Then at $N_{T P O}$ quarter-cycles before the end of cycle 2 , the control reference of throttle opening is 
set to $100 \%$, and the throttle plate starts to move to wide open throttle (WOT) position. At the end of cycle 2, the control references of the other parameters are set to their target values as given in Tab. 1; the spark ignition is eliminated and the engine operation is transited into the HCCI combustion mode. Notice that the SI-HCCI hybrid combustion cycles in Fig. 5 are not used in this case and they will be used in the next strategy.

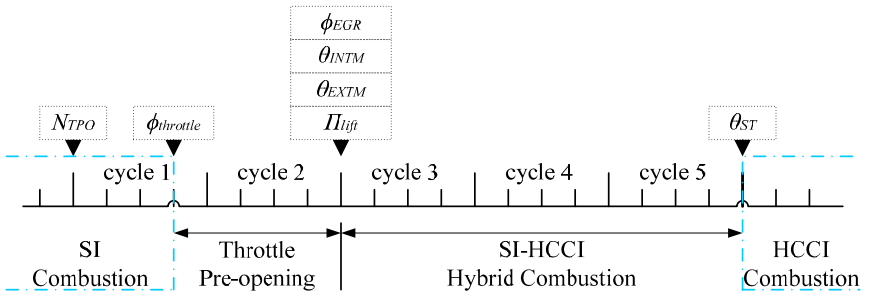

FIGURE 5. SCHEDULE OF ENGINE CONTROL EVENTS FOR MULTISTEP MODE TRANSITION STRATEGIES

By comparing the simulation results in Fig. 4 and Fig. 6, one can see that the throttle pre-opening helps to increase the fresh charge at the start of transition since the engine MAP is increased before the valve lift switch; and it also reduces the engine torque fluctuation, see Fig. 11 for the comparison with the one-step mode transition. However the performance is still not acceptable since the large engine torque fluctuation indicates degraded combustions during the mode transition.

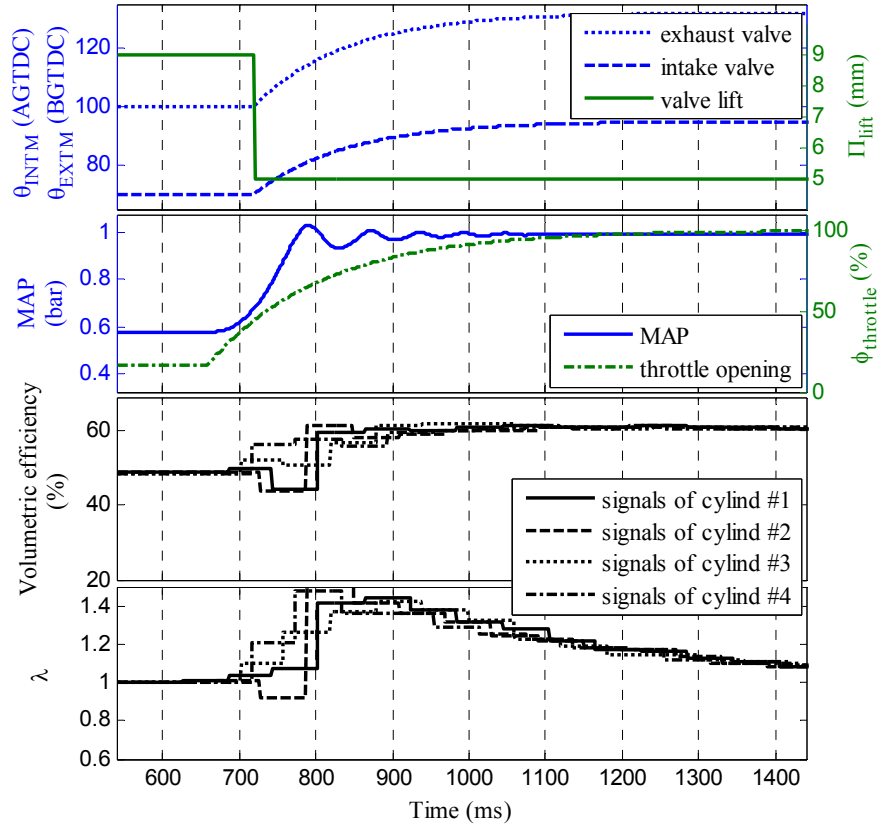

FIGURE 6. ENGINE RESPONSE OF MULTISTEP MODE TRANSITION WITH THROTTLE PRE-OPENING $\left(N_{T P O}=4\right)$

In order to investigate the cause of the degraded combustions during the mode transition, more combustion related engine variables are plotted in Fig. 7. One can see that $T_{I V C}$ is increased after the valve lift switch, but still far below the temperature required for steady state HCCI combustion.
This is mainly due to the response delays of the intake/exhaust valve timings, which lead to insufficient residue gas charge. The reduced charge temperature leads to retarded HCCI combustion timing and degraded IMEP. Furthermore, as discussed in [11], late combustion timing is accompanied with large variation of $\theta_{\text {SOHCCI }}$ and IMEP variables for the HCCI combustion mode. This means unstable combustions. Misfire might happen when the combustion is not stable. And misfire is disastrous for HCCI combustion mode since it might trigger misfire for the succeeding engine cycles.

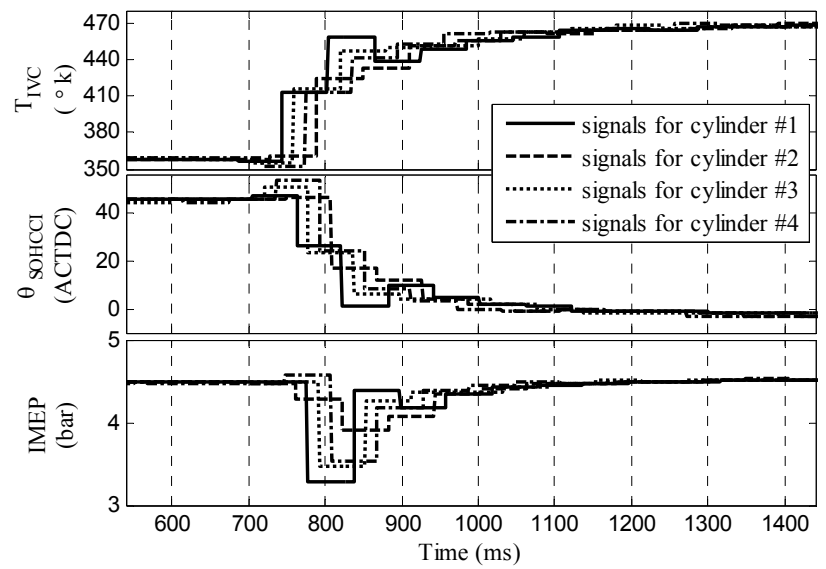

FIGURE 7. COMBUSTION PERFORMANCE OF MULTISTEP MODE TRANSITION WITH THROTTLE PRE-OPENING

In order to avoid misfire and unstable combustions during the mode transition, three engine cycles of the SI-HCCI hybrid combustions are applied in addition to the throttle pre-opening. As illustrated in Fig. 5 the spark ignition is kept for three engine cycles after the valve lift switch and it is eliminated at the end of cycle 5. With the spark ignition, the combustions of cycles 3, 4 and 5 are initiated by spark ignition in the SI mode but ended in the HCCI mode, and they are called SI-HCCI hybrid combustions. More details about this hybrid combustion mode can be found in [1] and [12].

The SI-HCCI hybrid combustions only occur under certain thermo conditions. The in-cylinder gas-fuel mixture needs to be lean, but if it is too lean the spark ignition might not be able to initiate the SI combustion. The charge temperature needs to be higher than what SI combustion requires but lower than that of the HCCI combustions. Only under this condition the unburned gas-fuel mixture during the initial SI combustion phase can meet the start of HCCI combustion criterion to initiate the HCCI combustion in the unburned zone. These thermo conditions are satisfied for cycles 3, 4, and 5. Accordingly the combustions during these engine cycles are in the SI-HCCI hybrid combustion mode. Once the hybrid combustion is engaged, misfire will not occur even with very late SOHCCI timing, since a portion of gas-fuel mixture has already been burned in the SI mode before $\theta_{\text {SOHCCI}}$. Moreover, under these special thermo conditions, engine IMEP output is highly correlated with the DI fuel quantity, so it is possible to regulate the engine IMEP (or torque) by controlling the DI fuel quantity. 
Whereas for the pure HCCI combustion mode the increased cooling effect due to increment of the DI fueling reduces the charge temperature, leading to degraded HCCI combustions.

The HIL simulation results of the multistep combustion mode transition are plotted in Fig. 10. One can see that $T_{I V C}$ of the first engine cycle after the valve lift switch is still low; but $\theta_{\text {SOHCCI }}$ is advanced for a few crank degrees, and the engine torque fluctuation is smaller than those without the hybrid combustions. In the HIL simulations, the DI fuel quantity and spark timing are controlled using the values for steady state engine operating condition. They are not optimal since the engine flow dynamics and the cycle-to-cycle residue gas dynamics make the thermo conditions different from those of the steady state. More advanced control strategy is required to cope with the thermo dynamics.

\section{MULTISTEP COMBUSTION MODE TRANSITION WITH ITERATIVE LEARNING CONTROL}

By investigating the simulation results plotted in Fig. 7 and Fig. 10. One can see that significant cycle-to-cycle and cylinder-to-cylinder variances occurred in most combustion related variables, such as $T_{I V C}$ and $\theta_{S O H C C I}$. This is mainly contributed by the fact that the change of the engine actuator positions affects the individual cylinder thermo condition differently. As a result, the IMEP and engine toque output also show significant variations. To reduce the variations of engine IMEP and torque, a few control schemes were tried. Feedback control was firstly tried, but since the mode transition only happens for several engine cycles, any feedback controls might not have time to improve the performance. Feedforward control was then considered. Through an iterative learning control (ILC) approach, the Feedforward DI fuel quantity can be regulated to make the IMEP track the control reference (4.5bar in this case). The smooth combustion mode transition was finally achieved in the HIL engine simulation environment with the help of ILC.

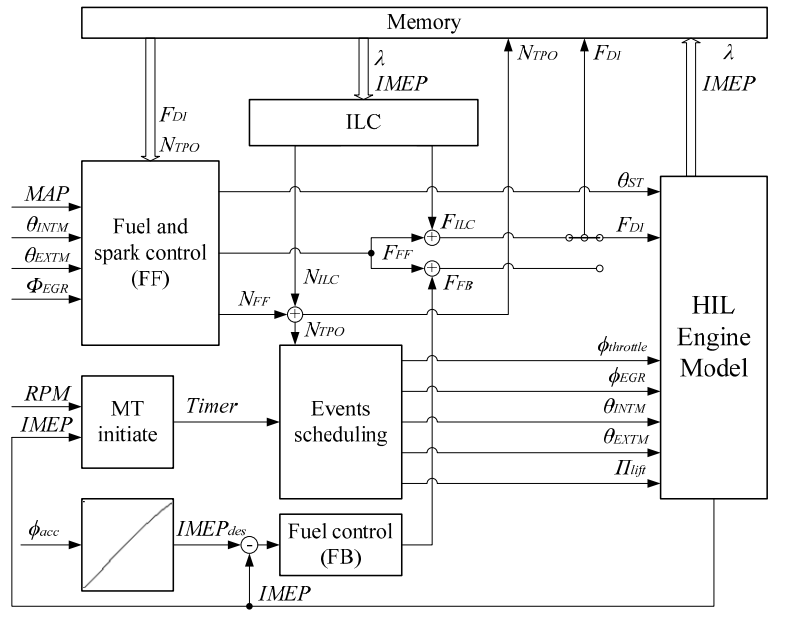

FIGURE 8. MULTISTEP COMBUSTION MODE TRANSITION CONTROL DIAGRAM
As illustrated in Fig. 8, the Feedforward controller is initialized using the steady state control values as described in the last section. Two control parameters are directly adjusted by the ILC during each learning iteration. They are $N_{T P O}$ and $F_{D I}$. For $N_{T P O}$ the learning algorithm is

$$
\begin{array}{ll} 
& N_{T P O}(i+1)=N_{F F}(i)+N_{L C C}(i)=N_{F F}(i)+\delta(\lambda(i, k, n)) \\
\text { and } & N_{F F}(i+1)=N_{T P O}(i+1)
\end{array}
$$

where

$$
\begin{cases}\delta(\lambda(i, k, n))=-1 & \text { if }: \operatorname{Max}(\lambda(i, k, n))>\lambda_{\text {nigh }} \\ \delta(\lambda(i, k, n))=1 & \text { if }: \operatorname{Min}(\lambda(i, k, n))<\lambda_{\text {low }} \\ \delta(\lambda(i, k, n))=0 & \text { otherwise }\end{cases}
$$

Through this learning algorithm, the normalized air-to-fuel ratios of each cylinder during the SI-HCCI hybrid combustion cycles are limited to between $\lambda_{\text {low }}$ and $\lambda_{\text {high }}\left(\lambda_{\text {low }}=1\right.$ and $\lambda_{\text {high }}=1.4$ in this case). Under this air-to-fuel ratio, IMEP is highly correlated to $F_{D I}$. Accordingly IMEP can be regulated by controlling $F_{D I}$ through the following formula,

$$
\begin{aligned}
& F_{D I}(i+1, k, n)=F_{F F}(i, k, n)+F_{I L C}(i, k, n) \\
& \text { and } F_{F F}(i+1, k, n)=F_{D I}(i+1, k, n)
\end{aligned}
$$

The iterative learning control term $\left(F_{I L C}\right)$ in Eq. (6) is calculated by a typical $P$ type self learning algorithm [13]. Notice that in Eq. (6) $F_{D I}$ is controlled with respect to each individual cylinder, due to the cylinder-to-cylinder variation as discussed. The trained control values are stored in memory after each learning iteration. They will be used as Feedforward terms for next learning iteration or torque tracking control that is going to be discussed in the next section. Figure 9 shows that the iterative learning control variables converge after a few iterations of learning. The first engine cycle (cycle 3) after the intake/exhaust valve lift switch experiences the largest improvement, leading to significant correction of $F_{D I}$. The largest cylinder-to-cylinder variation of $F_{D I}$ can also be seen at cycle 3 . The variation of $F_{D I}$ counteracts the variance of IMEP, and makes IMEP smooth.

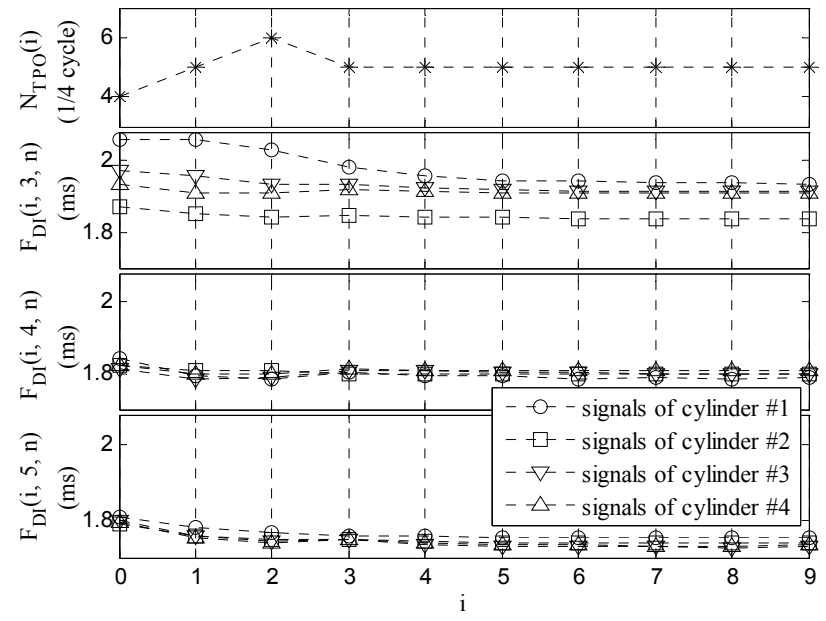

FIGURE 9. ITERATIVE LEARNING CONTROL SIGNALS 


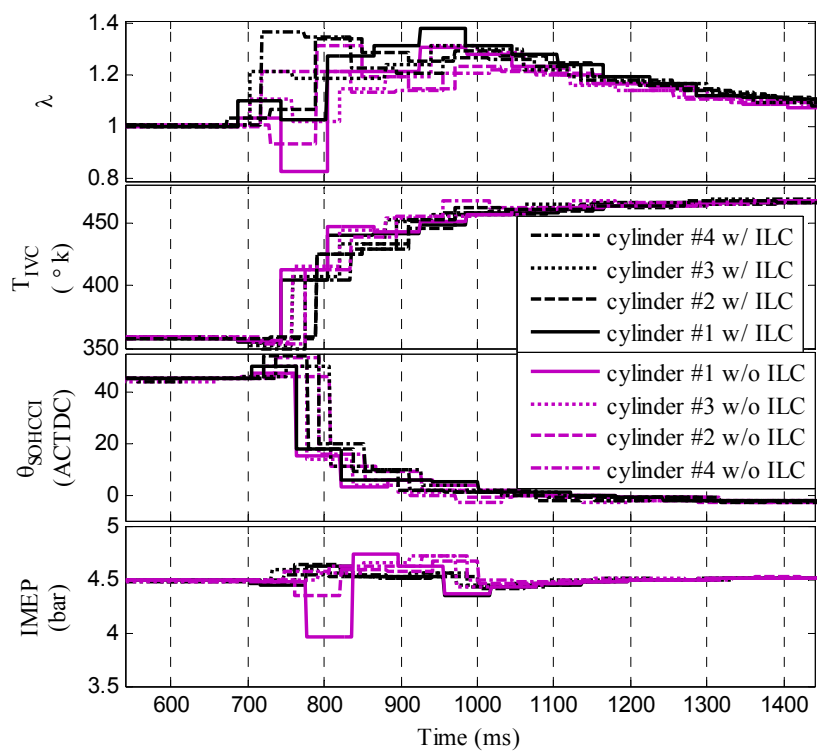

FIGURE 10. COMBUSTION PERFORMANCE OF MULTISTEP MODE TRANSITION

Some engine variables are plotted in Fig. 10 for both with and without the iterative learning control. With ILC of $N_{T P O}$, the normalized air-to-fuel ratios of all cylinders during the hybrid combustion cycles are limited in the desired range, while without it rich gas-fuel mixture can still be found during the combustion mode transition. Same as in the mode transition without ILC, the variables $\lambda, T_{I V C}$ and $\theta_{S O H C C I}$ during the mode transition with ILC also show large cycle-to-cycle and cylinderto-cylinder variations. The only signal with the reduced variation is the IMEP signal for the mode transition with ILC. This demonstrates the effectiveness of the ILC strategy.

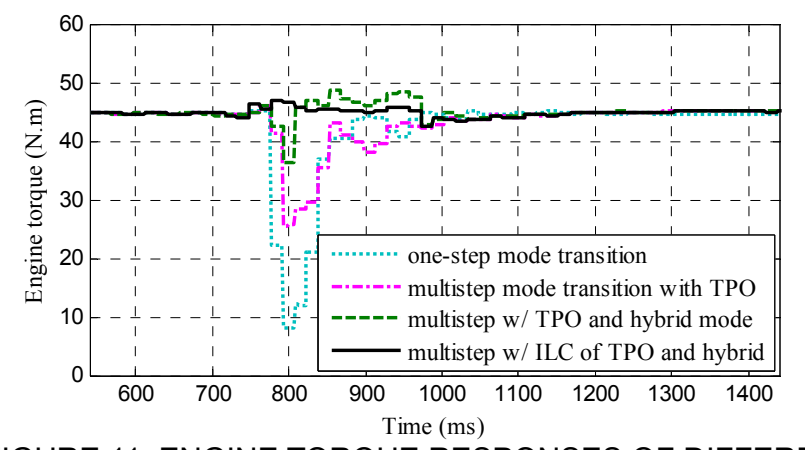

FIGURE 11. ENGINE TORQUE RESPONSES OF DIFFERENT COMBUSTION MODE TRANSITION STRATEGIES

TABLE 2. ENGINE KNOCK INDEX AND MAXIMUM ERROR OF IMEP AND ENGINE TORQUE SIGNALS

\begin{tabular}{|l|c|c|c|}
\hline & $\begin{array}{c}\text { IMEP error } \\
(\%)\end{array}$ & $\begin{array}{c}\text { Max dp/d } \\
(\text { bar/deg) }\end{array}$ & $\begin{array}{c}\text { Torque error } \\
(\%)\end{array}$ \\
\hline One-step & 51.6 & 3.4 & 81.8 \\
\hline TPO & 26.9 & 2.82 & 42.5 \\
\hline TPO + Hybrid & 12 & 2.65 & 18.6 \\
\hline $\begin{array}{l}\text { (TPO + Hybrid) } \\
\text { with ILC }\end{array}$ & 3.3 & 2.55 & 4.5 \\
\hline
\end{tabular}

Engine torque responses during the combustion mode transitions using different strategies are plotted in Fig. 11. Their statistics are listed in Tab. 2 for comparison. Smooth combustion mode transition can be realized by combining the throttle pre-opening, using the SI-HCCI hybrid combustions and the iterative learning control strategy. It shows only $4.5 \%$ of engine torque fluctuation when the engine combustion mode transits from the SI to HCCI combustion mode in constant load operation. A significant reduction of engine knock index $(d p / d \theta$ is a good indicator of engine knock [14]) can also be found during the mode transition. This indicates that the proposed strategy can suppress engine knock during the mode transition.

Notice that the iterative learning control is only activated when engine is operated at 4.5bar IMEP constant load condition. Once the ILC is activated, the feedback control needs to be deactivated, as illustrated in Fig. 8. Accordingly the ILC is only conducted in engine calibration during the product development phase, or during the constant load operation, such as cruise operation. This also makes the engine mode transition robust against the engine aging. Under transient engine conditions, the ILC is deactivated and the engine will be controlled in different way.

\section{COMBUSTION MODE TRANSITION DURING ENGINE TIP OUT OPERATION}

During the transient engine operation, the ILC term is deactivated and the feedback control will be activated, as illustrated in Fig. 8. The combustion mode transition process is controlled by a typical feedforward plus feedback control scheme. It is represented as

$$
F_{D l}=F_{F F}+F_{F B}
$$

The feedforward term $\left(F_{F F}\right)$ in Eq. (7) is the trained value after $i$ iterations of learning. The feedback term $\left(F_{F B}\right)$ in Eq. $(7)$ is the output of a PI controller, and it is used not only during the mode transition but also in the HCCI combustion mode.

In order to test the control strategy under the transient engine operating condition, an HIL simulation was conducted to simulate the engine throttle tip out operation. In the simulation, the engine was initially operated in the SI mode. As a step input is applied to the acceleration pedal, the pedal position decreased from $40 \%$ to $15 \%$. As a result, the engine IMEP reduced from 8.1 bar to 4 bar, which crosses the combustion mode transition threshold ( 4.5 bar). Accordingly the mode transition was triggered. As shown in Fig. 12, both $\phi_{\text {throttle }}$ and MAP signals were decreased at first, and increased due to the combustion mode transition. Slightly rich combustions can be seen during the early stage of the tip out operation, since the throttle opening was decreased in the SI mode; once the mode transition started, the in-cylinder gas mixture was lean. In Fig. 12, one can also see that the engine IMEP tracks the desired IMEP during the entire tip out operation with the feedforward control trained by the ILC. In contrary, the IMEP signal shows about $\pm 10 \%$ fluctuation during 
the combustion mode transition when the feedforward term was not trained. It can be concluded that the combination of feedback control and ILC trained feedforward control can lead to smooth combustion mode transition even under transient engine operating conditions.

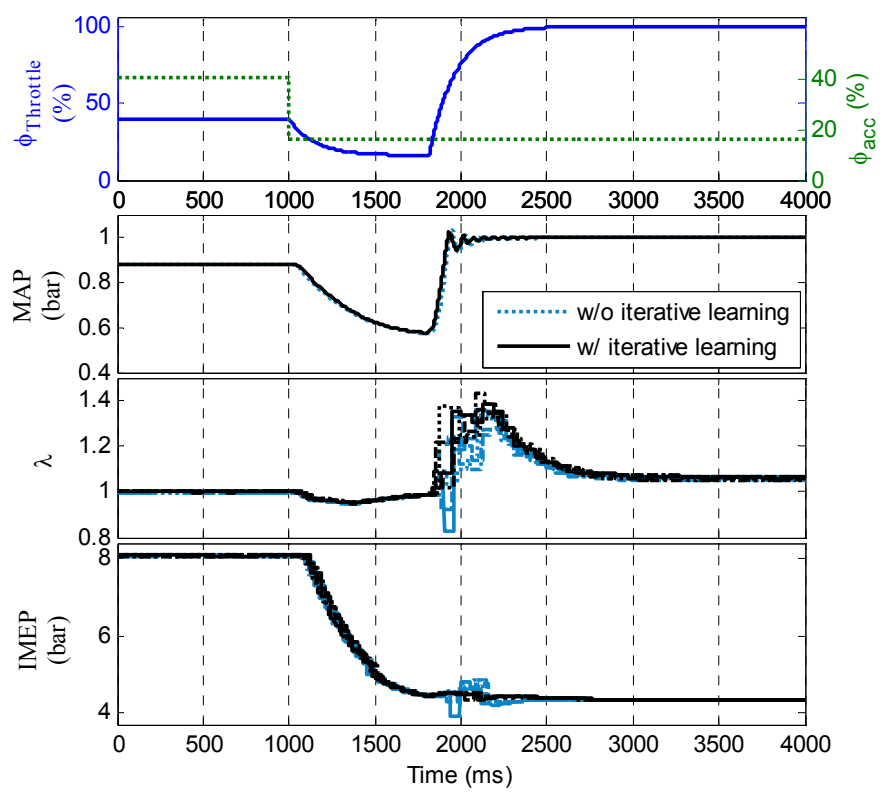

FIGURE 12. ENGINE RESPONSES DURING TIP OUT

\section{CONCLUSION}

In this paper, different control strategies are studied to make the SI to HCCI combustion mode transition smooth. It is gradually discovered that the smooth SI to HCCI combustion mode transition can be realized by combining the throttle preopening, utilizing SI-HCCI hybrid combustion phases, and the iterative learning control of the throttle pre-opening timing and DI fuel quantity. The entire control strategy is validated for the constant load engine operating condition in a hardware-in-theloop simulation environment. Furthermore, the control strategy can also be used during engine calibration process. When the trained feedforward control is combined with the PI feedback control, smooth SI to HCCI combustion mode transition can also be realized during transient engine operations.

\section{ACKNOWLEDGMENTS}

This work is supported by the U.S. Department of Energy under Grant DE-EE0000211.

\section{REFERENCES}

[1] Yang, X., and Zhu, G., “A Two-Zone Control Oriented SIHCCI Hybrid Combustion Model for the HIL Engine Simulation," Proceedings of 2011 American Control Conference, San Francisco, California, USA, 2011.
[2] Yang, X., Zhu, G., Sun, Z., "A Control Oriented SI and HCCI Hybrid Combustion Model for Internal Combustion Engines," Proceedings of 2010 ASME Dynamic Systems and Control Conference, Cambridge, MA, 2010.

[3] Zhao, F., Asmus, T., Assanis, D., et al, "Homogeneous Charge Compression Ignition (HCCI) Engines Key Research and Development Issues," 2003, Warrendale, Pennsylvania: Society of Automotive Engineers.

[4] Kong, S. C., and Reitz, R. D., "Application of Detailed Chemistry and CFD for Predicting Direct Injection HCCI Engine Combustion and Emission," Proceedings of the Combustion Institute, Vol. 29, 2002, pp. 663-669.

[5] Ravi, N., Roelle, M. J., et al, "Model-Based Control of HCCI Engines Using Exhaust Recompression," IEEE Transactions on Control Systems Technology, Vol. 18, No. 6, November 2010, pp. 1289-1302.

[6] Kang, J., Chang, C., and Kuo, T., "Sufficient Condition on Valve Timing for Robust Load Transients in HCCI Engines," SAE International, 2010-01-1243.

[7] Shaver, G. M., "Physics based modeling and control of residual-affected HCCI engines using Variable Valve Actuation," Ph.D. thesis, Stanford University, Sep, 2005.

[8] Roelle, M. J., Shaver, G. M., and Gerdes, J. C., "Tackling the Transition: A Multi-Mode Combustion Model of SI and HCCI for Mode Transition Control," Proceedings of IMECE 2004 International Mechanical Engineering Conference and Exposition, Anaheim, CA, Nov. 2004.

[9] Zhang, Y., Xie, H., et al, "Study of SI-HCCI-SI Transition on a Port Fuel Injection Engine Equipped with 4VVAS", SAE, 2007-01-0199.

[10] Wu, H., Kraft, M., et al, "Experimental Investigation of a Control Method for SI-HCCI-SI Transition in a MultiCylinder Gasoline Engine," SAE, 2010-01-1245.

[11] Liao, H. H., and Ravi, N., "Representing Recompression HCCI Dynamics with a Switching Linear Model," Proceedings of 2010 American Control Conference, Baltimore, MD, USA.

[12] Wagner, R. M., Edwards, K. D., et al, "Hybrid SI-HCCI Combustion Modes for Low Emissions in Stationary Power Applications," 3rd Annual Advanced Stationary Reciprocating Engines Meeting, Argonne National Laboratory, Argonne, IL June 28-30, 2006.

[13] Ouyang, P. R., Petz, B. A., and Xi, F. F., "Iterative Learning Control with Switching Gain Feedback for Nonlinear Systems," Journal of Computational and Nonlinear Dynamics, January 2011, Vol. 6.

[14] Cheng, W. K., Kenney T., and Yang, J., “On HCCI engine Knock," SAE 2007-01-1858, 2007.

[15] Yang, X., and Zhu, G., "A Mixed Mean-Value and Crankbased Model of a Dual-Stage Turbocharged SI Engine for Hardware-In-the-Loop Simulation”, Proceedings of 2010 American Control Conference, Baltimore, MD, 2010. 
9.10 Published JC Paper \#10

\section{A Two-Zone Control-Oriented SI-HCCI Hybrid Combustion Model for the HIL Engine Simulation}




\title{
A Two-Zone Control Oriented SI-HCCI Hybrid Combustion Model for the HIL Engine Simulation
}

\author{
Xiaojian Yang and Guoming G. Zhu
}

\begin{abstract}
To make HCCI (Homogeneous Charge Compression Ignition) combustion feasible in a SI (Spark Ignition) engine, it is necessary to have the smooth mode transition between SI and HCCI combustions. The SI-HCCI hybrid combustion studied in this paper describes the combustion mode that starts with SI and ends with HCCI combustions. The main motivation of studying the hybrid combustion mode is that the percentage of the HCCI combustion is a good indicator for combustion mode transition control when the hybrid combustion mode is used during the transition. This paper presents a control oriented model for the SI-HCCI hybrid combustion mode, where the SI combustion phase is modeled under a two-zone assumption and the HCCI combustion phase under a one-zone one. Note that SI and HCCI combustions are special cases in this model. The developed model is capable of simulating the entire engine operating range, and it was validated in a HIL (Hardware-In-the-Loop) simulation environment. The simulation results were compared with those of the corresponding GT-Power model, and good correlations were found for both SI and HCCI combustions.
\end{abstract}

\section{INTRODUCTION}

$\mathrm{T}$ HE continuing pursuit of improving fuel economy and the increasingly stringent emission regulations rekindle research interest in the homogeneous charge compression ignition (HCCI) combustion in recent years. The flameless nature of the HCCI combustion and its high dilution operation capability lead to low combustion temperature. As a result, the formation of NOx (Nitrogen Oxides) can be significantly reduced. Furthermore HCCI engine is capable of un-throttled operation that greatly reduces pumping loss and improves fuel economy [1].

On the other hand, HCCI combustion has its own limitations. The HCCI combustion is limited at high engine load due to the audible knock, and at low load due to misfire caused by lack of sufficient thermal energy to trigger the auto-ignition of the gas-fuel mixture late in the compression stroke [2]. In order to take advantages of the HCCI combustion mode in an internal combustion engine, other combustion mode, such as SI (Spark Ignition) combustion, is needed at high load, at ultra-low load (such as idle), and at certain operational conditions such as cold start and at high engine speed. However, it is fairly challenging to operate the engine in two distinct combustion modes, and it is even more

X. Yang and G. Zhu are with the Mechanical Engineering of Michigan State University, East Lansing, MI 48824, USA (yangxia2@egr.msu.edu and zhug@egr.msu.edu, respectively). difficult to have smooth combustion mode transition between SI and HCCI combustions [3], since the favorable thermo conditions for one combustion mode are always adverse to the other one. Due to the significant response delay of the hydraulic and electric variable valve timing (VVT) system, cycle-to-cycle residue gas dynamics and response delay in engine air handling system, it is almost impossible to achieve desired thermo conditions for combustion mode switch in one engine cycle [4].

To address this issue, SI-HCCI hybrid combustion mode has been proposed in [1], [5], and [6]. The hybrid combustion starts in SI combustion mode with a relatively low heat release rate; and once the thermo and chemical conditions of the unburned gas satisfy the start of HCCI (SOHCCI) combustion criteria, the combustion continues in HCCI combustion mode, which is illustrated by the dashed curves of mass fraction burned (MFB) shown in Figure 1. During an ideal SI to HCCI combustion transition process, the percentage of SI combustion (ST to SOHCCI, see Figure 1) decreases gradually while the HCCI combustion percentage (SOHCCI to end of combustion or MFB = 1) increases gradually. For the HCCI to SI combustion transition, the process is reversed. This hybrid combustion mode transition process allows using conventional cam phase system.

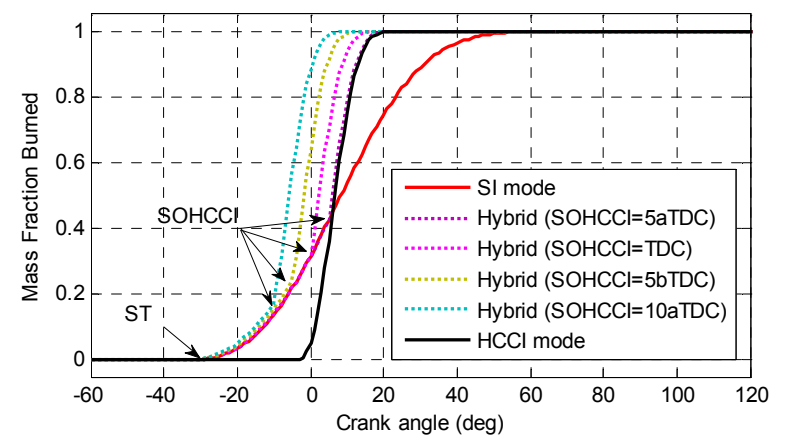

Figure 1: MFB in SI-HCCI hybrid combustion mode transition process

Using the SI-HCCI hybrid combustion mode, smooth mode transition can be realized along with the appropriate control strategy. To accurately control the mode transition between SI and HCCI combustions, a precise combustion model is required, and it needs to be control oriented. Multi-zone, three dimensional CFD models with detailed chemical kinetics are presented in [7], and they are able to precisely describe the thermodynamic, fluid-flow, heat transfer, and pollutant formation phenomena of the HCCI combustion. Similar combustion models have also been 
implemented into commercial codes such as GT-Power and Wave. However, these models with high fidelity cannot be used for control strategy development since they are too complicated to be used for real-time simulation, but they can be used as reference models for developing simplified (or control oriented) combustion models.

In this paper, similar to [1] and [8], mean-value method is used to model averaged chemical kinetics and thermodynamic properties, and the model calculation is updated at every crank degree to have the resolution high enough for control strategy development. In [1], the SI combustion process was modeled using a one-zone approach, which introduces large unburned gas temperature estimation error. To address the issue, this paper presents a control oriented model for the SI-HCCI hybrid combustion mode, where the SI combustion phase is modeled under a two-zone assumption and the HCCI combustion phase under a one-zone one. Simulation results show significant modeling error reduction, comparing with that of the one-zone SI combustion model. Also, the developed hybrid combustion model can be executed in real-time due to the low computational requirement.

\section{MODEL ARCHITECTURE}

The purpose of the combustion modeling is to correlate the trapped in-cylinder gas properties, such as air-to-fuel ratio, in-cylinder gas pressure and temperature, to the combustion characteristics such as misfire, knock, burn duration, and IMEP (Indicated Mean Effective Pressure). The developed combustion model will be used for model-based control strategy development and validation of the mode transition between SI and HCCI combustions into the real-time HIL simulation environment.

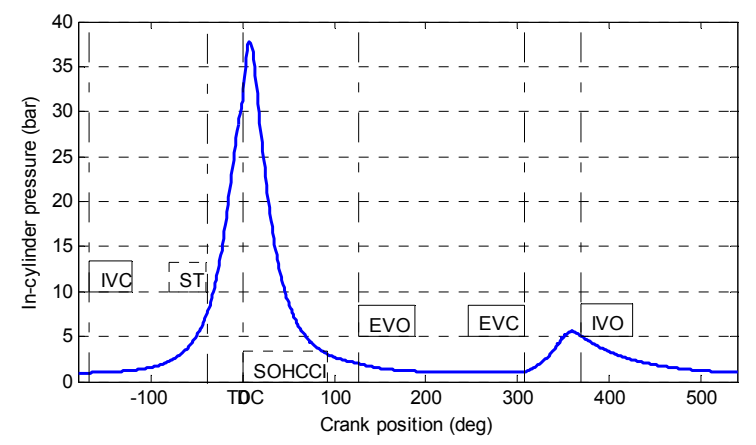

Figure 2: Combustion related events and phases

As mentioned in the last section, the combustion model is updated every crank degree, which is different from other modeling approaches presented in [8] and [9], where the model is updated at a fixed time step. There are many motivations of using the crank-based approach. The first is due to the fact that most combustion characteristics are usually function of the crank angle, such as burn duration and peak pressure location; Secondly, the entire combustion process is divided into several combustion phases related to these events associated with crank position. As shown in
Figure 2, these events are intake valve closing (IVC), spark timing (ST), SOHCCI, exhaust valve opening (EVO), exhaust valve closing (EVC), and intake valve opening (IVO). The in-cylinder behaviors (such as pressure, temperature, etc.) are modeled differently during each combustion phase that is defined between two combustion events.

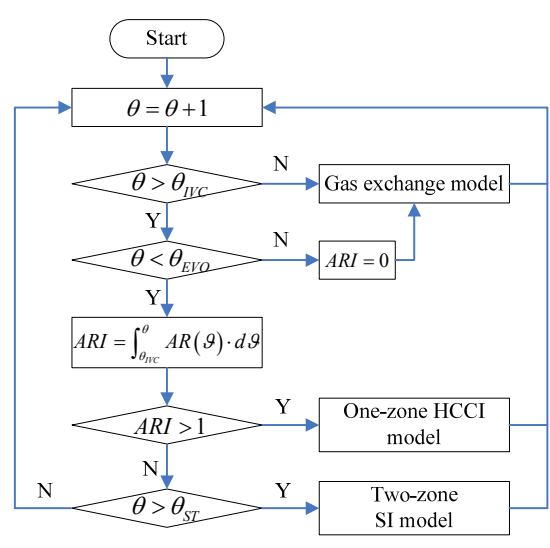

Figure 3: Combustion modeling diagram

The crank-based modeling approach has its own limitation, too. During the real-time simulation the entire model needs to be executed within the time period associated with one crank degree. This leads to short computational time window at high engine speed. For example, at $6000 \mathrm{rpm}$ of engine speed one crank degree corresponds to 28 micro seconds. In order to avoid the overrun in the simulation, the combustion model must be as simple as possible but with required accuracy.

In Figure 2, ST and SOHCCI are marked in the boxes with dashed box to distinguish them from other events, since the occurrences of these two events depend on combustion mode. The rest of events exist for any 4-stroke internal combustion engine. Accordingly, the main difference among each combustion mode lies in the phase between IVC and EVO. When the SOHCCI event does not occur between IVC and EVO events, the engine is operated at the SI combustion mode; and when the SOHCCI event occurs before the SI event, the engine is operated at the HCCI combustion mode; while the SI-HCCI hybrid combustion occurs inter-between the HCCI and SI combustion modes. Note that both SI and HCCI combustion modes are special cases of the SI-HCCI hybrid combustion mode. The combustion modeling architecture is illustrated in the diagram shown in Figure 3. It illustrates the relationship among all sub-system models. The detailed model of each combustion mode and gas exchange will be discussed in the following sections.

\section{Two-ZOnE SI COMBUSTION MODEL}

During the combustion the spark ignited flame front divides the in-cylinder gas mixture into two zones, the burned and unburned zones as shown in Figure 4. The temperature of the unburned zone is quite different from that of burned zone as seen in Figure 6 and Figure 7. However, this difference is 
ignored in one-zone SI combustion model discussed in [1], and an averaged value of temperature is used for both zones. This may not lead to large modeling error for SI combustion, in which the temperature difference does not impact much on the combustion model parameters such as in-cylinder pressure. However, for the SI-HCCI hybrid combustion, the unburned zone temperature is a key parameter for predicting the start of HCCI combustion, and it is the motivation for developing this two-zone combustion model.

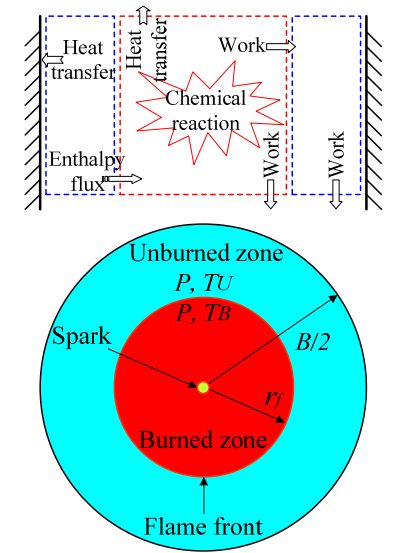

Figure 4: Two-zone combustion model

For simplicity, in the two-zone model, gas mixture pressure is assumed to be evenly distributed throughout both burned and unburned zones. Inertial gas is assumed to remain in the unburned zone after spark ignition. The fuel, air, and residue gas charge are uniformly premixed. The fuel burn rate is approximated by the Wiebe function [10]:

$$
x\left(\theta_{i}\right)=1-e^{-a\left(\frac{\theta-\theta_{S T}}{\Delta \theta}\right)^{m+1}}
$$

where $x$ is the mass fraction burned (MFB); $\theta$ is the current crank position; the predicted burn duration $\Delta \theta$ and Weibe exponent $m$ are calibration parameters of engine speed, load and coolant temperature. Coefficient $a$ depends on how the burn duration $\Delta \theta$ is defined. If $\Delta \theta$ is specified as $10 \%$ to $90 \%$ MFB, $a$ is calculated by

$$
a=\left\{[-\ln (1-0.9)]^{\frac{1}{m+1}}-[-\ln (1-0.1)]^{\frac{1}{m+1}}\right\}^{m+1}
$$

During the combustion phase between IVC and ST, in-cylinder gas is under isentropic compression, and the governing equations can be found in [1] and [11]. After SI combustion starts, the mass of burned zone is calculated based upon the mass of burned fuel that can be calculated from (1), assuming that the burned zone air-to-fuel ratio is known. According to the first law of thermodynamics [17], the energy balance equation of burned zone is represented by

$$
\frac{d\left(M_{B} \cdot e_{B}\right)}{d \theta}+P \frac{d V_{B}}{d \theta}+Q_{B}=M_{f} \frac{d x}{d \theta} h_{L H V} \cdot \eta_{S I}+\frac{d M_{B}}{d \theta} h_{U}
$$

where $M_{B}, V_{B}$, and $e_{B}$ are the mass, volume, and internal energy of burned zone; $Q_{B}$ is the heat transfer from the burned zone; $M_{f}$ is the mass of fuel injected into each cylinder for every engine cycle; $P$ is the gas pressure of both zones; $h_{L H V}$ is the lower heat value of fuel; $h_{U}$ is the specific enthalpy of unburned zone; and $\eta_{S I}$ is the combustion efficiency due to incomplete combustion.

The energy balance equation of unburned zone is

$$
\frac{d\left(M_{U} \cdot e_{U}\right)}{d \theta}+P \frac{d V_{U}}{d \theta}+Q_{U}=\frac{d M_{U}}{d \theta} h_{U}
$$

where $M_{U}, V_{U}$, and $e_{U}$ are the mass, volume and internal energy of burned zone, respectively; and $Q_{U}$ is the heat transfer from the burned zone.

Moreover, the gas of both burned and unburned zones can be considered as ideal gas [12], therefore ideal gas law holds for both zones. For burned zone, we have

$$
\frac{P \cdot V_{B}}{R \cdot T_{B}}=M_{B}=x \cdot M_{f} \cdot\left(1+\sigma_{0}\right)
$$

where $\sigma_{0}$ is the stoichiometric air-to-fuel ratio of the fuel; $T_{B}$ is the burned zone gas temperature; and $R$ is gas constant.

For unburned zone, the ideal gas law can be represented by

$$
\frac{P \cdot V_{U}}{R \cdot T_{U}}=M_{U}=M_{I V C}-x \cdot M_{f} \cdot\left(1+\sigma_{0}\right)
$$

where $T_{U}$ is the unburned zone gas temperature; $M_{I V C}$ is the gas mass at the crank position of IVC. Note that it is the total gas mass during the phase of IVC-EVO.

Additionally, the cylinder geometry yields

$$
V_{B}+V_{U}=V
$$

where $\mathrm{V}$ is the cylinder volume.

For a SI gasoline engine the heat transfer due to radiation is relatively small in comparison with convective heat transfer [10]. Thereby only convective heat transfer is counted in the energy balance equations, and Woschni correlation model [14] is used to calculate $Q_{B}$ and $Q_{U}$ in (3) and (4).

$$
Q=3.26 \cdot B^{m-1} \cdot P^{m} \cdot w^{m} \cdot T^{0.75-1.62 m} \cdot A \cdot\left(T-T_{W}\right)
$$

where $B$ is cylinder bore; $w$ is gas flow speed that is a function of engine speed; $A$ is the contact area between gas and cylinder wall; $T_{W}$ is average temperature of cylinder wall; and exponent $m$ is used for correlation, where $m=0.8$ gives best correlation for the combustion mode.

In the five equations from (3) to (7), there are five unknowns: $T_{B}, T_{U}, V_{B}, V_{U}$, and $P$. Due to the nonlinearity in equations (5) and (6), the solutions are not unique and the rational solution is adopted in the model simulation.

\section{OnE-ZONE COMBustion MOdel For HCCI Mode}

The most common practice in control oriented modeling of HCCI combustion is to assume the combustion process, that converts the fuel and oxidizer into combustion products, is governed by single rate equation of an Arrhenius form [10]

$$
A R=A \cdot x_{f}^{a} \cdot x_{o x}^{b} \cdot e^{\left(\frac{E_{a}}{R \cdot T}\right)}
$$


where $A R$ is the rate of disappearance of unburned fuel; $x_{f}$ and $x_{o x}$ are unburned fuel and oxidizer mass fractions; exponents $a$ and $b$ are usually selected to be unity; multiplier $A$ and Arrhenius activation energy $E_{a}$ can be obtained by matching the experimentally determined rates of burning.

Based on [9] and [13], the integral of the Arrhenius function can be used to estimate the crank position, $\theta_{\mathrm{SOHCCI}}$, of SOHCCI, The SOHCCI crank position is defined as the crank angle for $1 \%$ fuel burned under HCCI combustion, and it can be represented by

$$
A R I=\int_{\theta_{I V C}}^{\theta_{i}} A \cdot x_{f}^{a} \cdot x_{o x}^{b} \cdot e^{\left(-\frac{E_{a}}{R \cdot T}\right)} d \vartheta
$$

The Arrhenius integration in (10) starts at $\theta_{I V C}$, and is reset at $\theta_{E V O}$. During this period, if spark ignition never happens, the variable $T$ in (10) equals to in-cylinder gas temperature; during the SI combustion phase of the SI-HCCI hybrid combustion mode, only unburned zone temperature is used for $T$ after spark ignition. Once the HCCI combustion is triggered, the fuel burn rate is also modeled using Wiebe function in (1), but exponent $m$ and predicted burn duration $\Delta \theta$ will switch to the calibrations of HCCI mode.

The HCCI combustion is modeled under the one-zone assumption due to its flameless nature. Fuel and air are assumed to be premixed homogeneously throughout the whole cylinder; thermodynamic characteristics such as pressure and temperature are uniformly distributed in the cylinder, and they can be calculated using the simplified dynamics shown in equations (11) and (12) below.

$$
T\left(\theta_{i}\right)=T\left(\theta_{i-1}\right) \cdot\left(\frac{V\left(\theta_{i-1}\right)}{V\left(\theta_{i}\right)}\right)^{(\kappa-1)}+\eta_{H C C I} \cdot \frac{M_{f} \cdot h_{L H V} \cdot\left[x\left(\theta_{i}\right)-x\left(\theta_{i-1}\right)\right]}{M_{I V C} \cdot C_{v}}
$$

and

$$
P\left(\theta_{i}\right)=P\left(\theta_{i-1}\right) \cdot \frac{V\left(\theta_{i-1}\right)}{V\left(\theta_{i}\right)} \cdot \frac{T\left(\theta_{i}\right)}{T\left(\theta_{i-1}\right)}
$$

where $\eta_{H C C I}$ is a scaling factor due to incomplete combustion; $\kappa$ is the average heat capacity ratio of the cylinder charge.

There are two terms in the right hand of equation (11). The first term represents an isentropic compressing or expanding process. While the second term calculates the temperature rise due to the heat released during the combustion. Therefore, the complicated thermodynamic process of the combustion is simplified into an isentropic volume change process without heat exchange in one crank degree period with heat absorption from combusted fuel without volume change in an infinitely small time period.

\section{Models for Gas Exchange Phases}

The gas exchange process (from EVO to IVC in Figure 2) was modeled by combined correlation and physical modeling approach. It's simple but with fairly high fidelity.

\section{A. Gas Exhaust Process Mode}

Assume that the gas exit from cylinder to the exhaust manifold during the EVO-EVC phase for the negative valve overlap (NVO) or during the EVO-IVO for the positive valve overlap (PVO). In this phase, the in-cylinder gas mixture is assumed to isentropically expand in the cylinder, exhaust runner and manifold. The in-cylinder pressure drops quickly but not instantaneously down to the level of the exhaust manifold pressure. It normally takes a few crank degrees for the in-cylinder pressure to approach the exhaust manifold pressure. It is difficult to model this dynamics using simple dynamic equations for real-time simulations. For simplicity, a first order transfer function is used to approximate this dynamic process as:

$$
P(z)=\frac{1-\tau_{E V O}}{1-\tau_{E V O} \cdot z^{-1}} \cdot P_{E M}(z)
$$

where $z$ is the unit delay operator; $\tau_{E V O}$ is the transition time constant for exhaust valve opening; and $P_{E M}$ is the exhaust manifold absolute pressure.

As an isentropic process, the in-cylinder gas temperature can be calculated as a function of the pressure drop. That is:

$$
T\left(\theta_{i}\right)=T\left(\theta_{i-1}\right) \cdot\left[\frac{P\left(\theta_{i}\right)}{P\left(\theta_{i-1}\right)}\right]^{\frac{\kappa-1}{\kappa}}
$$

\section{B. Recompression Model for Negative Valve Overlap}

Negative valve overlap (NVO) is often used to regulate the HCCI combustions. There are two main advantages. One is to decompose the pilot fuel injected in this phase (EVC-IVO in Figure 2) into short carbon chain molecules [15]; and the other is to adjust the residue gas temperature. As a result the in-cylinder gas temperature at IVC can be optimized for desired SOC of the HCCI combustion. The first effect can hardly be modeled using governing equations. In [7] and [15], the Arrhenius integration is correlated to the experimental data. This paper ignores this effect until experimental data is available. For the second one, it can be approximated to an isentropic volume change process of ideal gas in a closed system. Temperature and pressure are calculated by

$$
T\left(\theta_{i}\right)=T\left(\theta_{i-1}\right) \cdot\left(\frac{V\left(\theta_{i-1}\right)}{V\left(\theta_{i}\right)}\right)^{(\kappa-1)}
$$

and

$$
P\left(\theta_{i}\right)=P\left(\theta_{i-1}\right) \cdot\left(\frac{V\left(\theta_{i-1}\right)}{V\left(\theta_{i}\right)}\right)^{\kappa}
$$

Another important parameter calculated in this phase is the residue gas mass. It is calculated based upon ideal gas law, and updated once per engine cycle at EVC for NVO.

$$
M_{r}=M\left(\theta_{E V C}\right)=\frac{P\left(\theta_{E V C}\right) \cdot V\left(\theta_{E V C}\right)}{R \cdot T\left(\theta_{E V C}\right)} \cdot \eta_{r}
$$

where $\eta_{r}$ is the discharge coefficient.

\section{Gas Exchange for Positive Valve Overlap}

The gas exchange behavior during the phase from IVO to EVC for the PVO is also very complicated. Since both intake and exhaust valves are partially opened in this phase, residue gas and fresh charge can flow in many ways depending on the 
pressure ratio across each valve. For simplification, assume gas exchange across exhaust valve is terminated in this phase; part of residue gas can flow to intake port, but finally it flows back into cylinder during the rest of the intake process. Based upon this assumption, the mass of residue gas for PVO can be calculated by equation (18) at IVO instead of EVC for NVO, and gas exchange of this phase is actually a part of the air intake process.

$$
M_{r}=M\left(\theta_{I V O}\right)=\frac{P\left(\theta_{I V O}\right) \cdot V\left(\theta_{I V O}\right)}{R \cdot T\left(\theta_{I V O}\right)} \cdot \eta_{r}
$$

This modeling approach may result in slight error for $M_{r}$. Fortunately, the PVO only occurs in case of SI combustion process, in which the influence of residue gas to the whole engine performance is much less than that during the HCCI combustion process which usually involves the NVO strategy. On the other hand, the multiplier $\eta_{r}$ can also be calibrated to match the test data or the high resolution simulation data. For this paper the GT-Power simulation data is used to calibrate the entire combustion model.

\section{Air-intake Process Model}

The air-intake process from IVO to IVC is also a process of in-cylinder gases mixing. During this phase the fresh charged air, injected fuel vapor, and residue gas are assumed to be mixed homogeneously, which is an assumed condition for combustion. At the same time, the in-cylinder pressure drops to the pressure level of intake manifold [16]. A first order transfer function is also used in this model.

$$
P(z)=\frac{1-\tau_{I V O}}{1-\tau_{I V O} \cdot z^{-1}} \cdot P_{I M}(z)
$$

and

$$
T\left(\theta_{i}\right)=\frac{M_{f} \cdot T_{f}+M_{a} \cdot T_{I M}+M_{r} \cdot T_{r}}{M_{f}+M_{a}+M_{r}}
$$

where $\tau_{E V O}$ is the transition time constant for intake valve opening; $P_{E M}$ is the intake manifold absolute pressure; $M_{a}$ is the fresh charge mass (note that it could be the mixture of fresh air and external EGR gas); $T_{r}$ is the temperature of residue gas. Assuming that the residue gas expanses isentropically throughout the cylinder and intake port it can be calculated using equation (14).

At the end of air-intake process, which is at IVC, the total in-cylinder gas mass $M_{t}$ needs to be calculated. That is,

$$
M_{t}=M\left(\theta_{I V C}\right)=\frac{P\left(\theta_{I V C}\right) \cdot V\left(\theta_{I V C}\right)}{R \cdot T\left(\theta_{I V C}\right)} \cdot \eta_{t}
$$

\section{Model Calibration And VALidation}

Equations (1) to (21) were programmed into an $S$-function in Simulink using $\mathrm{C}$ language. The developed Simulink model was calibrated by the simulation data of the GT-Power combustion model. The setup of the GT-Power model is the same in the Simulink model. For both models the combustion related engine parameters are the same and listed in Table 1.

Calibrations were generated by sweeping the engine speed and load within the engine operating range. For each test condition, GT-Power simulation results were used as the baseline. Firstly, $\eta_{r}$ and $\eta_{t}$ were calibrated to make the residue gas and total charge quantities match the GT-Power simulation results; secondly, coefficients such as $m$ and $k$ were calibrated to make MFB profile match; at last, $\eta_{S I}$ or $\eta_{H C C I}$ were adjusted to make IMEP match.

\begin{tabular}{l|l}
\multicolumn{2}{c}{ Table 1: Combustion related engine specification } \\
\hline \hline \multicolumn{1}{c|}{ Parameter } & \multicolumn{1}{c}{ Model value } \\
\hline bore/stroke/con-rod length & $86 \mathrm{~mm} / 86 \mathrm{~mm} / 143.6 \mathrm{~mm}$ \\
\hline compression ratio & $9.8: 1$ \\
\hline intake valve/exhaust valve diameters & $31.4 \mathrm{~mm} / 22.5 \mathrm{~mm}$ \\
\hline Intake / exhaust valve lifts of high stage & $8.8 \mathrm{~mm} / 8.75 \mathrm{~mm}$ \\
\hline Intake / exhaust valve lifts of low stage & $4.8 \mathrm{~mm} / 4.75 \mathrm{~mm}$
\end{tabular}

The one-zone combustion model for SI and HCCI combustion has been validated during previous work in [1] and [11]. In this paper, the two-zone SI combustion model is mainly investigated. Key combustion related parameters obtained from both GT-Power and the two-zone models were plotted for comparison and shown in Figure 5 to Figure 7.

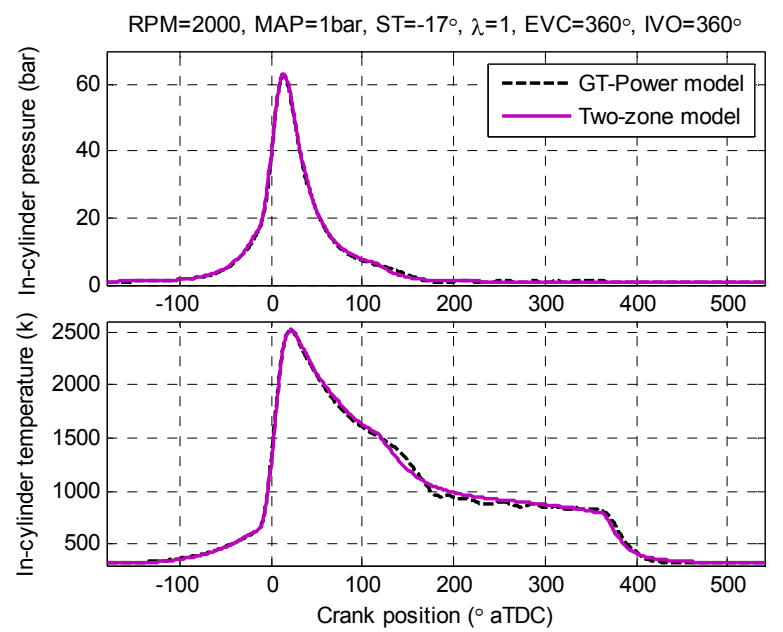

Figure 5: In-cylinder pressure and temperature traces of SI combustion

Good correlations have been demonstrated in Figure 5 for the in-cylinder pressure and temperature. Note that the temperature in Figure 5 is the average temperature of both burned and unburned zone temperatures. In Figure 6 and Figure 7, the burned and unburned zone temperatures are plotted separately for both GT-Power and two-zone models and the correlations are also fairly good.

In Figure 7 , after spark ignition $\left(-17^{\circ}\right.$ aTDC in this case) the unburned zone temperatures of both GT-Power model and the two-zone model are higher than the in-cylinder gas temperature without combustion, which is calculated by setting $T_{I V C}$ to be same as that in the simulation with combustion and injecting no fuel. This indicates that the burned zone is always pushing the unburned zone during the SI combustion phase and applying work to it, resulting fast increment of the unburned zone temperature. Based on equation (10), the temperature increment advances SOHCCI and triggers HCCI combustion in it. This is the main objective of the SI-HCCI hybrid combustion mode. 


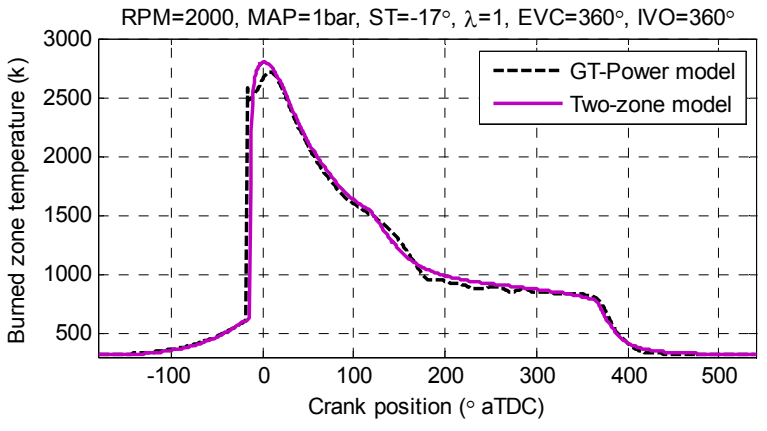

Figure 6: Burned zone temperature traces

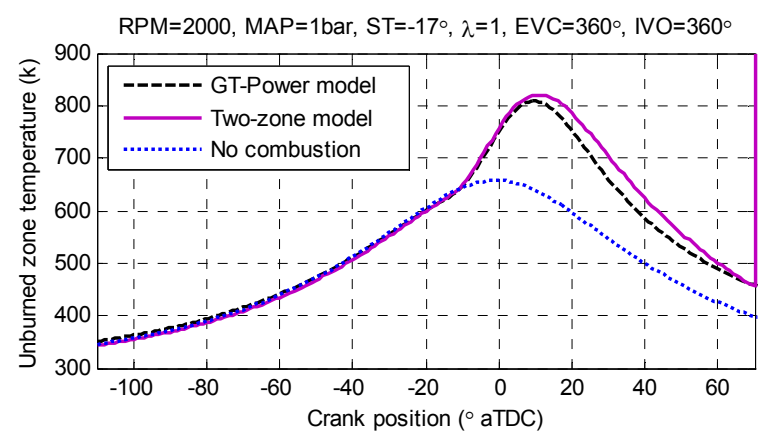

Figure 7: Unburned zone temperature trace

The comparison data shown in Figure 5 to Figure 7 are for one engine operating condition. For the entire engine operating range, several key combustion parameters were selected for comparison in Figure 8. Two load conditions are investigated at MAP $=0.4$ bar for low load condition and MAP $=1$ bar for full load condition. For each condition, engine speed sweeps from $1000 \mathrm{rpm}$ to $5000 \mathrm{rpm}$ with 1000 rpm increment interval. Fairly good correlations are shown in Figure 8, which indicates that the two-zone model is accurate for modeling SI combustion process.
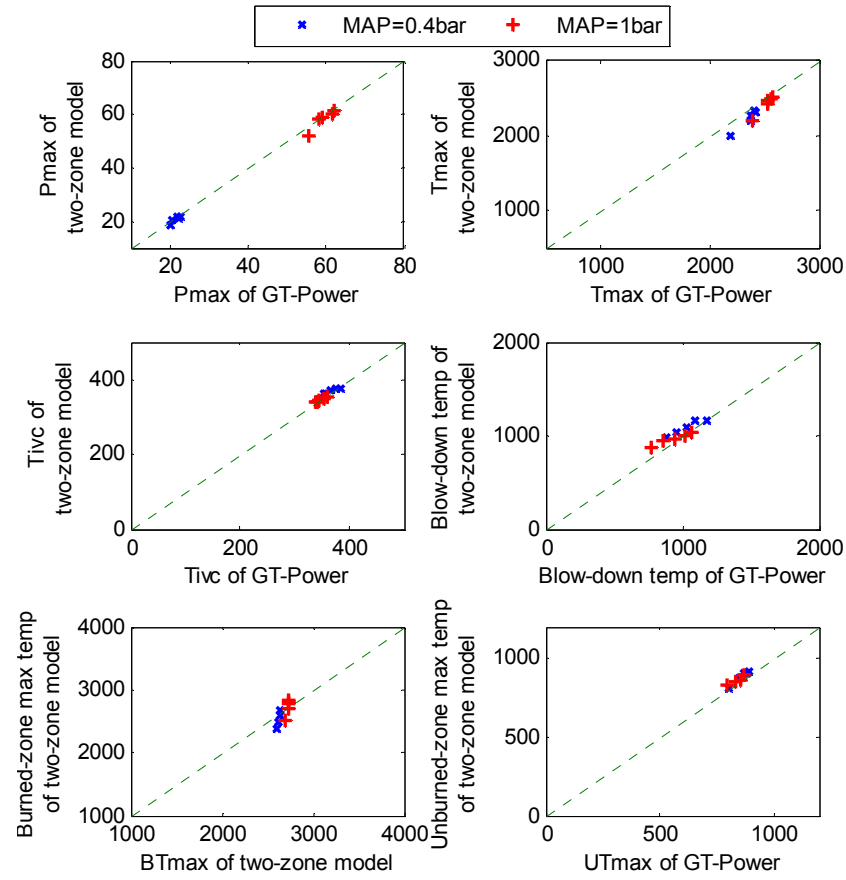

Figure 8: Correlation plots of GT-Power and the two-zone SI model

\section{CONCLUSIONS}

This paper presents a control oriented combustion model for a HCCI (homogeneously charge compression ignition) capable SI (spark ignited) engine. The SI combustion was modeled using the two-zone method and HCCI combustion is under one-zone assumption. The developed control oriented model can be used to simulate not only the SI and HCCI combustions but also the spark assisted HCCI combustion or the hybrid combustion. For SI and HCCI combustion modes, the model has also been calibrated and validated using the corresponding GT-Power simulation results.

\section{REFERENCES}

[1] X. Yang, G. Zhu, and Z. Sun, "A Control Oriented SI and HCCI Hybrid Combustion Model for Internal Combustion Engines", Proceedings of 2010 ASME Dynamic Systems and Control Conference, Cambridge, MA, 2010.

[2] F. Zhao, T. Asmus, D. Assanis, J. E. Dec, J. A. Eng, and P. M. Najt, Homogeneous Charge Compression Ignition (HCCI) Engines Key Research and Development Issues, 2003, Warrendale, Pennsylvania: Society of Automotive Engineers.

[3] H. Wu and M. Craft, "Experimental Investigation of a Control Method for SI-HCCI-SI Transition in a Multi-Cylinder Gasoline Engine," SAE International 2010-01-1245, 2010.

[4] J. Etheridge, S. Mosbach, and M. Kraft, "A Fast Detailed-Chemistry Modeling Approach for Simulating the SI-HCCI Transition," SAE International 2010-01-1241, 2010.

[5] Y. Zhang, H. Xie, N. Zhou, T. Chen, and H. Zhao, "Study of SI-HCCI-SI Transition on a Port Fuel Injection Engine Equipped with 4VVAS," SAE International 2007-01-0199, 2007.

[6] A. Cairns and H. Blaxhill, "The Effects of Two-Stage Cam Profile Switching and External EGR on SI-CAI Combustion Transitions," SAE International, 2007-01-0187, 2007.

[7] S. M. Aceves, D. L. Flowers, R. W. Dibble, and A. Babajimopoulos, Overview of Modeling Techniques and their Application to HCCI/CAI Engines, in HCCI and CAI Engines for the Automotive Industry, $\mathrm{H}$. Zhao, Editor. 2007, Woodhead Publishing: Cambridge.

[8] D. J. Rausen, et al, "A mean value model for control of homogeneous charge compression ignition (HCCI) engines," ASME Journal of Dynamics, Measurement, and Control, Vol. 127, Sep, 2005.

[9] M. Canova and S. M. Mohler, "Mean Value Modeling and Analysis of HCCI Diesel Engines With External Mixture Formation," ASME Journal of Dynamics, Measurement, and Control, Vol. 131, Jan, 2009.

[10] J. B. Heywood, Internal Combustion Engine Fundamentals, McGraw-Hill, Inc., 1988.

[11] X. Yang and G. Zhu, "A Mixed Mean-Value and Crank-based Model of a Dual-Stage Turbocharged SI Engine for Hardware-In-the-Loop Simulation", Proceedings of 2010 American Control Conference, Baltimore, MD, 2010.

[12] L. Guzzella and C. H. Onder, Introduction to Modeling and Control of Internal Combustion Engine Systems, Springer, Inc., 2004.

[13] C. J. Chiang and A. G. Stefanopoulou, "Stability Analysis in Homogeneous Charge Compression Ignition (HCCI) Engines With High Dilution," IEEE Transactions on Control System Technology, Vol. 15, No. 2, March 2007.

[14] G. M. Shaver, M. J. Roelle and J. C. Gerdes, "Modeling cycle-to-cycle dynamics and mode transition in HCCI engines with variable valve actuation," Control Engineering Practice, Vol. 14. 2006, pp. 213-222.

[15] N. Ravi, H. H. Liao, and A. F. Jungkunz, "Modeling and control of exhaust recompression HCCI using split injection," Proceedings of 2010 American Control Conference, Baltimore, MD, 2010.

[16] M. McCuen, Z. Sun, and G. Zhu, "Control-oriented mixing model for homogeneous charge compression ignition engine," Proceedings of 2010 American Control Conference, Baltimore, MD, June 2010.

[17] Y. A. Cengel and M. A. Boles, Thermodynamics: An Engineering Approach, 5th edition, McGraw-Hill, 2006. 
9.11 Published JC Paper \#11

Modeling and Control of an Electric Variable Valve Timing System for SI and HCCI Combustion Mode Transition 


\title{
Modeling and Control of an Electric Variable Valve Timing System for SI and HCCI Combustion Mode Transition
}

\author{
Zhen Ren and Guoming G. Zhu
}

\begin{abstract}
This paper models an electric variable valve timing (VVT) system and develops the corresponding controller for the electric VVT system. The studied electric VVT uses a planetary gear system for engine cam timing control; and a cyclic torque disturbance is applied to the cam shaft. The main motivation of utilizing the electric VVT system is for the mode transition control between the spark ignited (SI) and homogeneous charge compression ignition (HCCI) combustions due to its fast response time. During the combustion mode transition between SI and HCCI operations, it is required for the engine cam timing to follow a desired trajectory to make the smooth combustion mode transition possible. This is mainly due to the fact that the engine valve timings effect the engine recompression operation that is directly associated with the start of HCCI combustion. A control oriented electric VVT model was developed and closed-loop control strategies were developed to maintain the cam phase at a desired level, as well as to follow a desired trajectory during the combustion mode transition. Simulation results are included.
\end{abstract}

Key words: Variable valve timing system; HCCI Combustion control, Powertrain control

\section{INTRODUCTION}

$\mathrm{C}$ ONTINUOUSLY variable valve timing (VVT) systems used in internal combustion engines were developed in nineties [1] and have since been widely used due to the growing fuel economy demands and emission regulations. VVT system improves fuel economy and reduces emissions at low engine speed, as well as improves engine power and torque at high engine speed. Conventional electronic-hydraulic VVT ([1] and [2]), also called hydraulic VVT, is the most widely used in the industry today. The hydraulic VVT systems require minor changes when applied to a previously non-VVT valve-train [1], which makes design and engineering relatively easy. However due to its mechanism, the hydraulic VVT system also has its limitations [3]. The response and performance of the hydraulic VVT system are significantly affected by the engine operating conditions such as engine oil temperature and pressure. For instance, at low engine temperature, the hydraulic VVT system cannot be activated and has to remain at its default position so that the cold start performance and emissions cannot be improved [3]. This leads to the study of other variable valve-train system, such as electromagnetic [4], hydraulic [5], electro-pneumatic [6], and electrical motor driven planetary gear systems ([7] and [8]). Electric motor

Both Ren and Zhu are with Mechanical Engineering of Michigan State University, 2555 Engineering Building, East Lansing, MI 48824, USA (e-mails: renzhen@msu.edu for Ren and zhug@msu.edu for Zhu). driven VVT operational performance is independent of engine oil temperature and pressure [3]. Comparing to hydraulic VVT system, electric motor driven VVT system is less limited to engine operating conditions and therefore gives better performance and better emission in a wider operational range. Especially, since the electrical VVT is independent of the engine oil pressure, the response time is greatly improved.

The major advantage of HCCI (homogeneous charge compression ignition) combustion is realized by eliminating the formation of flames. That results in much lower combustion temperature. As a consequence of the low temperature, the formation of NOx (nitrogen oxides) is greatly reduced. The lean burn nature of the HCCI engine also enables un-throttled operation to improve engine fuel economy. Unfortunately, HCCI combustion is feasible only over a limited engine operational range due to engine knock and misfire. To make a HCCI engine work in an automotive internal combustion engine, it has to be capable of operating at both SI combustion mode at high load and HCCI combustion mode at low and mediate load ([9] and [10]). This makes it necessary to have a smooth transition between SI and HCCI combustion modes.

Achieving the HCCI combustion and controlling the mode transition between SI and HCCI combustions in a practical engine require implementation of enabling devices and technologies. There are a number of options, and the necessary prerequisite for considering any of them is their ability to provide control of thermodynamic conditions in the combustion chamber at the end of compression. The range of devices under consideration includes variable valve actuation (cam-based or camless), variable compression ratio, dual fuel systems (port and direct fuel injection with multiple fuel injections), supercharger and/or turbocharger, exhaust energy recuperation and fast thermal conditioning of the intake charge mixture, spark-assist, etc. Variable Valve Actuation can be used for control of the effective compression ratio (via the intake valve closing time), the internal (hot) residual fraction via the negative valve overlap (recompression) ([11] and [12]), or secondary opening of the exhaust valve (residual re-induction) ([11] and [12]). In addition to providing the basic control of the HCCI combustion, i.e., ignition timing and burn rate or duration, the VVT systems plays a critical role in accomplishing smooth mode transitions from SI to HCCI and vice versa ([13], [14] and [15]). In this paper, the electrical VVT system is selected to control the engine valve timings when it is operated at SI and HCCI combustion modes, and during the combustion mode transition the electrical VVT is controlled to track a desired trajectory. 
In order to control the electric planetary VVT system, a feedback controller was introduced in [8]. Due to the steady state and transient control accuracy requirements of the HCCI combustion, the closed-loop electric VVT system needs not only to meet steady-state performance requirement but also to track a desired trajectory during the combustion mode transition. Therefore, a feedback controller with feedforward control is developed in this paper. In the studied VVT system, the cam phase is the integration of speed difference between the electric VVT motor and crankshaft. This leads to using the rate of the reference cam phase as feedforward command. Output covariance control (OCC) ([16][17], and [18]), an $H_{2}$ controller, is used in feedback to reduce the tracking error. Performance of the OCC controller is compared with well-tuned proportional-derivative (PD) controllers, and the OCC with feedforward provides better cam phase tracking performance than PD controllers. Different cam phase sample rates are also studied and results show that 4 samples per engine cycle are sufficient for OCC feedback.

The paper is organized as following. Section II describes the electric VVT model and system architecture. Section III introduces OCC controller framework. Section IV presents the feedforward control strategy and the closed-loop controller design. Section $\mathrm{V}$ provides the simulation results. Conclusion is drawn in Section VI.

\section{MODELING}

\section{A. Planetary VVT components}

The planetary gear VVT system studied in this paper consists of four major components (see Fig. 1). Ring gear, serves as VVT pulley, is driven directly by crankshaft through a timing belt at half crankshaft speed. Planet gear carrier is driven by an electric VVT motor. Planet gears engage both ring and sun gears. Sun gear is connected to the camshaft. The sun and planet gears are passive components that obtain kinetic energy from carrier and ring gears. Comparing to other components, the inertia of engine fly wheel and crank shaft is very large. As a result, dynamics of the ring gear is ignored in this study. All other components have known mechanical properties and their dynamics are considered in the modeling.

\section{B. Planetary Gear System Kinematics}

In a planetary gear system [19] shown in Fig. 1, angular velocities of components are determined by

$\frac{\omega_{s}(t)-\omega_{c}(t)}{\omega_{r}(t)-\omega_{c}(t)}=-\frac{n_{r}}{n_{s}}$

where $\omega_{s}, \omega_{c}$ and $\omega_{r}$ are angular velocities of the sun, carrier, and ring gears, respectively. $n_{r}$ and $n_{s}$ are the teeth numbers of ring and sun gears. Laplace transformation of (1) can be expressed as

$\Omega_{s}(s)=-\frac{n_{r}}{n_{s}} \Omega_{r}(s)+\frac{n_{r}}{n_{s}} \Omega_{c}(s)+\Omega_{c}(s)$

The half of cam phase angle $\phi$ is the integration of the difference between camshaft and crankshaft speeds. That is,

$\phi=2 \int_{0}^{t}\left[\omega_{s}(\tau)-\omega_{r}(\tau)\right] d \tau$

and its Laplace transformation is

$\Phi(s)=\frac{2}{s}\left[\Omega_{s}(s)-\Omega_{r}(s)\right]$

Substituting (2) into (4), we have

$\Phi=\frac{2}{s}\left(\frac{n_{s}+n_{r}}{n_{s}}\right)\left(\Omega_{c}-\Omega_{r}\right)$

Equation (5) shows that the cam phase is an integral function of speed difference between carrier and ring gears. In other word, by controlling the VVT motor speed with respect to the engine speed, cam phase can be adjusted. When the carrier speed is equal to the ring speed, cam phase is held; when the carrier speed is greater than the ring speed, cam phase is advancing; and when the carrier speed is slower than the ring speed, cam phase is retarding. Notice that equation (5) contains an integrator, and target cam phase reference cannot be used as feedforward control directly.

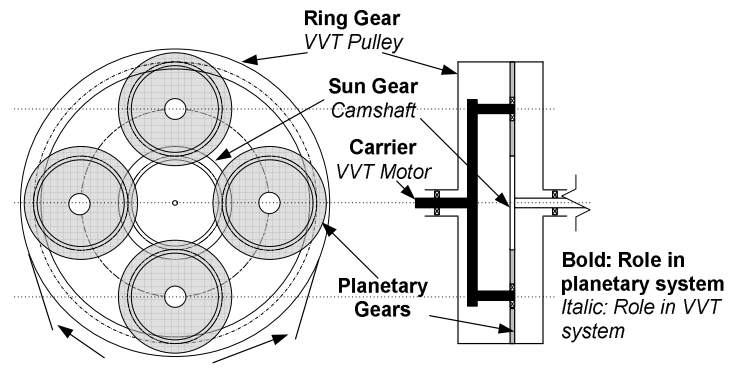

Fig. 1: Electric planetary gear VVT system

\section{Planetary Gear System Dynamics}

Planetary gear system dynamics with an electric motor are modeled in this section. In this study, the gear system friction is ignored. Fig. 2 shows free body diagrams of planetary gear components.

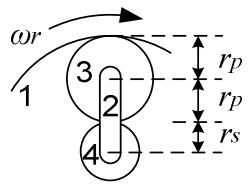

a) Planetary System

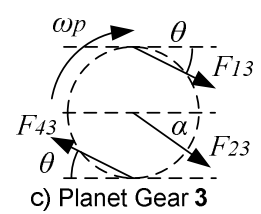

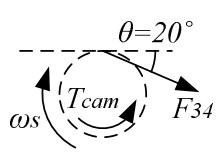

b) Sun Gear 4

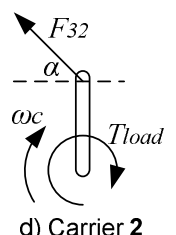

Fig. 2: Free body diagrams of planetary gear components

Without loss of generality comparing with the system in Fig. 2, the system is treated as having only one planet gear (Fig. 2a). Since all the gears are properly engaged, we have

$\frac{n_{s}}{r_{s}}=\frac{n_{p}}{r_{p}}=\frac{n_{r}}{r_{r}}, \quad r_{r}=2 r_{p}+r_{s}$

where $n_{p}$ is planet gear number of teeth. $r_{s}, r_{p}$, and $r_{r}$ are pitch circle radius of sun, planet, and ring gears. In this study, the gears use a standard pressure angle $\theta$ of 20 degrees. Since the ring has a very large inertia comparing to other 
components, angular velocity of the ring $\omega_{r}$ is assumed to be constant during the phase shift. From (2):

$\dot{\omega}_{s}=\frac{n_{r}+n_{s}}{n_{s}} \dot{\omega}_{c}$

There are two torques applied to the sun gear (Fig. 2b). They are camshaft load $T_{\text {cam }}$ and torque from tooth force $F_{34}$.

$F_{34} \cdot r_{s} \cdot \cos \theta-T_{\text {cam }}=J_{s} \dot{\omega}_{S}$

where $J_{s}$ is sun gear's moment of inertia with respect to its center of gravity.

Two tooth forces $\left(F_{43}\right.$ and $F_{13}$ ) and one bearing force from carrier $F_{23}$ are applied to planet gear (Fig. 2c) that rotates around the bearing on the carrier at $\omega_{p}$ :

$\frac{\omega_{p}(t)-\omega_{c}(t)}{\omega_{s}(t)-\omega_{c}(t)}=-\frac{n_{s}}{n_{p}}$

and from torque balance with respect to bearing point:

$\left(F_{43}+F_{13}\right) \cdot r_{p} \cdot \cos \theta=J_{p} \dot{\omega}_{p}$

where $J_{p}$ is planet gear's moment of inertia with respect to its center of gravity.

The planet gear also rotates about the center of sun gear. Therefore

$\left[F_{13}\left(2 r_{r}\right)-F_{43} r_{s}\right] \cos \theta+F_{23}\left(r_{p}+r_{s}\right) \cos \alpha=J_{p s} \dot{\omega}_{c}$

where the direction and magnitude of bearing force $F_{23}$ are unknown. The planet gear's moment of inertia with respect to the center of sun gear $J_{p s}$ can be calculated by

$J_{p s}=J_{p}\left[1+m_{p}\left(r_{s}+r_{p}\right)^{2}\right]$

Since the carrier is driven directly by the motor shaft, its inertia is also considered as part of motor shaft inertia, and modeled in the next sub-section. Torque balance of carrier is $F_{32} \cos \alpha\left(r_{p}+r_{s}\right)=T_{\text {load }}$

where $T_{\text {load }}$ is the mechanical load to the motor shaft and $F_{32}$ is the bearing force from planet gear.

Equations (6-13) can be simplified as follows.

$T_{\text {load }}=J_{\text {gears }} \dot{\omega}_{c}+k T_{\text {cam }}$

where constant $J_{\text {gears }}$, equivalent inertia of the planetary gear system, and $k$, factor of gear ratio, can be calculated:

$$
\begin{aligned}
J_{\text {gears }} & =J_{p}\left[1+m_{p}\left(r_{s}+r_{p}\right)^{2}-\frac{2 n_{r}}{n_{p}} \frac{-n_{r}+n_{p}}{n_{s}}\right] \\
& +J_{s}\left(\frac{n_{r}+n_{s}}{n_{s}} \frac{2 n_{r}}{n_{s}}+1\right)
\end{aligned}
$$

and

$$
k=\left(1+\frac{2 n_{r}}{n_{s}}\right)
$$

\section{Electric VVT Motor Dynamics}

An electric motor is used to drive carrier in the planetary system. A local closed-loop speed governor is used to control both the motor speed and direction. The input to the local motor controller is the reference speed and direction. In this study, the motor and its controller are treated as an actuator (Fig. 3). It is modeled with two inputs of motor velocity command and cam load, and one output of motor shaft speed.

The mechanical load of the motor can be modeled [20] as

$J_{c} \dot{\omega}_{c}=\tau-B \omega_{c}-T_{\text {load }}$

where $J_{c}$ is the moment of inertia of motor shaft and carrier,

$B$ is the friction coefficient, and $\tau$ is the motor torque. Substituting (14) into (15) leads to

$\left(J_{c}+J_{\text {gears }}\right) \dot{\omega}_{c}+B \omega_{c}=\tau-k T_{\text {cam }}$

and the associated transfer function can be written as

$\Omega_{c}(s)=\frac{1}{\left(J_{c}+J_{\text {gears }}\right) s+B}\left[T(s)-k T_{\text {cam }}(s)\right]$

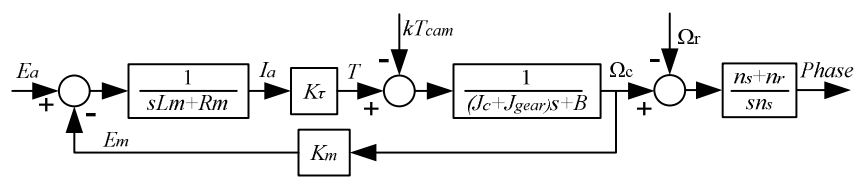

Fig. 3: Block diagram of electric motor with planetary gear system Modeling procedure of the electrical portion can be found in [20]. Let $J=J_{c}+J_{\text {gears }}$, the electric motor with planetary gear load (Fig. 3) can then be represented by

$\Omega_{c}(s)=G_{e}(s) E_{a}(s)+G_{m}(s) T_{c a m}(s)$

where the voltage input transfer function is

$G_{e}=\frac{K_{\tau}}{\left(L_{m} s+R_{m}\right)(J s+B)+K_{\tau} K_{m}} \cong \frac{K_{\tau}}{R_{m}(J s+B)+K_{\tau} K_{m}}$

and the mechanical input transfer function is

$G_{m}=\frac{-\left(L_{m} s+R_{m}\right)}{\left(L_{m} s+R_{m}\right)(J s+B)+K_{\tau} K_{m}} \cong \frac{-R_{m} k}{R_{m}(J s+B)+K_{\tau} K_{m}}$

and $K_{\tau}, K_{m}, L_{m}, R_{m}$ are the motor parameters representing motor torque constant, back EMF (electric magnetic field) constant, armature inertia and resistance, respectively [20].

\section{OUTPUT COVARIANCE CONTROL (OCC)}

Consider the following linear time-invariant system

$$
\begin{array}{ll}
\mathbf{x}_{\mathbf{p}}(k+1) & =\mathbf{A}_{\mathbf{p}} \mathbf{x}_{\mathbf{p}}(k)+\mathbf{B}_{\mathbf{p}} \mathbf{u}(k)+\mathbf{D}_{\mathbf{p}} \mathbf{w}_{\mathbf{p}}(k) \\
\mathbf{y}_{\mathbf{p}}(k) & =\mathbf{C}_{\mathbf{p}} \mathbf{x}_{\mathbf{p}}(k) \\
\mathbf{z}(k) & =\mathbf{M}_{\mathbf{p}} \mathbf{x}_{\mathbf{p}}(k)+\mathbf{v}(k)
\end{array}
$$

where $\mathbf{x}_{p}, \mathbf{u}, \mathbf{w}_{\mathbf{p}}, \mathbf{v}$ represent state, control, process noise, and measurement noise, respectively. Vector $\mathbf{y}_{\mathbf{p}}$ contains all variables whose dynamic responses are of interest. The vector $\mathbf{z}$ is a vector of noisy measurements. Suppose that a strictly proper output feedback stabilizing control law below is employed for plant (23).

$$
\begin{array}{ll}
\mathbf{x}_{\mathbf{c}}(k+1) & =\mathbf{A}_{\mathbf{c}} \mathbf{x}_{\mathbf{c}}(k)+\mathbf{F z}(k) \\
\mathbf{u}(k) & =\mathbf{G} \mathbf{x}_{\mathbf{c}}(k)
\end{array}
$$


Then the resulting closed-loop system is

$\mathbf{x}(k+1)=\mathbf{A x}(k)+\mathbf{D} \mathbf{w}(k)$

$\mathbf{y}(k)=\left[\begin{array}{c}\mathbf{y}_{\mathbf{p}}(k) \\ \mathbf{u}(k)\end{array}\right]=\left[\begin{array}{c}\mathbf{C}_{\mathbf{y}} \\ \mathbf{C}_{\mathbf{u}}\end{array}\right] \mathbf{x}(k)=\mathbf{C x}(k)$

where $\mathbf{x}=\left[\begin{array}{ll}\mathbf{x}_{\mathbf{p}}^{\mathbf{T}} & \mathbf{x}_{\mathbf{c}}^{\mathbf{T}}\end{array}\right]^{\mathbf{T}}$ and $\mathbf{w}=\left[\begin{array}{ll}\mathbf{w}_{\mathbf{p}}^{\mathbf{T}} & \mathbf{v}^{\mathbf{T}}\end{array}\right]^{\mathbf{T}}$. Formulas for

A , C , and D can be obtained based upon (23) and (24).

Consider the closed-loop system (25). Let $\mathbf{W}_{\mathbf{p}}$ and $\mathbf{V}$ denote positive definite symmetric matrices with dimensions equal to these of the process noise $\mathbf{w}_{\mathbf{p}}$ and measurement vector $\mathbf{z}$, respectively. Define $\mathbf{W}=$ block diag $\left[\begin{array}{ll}\mathbf{W}_{\mathbf{p}} & \mathbf{V}\end{array}\right]$ and let $\mathbf{X}$ denote the closed-loop controllability Gramian from the input $\mathbf{W}^{-\frac{1}{2}} \mathbf{w}$. Since $\mathbf{A}$ is stable, $\mathbf{X}$ satisfies $\mathbf{X}=\mathbf{A X} \mathbf{A}^{\mathbf{T}}+\mathbf{D W D} \mathbf{D}^{\mathbf{T}}$

In this paper we are interested in finding controllers of form (12) that minimize the (weighted) control energy trace $\left(\mathbf{R C}_{\mathbf{u}} \mathbf{X} \mathbf{C}_{\mathbf{u}}^{\mathbf{T}}\right)$ with $\mathbf{R}>0$ subject to the following constraint

$\mathbf{Y}=\mathbf{C X C} \mathbf{C}^{\mathbf{T}} \leq \overline{\mathbf{Y}}$

where $\overline{\mathbf{Y}} \geq 0$ are given and $\mathbf{X}$ solves (14). This problem, called the output covariance constraint (OCC) problem, is defined as finding a full-order dynamic output feedback controller (24) for system (11) that minimizes the OCC cost

$J_{\text {occ }}=\operatorname{trace}\left(\mathbf{R C}_{\mathbf{u}} \mathbf{X C}_{\mathbf{u}}^{\mathbf{T}}\right), \quad \mathbf{R}>0$

subject to (24) and (25).

The OCC problem has several interesting interpretations. For instance, assume first that $\mathbf{w}_{\mathbf{p}}$ and $\mathbf{v}$ are uncorrelated zero-mean white noises with intensity matrices $\mathbf{W}_{\mathbf{p}}>0$ and $\mathbf{V}>0$. Let $E$ be an expectation operator, and:

$$
\begin{aligned}
E\left[\mathbf{w}_{\mathbf{p}}(k)\right] & =0 ; E\left[\mathbf{w}_{\mathbf{p}}(k) \mathbf{w}_{\mathbf{p}}^{\mathbf{T}}(k-n)\right]=\mathbf{W}_{\mathbf{p}} \delta(n) \\
E[\mathbf{v}(k)] & =0 ; E\left[\mathbf{v}(k) \mathbf{v}^{\mathbf{T}}(k-n)\right]=\mathbf{V} \delta(n)
\end{aligned}
$$

Define $E_{\infty}[\cdot]:=\lim _{k \rightarrow \infty} E[\cdot]$ and $\mathbf{W}=$ block diag $\left[\begin{array}{ll}\mathbf{W}_{\mathbf{p}} & \mathbf{V}\end{array}\right]$, it is easy to see that the OCC is the problem of minimizing $E_{\infty} \mathbf{u}^{\mathbf{T}} \mathbf{R u}$ subject to the OCC constraint $\mathbf{Y}:=E_{\infty} \mathbf{y}(k) \mathbf{y}^{\mathbf{T}}(k) \leq \overline{\mathbf{Y}}$. As is well known, the constraint may be interpreted as constraint on the variance of the performance variables or lower bounds on the residence time (in a given ball around the origin of the output space) of the performance variables [21].

The OCC problem can also be interpreted from a deterministic point of view: define the $\ell_{\infty}$ and $\ell_{2}$ norms:

$\|\mathbf{y}\|_{\infty}^{2}:=\sup _{k \geq 0} \mathbf{y}^{\mathbf{T}}(k) \mathbf{y}(k)$

$\|\mathbf{w}\|_{2}^{2}:=\sum_{k=0}^{\infty} \mathbf{w}^{\mathbf{T}}(k) \mathbf{w}(k)$ and define the (weighted) $\ell_{2}$ disturbance set

$\mathscr{W}:=\left\{\mathbf{w}: \mathbf{R} \rightarrow \mathbf{R}^{n_{w}}\right.$ and $\left.\left\|\mathbf{W}^{-1 / 2} \mathbf{w}\right\|_{2}^{2} \leq 1\right\}$

where $\mathbf{W}>0$ is a real symmetric matrix. Then, for any $\mathbf{w} \in \mathscr{W}$, we have (see [22] and [23] for details)

$\|\mathbf{y}\|_{\infty}^{2} \leq \bar{\sigma}[\mathbf{Y}]$, and $\left\|\mathbf{u}_{i}\right\|_{\infty}^{2} \leq\left[\mathbf{C}_{\mathbf{u}} \mathbf{X C}_{\mathbf{u}}^{\mathbf{T}}\right]_{i i}, i=1,2, \ldots n_{u}$

where $n_{u}$ is the dimension of $\mathbf{u}$. (Here, $\bar{\sigma}[\cdot]$ denotes the maximum singular value and $[\cdot]_{i i}$ is the $i$-th diagonal entry.) Moreover, references [22] and [23] show that the bounds in (32) are the least upper bounds that hold for any signal $\mathbf{w} \in \mathscr{W}$.

Thus, if we define $\overline{\mathbf{Y}}:=I \epsilon^{2}$ in (27) and $\mathbf{R}=\operatorname{dial}\left[r_{1}, r_{2}, \ldots, r_{n_{u}}\right]$ in (28), the OCC is the problem of minimizing the (weighted) sum of worst-case peak values on the control signals given by

$J_{\text {occ }}=\sum_{i=1}^{n_{u}} r_{i}\left\{\sup _{\mathbf{w} \in \mathscr{W}}\left\|\mathbf{u}_{i}\right\|_{\infty}^{2}\right\}$

subject to constraints on the worst-case peak values of the performance variables of the form:

$\sup _{\mathbf{w} \in \mathscr{W}}\|\mathbf{y}\|_{\infty}^{2} \leq \epsilon^{2}$

This interpretation is important in applications where hard constraints on responses or actuator signals cannot be ignored such as space telescope pointing error and machine tool control problems. Detailed proof can be found in [18]. The controller system matrices $\mathbf{A}_{\mathbf{c}}, \mathbf{F}$, and $\mathbf{G}$ can be calculated using an iterative algorithm introduced in [16] and [18].

\section{CONTROLler DESIGN}

\section{A. Control Design Parameters}

The electric motor VVT system model includes the VVT controller, the local motor controller, motor/planetary dynamics, and planetary kinematics (see Fig. 4). The system parameters are listed as following and the controllers were designed based on these assumed parameters.

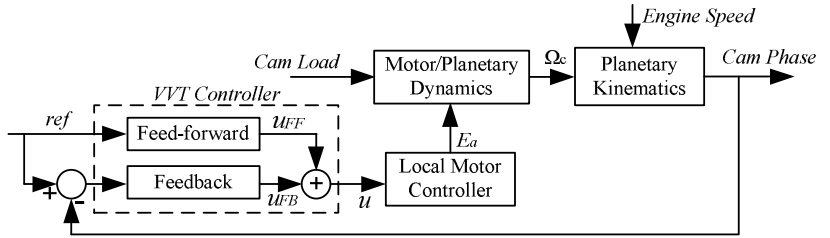

Fig. 4: Electrical motor VVT control framework

It is assumed that the voltage input transfer function is:

$G_{e}=\frac{45}{0.2 s+1}$

the mechanical input transfer function is:

$G_{m}=\frac{-5}{0.2 s+1}$

and the motor has a local PI controller defined by: 


$$
K_{\text {motor }}=\frac{s+0.1}{s}
$$

\begin{tabular}{|c|c|c|c|}
\hline Component & Sun & Ring & Planet \\
\hline Number of teeth & 30 & 60 & 15 \\
\hline
\end{tabular}

Teeth numbers of the gear train is listed in Tab. 1. Substituting these values into (5), planetary kinematics is

$\Phi=\frac{6}{s}\left(\Omega_{c}-\Omega_{r}\right)$

The cam torque load for each cylinder (Fig. 5) consists of three portions: constant friction load, sinusoidal load representing cam profile, and steps represent the valve spring pre-load. In the study, a 4 cylinder engine is simulated. The total load is a combination of 4 single cylinder loads with 180 degree phase shift for each cylinder.

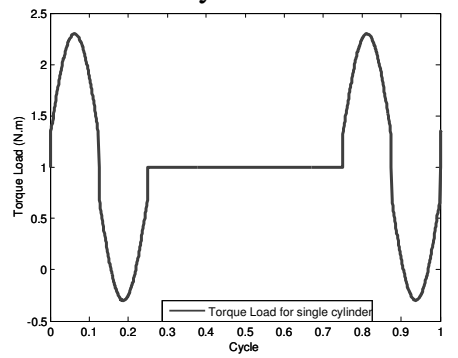

Fig. 5: Torque load for single cylinder

\section{B. Feedforward Controller}

In order to improve the system response, a feedforward controller was employed in the control design. Due to the physical characteristics of the electrical VVT system stated in the previous section, the reference signal was not used directly as feedforward; instead, the derivative of the cam phase reference signal was used as feedforward controller.

The feedforward gain was determined by the ratio between desired cam phase slope and the motor speed. Using inverse kinematics of (38), feedforward gain $K_{F F}$ can be determined as:

$u_{F F}=K_{F F} r \dot{e} f+\omega_{r}=\frac{1}{6} r \dot{f} f+\omega_{r}$

where $u_{F F}$ is the feedforward control effort. ref is the filtered derivative of the reference signal $r e f$

$$
r e f=\frac{s}{0.05 s+1} r e f
$$

\section{Baseline Controllers}

Since the electrical phase actuator plant contains an integrator, PD controllers were used as our baseline ones. Two baseline feedback controllers were tuned as performance comparison, where $K_{1}$ was tuned without feedforward and $K_{2}$ was tuned with feedforward, and they are:

$K_{1}=7+0.03 s$ and $K_{2}=1+0.005 s$

\section{OCC feedback Controller}

For OCC design, considering mechanical cam load as a disturbance, VVT controller output as a plant input, and the cam phase as an output, system matrices of the electric VVT system (Fig. 4) can then be written as

$$
\begin{aligned}
& \mathbf{A p}=\mathbf{A}=\left[\begin{array}{cccc}
0 & 225 & 0 & -25 \\
0 & -230 & 0.1 & 0 \\
0 & -225 & 0 & 0 \\
0 & 0 & 0 & -5
\end{array}\right], \quad \mathbf{B p}=\mathbf{B}=\left[\begin{array}{l}
0 \\
1 \\
1 \\
0
\end{array}\right] \\
& \mathbf{C p}=\mathbf{M p}=\mathbf{C}=\left[\begin{array}{llll}
6 & 0 & 0 & 0
\end{array}\right], \mathbf{D}_{\mathbf{p}}=\left[\begin{array}{llll}
0 & 0 & 0 & 1
\end{array}\right]^{\mathbf{T}}
\end{aligned}
$$

The control design parameters were chosen as

$$
\mathbf{W}_{\mathbf{p}}=2, \quad \mathbf{V}=0.01, \quad \mathbf{R}=[1]
$$

Using the control design algorithm introduced in [18], the resulting OCC controller is

$$
K=\frac{-164 s^{3}-3.9 \times 10^{4} s^{2}-2.9 \times 10^{5} s-2.8 \times 10^{4}}{s^{4}+298.8 s^{3}+1.8 \times 10^{4} s^{2}+3.27 \times 10^{5} s+3.25 \times 10^{4}}
$$

\section{SimUlation AND Results}

Simulations were conducted in Simulink. To simulate the engine valve operation under SI and HCCI transition, the reference signal was selected as a 40 crank degree phase retard that completes in 3 engine cycles. For simplicity, the transition reference signal is divided into three stages with a constant slope. For the first engine cycle the retard phase is $50 \%$ (20 degrees), the second cycle is $33.3 \%$ ( $40 / 3$ degrees), and the third $16.7 \%$ (20/3 degrees). The phase controller output signal was sampled every $5 \mathrm{~ms}$ and the feedback signal is updated 4 times per engine cycle. The closed-loop system performance at $1500 \mathrm{rpm}$ and $2000 \mathrm{rpm}$ were evaluated.

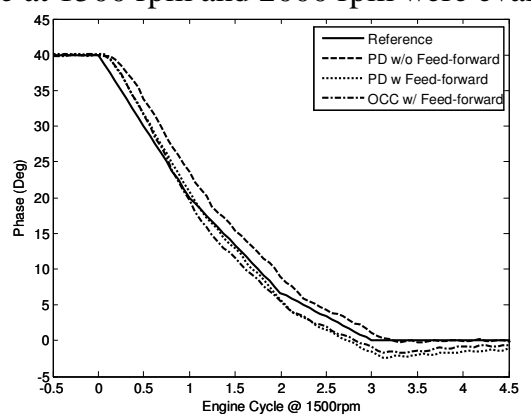

Fig. 6: Output comparison at 1500rpm

Fig. 6 compares the cam phase responses between three controllers: OCC, PD with and without feedforward, at 1500 $\mathrm{rpm}$. It shows that the initial response of the PD controller with feedforward is much faster than the PD controller without feedforward. However, due to the relatively low gain of the PD controller with feedforward, after the second cycle, it has a larger overshoot with longer settling time than the PD controller without feedforward. The OCC controller has the advantage of fast response with small overshoot. Table 2 shows output phase angles at the end of each engine cycle after the SI and HCCI transition starts. OCC controller with feedforward has the lowest overall tracking errors. It is noticed that performance is quite different at different engine speeds of $1500 \mathrm{rpm}$ and $2000 \mathrm{rpm}$ (Fig. 7 and Tab. 2) due to different feedback sampling rate at different engine speeds. 


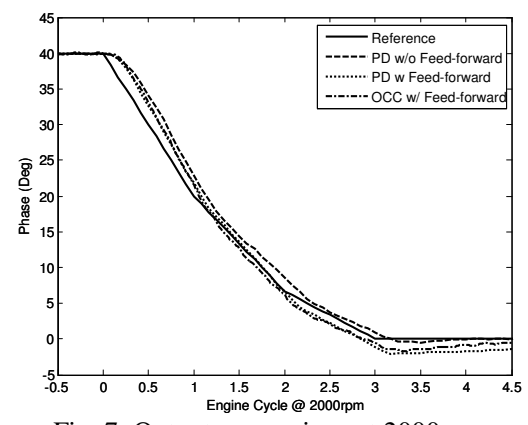

Fig. 7: Output comparison at 2000rpm

Tab. 2: Output comparison at end of each cycle

\begin{tabular}{|c|c|c|c|c|}
\hline \multirow{2}{*}{$\begin{array}{c}\text { Engine } \\
\text { speed }\end{array}$} & $\begin{array}{c}\text { Cycle } \\
\text { Number }\end{array}$ & \multicolumn{3}{|c|}{ Error (Deg) } \\
\cline { 2 - 5 } & 1 & +3.5 & +0.9 & -0.5 \\
\hline \multirow{4}{*}{$1500 \mathrm{rpm}$} & 2 & +2.3 & -0.8 & -1.0 \\
\cline { 2 - 5 } & 3 & +1.1 & -1.5 & -0.9 \\
\cline { 2 - 5 } & 4 & -0.1 & -1.5 & -0.8 \\
\cline { 2 - 5 } & 1 & +2.8 & +1.6 & +1.3 \\
\cline { 2 - 5 } & 2 & +1.8 & -0.2 & -0.5 \\
\cline { 2 - 5 } & 3 & +0.8 & -1.2 & -0.6 \\
\cline { 2 - 5 } & 4 & -0.1 & -1.5 & -0.8 \\
\hline
\end{tabular}

Tab. 3: Output comparison at 1500rpm with different sample rate

\begin{tabular}{|c|c|c|c|c|}
\hline \multirow{2}{*}{$\begin{array}{c}\text { Sample } \\
\text { Rate }\end{array}$} & \multirow{2}{*}{$\begin{array}{c}\text { Cycle } \\
\text { Number }\end{array}$} & \multicolumn{3}{|c|}{ Error (Deg) } \\
\cline { 2 - 5 } & PD & PD w/ ff & OCC w/ ff \\
\hline \multirow{4}{*}{8 / cycle } & 1 & +2.6 & +1.2 & +0.3 \\
\cline { 2 - 5 } & 2 & +1.7 & +0.0 & -0.3 \\
\cline { 2 - 5 } & 3 & +0.9 & -0.6 & -0.4 \\
\cline { 2 - 5 } & 4 & +0.1 & -0.9 & -0.4 \\
\cline { 2 - 5 } & 1 & +2.6 & +1.8 & +1.0 \\
\cline { 2 - 5 } 16/ cycle & 3 & +1.8 & +0.7 & +0.5 \\
\cline { 2 - 5 } & 4 & +0.9 & -0.1 & +0.2 \\
\cline { 2 - 5 } & & +0.1 & -0.5 & +0.0 \\
\hline
\end{tabular}

The tracking performances with higher feedback sampling rates are also studied (Tab. 3). The simulation data show that the tracking error reduces when the samples per engine cycle increased from 4 to 8 , but further increment of sample number does not reduce the tracking error significantly. Especially, with the OCC design, the tracking error is fairly small with 4 samples per cycle. Therefore, considering the limited tracking error reduction and increased computational requirement, 4 samples per cycle of the cam phase signal is proper for this application.

\section{CONCLUSION}

An electric VVT system with planetary gear train was modeled based upon individual component dynamics and kinematics. A closed-loop OCC (output covariance constraint) control with feedforward control is proposed to reduce the cam phase tracking error during SI (spark ignited) and HCCI (homogeneous charge compression ignition) combustion mode transition. Due to the physical characteristics of the electric VVT system, the filtered derivative of the cam phase reference is used as the feedforward control. Comparing with the well tuned PD controllers, simulation results show the OCC controller provides fast response with low overshot and low tracking error. With the OCC controller the cam phase signal sampled at 4 times per engine cycle is sufficient to meet the maximum tracking error requirement of less than 1.5 degree.

\section{ACKNOWLEDGMENT}

This paper is based upon the research supported by the Department of Energy under award number DE-EE0000211.

\section{REFERENCES}

[1] Y. Moriya, A.Watanabe, H. Uda, H. Kawamura, M. Yoshiuka, M. Adachi, "A Newly Developed Intelligent Variable Valve Timing System - Continuously Controlled Cam Phasing as Applied to New 3 Liter Inline 6 Engine”, SAE paper 960579, 1996.

[2] P. H. Dugdale, R. J. Rademacher, B. R. Price, J. W. Subhedar, R. L. Duguay, "Ecotec 2.4L VVT: A Variant of GM's Global 4-Cylinder Engine", SAE paper 2005-01-1941, 2005.

[3] M. Hattori, T. Inoue, Z. Mashiki, A. Takenaka, H. Urushihata, S. Morino, T. Inohara, "Devalopment of Variable Valve Timing System Controlled by Electric Motor", SAE paper 2008-01-1358, 2008.

[4] M. Theobald, B. Lequesns, and R. Henry, "Control of Engine Load via Electromagnetic Valve Actuators," SAE paper 940816, 1994.

[5] Z. Sun, and T. Kuo, "Transient Control of Electro-Hydraulic Fully Flexible Engine Valve Actuation System", IEEE Transactions on Control Systems Technology, Vol. 18, No. 3, May, 2010, pp 613-621.

[6] J. Ma, G. Zhu, and H. Schock, "A dynamic model of an electro-pneumatic valve actuator for internal combustion engines," ASME Journal of Dynamic Systems, Measurement and Control, Vol. 132, March, 2010.

[7] R. J. Pierik, J. O. Wilson, "Engine Timing Drive with Fixed and Variable Phasing", U.S. Patent 5,327,859, 1994.

[8] H. Urushihata, H. Iida, "Variable Valve Timing Control Device of Internal Combustion Engine", U.S. Patent 7,363,896 B2, 2008.

[9] Zhang, Y., H. Xie, N. Zhou, T. Chen, and H. Zhao, "Study of SI-HCCI-SI Transition on a Port Fuel Injection Engine Equipped with 4VVAS," SAE 2007-01-0199, 2007.

[10] A. Cairns and H. Blaxill, "The Effects of Two-Stage Cam Profile Switching and External EGR on SI-CAI Combustion Transitions," SAE Technical Paper, 2007-01-0187, 2007.

[11] G. M. Shaver, et al, "Dynamic modeling of residual-affected homogeneous charge compression ignition engines with Variable Valve Actuation," ASME Journal of Dynamics, Measurement, and Control, Vol. 127, September, 2005, pp. 374-381.

[12] G. M. Shaver, Physics based modeling and control of residual-affected HCCI engines using Variable Valve Actuation, $\mathrm{PhD}$ thesis, Stanford University, September, 2005.

[13] D. Law, D. Kemp, J. Allen, G. Kirkpatrick, T. Copland, "Controlled Combustion in an IC-Engine with a Fully Variable Valve Train”, SAE paper 2001-01-0251, 2001.

[14] N. Milovanovic, R. Chen, J. Turner, "Influence of the Variable Valve Timing Strategy on the Control of a Homogeneous Charge Compression (HCCI) Engine", SAE paper 2004-01-1899, 2004.

[15] F. Agrell, H. Angstrom, B. Eriksson, J. Wikander, J. Linderyd, "Integrated Simulation and Engine Test of Closed Loop HCCI Control by Aid of Variable Valve Timings", SAE paper 2003-01-0748, 2003.

[16] G. Zhu, R. E. Skelton, "Integrated Modeling and Control for the Large Spacecraft Laboratory Experiment Facility", Journal of Guidance, Control and Dynamics, Vol. 17, No. 3, pp. 442-450, 1994.

[17] G. Zhu, K. M. Grigoriadis, R. E. Skelton, "Covariance Control Design for Hubble Space Telescope", Journal of Guidance, Control and Dynamics, Vol. 18, No. 2, pp. 230-236, 1995.

[18] G. Zhu, M. A. Rotea, R. Skelton, "A Convergent Algorithm for the Output Covariance Constraint Control Problem”, SIAM J. Control Optim., Vol. 35, No.1, pp. 341-361, 1997.

[19] J. E. Shigley, and C. R. Mischke, Mechanical Engineering Design, $6^{\text {th }}$ Edition, McGraw-Hill, 2001.

[20] C. L. Phillips, and R. D. Harbor, Feedback Control System, $4^{\text {th }}$ Edition, Prentice Hall, 2000.

[21] S. Meerkov, T. Runolfsson, Output residence time control, IEEE Trans. Automat. Control, 34, pp. 1171-1176, 1989.

[22] D. A. Wilson, Convolution and Hankel operator norms for linear systems, IEEE Trans. Automat. Control, 34, pp. 94-97, 1989.

[23] G. Zhu, M. Corless, R. Skelton, Robustness properties of covariance controllers, in Proceedings of Allerton Conf., Monticello, IL, 1989. 
9.12 Published JC Paper \#12

A Control-Oriented SI and HCCI Hybrid Combustion Model for Internal Combustion Engines 


\section{A CONTROL ORIENTED SI AND HCCI HYBRID COMBUSTION MODEL FOR INTERNAL COMBUSTION ENGINES}

\author{
Xiaojian Yang \\ Mechanical Engineering \\ Michigan State University \\ East Lansing, MI 48824 \\ yangxia2@msu.edu
}

\author{
Guoming G. Zhu \\ Mechanical Engineering \\ Michigan State University \\ East Lansing, Ml 48824 \\ zhug@egr.msu.edu
}

\author{
Zongxuan Sun \\ Mechanical Engineering \\ University of Minnesota \\ Minneapolis, MN 55455 \\ zsun@umn.edu
}

\begin{abstract}
The combustion mode transition between SI (spark ignited) and HCCI (Homogeneously Charged Compression Ignition) of an IC (Internal Combustion) engine is challenge due to the thermo inertia of residue gas; and model-based control becomes a necessity. This paper presents a control oriented two-zone model to describe the hybrid combustion that starts with SI combustion and ends with HCCI combustion. The gas respiration dynamics were modeled using mean-value approach and the combustion process was modeled using crank resolved method. The developed model was validated in an HIL (Hardware-In-the-Loop) simulation environment for both steady-state and transient operations in SI, HCCI, and SIHCCI hybrid combustion modes through the exhaust valve timing control (recompression). Furthermore, cooled external EGR (exhaust gas re-circulation) was used to suppress engine knock and enhance the fuel efficiency. The simulation results also illustrates that the transient control parameters of hybrid combustion is quite different from these in steady state operation, indicating the need of a control oriented SI-HCCI hybrid combustion model for transient combustion control.
\end{abstract}

\section{INTRODUCTION}

To obtain the benefit of the high efficiency of compression ignition (CI) engines and the low emissions of spark ignition (SI) engines, there has been a rekindled interest in the homogeneous charge compression ignition (HCCI) combustion in recent years. The major advantage of HCCI combustion is realized by eliminating the formation of flames and results in a much lower combustion temperature. As a consequence of the low temperature, the formation of $\mathrm{NOx}$ (nitrogen oxides) is greatly reduced. The lean burn nature of the HCCI engine also enables un-throttled operation to improve vehicle fuel economy. Unfortunately, HCCI combustion is feasible only over a limited engine operational range due to knock and misfire. To make a HCCI engine work in an automotive, the internal combustion engine has to be capable of operating at both SI combustion mode at high load and HCCI combustion mode at low and mediate load ([13] and [20]). This makes it necessary to have a smooth combustion mode transition between SI and HCCI combustion modes.

Achieving the HCCI combustion and controlling the mode transition between SI and HCCI combustions in a practical engine require implementation of enabling devices and technologies. There are a number of options, and the necessary prerequisite for considering any of them is their ability to provide control of thermodynamic conditions in the combustion chamber at the end of compression. The range of devices under consideration includes variable valve actuation (cam-based or camless), variable compression ratio, dual fuel systems (port and direct fuel injection with multiple fuel injections), supercharger and/or turbocharger, exhaust energy recuperation and fast thermal conditioning of the intake charge mixture, spark-assist, etc. Variable Valve Actuation can be used for control of the effective compression ratio (via the intake valve closing time), the internal (hot) residual fraction via the negative valve overlap (recompression) ([11] and [12]), or secondary opening of the exhaust valve (residual re-induction) ([11] and [12]). In addition to providing the basic control of the HCCI combustion, i.e., ignition timing and burn rate or duration, the selected devices will play a critical role in accomplishing smooth mode transitions from SI to HCCI and vice versa.

The main challenge for the mode transition between SI and HCCI combustions is that a stable steady state HCCI operating condition is not stable and robust during the mode transitions. This is primarily due to the fact that the HCCI combustion is heavily influenced by the residual gas properties (quantity, temperature, and its composition) from the previous cycle and 
its mixing characteristics with the fresh intake air. Also given the practical constraints of short transition period (5 to 6 combustion cycles) and smooth transition torque, a cycle-tocycle intervention of the combustion process is a necessity. In reference [13], it shows that the transition from SI to HCCI is more difficult to achieve than the switch from HCCI to SI. This is due to the fact that the residual gas fraction management is required for transition from HCCI to SI; while the transition from SI to HCCI needs to take account the additional influence of in-cylinder thermal inertia. It also found that there was a hybrid combustion mode [13] that occurred during both the SI to HCCI and the HCCI to SI transitions. This hybrid combustion mode consists of both SI and HCCI combustions, as shown in Figure 1. It can be seen that the hybrid combustion starts as SI combustion with a low heat release rate (section ST to SOHCCI (start of HCCI combustion) in Figure 1), and then HCCI conditions are achieved to trigger HCCI combustion (section after SOHCCI). Through the transition from SI to HCCI combustions, the duration between ST and SOHCCI reduces and the HCCI combustion duration increases gradually, within a few cycles of the hybrid combustion mode.

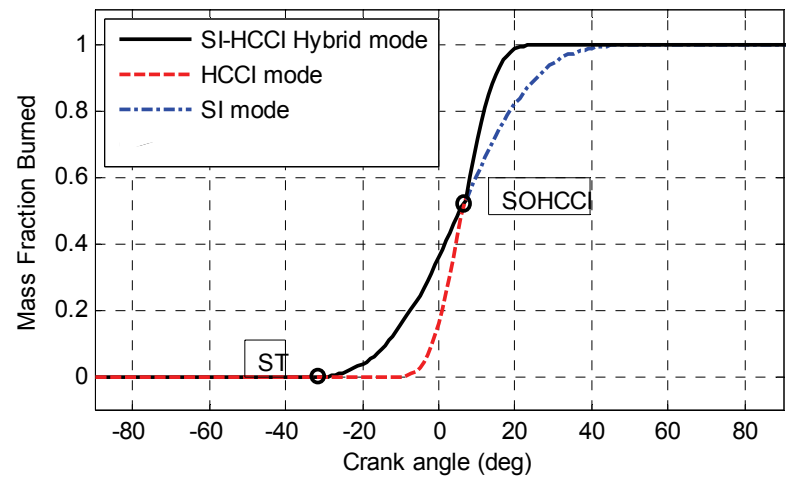

\section{FIGURE 1: MFB IN SI-HCCI HYBRID COMBUSTION MODE TRANSITION PROCESS}

To accurately control the HCCI combustion process, especially the mode transition between $\mathrm{SI}$ and $\mathrm{HCCI}$ combustions, a precise combustion model is required. This is mainly due to the fact that not all key control parameters of a HCCI combustion process can be measured directly. For instance, the temperature of the in-cylinder gas mixture after the intake valve is closed, which is a key parameter for estimating start of HCCI combustion (SOHCCI), cannot be measured using existing sensor technology. It is well known that the existing engine modeling tools, such as GT-Power and WAVE are only good at off-line simulations, and therefore, cannot be used for model-based control. The other HCCI combustion models ([1], [11], and [12]) describe either SI or HCCI combustions. As discussed, to control the mode transition between SI and HCCI combustion modes, we need to have a mixed mean-value and crank resolved SI-HCCI hybrid combustion model that can be used for model-based control. This model shall be able to be executed in real-time for HIL
(Hardware-In-the-Loop) simulations. This is the modeling work studied in this paper.

Also, to have smooth transition between SI and HCCI combustions, accurate intake flow with good mixing is the key. Reference [21] provides a two-zone intake mixing model that describes intake mixing process. References [14] and [15] present an intake strategy entailing opening the intake valves at different timings to improve the flow characteristics and provide better mixing. The advantage of this strategy is the ability to use NVO (negative valve overlap) or recompression while avoiding the inherent mixing problems associated with it. Since the NVO strategy alters the existing valve timings, it is much simpler than re-induction strategies. It needs to open the exhaust valves at non-traditional timings, leading to complication of the control system. Furthermore, since the exhaust gas does not leave the cylinder (as it does with reinduction strategies), there is less heat lost which improves the overall engine efficiency. To fully utilize this method, we must have accurate information about the system behavior, particularly, the fluid flow during the mixing process. The developed hybrid combustion model will be integrated with the intake mixing model described in [21] in future.

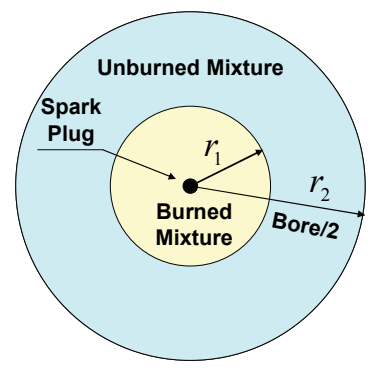

\section{FIGURE 2: TWO-ZONE SI-HCCI HYBRID COMBUSTION} MODEL

The main goal of mode transition control is to ensure smooth and robust mode transition between SI and HCCI combustions to avoid engine misfire at low load and knocking at mediate load. For model-based mode transition control, we propose to develop a two-zone SI-HCCI combustion model, shown in Figure 2, where SI and HCCI combustion modes are its special cases. The modeling purpose is to correlate the trapped in-cylinder gas properties (such as air-to-fuel ratio, trapped gas re-circulation, and temperature) to the combustion characteristics such as misfire, knock, SOHCCI, HCCI burn duration, and IMEP (Indicated Mean Effective Pressure). The developed model will be used for model-based transition operation from SI to HCCI or vice versa. Figure 2 illustrates the two-zone hybrid SI-HCCI combustion model architecture. For the engine operated at a SI-HCCI hybrid combustion mode, the combustion is initiated by the ignition system, leading to increased burned zone radius $r_{1}$ until the auto-ignition condition of HCCI combustion is achieved where the burned zone radius $r_{l}$ equals to $r_{S O H C C I}$. This is the burned zone radius at the timing of SOHCCI, see Figure 1 and Figure 2. The 
combustion continues with HCCI combustion in the unburned zone. There are two special cases: HCCI combustion is achieved when $r_{S O H C C I}$ equal to zero and SI combustion occurs when $r_{\mathrm{SOHCCI}}$ equal to $r_{2}$. Therefore, the SI-HCCI hybrid combustion mode is a generalization of both SI and HCCI combustion modes.

The paper is organized as follows. The dynamic equations used for modeling the proposed hybrid combustion were presented and discussed in great detail; the developed model was implemented into a HIL simulator for an I4 engine; and both steady state and dynamic simulation results were presented and discussed. At last, conclusions are drawn.

\section{HYBRID SI AND HCCI COMBUSTION MODEL}

The thermodynamic characteristics of in-cylinder gas, such as in-cylinder pressure and temperature, are of great interest in the SI-HCCI combustion modeling. This is especially important at certain critical combustion events such as the intake valve closing.

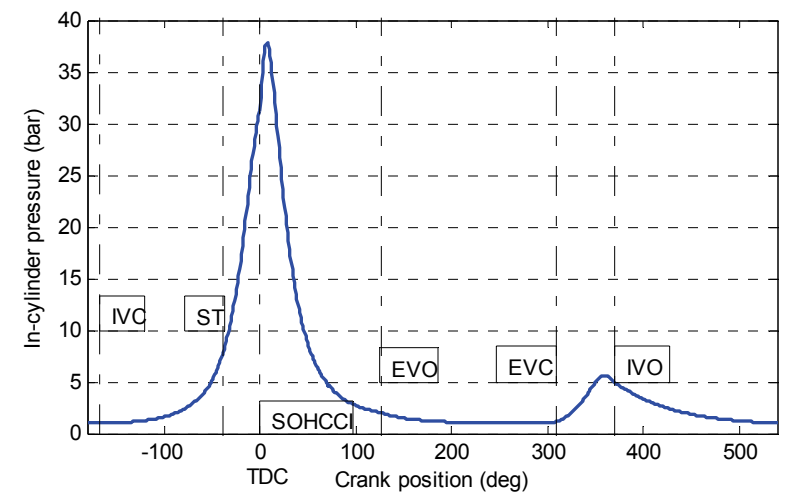

\section{FIGURE 3: COMBUSTION EVENTS WITHIN AN ENGINE CYCLE}

Figure 3 shows six combustion events of a SI-HCCI dualstage combustion process within one engine cycle. They are intake valve closing (IVC), spark timing (ST), start of HCCI combustion (SOHCCI), exhaust valve opening (EVO), exhaust valve closing (EVC), and intake valve opening (IVO). For each combustion phase between two combustion events, the incylinder behaviors (such as pressure, temperature, etc.) were modeled using thermodynamic governing equations with initial conditions derived from last combustion event. In the rest of this paper, crank resolved models of each combustion phase will be discussed.

\section{Phase I: IVC to ST}

Without combustion, the compression process from IVC to ST can be approximated as an isentropic process. In-cylinder pressure and temperature vary as functions of in-cylinder volume. Equations (1) and (2) describe the crank resolved in- cylinder pressure and temperature with initial conditions $T\left(\theta_{I V C}\right)=T_{I M}$ and $P\left(\theta_{I V C}\right)=P_{I M}$.

$$
T\left(\theta_{i}\right)=T\left(\theta_{i-1}\right) \cdot\left(\frac{V\left(\theta_{i-1}\right)}{V\left(\theta_{i}\right)}\right)^{(\kappa-1)}, T\left(\theta_{0}\right)=T\left(\theta_{I V C}\right)=T_{I M}
$$

and

$$
P\left(\theta_{i}\right)=P\left(\theta_{i-1}\right) \cdot\left(\frac{V\left(\theta_{i-1}\right)}{V\left(\theta_{i}\right)}\right)^{\kappa}, P\left(\theta_{0}\right)=P\left(\theta_{I V C}\right)=P_{I M}
$$

where $\kappa$ is the average heat capacity ratio of the gas charged into the cylinder; $\theta$ is crank position; $T_{I M}$ and $P_{I M}$ are the intake manifold temperature and absolute pressure respectively; and $\theta_{I V C}$ is the crank angle at intake valve closing.

\section{Phase II: ST to SOHCCI}

Based upon references [1], [2], [4], and [5], the Arrhenius integral can be used to estimate the crank position of start of HCCI combustion (SOHCCI) $\theta_{\text {SOHCCI }}$ The SOHCCI crank position, defined as the crank angle for $1 \%$ fuel burned under HCCI combustion, can be determined as the crank angle when the following integral reaches one.

$$
A R(\theta)=\int_{\theta_{I V C}}^{\theta} \frac{A}{N_{e}} e^{-\frac{E_{a}}{R \cdot T(\vartheta)}} d \vartheta
$$

where $\theta$ is the current crank angle; $N_{e}$ is engine speed; $A$ is a scaling constant; $E_{a}$ is the activation energy for the autoignition reaction; $R$ is the in-cylinder gas constant and $T$ is the in-cylinder temperature of the unburned zone.

The SI combustion begins after spark ignition event. The combustion process in this phase is modeled using the Wiebe function ([3] and [9]) below, see equation (4).

$$
x(\theta)=1-e^{-a\left(\frac{\theta-\theta_{S T}}{\Delta \theta}\right)^{m+1}}
$$

where the predicted burn duration $\Delta \theta$, Weibe coefficients $a$ and $m$ are functions of the normalized air-to-fuel ratio, engine speed, and load; and $x$ is mass fraction burned (MFB).

In this combustion phase, it is assumed that the combustion progresses from the burned zone to the unburned zone (see Figure 2), and both pressure and temperature are evenly distributed across both zones inside the engine cylinder. Note that in future research the two-zone temperatures will be modeled separately. Under the above assumptions, the incylinder temperature is a function of cylinder volume and MFB, as shown in equation (5); and the corresponding incylinder pressure can be calculated based upon the in-cylinder temperature and volume under ideal gas assumption.

$$
T\left(\theta_{i}\right)=T\left(\theta_{i-1}\right) \cdot\left(\frac{V\left(\theta_{i-1}\right)}{V\left(\theta_{i}\right)}\right)^{(\kappa-1)}+\eta \cdot(1-\alpha) \cdot \frac{Q_{L H V}\left[x\left(\theta_{i}\right)-x\left(\theta_{i-1}\right)\right]}{C_{v} \cdot(1+\lambda)}
$$


and

$$
P\left(\theta_{i}\right)=P\left(\theta_{i-1}\right) \cdot \frac{V\left(\theta_{i-1}\right)}{V\left(\theta_{i}\right)} \cdot \frac{T\left(\theta_{i}\right)}{T\left(\theta_{i-1}\right)}
$$

where $\eta$ is the combustion efficiency due to heat loss across cylinder wall and head, and it's a calibration of coolant temperature, engine speed and load; $\alpha$ is the inertia gas fraction of the in-cylinder gas mixture; $C_{v}$ is the specific heat of incylinder gas mixture; $Q_{L H V}$ is the low heating value of the fuel; $\lambda$ is the normalized air-to-fuel ratio of the in-cylinder gas mixture.

During this combustion phase, the Arrhenius integral, see equation (3), continues its integration. The in-cylinder temperature and pressure increase rapidly due to the SI combustion. As a result, the Arrhenius integral increases sharply as well, and when it reaches the criteria of the start of HCCI combustion $(A R \geq 1)$ the HCCI combustion starts in the unburned gas mixture zone, see Figure 2, which leads to the next combustion phase.

\section{Phase III: SOHCCI to EVO}

In this combustion phase the in-cylinder pressure and temperature are modeled based upon equations (5) and (6) with the same form of MFB calculation as equation (4). However the predicted burn duration $\Delta \theta$, Weibe coefficients $a$ and $m$ are quite different. Especially, the HCCI combustion burn duration $\Delta \theta$ is obtained by the following equations [2]:

$$
\Delta \theta=k \cdot T\left(\theta_{\text {SOHCCI }}\right)^{-\frac{2}{3}} \cdot T_{H C C I}^{\frac{1}{3}} \cdot e^{\frac{E_{c}}{3 R \cdot T_{H C C I}}}
$$

and

$$
T_{H C C I}=T\left(\theta_{\text {SOHCCI }}\right)+\eta \cdot(1-\alpha) \cdot \frac{Q_{L H V}\left[1-x\left(\theta_{\text {SOHCCI }}\right)\right]}{C v \cdot(1+\lambda)}
$$

where $k$ is a scaling constant; $E_{c}$ is the activation energy of global reaction; $T_{H C C I}$ is the average in-cylinder gas temperature during HCCI combustion phase; $\alpha$ is the inertia gas fraction of the in-cylinder gas mixture and it is calculated by equation (15).

After HCCI combustion, the in-cylinder gas performs isentropic expansion, and the calculations of in-cylinder pressure and temperature follow equations (5) and (6), but note that in this case equations (5) and (6) are same as (1) and (2) since MFB remains at a constant level after the HCCI combustion is finished.

\section{Phase IV: EVO to EVC}

After the exhaust valve is opened, the in-cylinder gas isentropically expands in the engine cylinder and exhaust manifold. The in-cylinder pressure drops quickly but not instantaneously, it normally takes a few crank degrees for the in-cylinder pressure to approach the pressure in the exhaust manifold. It is difficult to model this dynamics using simple dynamic equations for real-time simulations. For simplicity, a first order transfer function is used to approximate this dynamic process.

$$
P(z)=\frac{1-\tau_{E V O}}{1-\tau_{E V O} \cdot z^{-1}} \cdot P_{E M}(z)
$$

where $P_{E M}$ is the exhaust manifold absolute pressure; and $\tau_{E V O}$ is the transition time constant. Note that, for simplification, $\tau_{E V O}$ together with $\tau_{P C}$ and $\tau_{T C}$ from equations (12) and (13) are set to be constant calibrations. However, these time constants are fixed in engine cycle domain but are varying in time domain as functions of engine speed.

Then, in-cylinder temperature can be calculated as a function of the in-cylinder pressure as follows.

$$
T\left(\theta_{i}\right)=T\left(\theta_{i-1}\right) \cdot\left[\frac{P\left(\theta_{i}\right)}{P\left(\theta_{i-1}\right)}\right]^{\frac{\kappa-1}{\kappa}}
$$

where $\kappa$ is the average heat capacity ratio. By solving equations (9) and (10) iteratively, the thermodynamic properties (pressure and temperature) at EVC can be obtained. Therefore, the mass of residue gas $m_{R}$ can be derived as function of exhaust valve closing assuming it is ideal gas.

$$
m_{R}=m\left(\theta_{E V C}\right)=\frac{P\left(\theta_{E V C}\right) \cdot V\left(\theta_{E V C}\right)}{R \cdot T\left(\theta_{E V C}\right)}
$$

\section{Phase V: EVC to IVO}

This phase is called recompression or negative valve overlap ([17], [18], and [19]). During this phase engine cylinder is sealed as a closed system again, and the in-cylinder gas is isentropically compressed or expanded, so equations (1) and (2) are used to calculate both temperature and pressure. Through the recompression the thermodynamic properties of the residue gas are changed to match the required combustion characteristics for the next engine cycle.

\section{Phase VI: IVO to IVC}

The air charging process between IVO to IVC is also a process of in-cylinder gases mixing. During this phase the fresh charged air, injected fuel vapor, and residue gas are assumed to be mixed homogeneously, which is an assumed condition for HCCI combustion. The in-cylinder gas characteristics at IVC are of great importance to the start of HCCI combustion. Same approach as those used in Phase IV is used for both in-cylinder pressure and in-cylinder temperature calculations.

$$
P(z)=\frac{1-\tau_{P C}}{1-\tau_{P C} \cdot z^{-1}} \cdot P_{I M}(z)
$$

and 


$$
T(z)=\frac{1-\tau_{T C}}{1-\tau_{T C} \cdot z^{-1}} \cdot T_{m i x}(z)
$$

where $P_{I M}$ is the intake manifold absolute pressure; $\tau_{P C}$ and $\tau_{T C}$ are the transition time constants of pressure and temperature; and $T_{m i x}$ is the in-cylinder gas mixture temperature at IVC. It is calculated by equation (14);

$$
T_{\text {mix }}=\frac{m_{F} \cdot C v_{F} \cdot T_{F}+m_{G} \cdot C v_{G} \cdot T_{G}+m_{R} \cdot C v_{R} \cdot T_{R}}{m_{F} \cdot C v_{F}+m_{G} \cdot C v_{G}+m_{R} \cdot C v_{R}}
$$

where $m_{F}, C v_{F}$, and $T_{F}$ are the mass, specific heat, and temperature of fuel vapor trapped in cylinder respectively; $m_{G}$ is the mass of gas mixture charged into cylinder, which consists of fresh air and cooled EGR gas; $C v_{G}$ and $T_{G}$ are the specific heat and temperature of gas mixture; $C v_{R}$ and $T_{R}$ are the specific heat and temperature of the residue gas left from last engine cycle.

Both cooled EGR gas and the residue gas are regarded as inertia gas, so the total inertia gas fraction of the in-cylinder gas mixture is calculated as:

$$
\alpha=\frac{m_{G} \cdot x_{E G R}+m_{R}}{m_{G}+m_{R}}
$$

where $x_{E G R}$ is the cooled EGR fraction in the gas mixture inside the intake manifold.

\section{SIMULATIONS OF SI-HCCI HYBRID COMBUSTIONS}

The SI and HCCI combustion modes and the SI-HCCI hybrid mode were simulated based upon the engine configuration shown in Figure 4, where the engine parameters are given in Table 1.

This engine was equipped with an EGR cooler and valve to provide cooled external EGR gas, which is important to provide an additional degree of freedom to adjust the incylinder EGR fraction independent of the in-cylinder gas mixture temperature. The engine also features dual intake and exhaust valves with independent variable valve timing (VVT) control for both intake and exhaust valves. The exhaust and intake VVT controls are mainly used for recompression of the residue gas (see the recompression process between EVC and IVO shown in Figure 3). The residue gas recompression changes the in-cylinder gas mixture temperature at IVC $\left(T\left(\theta_{I V C}\right)\right)$ significantly, and $T\left(\theta_{I V C}\right)$, along with the IVC timing, determines the SOHCCI timing. Higher compression ratio is also selected to make the HCCI combustion possible at low engine load condition. For this simulation, the engine compression ratio is $11: 1$, see Table 1 .

The entire combustion process was modeled based upon equations (1) to (15) in Matlab/Simulink as a function of the engine crank angle, and the engine air handling sub-systems, such as intake and exhaust manifolds, were modeled using the mean-value technique described in [8] and [16]. The entire engine model consists two portions: crank resolved model for in-cylinder parameters such as pressure, temperature, etc. and mean-value model for external ERG fraction, intake and exhaust manifold dynamics, engine speed, etc. The developed engine model was implemented into a dSPACE based Hardware-In-the-Loop (HIL) simulator. The mean-value engine model was updated every millisecond and the crank resolved model is updated every crank degree.

Both steady-state and transient simulations performed in this paper were completed at $2000 \mathrm{rpm}$ engine speed with 3.7 bar IMEP. Engine operational condition selected for the combustion mode transition is critical. Engine could have heavy Knock if the engine load is relatively high. The rate of the in-cylinder pressure rise is often used as an indication of engine knock and is adopted in this paper. A rising rate of more than 3 bar per crank degree leads to unacceptable engine knock ([6] and [10]). During the mode transition, properly controlling the SOHCCI timing can also help preventing the engine knock.

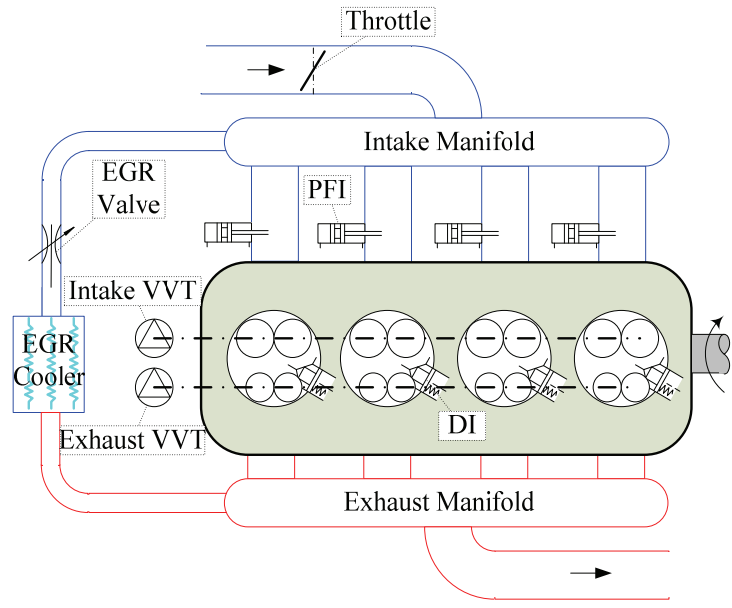

FIGURE 4: ENGINE CONFIGURATION

TABLE 1: ENGINE SPECIFICATIONS

\begin{tabular}{l|l}
\hline \hline Parameter & Model value \\
\hline bore/stroke/con-rod length & $88 \mathrm{~mm} / 82.2 \mathrm{~mm} / 132 \mathrm{~mm}$ \\
\hline compression ratio & $11: 1$ \\
\hline intake valve opening duration & $180 \mathrm{crank}$ degree \\
\hline exhaust valve opening duration & 180 crank degree \\
\hline Intake/exhaust manifold volume & $2.5 \mathrm{~L} / 2.3 \mathrm{~L}$
\end{tabular}

\section{SIMULATION RESULTS AND DISCUSSION}

HIL simulations based upon the engine model were conducted in the dSPACE HIL simulation environment to simulate the SI-HCCI hybrid combustions. Note that both SI and HCCI combustion modes are special cases of the SI-HCCI combustion. When the HCCI combustion did not occur, the engine is operated at the SI combustion mode; and when the 
HCCI combustion occurs at the start of SI combustion, the engine can be considered to be operated at the HCCI combustion mode; while the SI-HCCI hybrid combustion occurs inter-between the HCCI and SI combustion modes.

\section{Steady-state performance}

Steady-state combustions of SI, HCCI, and the hybrid mode with different SOHCCI timings were studied first to find how engine valve timing affects the combustion mode switch. Figure 5 to Figure 7 show the in-cylinder profiles of pressure, temperature, and MFB.

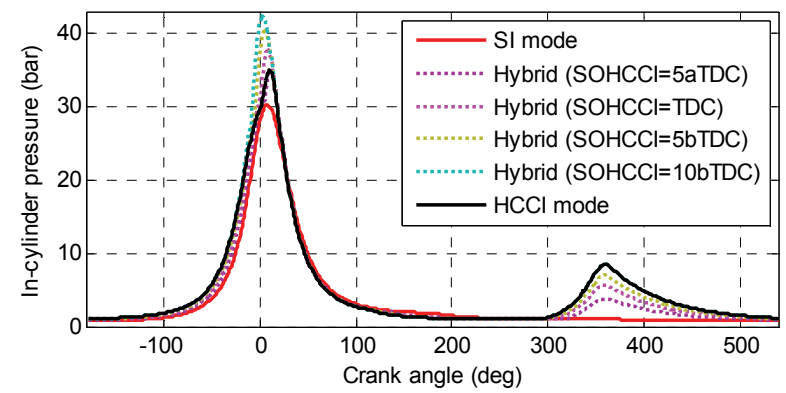

FIGURE 5: IN-CYLINDER PRESSURE PROFILES OF DIFFERENT COMBUSTION MODES

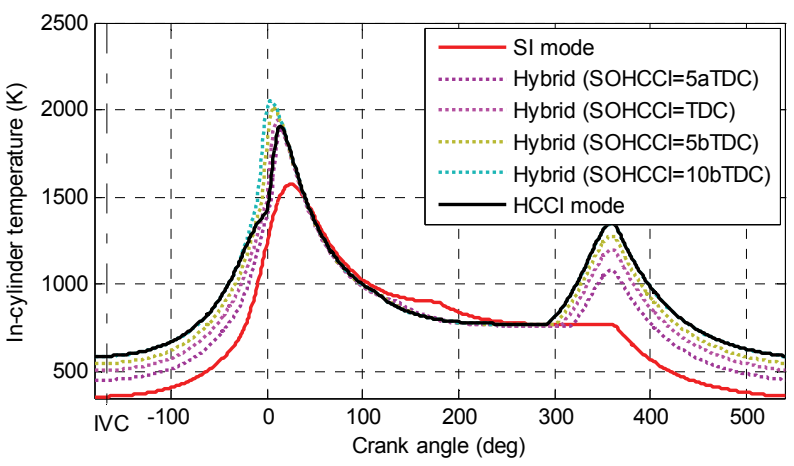

FIGURE 6: IN-CYLINDER TEMPERATURE PROFILES OF DIFFERENT COMBUSTION MODES

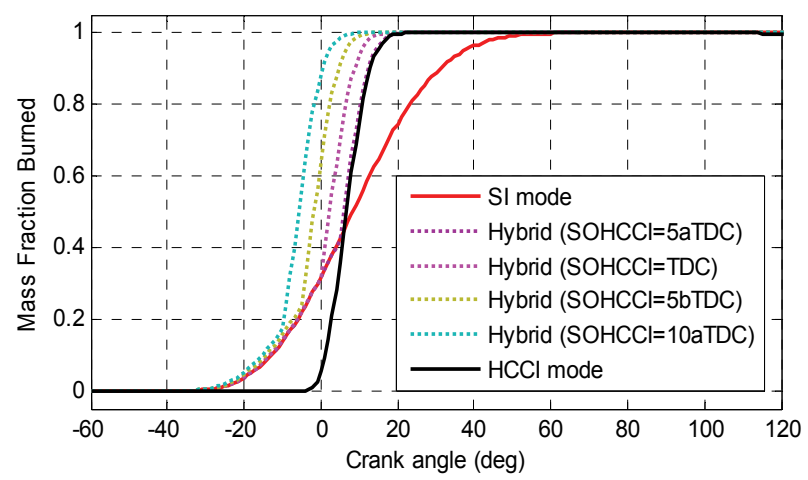

FIGURE 7: MFB PROFILES OF DIFFERENT COMBUSTION MODES
As the engine exhaust valve timing was advanced, the effect of recompression increases, as shown in Figure 5 and Figure 6. The increased recompression increases in-cylinder mixture temperature, which makes HCCI combustion possible after spark ignition. This is so called SI-HCCI hybrid combustion mode. Meanwhile the in-cylinder gas mixture temperature at intake valve closing $T\left(\theta_{\mathrm{IVC}}\right)$ increases, see Figure 6, and the SOHCCI timing advances as the temperature increases. This can be observed in Figure 7, where after SI combustion initiated, the start of HCCI (SOHCCI) combustion advanced, leading to increased fraction of fuel burned in the HCCI mode. When the exhaust valve timing was advanced to certain location, it is not necessary to ignite the in-cylinder gas mixture by a spark; and it can be auto-ignited through gas compression, achieving the HCCI mode.
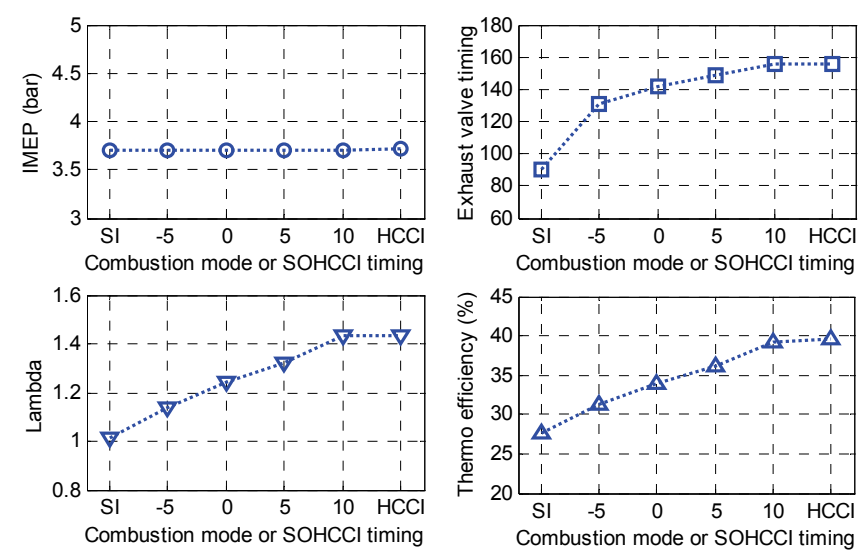

\section{FIGURE 8: ENGINE PERFORMANCE OF DIFFERENT COMBUSTION MODES}

In these simulations, engine IMEP was maintained at 3.7 bar as shown in Figure 8; the maximum lift of intake valve was also held at 95 crank degrees after gas exchange TDC. In order to have a constant IMEP (3.7 bar) when the exhaust valve timing advanced, the amount of fuel injected was decreased, accordingly to maintain a constant IMEP. This leads to increased air-to-fuel ratio with improved combustion efficiency (fuel economy). From these simulations, we found that the HCCI fuel consumption is about $30 \%$ less than that of SI combustion when the IMEP was held constant.

The influence of EGR gas to the hybrid combustion modes was also investigated through steady-state simulations. HCCI combustion has much shorter burn duration than that of SI combustion. This leads to high peak in-cylinder pressure and temperature, as shown in Figure 5 and Figure 6. However, HCCI combustion is capable of operating with higher EGR rate than that of SI combustion. Figure 9 demonstrates how the cooled EGR rate affects the in-cylinder gas temperature. The simulations in Figure 9 were completed with 3.7 bar IMEP, where the exhaust valve timing and fuel quantity were adjusted to maintain a constant IMEP (3.7 bar) for each simulation. 
Simulation results show that the higher EGR rate the lower the peak in-cylinder gas temperature and the higher fuel efficiency, see Figure 10.

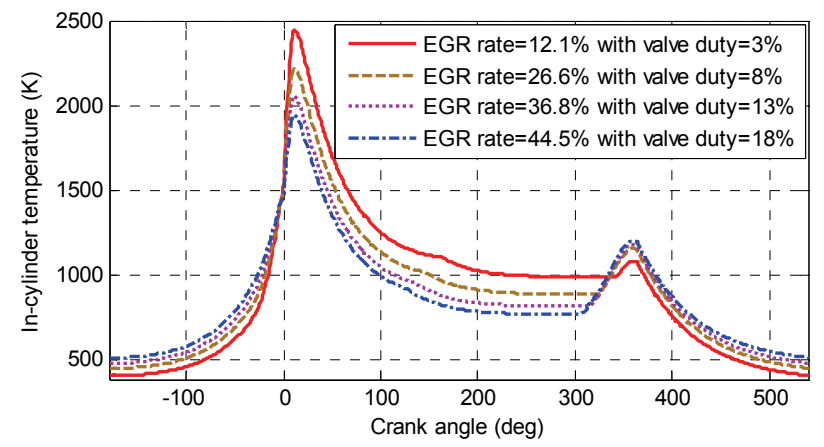

FIGURE 9: IN-CYLINDER TEMPERATURE VARIES WITH EXTERNAL EGR RATE
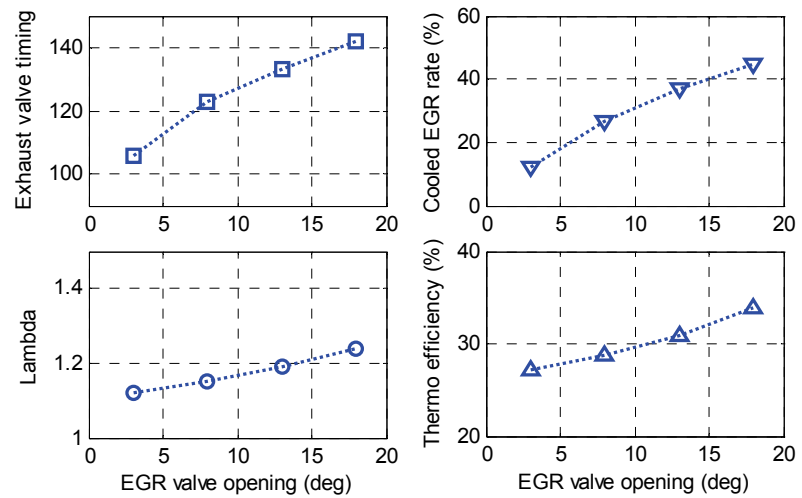

FIGURE 10: ENGINE PERFORMANCES VARY WITH
EXTERNAL EGR RATE

\section{Transient performance}

The combustion mode transient performance is of great interest in the modeling. The SI to HCCI mode transition with hybrid mode was simulated. The exhaust valve timing and fuel quantity were adjusted every consecutive engine cycle (cycles 1 to 6 in Figure 11). Two different strategies were adopted for the hybrid combustion mode control. One used the same control parameters as in the steady-state simulations; the other used control parameters adjusted for transient operation. For comparison, the SI to HCCI mode transition without the hybrid mode was also simulated. For all cases, the engine spark was cut at cycle 7 to achieve HCCI combustion. Figure 12 shows the engine transient responses of IMEP and SOHCCI timing for 20 consecutive engine cycles. Since the engine is running at 2000rpm, 20 engine cycles cover 1.25 second time period.

From Figure 12, the engine transient performance was improved by operating the engine at the hybrid combustion mode during mode transition. Without hybrid combustion mode, the IMEP dropped far below the target value (3.7bar) and SOHCCI was dramatically retarded to almost 80 crank degrees after gas-exchange TDC, which indicate partial-burn combustion at cycle 7 . In both cases with hybrid combustion mode, the one using the ad hoc transient control parameters provides smaller IMEP and SOHCCI variations during the transient operation than these using steady state parameters. This indicates that using steady-state mapping parameters during the combustion mode transition cannot provide the best performance during the mode transition and model-based transient control strategy has potential to provide optimal mode transition. Notice that the engine IMEP using the ad hoc transient control parameters was kept close to 3.7 bar during the transient operation and SOHCCI was smoothly transient from 14 degrees before TDC to 3 degrees after TDC.

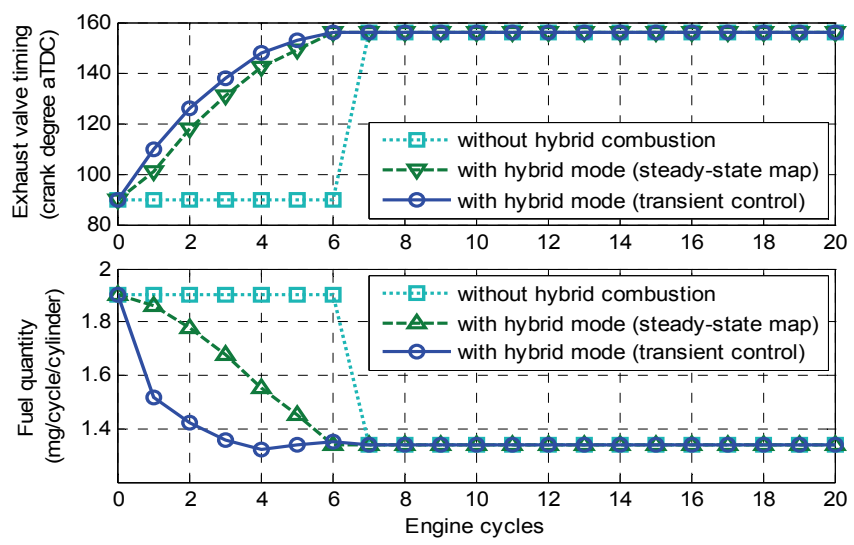

FIGURE 11: ENGINE SETTING FOR MODE TRANSITION
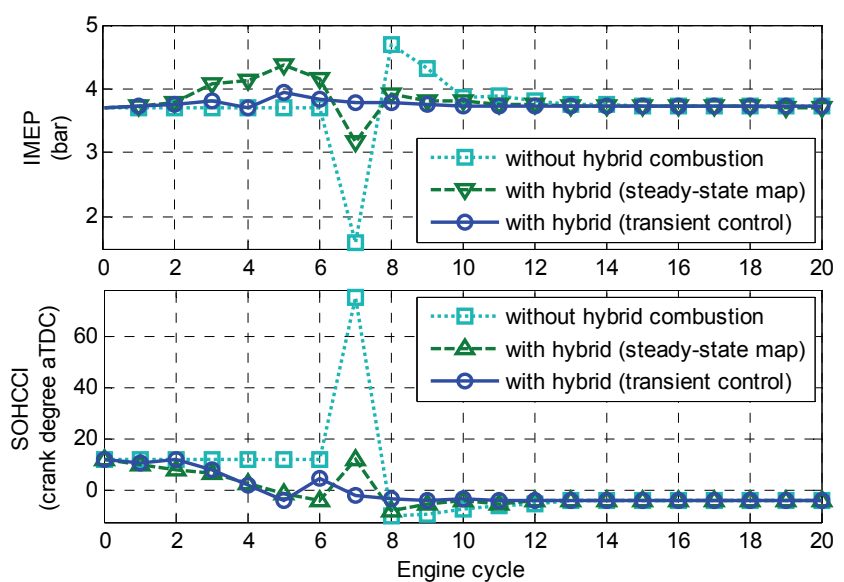

FIGURE 12: ENGINE TRANSIENT PERFORMANCE AT THE MODE TRANSTION FROM SI TO HCCI

\section{CONCLUSION}

A crank resolved hybrid combustion model of SI (spark ignited) and HCCI (Homogeneously Charge Compression Ignition) was developed over an engine cycle. Combined with the mean-value engine gas handling models from previous research, the hybrid combustion model was implemented in 
Simulink and validated in a HIL (Hardware-In-the-Loop) simulator. The simulation results show that the hybrid combustion SOHCCI (Start of HCCI) timing is highly related to the exhaust valve timing used for recompression. Therefore, a smooth combustion mode transition can be achieved by adjusting the exhaust valve timing. The cooled EGR (Exhaust Gas Recirculation) was also used to control both engine charge temperature and EGR rate. The transient performances of the mode transition simulation presented in this paper demonstrated the significance of the hybrid SI and HCCI combustion mode and its control oriented model. This is mainly due to the fact that the steady state control parameters for SIHCCI hybrid combustion are no longer optimal during the transient SI-HCCI combustion and a model-based control is a necessity.

\section{ACKNOWLEDGMENTS}

The U.S. Department of Energy is thanked for the funding of the project under Grant DE-EE0000211

\section{REFERENCES}

[1] D. J. Rausen, et al, "A mean value model for control of homogeneous charge compression ignition (HCCI) engines," ASME Journal of Dynamics, Measurement, and Control, Vol. 127, September, 2005, pp. 355-362.

[2] C. J. Chiang and A. G. Stefanopoulou, "Stability Analysis in Homogeneous Charge Compression Ignition (HCCI) Engines With High Dilution," IEEE Transactions on Control System Technology, Vol. 15, No. 2, March, 2007.

[3] J. B. Heywood, Internal Combustion Engine Fundamentals, McGraw-Hill, Inc., 1988.

[4] J. Bengtsson, M. Gafvert, and P. Strandh, "Modeling of HCCI Engine Combustion for Control Analysis," 43rd IEEE Conference on Decision and Control, December 14 - 17, Dec., 2004.

[5] M. Hillion, J. Chauvin, and N. Petit, "Controlling the Start of Combustion on an HCCI Diesel Engine," American Control Conference, June, 2008.

[6] C. F. Daniels, G. G. Zhu, and J. Winkelman, "Inaudible Knock and Partial Burn Detection Using In-Cylinder Ionization Signal," SAE 2003-01-3149, 2003.

[7] I. Haskara, G. Zhu, C. Daniels, and J. Winkelman, “Closed Loop Maximum Dilution Limit Control using In-Cylinder Ionization Signal," SAE 2005-01-3751, SAE Powertrain and Fluid Systems Conference \& Exhibition, San Antonio, Texas, October, 2005.

[8] L.Guzzella and C.H.Onder, Introduction to Modeling and Control of Internal Combustion Engine Systems, Springer, Inc., 2004.

[9] M. Mittal, G. G. Zhu, and H. J. Schock, "Fast mass fraction burned calculation using net pressure method for real-time applications," Proceedings of the Institution of Mechanical Engineers, Part D, Journal of Automobile Engineering, Vol. 223, pp389-394, 2009.

[10] Andreae, M.M., W.K. Cheng, T. Kenney, and J. Yang, On HCCI engine Knock. SAE 2007-01-1858, 2007.

[11] G. M. Shaver, et al, "Dynamic modeling of residual-affected homogeneous charge compression ignition engines with Variable Valve Actuation," ASME Journal of Dynamics, Measurement, and Control, Vol. 127, September, 2005, pp. 374-381.

[12] G. M. Shaver, "Physics based modeling and control of residualaffected HCCI engines using Variable Valve Actuation," $\mathrm{PhD}$ thesis, Stanford University, September, 2005.

[13]Zhang, Y., H. Xie, N. Zhou, T. Chen, and H. Zhao, "Study of SIHCCI-SI Transition on a Port Fuel Injection Engine Equipped with 4VVAS," SAE 2007-01-0199, 2007.

[14]A-F M. Mahrous, M. L. Wyszynski, H. Xu, A. Tsolakis, and J. Qiao, " Effect of Intake Valves Timings on In-Cylinder Charge Characteristics in a DI Engine Cylinder with Negative Valve Overlapping," SAE Technical Paper Series (SAE International), SAE 2008-01-1347, 2008.

[15] A-F. M. Mahrous, A. Potrzebowski, M. L. Wyszynski, H. M. Xu, A. Tsolakis, and P. Luszcz, "A Modeling Study into the Effects of Variable Valve timing on the Gas Exchange Process and Performance of a 4-valve DI Homogeneous Charge Compression Ignition (HCCI) Engine," Energy Conversion and Management 50, 2009, pp. 393-398.

[16]X. Yang and G. G. Zhu, "A Mixed Mean-Value and Crank-based Model of a Dual-Stage Turbocharged SI Engine for Hardware-Inthe-Loop Simulation", Proceedings of 2010 American Control Conference, Baltimore, MD, 2010.

[17] T. Kuo, "Valve and Fueling Strategy for Operating a Controlled Auto-ignition Combustion Engine," SAE 2006 Homogeneous Charge Compression Ignition Symposium, SAE International, San Ramon, CA, pp. 11-24, 2006.

[18] T. Kuo, Z. Sun, J. Kang, J. Eng, C. Chang, B. Brown, P. Najt, and M. Chang, "Method for Transition between Control Auto-Ignition and Spark Ignition Mode in Direct Fuel Injection Engines", United States Patent 7,370,616, 2008.

[19] P. Tunestal and B. Johansson, "HCCI Control, in HCCI and CAI Engines for the Automotive Industry," H. Zhao, Editor, Woodhead Publishing: Cambridge, 2007.

[20]A. Cairns and H. Blaxill, "The Effects of Two-Stage Cam Profile Switching and External EGR on SI-CAI Combustion Transitions," SAE 2007-01-0187, 2007.

[21] M. McCuen, Z. Sun, and G. Zhu, "Control-oriented mixing model for homogeneous charge compression ignition engine," Proceedings of 2010 American Control Conference, Baltimore, MD, June 2010. 